\title{
S-wave splitting in the transpressional zone of New Zealand plate-boundary: implications for deformation and dynamics
}

by

Sapthala Karalliyadda

\section{Victoria \\ UNIVERSITY OF WELLINGTON \\ Te Whare Wānanga \\ o te Ūpoko o te Ika a Māui}

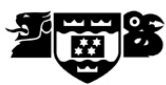

\author{
A thesis \\ submitted to Victoria University of Wellington \\ in fulfilment of the \\ requirements for the degree of \\ Doctor of Philosophy \\ in Geophysics.
}





\section{Abstract}

Seismic anisotropy in the transpressional plate-boundary zone in New Zealand is investigated with shear-wave splitting to gain insights into lithospheric deformation and mantle flow. Constraints on plate-boundary deformation in the lithosphere of the oblique-collision and subduction regimes in South Island have been estimated from the local and regional shear-wave splitting parameters that are made at both inland and offshore seismographs. Mantle and lithospheric anisotropy of the southernmost Hikurangi subduction zone in the southern North Island is examined from SKS, ScS and teleseismic S-phases. The splitting of these phases measured on a recent transect crossing the Wellington region is analyzed to understand the lateral anisotropic structure of the fore-arc Hikurangi subduction zone.

Local and regional splitting reveal both laterally and depth varying anisotropy in South Island. The scatter in splitting parameters at individual stations suggests the splitting of high-frequency S-phases is mainly controlled by heterogeneous anisotropic structure and S-wave propagation direction within those heterogeneities. When the average results are examined as a whole through 2-D delay time tomographic inversion and spatial averaging, consistent patterns in delay times and fast azimuths exist. Spatially averaged fast azimuths indicate a localized high strain zone in the southern central region of the South Island. Based on fast azimuths observed above $100 \mathrm{~km}$ depth, we suggest that the plate-boundary sub-parallel anisotropy that is produced by pervasive shear is mainly distributed within a zone extending $\sim 130 \mathrm{~km} \mathrm{SE}$ of the Alpine fault in the southern South Island and is widely distributed (at least $200 \mathrm{~km}$ wide) in the northern South Island. Average station delay times $(\delta t)$ of $\sim 0.1-0.4 \mathrm{~s}$ compared to $1.7 \mathrm{~s}$ SKS $\delta t$ from previous studies in South Island further suggest a deep seated anisotropic zone or sensitivity of S-wave splitting to the layered and/or heterogeneous anisotropic structure of the plateboundary zone in the inland South Island. The heterogeneous anisotropic structure further suggests that the lithosphere is not only characterized by the plate-boundary parallel shear related to Cenozoic deformation, but is also affected by anisotropic imprints from the other tectonic episodes and anisotropy that is governed by the contemporary stress.

A shear-wave splitting anisotropy investigation in the offshore South Island regions is an extended study of the inland experiment and aims to provide a broad-scale understanding of the plate-boundary deformation. Individual splitting of local and regional S-phases yield a range of $\delta t$ that varies between very small $\delta t(\sim 0.02$ $\mathrm{s})$, which may represent a nearly isotropic medium, and large $\delta t(\sim 0.6 \mathrm{~s})$, which corresponds to lithospheric anisotropy. The average station $\delta t$ of $\sim 0.25 \mathrm{~s}$ and variable delays of the individual splitting measurements imply that the observed splitting is most likely controlled by the geometry of the ray paths. Long ray paths that are detected at the stations further away from the plate-boundary appear to penetrate to deeper lithosphere and capture a significant portion of the upper-mantle anisotropy to produce fast azimuths parallel to the plate-boundary shear (NE-SW). Thus, the long and deep ray paths respond to the deeper structure, but may not be 
re-split by the upper-most crustal structures. However, the observed variable delays suggest that changes in ray propagation direction with respect to the orientation of symmetry axes of the anisotropic media may have an effect on the measured anisotropy. Offshore measurements that are close to the land are consistent with the inland measurements and appear to be controlled by the regional stress field. This implies that short and shallow ray paths are mostly sensitive to the crustal anisotropy. The uneven distribution of ray paths from the shallow and deep events, therefore, plays a dominant role in controlling the observed splitting depending on their depth sensitivity and/or extent of anisotropy. Consequently, when fast directions are spatially averaged along with the inland measurements consistent patterns appear to correlate with the possible depth contribution of anisotropy in the region. We are unable to provide accurate constraints on the offshore extent of plate-boundary parallel shear because of the shallow stress-controlled anisotropy that likely overlies the mantle-shear zone. However, the splitting parameters from long and deep ray paths suggest a deep-seated, plate-boundary sub-parallel shear in a broad zone at least in the northern and upper-central South Island.

Mantle anisotropy detected from teleseismic earthquakes recorded across the southern North Island displays NE-SW fast axis alignment, consistent with the strike of the Hikurangi trench and the predominant upper-plate faulting trends, with a range of $\delta t(\sim 0.5-3.0 \mathrm{~s})$ and small-scale variation in NE-SW fast azimuths. When combined with the previous measurements in the western side of the array, $\delta t$ from long period $(>7 \mathrm{~s})$ S-phases indicate an abrupt lateral variation across the fore-arc Hikurangi subduction zone. This lateral variation together with frequency dependence suggest that the shear wave splitting in the fore-arc of the Hikurangi subduction zone in the southern North Island is governed in part by the laterally varying crustal contribution of anisotropy or isotropic velocity variations within the shallow crust. Frequency dependent splitting also suggests that the anisotropic structure is governed by either multilayer or more complex anisotropy perhaps due to the combined effects of laterally varying multilayer structure. If the variations are due to lateral changes in crustal anisotropy, then mantle and crustal deformation are most likely coupled in the east of the Wairarapa fault where there is a possibility of strong crustal contribution. 


\section{Acknowledgments}

My PhD journey was full of challenges and rewarding experiences. I am thankful to a number of people who have supported me throughout this journey. First and foremost I would like to express my deepest gratitude to both my supervisors - thank you Martha Savage and Tim Stern for your support from the beginning to the end of my $\mathrm{PhD}$. Especially, thank you Martha for being an encouraging and cheerful supervisor. This thesis would not have been possible without your guidance and countless recommendations, which I considered as medicine. To me my weekly meeting time with you was like a doctor's appointment and I was the patient who absorbed all the medicine that you recommended to cure my problems. Thank you Tim for your insight and for teaching me to be very concise.

I am grateful to several people who have supported me with my field work and data processing in the early stage of my research project. A huge thanks to Mark Henderson, Anya Seward, and Carolin Boese for their help with the field deployments and field instructions. A special thanks also to Sonja Greve for her assistance with seismic data processing. Thank you Andrew and Alex for solving all the computer problems as much as possible. I wish I had talked to Adrian Benson two years earlier - all my research work would have been much easier and faster if I did. You are like a walking encyclopedia and I certainly learned quite a lot of tricks that are related to all sorts of computer matters and open-source softwares. Thank you so much for your help with fixing some of my burning computer issues.

Financial support for the projects was given by the VUW doctoral scholarship, NSF, FRST, and Victoria finishing scholarship. All three research projects would not have been possible without the data and instruments provided by New Zealand GeoNet project (www.geonet.org.nz), IRIS, and PASSCAL. I acknowledge the support of collaborators from Colorado State University during the on-land and OBS experiments. Thank you A. Sheehan, P. Molnar, J. Collins, D. Zietlow, J. Stachnick and OBS cruise for providing OBS orientations, OBS data, planning the OBS experiment and giving suggestions on how to improve the quality of the offshore study.

My PhD work was greatly supported with several softwares/packages: Mfast, GMT, SAC, MATLAB, TESSA, SWST, SPLITSHEAR, MSAT and TAUP. I would like to thank the authors of these codes/softwares for making them publicly available for use. $\mathrm{LAT}_{\mathrm{E}} \mathrm{X}$ utility was used to typeset the thesis.

I would like to thank Euan Smith and John Townend for their comments on the initial proposal of my research project. All your comments enabled me to improve the quality of my work. I'm grateful to G. Rumpker, D. Okaya and Fan-Chi Lin, with whom I had many useful discussion on my results and research work during their visits to VUW. A thank you to the Geophysics discussion group for sharing Geophysics knowledge in various disciplines as well as for birthday treats and other celebrations. Thank you Jess Johnson and Adrian Shelly for being my anisotropy office buddies in the last couple of years, sharing all your anisotropy knowledge. A very special thank you to my friends Katie Jacobes and Jo Leavesly, who helped me in every possible way. Katie, you have such a good sense of humor, thank you for being such a wonderful officemate and for helping with my MATLAB questions and coding problems. I really enjoyed our gossip times, baking, 
fishing, concerts and all the fun times together. Thank you Anya, Carolin, Denise, Kathy, Adrian, Katharine, Richard and Pegha for dinners, lunches and board/card-games. Thank you Caroline for sharing information about the SAHKE deployment.

I have also been blessed with lovely flatmates throughout my journey. Most of them were international friends, who gave me an opportunity to experience various cultures. Many thanks to Conor for reading through some of my thesis chapters. Thank you Jamie, Floor, Sineth, Kalpani, Shanika, Aminda, Indika, Kosala for keeping in touch with me whenever it was possible and helping me in various situations. I am grateful to all of you for adding a smile to my sometimes stressful life and your generosity. A huge thank you to aunt Seetha for making me feel welcome and providing me with a comfortable home-like environment during my stays in Wellington in the last couple of months of my $\mathrm{PhD}$. Thank you Chami, Chathu, Sanju and Uthpala for being besties forever, and especially for all your help when my father was ill.

I experienced both extremely happy and sad moments during my $\mathrm{PhD}$. I am grateful to my family: my mother, Vishan, Ruchi and my mother-in-law for being patient with my busy PhD life, encouraging me morally and emotionally especially during my father's loss. My father (appachi) was a huge part of my life and I was quite unfortunate to lose him in the early stages of my PhD. There were a million times when I felt guilty and missed him so badly. Dear appachi, You were my inspiration and motivation to continue on to be strong and complete my PhD. May you Attain the Supreme Bliss of Nibbana! I'm thankful to my parents for their guidance throughout my education career. I'm grateful to Vishan for believing in me, for being responsible and flexible with everything and for traveling between Wellington and Hamilton. Although we stayed apart, I was so happy that you were here in New Zealand, supporting me in every way and allowing me to do what I wanted to do.

Last but not least, I want to acknowledge everyone who gave me a solid background to begin this journey. Many thanks to my former advisors, especially to L. Perera, for giving me a chance to be involved with research in seismology. A heartfelt special thanks to Dayanthie Weerarathne for her guidance and for encouraging me to continue on with my higher studies. I would not have come to beautiful New Zealand without the travel and research opportunities given by Carnegie Institute of Washington and AGU-2007 student travel grant, Thank you. AGU-2007 is where I found information about the VUW PhD program. Finally, thank you VUW and VUW-international - it has been an amazing and challenging $\mathrm{PhD}$ journey that enabled me to do seismic research whilst experiencing earthquakes! 


\section{Contents}

Abstract $\quad$ iii

Acknowledgments $\quad$ v

Table of Contents vii

List of Figures xiii

List of Tables $\quad$ xvii

1 Overview 1

1.1 Introduction to study . . . . . . . . . . . . . . . . 1

1.1.1 Thesis structure ................... 2

1.2 Deformation and dynamics . . . . . . . . . . . 4

1.2.1 Deformation at continental zones .......... . 6

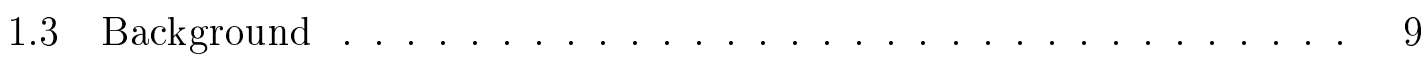

1.3.1 Tectonic synthesis . . . . . . . . . . . . 10

1.4 Geology and structure . . . . . . . . . . . . . . . . 13

1.4 .1 Geology .......................... 13

1.4.2 Geophysical implications . . . . . . . . . . . 18

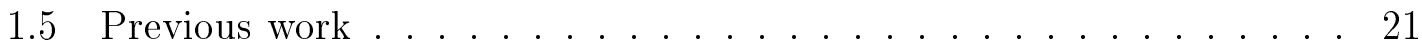

1.6 Previous anisotropy studies . . . . . . . . . . . 33

$\begin{array}{lll}2 & \text { Seismic anisotropy and deformation } & 37\end{array}$

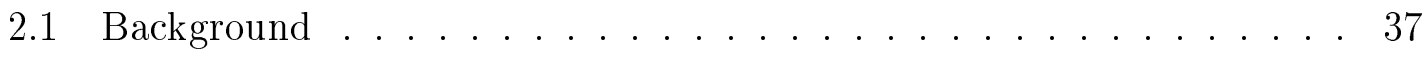

2.2 Concepts of wave propagation $\ldots \ldots \ldots \ldots$. . . . . . . 37 
2.2.1 Elastic tensors . . . . . . . . . . . . . . 38

2.2.2 Elastic waves in homogeneous anisotropic media . . . . . . . 40

2.2.3 Plane waves in hexagonal anisotropy systems . . . . . . . . . . 41

2.3 Shear-wave splitting and splitting parameters . . . . . . . . . 45

2.4 Origin of anisotropy . . . . . . . . . . . . . . . . 47

2.4.1 Extrinsic anisotropy .................. 47

2.4 .2 Intrinsic anisotropy $\ldots \ldots \ldots . \ldots \ldots$

2.5 Deformation kinematics and anisotropy . . . . . . . . . . 52

2.5.1 Dislocation creep and diffusion creep . . . . . . . . . 54

2.5.2 Factors controlling deformation ........... 57

2.6 Sampling anisotropy _. . . . . . . . . . . . . 60

2.7 Characterizing tectonic regimes . . . . . . . . . . . 61

2.7.1 Transpressional zones: S-wave splitting . . . . . . . . . 62

2.7.2 Subduction zones: S-wave splitting and dynamics . . . . . . 63

$\begin{array}{lll}3 & \text { Data and methodology } & 71\end{array}$

3.1 Experiment and Data . . . . . . . . . . . . 71

3.1 .1 On-land experiment . . . . . . . . . . . 73

3.1 .2 Offshore SI experiment . . . . . . . . . . . 77

3.1 .3 Southern NI experiment . . . . . . . . . . . . 79

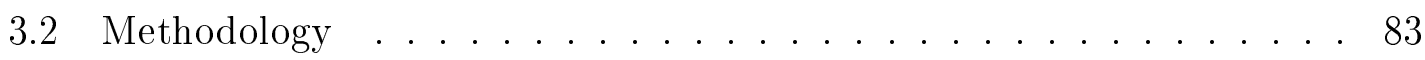

3.2.1 Summary of splitting analysis methods . . . . . . . . . . 83

3.2.2 Transverse component and eigenvalue minimization . . . . . 86

3.2 .3 Mfast .............................. 92

3.3 Limitations, quality and statistics . . . . . . . . . . . 98 
3.3.1 Diagnosing robust splitting measurements . . . . . . . . 99

3.4 Direct estimations . . . . . . . . . . . . . . . . . . 108

3.5 Characterizing splitting parameters . . . . . . . . . . . 110

3.5.1 2-layer and multiple layer modeling . . . . . . . . . . 110

3.5.2 Frequency dependent splitting . . . . . . . . . . . 112

3.5.3 Dipping anisotropic layers . . . . . . . . . . . 114

3.6 Shear-wave splitting tomography _. . . . . . . . . . 116

3.6.1 2-D delay time tomography and spatial averaging . . . . 116

3.6.2 3-D splitting inversion . . . . . . . . . . . . 119

4 On-land anisotropy in SI

4.1 Introduction . . . . . . . . . . . . . . 126

4.2 Data and method . . . . . . . . . . . . . . . . 131

4.2.1 Limitations and statistical analysis ........... 138

4.2.2 Resolving the spatial heterogeneity . . . . . . . . . . . 140

4.3 Results . . . . . . . . . . . . . . . . . . 142

4.4 Interpretations and discussion . . . . . . . . . . . . . . . . 147

4.4.1 Depth extent and backazimuthal variations . . . . . . . . 147

4.4.2 Evidence for heterogeneous anisotropic structure . . . . . . . . 154

4.4.3 Interpretations from TESSA . . . . . . . . . . . . . 159

4.4.4 Comparison with previous anisotropy studies . . . . . . . 164

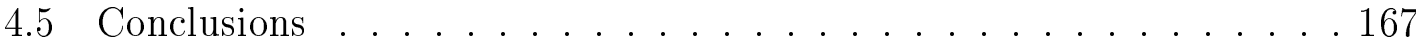

5 S-wave splitting in the offshore SI

5.1 Introduction . . . . . . . . . . . . . . . 169

5.1.1 Constraining plate-boundary deformation . . . . . . . . . 170 
5.2 Splitting parameters from OBS data . . . . . . . . . . 174

5.2.1 Data and method .................. 174

5.2 .2 Results and interpretations . . . . . . . . . . 176

5.2.3 Comparison with SKS splitting measurements . . . . . . 183

5.3 Integration . . . . . . . . . . . . . . . . . . 185

5.3.1 Additional land measurements . . . . . . . . . . . . 186

$5.3 .2 \quad 2-\mathrm{D} \delta t$ tomography and spatial averages of $\phi \ldots 191$

5.3.3 Preliminary results from 3-D tomography . . . . . . . . . 197

5.4 Discussion and conclusions . . . . . . . . . . . . . . . 203

6 Deep anisotropy in southern NI 207

6.1 Splitting of SKS, ScS and Tel. S . . . . . . . . . . 207

6.2 Synthesis of splitting parameters . . . . . . . . . . . . . 214

6.2.1 Frequency dependence . . . . . . . . . . . . . 215

6.2.2 Lateral variations of splitting along the transect . . . . . . 219

6.2.3 Possibility of dipping anisotropic layers? . . . . . . . . . . . 231

6.3 Conclusions . . . . . . . . . . . . . . . . 236

7 Concluding remarks $\quad 239$

7.1 South Island on-land and offshore experiment . . . . . . . . . 239

7.2 Southern North Island experiment . . . . . . . . . . . . . . . . . 243

$\begin{array}{ll}\text { A On-land investigation } & 245\end{array}$

A.1 Station information . . . . . . . . . . . . 245

A.2 Events information . . . . . . . . . . . . 247

A.3 chapter 4: Supplementary figures . . . . . . . . . . 265 
B Offshore investigation

B.1 Station information . . . . . . . . . . . . . . 269

B.2 Measurements selection . . . . . . . . . . . . 270

B.3 Event information . . . . . . . . . . . . . . . 270

B.4 Combined analysis . . . . . . . . . . . . . . 277

C Southern NI investigation $\quad 279$

C.1 Station information . . . . . . . . . . . . . . 279

C.2 Event information . . . . . . . . . . . . . . . 279

C.3 Null-measurement information . . . . . . . . . . . . . . . . . 282

C.4 Multilayer analysis . . . . . . . . . . . . . . . . 284

C.4.1 Layered anisotropic structure in region C . . . . . . . . 286

C.4.2 Layered anisotropic structure in region $\mathbf{E}$. . . . . . . . . 290

C.4.3 Layered anisotropic structure in region $\mathbf{S}$. . . . . . . . . . 292

C.4.4 Overall structure . . . . . . . . . . . . . 293

C.5 Combined analysis of frequency-dependent splitting . . . . . . . . . 294

$\begin{array}{ll}\text { D Data-processing and archiving information } & 297\end{array}$

Bibliography 



\section{List of Figures}

1.1 Tectonic setting of New Zealand . . . . . . . . . . . . . 5

1.2 The two end-member deformation models of central South Island. . . 9

1.3 The tectonic evolution of the New Zealand plate-boundary . . . . . 12

1.4 The geology map of New Zealand . . . . . . . . . . . . . . . 13

1.4 The basement terrane map of New Zealand . . . . . . . . . . . . . . 14

1.5 Summary of the previous seismic anisotropy investigations . . . . . 23

1.6 Seismic anisotropy in Hasst schist from petrophysical studies . . . . . 27

1.7 Dynamic model by Duclos et al. [2005] . . . . . . . . . . . . . 29

1.8 Comparison of GPS velocity data and the predicted horizontal surface velocities . . . . . . . . . . . . . . . 31

2.1 An anisotropic system with transverse isotropy . . . . . . . . . . . 43

2.2 Different anisotropic media and shear-wave splitting. . . . . . . . . 45

2.3 Deformation kinematic diagram . . . . . . . . . . . . 54

2.4 Velocity anisotropy of olivine crystal. . . . . . . . . . . . 55

2.5 Sampling anisotropy at depths using various S-phases. . . . . . . . 61

2.6 Subduction zone flow models . . . . . . . . . . . . . . . 69

3.1 The comparison between filtered and unfiltered S phases. . . . . . . . 72

3.2 Temporary deployments . . . . . . . . . . . . . . . . 74

3.3 Earthquakes used for the on-land experiment. . . . . . . . . . 75

3.4 Events used for schist anisotropy analysis . . . . . . . . . . . . 77

3.5 MOANA OBS network and the events distribution . . . . . . 78

3.6 Transect (SAHKE) locations in the south of NI . . . . . . . 81

3.7 Different Tel./regional S-wave ray paths and event distribution . . . . 82 
3.8 The main steps in Mfast . . . . . . . . . . . . . . . 89

3.9 Processing steps of the Mfast . . . . . . . . . . . . . . 93

3.10 Defining a set of S-wave splitting analysis widows . . . . . . . 96

3.11 Best cluster estimation . . . . . . . . . . . . . . . 97

3.12 An example of cycle skipped measurement . . . . . . . . . . . . . 102

3.13 An example of a null measurement . . . . . . . . . . . . . . . . 104

3.14 Olivine deformation . . . . . . . . . . . . . . 109

3.15 Apparent splitting parameters in 2-layer media . . . . . . . . . . 111

3.16 Splitting parameters in systems with horizontal and plunging symmetry axes . . . . . . . . . . . . . . . . 114

3.17 Splitting parameter in the LQT-coordinate system . . . . . . . . . 115

3.18 Schematic shows the calculation of total anisotropy along a given ray

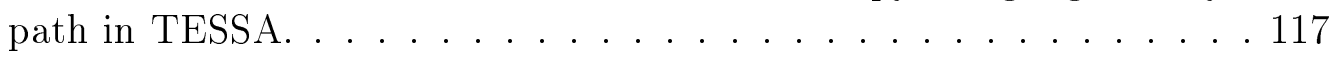

3.19 Model space and ray paths used for 3-D tomography. . . . . . . . . 120

4.1 Tectonic setting of the South Island, New Zealand . . . . . . . . . . 128

4.1 Schematic diagrams showing the proposed models of deformation for central South Island. . . . . . . . . . . . . . . . . . . 129

4.2 Maps showing the distribution of earthquakes that yield $\mathrm{A}$ and $\mathrm{B}$ quality splitting measurements . . . . . . . . . . . . 132

4.3 Outputs from the S-wave splitting analysis using Mfast . . . . . . . 134

4.3 Mfast-A grade measurement . . . . . . . . . . . . . . . 135

4.3 Mfast-B grade measurement . . . . . . . . . . . . . 136

4.4 Straight-line ray paths in two subduction zones . . . . . . . . . . 139

4.5 The spatial distribution of $\phi$ (A grade) plotted as circular histograms 146

4.6 Changes in splitting parameters with depth in northern SI. . . . . . 148

4.6 Changes in splitting parameters with depth in southern SI. . . . . . . 149 
4.7 Anisotropy in the two subduction systems. . . . . . . . . . . . 153

4.8 Changes in $\delta t$ with $\phi_{p}$ and depth at DSZ . . . . . . . . . . 156

4.9 Changes in $\delta t$ with period of the S-waves . . . . . . . . . . 158

4.10 2-D delay-time tomography from TESSA . . . . . . . . . . 162

4.11 Spatially averaged fast azimuths from TESSA. . . . . . . . . 163

5.1 Study region, network and S-wave splitting measurements . . . . . 173

5.2 Changes in splitting parameter with distance . . . . . . . 178

5.3 Individual measurements: eastern offshore . . . . . . . . . . . 182

5.4 A comparison of local splitting and SKS splitting measurements onland and offshore. . . . . . . . . . . . . . . . . 184

$5.5 \phi$ from the shallow S-phases in the central SI . . . . . . . . . . 188

5.6 Average $\delta t$ variations across the schist rock in central SI . . . . . . . 190

5.7 Delay time tomography of the offshore and on-land splitting. . . . . . 194

5.8 Spatial averages of offshore and on-land splitting measurements. . . . 195

5.9 Spatially averaged $\phi$ from quadtree griding. . . . . . . . . . . 196

5.10 3D-tomographic solutions. . . . . . . . . . . . . . 200

5.112 -D depth layers through the 3 -D model . . . . . . . . . . 201

5.12 Schematic of the ray configuration and sensitivity of ray paths to different anisotropic zones. . . . . . . . . . . . . . . 204

6.1 Examples of the splitting measurements from SKS, ScS and Tel. S

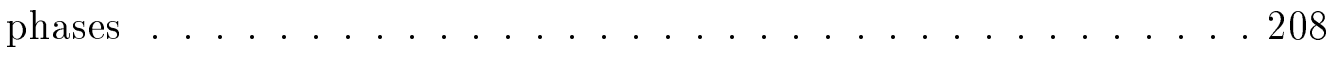

6.2 SAHKE network, tectonic settings and splitting parameters . . . . . 209

6.32 -D view of the slab geometry and ray-traced arrivals. . . . . . . . 210

$6.4 \delta t$ versus BAZ (a) and $\phi$ versus BAZ . . . . . . . . . . . 211

6.5 $\delta t$ as a function of the period of the different S-phases . . . . . . 218 
6.6 Record sections of the Tel. S and SKS arrivals. . . . . . . . . . . . 219

6.7 Map views of the splitting measurements plotted at the stations. . . . 221

6.8 Lateral variation in splitting parameters along the transect. . . . . . 223

6.9 The average SKS $\delta t$ variation along the SHAKE transect. . . . . . . . 226

$6.10 \delta t$ variation along the profile from Tel.S and SKS phases. . . . . . . 226

6.11 Comparison of splitting measurements from RTZ and LQT coordinate systems. . . . . . . . . . . . . . . . . . 232

6.12 Splitting measurements in the LQT coordinate system. . . . . . . . 233

6.13 Comparison of observed splitting with the predicted splitting parameters from slab-coupled flow model. . . . . . . . . . . . . 235

6.14 Schematic diagram showing the possible anisotropic structure in the region. . . . . . . . . . . . . . . . 237

A.1 The comparison of $\phi$ change due to polarity flip at DCZ . . . . . 245

A.2 Examples of the equal area sterographs display variations in station splitting parameters with BAZ and incidence angle. . . . . . . . 266

A.3 Velocity anisotropy is calculated from shallow slab events in the northern SI. . . . . . . . . . . . . . . 267

A.4 Examples of scatter in $\delta t$ with depth observed at some stations. . . . 267

C.1 Map showing the spatial division of stations used for two/three layer analysis. . . . . . . . . . . . . . . . 285

C.2 2-layer models for the central part of SHAKE profile . . . . . . 287

C.3 3-layer models for the central part of SHAKE profile . . . . . . . 287

C.4 2-layer models for the east of the SHAKE profile . . . . . . . . . . . 291

C.5 3-layer models for the eastern part of SHAKE profile . . . . . . . . 291

C.6 3-layer and 2-layer models for the southwest region. . . . . . . . . . 292

C.7 Integration of frequency-dependent splitting . . . . . . . . . . . 294

C.8 $\delta t$ versus $\mathrm{T}$ and comparison with the previous models $\ldots \ldots . . .295$ 


\section{List of Tables}

2.1 An average differential SWA measured at different geological terranes 49

2.2 Single crystal and polycrystal velocity anisotropy $\left(V_{p}\right) \ldots \ldots$. . . . 52

2.3 Flow law parameters of the olivine deformation . . . . . . 57

3.1 Event selection criteria and event/measurement information. . . . . 76

3.2 Event selection criteria for SKS phases and measurement information. 81

4.1 (a) Summary statistics (see p105) of the splitting parameters from shallow $(\leq 100 \mathrm{~km})$ events. . . . . . . . . . . . . . 143

4.1 (b) Summary statistics of the splitting parameters from deep (>100 $\mathrm{km})$ events. . . . . . . . . . . . . . . . . . . . 144

4.2 Regression coefficients and uncertainties of the Figure $4.7 \ldots$. . . 152

5.1 The number of measurements from different selection criteria. . . . 176

5.2 Measurements and statistics of offshore splitting measurements shown in figure $5.2 \ldots \ldots \ldots$. . . . . . . . . . . . . . . . . . . . . . . . . . . . .

5.3 All measurements and statistics of the shallow $(\leq 40 \mathrm{~km})$ splitting measurements in central SI. . . . . . . . . . . . . . 189

5.4 Parameters used for the delay-time tomography and/or spatial averaging . . . . . . . . . . . . . . . . 192

5.5 Model-space, model and inversion parameters . . . . . . . 198

6.1 Measurement information of the SKS, ScS and Tel. S splitting . . . . 212

6.2 Fresnel zone approximations for the average frequencies of ScS, Tel. S and SKS phases at different depths . . . . . . . . . 2 216

A.1 Station information of the on-land experiment . . . . . . . . 246

A.2 Events yield A-grade best filter measurement at least at one station. . 247

B.1 Station information of the OBS deployments . . . . . . . 269 
B.2 Events with AB quality high-grade measurements. . . . . . . . . 270

B.3 Measurement information . . . . . . . . . . . . 277

C.1 Station information of the SAHKE transect . . . . . . . . . 279

C.2 Event information . . . . . . . . . . . . . . . . 280

C.3 Information of the null-splitting . . . . . . . . . . . . 282

C.4 Search ranges of $\phi$ and $\delta t \ldots \ldots . \ldots . \ldots . \ldots 286$

C.5 Misfits of the best-fit 2-layer and selected 3-layer models. . . . . . . . 288

C.6 Comparison between predicted and observed SKS $\delta t$ in SI . . . . . . 295 


\section{Chapter 1}

\section{Overview}

\subsection{Introduction to study}

This thesis reports an investigation of seismic anisotropy measured by shear-wave splitting in the transpressional domain of the plate-boundary zone in New Zealand to understand the deformation and dynamics of the system. This domain includes a continental oblique-collisional setting and two subduction systems. The first phase of study is designed to investigate anisotropic structure in the transpression-subduction domain in South Island from local/regional shear-waves. This is carried out as two sub-studies, namely an on-land experiment and an off-shore experiment. These two experiments provide a unique splitting parameter data-base and an extensive splitting analysis that allow us to seek answers to address the following questions:

1. How does anisotropy distribute laterally and vertically in and around the plate boundary zone at upper-lithospheric depths in South Island? Where does the majority of anisotropy reside?

2. What are the different sources of observed anisotropy? Is this zone governed only by plate-boundary shear?

3. If there is a plate-boundary shear induced deformation, can we resolve the depth extent of that anisotropy from local/regional S-splitting?

4. How do S-wave splitting parameters vary in off-shore South Island?

5. Does the mantle-shear zone beneath the transpressional Alpine fault extend over a wide region? Or is it localized in the lithospheric upper-mantle and distributed in the asthenosphere? 
6. Can we delineate the possible deformation kinematics (e.g. dislocation creep, diffusion creep, and dynamic recrystallization etc.) from the observed anisotropy to understand the rheology of the system?

Local splitting parameters on land have been previously investigated by small-scale studies in some localized areas, especially in the northern South Island. Our attempt is to carry out a full-scale splitting analysis in the entire South Island including offshore regions using a new seismic data set from an enhanced station network in South Island.

The second phase of the thesis investigates the subduction dynamics and lithospheric deformation in the south-east Hikurangi subduction zone of the North Island from teleseismic S-wave (i.e. SKS, ScS and regional S) splitting. Earthquake records from the broad-band transect (SAHKE transect) across the previously unexplored region of the south-east Hikurangi subduction zone enable us to examine:

1. Variations in anisotropic structure across the fore-arc region

2. Lithospheric-mantle wedge complexities and upper-plate deformation in response to oblique subduction

\subsubsection{Thesis structure}

The thesis consists of seven chapters. The first three chapters present background information and methodology. The next three chapters include results and interpretations. The final chapter summarizes the key findings of this study. The summary of the content in each chapter is given in the next page: 
Chapter 1: Discusses the tectonic character and deformation at transpressional and subduction settings, and introduces New Zealand's tectonic setting, geology and geophysical structure. It also summarizes previous work that is related to this study.

Chapter 2: Seismic anisotropy and theories behind shear-wave splitting are explained. Different sources of anisotropic fabrics, their origin and tectonic significances are discussed to explain how deformation/dynamics can be investigated from splitting parameters.

Chapter 3: Contains information about the data utilized, experiment set-up and methodology. It also includes information on other different phenomena that can be used to characterize the observed splitting parameters.

Chapter 4: is a published journal article on splitting parameter analysis from land stations. Note that some parts of the introduction and methodology of this chapter are repetitive. Work related to this section, but completed after this manuscript publication, has been included appendix 5.3.1.

Chapter 5: This chapter includes results from the offshore experiment and this is also in the process of preparation for journal publication. Therefore, some introduction parts can be repetitive from previous chapters.

Chapter 6: Presents results from the study of deep anisotropy in the south-east Hikurangi subduction zone.

Chapter 7: Concludes the key findings and discusses limitations and future directions. 


\subsection{Deformation and dynamics at convergent margins}

Convergent plate boundaries are the locus for subduction and/or mountain-building. Oceanic-continental plate convergence results in the subduction of the denser oceanic lithosphere underneath the continental lithosphere, whereas regions of continentalcontinental convergence, which are also known as 'collisional zones', can result in two lithospheric blocks sliding past each other, resulting in transform faulting and/or orogeny. Formation of these end products at continental convergent zones greatly depends on the obliquity of convergence. Both plate-boundary parallel and orthogonal plate movements occur at oblique-convergent zones; therefore, such zones are called 'transpressional zones'. Transpressional boundaries can be characterized by both oblique-transform faulting and orogenesis as in the case of South Island, New Zealand. A significant portion of the plate-boundary that traverses New Zealand is considered to have a transpressional character and this transpressional zone contains to two subduction systems at the northern (Hikurangi) and southern (Puyseger) ends, in which the Pacific plate subducts beneath the Australian plate and visa versa (Fig. 1.1). The plate-boundary in New Zealand is a unique example of where subduction, transform faulting, and orogenesis can be observed along a single boundary-zone and provides an opportunity to seek solutions for the collision and subduction related questions.

One of the main concerns in both collision and subduction systems is how lithospheric blocks deform when they interact at plate-boundaries. The rheology of the system controls the distribution of the deformation that is produced by the plate-boundary forces [Thatcher, 2009]. Rheology primarily depends on the lithology (i.e. mineralogy and rocks), temperature, fluid involvement and tectonic environment [Burgmann and Dresen, 2008]. Unlike rigid oceanic plates, weak continental lithosphere is more susceptible to deform intensely at the plateboundaries and is often associated with large-scale faulting [Molnar and Tapponnier, 


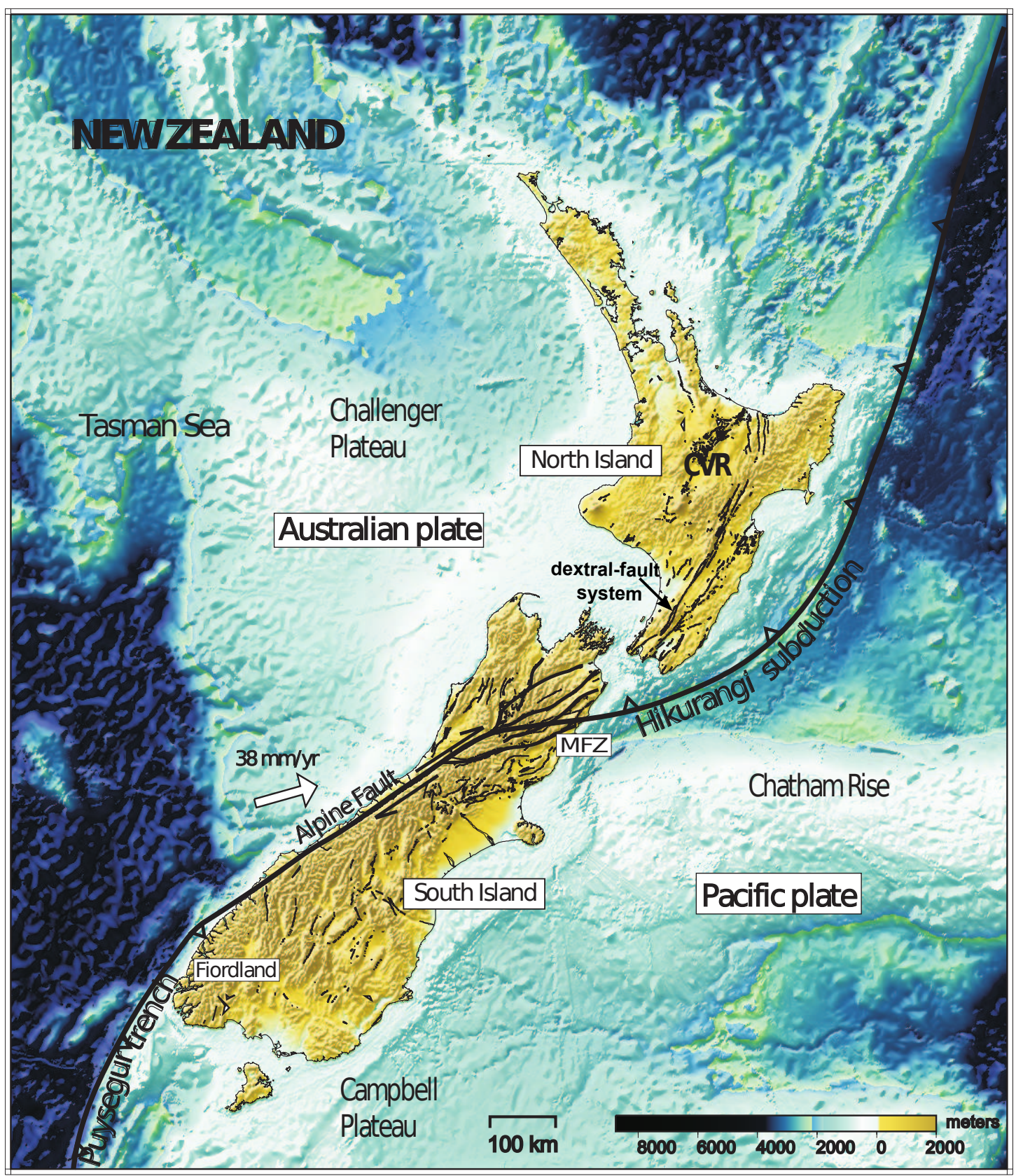

Figure 1.1 Tectonic setting of New Zealand (MFZ: Marlborough Fault Zone, CVR: Central Volcanic Region). The relative plate motion (vector) between Australian and Pacific plates is denoted by the arrow with $38 \mathrm{~mm} / \mathrm{yr}$.

1975, England and Jackson, 1989]. The study of deformation distribution in such large-scale plate-boundary faults helps in understanding the strength of the fault at depth, delineating possible deformation mechanisms, and perhaps in forecasting the locations of mega-scale plate-boundary earthquakes. An architecture of such a deformation distribution around plate-boundaries can be explored via structuralgeological, geodetic, petrophysical and geophysical investigations. 


\subsubsection{Deformation at continental zones}

\section{Subduction, collision and mountain-building}

In certain tectonic settings (e.g. plate-boundary zone in South Island), subduction of the oceanic lithosphere at convergent margins leads to the collision of the two continental lithospheric blocks soon after consuming the oceanic lithosphere. Sediments extracted from the ocean basin are pushed up as two continental blocks converge, initiating mountain belts. In the long term, continental-collision results in the accumulation of deformation/strain that is possibly distributed in wide regions of the adjacent plate-boundary zone as a result of crustal shortening with folding, thrusting and continuous growth of the mountain belts (e.g. Asia-Eurasia collisional zone; Molnar and Tapponnier [1975]). As argued in several tectonic models [Toussaint et al., 2004], structural evolution during transition from subduction to collision may occur either as continuous subduction of the lithosphere or lithospheric delamination, which leaves the whole crust or part of the crust at the surface. In the latter case, crust at the surface can be subjected to intense deformation (e.g. folding, faulting, crustal thickening) as the plates collide. Then the detached mantlelithosphere may [Toussaint et al., 2004]:

1. continue its subduction

2. break off and sink into the convecting mantle

3. thicken until it becomes unstable and drips down

4. shorten by folding

Which process is favored is controlled by factors such as rheology, thermal structure, composition of the lithosphere, rate of convergence, and the thickness of the lithospheric blocks [van den Beukel and Wortel, 1987]. 


\section{Plate-boundary faults}

At continental-collisional zones the deformation of the adjacent lithospheric blocks via large-scale faulting plays a dominant role in the dynamics of the entire system. In the suture zone, where two blocks of continental lithosphere collide, large-scale plateboundary faults evolve depending on the relative motion of the two plates [Vauchez and Nicolas, 1991]. Forces acting on such faults are large enough to develop a strong structural discontinuity that extends from the surface through the brittle/semibrittle crust and into the ductile upper-mantle or asthenosphere. Fault properties and behavior change from top to bottom, depending on the stress conditions and rheology of the system [Otsuki, 1978, Thatcher, 2009]. The depth extent of such large-scale faults is poorly constrained, since seismic slip is mostly confined to the first 10-20 km of the crust [Bourne et al., 1998]. Two end member scenarios explain the discontinuous deformation and character of the faults in the brittle crust and the ductile continental lithosphere. One end member scenario is mainly focused on the character of the faults in the brittle upper crust [Bourne et al., 1998]. This model emphasizes the block-like behavior of the regions between faults in the brittle crust and follows the plate-kinematic theory. Here, faulting areas are considered to be relatively weak compared to the host rock, dividing crust into blocks that are smaller than tectonic-plates. In contrast, the other end member scenario explains the continuous deformation of the entire lithosphere, assuming that deformation occurs on thin viscous sheets (e.g. vertical shear planes) with little or no depth variation in strain rates [England et al., 1985, England and Jackson, 1989]. Thus, it does not consider the discontinuous slip behavior on faults as in the block model [Savage and Burford, 1970].

It is important to consider how large-scale faults extend horizontally and in depth by means of the above two scenarios. Is the deformation at faults biased towards one model? Or can faults accommodate the combined effects of both models? These 
are some of the important questions yet to be answered from case studies. How plate boundary faults evolve in the ductile lithosphere and/or in the asthenosphere has been demonstrated by two models that incorporate the above two end-member scenarios (Fig. 1.2). As described in one model (figure 1.2-ii), narrow faults that exist in the brittle crust can extend into the lower-crust and to the uppermantle as a narrow shear zone until they find a mechanically weak domain where they could attain distributed shear deformation [Savage and Burford, 1970]. In this case typically the semi-brittle lower-crust and ductile upper-mantle is strong enough to be cut by the narrow localized shear zones and distributed deformation only occurs in the asthenosphere, where dislocation creep deformation dominates (section 2.5). Although the preferred deformation mechanism in the lower-crust and upper-mantle is dislocation creep, other mechanisms (e.g. dynamic recrystallization, diffusion creep) can become prominent depending on the stress/strain, temperature conditions, mineralogy and grain size of the fault zone (section 2.5). For instance, grain size reduction in highly strained (shear strain 1-10) rock would produce fine grained mylonite/ultramylonite layers (high-strain shear zones) that indicate strain localization. In such regions the width of the shear zone can be as small as a few millimeters [Burgmann and Dresen, 2008]. Narrow (1-2 km) mylonite zones that were formed in the mid-crust and exposed at the exhumed sections of the Alpine fault are evidence for such narrow localized shear-zones at depth [Little et al., 2002a].

The other model (model (i) in figure 1.2) consists of a weak lower crust and upper mantle that deform via dislocation creep (section 2.5), allowing distributed deformation beneath the brittle faults [McKenzie and Jackson, 1983] similar to thin-viscous sheet type deformation. Intrinsically anisotropic minerals in the lowercrust (e.g. mica and amphiboles) and the upper-mantle (e.g. olivine) will produce an anisotropic medium when they deform via dislocation creep in such shear zones [Nicolas, 1993]. As inferred by seismic anisotropy studies (SKS shear-wave splitting, petrophysics and Pn anisotropy) in plate boundary fault zones (e.g. Alpine fault 
i. Distributed deformation / Thin viscous model

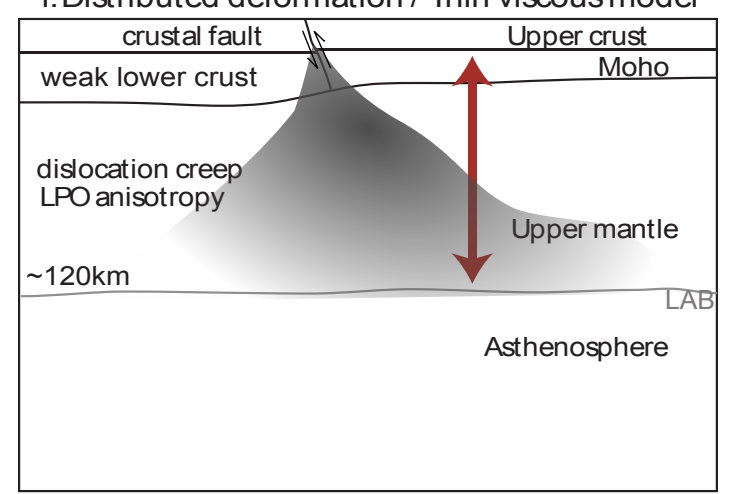

ii. Localized deformation / Fan shape model

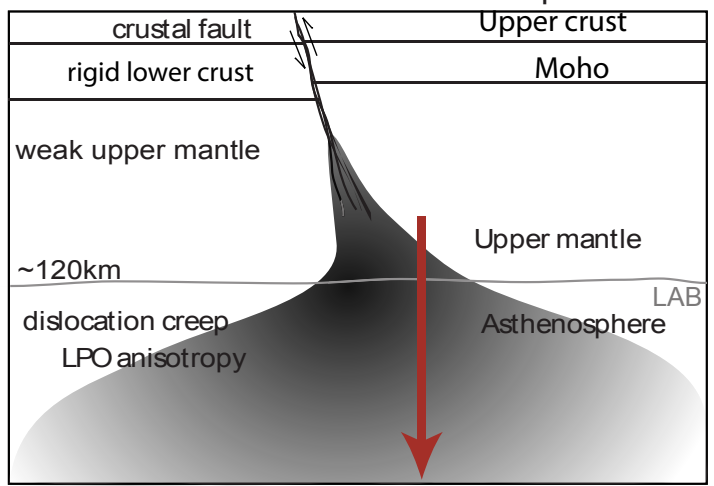

Figure 1.2 The two end-member models of distributed shear (i) and localized (ii) deformation that explain the deformation in the plate-boundary zone of central South Island. Gray-shaded region indicates the shear zone and dark to light gray shading marks the distribution of strain (high to low shear strain) around the Alpine fault. The red arrow denotes the predicted depth distribution of shear zone below the fault.

zone, San Andreas fault zone), those shear deformation zones can be distributed in 200-400 km wide areas around the fault [Klosko et al., 1999, Vauchez and Tommasi, 2003]. Nevertheless, in some cases such observations contradict the geodetic strain estimations and structural geology observations, suggesting either variations in deformation conditions (or the deformation mechanisms) within the shear zone or temporal variations in stress-strain conditions that may effect the state of deformation resulting in complex structure or that there is evolution from model(i) to model(ii) (see Fig. 1.2) [Houlié and Stern, 2012, Burgmann and Dresen, 2008].

\subsection{Background: tectonic setting of New Zealand}

New Zealand's land mass consist of two Islands (North and South), which are emergent segments (10\% of the total land mass) of the mostly submerged continental micro-plate 'Zealandia'. It was detached from the Gondwana supercontinent by sea floor spreading and oceanic-crust formation during the late Cretaceous (Fig. 1.1; Cox and Sutherland [2007]). Since then the geometry of the land mass has been reshaped through major tectonic episodes (section 1.3.1). The present-day setting of 
New Zealand is tectonically significant as it lies along the active plate-boundary zone of the Australia-Pacific (AUS-PAC) plates. Tectonic activity along this boundary is marked by the two oblique subduction systems (Pysugur and Hikurangi subduction zones) with opposite convergence polarity, the central transpressional/collisional zone with major oblique-strike slip fault (i.e. the Alpine Fault) and Southern Alps mountain range, and Marlborough fault zone (MFZ) that indicates the transition from subduction to collision (Fig. 1.1).

\subsubsection{Tectonic synthesis}

New Zealand has evolved since the late Cretaceous (80 Ma) and undergone various tectonic processes. In the early Cretaceous, New Zealand was a part of the eastern Gondwana subduction system [Mortimer, 2004]. The crustal component of New Zealand is derived from the accretionry tectonic processes that took place during the Paleozoic and Mesozoic (Fig. 1.3-A; Cox and Sutherland [2007]). Late Cretaceous rifting that took place in the Tasman and Pacific sea isolated the continental fragment with New Zealand's landmass from the Gondwana supercontinent, resulting in basin and oceanic crust formation in the southern ocean (Fig. 1.3-B). The plate-boundary through New Zealand started to develop during Eocene ( $\sim 40-45 \mathrm{Ma}$ ) approximately during the same time that the Resolution rift boundary and passive margin formed to the south and southwest of New Zealand respectively [Sutherland et al., 2000].

Until the mid Oligocene, the deformation within the New Zealand plate-boundary zone was not that prominent, and the relative motion of AUS-PAC plates was relatively slow due to close proximity of the pole of rotation between the AUS-PAC plates and New Zealand [Cox and Sutherland, 2007]. However, in the late Oligocene $(\sim 25 \mathrm{Ma})$ the rate of relative motion of the AUS-PAC plate increased and the plateboundary attained a dominant strike-slip behavior in the central collisional zone as 
a result of the southward movement of the pole of rotation [Cande and Stock, 2004, Cox and Sutherland, 2007]. This change enhanced the strike-slip movement and caused the Australian passive-margin, which was formed during Eocene rifting, to migrate towards the central collisional zone (Fig. 1.3-C). The Eocene rift margin that intruded in to the New Zealand plate boundary zone in the late Oligocene had a direct impact on the late Cenozoic evolution of the plate-boundary and the deformation associated with the Alpine Fault especially in the South Island [Sutherland et al., 2000]. Subduction along the northern part of the plate-boundary was initiated during $20-25 \mathrm{Ma}$ at the Hikurangi margin in the northern part of the strike-slip plate-boundary zone (Fig. 1.3-D). Subduction at the Puysegur margin, which is in the south of the strike-slip zone, has a relatively young origin history ( 7-10 Ma) compared to the Hikurangi subduction, however both systems started to evolve during the time when the central continental-continental margin was mostly strike-slip.

In central South Island, the pure strike-slip nature of the plate-boundary changed to oblique convergence around $\sim 6 \mathrm{Ma}$, resulting in significant compression [Walcott, 1998]. According to Cande and Stock [2004], this convergence initiated $\sim 11 \mathrm{Ma}$ in the southern South Island (lat:44.5º. The present-day relative motion vector can be decomposed into a $\sim 36 \mathrm{mma}^{-1}$ plate-boundary parallel component and $\sim 11$ $\mathrm{mm} \mathrm{a}^{-1}$ fault-normal components [Norris et al., 1990]. Present-day GPS inversion suggests that $\sim 70 \%$ of the total plate displacement is taken up by the Alpine fault [Beavan et al., 1999, Sutherland et al., 2000], and most of the rest is accommodated in the lithosphere as distributed deformation [Norris et al., 1990]. The fault-normal component is responsible for $\sim 100 \mathrm{~km}$ of crustal shortening since the origin of the oblique-convergence. The plate-parallel component governs the strike-slip movement of the Alpine Fault. Because of both strike-slip motion and the compressional character of the central South Island, this zone is well-described as a transpressional zone. The transpressional zone is linked with the Hikurangi subduction system 


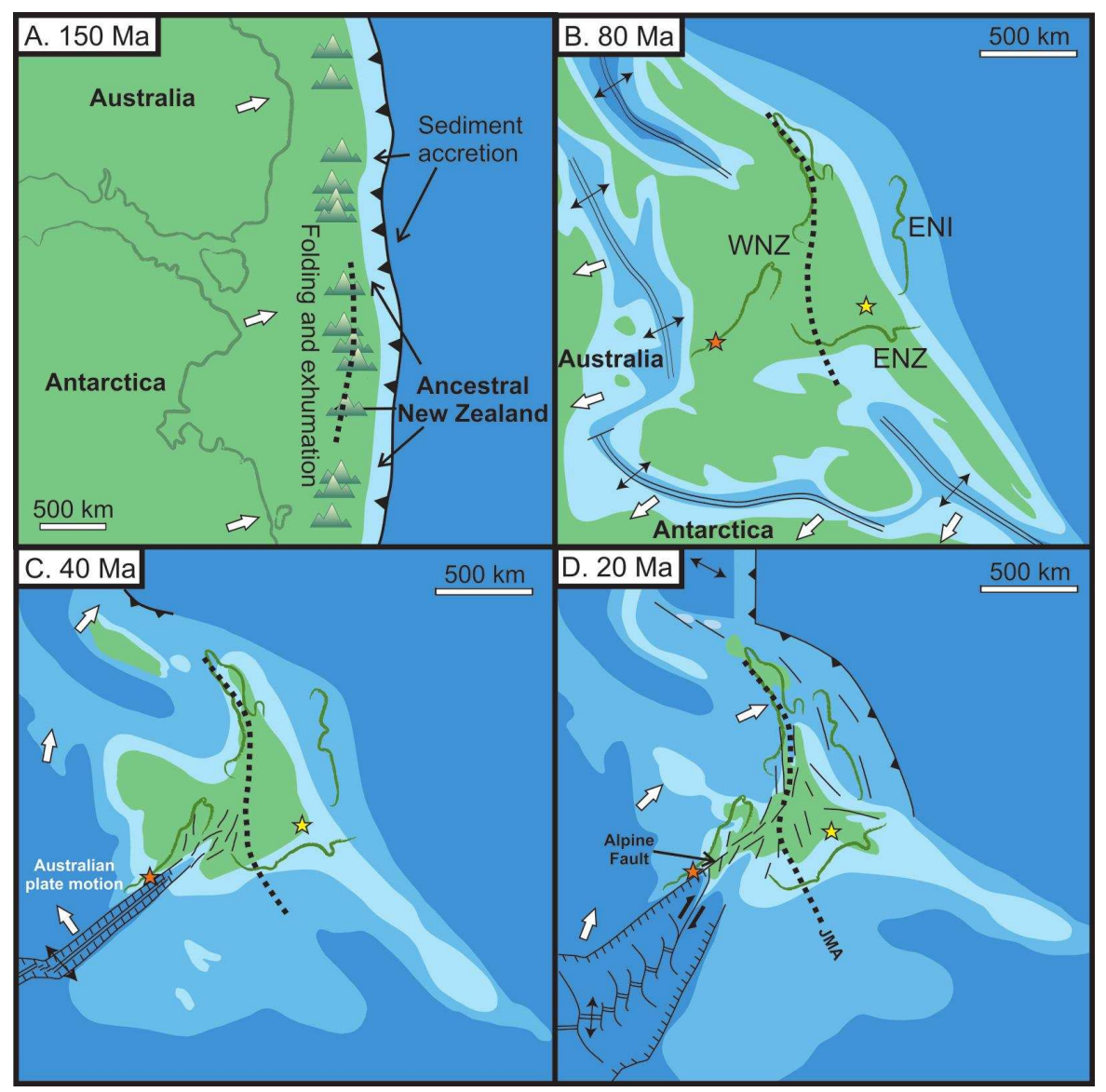

Figure 1.3 The tectonic evolution of New Zealand plate-boundary (after Cox and Sutherland [2007]). A dashed line marks the position of the Junction Magnetic Anomaly. Western, Eastern provinces and eastern North Island are denoted by WNZ, ENZ, and ENI respectively. Red and yellow stars show the positions of the two points on either side of the Alpine fault that became juxtaposed at the central Alpine fault as result of the plate-boundary evolution. A) ancient Gondwana subduction. B) rifting in the Tasman sea/south of New Zealand and separation from Gondwanaland. C) migration of the Australian Passive margin towards the strike-slip fault zone in Central South Island. D) a change in fault character from pure strike-slip to oblique strike-slip movement and subduction initiation.

(which is located in the northeast of the South Island and the east of North Island) by the transitional fault zone (Marlborough fault system) in the north/northeast of the South Island (Fig. 1.1). In the North Island, the Hikurangi subduction and the associated structures dominate over a $\sim 250 \mathrm{~km}$ broad zone of plate margin [Lamb, 2011]. This subduction zone is characterized by the back-arc spreading in central-north, Central Volcanic Region (CVR), and the dextral-faulting system in the overriding plate, accommodating transpression (Fig.1.1). 


\subsection{Geology and structure of the South Island}

The geology and geophysical structure of the area have been explored thoroughly in some localized areas (e.g. SIGHT and SAPSE experiments). In map view, the geology and geomorphology of New Zealand simply reflect the oblique-transform nature of the present-day plate-boundary zone (Fig. 1.4a).

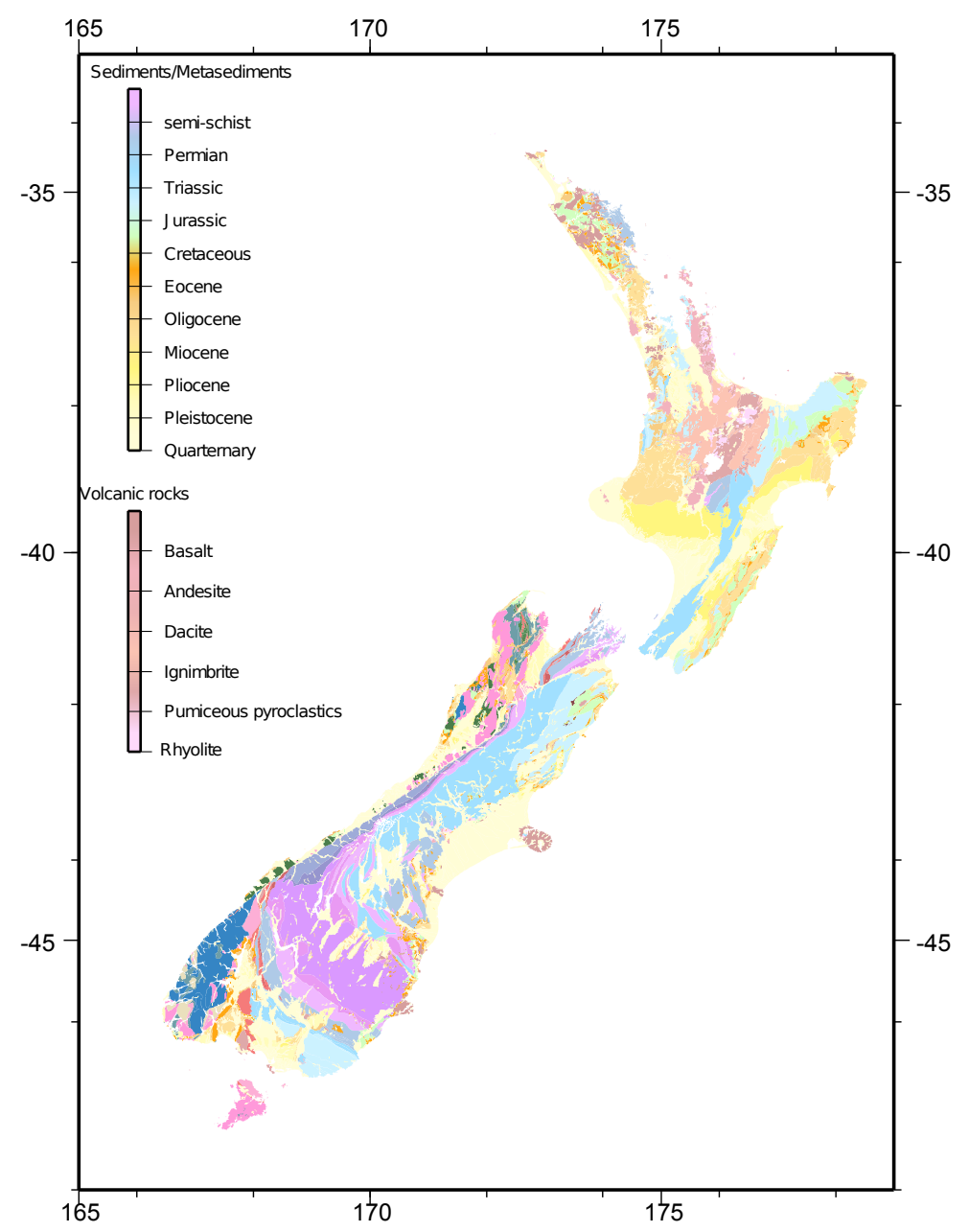

Figure 1.4 (a) The geology map of New Zealand (data source: GNS science www.gns.cri.nz.)

\subsubsection{Geology}

According to Mortimer [2004], New Zealand basement geology is mainly characterized by nine major volcanic-sedimentary terranes (from west to east: Buller, Takaka, 


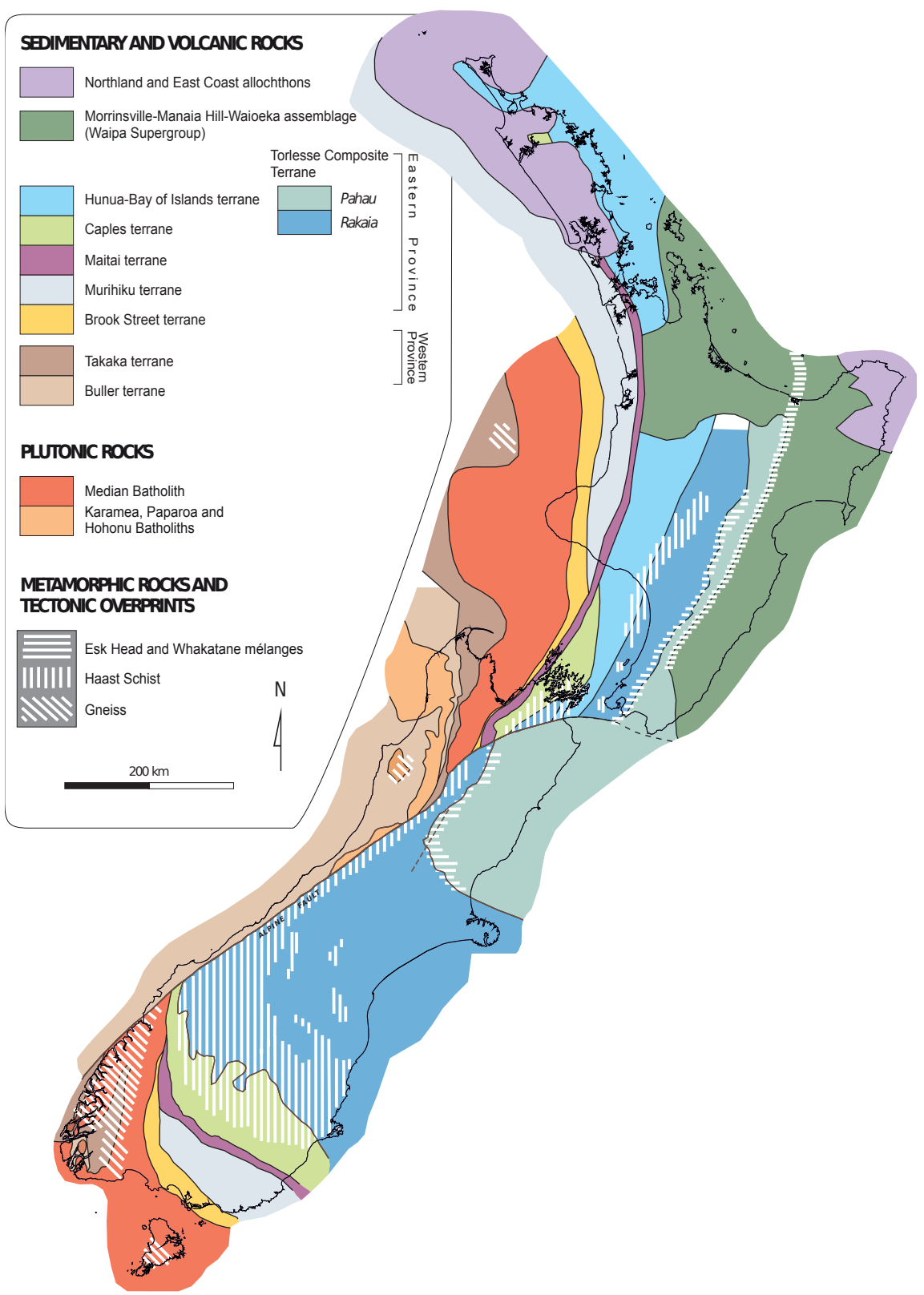

Figure 1.4 (b) The basement terrane map of New Zealand (after Mortimer [2004]).

Brook Street, Murihiku, Maitai, Caples, Bay of Islands, Rakaia, and Pahau), and minor igneous intrusions (three batholiths units: Median, Karamea-Paparoa and Hohonu) and overprinted metamorphic belts (Haast schist, gneiss and melange) in the volcanic-sedimentary terranes (Fig. 1.4b). Exposed basement rocks do not contain any Precambrian rock units and rock age varies from middle-Cambrian to late Early Cretaceous. These rocks are weakly metamorphosed and foliations are not intense. The parent source of the basement rock is the eroded sediments from the mountain belts on the subduction margin of eastern Gondwanaland (section 
1.3.1; Mortimer [2004]). About $100 \mathrm{~km}$ away from the present-day plate-boundary, the alignment of volcanic-sedimentary belts and the lithologic boundaries that were built eastward represent the orientation of the ancient Gondwana margin, but close to the plate margin this orientation changes from fault-oblique to fault-subparallel with an apparent oroclinal bend that is likely associated with the dextral sense of motion during Cenozoic deformation [Cox and Sutherland, 2007]. Sedimentary terranes are divided into Western and Eastern provinces taking into account the position of Median Batholith that was formed $~ 375-110$ Ma (long-lived composite batholith) from subduction process, and considering the age of the rock units [Cox and Sutherland, 2007]. Eastern province rocks are generally younger than the western province. The following section summarizes the important information about those geologic units (Fig. 1.4b) and most of the details are adapted from Mortimer [2004] and Cox and Sutherland [2007].

\section{Volcanic-sedimentary terranes}

Volcanic-sedimentary terranes display weak metamorphism. Buller terrane, which is the most prominent lithology in the west of the South Island, has metamorphosed siliciclastic sandstone and mud stones. The rocks in this terrane generally display upright folds and steep cleavage, and belong to lower Paleozoic Gondwana sedimentary units. In northwestern South Island, the rock unit consists of granitic intrusions. Takaka terrane, which spreads across the western North Island, contains siliciclastic, carbonate and volcanic rocks of the Cambrian to Early Devonian age. This is a lithologically stratified complex rock unit, which is separated from the Brook Street terrane by the intrusion of median Batholith. The main rock type in the eastern province ( $\sim 60 \%$ of the New Zealand rock) is quartzofeldspathic greywacke. Pyroxene-rich basaltic volcanic rocks dominate in the Brook Street terrane. The contact between Brook Street terrane and Murihiku terrane in the northwestern North Island is marked by the Letham Ridge Thrust. Murihiku terrane consists 
of volcanoclastic rock rich with marine sandstone. Maitai terrane contains the eastern Dun Mountain Ophiolite Belt that is used to constrain the plate-boundary deformation due to strike-slip motion and the Alpine fault offset [Wellman, 1979]. It is a well-stratified volcanoclastic sedimentary rock unit with spatially correlated magnetic properties (e.g. Junction magnetic anomaly).

Weakly metamorphosed volcanoclastic greywacke and argillite are stratified in the Caples terrane. The lithologic boundary between the Caples and Rakaia terranes is considered to be overprinted by the Otago (Haast) schist. The Rakaia terrane, which is in the eastern South Island and central North Island, is the largest terrane by area. This unit consists of submarine quartzofeldspathic sandstone-mudstone. In most of the places, steeply dipping greywacke (Torlesse greywacke) bedding planes $(>60$ $\left.90^{\circ}\right)$ with rapid changes in strike of the bedding is significant. In some locations, shallowly dipping bedding planes $\left(<30^{\circ}\right)$ are overturned to form folds with steeply dipping fold axes. Within the rock unit, metamorphic grade increases from east towards the overprinted Haast schist in west. The Bay of Island terrane also has a complex internal structure. Sandstone fabric in this terrane is similar to the petrofabric of the Rakaia terrane. Lithology of the Pahau terrane is consistent with the Rakaia terrane, but it is a younger Torlesse terrane compared to Rakaia terrane that consists of clastic detritus and trench deposits. Clastic detritus in this terrane is assumed to be recycled material from the Rakaia terrane [Wandres and Bradshaw, 2005, Mortimer, 2004].

\section{High-grade metamorphic rocks}

Geologically significant rock units in New Zealand are the three types of overprinted high-grade rocks (gneisses, Haast schist and melanges) with regional metamorphism. These metamorphosed rock units are linked with different deformational processes (e.g.subduction, oblique-convergence related) associated with the plate- 
boundary evolution. Gneisses are considered to be the product of an amphibolitegranulite facies recrystallization during the Devonian to Cretaceous period [Cox and Sutherland, 2007], and are commonly seen in the western province. Older Torlesse quartzofeldspathic greywake (Rakaia terrane) is considered to be the protolith of the Hasst schist. Hasst schist in South Island represents the distinct schist units (Otago, Marlborough and Alpine schists) with different history of origin and metamorphic characteristics. Otago schist (garnet-biotite-albite zone) is a north-west trending anticlinorium with sub-horizontal foliations, which spreads $\sim 150 \mathrm{~km}$ in area. The schist is dominated by fold, shear zones and clear stretching lineations [Cox and Sutherland, 2007]. Although Marlborough schist is located further north, structures within that unit are similar to Otago schist in the south. Marlborough and Otago schists are interconnected by elongated, narrow schist bands of Alpine schist in the central South Island. The Alpine schist had been subjected to both amphibolitefacies peak metamorphism and ductile-brittle deformation during exhumation [Little et al., 2005, Cox and Sutherland, 2007]. Depending on the metamorphic grade, Hasst schist can also be sub-grouped into several deformation zones. Petrophysical studies have shown that these zones also exhibit a high degree of anisotropy [Godfrey et al., 2000, Okaya et al., 1995]. The outer-most zone is a chlorite zone with low grade metamorphism. Biotite and garnet-oligoclase zones reflect the increase in metamorphic grade towards the Alpine fault (and the plate boundary). These zones are also characterized by well developed foliations. Unlike Haast schist, melanges do not show pronounced foliations and they represent the deformation in the shallower zone of the Mesozoic accretionory prism. 


\subsubsection{Geophysical implications of the present-day structure}

This section summarizes the important geophysical observations from previous experiments. The key questions that those studies aimed to address in this region are: How do plate-boundary faults (e.g. Alpine fault) evolve at depth? What is the crust-mantle character in response to transpression? What type of crustmantle structure would best explain the observed geophysical, structural geology and geodetic observations? An on-land plate-boundary configuration, a young orogen with an active deformation and rapid exhumation, and ongoing tectonic processes with enhanced seismicity are some of the advantages that make this plate-boundary domain an exceptional place to seek answers for the above questions. Presentday structures in the study region can be divided into three main domains from north to south: 1) Hikurangi subduction zone, 2) Central transpressional zone, and 3) Fiordland subduction zone. Most of the geophysical experiments to date were focused on the structure beneath the continental collision zone with transpressional character.

\section{Transpressional zone}

The central transpressional zone is characterized by structures resulting from both collision and oblique strike-slip motion between the plates. The Alpine fault, which is the main surface manifestation of the plate-boundary, extends for about $800 \mathrm{~km}$, striking $055^{\circ}$ in the South Island [Sutherland, 1999]. It accommodates $70 \%$ of the current plate motion [Norris et al., 1990] and the rate of relative plate motion is $\sim 38 \mathrm{~mm} /$ yr with $\sim 36 \mathrm{~mm} /$ yr plate-parallel and $\sim 11 \mathrm{~mm} /$ yr plateperpendicular components [DeMets et al., 1994a]. Because of the right-lateral strikeslip motion, geologic terranes in South Island display a clear $\sim 400-460 \mathrm{~km}$ dextral offset. According to [Little et al., 2002b], the bending related deformation of geologic units and other structures in the central South Island (e.g. fold and faults) is mainly 
governed by the dextral-transpression and the shortening in the crust $(\sim 100 \mathrm{~km}$ shortening across the plate-boundary [Norris et al., 1990]) during the last $10 \mathrm{My}$.

The transpressional character of the central Alpine fault has resulted in crustal thickening. Consequently, the Southern Alps (peak hight of $3700 \mathrm{~m}$ ) is being uplifted at a rate of approximately $7-10 \mathrm{~mm} / \mathrm{yr}$ and the shallow-mid crust of the Pacific plate is being exhumed along the Alpine fault [Davey et al., 2007, Little et al., 2005]. $\mathrm{P}_{n}$ travel times estimated from the waves refracted along the root of the Southern Alps in the central South Island suggest the crustal thickness of the South Island is highest ( $\sim 48 \mathrm{~km}$ ) about $80 \mathrm{~km}$ SE of the Alpine fault [Bourguignon et al., 2007a]. As inferred by the SIGHT experiment, crustal thickening $(\sim 17 \mathrm{~km})$ due to shortening is asymmetrically distributed and mostly concentrated in the central-eastern South Island beneath the Southern Alps. The thickness of the lithospheric root is about $100 \mathrm{~km}$ and this zone is also characterized by a mantle high-speed (and high density) body at depths of $\sim 80-100 \mathrm{~km}$ [Stern et al., 2000]. Scherwath et al. [2003] noticed a negative Bouguer gravity anomaly that fits well with the location of the thick crustal root, which is isostatically imbalanced with negative buoyancy. This negativelybuoyant crustal root is assumed to be supported by the high density mantle body (i.e. the mantle high-speed zone discussed above) [Stern et al., 2000]. Based on these findings along with SKS and $\mathrm{P}_{n}$ anisotropy estimates, Stern et al. [2000] proposed a lithospheric-thickening model with distributed deformation to explain the crustmantle dynamics beneath the transpressional zone. Lithospheric deformation in this region has also been explained using the intracontinental subduction model [Wellman, 1979] with localized deformation. This model is also supported by numerical modeling in the central South Island [Beavan et al., 2004, Ellis et al., 2006]. 


\section{Fiordland subduction}

The central transpressional zone is linked to Puysegur subduction in the southwest offshore. Oceanic lithosphere that was formed at the AUS-PAC boundary about 40-25 Ma from rifting processes is being subducted at the Puysegur Trench [Davey et al., 2007]. This subduction migrates into the Fiordland region (Fig. 1.1) due to transcurrent plate-motion of the inland pate-boundary [Lebrun et al., 2003]. In Fiordland, the plate motion is partitioned between subduction and strike-slip motion. The Alpine fault in this region tends to be sub-vertical and accommodates $\sim 55-85 \%$ of the total plate motion [Sutherland et al., 2000]. Significant crustal deformation is assumed to occur in the region's eastern part because of the strong strike-slip behavior of the fault in the southern South Island, extending offshore. Studies also suggested an uplifted lower-crust during the Cretaceous in western Fiordland [Davey and Smith, 1983] and a high-velocity zone in the upper-mantle of the Pacific plate [Eberhart-Phillips and Reyners, 2001]. This high-velocity zone is attributed to a mechanically strong region in the upper-mantle, which may cause the subducting slab to change its strike (from $\sim 70^{\circ}$ to $15^{\circ}$ ) and dip from moderately to steeply dipping at the north end [Reyners et al., 2002, Eberhart-Phillips and Reyners, 2001]. Yet, this subduction is relatively young ( $10 \mathrm{My})$ with a maximum slab extension of $\sim 150 \mathrm{~km}$ [Davey and Smith, 1983]. High SKS delays (2.5 s) recorded in this region have been explained as a coupling of trench-parallel flow in the subduction settings and the plate-boundary parallel shear deformation associated with the transpressional zone [Duclos et al., 2005].

\section{Hikurangi subduction and Marlborough faults}

The northern termination of the transpressional zone is marked by the transitional fault zone (i.e. Marlborough fault zone-MFZ) in north/northeast South Island (Fig. 1.1). This zone then extends into the Hikurangi subduction zone with an 
oblique subduction. The northern South Island is, therefore, subjected to both subduction related deformation and deformation associated with transform faults. The MFZ consists of four main strike-slip faults (Hope, Clarence, Awatere, and Wairau faults), and the northernmost Wairau fault is considered to be the northward continuation of the Alpine fault (section 4.1). As suggested by receiver function studies, unbroken Moho (depth $\gtrsim 15 \mathrm{~km}$ ) beneath this zone is compatible with pervasive shear deformation in the lithosphere below the crustal faults [Wilson et al., 2004]. This shear deformation appears to control the rotational-type deformation of the crustal fault blocks that are presumably aligned with the shear fabric below [Hall et al., 2004]. Oblique-subduction dominates in the southern North Island. Northwest-dipping subducting Pacific crust first encounters the overlying submerged accretionry prism and then the overriding Australian plate. The relative plate motion of $\sim 42 \mathrm{~mm} / \mathrm{yr}$ in this region is partitioned into plate-boundary parallel and orthogonal components, resulting in offshore thrust faulting, dextral faulting in the upper-plate and fault-block rotation [Henrys et al., 2013].

\subsection{Previous work: seismic anisotropy and deformation in South Island}

Various techniques (e.g. Pn anisotropy, shear-wave splitting, P-wave delays and GPS/structural studies) have been implemented to understand the deformation and dynamic structure of the plate-boundary zone in the South Island. When consider the plate motion history (section 1.3.1), this plate-boundary was mainly pure strikeslip during $\sim 30$ - $6 \mathrm{Ma}$ and it attained transpressional character only in the last $\sim 6$ Ma. An SKS splitting study by Klosko et al. [1999] pointed out the possibility of broad-scale deformation that results from the plate-boundary shear, however the spatial and depth extent of this pervasive shear zone was not accurately resolved by this study. Plate-boundary parallel to sub-parallel SKS-splitting azimuths through 
out the entire South Island have been interpreted as a large-scale strike-slip straining in the mantle that is possibly associated with the shear zone [Klosko et al., 1999, Molnar et al., 1999]. Such distributed shear deformation had also been predicted by geodetic studies [Walcott, 1998, Beavan et al., 1999].

Finite strain governs the preferred alignment of intrinsically anisotropic minerals and creates an anisotropic medium in which S-waves can split into two mutually perpendicular quasi S-waves with different speeds [Savage, 1999]. For large strains in olivine dominant systems, the polarization direction of the fast S-wave reflects the maximum extension (or shear plane), whereas the polarization direction of the slow wave corresponds to the axis of maximum shortening [Ribe, 1992, Molnar et al., 1999]. Taking into account the mantle strain that is constrained by SKS splitting measurements [Klosko et al., 1999], geodetic and structural constraints [Walcott, 1998, Norris et al., 1990] and the distributed lateral heterogeneity as detected from P-wave delays across the central plate-boundary zone, Molnar et al. [1999] suggested that the mantle lithosphere of the South Island is most likely characterized by distributed deformation as demonstrated in a thin-viscous sheet model and does not agree with the localized deformation model (Fig. 1.2-ii and section 1.2.1). Constraints from mantle rheology studies and strain modeling further suggest that pervasive shear at depths is most likely accommodated as vertical shear planes (fan shape) that reside within the lower-crust and the uppermost $100 \mathrm{~km}$ of lithospheric mantle [Collins et al., 2010]. In the transition fault zone (Marlborough faults), interpretation of anisotropic receiver functions suggests a continuous Moho with spatial variations in crustal thickness that indicates the presence of distributed strain in the middle to lower crust just beneath the strain-localized segment of the fault [Wilson et al., 2004]. This study suggested a large-scale pervasive shear zone at deeper parts (deeper than $\sim 15 \mathrm{~km}$ ) possibly extending from the lower-crust [Wilson et al., 2004]. In agreement with these observations, local shear-wave splitting studies in the same region found fault-parallel (NE-SW) fast direction, possibly resulting 
from this pervasive shear [Balfour et al., 2005, Audoine et al., 2000]. An anisotropic velocity inversion by Eberhart-Phillips and Henderson [2004] found a high anisotropy zone between the Awatere and Wairau faults (north-west of the Marlborough faults) that was interpreted as distributed aligned fractures, which resulted from the longterm shear strain in the brittle crust.

The following subsections summarize some of the other geophysical constraints on the plate-boundary deformation especially in the central South Island to test the competing models (Fig. 1.2) that with whether the deformation in this plateboundary zone either localized at the plate-boundary fault (i.e Alpine Fault), extending into deep lithosphere, or distributed as a large-scale shear zone at depths.

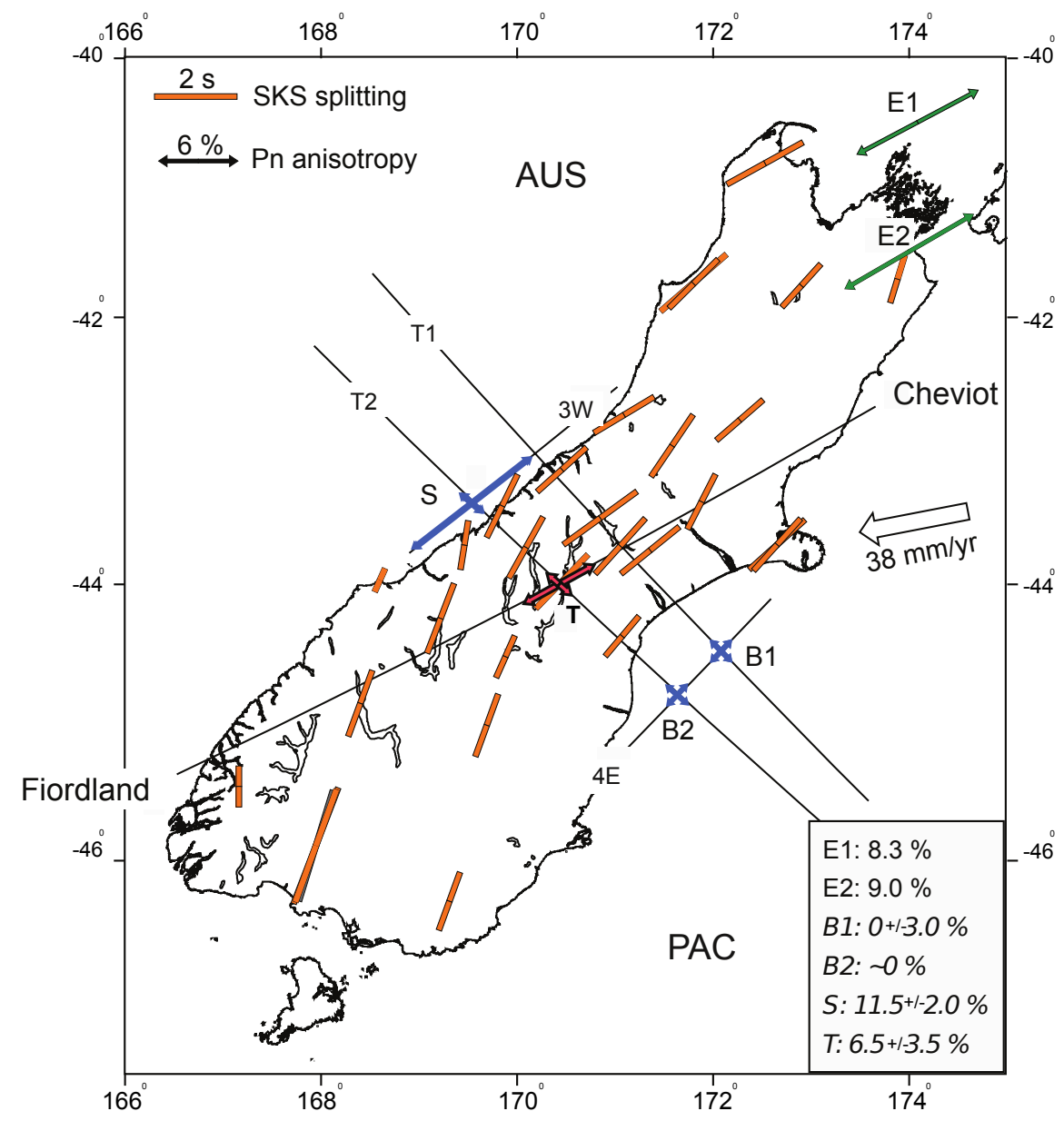

Figure 1.5 Summary of the previous seismic anisotropy investigations in South Island (after Bourguignon et al. [2007b]). Red $(\mathrm{T})$, blue $\left(\mathrm{B}_{1}, \mathrm{~B}_{2}\right.$ and $\left.\mathrm{S}\right)$ and green $\left(\mathrm{E}_{1}\right.$ and $\mathrm{E}_{1}$ ) bars indicate the $P_{n}$ anisotropy measurements. SKS measurements are denoted by the orange bars. $T_{1}, T_{2}, 3 \mathrm{~W}$ and $4 \mathrm{E}$ show the SIGHT transects across the South Island. 


\section{Constraints from $P_{n}$ anisotropy studies}

$P_{n}$ phases that are refracted in the upper-mantle have been used to study crustal and upper-mantle anisotropy structure. Two parallel studies of Pn anisotropy across the South Island were carried out as a part of the South Island Geophysical Transect (SIGHT) experiment [Stern et al., 2007]. $P_{n}$ anisotropy investigated at the intersection between transect one $\left(\mathrm{T}_{1}\right)$ and line $3 \mathrm{~W}$ west $(\sim 30 \mathrm{~km})$ of the Alpine fault ranges between 11 - $13 \%$ (Scherwath et al. [2002]; Fig. 1.5).

Assuming that faster Pn orientation is caused by lattice preferred orientation and reflects the same anisotropy detected by SKS splitting, the angle between maximum extension with respect to the shear plane $(\Phi)$ can be related to width of shear deformation (W) and the amount of displacement due to shear (d) via:

$$
\tan (2 \Phi)=2 W / d \quad[\text { Molnar et al., 1999] }
$$

(also see section 3.4). Pn velocities estimated on transect $2\left(\mathrm{~T}_{2}\right)$ and $\mathrm{T}_{1}$ across the central South Island by Scherwath et al. [2002] and Van Avendonk et al. [2000] respectively found low $\mathrm{Pn}$ velocities compared to typical mantle velocities of $8 \mathrm{~km} / \mathrm{s}$ within a zone distributed over $100 \mathrm{~km}$ west of the Alpine fault, suggesting shearstrain is distributed in the west offshore, producing mantle anisotropy. Assuming the ratio of $\mathrm{P}$-wave to $\mathrm{S}$-wave anisotropy is equal to $\sim 1.4$ (from mantle xenoliths, Ismail and Mainprice [1998]), the percent S-wave anisotropy associated with this distributed shear zone ranges from $\sim 7 \pm 3 \%$ to $9.5 \pm 5 \%$. If 1.8 s shear-wave splitting is assumed [Klosko et al., 1999], then the thickness (see equation 3.17, Vinnik et al. [1989a]) of this anisotropic layer may range either from 85 to $212 \mathrm{~km}$ or from 58 to $188 \mathrm{~km}$. This implies that the majority of the anisotropy could plausibly reside in the mantle lithosphere. Usually dislocation creep governs development of lattice preferred orientation in the mantle material. However dynamic recrystallization can be activated under favorable conditions (see section 2.5.1), and fabrics produced via 
dynamic recrystallization is highly anisotropic (>10\%). Resulting fast alignment is more consistent with the shear plane compared to anisotropy produced via dislocation creep [see Zhang and Karato, 1995, Fig. 4]. Thus, inferred higher Pn anisotropy (11 -13\%) and higher shear strains (2.5 - 4.6) in the west coast suggest that the anisotropy in the mantle lithosphere in the central plate-boundary zone may have been influenced by temperature-dependent dynamic recrystallization.

Pn anisotropy estimation at the intersections of $4 E$ with $\mathrm{T}_{1}$ and $\mathrm{T}_{2}$ (Baldock and Stern [2005]; Fig. 1.5) suggested apparent changes in Pn velocities. On $T_{2}$ velocities change from $\sim 7.95 \pm 0.05 \mathrm{~km} / \mathrm{s}$ to typical mantle Pn velocities of $8.45 \pm 0.05 \mathrm{~km} / \mathrm{s}$ about $235 \mathrm{~km}$ east of the plate-boundary. This change has been interpreted as the limit of the plate-boundary induced lithospheric shear. Notably, the intersection of $4 E$ with $\mathrm{T}_{1}$ and $\mathrm{T}_{2}$ yield null anisotropy and the most likely explanation for such a change is the absence of strain-induced anisotropy in the mantle lithosphere in the east. However, null anisotropy is also possible if the upper-mantle is laterally heterogeneous or if the source of anisotropy is deeper than the $P_{n}$ paths. The width of the shear deformation in the central South Island (region north of $\mathrm{T}_{2}$ ) is, therefore, most likely to be within $\sim 200 \mathrm{~km}$ east of the plate-boundary.

Both Scherwath et al. [2002] and Baldock and Stern [2005] concluded that some degree of dynamic recrystallization in the mantle lithosphere of the central South Island occurs because of the narrower deformation zone $(<200 \mathrm{~km})$ in the central South Island, higher Pn anisotropy $30 \mathrm{~km}$ west offshore [Scherwath et al., 2002] and changes in SKS fast orientations from the Alpine fault subparallel ( $30^{\circ}$ counter clockwise from the Alpine fault) to more fault-parallel orientation in the central South Island. Bourguignon et al. [2007b] found maximum 7-13\% Pn anisotropy 80 $\mathrm{km}$ east of the Alpine fault on the Fiordland-Cheviot profile (Fig. 1.5) compared to higher (11-13\%) Pn and null measurement $30 \mathrm{~km}$ west of the Alpine fault [Scherwath et al., 2002] and $\sim 235 \mathrm{~km}$ east of the fault respectively [Baldock and Stern, 2005]. As constrained by Bourguignon et al. [2007b], if anisotropy is homogeneous and is 
7-13\% $P_{n}$ anisotropy, a $\sim 100 \mathrm{~km}$ thick anisotropic layer is needed to explain the SKS splitting in South Island.

\section{Petrophysical constraints}

Because of the high seismic anisotropy inferred from in-situ studies, several laboratory experiments were carried out to study petrophysics of the crust and mantle rocks in South Island. Compressional and shear-wave anisotropy measurements in the Alpine Fault Zone, the Haast schist terrane, and the Torlesse greywacke terrane (Figures 1.4a) determined an increase in anisotropy towards the Alpine fault, implying the rocks with stronger metamorphic foliations could produce a significant anisotropy at crustal depths [Okaya et al., 1995]. This study also noticed that anisotropy in the Alpine fault mylonites and adjacent Haast schist can be as high as $17 \%$ (compressional wave) at mid-crustal pressures and concluded that structural orientation (e.g. changes in orientation of the foliations from vertical to sub-horizontal from the Alpine Fault towards Canterbury plains) with respect to ray propagation direction may have a considerable effect on the amount of anisotropy observed.

A more focused petrophysical analysis of the Haast schist was done by Godfrey et al. [2000], comparing the Hasst schist measurements with other global schist measurements. The study suggested that significant crustal anisotropy can be contributing to SKS splitting, if near-vertical ray paths of SKS phases propagate through the anisotropic material with a sub-horizontal/horizontal symmetry axis and sample the whole thickness. This study clearly demonstrated how split delays can vary as a function of angle $(\psi)$ between ray path (in this case vertical ray paths) and the axis of symmetry in $5 \mathrm{~km}, 10 \mathrm{~km}$ and $20 \mathrm{~km}$ anisotropic layer thicknesses (Fig. 1.6).

Curve A-1 in figure 1.6 represents the predicted delay time variation in the Haast 


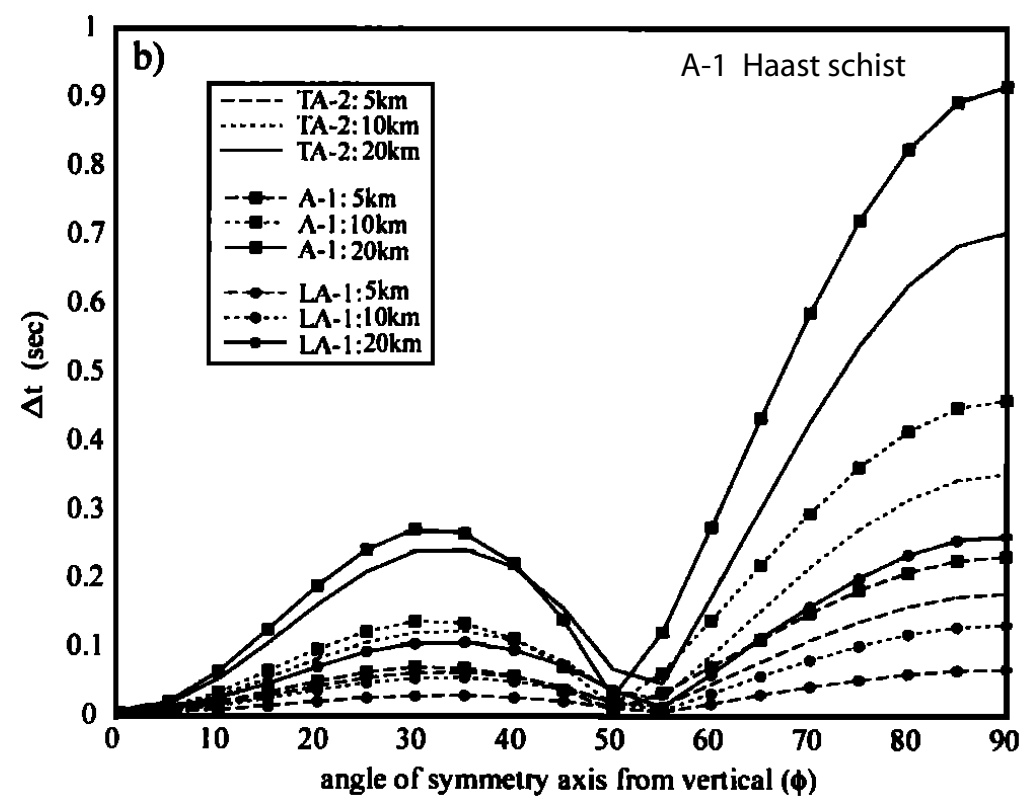

Figure 1.6 Expected delay times from the split SKS waves that travel vertically through schist samples with varying orientations of symmetry axis from the vertical [Godfrey et al., 2000]). The lines with square symbols (A-1) show the estimated delay times in the Haast schist in South Island. The other two lines represent the expected delay times in similar schist terranes in Alaska (TA-Coldfoot Schist) and California (LA-Pelona Schist). Three measurements at each site indicate the estimations at depths $5 \mathrm{~km}, 10 \mathrm{~km}$ and $20 \mathrm{~km}$. Note that vertical ray paths (Hasst schist: A-1) yield the highest delay times when the axis of symmetry is subhorizontal/horizontal (i.e. $90^{\circ}$ from the vertical).

schist. The rays traveling vertically through $20 \mathrm{~km}$ anisotropic layer thickness can produce $\sim 0.5-0.8 \mathrm{~s}$ splitting delay time if the symmetry axis is horizontal to subhorizontal $\left(\psi>70^{\circ}\right.$; i.e. sub-/verticle foliation), whereas rays can yield little $(<\sim 0.2$ s) or no splitting if the symmetry axis is sub-vertical to vertical [Godfrey et al., 2000]. These observations emphasize the importance of considering ray propagation direction with respect to the symmetry axis when evaluating splitting measurements, especially in the case of local and regional shear-wave splitting that involve wide angle ray paths.

S-wave anisotropy determined from the petrophysical analysis of mantle xenoliths in South Island is about $5 \%$ for waves traveling in the foliation plane perpendicular to the flow direction [Duclos et al., 2005]. Lack of splitting is also possible if the S-wave propagation is at $45^{\circ}$ to the foliation plane [Duclos et al., 2005]. The inferred layer thickness of anisotropy is about $150 \mathrm{~km}$ if we assume $1.5 \mathrm{~s}$ average S-wave splitting for 
South Island [Duclos et al., 2005]. Petrologial information acquired from peridotites indicated high-temperature deformation of olivine with pronounced foliation and lineation. Lineations are aligned with the [100] axis and foliations are normal to [010] axis of the olivine crytals, suggesting the dominant slip system is (010)[100] at high temperatures. However, at high temperatures (100)[001] slip systems have also been observed from orthopyroxene in some samples. Duclos et al. [2005] noticed stronger lithospheric anisotropy in South Island $(\sim 5 \%$ S-wave anisotropy with $\sim 1.5$ s splitting) compared to $\sim 3.5 \%$ S-wave anisotropy (0.8 s splitting) in North Island. A possible explanation given for this discrepancy is that intense deformation and vertical foliations around the Alpine fault result from dextral-shear between the AU and PAC lithospheric blocks.

Figure 1.7(a,b,c) shows the dynamic model proposed for the central South Island that Duclos et al. [2005] used to explain the petrophysical measurements of mantle Xneoliths, Pn anisotropy and SKS splitting observations. The model consists of a narrow zone of shear to Moho depths that explains the dextral shearing of the Alpine fault, but a broad zone (as wide as the entire South Island) of shear deformation in the deep mantle-lithosphere as in the case of 'wrench-fault type' deformation [Vauchez and Tommasi, 2003]. Present day structure consists of an asymmetric anisotropic body as a result of the change in fault movement (pure strike-slip to oblique strike-slip) and collision in the central part. During strike-slip motion of the Alpine fault, this body was symmetric (1.7-a). But now it has a shallower upperlimit on the Australian side, and deeper upper-surface (deeper than Moho depth) and more distributed configuration on the Pacific side (1.7-c). This present-day model explains the higher $P_{n}$ anisotropy on Australian side [Scherwath et al., 2002], null Pn anisotropy [Baldock and Stern, 2005] about $235 \mathrm{~km}$ east from the plateboundary, and the distributed zone of plate-boundary parallel shear as determined from SKS splitting [Klosko et al., 1999]. The model further suggests that the plateboundary (the Alpine fault) parallel shear is mostly in the upper-mantle lithosphere, 
but can extend into the asthenosphere.
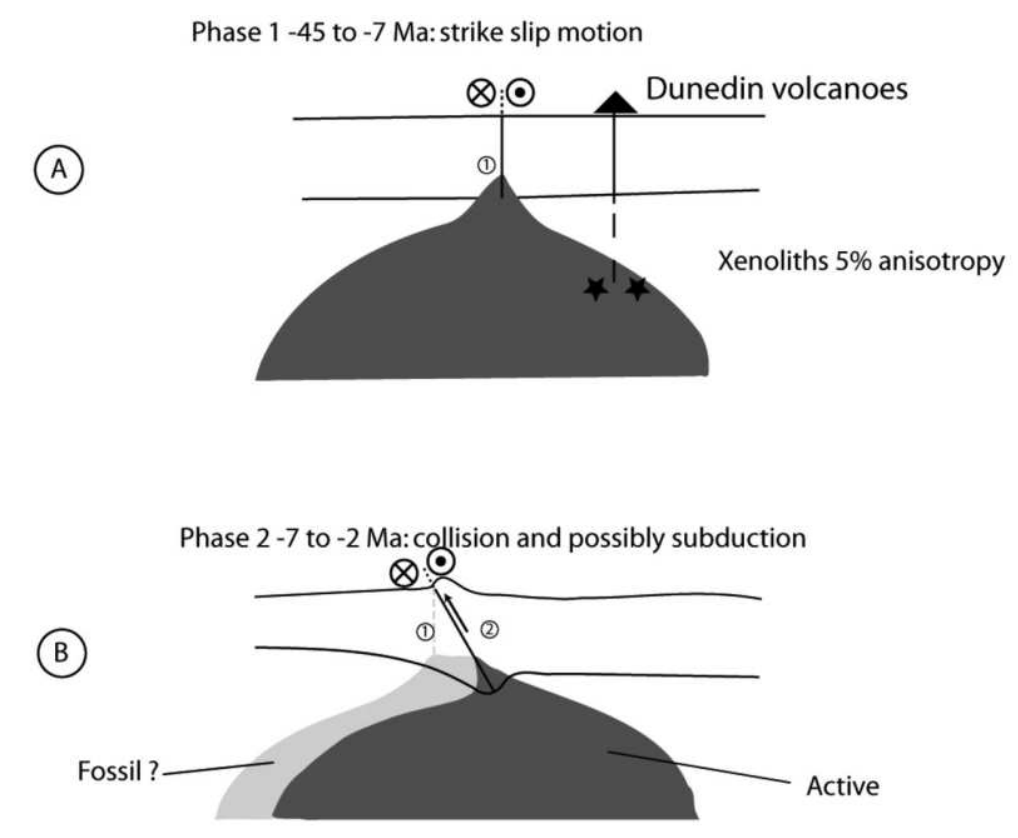

Phase 3:-2 to present : collision and lower crust subduction

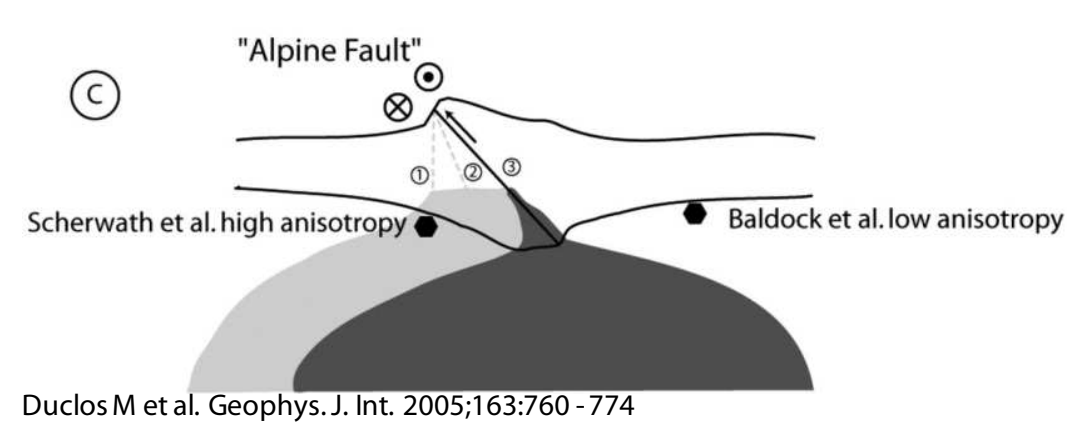

Figure 1.7 Dynamic model proposed by Duclos et al. [2005] to explain the seismic anisotropy measurements in central South Island. (A) Symmetric anisotropic body due to wrench-fault type deformation when the fault was pure strike-slip (before $\sim 7 \mathrm{Myr}$ ). (B) Accumulation of asymmetric nature of the anisotropic body as a result of the oblique collision and subduction processes. (C) Present-day asymmetric and distributed anisotropic body. Note that the upper-surface of this body is shallower on the Australian side than in the Pacific plate. 1, 2, and 3 indicate the different stages of the Alpine fault evolution. 


\section{Constraints from crustal finite strain estimations and geodetic data}

How the shallow-crust and surface deform in response to plate-boundary deformation in the mantle-lithosphere has been investigated quantitatively by geodetic studies (e.g. velocity field determination from GPS) and structural analysis (e.g. finite strain estimation from geological markers). Unlike the GPS-based geodetic studies that consider the present-day deformation patterns on the Earth's surface, bending related geological markers may have recorded multiple deformation histories and therefore provide more accurate and detailed constraints on the strain history and associated mantle fabrics [Little et al., 2002b]. Bending-related geological markers in four domains that are defined with an increase in distance from the Alpine fault [see D, C, B, and A domains in Little et al., 2002b, Fig. 4] indicated an increase in finite strain towards the fault. According to this study, little or no bending-related finite strain is found in the farthest domain from the Alpine fault (domain A), where there is still a considerable shear-wave splitting, suggesting weak coupling of shallowcrustal and upper-mantle deformation. Maximum horizontal stretch calculated from the domains that are close to the Alpine fault are consistent with the SKS-splitting directions (i.e. $\sim 30^{\circ}$ anticlockwise from the Fault). This suggests that crust and mantle deform coherently towards the fault in this region, where SKS splitting is assumed to follow finite strain due to shear at depth. According to structural geology estimates, a low shear strain value of $\sim 1-2$ [Little et al., 2002b] is too low to promote deformation via dynamic recrystallization and, thus, whether dynamic recrystallization itself explains the high $P_{n}$ anisotropy and the deflection of SKS fast azimuth towards the fault in the central South Island is unresolved.

Strain estimations from geodetic velocities at the surface are assumed to provide inconsistent constraints on the deformation distribution at depths as they reflect the elastic strain accumulation due to continuous slip at deeper part of the fault [Ellis et al., 2006, Moore et al., 2002]. Most of these studies are based on the assumption 

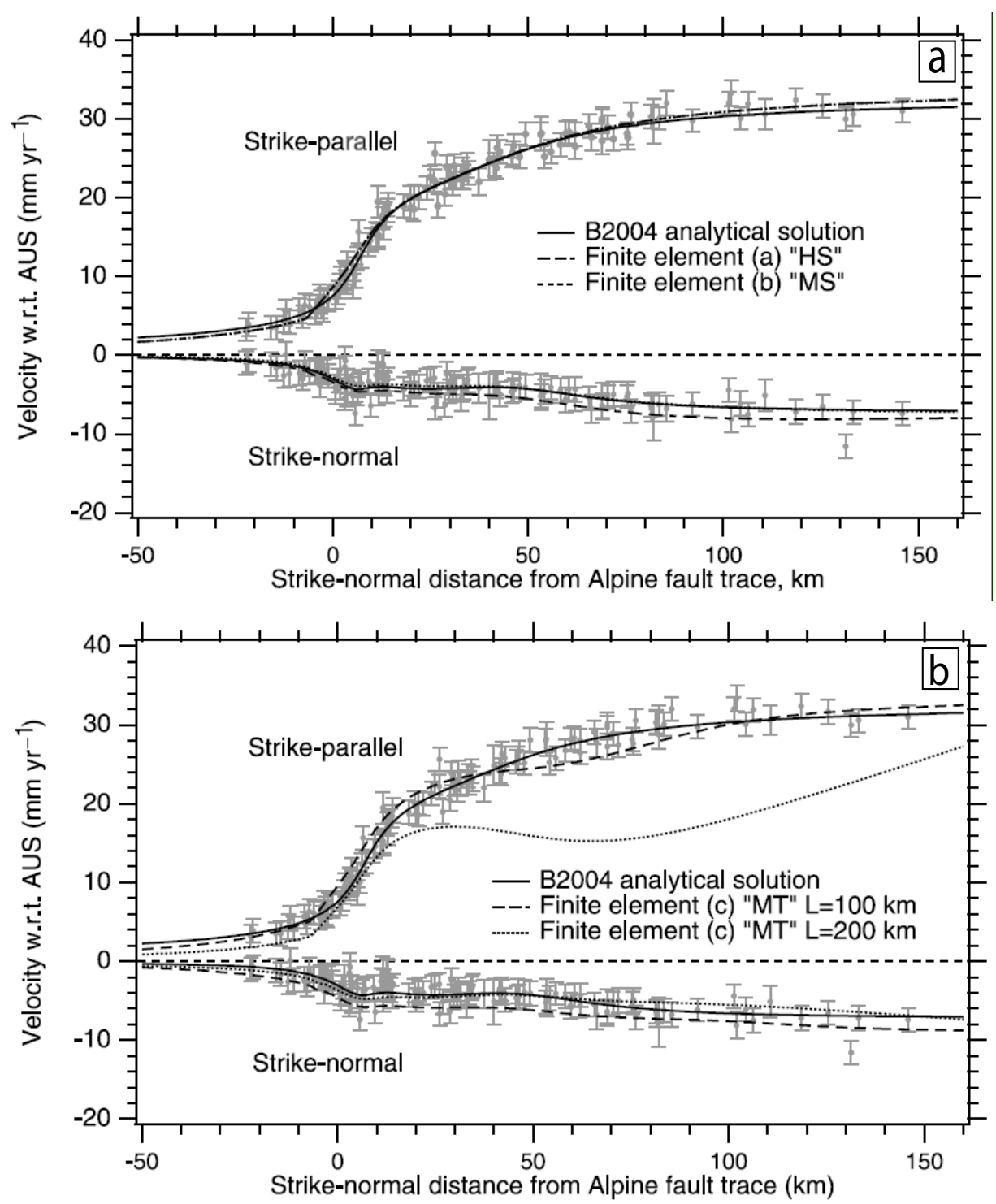

Figure 1.8 (a) Comparison of GPS velocity data (gray symbols) and the predicted horizontal surface velocities using the analytical solution by Beavan et al. [2004] and models with boundary conditions that satisfy the elastic half-space (HS) and mantleshortening (MS). (b) The same comparison using a model with boundary conditions of mantle-thickening (MT) model [Stern et al., 2000]. Velocity predictions from MT model with a width of distributed deformation $(\mathrm{L})=100 \mathrm{~km}$ fit well with the observed data compared to a model with $\mathrm{L}=200 \mathrm{~km}$. Figure after Ellis et al. [2006].

that strain estimations from the present day velocity field is representative of the crustal deformation during My time-scales [Beavan et al., 1999, Houlié and Stern, 2012]. Strain estimations from GPS velocity measurements in the central South Island indicate surface deformation due to relative plate motion of AUS-PAC plates is distributed in a zone of $\sim 80 \mathrm{~km}$ width [Moore et al., 2002]. Campaign GPS surveys in the central South Island also suggested a narrow zone of shear-strain parallel to 
the Alpine fault in the upper-mid crust and a distributed zone ( 100 km east) of fault-perpendicular deformation [Beavan et al., 2004, 1999]. Both Moore et al. [2002] and Beavan et al. [2004] predicted the possibility of distributed fault-parallel shear deformation over a $36 \mathrm{~km}$ wide region at deeper depths ( $\gtrsim 18 \mathrm{~km})$, however the oppositely dipping slip patch, which has been modelled in the deeper section, is attributed to either subducted Pacific mantle-lithosphere or an artifact of the distributed shear [Beavan et al., 2004, 1999]. Horizontal surface velocities predicted from the finite-element modeling agree well with the interseismic velocities estimated from the GPS data regardless of the model assumption (i.e. distributed shear or localized models-Fig. 1.8). The present-day deformation is limited to $100 \mathrm{~km}$ distance from the Alpine fault as implied by both GPS estimations and finite element modeling. Those predictions do not agree with the model with $\sim 200 \mathrm{~km}$ width of distributed lithospheric shear that was constrained by the shear-wave splitting and Pn anisotropy studies [Klosko et al., 1999, Scherwath et al., 2002]. Nevertheless, the recent study by Houlié and Stern [2012] used the direction of extension axis derived from GPS data (i.e. direction perpendicular to the compression direction) as a proxy for the direction of shear to measure the angle between SKS splitting directions and the shear plane $(\Phi)$, and to determine the width of deformation using the relationship given in equation 1.1. This study divided deformation in South Island into two distinct domains according to $\Phi$. In the northern and upper central South Island, $\Phi$ is estimated to be $28^{\circ}$ and interpreted as a product of finite strain during simple shear. Here, shear deformation is estimated to distribute in at least in a $\sim 200 \mathrm{~km}$ wide zone. $\Phi$ is found to be smaller $\left(\Phi=3^{\circ}\right)$ in the southern and lower-central South Island than the northern South Island and is attributed to either much higher strain or the combined effects of pure shear and simple shear deformation [Houlié and Stern, 2012]. 


\subsection{Previous anisotropy studies in the southern Hikurangi subduction zone}

Previous studies by Gledhill and Stuart [1996] and Gledhill and Gubbins [1996] have investigated shallow and deep anisotropy respectively in the eastern part of the southernmost North Island using the data recorded on the Tararua array (Fig. 3.6). Gledhill and Stuart [1996] found little crustal anisotropy ( 0.2 s) in the overriding Australian plate, but a clear increase in delay time with depth ( 1.4\% S-wave velocity anisotropy). The $1.5 \pm 0.4 \mathrm{~s} \delta t$ and $28^{\circ} \pm 4^{\circ} \phi$ of SKS splitting anisotropy calculated from the same station network [Gledhill and Gubbins, 1996] suggested trench parallel flow governs the anisotropy in this region, and backazimuthal dependence suggested a dipping axis of symmetry or multilayer anisotropy. Follow-up studies by Marson-Pidgeon and Savage [1997], MarsonPidgeon and Savage [2004a], Marson-Pidgeon et al. [1999], and Marson-Pidgeon and Savage [2004b] provide an expanded set of splitting measurements from various S-phases (SKS, ScS and teleseismic S) recorded on the SNZO station in Wellington, the POMSII-array that was distributed in the lower-central North Island and the Tararua array (Fig 6.2). The POMSII-array only had three stations (LBLU, LKIR and LMAU) in our region of interest. Splitting anisotropy studies at the SNZO found frequency dependent splitting from SKS, teleseismic S and ScS. Small delays observed from ScS phases from both the SNZO [Marson-Pidgeon and Savage, 1997] and the Tararua array [Marson-Pidgeon et al., 1999] were attributed to small-scale heterogeneities. Consistent orientation of $\phi$ (NE-SW) through out the eastern part of the lower North Island suggest that a similar process controls the anisotropy in the mantle wedge and sub-slab region. It is possibly caused by trench-parallel flow.

Comparing S-wave splitting with the maximum P-velocity directions of NE-SW $\left(\sim 30^{\circ}\right)$ in the southwest Pacific [Galea, 1993, Shearer and Orcutt, 1985], MarsonPidgeon et al. [1999] argued for the possibility of observed splitting to be occurring 
within the subducting oceanic lithosphere as a result of the fossil anisotropy at the ancient spreading margins in the Pacific ocean. However, modeling of splitting parameters with respect to anisotropic thicknesses in the slab (or the length of the ray path within the slab) in different regions found that the model predictions do not match the observed splitting, suggesting anisotropy does not entirely reside in the slab, but may contribute significant amounts to the total anisotropy [Faccenda et al., 2008]. Modeling of P-wave dispersion in Wellington region [Gubbins and Snieder, 1991] suggested a thin $(6-15 \mathrm{~km})$ high-velocity lid on top of the subducting lithosphere that possibly resulted from the ecologitization of the subducting gabbroic crust. Deformed egologite rocks are considered to be strongly anisotropic [Zhang et al., 2008]. According to Gledhill and Gubbins [1996], stress-induced fluid filled cracks may also play a dominant role in the Hikurangi subducting slab. Matcham et al. [2000] found $4.4 \pm 0.99 \%$ velocity anisotropy with $\phi$ of $29^{\circ}$ from the subducting slab earthquakes between $20-70 \mathrm{~km}$ depth range in the Wellington region, supporting the idea of crack-induced anisotropy in the slab or implying high anisotropy in the slab. This observation also supports the previous findings of increase in $\delta t$ with depth in the same region by Gledhill and Stuart [1996] and Brisbourne et al. [1999].

In general, the splitting observations in this region are assumed to agree with the trench parallel flow model (see section 2.7.2). Consistent NE-SW $\phi$ from both shallow and deep anisotropy investigations parallel to structural and geological trends and strike of the subduction zone further imply that this region most likely follows the vertical coherent deformation model [Silver, 1996]. Nevertheless, the observed small-scale variations in $\delta t$ and $\phi[$ Marson-Pidgeon et al., 1999, MarsonPidgeon and Savage, 2004a] with azimuth have been attributed to a structure with more than a single layer of anisotropy or plunging symmetry axis [Gledhill and Gubbins, 1996]. Modeling of seismic anisotropy measurements from shear wave splitting (both local and SKS) and anomalous surface wave polarizations 
(fundamental modes of Love waves) observed at the Tararua array, POMS II array and at SNZO in the south of the North Island found a best-fit model with plunging hexagonal symmetry axis $\left(\sim 30^{\circ}\right.$ SE to fit surface wave measurement and $\sim 15^{\circ}$ SE to fit local S-wave splitting measurements) in the overriding Australian plate [Brisbourne et al., 1999]. The discrepancy between surface wave anisotropy and S-wave splitting has been explained in terms of frequency dependent splitting that was determined by Marson-Pidgeon and Savage [1997] at SNZO. Slab and sub-slab anisotropy in the proposed model are assumed to have horizontal symmetry axes to explain the increase in $\delta t$ with depth. A receiver function study by Savage et al. [2007], however, used an upper anisotropic layer with horizontal symmetry axis and a metasediment layer with vertical symmetry axis in the lithospheric mantle-wedge to explain the delay in Ps arrival with a polarity change.

In contrast to consistent splitting parameters observed from previous SKS splitting investigations at Tararua array, Marson-Pidgeon and Savage [2004b] found noticeable lateral variations in the splitting parameters from $\mathrm{ScS}$ and teleseismic $\mathrm{S}$ waves along the profile that is aligned in NE-SW arm of the Tararua array. This study also noticed variable splitting parameters from different events recorded at the same station. These variations have been integrated with the backazimuthal variations in $\delta t$ to show that lateral variations in anisotropic structure exist across the array. They suggest a distinct anisotropic boundary that runs in a $\sim$ NE-SW direction from $\sim 40$ $\mathrm{km}$ east of the Tararua array towards Kapiti Island in the western offshore region. The study also pointed out that it is important to investigate seismic anisotropy in the eastern side of the Tararua array to provide more constraints on the spatial extent of this anisotropic body. 


\section{Chapter 2}

\section{Seismic anisotropy and its relationship to tectonic deformation and dynamics}

\subsection{Background}

Seismic anisotropy can be defined as directionally dependent elastic response of seismic waves to the anisotropic nature of the Earth's interior. The properties of seismic waves are primarily controlled by the elastic properties of the Earth's materials. Hess [1964] first used azimuthal variations in $P_{n}$ velocities (also known as propagation anisotropy) to detect seismic anisotropy in the ocean lithosphere. Afterwards, several other methods were introduced to investigate anisotropy using shear-waves and surface waves. These approaches are sensitive to different vertical and lateral scales of anisotropy. Among these, shear-wave splitting is a well-established and commonly used technique in exploring seismic anisotropy. Compared to other methods (e.g. P-wave velocity anisotropy, surface-wave polarization anisotropy), this method is less influenced by lateral heterogeneities [Long and Silver, 2009].

\subsection{Concepts of wave propagation in anisotropic media}

This section provides some of the important aspects of elastic wave propagation in homogeneous anisotropic media and explains the shear-wave splitting phenomenon. In an anisotropic medium, behavior and properties of the seismic waves strongly depend on the direction of propagation and the elastic properties of the material [Babuska and Cara, 1991]. Seismic waves attain different speeds depending on the 
propagation direction of the waves in such a medium. To fully understand the seismic wave propagation and polarization in anisotropic media, it is important to understand the elastic properties of the media. The annotations and formulas discussed in the subsections of section 2.2 are taken from Babuska and Cara [1991] and Cerveny [2001].

\subsubsection{Elastic tensors}

When materials are subjected to stress, they are strained (or deformed). For small elastic deformation, the generalized Hooke's law (equation 2.1) gives the linear relationship between stress and strain in an anisotropic medium:

$$
\boldsymbol{\sigma}_{i j}=c_{i j k l} \varepsilon_{k l},
$$

where $\boldsymbol{\sigma}_{i j}$ and $\varepsilon_{k l}$ are Cartesian components of stress and strain tensors. $c_{i j k l}$ is the component of the elasticity tensor, which is also known as a stiffness tensor. The Einstein summation convention is used, in which repeated indices represent summation (e.g. $\boldsymbol{\sigma}_{i j}=\sum_{k, l=1}^{3} c_{i j k l} \boldsymbol{\varepsilon}_{k l}$ ).

\section{Stress, strain and stiffness tensors}

Both stress and strain tensors are symmetric (i.e. $\boldsymbol{\sigma}_{i j}=\boldsymbol{\sigma}_{j i}$ and $\boldsymbol{\varepsilon}_{k l}=\boldsymbol{\varepsilon}_{l k}$ ). For small strains, a strain tensor can be expressed using a displacement vector $\left(\mathbf{u}_{r}\right)$ which is a function of the position of the medium (equation 2.2):

$$
\varepsilon_{k l}=\frac{1}{2}\left(\partial_{l} \mathbf{u}_{k}+\partial_{k} \mathbf{u}_{l}\right),
$$

where $\partial_{l} \mathbf{u}_{k}$ and $\partial_{k} \mathbf{u}_{l}$ are spatial derivatives with respect to $x_{l}$ and $x_{k}$ coordinates, respectively. 
A stiffness tensor $\left(c_{i j k l}\right)$ is a $4^{\text {th }}$ order tensor with $81\left(3^{4}\right)$ elastic coefficients. However, it can be denoted using 36 coefficients (see $c_{i j k l}$ matrix) due to the fact that stress and strain tensors are symmetric (i.e. $\boldsymbol{\sigma}_{i j}=\boldsymbol{\sigma}_{j i}$ and $\varepsilon_{k l}=\varepsilon_{l k}$ ).

$$
\begin{gathered}
c_{i j k l}=c_{j i k l}, \\
c_{i j k l}=c_{j i l k}, \\
\left(\begin{array}{ccccccc}
c_{1111} & c_{1122} & c_{1133} & c_{1123} & c_{1113} & c_{1112} \\
c_{2211} & c_{2222} & c_{2233} & c_{2223} & c_{2213} & c_{2212} \\
c_{3311} & c_{3322} & c_{3333} & c_{3323} & c_{3313} & c_{3312} \\
c_{2311} & c_{2322} & c_{2333} & c_{2323} & c_{2313} & c_{2312} \\
c_{1311} & c_{1322} & c_{1333} & c_{1323} & c_{1313} & c_{1312} \\
c_{1211} & c_{1222} & c_{1233} & c_{1223} & c_{1213} & c_{1212}
\end{array}\right)
\end{gathered}
$$

Stress $\left(\boldsymbol{\sigma}_{i j}\right)$ can be expressed by means of a derivative of strain energy density (i.e. work done per unit volume to deform a material from a stress free state to stressed state; $U$ ) with respect to strain, and can be related to $c_{i j k l}$ using the following relationships:

$$
\begin{gathered}
\boldsymbol{\sigma}_{i j}=\partial U / \partial \boldsymbol{\varepsilon}_{i j} \\
c_{i j k l}=\partial \boldsymbol{\sigma}_{i j} / \partial \boldsymbol{\varepsilon}_{k l}
\end{gathered}
$$

$c_{i j k l}$, thus, can be expressed via partial derivatives of $U$ and strain:

$$
\begin{gathered}
c_{i j k l}=\partial^{2} U / \partial \varepsilon_{k l} \partial \varepsilon_{i j} \\
\partial^{2} U / \partial \varepsilon_{k l} \partial \varepsilon_{i j}=\partial^{2} U / \partial \varepsilon_{i j} \partial \varepsilon_{k l} \\
c_{i j k l}=c_{k l i j}
\end{gathered}
$$


Considering the above symmetry condition $\left(c_{i j k l}=c_{k l i j}\right)$, components of the stiffness tensor will be reduced further into 21 elastic coefficients. Then the resultant matrix $\left(c_{m n}\right)$ with elastic coefficients can be written as:

$$
c_{m n}=\left(\begin{array}{cccccc}
c_{1111} & c_{1122} & c_{1133} & c_{1123} & c_{1113} & c_{1112} \\
& c_{2222} & c_{2233} & c_{2223} & c_{2213} & c_{2212} \\
& & c_{3333} & c_{3323} & c_{3313} & c_{3312} \\
& & & c_{2323} & c_{2313} & c_{2312} \\
& & & & c_{1313} & c_{1312} \\
& & & & & c_{1212}
\end{array}\right)
$$

These 21 elastic coefficients in the stiffness tensor describe the elastic behavior of the most general anisotropic solid that has the lowest degree of symmetry (i.e. tryclinic).

\subsubsection{Elastic waves in homogeneous anisotropic media}

Spatial variations in stress $\left(\partial_{j} \boldsymbol{\sigma}_{i j}\right)$ and time variations in displacement $(\ddot{\boldsymbol{u}}=$ $\left.\partial^{2} \boldsymbol{u}_{i} / \partial t^{2}\right)$ are related to each other via an elastodynamic equation [Cerveny, 2001]:

$$
\partial_{j}\left(\boldsymbol{\sigma}_{i j}\right)+\boldsymbol{f}_{i}=\rho \ddot{\boldsymbol{u}}_{i}
$$

where $\boldsymbol{f}$ is a vector that defines the force per unit volume. If no external forces are acting on the medium, $\boldsymbol{f}_{i}=0$. Combining equation 2.3 with equations 2.1 and 2.2, the elastodynamic equation for homogeneous anisotropic media can be expressed as:

$$
c_{i j k l} \partial_{j} \partial_{k}\left(\boldsymbol{u}_{l}\right)=\rho \ddot{\boldsymbol{u}}_{i},
$$

Next, we will consider the plane wave, which is the most fundamental solution of the elastodynamic equation, to explain the properties of elastic waves in the 
homogeneous anisotropic media. The plane wave solution in a homogeneous anisotropic medium can be expressed as the functions of space $(\overrightarrow{\boldsymbol{x}})$ and time (t):

$$
\boldsymbol{u}(\overrightarrow{\boldsymbol{x}}, t)=\mathbf{A} f(t-\overrightarrow{\boldsymbol{N}} \cdot \overrightarrow{\boldsymbol{x}} / \boldsymbol{\nu})
$$

where $\mathrm{A}$ is the amplitude of particle motion, $\vec{N}$ is the unit vector normal to wave propagation direction and $\boldsymbol{\nu}$ is the phase velocity. Partial derivatives of time and space obtained from plane wave solution (equation 2.5) are then combined with equation 2.4 to examine the properties of plane waves in homogeneous, elastic anisotropic media (equation 2.6).

$$
c_{i j k l} \boldsymbol{N}_{j} \boldsymbol{N}_{k} \boldsymbol{a}_{l}=\rho \boldsymbol{\nu}^{2} \boldsymbol{a}_{i}
$$

Defining a density normalized stiffness tensor $\left(c_{i j k l} / \rho\right)$ as $C_{i j k l}$, the Christoffel equation (equation 2.7) with the Christoffel matrix $\Gamma_{i l}\left(\Gamma_{i l}=C_{i j k l} \boldsymbol{N}_{j} \boldsymbol{N}_{k}\right)$ can be obtained from the equation 2.6.

$$
\Gamma_{i l} \boldsymbol{a}_{l}=\boldsymbol{\nu}^{2} \boldsymbol{a}_{i}
$$

Here, $\boldsymbol{\nu}^{2}$ and $\boldsymbol{a}_{i}$ are the eigenvalues and the eigenvectors of $\Gamma_{i l}$. From this equation we can obtain three eigenvalues and three mutually perpendicular eigenvectors that correspond to the three different wave types in a homogeneous anisotropic medium.

\subsubsection{Plane waves in hexagonal anisotropy systems}

Transversely isotropic materials that are characterized by a single plane of isotropy and one axis of rotational symmetry exhibit hexagonal symmetry. Thus, such systems are also known as hexagonal anisotropy systems. The 21 elastic coefficients discussed above in a general anisotropic medium are further reduced depending on 
the symmetry of the system. In an isotropic case, only two elastic coefficients (also known as Lamé elastic moduli) are enough to fully describe the elastic properties of the medium. Hexagonal anisotropy symmetry is the most common system that is used to understand seismic wave propagation in different Earth media. Therefore, we will consider the plane wave propagation in a hexagonal anisotropy system in detail. Horizontal lithologic layering with a vertical symmetry axis (Figure 2.1) and vertically aligned cracks with a horizontal symmetry axis (Figure 2.2a) are two natural examples of hexagonal symmetry. To characterize the elastic properties of such a system, one must have five elastic coefficients (A, F, C, L, and N) [Love, 1944]. The stiffness tensor of the hexagonal anisotropy system can be denoted using the above coefficients as given below:

$$
c_{m n}=\left(\begin{array}{ccccccc}
A & A-2 N & F & & \\
A-2 N & A & F & & & \\
F & F & C & & & \\
& & & & L & & \\
& & & & & L & \\
& & & & & N
\end{array}\right)
$$

where, A, F, C, L, N and $A-2 N$ are also known as Love's coefficients after Love [1944].

Let us characterize the properties of plane waves that propagate in two main directions: (a) Plane wave propagating in the direction of a plane perpendicular to the symmetry axis (blue arrow in figure 2.1) (b) Wave propagating in the direction parallel to the symmetry axis (red arrow in figure 2.1) using the above $c_{m n}$ matrix of the hexagonal system.

\section{a. Wave propagating in the plane perpendicular to the symmetry axis:}

For this type of wave propagation system, the Christoffel matrix $\left(\Gamma_{i l}\right)$ yields: 


$$
\Gamma_{i l}=\frac{1}{\rho}\left(\begin{array}{ccc}
A & 0 & 0 \\
0 & N & 0 \\
0 & 0 & L
\end{array}\right)
$$

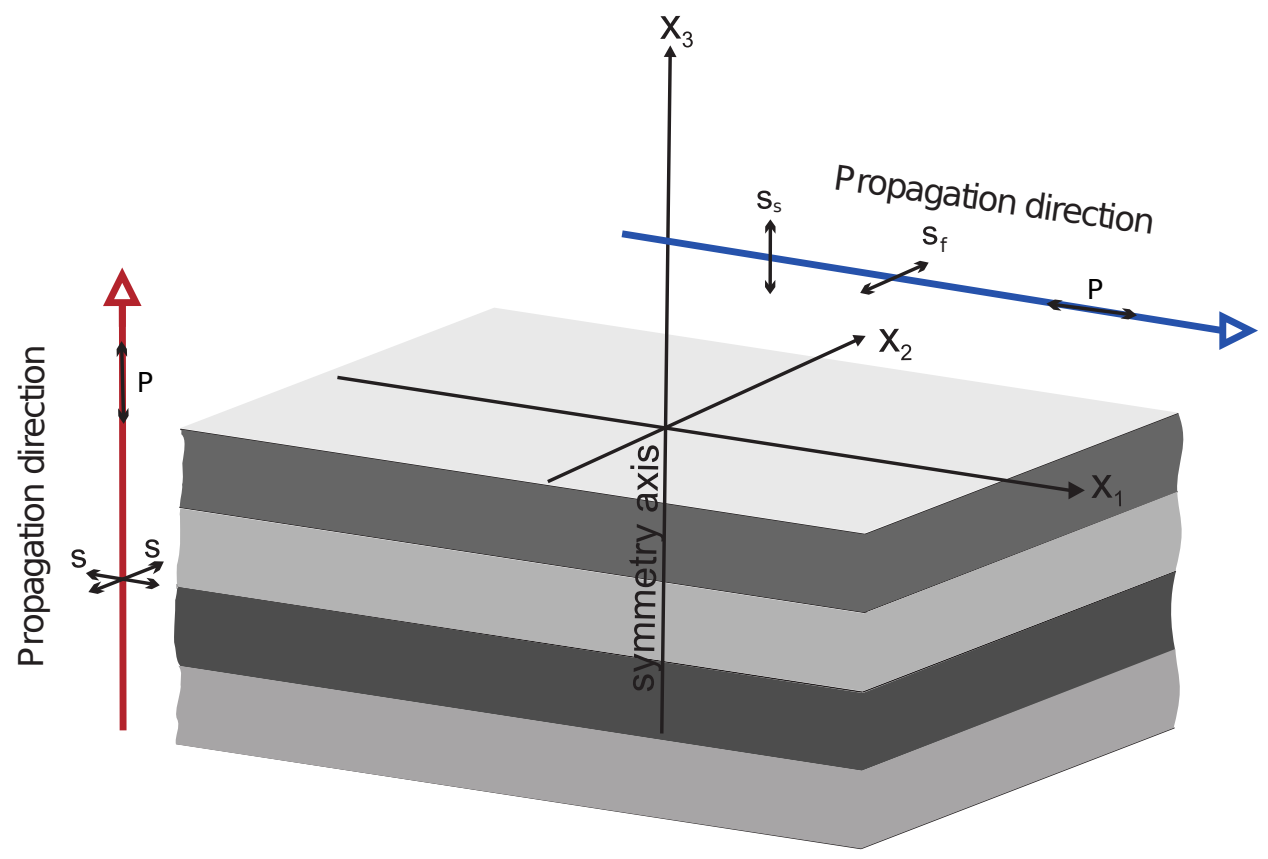

Figure 2.1 Layered anisotropic system with hexagonal symmetry (also equivalent to transverse isotropy with vertical axis of symmetry). $x_{3}$ axis denotes the symmetry axis. Blue and red arrows indicate the $\mathrm{S}$-wave propagation parallel to the plane perpendicular to the symmetry axis and parallel to the symmetry axis, respectively. The presence of quasi $\mathrm{S}$ phases upon propagating through the media is denoted by $S_{f}$ (fast) and $S_{s}$ (slow).

This matrix has three different eigenvalues $\lambda_{1}{ }^{2}, \lambda_{2}{ }^{2}$ and $\lambda_{3}{ }^{2}$ that correspond to the three eigenvectors $a_{1}, a_{2}$ and $a_{3}$, respectively. These eigenvectors indicate the polarization directions of the three different wave types with varying velocities along $x_{1}$ direction:

$$
\left(a_{1}\right)=\mid \begin{aligned}
& 1 \\
& 0 \\
& 0
\end{aligned}
$$$$
\left(a_{2}\right)=\mid \begin{aligned}
& 0 \\
& 1 \\
& 0
\end{aligned}
$$$$
\left(a_{3}\right)=\mid \begin{aligned}
& 0 \\
& 0 \\
& 1
\end{aligned}
$$

The phase velocities of the waves are given by: 


$$
\lambda_{1}=\sqrt{\frac{A}{\rho}} \quad \lambda_{2}=\sqrt{\frac{N}{\rho}} \quad \lambda_{3}=\sqrt{\frac{L}{\rho}}
$$

Here, $\lambda_{1}$ is the velocity of the fastest wave, which is polarized parallel to the propagation direction. Thus, this wave denotes the $\mathrm{P}$ wave. The other two mutually perpendicular phases are polarized perpendicular to the wave propagation direction (S waves) and travel with different velocities $\left(\lambda_{2}\right.$ and $\left.\lambda_{3}\right)$. In the absence of anisotropy (or isotropic case), $\lambda_{2}=\lambda_{3}$ and there will only be a single phase that is polarized in the direction perpendicular to the direction of wave propagation. This phase represents the unsplit shear (S-wave) wave.

Thus, if a wave propagates perpendicular to the symmetry axis there will be two mutually perpendicular quasi $\mathrm{S}$ phases $\left(S_{f}\right.$ and $S_{s}$ in figure 2.1$)$ that are polarized perpendicular to the wave propagation direction. This phenomenon is known as shear-wave splitting.

\section{b. Wave propagation in the plane parallel to the symmetry axis:}

The Christoffel matrix $\left(\Gamma_{i l}\right)$ for the wave propagated in $x_{3}$ direction is given by:

$$
\begin{aligned}
& \Gamma_{i l}=\frac{1}{\rho}\left(\begin{array}{ccc}
L & 0 & 0 \\
0 & L & 0 \\
0 & 0 & C
\end{array}\right)
\end{aligned}
$$

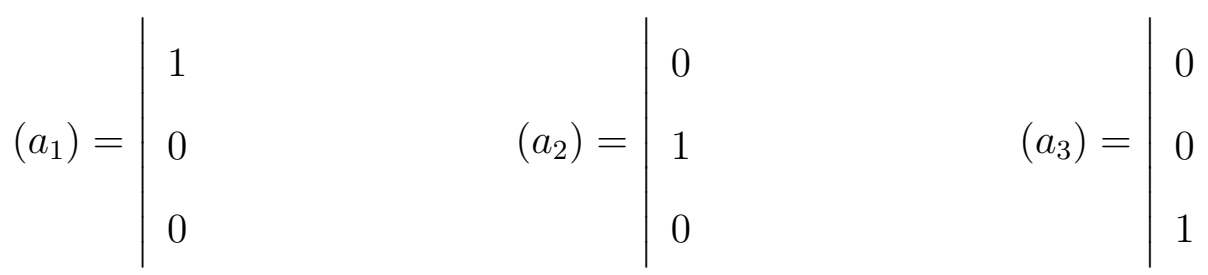

The phase velocities of the waves in each direction are given by: 


$$
\lambda_{1}=\sqrt{\frac{L}{\rho}} \quad \lambda_{2}=\sqrt{\frac{L}{\rho}} \quad \lambda_{3}=\sqrt{\frac{C}{\rho}}
$$

There are two degenerate eigenvalues $\left(\lambda_{1}{ }^{2}\right.$ and ${\lambda_{2}}^{2})$, which represent the velocity of the unsplit S-wave that is polarized perpendicular to the symmetry axis $(S$ in figure 2.1). The other eigenvalue $\left(\lambda_{3}\right)$ is the velocity of the $\mathrm{P}$-wave that is polarized parallel to the propagation direction or the symmetry axis $\left(x_{3}\right)$. Except when a wave's propagation direction is along the symmetry axis, the other propagation directions in the anisotropic medium will display S-waves splitting, but the propagation velocities of split S-waves may vary depending on the angle between symmetry axis and the ray propagation direction (Fig. 2.2 and Fig. 1.6).

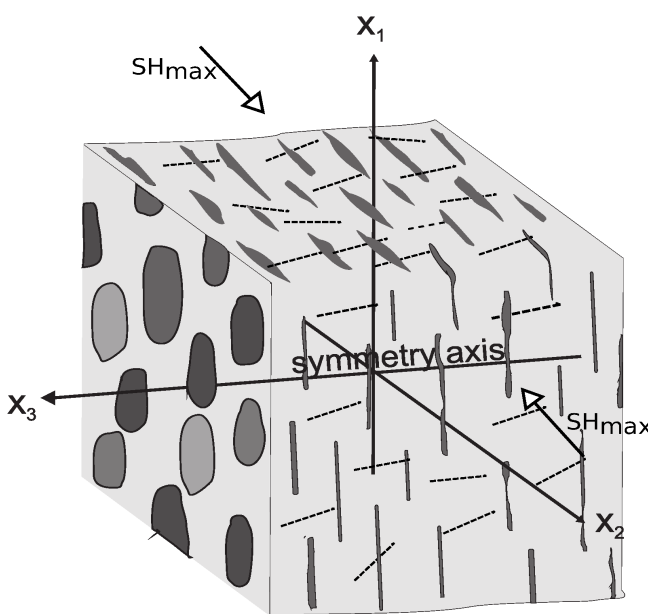

(a)
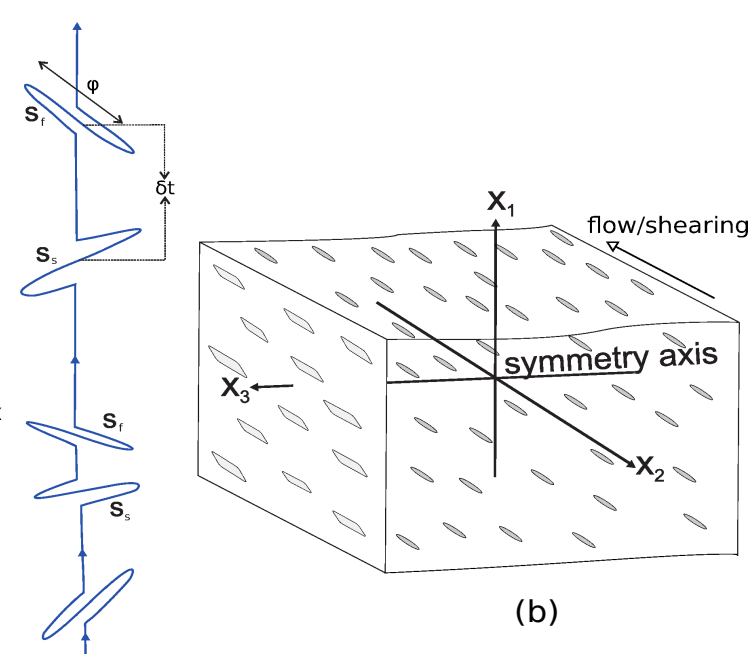

(b)

Figure 2.2 S-wave splitting in different anisotropic media when propagation in the plane perpendicular to the symmetry axis $\left(x_{3}\right)$. Splitting of $\mathrm{S}$-wave is marked in blue. $\delta t$ indicates the time lag between fast $\left(S_{f}\right)$ and slow $\left(S_{s}\right)$ split S-waves, and $\phi$ denotes the polarization direction of the fast split wave. (a) a system with vertically aligned cracks. $S H_{\max }$ denotes the maximum compressive direction. (b) mineral alignment due to flow or shear.

\subsection{Shear-wave splitting and splitting parameters}

The presence of quasi shear-waves is a distinct property of elastic waves traversing an anisotropic medium, satisfying the relationship between the direction of propagation and symmetry of the medium. Most importantly, this property can be used to 
distinguish anisotropy from isotropy and provide detailed information about the anisotropic medium. Because polarization directions of the split waves are controlled in part by the alignment of the anisotropic medium, this is also known as polarization anisotropy.

As highlighted in section 2.2.3, fast $\left(S_{f}\right)$ and slow $\left(S_{s}\right)$ split S-waves are polarized in mutually perpendicular directions depending on the alignment of the anisotropic medium and the wave propagation direction (Figure 2.2). Usually, the polarization direction of the $S_{f}$ wave $(\phi)$ is related to the alignment of the anisotropic medium, and the delay time between $S_{f}$ and $S_{s}$ depends on the extent and/or degree of anisotropic medium and path length of the ray within the medium, but it also depends on the propagation direction [Savage, 1999]. When these two split waves propagate with two different velocities, $\beta_{f}$ (velocity of $S_{f}$ wave) and $\beta_{s}$ (velocity of $S_{s}$ wave), they will accumulate delay time $(\delta t)$ along the travel path. If the length of the travel path is $l$ and the isotropic velocity of the shear-wave is $\beta_{0}$, for small anisotropy the relationship between $\delta t$ and $l$ is given by the following linear equation [Silver and Chan, 1991]:

$$
\delta t=l \delta \hat{\beta} / \beta_{0}
$$

Here, $\delta \hat{\beta}$ is a fraction of velocity anisotropy calculated from the S-wave splitting (i.e. $\delta \hat{\beta}=\left(\delta \beta_{f}-\delta \beta_{s}\right) / \beta_{0} ; \beta_{f}=\beta_{0}+\delta \beta_{f}$ and $\left.\beta_{s}=\beta_{0}-\delta \beta_{s}\right)$. Accordingly, $\delta t^{\dagger}$ is proportional to $l$. Thus, $\delta t$ and $\phi^{\dagger}$ are considered as the main splitting parameters that could be determined from shear-wave splitting to investigate the subsurface anisotropic structure in homogeneous media.

\footnotetext{
${ }^{\dagger}$ hereafter, $\delta t$ denotes delay time/s and $\phi$ denotes fast direction/s.
} 


\subsection{Origin of anisotropy}

Anisotropy occurs at various depths at different length scales depending on the elastic properties of the materials and the processes associated with the formation (e.g. geodynamic and deformational process). Anisotropy has two main forms: 1) extrinsic and 2) intrinsic anisotropy. Extrinsic anisotropy is related to the structural arrangement of the Earth's material, therefore, it is also known as shape preferred orientation (SPO). Intrinsic anisotropy is predominantly caused by the lattice preferred orientation (LPO) of intrinsically anisotropic minerals [Kendall, 2000, Montagner and Guillot, 2002].

SPO fabrics are mainly observed at crustal depths and possess either stress or structural origin. Stress-induced anisotropy is observable under low confining pressure $(<200 \mathrm{Mpa})$ [Kern and Wenk, 1990] in the uppermost crust as a result of the preferential orientation of fluid-filled (usually water or melt) micro-cracks (Figure 2.2a). In addition to stress controlled sources, structural anisotropy is also present at various scales at crustal depths. Macro-scale structures such as faults, bedding planes and foliations are some of the examples for anisotropy caused by structure. Most of these structurally controlled fabrics are also associated with LPO fabrics at mid-lower crustal depths. LPO anisotropy is the dominant source of anisotropy in the upper mantle; it also has a significant effect on the anisotropy in the lower crust [Mainprice and Nicolas, 1989].

\subsubsection{Extrinsic anisotropy}

\section{a) Stress-induced anisotropy}

Stress-induced anisotropy is the most abundant source of anisotropy in the upper crust where there is a low confining pressure or high fluid pressure. Earlier studies on elastic properties of rocks under hydrostatic stress suggest that the elastic properties 
of rocks under low effective stress $(\lesssim 1 \mathrm{~kb})$ are primarily controlled by the properties of the micro-cracks. The effect of non-hydrostatic stress (or differential stress) on velocity anisotropy was first studied by Nur [1971]. This study showed that when rocks are subjected to non-hydrostatic stress, cracks can be closed preferentially, leaving some cracks that remain open in another direction. This mechanism may change the rock from its isotropic state to an anisotropic state as result of the change in elastic properties within a given direction of the medium. In such a crackinduced anisotropic medium shear-wave splitting is possible and depends on the crack alignment and the stress direction (Figure 2.2a). The polarization direction of the fast shear-wave $(\phi)$ in such an anisotropic medium is usually parallel to the alignment (or the strike) of the cracks remaining open and hence parallel or subparallel to the horizontal maximum compressive direction $\left(S H_{\max }\right)[$ Crampin, 1978, Crampin and Lovell, 1991]. Hudson [1980] provides the effective elastic constants of the cracked medium in terms of crack density, suggesting that the shear-wave splitting from cracked media does not depend on the crack dimensions, as the wavelength of the shear-waves are much higher than the crack dimensions. However, the crack geometry, crack density and degree of crack saturation (or pore fluid pressure) have significant effects on the shear-wave splitting and the differential shear-wave anisotropy (SWA) [Crampin, 1978, 1994].

Crack-induced anisotropy can have different forms such as fluid-filled cracks, microcracks and aligned pore spaces. In shallow sedimentary rocks, fluid-filled cracks are the main source of anisotropy [Mueller, 1991] and, in igneous and metamorphic rocks anisotropy is attributed to aligned dry cracks and micro-cracks [Crampin, 1994] (see table 2.1). It is usually assumed that the split shear-waves from steep arrivals (incidence angle $\lesssim 45^{\circ}$ ) are mainly due to vertically aligned cracks at shallow crustal depths [Leary et al., 1990]. This system (i.e. vertical crack planes) acts as a medium with transverse isotropy with horizontal symmetry axis (Figure 2.2a) [Crampin and Lovell, 1991, Crampin, 1994]. Because the alignment of vertical cracks is primarily 
Table 2.1 An average differential SWA measured at different geological terranes (adapted from Crampin [1994]).

\begin{tabular}{ccc}
\hline Rock type & Depth $(\mathrm{km})$ & Percent SWA (\%) \\
\hline Volcanic rocks & 10 & $4.0-5$ \\
Volcanic caldera & 10 & $\sim 6.5$ \\
Granite (geothermal area) & 2 & 8 \\
\hline Metamorphic rocks & 5 & 4 \\
Metamorphic rocks (after shocks area) & 20 & 7 \\
Granulite facies & 1 & 10 \\
\hline Shale and lime stone & $1.1-2$ & 2 \\
Fault zone (sedimentary) & $0.3-1.4$ & $7-8$ \\
Sand stone and clay & 0.04 (very shallow) & $12-14$ \\
\hline
\end{tabular}

controlled by the local stress field, shear-wave splitting in cracked media can be used as a tool to investigate the changes in the local stress field over time. This concept has been successfully tested as a stress monitoring tool at volcanoes [Miller and Savage, 2001, Gerst and Savage, 2004] and in various other tectonic domains [Crampin et al., 2004, Peacock et al., 1988].

\section{b) Structurally-induced anisotropy}

Structural anisotropy is mainly caused by the alignment of foliation planes, bedding planes or macro-scale faults (e.g. San-Andreas fault, Alpine fault). This has been frequently observed at crustal depths. Barruol et al. [1997] suggested that foliations control most of the splitting in the mid-lower crust. They explained this in terms of vertically aligned foliations that are developed in the middle to lower crust where there is a high enough temperature to develop foliations from vertically aligned phyllosilicates (e.g. Mica) in felsic rocks. An important observation of this study is that higher $\delta t$ are recorded from the S-waves that traverse parallel to the foliation plane, whereas no or little splitting occurs if the propagation direction is normal to the foliation planes. Vertical ray paths that travel in regions with steeply dipping foliations (e.g. strike-slip and convergent plate-boundaries) would produce $\delta t$ within the range of 0.3-0.5 s [Barruol et al., 1997]. This suggests that not only the mineral 
composition but also the orientation of foliation plane with respect to ray path of the S-wave, is crucial in producing splitting. Studies also showed that rocks with a single set of foliations produce more pronounced splitting compared to rocks with multiple sets of foliations [Lloyd et al., 2009]. Other than foliated structures, aligned melt-inclusions or melt-bands at the lower-crustal or the upper-mantle depths in the tectonic settings, such as mantle wedge and volcanic terranes, also play a dominant role in producing a structurally-induced anisotropy [Holtzman et al., 2003].

\subsubsection{Intrinsic anisotropy}

Unlike extrinsic anisotropy, intrinsic anisotropy is independent of the wavelength of the S-wave and results either from strain-induced mineral alignment (also known as lattice preferred orientation) or the preferred orientation of individual mineral grains during the rock's genesis [Kendall, 2000].

\section{Lattice preferred orientation (LPO)}

LPO is caused by the preferred orientation of intrinsically anisotropic crystals of rock-forming minerals (Figure 2.2b). Changes in the strain field due to plastic deformation mainly control the preferred orientation of the minerals and the strength of LPO depends on the finite strain. Dislocation creep and diffusion creep mechanisms govern the strain-induced deformation at high temperatures $(\sim 0.5 \mathrm{Tm}$, where Tm is melting temperature) in rocks (section 2.5; Kameyama et al. [1999]). Diffusion creep activates under low stress and/or in material with small grain size. This may lead to the development of randomly oriented fabric with no anisotropy or straining in a preferred direction. On the other hand, dislocation creep plays a dominant role in producing LPO fabrics in the upper-mantle and the lower-crust via strain-induced alignment. This mechanism operates under high stress conditions and/or in media with large grain sizes. However, the resultant anisotropic properties depend on the geometry of deformation and physical/chemical conditions during the 
deformation [Karato et al., 2008].

The majority of LPO anisotropy occurs in the upper-mantle and the lower-crust, where the rocks deform in a ductile manner and have anisotropic mineral aggregates. In addition to strain, Temperature $(\mathrm{T})$, pressure $(\mathrm{P})$ and mineralogy are also important factors that control the strength of the LPO fabric. In general, the strength of the LPO increases with increasing $\mathrm{T}$ and decreases with increasing P. One of the most important factors affecting the LPO strength is the mineralogy of rocks. Advances in mineral physics and petrographic analysis provide detailed information about the anisotropic properties of minerals (single crystals and polycrystals) and the overall anisotropy of rocks. The findings of these studies are also useful in interpreting an in-situ shear-wave splitting anisotropy to understand the LPO contribution to total splitting. Anisotropy of a single crystal is expressed by percentage anisotropy (A) as given below:

$$
\left.A=200\left(\frac{V_{\max }-V_{\min }}{V_{\max }+V_{\min }}\right)\right)
$$

where, $V_{\max }$ and $V_{\min }$ are the maximum and minimum velocities of either the $\mathrm{P}$ $\left(V_{p}\right)$ or $\mathrm{S}\left(V_{s}\right)$ phases. In the upper-mantle, olivine, enstatite (orthopyroxene) and diopside (clinopyroxenes) are considered to be the main intrinsically anisotropic minerals [Mainprice et al., 2000]. Olivine is the volumetrically most important mineral in the upper mantle rocks that could generate strong LPO fabrics. However, when olivine combines with orthopyroxene and clynopyroxene (e.g. in the uppermantle rocks), the resultant velocity anisotropy can vary depending on the mineral composition. Polycrystalline aggregates of olivine in the upper-mantle show the highest $V_{p}$ anisotropy although the single crystal anisotropy of lower-crustal minerals is higher than that of upper-mantle anisotropy [Mainprice et al., 2000]. In the lower-crust, hornblend, plagioclase, and diopside play a dominant role in the development of LPO fabrics (Table 2.2). As suggested by Mainprice and Nicolas 
Table 2.2 Single crystal and polycrystal velocity anisotropy $\left(V_{p}\right)$ of intrinsically anisotropic minerals of the lower-crust and the upper-mantle [Mainprice and Nicolas, 1989, Mainprice et al., 2000].

\begin{tabular}{ccc}
\hline \multicolumn{3}{c}{ Lower crust } \\
\hline Mineral & Single crystal $V_{p}(\%)$ & Polycrystal $V_{p}(\%)$ \\
\hline Qartz & 24.4 & 6 \\
Plagioclase & 27.4 & 13 \\
Diopside & 29 & 5 \\
Hornblend & 23.9 & 12 \\
\hline \multicolumn{3}{c}{ Upper mantle } \\
\hline Olivine & 25 & $\sim 15$ \\
Enstatite & $15-12$ & $\sim 5$ \\
Diopside & 29 & 5 \\
\hline
\end{tabular}

[1989], monomineralic plagioclase that deforms plastically in the lower crust can also act as a strong source of anisotropy.

\subsection{Deformation kinematics and anisotropy}

The elastic anisotropy measured from shear-wave splitting is indirect evidence for deformation of the Earth's interior. As explained in sections 2.4.1 and 2.4.2, rocks acquire anisotropic properties due to the preferential orientation of minerals at deep crustal or mantle depths and micro-cracks at shallow depths. Viscoelastoplastic rheology can be used to fully characterize the lithospheric deformation, assuming that the shallow brittle zone and mid-transition zone has elasto-plastic behavior and the mantle rocks follow the viscous behavior. The resultant total strain rate is expressed as the sum of elastic $\dot{\varepsilon}^{e l}$, plastic $\dot{\varepsilon}^{p l}$ and viscous $\dot{\varepsilon}^{v i s}$ strain rates [Kameyama et al., 1999].

$$
\dot{\varepsilon}=\dot{\varepsilon}^{e l}+\dot{\varepsilon}^{p l}+\dot{\varepsilon}^{v i s}
$$

Here, $\dot{\varepsilon}^{e l}=\frac{1}{\mu} \frac{\partial \sigma}{\partial t}$; where $\mu$ is shear modulus that represents the linear rheology (elastic deformation) in which the rate of deformation (or strain rate) linearly varies with 
the dynamic stress $(\sigma)$. In the elastic (brittle) domain, intercrystalline deformation kinematics (e.g. grain boundary sliding and fracturing) govern strain accumulation. $\dot{\varepsilon}^{p l}$ and $\dot{\varepsilon}^{v i s}$ terms denote the visco-plastic deformation in the transition zone (midlower crust) and the upper-mantle respectively. $\dot{\varepsilon}^{p l}$ indicates a low-temperature stress-controlled plastic deformation process that occurs via the Peierls mechanism as given in the equation 2.11 [Kameyama et al., 1999]. Peierls mechanism is attributed to a low-temperature $\left(\leq 0.3 T_{m}\right)$ dislocation glide (section 2.5.1) within the crystal lattice, which causes permanent strain in the lattice. Compared to power-law creep (below), Peierls mechanism is less dependent on the temperature, but greatly controlled by the differential stress $(\sim 500 \mathrm{MPa})$.

$$
\dot{\varepsilon}^{p l}=A \exp \left[-H / R T\left(1-\frac{\sigma}{\sigma_{p}}\right)^{q}\right]
$$

where $H$ is activation enthalpy of the glide motion, $\mathbf{A}$ and $\mathbf{q}$ are material constants, $R$ denotes the gas constant, and $T$ is temperature. $\sigma_{p}$ represents the Peierls stress (i.e. the minimum differential stress that is needed to activate dislocation glide at very low temperatures). This low-temperature plastic deformation activates at large differential stresses once the stress reaches the yield stress level.

$\dot{\varepsilon}^{v i s}$ is a product of high-temperature plastic deformation (viscous creep) that is usually produced via two creep mechanisms: diffusion creep and dislocation/powerlaw creep (equation 2.12), under upper-mantle conditions. Thus, $\dot{\varepsilon}^{v i s}=\dot{\varepsilon}^{d i s}+\dot{\varepsilon}^{d i f}$, where $\dot{\varepsilon}^{d i s}$ and $\dot{\varepsilon}^{d i f}$ are strain rates due to dislocation and diffusion creep respectively. The activation of different types of deformation kinematics primarily depends on the mineralogy and grain size of the rock fabric, the geothermal gradient, state of stress, and finite strain [Kirby and Kronenberg, 1987]. Figure 2.3 [Kameyama et al., 1999] explains which creep mechanism becomes more favorable in olivine grains (constant grain size of $0.1 \mathrm{~mm}$ ) under different temperature, stress, and strain conditions. 


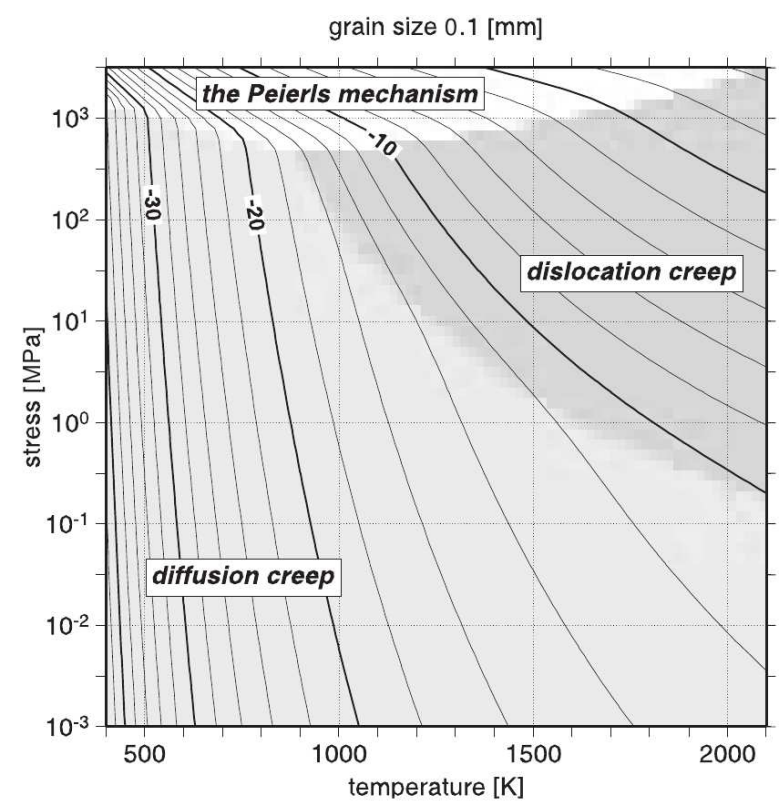

Figure 2.3 Deformation kinematic diagram as function of stress, temperature, and strain (after Kameyama et al. [1999]) when olivine grain size is equal to $0.1 \mathrm{~mm}$. Bold lines indicate constant strain rates $\left(s^{-1}\right)$ in log scale.

\subsubsection{Dislocation creep and diffusion creep}

The two forms of viscous deformation kinematics significantly alter the mantle fabrics, reflecting the most dominant kinematic under existing physical conditions. LPO anisotropy is such a deformed fabric that results from the dislocation creep of intrinsically anisotropic minerals such as olivine (section 2.4.2) in the uppermantle [Karato and Wu, 1993]. When minerals in rocks are subjected to dislocation creep, they will produce a preferred orientation through the mechanisms of 1) intracrystalline slip, 2) recrystallization, and 3) grain boundary sliding [Kirby and Kronenberg, 1987]. Dislocation mechanisms activate due to the stress fields around the crystal dislocations. Dislocations can move through the crystal lattice along the favorable slip plane towards the crystal boundaries, resulting in a strain localization along that slip plane. Because of the intrinsic anisotropy of some minerals (e.g. olivine, orthopyroxene), crystals may have a favorable and active slip plane along where dislocations can migrate easily. This process is known as dislocation glide (edge dislocation). For example, the [100](010) slip system in the orthorhombic 


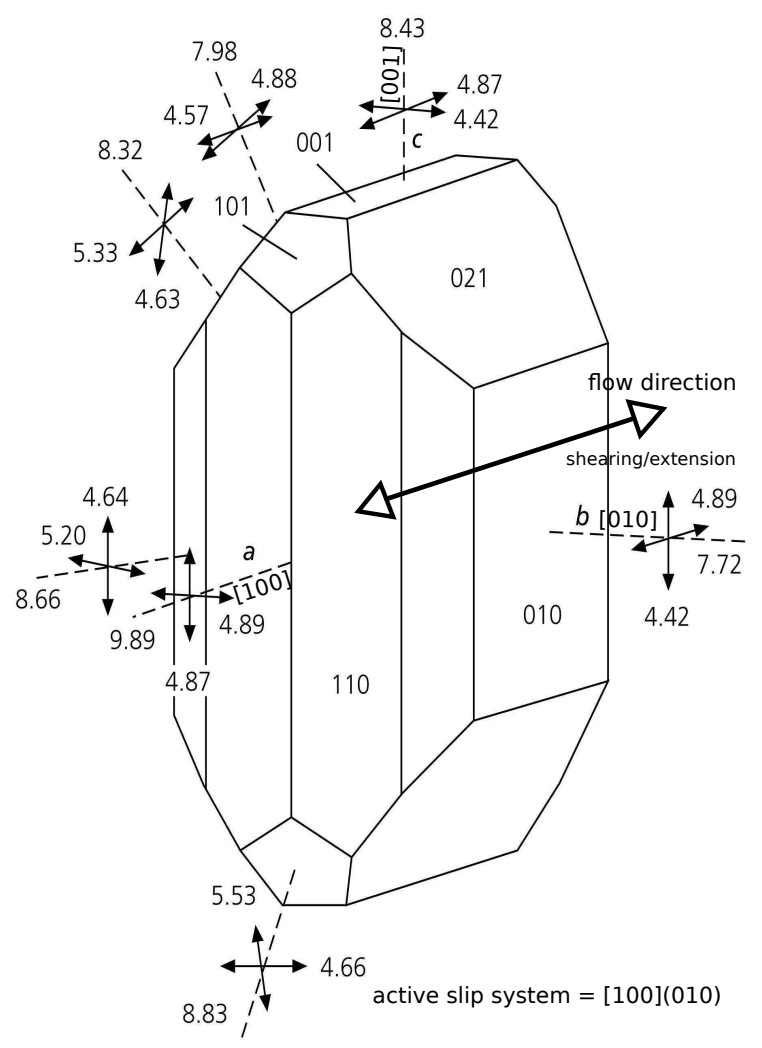

Figure 2.4 Velocity anisotropy of olivine crystal. Velocities of $\mathrm{S}$ and $\mathrm{P}$ waves along different propagation directions within the crystal are marked by double headed arrows and dotted lines, respectively. Note that the highest $\mathrm{P}$-wave velocity is along the direction of the a-axis [100]. Typical active slip system is [100](010) and this system aligns with the flow direction, shearing (or maximum extension). In this example, flow direction could be in either direction as indicated by two arrow heads parallel to [100] axis. Note b-axis and c-axis are marked by [010] and [001] respectively.

olivine crystal (Figure 2.4) is the most active slip system at high-temperature and at low differential stresses. Impurities and stress around the other dislocations in the lattice sometimes hinder the progression of dislocation glide towards the boundary. Consequently, the dislocation climb mechanism (screw dislocation) is activated by crossing the slip planes. Dislocation creep is defined as a combination of both dislocation glide and climb mechanisms. For naturally deformed olivine crystals, the viscous strain rate $\left(\dot{\varepsilon}_{s}\right)$ resulting from either dislocation creep or diffusion creep (grain-size dependent creep) can be expressed in the form of power law rheology that explains the non-linear viscous deformation (equation 2.12) [Karato and $W u$, 
1993]:

$$
\left.\dot{\varepsilon}_{v i s}=A(\sigma / \mu)^{n}(d / b)^{m} \exp \left[-\left(E^{*}+P V^{*}\right) / R T\right)\right] \text { Karato and } W u[1993]
$$

where $A$ is the material constant, $\sigma$ is the differential stress, $\mu$ is the shear modulus, $E^{*}$ and $V^{*}$ are activation energy and activation volume respectively, $P$ is the hydrostatic pressure, $\mathrm{R}$ is the gas constant, and $\mathrm{T}$ is the absolute temperature. $d, b, m$, and $n$ are the extra parameters that denote the grain size, the length of the Burgers vector, the grain-size exponent and the stress exponent respectively. $\mathrm{m}=$ 0 (grain size independent) for the dislocation creep mechanism, which is promoted at high-stress levels ( $\gtrsim 10 \mathrm{MPa}$ ) and at high temperatures (Fig. 2.3). However, dislocation creep deformation is likely influenced by the chemical environment (water content and mineral chemistry), hydrostatic pressure and dynamic recrystallization processes [Karato and Wu, 1993]. Dynamic recrystallization is a temperature dependent mechanism that is favorable under high-strain and high-temperature conditions [Bystricky et al., 2000]. It can produce more pronounced anisotropic fabrics ( $\sim 20 \%$ P-wave anisotropy) than dislocation creep, rotating the fast direction into the plane of shear or the flow direction [Ismail and Mainprice, 1998, Zhang and Karato, 1995]. This controls the grain size in the dislocation creep regime due to formation of new grain boundaries and/or change in existing grain-boundaries. Grain sized under dislocation creep conditions is given by:

$$
d=K b(\sigma / \mu)^{-q} \quad \text { Karato and } W u[1993]
$$

where $\mathbf{K}$ and $\mathbf{q}$ are temperature insensitive constants. According to equation 2.13, grain size reduces as the stress level increases. If significant grain size reduction occurs during the dynamic recrystallization, especially at low temperatures, then the grain-size sensitive creep (or diffusion) [Poirier, 1990] is enhanced and the transition from dislocation creep to diffusion creep takes place (Fig. 2.3). Most 
Table 2.3 Flow law parameters in equation 2.12 for olivine deformation in dislocation and diffusion creep regime under dry conditions [Karato and Wu, 1993]

\begin{tabular}{ccc}
\hline Parameter $^{\dagger}$ & Dislocation creep & Diffusion creep \\
\hline $\mathrm{A}\left(\mathrm{s}^{-1}\right)$ & $3.5 \times 10^{22}$ & $8.7 \times 10^{15}$ \\
$\mathrm{n}$ & 3.5 & 1.0 \\
$\mathrm{~m}$ & 0 & $\sim 2.5$ \\
$\mathrm{E}\left(\mathrm{kJ} \mathrm{mol}^{-1}\right)$ & 540 & 300 \\
$\mathrm{~V}\left(\mathrm{~cm}^{3} \mathrm{~mol}^{-1}\right)$ & $\sim 15-25$ & 6 \\
\hline${ }^{\dagger}$ Parameters A, n, $\mathrm{m}, \mathrm{E}$ and $\mathrm{V}$ are explained in section 2.5 .1
\end{tabular}

importantly, effective viscosity in the dislocation regime is a function of differential stress, whereas, in the diffusion creep regime, effective viscosity is sensitive to the grain size [Kirby and Kronenberg, 1987].

Materials with small grain sizes undergo diffusion creep at low stress levels and low temperatures. This deformation is caused by the diffusion of vacancies within the crystal lattice. An individual crystal strain is caused by the directional diffusion of vacancies and atoms. Diffusion rate is proportional to the applied differential stress. The vacancies usually diffuse from low to high pressure (stress) regions within the lattice, and in the opposite direction to the atomic flux. Unlike in dislocation creep, grain sizes in diffusion creep are controlled by both dynamic recrystallization and grain growth.

\subsubsection{Factors controlling deformation and implications for mantle dynamics}

As discussed in section 2.5.1, olivine can be subjected to either dislocation or diffusion creep, depending on the flow parameters in equation 2.12 (see table 2.3). Olivine deformation has the following characteristics [Karato, 1998, Karato et al., 2008]: 
1. Both dislocation and diffusion creep mechanisms can be enhanced and/or altered in the presence of water. Consequently, the activation of different slip systems (e.g. changing from the typical [100](010) system to [001](010)) and different diffusion mechanisms (e.g. wet diffusion) are possible [Karato et al., 2008]. However, dislocation to diffusion transition depths are independent of the water content.

2. Both creep mechanisms are sensitive to geothermal gradients, which determines the transition between the two creep mechanisms. For example, the upper-mantle beneath mid-oceanic ridges has a high geothermal gradient and, therefore, dislocation creep dominates. But the geothermal gradient in the cold (or old) upper-mantle is lower than that in the mid-oceanic ridges. Thus, diffusion creep dominates in such a domain.

\section{Implication for mantle dynamics}

The above controlling factors will affect the creep mechanisms, which in turn control mineral orientation and hence anisotropy. Thus, implications for mantle dynamics and factors controlling mantle deformation can be gained from the study of in-situ seismic anisotropy and laboratory studies of mantle-derived rocks (e.g. Xenoliths). Typical differential stress levels in the upper-mantle $(\lesssim 10 \mathrm{MPa})$ of tectonic domains away from the mid-oceanic ridges are not enough to activate the dislocation creep mechanisms, unless there are high-temperature conditions. Thus, in such systems, diffusion creep dominates in the shallow $(\sim 100 \mathrm{~km})$ upper-mantle (or in the deep lithosphere) and results in a rheologically weakened upper-mantle with little anisotropy [Karato and Wu, 1993]. The higher seismic anisotropy observed in such a setting is attributed to frozen LPO anisotropy that formed when this region was relatively hot and/or experiencing very high tectonic stresses [Silver, 1996]. Due to high temperatures at the intermediate depths $(\sim 100-150 \mathrm{~km})$ of the upper- 
mantle (i.e. shallow asthenosphere) compared to the shallow upper mantle $(\sim 40-100$ $\mathrm{km}$ ), dislocation creep becomes active and reflects the present-day asthenospheric flow or shear [Karato and Wu, 1993]. In the deep upper-mantle below the shallow asthenosphere, again diffusion mechanisms are favorable and more rapid as a result of grain boundary sliding mechanisms that are activated at relatively high-strain conditions compared to that in the usual diffusion creep conditions. This process may result in super-plasticity with little or no anisotropy in the deep mantle [Poirier, 1985].

Continental environments, such as collisional zones in plate-boundaries, are more likely to be characterized by large differential tectonic stresses $(\geq 100 \mathrm{MPa}$; Karato and $W u[1993]$ and Fig. 2.3). As a result, dislocation creep dominates in the shallowmantle. Increase in stress results in instantaneous increase in strain at a given grain size, but increase in stress will also reduce the grain size. Karato and Wu [1993, Fig. 4] demonstrated that this grain size reduction will promote diffusion creep if this process is close to the transition boundary between dislocation and diffusion creep. In summary, whether dislocation or diffusion creep is favorable depends on the tectonic setting and the physical/chemical conditions of the setting. As concluded by many studies, activation of these mechanisms at various depths may result in mechanical decoupling between Earth layers and deformation localization [Silver, 2002, Vauchez and Nicolas, 1991, Avouac and Tapponnier, 1993]. 


\subsection{Sampling anisotropy using different S-phases}

Anisotropy at different depths can be investigated through the shear-wave splitting of different S phases (e.g. SKS, teleseismic S, ScS and local/regional S) (Figure 2.5). SKS splitting gives constraints on the receiver side mantle anisotropy because of its phase conversions $(\mathrm{S}-\mathrm{P}(\mathrm{K})-\mathrm{S})$ at the core mantle boundary. This is the most commonly used and earliest-established method to characterize the receiver-side mantle dynamics and deformation [Silver, 1996]. Although its vertical resolution is poor, SKS splitting provides a good lateral resolution to constrain the dynamics and deformational patterns in the upper-mantle and asthenosphere. The study of local/regional S- wave splitting can resolve the vertical anisotropic structure beneath the station. Most of the SKS splitting studies assume that crustal and/or subcrustal anisotropy is negligible compared to mantle anisotropy. Several crustal anisotropic studies have noted that such an assumption is not valid in most of the active tectonic domains [Godfrey et al., 2000, Barruol, 1993]. Furthermore, to fully characterize the plate-boundary processes or deformation around large scale faults, both mantle and crustal deformation must be taken into account.

In tectonic domains (e.g. subduction zones, collisional and strike-slip plate boundaries etc.), it is also important to understand how the lithosphere responds to active tectonics and geodynamic processes. These domains are likely to experience significant deformation and, hence, be characterized by anisotropic structure. For example, investigation of seismic anisotropy via splitting of local and regional events recorded at varying depths in subduction zones (subduction zone in figure 2.5) allows the isolation of anisotropy in the overriding plate, mantle wedge, suducting-slab, and sub-slab. Such an investigation with good depth resolution enables capturing the subduction zone complexities [Long and Silver, 2009, Xie, 1992, Huang et al., 2011b]. Therefore, the combination of the splitting from different S-phases can be used to depict the 3-dimensional anisotropic structure of tectonic domains. However, 


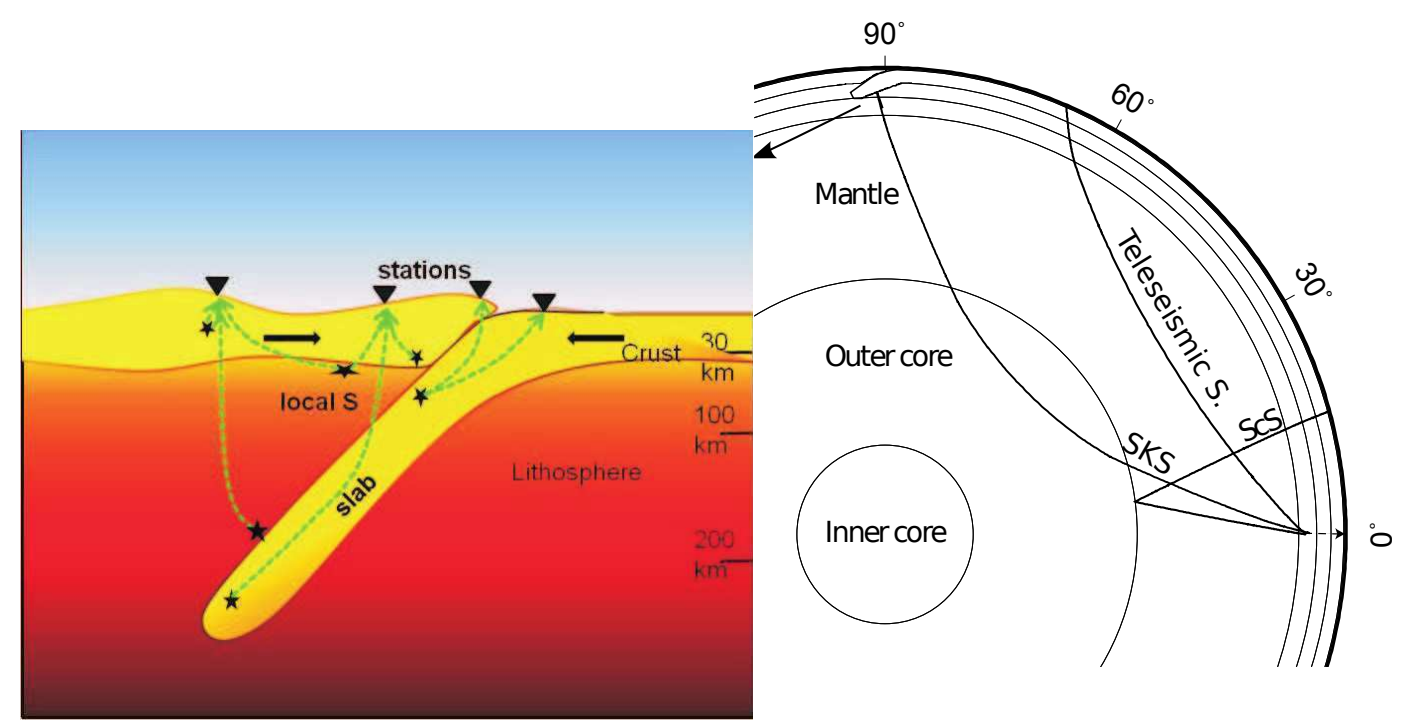

Figure 2.5 Different S-phases utilized in S-wave splitting to investigate the anisotropic structure at various depths. Ray paths of SKS, telesismic S-phases and regional S-phases are marked in the black color curved paths in right-side figure. The left-side figure illustrates the possible ray paths of local S-waves in a subduction setting.

frequency dependent effects (section 3.5.2) and ray-path ambiguity can make such combinations difficult to interpret.

\subsection{Characterizing tectonic regimes using S-wave splitting}

Patterns of S-wave splitting directions and the strength of anisotropy are characteristics of certain tectonic domains and their prevailing physical and chemical conditions. Our study area consists of both subduction and transpressional tectonic domains, where anisotropic properties may vary distinctly as predicted by shear-wave splitting studies in similar global-tectonic settings. Let us consider how S-wave splitting can be used to determine deformation and dynamics in these systems. 


\subsubsection{Transpressional zones: S-wave splitting}

The majority of the plate motion in the transpressional domain in New Zealand is predicted to occur parallel to the plate-boundary. As a result, transcurrent flow dominates around the Alpine fault, Marlborough fault zone, and North Island dextral fault-belt. Global studies have suggested that shear-wave splitting in such domains is governed by mantle-flow derived anisotropy at upper-mantle depths [Silver, 2002, Vauchez and Nicolas, 1991], structural anisotropy due to vertical foliations [Barruol, 1993] in the mid-lower crust, and stress-aligned cracks [Peacock et al., 1988] or sheared-cracks in the shallow crust. Lithospheric shear-zones associated with the large strike-slip faults (e.g. San-Andreas fault, Alpine fault), have the sense of motion parallel to the fault. In that case, intrinsically anisotropic minerals at mid-lower crustal depths may align parallel to the shear, forming vertical or horizontal foliations [Mehl et al., 2003]. Depending on the rock fabric and metamorphic conditions (e.g. high-grade metamorphism), such foliated fabrics could also be present in the upper crust (e.g. Schists). These crustal shear zones can extend into the upper-mantle and possibly down to the asthenosphere as mantle shear-zones. In the lower lithosphere, such shear zones would generate anisotropic mineral alignment that is parallel to the plate-boundary transcurrent movement. In both cases, vertically propagating S-waves can split by the fabric alignment and the faster S-wave will be polarized in the direction parallel to the transcurrent shear and the alignment of vertical foliations with high strength of anisotropy [Vauchez and Nicolas, 1991]. The strength and fast polarization direction may vary depending on the crust and mantle contributions [Barruol et al., 1997]. For instance, if the crustal foliations are not well developed or anisotropy is altered by other mechanisms to produce different geometry or different types of anisotropy, then crust-mantle decoupling that may result in complex shear-wave splitting is also possible.

Collision and orogensis in South Island, New Zealand are more recent tectonic events 
that initiated with the migration of the Australian passive-margin into transcurrent plate-boundary zone (section 1.3.1). According to [Nicolas, 1993], when passive margins are subjected to transcurrent motion as a result of plate collision, two types of anisotropic fabrics may occur: 1) frozen mineral alignment from asthenospheric flow parallel to rift axis and 2) mineral alignment parallel to transcurrent motion. Although these two anisotropies are expected to have similar orientations, changes in plate-boundary orientation during the two tectonic events may alter the general trend of fabric orientations, resulting in spatially or depth varying anisotropy. Thus, deflection of plate-boundary parallel fast azimuth of shear-wave splitting can be attributed to changes in tectonic environment over the geologic timescale [Nicolas, 1993]. Such variations have also been attributed to present-day stress governed anisotropy in the shallow crust by some local shear-wave splitting studies [Peacock et al., 1988]. In that case, stress-induced alignment of the cracks at shallow depths would produce vertical crack planes that are parallel to the maximum horizontal compressives direction $\left(S H_{\max }\right)$, so that the fast split $\mathrm{S}$-wave from a vertical ray path will be polarized parallel to the $S H_{\max }$.

\subsubsection{Subduction zones: S-wave splitting and dynamics}

Subduction zones, where the descending slab lithosphere interacts with the overriding lithosphere, are expected to be characterized by complex mantle dynamics and lithospheric deformation. The complexities can vary from one subduction zone to another depending on the deformation conditions (e.g. pressure, temperature, strain rate and/or stress), subduction zone parameters such as convergence velocity, slab dip, trench migration velocity [Long and Silver, 2009] and the properties of subduction zone material. The study of shear-wave splitting from core phases (e.g. SK(K)S, ScS) and teleseismic S-phases and geodynamic modeling allows us to constrain the mantle flow in the subduction systems. Mantle flow governs the 
development of LPO by aligning the crystallographic axes of minerals in a preferred direction, most commonly parallel to the mantle flow direction [Savage, 1999].

\section{Shear-wave splitting anisotropy in subduction systems}

Vertically traveling S-waves in a subduction zone can be split by anisotropy in the sub-slab region, subducting slab, mantle wedge and the overriding plate [Long and Silver, 2009]. These zones can be characterized by large-scale intrinsic anisotropy that is present at mantle depths and small-scale crustal anisotropy due to cracks/microcracks and melt/fluid-filled microcracks or pockets, faulting structures in the upper-plate and bending related cracks in the slab [Morley et al., 2006, Gledhill and Stuart, 1996]. Despite the complex nature of the local/regional S-wave splitting, the good depth resolution of those phases enables us to integrate them with the SKS splitting to isolate the anisotropic signatures in the sub-slab region, mantle wedge, slab and overlying plate [Savage, 1999]. If the length-scale of anisotropy and/or orientation of the alignments in those zones are significantly variable, frequency dependent splitting and/or multilayer/two-layer modeling approaches (sections 3.5.1 and 3.5.2) incorporated with splitting analysis can be used to resolve the complexities in subduction zones.

\section{Origin and factors controlling the slab, mantle wedge and sub-slab anisotropy}

In subduction zones, the mantle wedge and sub-slab regions are usually considered to be the dominant anisotropic zones. Nevertheless, recent studies have suggested that the slab itself also contributes a considerable amount to the total anisotropy in some systems [Faccenda et al., 2008, Morley et al., 2006]. Slab anisotropy can have a different origin of anisotropy and SKS/Tel.S wave splitting may not be capable of detecting such relatively small-scale localized anisotropic structures in the slab. 
As inferred by an integrated study of local S to $\mathrm{P}$ conversions, teleseismic receiver function studies and Pn azimuthal anisotropy studies [Song and Kawakatsu, 2012], the slab itself may consist of interior layering and the uppermost mantle region of the slab may be characterized by a localized anisotropic zone. Song and Kawakatsu [2012] attributed such a localization of anisotropy to the anisotropy preserved in the subducting ocean lithosphere from paleo-spreading. However, Long and Silver [2008] found no correlation between such fossil spreading directions and $\phi$ (polarization direction of the fast S-wave) from splitting anisotropy in the global subduction settings, and they concluded that there is no clear relationship between the age of the subducting slab (or thickness of the slab) and the amount of observed $\delta t$.

In addition to anisotropy derived from paleo-spreading, anisotropy in the slab or mantle wedge can be modified if the system is hydrated. As explained in Long [2013], the shallow slab is most likely to retain water in the form of hydrous minerals (e.g. Serpentine minerals like Antigorite and Lizardite, and clay minerals like talc and chlorite). Such a mineralization is possible during the formation of oceanic crust in the oceanic environments (spreading centers) and/or when the topmost layer of newly created crust is subjected to hydrothermal alterations [Boudier et al., 2010, Long, 2013] and/or hydration/serpentinization of the bending-induced faulting in the shallow layer of the subducting slab [Faccenda et al., 2008, Ji et al., 2013]. Compared to Antigorite, Talc is more stable under high temperature conditions and can be present from the sea-floor to $150 \mathrm{~km}$ in the subduction zone. Talc can be derived from the unstable Antigorite under high temperature/pressure conditions especially in the mantle wedge [Mainprice and Ildefonse, 2009]. These hydrous minerals are assumed to produce strongly anisotropic media because of their relatively high single crystal anisotropy (e.g. SWA of antigorite and talc $\simeq 68 \%$ and Lizardite $~ 81 \%$ ) [Long, 2013].

The majority of studies agree with trench parallel flow below and above the slab (close to trench) as predicted by the slab-entrained flow (section 2.7.2) . However, a 
few other studies have noticed trench perpendicular/oblique $\phi$ in the back-arc region (mantle wedge) [Morley et al., 2006]. Fischer et al. [2000] proposed the 2D-corner flow model to explain the trench perpendicular splitting observed in the back-arc regions (section 2.7.2). Splitting estimations from the local shear-wave splitting and some SKS-splitting studies have determined that anisotropy in the wedge is more complex than anywhere else in the subduction zone [Wirth and Long, 2010, Abt and Fischer, 2008]. Several studies argue that the complex anisotropy in the mantle-wedge above the slab can also be due to B-type olivine (010)[001] as result of the high $\mathrm{H}_{2} \mathrm{O}$ content under low temperature and high stress conditions [Karato et al., 2008], or high pressure conditions (deeper than $\sim 90 \mathrm{~km}$, or pressure around 3 GPa) [Jung et al., 2009, Long, 2013]. Serpentinization or mineralization of clay minerals can occur in the mantle wedge as a result of the water-rich sediments or dehydration of the slab-mineral. In that case, the wedge may be characterized by highly anisotropic minerals other than olivine (e.g. antigorite, talc) with variable anisotropic symmetry properties. Mehl et al. [2003] demonstrated that presence of high $\mathrm{H}_{2} \mathrm{O}$ content could result in the activation of (001)[100] slip in C-type olivine, producing horizontal foliations rather than typical vertical foliations that are caused by the (010)[100] slip. In both cases, the S-wave splitting will yield trench parallel $\phi$, but $\delta t$ resulting from the horizontal foliations can be higher than $\delta t$ resulting from the vertical foliations. Holtzman et al. [2003] ascribed a complex splitting in the wedge to the effect of melt on LPO of olivine. Deviatoric stress can create an anisotropic alignment of the melt pockets and this alignment may cause girdles in a- and c-axes of olivine in the absence of melt segregation. However, if the melt is segregated into melt rich shear zones, then the olivine a-axis can be rotated $90^{\circ}$ from the shear plane, resulting in trench perpendicular or oblique $\phi$ [Holtzman and Kendall, 2010].

Sub-slab region anisotropy can be approximately estimated by either a combined study of the splitting of SKS and local S-phases that originate in the slab, isolating 
the splitting in the mantle below the slab [Long and Silver, 2009] from the mantlewedge/slab anisotropy or by source-side splitting of down-going teleseismic S and correction for receiver-side splitting [Russo, 2012, Lynner and Long, 2013]. Long and Silver [2009] showed that most of the suduction systems are characterized by trench-parallel flow beneath the slab, and that the observed splitting delays agree with the anisotropy due to flow in the upper-mantle and/or in asthenosphere. Song and Kawakatsu [2012] proposed a different hypothesis to characterize the observed splitting in sub-slab region. The proposed idea is that the sub-slab oceanic asthenosphere is coupled with the subducting slab. As a result of this slab-coupled suducting asthenosphere, the sub-slab region can be characterized by orthorombic symmetry as predicted in the oceanic asthenosphere, but the symmetry axis is tilted because of the subduction. Inferred variations of splitting parameters from this method tend to agree well with splitting observations in shallow (dip $<15^{\circ}$ ) subduction zones [see Song and Kawakatsu, 2012, Fig. 2]. However, in steep subdution settings the predicted $\phi$ with this model are parallel or subparallel to the trench as predicted by the trench parallel flow model (next section and Fig. 2.6) and, therefore, it is hard to discriminate between the two models.

\section{Subduction zone flow models}

If the anisotropy in subduction settings is mainly governed by mantle flow, the $\phi$ derived from the S-wave splitting must be either parallel to the relative plate motion direction (RPM) and/or to the absolute plate motion direction (APM). Deviation from these simple trends implies that there are other mechanisms that could be affecting the anisotropy or flow pattern in subduction settings. Based on the splitting parameters from various subduction settings, several flow models have been proposed to describe the flow geometries in those settings. Some models are focused on the flow patterns in certain regions (e.g. subslab, mantle-wedge, back-arc). Russo and Silver [1994] proposed the first suduction zone flow model to 
explain the trench parallel splitting from the SKS phases. This model consists of trench-parallel flow just beneath the slab that results from the retrograde motion of the slab relative to mantle flow. This model is comparable to entrained flow around the slab that is proposed by other recent studies (Fig. 2.6). The trench parallel flow is, however, assumed to decouple from the upper-mantle asthenospheric flow, which represents the APM direction at depths or away from the subduction setting.

Trench parallel $\phi$ above the slab, which is determined from large $\delta t$ ( $\gtrsim 1 \mathrm{~s}$ ) of local S-wave splitting [Smith et al., 2001], is assumed to result from the along-arc mantle flow above the slab. Such a flow pattern can be generated from the slab rollback effect and/or a trench parallel component of the APM/RPM direction in oblique subduction domains. In addition to that, if the overriding plate consists of large scale strike-slip faulting parallel to the trench due to RPM in the forearc region (e.g. southern Hikurangi subduction zone), then mantle shear zones beneath those faults could also produce splitting that is parallel to the faulting and the trench. The two-dimensional back-arc flow (also known as 2-D corner flow) model explains trench perpendicular $\phi$ with strong splitting in the back-arc regions [Fischer et al., 2000]. Furthermore, models with olivine transitioning from A- to B-type in the mantle wedge, models with horizontal and vertical foliations that result from trench parallel flow in the transpressional subduction settings [Mehl et al., 2003] and the recent model of slab-coupled asthenospheric flow by Song and Kawakatsu [2012] have been proposed to explain the variable splitting parameters in subduction zones by recent studies (Long [2013]; see Fig. 2.6).

Because of the complexities in the different subduction settings it is impossible to provide a distinct flow model that generalizes the flow patterns in various subduction regimes. Long and Silver [2008] utilized a combined flow model (Fig. 2.6-C) that is characterized by 2D corner flow in the back-arc region and 3D flow around the slab (similar to trench parallel slab-entrained flow) to test whether there is a relationship between splitting parameters from various subduction zones and subduction zone 
A. sub-slab trench parallel flow

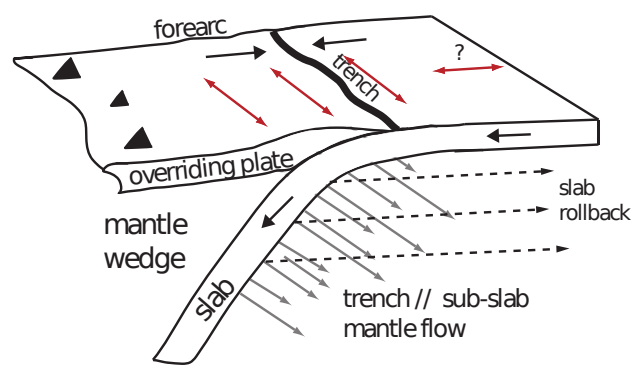

C. Combined model (2-D +3-D flow)

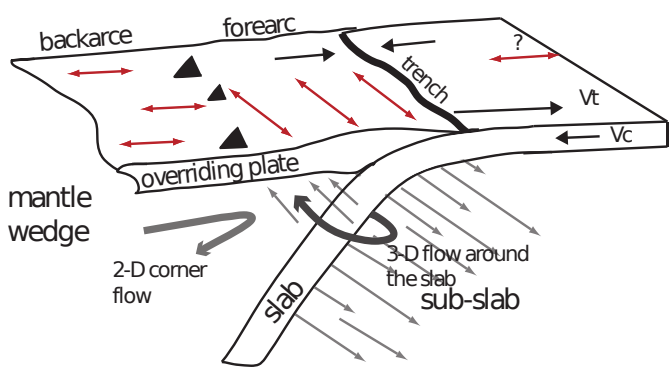

B. subslab and forearc -wedge trench parallel flow

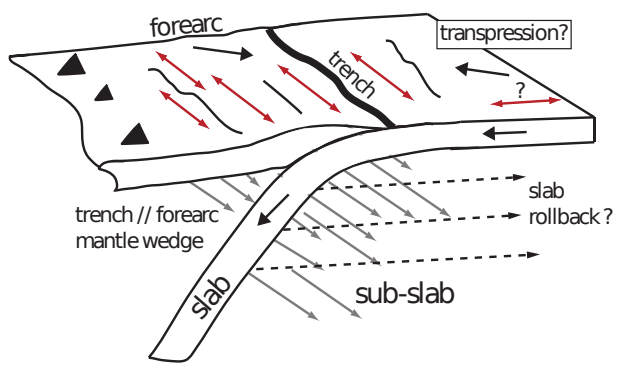

D. slab-coupled asthenodpheric flow

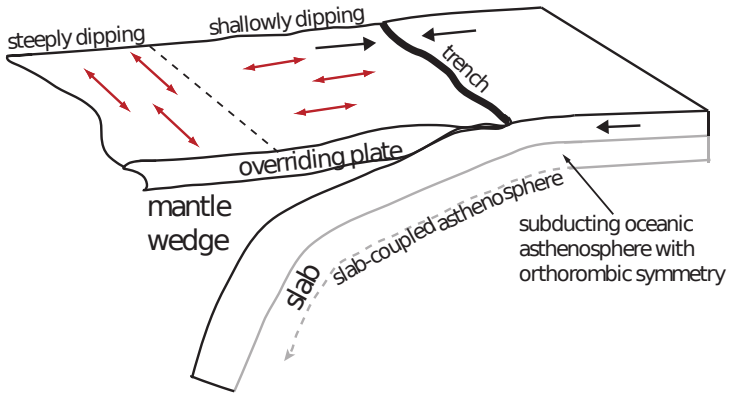

Figure 2.6 Schematics display the main flow models proposed for subduction zones. (a,b) Trench parallel flow in the sub-slab and above the slab [Russo and Silver, 1994, Smith et al., 2001]. (c) Combined flow model Long and Silver [2008] (2-D corner flow model [Fischer et al., 2000] and 3-D slab entrained flow). (d) Slab-coupled asthenospheric flow [Song and Kawakatsu, 2012].

parameters (see 2.7.2). 2D corner flow model is assumed to derive from the down dip motion of the slab, which possibly controls the trench perpendicular $\phi$ in the back-arc region as noticed by many studies. Conversely, 3D flow or slab entrained flow can be approximated by trench parallel flow below and above the slab and is mainly governed by the trench migration velocity relative to the hot spot reference frame. The trench parallel flow in the wedge, however, can be altered by the trench perpendicular or oblique $\phi$ in the presence of B-type olivine fabrics. Long and Silver [2009] concluded that trench parallel flow in the sub-slab is mainly governed by the trench migration velocity, and trench parallel flow (relative to surrounding mantle) of the fast-moving trenches is more likely to produce stronger anisotropy than that of the slow or nearly stationary trenches. As estimated by Gledhill and Gubbins [1996], sub-slab splitting anisotropy of the subduction zone in New Zealand $(\sim 1.5$ s) is also higher than most of the global subduction zones and, therefore, is expected to be characterized by a significant trench parallel flow. 


\section{Chapter 3}

\section{Data and methodology}

This chapter discusses the instruments, quality of the S-phases and the main methods that have been utilized in this study. In some sections theories related to methodology are discussed in detail. Hereafter South Island and North Island are denoted by the acronyms of SI and NI, respectively.

\subsection{Experiment and Data}

The thesis consists of three main research projects (1) on-land SI, 2) offshore SI, and 3) southern NI). All three projects were focused on investigating S-wave splitting anisotropy. The on-land and offshore SI experiments utilized local and regional high frequency $\mathrm{S}$ phases to explore S-wave splitting parameters in the plate-boundary zone in SI, New Zealand, whereas the southern NI experiment utilized low frequency SKS, ScS and teleseismic S-phases to examine the subduction zone anisotropy. In all three studies, seismic records from broad-band seismographs are used to determine S-wave splitting. Local/regional S arrivals recorded on Ocean Bottom Seismographs (OBS) are used to investigate S-wave splitting anisotropy in the offshore, SI.

\section{S-wave signals and quality of the seismograms}

Shear-wave splitting analysis window must be free from noise and a clear S wave onset is preferred for reliable splitting measurements. To minimize the distortion of S-waves at layer boundaries and/or at the free surface, S-wave splitting analysis is usually carried out using the S-waves that are within the incidence angle $\left(i_{c}\right)$ of $35^{0}$ degrees (See section 3.3) [Nuttli, 1961]. S-waves are also filtered to enhance the S-arrival and to increase the Signal-to-Noise ratio (SNR) by removing unnecessary 
noise. Thus, all three studies used suitable band pass filters, which were two-way 4-pole Butterworth filters. The applied filter frequency bands were dependent on the different types S-phases that have been used to examine splitting (see sections 3.1.1, 3.1.2, and 3.1.3). Figure 3.1 shows the comparison between unfiltered (top two) and filtered (bottom two) S phases (local S and SKS). The filter bandwidth was selected so that the filtering only enhanced the S-wave onset without distorting the S-phase.
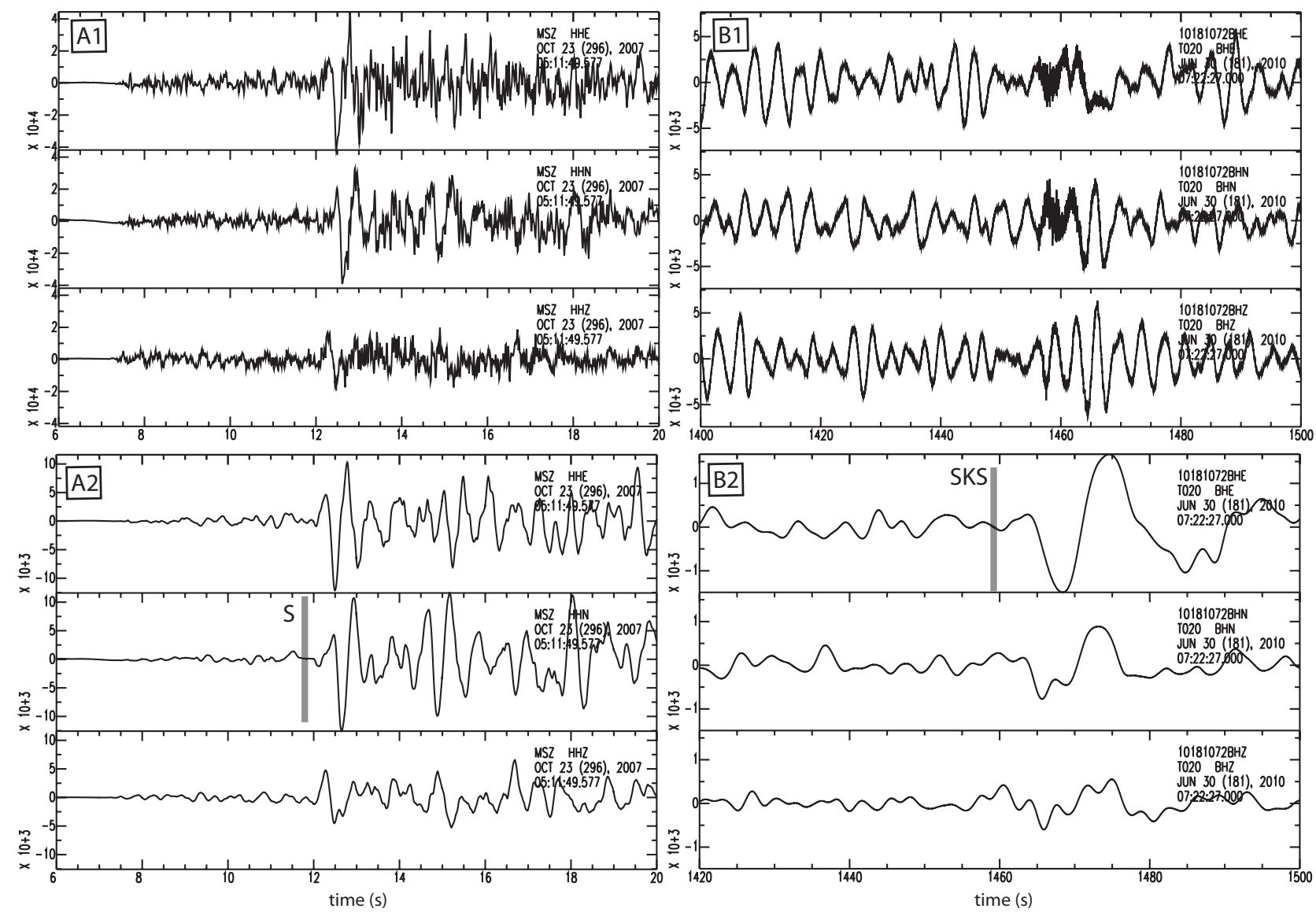

Figure 3.1 The comparison between filtered and unfiltered S phases. A1 and A2 are the unfiltered and filtered (band pass between 0.2 and $3 \mathrm{~Hz}$ ) high frequency local S phases. B1 and B2 shows the SKS phases unfiltered and band pass filtered between 0.04 and $0.1 \mathrm{~Hz}$. Note the enhanced S-wave onset in the filtered records. 


\subsubsection{On-land SI: anisotropy of the on-land plate-boundary zone}

Focus: We Investigate shear-wave splitting in SI New Zealand to understand the spatial and depth distribution of S-wave splitting anisotropy and to consider implications for the distribution of deformation in the plate-boundary zone.

\section{Experiment}

Local S-phases that were recorded on both permanent and temporary broadband stations in SI are used to investigate seismic anisotropy in the lithosphere using the shear-wave splitting technique. We used thirty 3-component broad band stations. Twenty six of them were GeoNet permanent stations and the other four stations were temporary PASSCAL seismographs that were deployed by Victoria University of Wellington in collaboration with the University of Colorado to enhance the seismic coverage. Both sets of instruments consisted of Guralp-3ESP and Kinematrics FBAES broad-band seismographs (Fig. 3.2). The PASSCAL utility package and Antelope software are used to convert RT130 data into SAC format (appendix D).

\section{Data selection}

We used local and regional S-waves recorded for the period of 2004-2010 on GeoNet network stations and events recorded for an eleven month period (2009 February 2010 January) on the temporary stations in the northern SI (Fig. 3.2 and squares in Fig. 3.3). 

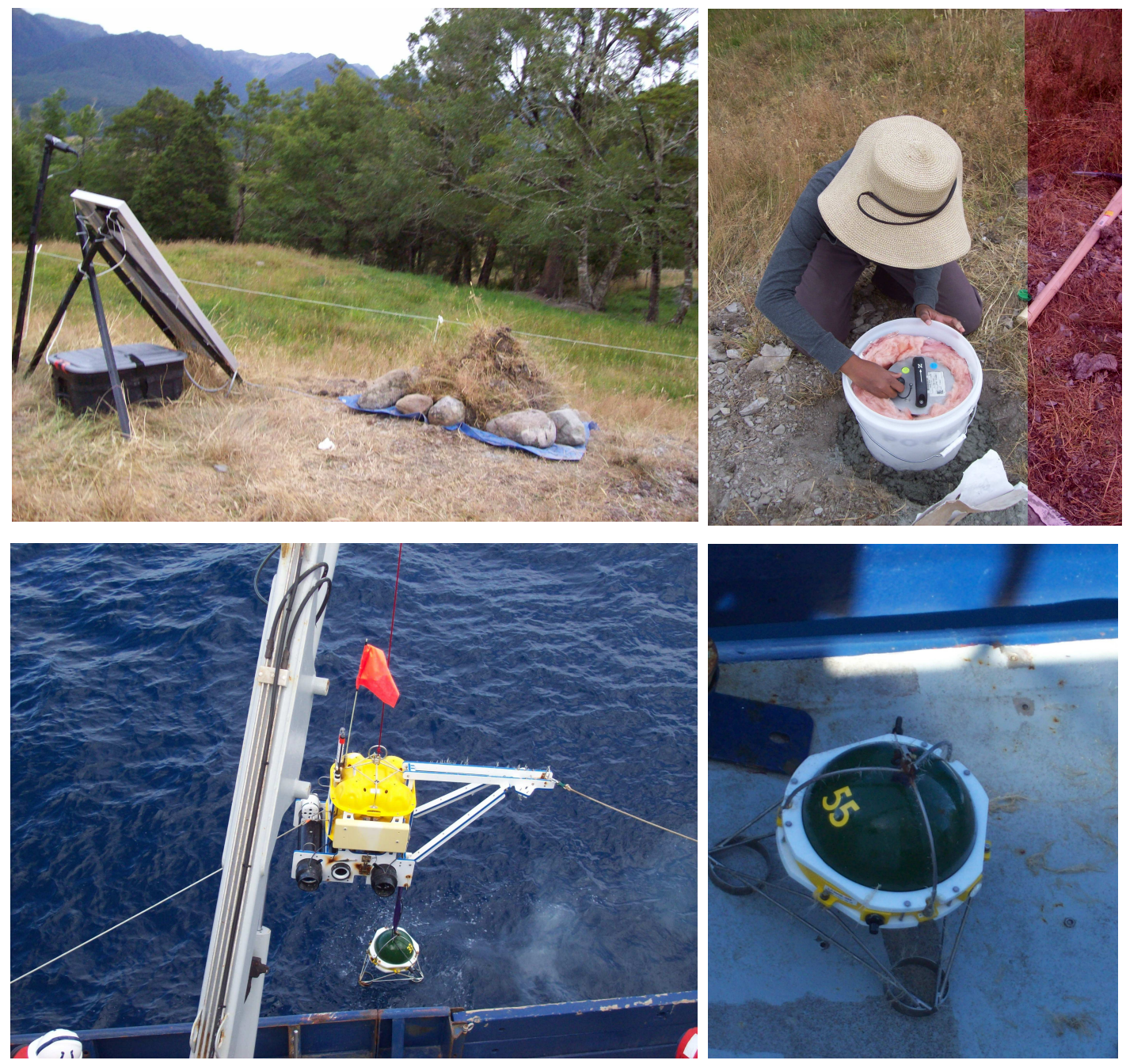

Figure 3.2 Temporary deployments (2009-10) and MONA OBS recovery in 2010. 


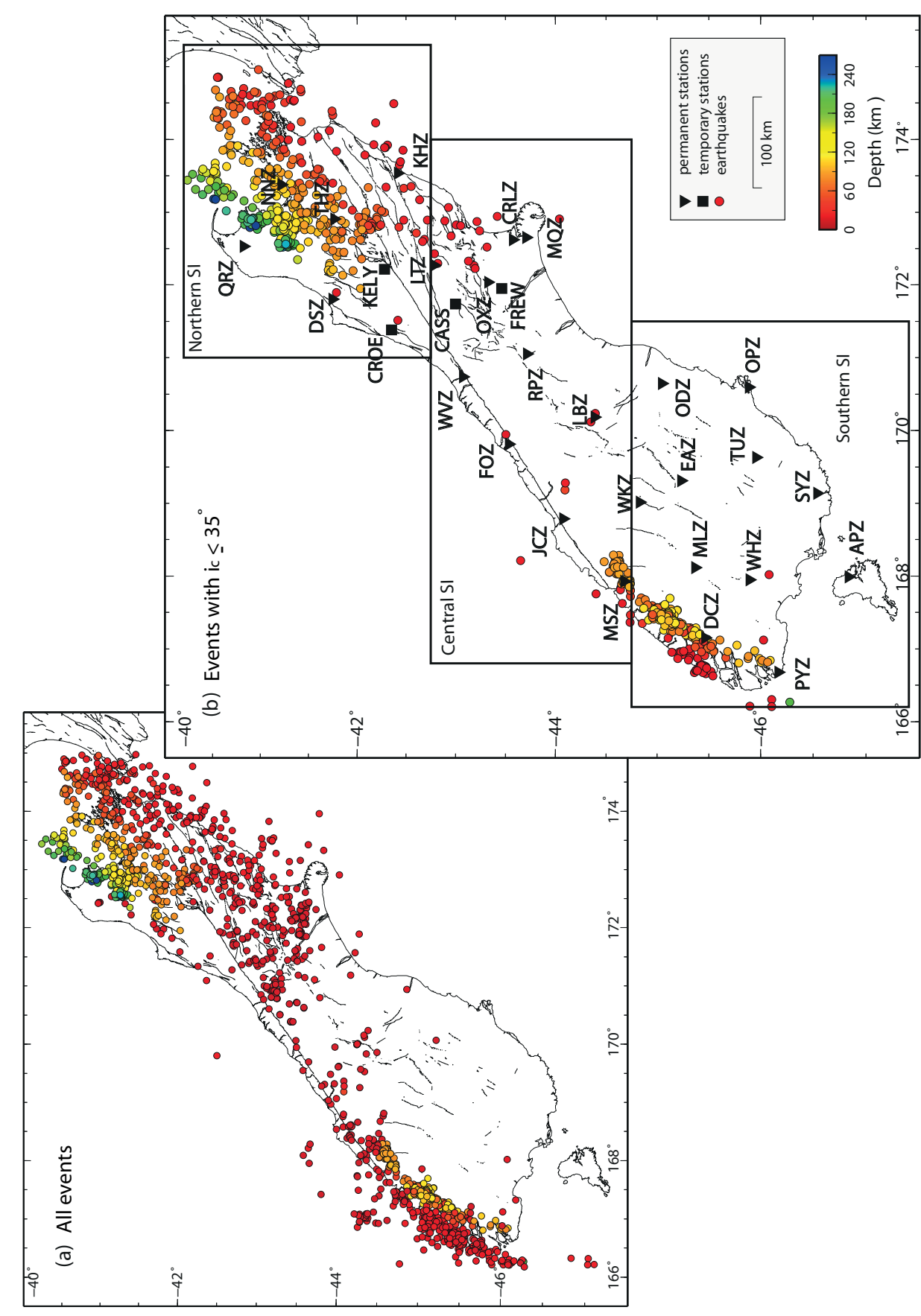

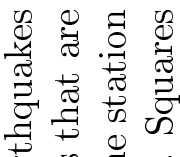

कृ

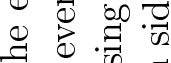

$F \otimes \stackrel{7}{=}$

ज焉盯

$\exists$ :

蛋

氙 $\frac{\overrightarrow{0}}{0}$

च

जี

$\stackrel{0}{80} \widehat{\sigma}$

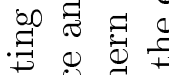

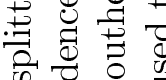

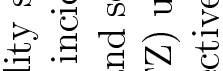

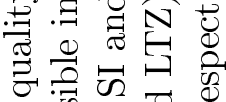

उ 0 ह

82 苟

구

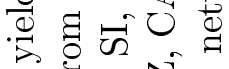

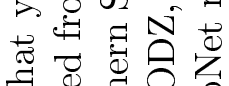

की

我过

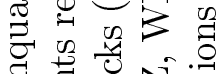

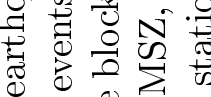

- o \& $巳$

पे

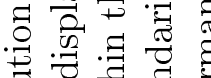

话寻寻

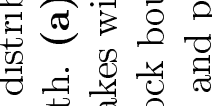

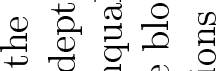

$\infty 0 \underset{\pi}{\square}$

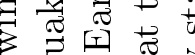

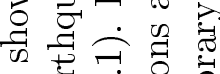

品

छ

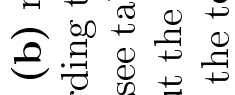

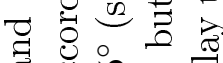

ส

(ิ)

m $0.0 \%$

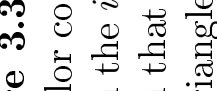

๖

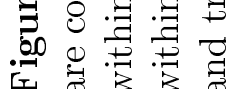


Table 3.1 Event selection criteria and event/measurement information.

\begin{tabular}{|c|c|c|c|c|c|}
\hline Block & $\begin{array}{l}\text { min. magni- } \\
\text { tude }\end{array}$ & tot.events & $\begin{array}{l}\text { max.event depth } \\
(\mathrm{km})\end{array}$ & $\begin{array}{l}\text { no. } \\
\text { meas. }\left(\mathrm{AB}^{*}\right)\end{array}$ & $\mathbf{A B}^{*} / i_{c} \leq 35^{0}$ \\
\hline ALL & - & 2851 & 244.00 & 8336 & 3183 \\
\hline Northern SI & 3.5 & 1070 & 243.85 & 1925 & 1167 \\
\hline Central SI & 3.0 & 470 & $55.5^{* *}$ & 1898 & 189 \\
\hline Southern SI & 3.5 & 769 & 177.42 & 2910 & 1213 \\
\hline
\end{tabular}

*AB grade measurements (section 3.2.3)

** depth indicates event depths of the majority of events in the central SI, but a few subduction zone events that were recorded on the central SI stations had the maximum depth of $\sim 90 \mathrm{~km}$.

The complete region was divided into three geographic units: northern, central and southern SI and events in each block were collected from the stations in that particular block during the initial retrieval of data (Fig. 3.3b). Stations that are close to the borders (WKZ, MSZ, ODZ, CASS and LTZ) of a block used the events recorded from either side. This criterion is used to control the epicentral distances and to avoid analyzing unnecessary waveforms at each station.

The number of events that give acceptable quality splitting measurements was further reduced by limiting events that have incidence angles less than $35^{0}$ (Fig. 3.3b, section 3.3). To obtain good signal-to-noise ratio, we used a threshold magnitude of 3.5 in northern and southern SI. Due to the lack of seismicity and deep events in central SI, the threshold magnitude of 3.0 was chosen to obtain more events (table $3.1)$.

In addition to the above analysis, splitting parameters from smaller earthquakes $(2.0$ $\leq$ magnitude $<3.0$ ) are combined with the above measurements to investigate the in-situ seismic anisotropy of the Haast schist belt in central-southern SI. For this analysis, earthquakes that are within $60 \mathrm{~km}$ epicentral distances and depths down to $40 \mathrm{~km}$ are extracted from 10 stations (WKZ, EAZ, TUZ, JCZ, FOZ, WVZ, LBZ, ODZ, MLZ, and OPZ) in or near by regions of the schist (Fig. 3.4). 


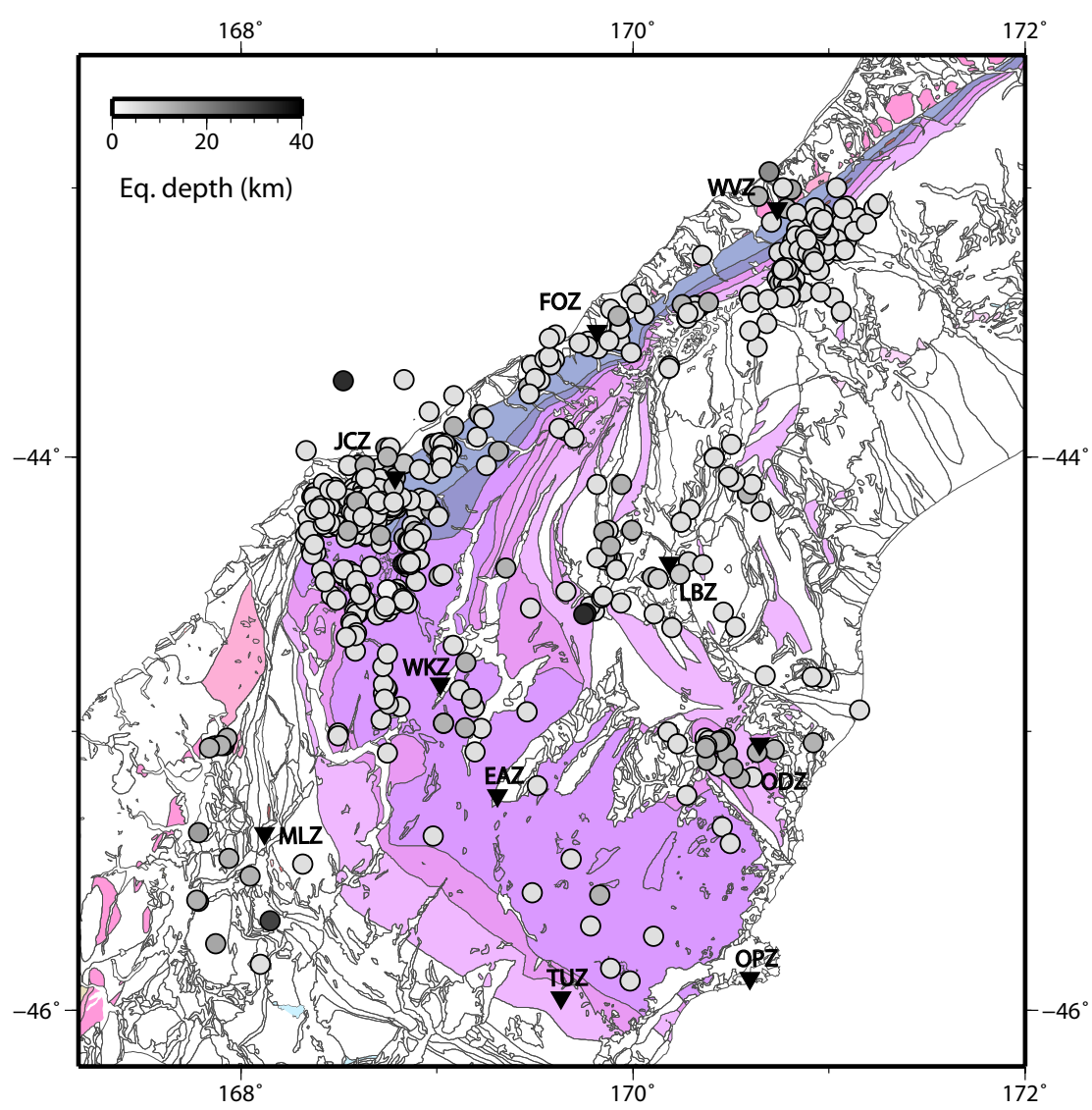

Figure 3.4 Events (mag. $\geq 2.0$ ) used to estimate splitting anisotropy of the schist rock in Central SI. Pink to purple colors indicate the schist rock of low to high grade metamorphism.

\subsubsection{Offshore SI: Local and regional shear-wave splitting in the offshore SI}

Focus: We examine the anisotropic structure in the offshore SI to study deformation distribution around the plate-boundary zone. This is an extension of the on-land study.

\section{Experiment}

Local and regional S phases recorded on the MOANA (Marine Observation of Anisotropy Near Aotearoa) Ocean Bottom Seismographic (OBS) network (Fig 3.5) are use to determine splitting parameters in the eastern and western offshore regions 
of the SI [Collins et al., 2010]. The initial deployment consisted of 30 OBS and data were collected from Januray 2009 to February 2010. OBS instruments used for the experiment belong to U.S. Ocean Bottom Seismic Instrument Pool. Twenty-nine OBS seismographs were Nanometric Trillium 240 and one was a Nanometric Trillium 40 seismograph. They were set up to record continuous wave-form data with a 50 sps sampling rate [Collins et al., 2010, Yang et al., 2012]. The seismometer orientations of the OBS on the seafloor were estimated from the Rayleigh wave arrival azimuths that are determined from the polarization analysis of the Rayleigh waves [Stachnik et al., 2012]. All the OBS seismograms have been corrected for sensor orientations that were determined by Stachnik et al. [2012] using SAC software prior to processing data for splitting. Except for NZ01 and NZ17, all the other stations recorded good quality waveforms that can be used for observing splitting.

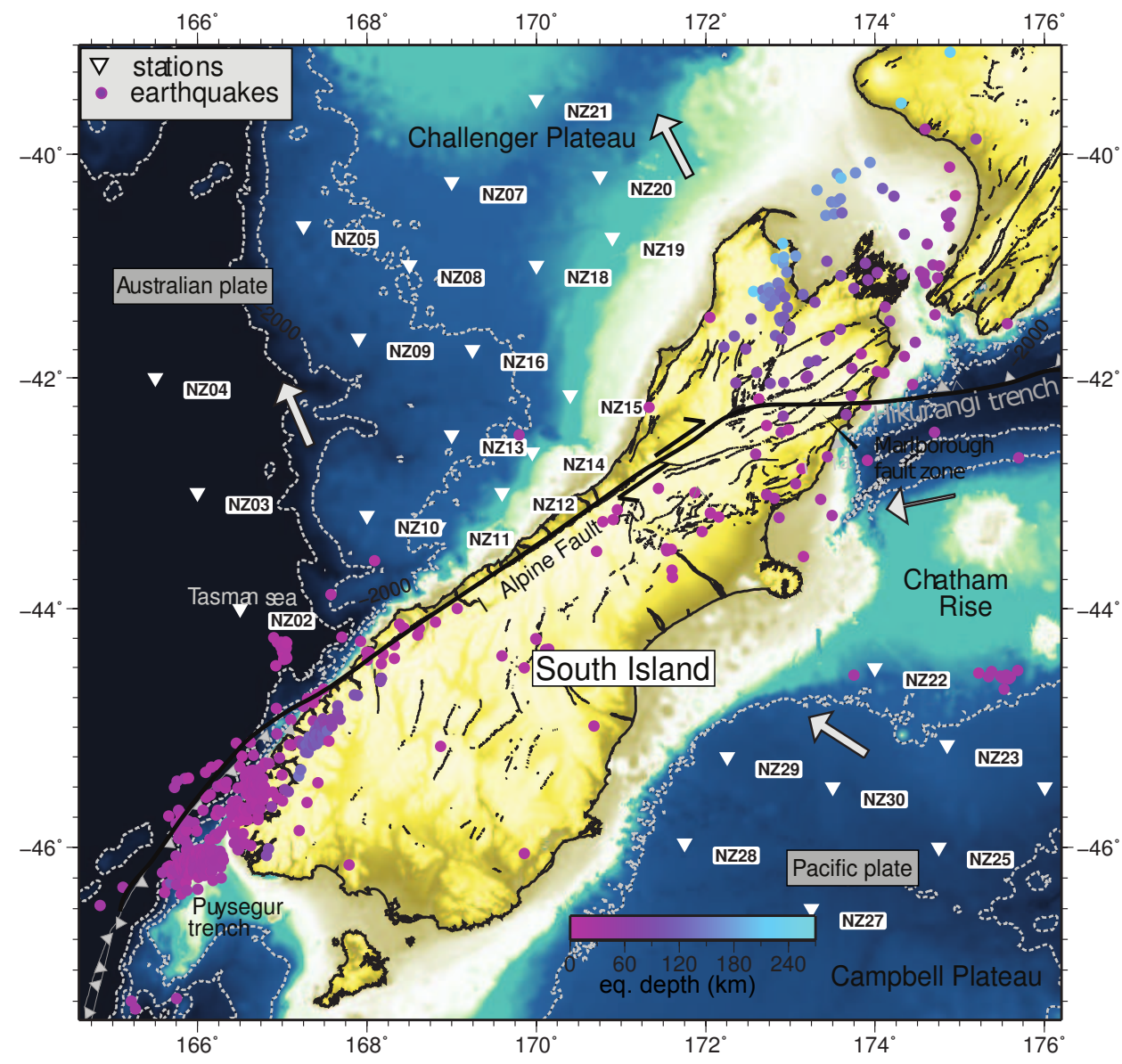

Figure 3.5 MOANA OBS network (white triangles). Circles indicate the events with AB quality splitting measurements and they are colored according to event depth. 


\section{Data selection}

Seismicity in the area is mostly distributed along the plate-boundary outside of the OBS network, roughly centering between the western and the eastern OBS deployments (Fig. 3.5). We used $\sim 2000$ S-phases from events with magnitude (M) $\geq$ 3 in western SI and $\mathrm{M} \geq 3.5$ in the eastern South Island. The event information in the GeoNet catalog from February 2009 - January 2010 was used to identify the events in OBS records. We have eliminated poor quality events and/or measurements when picking the S-phases. Because of the noisy nature of the OBS seismograms, S-waves were subjected to filtering using a range of two-pole Butterworth bandpass filters. In general, the majority of wave-forms consisted of high frequency $(0.5-10 \mathrm{~Hz})$ S-phases.

\subsubsection{Southern NI: Deep anisotropic structure beneath the Hikurangi subduction zone}

Focus: This study aims to expand the understanding of the deep anisotropic structure beneath the southern Hikurangi subduction zone using telesisemic S-phases recorded on the SAHKE broadband-transect across the southeastern NI.

\section{Experiment}

The main aim of the Seismic Array Hikurangi Experiment (SAHKE) is to investigate the tectonics and subduction interface processes at the southernmost part of the Hikurangi subduction margin. The project had both onshore (ARRAY, TRANSECT, WANGANUI, and NORTHERN lines) and offshore (OBSTRANSECT) deployments and aimed to record local, regional and global passive seismicity and regional on-land and offshore active source signals [Seward et al., 2010, 
Henrys et al., 2013]. For our study, we only use the teleseismic (Tel.) and regional passive seismic data recorded on the broadband transect (SAHKE transect) that extends from the center to the east-coast of the southern NI (Fig. 3.6). Shear-wave splitting is used to determine the anisotropy in this region. Tel. S, direct S, SKS, and ScS splitting was not well explored in the southeast of North Island by previous studies. Therefore, this study enables us to obtain a complementary set of splitting data to expand our knowledge on the anisotropic structure in Hikurangi subduction zone. Combining our measurements with the existing splitting measurements for the western region of the lower NI, we examine splitting anisotropy variations across the Hikurangi subduction zone.

The northwest-southeast oriented broadband transect consisted of 10 3-component seismographs. Most of these sites had CMG40T sensors, but two sites had CMG3ESP sensors (Fig. 3.6 and appendix C.1). Either Reftek-130s or Earth Data PR6-24 data loggers were used at each site, therefore both the PASSCAL utility package and integrated methods were used to convert RT130/mseed data to SAC format (appendix D). The broadband stations were installed between November 2009 and December 2010. Stations had variable and non-continuous recording periods $(\sim$ 6 -18 months). As a reference set of data, we have also investigated splitting from the teleseismic S-waves recorded between 2009 November and 2011 November at the permanent GeoNet broadband station (WEL), which is located in the Wellington region.

\section{Data selection}

Global events with magnitudes $\geq 6$ that were obtained from the IRIS earthquake catalog have been used to extract $\mathrm{SK}(\mathrm{K}) \mathrm{S}$, ScS and Tel. direct $\mathrm{S}$ arrivals from the seismograms. We used previously established event selection and filtering criteria from Marson-Pidgeon and Savage [1997] (table 3.2). These criteria have been 


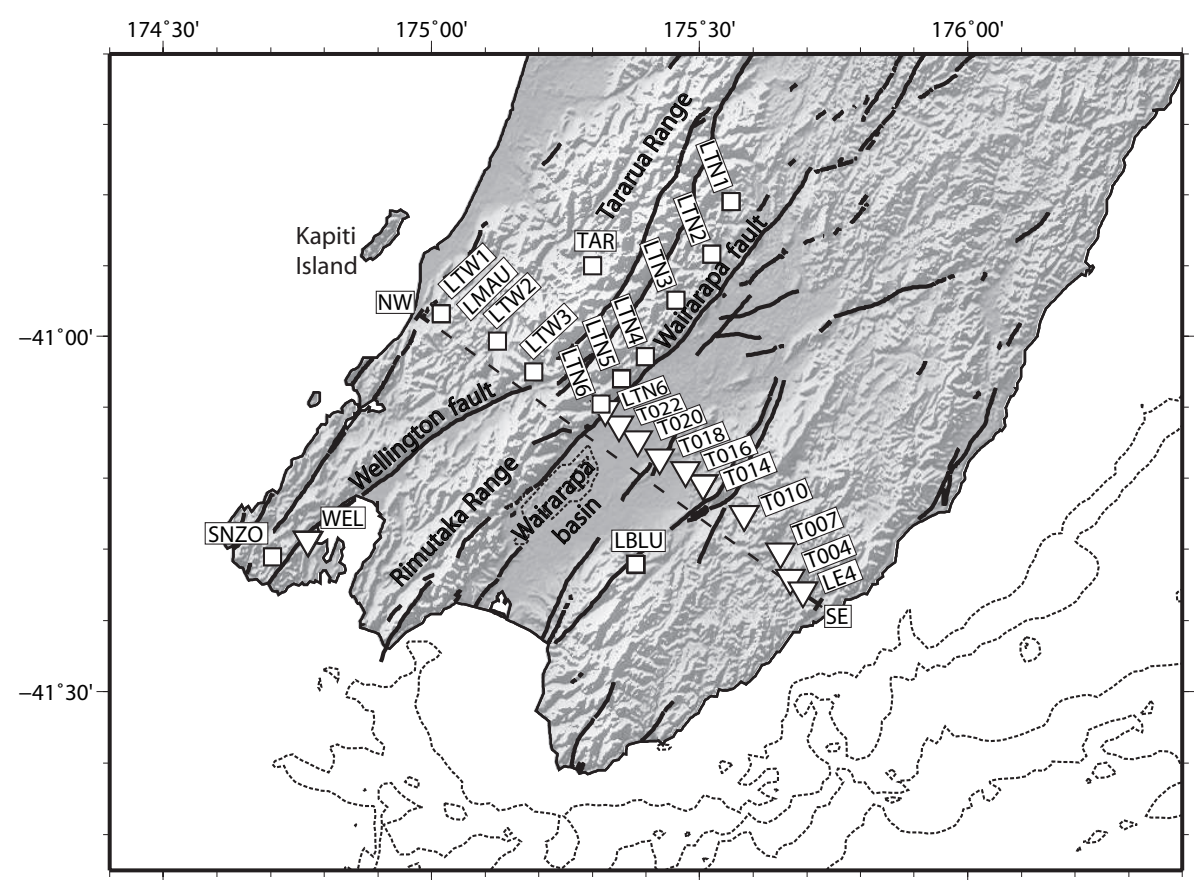

Figure 3.6 Locations of the SAHKE transect and WEL-GeoNET permanent station (white triangles). White squares display the locations (Tararua array and SNZO permanent station) of previous teleseismic-splitting studies in the southern NI. Black lines and dotted lines display the active faults and bathymetry respectively.

applied to examine the S-wave splitting anisotropy in Wellington region before and such selection criteria allow us to extract unambiguous phases and nearly vertical incident waves with less noise contamination than the raw records. The event selection, filtering criteria, and measurement information are given in the table 3.2.

The expected phase arrival times are identified using the Taup toolkit [Crotwell et al., 1999] and the actual arrivals are picked manually. Figure 3.7 displays ray paths from teleseismic events that produce good quality splitting parameters in the

Table 3.2 Event selection criteria for SKS phases and measurement information.

\begin{tabular}{|c||lllll|}
\hline phase & $\begin{array}{l}\text { searched } \\
\text { depths* }\end{array}$ & distance $\left(^{\circ}\right)$ & filter $(\mathbf{H z})$ & $\begin{array}{l}\text { no of mea- } \\
\text { surements }\end{array}$ & $\begin{array}{l}\text { min.depth } \\
(\mathbf{k m})\end{array}$ \\
\hline \hline SKS & all depths & $85-125$ & $0.04-0.1$ & 48 & - \\
ScS & $>390$ & $\leq 35$ & $0.1-0.3$ & 21 & $>400 \mathrm{~km}$ \\
Tel. S & $>200$ & $60-80$ & $0.04-0.1$ & 33 & $>450 \mathrm{~km}$ \\
\hline
\end{tabular}

*Search depth range for source side 
analysis. We used the transverse component minimization technique [Silver and Chan, 1991] to determine splitting parameters from SKS phases (see section 3.2.2). Splitting parameters of ScS and Tel. S phases are determined from the eigenvalue minimization technique (section 3.2.3).

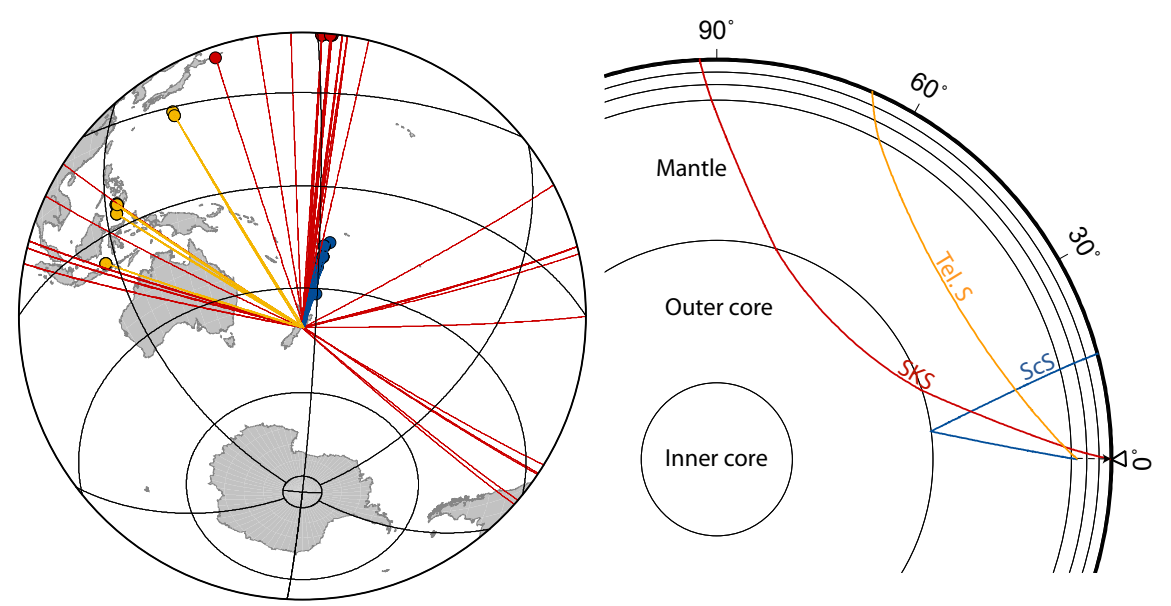

Figure 3.7 Ray paths distribution of SKS (red), ScS (blue), telesisemic S (yellow) around the SAHKE transect in Fig. 3.6 


\subsection{Methodology}

Shear-wave splitting is used to investigate seismic anisotropy in all three case studies. Different types of S-phases (local/regional S, teleseismic S, ScS and SKS) have been utilized to investigate anisotropy and, therefore, different S-wave splitting techniques (e.g. Transverse and eigenvalue minimization) are implemented to measure splitting parameters.

\subsubsection{Summary of different S-wave splitting analysis methods and their applications}

Seismic shear-wave measurements contain a range of frequencies and therefore Swave splitting can be sensitive to the structures of different scales-lengths [ $W u, 1982$, Long and Silver, 2009]. Both high-frequency and broad-band seismograms (Fig. 3.1) can be used to estimate shear-wave splitting parameters, but the splitting of highfrequency phases are more sensitive to the upper-most crustal anisotropy [Rumpker and Silver, 1998]. If the split S-phases exhibit a clear separation in the seismogram, splitting parameters can be easily estimated using a simple particle motion analysis [Vidale, 1986]. This is possible with most of the high-frequency S-phases, however such a simple approach is not suitable to determine splitting parameters from noisy

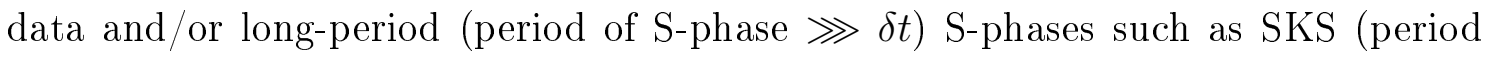
$>10$ s) [Vecsey et al., 2008, Long and Silver, 2009]. The various techniques of Swave splitting are available to study splitting parameters depending on the different types of S-phases used in the experiment (e.g. SKS, local/regional, teleseismic S and $\mathrm{ScS}$ etc.). Here we discuss some of the commonly used methods. Eigenvalue minimization and transverse component minimization techniques are discussed in detail in section 3.2.2 as the work presented here is primarily based on those two techniques. 


\section{Transverse component and eigenvalue minimization methods}

The transverse component and eigenvalue minimization methods are two of the commonly used techniques to determine shear-wave splitting parameters. The transverse component minimization technique is commonly used with SKS splitting analysis, whereas eigenvalue minimization is most often utilized in local and regional splitting analysis and is also applicable to teleseismic splitting. Commonly used splitting techniques, such as SplitLab and Mfast, are based on the transverse component and eigenvalue minimization methods. Although these two methods are first introduced in some early literature (e.g., Silver and Chan [1988]), detailed information on the methodology is given in Silver and Chan [1991]. Both methods use grid searches over splitting parameters $(\delta \mathrm{t}$ and $\phi)$ to find the pair that best minimize the energy on the transverse component or the pair that best linearize the corrected particle motion [Long and Silver, 2009]. The theoretical explanation of these two methods is given in section 3.2.2.

\section{Cross-correlation method}

This method assumes that the two split S-phases in anisotropic media exhibit similar shape waveforms. As explained by Bowman and Ando [1987], the method involves usage of particle motion plots of horizontal components, the rotation of horizontal components and calculation of the cross correlation coefficient between those two components for time lags with constant intervals (usually ranging from +1 to $-1 \mathrm{~s}$ ). The angle and time lag that gives the maximum correlation coefficients determine the polarization direction of the fast S-wave and delay time between two split phases [Fukao, 1984]. Similar to the eigenvalue minimization method, this method utilizes horizontal particle motion plots, which are created in the geographic coordinate system to check the linearity of particle motion when the correlation coefficient is maximized. 


\section{Splitting intensity method}

Chevrot [2000] developed this technique to analyze splitting parameters from multi-events and this method is complementary to the transverse minimization technique [Silver and Chan, 1988, 1991], which applies to single records. The method defines a splitting intensity $(\mathbf{I})$, which is a measure of amplitude of the transverse component relative to the derivative of the radial component. I can be derived from the relationship given in equation 3.10 [Vinnik et al., 1989b] for an individual record, defining splitting intensity as: $\mathbf{I}=\delta t \sin 2 \beta$, where $\beta$ is the angle between $\phi$ and initial polarization direction $\left(\phi_{p}\right)$. I is then determined from the singular value decomposition (SVD) procedure discussed in Chevrot [2000]. It requires a wide range of $\phi_{p}$ (BAZ in the case of SKS splitting investigations) to determine splitting parameters accurately. $\mathbf{I}$ is plotted with respect to $\phi_{p}$ (also known as the splitting vector; Long and Silver [2009]) to find the best fitting $\sin 2 \beta$ curve and to determine $\delta t$ and $\phi$. The amplitude of this sinusoidal curve is equivalent to $\delta t$ and $\phi$ can be determined from the phase at the origin. The main advantage of the splitting intensity method is that unlike the splitting operator [Silver and Chan, 1991], it is commutative and thus it follows the main assumption of single homogeneous anisotropy approximation. As shown by Silver and Long [2011], splitting intensity, therefore, will not provide information on the apparent splitting parameters (or the complex anisotropy) as a splitting operator does (section 3.2.2). This method is ideal for tomography inversions as it assumes the homogeneous single layer anisotropy [Silver and Long, 2011], but results could be ambiguous because the splitting intensity method cannot distinguish in which order layers appear. 


\subsubsection{Transverse component and eigenvalue minimization: theoretical overview}

Eigenvalue minimization and transverse component minimization methods by [Silver and Chan, 1991] (here after SC91) assume that the shear-wave splits into two orthogonally polarized (radial and transverse or fast and slow) quasi S-phases as it traverses through an anisotropic medium and therefore, exhibits elliptical particle motion, indicating energy on both horizontal components.

\section{Eigenvalue minimization method:}

This method uses the same approach implemented in Vidale [1986], which analyzes the polarization directions of three component seismographs using a covariance matrix of the analytic signals and calculates eigenvectors associated with three eigenvalues that corresponds to the particle motion directions of each component. But in the splitting analysis, only two orthogonally polarized horizontal-components are considered.

If a shear wave $(\tilde{u}(\omega))$ propagated through length $L$ in an isotropic, homogeneous medium and reaches the surface at $T_{0}$ time, its propagation can be represented mathematically as (SC91):

$$
\tilde{u}(\omega)=\mathbf{A} \Psi(\omega) \exp \left[-i \omega T_{0}\right]
$$

where, A and $\Psi(\omega)$ are the complex vector amplitude and the wavelet function, which is a function of frequency $(\omega)$, respectively. A can be approximated by complex scaler $(A)$ and real unit vector $(\hat{p})$ that indicates the polarization direction of the S-wave (i.e. $\hat{\mathbf{p}}$ is perpendicular to the wave propagation direction). By replacing $\mathbf{A}$ with $A \hat{\mathbf{p}}$, equation 3.1 can be rearranged as: 


$$
\tilde{u}(\omega)=\Psi(\omega) \exp \left[-i \omega T_{0}\right] \hat{\mathbf{p}}
$$

Then, the covariance matrix $\left(c_{i j}(\phi, \delta t)\right)$ between the two split S-waves $\left(u_{i}(t)\right.$ and $\left.u_{j}(t-\delta t)\right)$ in an anisotropic medium, arriving at the surface with the time lag $(\delta t)$ is given by:

$$
c_{i j}(\phi, \delta t)=\int_{-\infty}^{\infty} u_{i}(t) u_{j}(t-\delta t) d t
$$

Here, $\phi$ defines the fast polarization direction with respect to $\hat{\mathbf{p}}$. SC91 method defines an inverse splitting operator $\left(\Gamma^{-1}\right)$ which reverses the split phases into unsplit stage. Splitting operator $\Gamma$ denotes the splitting of S-wave into fast and slow waves with time lag of $\delta t$ and mutually perpendicular polarization directions of $\hat{\mathbf{f}}$ and $\hat{\mathbf{s}}$. The mathematical explanation of the $\Gamma$ as given in SC91:

$$
\Gamma_{(\phi, \delta t)} \equiv \exp [i \omega \delta t / 2] \hat{\mathbf{f}} \hat{\mathbf{f}}+\exp [-i \omega \delta t / 2] \hat{\mathbf{s}} \hat{\mathbf{s}}
$$

Equation 3.2 can, therefore, be redefined incorporating $\Gamma$ to denote a split shear wave $\left(u_{s}(\omega)\right)$ as:

$$
u_{s}(\omega)=\Psi(\omega) \exp \left[-i \omega T_{0}\right] \Gamma_{(\phi, \delta t)} \hat{\mathbf{p}}
$$

To compact the splitting operator, a time difference tensor is defined as [Silver and Chan, 1991, Walsh, 2012]: $\delta T=\delta t / 2(\hat{\mathbf{f}} \hat{\mathbf{f}}-\hat{\mathbf{s}} \hat{\mathbf{s}})$ and, thus, the equation 3.4 can be re-written as:

$$
\Gamma_{(\phi, \delta t)} \equiv \exp \left[i \omega \delta T_{(\phi, \delta t)}\right]
$$


Correction for splitting is done by searching for the inverse splitting operator that successfully reverses splitting to its unsplit stage $(\tilde{u}(\omega))$ :

$$
\tilde{u}(\omega)=\Gamma^{-1} * u_{s}(\omega)
$$

In the absence of anisotropy or if the the seismogram is fully corrected for splitting, $c_{i j}(\phi, \delta t)$ (equation 3.3) will only have one eigenvalue and its eigenvector is equivalent to the initial polarization direction $\left(\hat{p}=\phi_{p}\right) . c_{i j}(\phi, \delta t)$ will have two eigenvalues, if there are two split shear-waves and the eigenvector associated with the smaller eigenvalue $\left(\lambda_{2}\right)$ is equal to the $\phi$. The best $\Gamma^{-1}$ is determined from the eigenvalues of the $c_{i j}(\phi, \delta t)$ that are calculated from the rotated and shifted seismograms over every possible pair of $\phi$ and $\delta t$ using the grid search approach (Fig. 3.8-3).

Because of the noise associated with seismic records, this method looks for the pair $\left(\lambda_{1}\right.$ and $\left.\lambda_{2}\right)$ with the smallest $\lambda_{2(\min )}$ (or the most nearly singular matrix) to define the best splitting parameters $(\phi, \delta t)$ and the inverse splitting operator that best resolves for splitting (Fig. 3.8). Unlike other methods, this method can be used to estimate splitting parameters even if the initial polarization direction $\left(\phi_{p}\right)$ is not known and it is commonly used to examine splitting parameters from local and regional shear-waves. The minimization of $\lambda_{2}$ is equivalent to maximizing the $\lambda_{1} / \lambda_{2}$ as in the aspect ratio method [Shih et al., 1989]. Maximizing correlation coefficients of the cross-correlated S-waves as in Bowman and Ando [1987] is also equivalent to maximizing the determinant of the $c_{i j}(\phi, \delta t)$ [Silver and Chan, 1991]. The corrected S-wave is rotated into its initial polarization direction to achieve the original state of the S-wave. Unlike in SKS phases, $\phi_{p}$ direction of the local and regional S-waves is not known. The eigenvector of the larger eigenvalue, when the other one is minimized, gives the $\phi_{p}$. 


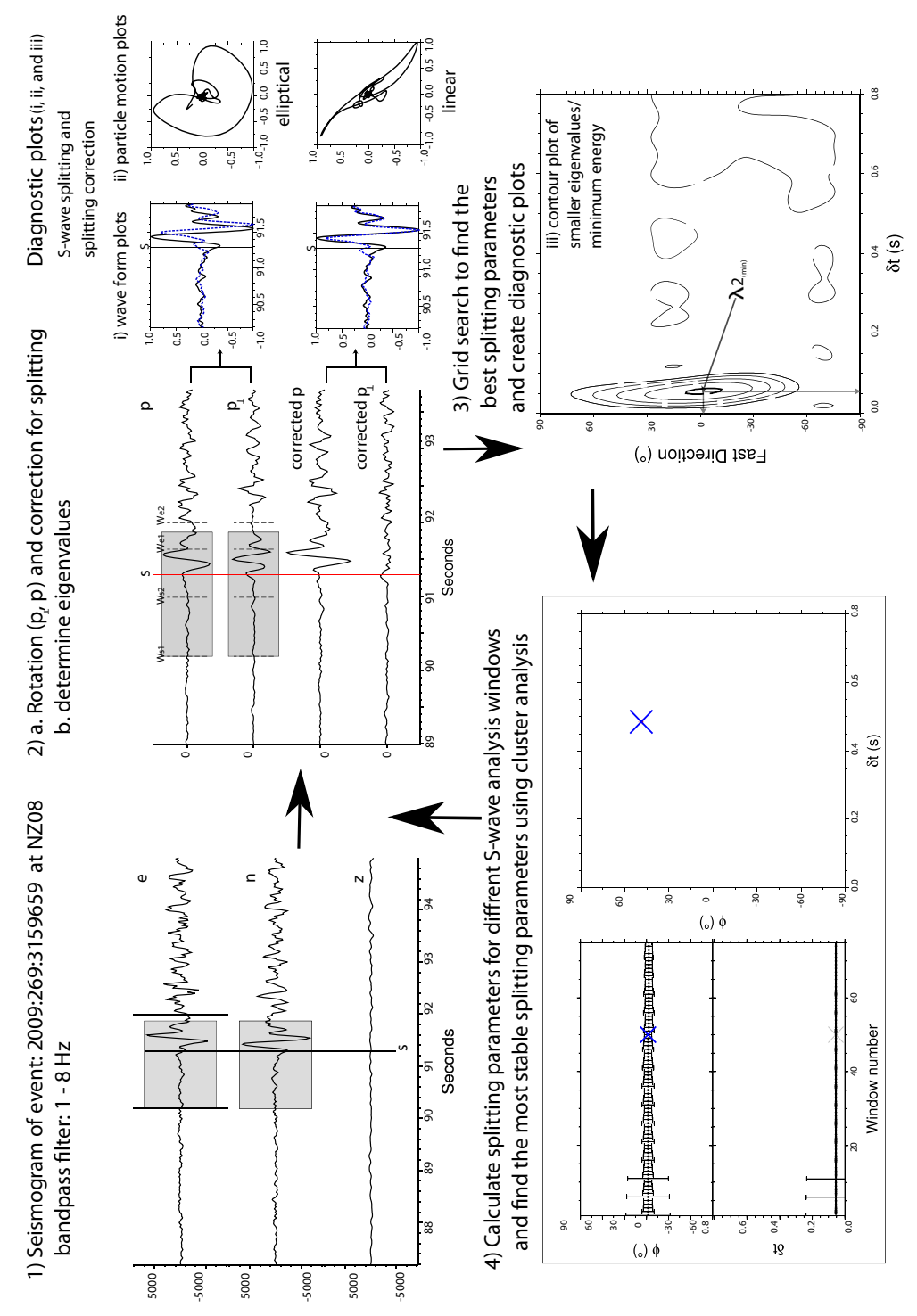

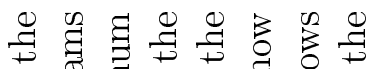
品

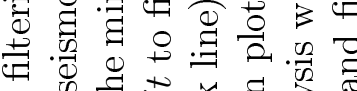

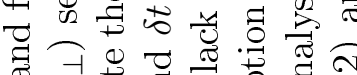
है

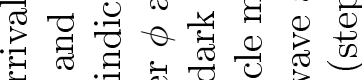

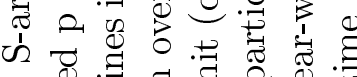

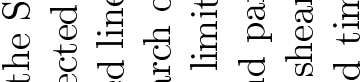
so

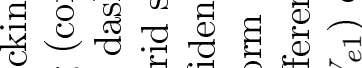

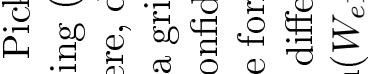

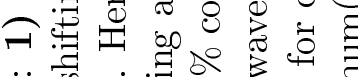

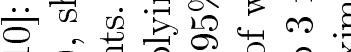

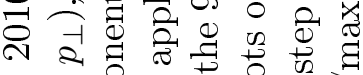
उं चै $N . 尹$

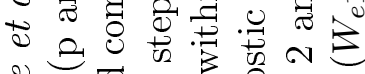
ऽ) ह 100

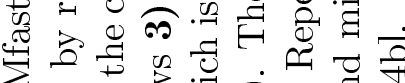

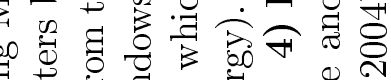

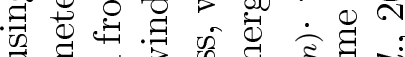
का व

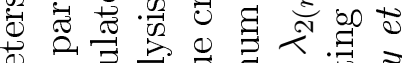

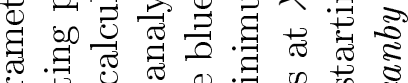
运. bo की

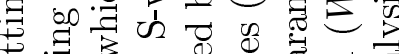

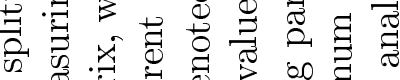

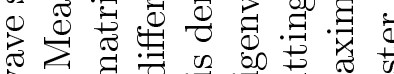
年 कर

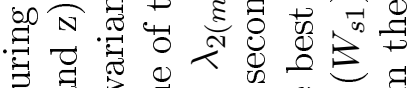
कू ध

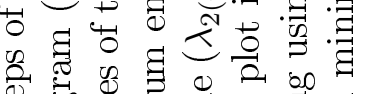
फ

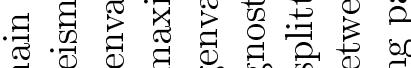
a क .00 .000 की 苛

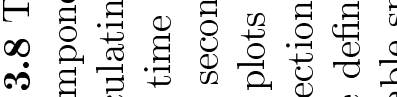

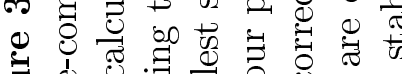

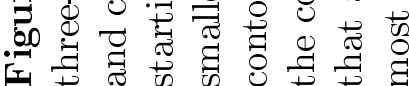




\section{Transverse component minimization method:}

Transverse component minimization is a frequently used approach to determine splitting parameters from core phases, such as SKS and SKKS. As a result of the P-to-S conversion at the core-mantle boundary (CMB), when SK(K)S phases propagate through the receiver side CMB, the phases exhibit no splitting or energy on the transverse component that remains from the source side anisotropy. Thus, in the case of $\mathrm{SK}(\mathrm{K}) \mathrm{S}$ splitting $\phi_{p}$ is already known and it is equivalent to the direction of radial polarization.

If a radially polarized S-wave traverses through an anisotropic medium, S-wave splitting results in energy on the both radial $\left(u_{r}\right)$ and transverse $\left(u_{t}\right)$ components. As describe in [Silver and Chan, 1988], the horizontal projection of these two components on the RTZ coordinate system can be expressed as a functions of the radial wave in the unsplit stage $\left(\tilde{u}_{r}\right), \delta t$ and $\phi$ as:

$$
\begin{array}{r}
u_{r}(t)=\tilde{u}_{r}(t) \cos ^{2} \phi+\tilde{u}_{r}(t-\delta t) \cos ^{2} \phi \\
u_{t}(t)=-\frac{1}{2} \sin 2 \phi\left[\tilde{u}_{r}(t)-\tilde{u}_{r}(t-\delta t)\right]
\end{array}
$$

As derived by Vinnik et al. [1989a], time derivative of $u_{r}(t)\left(=\grave{u}_{r}(t)\right)$ is a function of $u_{t}(t)$.

$$
u_{t}(t) \simeq-\frac{1}{2} \delta t \sin (2 \phi) \grave{u}_{r}(t)
$$

$\delta t$ will be zero in the absence of anisotropy and thus component $u_{t}(t)$ does not exist and the energy on the $u_{t}(t) \simeq 0$. Before splitting, the initial polarization direction $\left(\phi_{p}\right)$ of SKS phase can be approximated as the radial polarization direction and it is equal to BAZ direction. Therefore, the seismogram is first rotated to radial $(\mathrm{R})$ and transverse $(\mathrm{T})$ directions and a grid search is performed to minimize the energy on the corrected $u_{t}(t)$. Since SKS splitting usually produces large $\delta t$ compared to local and regional splitting, the grid search of $\phi$ and $\delta t$ is done with $1^{\circ}$ and $0.05 \mathrm{~s}$ 
increments respectively. Instead of minimizing $\lambda_{2}$ as in the eigenvalue minimization, one can perform a grid search to find the minimum energy on the corrected transverse component $\left(\tilde{u}_{t}(t)\right)$, calculating the energy $E_{t}(\phi, \delta t)$ on th $\left(\tilde{u}_{t}(t)\right)$ for each $\phi, \delta t$-pair using the following estimation:

$$
E_{t}(\phi, \delta t)=\frac{1}{T} \int_{0}^{T} \tilde{u}_{t}^{2}(t) d t
$$

\section{Error estimation}

Errors of the best splitting parameters $(d \phi$ and $d \delta t)$ are determined from the $95 \%$ confidence region in the $2-\mathrm{D}$ contour plots $(\phi$ and $\delta t)$ of $\lambda_{2(\min )}$ or minimum energy $\left(E_{t}^{\min }(\phi, \delta)\right)$. Silver and Chan [1991] demonstrated that $\lambda_{2}$ is a measure of variance of the noise. Thus, the confidence region of the $\lambda_{2(\min )}$ or minimum energy can be used to constrain the errors of the splitting parameters. $\lambda_{2(\min )}$ values within the $95 \%$ confidence region satisfy the following relationship, determined from the F-distribution [Silver and Chan, 1991].

$$
\frac{\lambda_{2}}{\lambda_{2(\min )}} \leq 1+\frac{k}{\nu-k} F_{k, \nu-k}(1-\alpha)
$$

Where, $\nu$ is the degrees of freedom, $\alpha$ is the $95 \%$ confidence interval $(=0.05)$, and $k$ is the number of free parameters $(\mathrm{k}=2: \phi$ and $\delta t) . F_{k, \nu-k}(1-\alpha)$ is the inverse of the F-distribution. $\nu$ depends on the instrument response and noise spectrum and it can be determined from the data as it is a measure of number of uncorrelated time points in the digital-time series [Silver and Chan, 1988]. Errors of the best $\phi$ and $\delta t$, are the maximum lengths of the $95 \%$ confidence interval along the $\phi$ and $\delta t$ axes respectively (contour plot in Fig. 3.8). A detailed explanation on this error estimation is given in Walsh [2012]. 
In the case of transverse energy minimization, $95 \%$ confidence region of the $\phi$ and $\delta t$ values agrees with the relationship: $E_{t}(\phi, \delta t) / E_{t}^{\min }(\phi, \delta t)<F_{\alpha}(\nu, \nu)$, where, $E_{t}^{\min }(\phi, \delta t)$ is the threshold limit of the $95 \%$ confidence $(\alpha=0.05)$ and $\nu$ is the degrees of freedom [Silver and Chan, 1988]. This error calculation of SC91 is being re-evaluated by [Walsh et al.], but this thesis uses the original error estimation procedure.

\subsubsection{Mfast}

The Mfast technique [Wessel, 2010, Savage et al., 2010] is a fully-automated shear-wave splitting software, which was developed on the basis of the eigen-value minimization method [Silver and Chan, 1991] and the cluster analysis method [Teanby et al., 2004b]. The method is suitable for the routine analysis of splitting measurements on a large set of local and regional events.

Before processing data with Mfast, S arrivals are hand picked on the 3-component seismograms with the help of expected arrival times that are determined using the Taup toolkit [Crotwell et al., 1999]. During picking, clearly visible poor quality seismograms can be eliminated. The first step of Mfast involves searching over a given set of 14 band pass filters, which are two-pole Butterworth filters with frequency band-widths within the range of $0.4 \mathrm{~Hz}$ to $10 \mathrm{~Hz}$, and calculating the signal-to-noise ratio (SNR) to find filters that give the highest SNR-bandwidth product $(\geq 3)$ for each earthquake-station pair.

- SNR: The SNR of the filtered wave is determined from the 3 second window lengths relative to the $\mathrm{S}$ pick $(\mathrm{t}=0 \mathrm{~s}$ ) before (noise) and after (signal). Mfast assumes $0.05 \mathrm{~s} \mathrm{~S}$ arrival errors and, thus, noise window ranges from -3.05 to $-0.05 \mathrm{~s}$ and signal window ranges from 0.05 to $3.05 \mathrm{~s}$. SNR is calculated taking the minimum amplitude ratios of the Fast Fourier Transform of the noise and 
the signal windows.

Then the signals obtained from the selected filters (maximum up to three) are used to estimate the splitting parameters. The dominant period of each S-phase is determined from the frequency that corresponds to the frequency with the highest amplitude of the amplitude spectrum $\left(f_{d}\right)$. The amplitude spectrum is calculated from the three second window directly after the S-pick. This period is then used to determine the minimum and maximum cutoff limits of the shear-wave analysis window. The main steps associated with the Mfast are shown in Fig. 3.9.

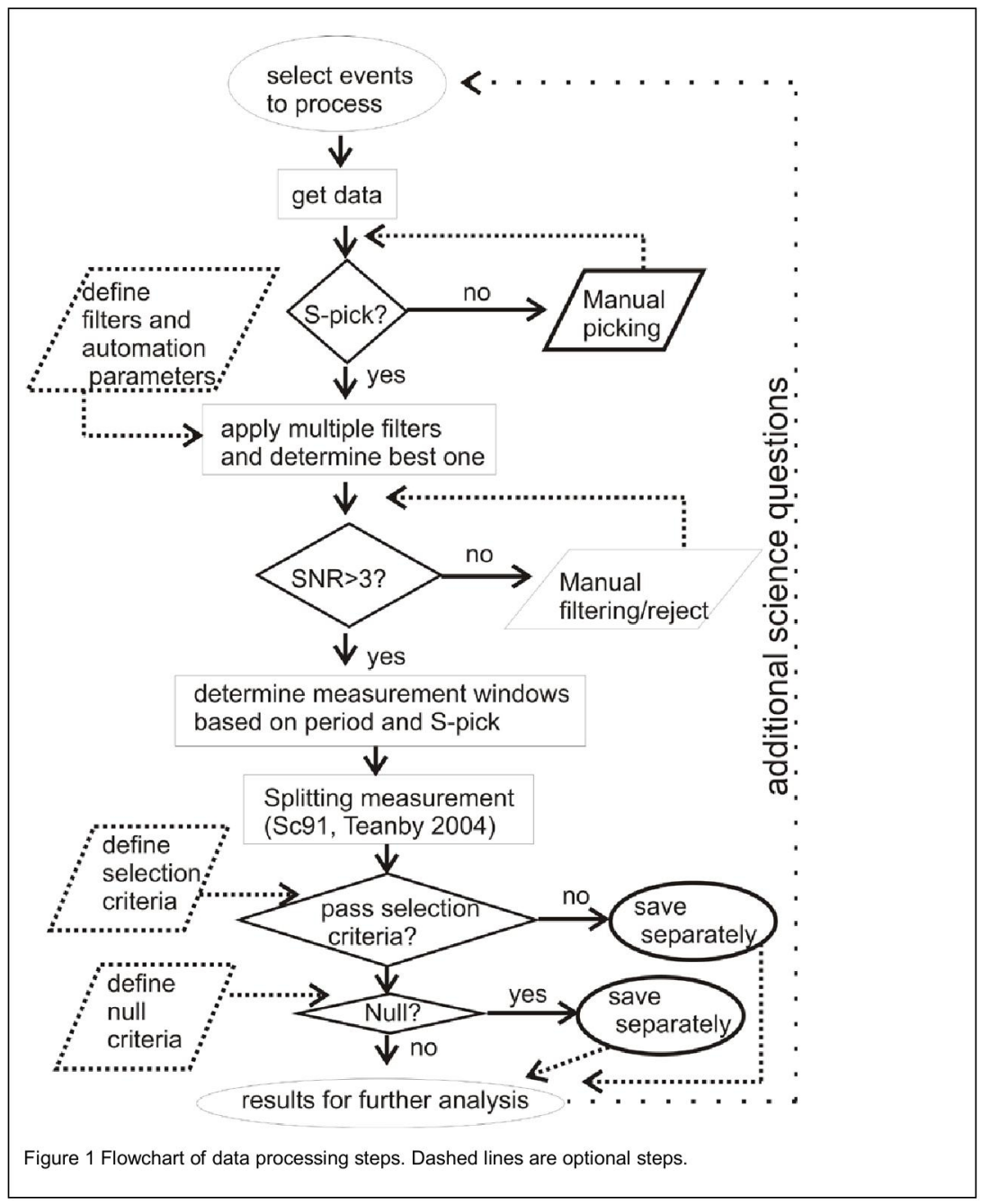

Figure 3.9 Processing steps of the Mfast program [after Savage et al., 2010].

Cluster analysis minimizes uncertainties in the splitting parameters that are caused by the selection of S-wave analysis windows [Teanby et al., 2004b]. This method 
finds the analysis window that gives the most stable splitting parameters with small errors by performing splitting measurements through a given set of analysis windows that are defined between minimum $\left(W_{s 1}\right) /$ maximum $\left(W_{s 2}\right)$ starting times and minimum $\left(W_{e 1}\right) / \operatorname{maximum}\left(W_{e 2}\right)$ end times (Fig. 3.10). Then the most stable splitting parameters are chosen by applying the cluster grading criteria.

Mfast uses several grading criteria to extract good quality splitting measurements, to minimize the filtering effects on the splitting measurements, and to minimize the scatter in splitting parameters at a single station. Depending on the purpose of analysis, it is possible to use one or more grading schemes for better evaluation of the splitting measurements. The grading criteria used in this study are discussed below.

\section{Grading based on the cluster grading and errors associated with splitting measurements}

AB graded measurements: The splitting parameters that are given as A and/or B grade criterion (here after AB grade), which is defined in Savage et al. [2010], are considered as good quality splitting measurements. Both cluster parameters [Teanby et al., 2004b] and measurement errors have been used to define these grades. We only used $\mathrm{AB}$ grade measurements to investigate the anisotropic structure. $\mathrm{AB}$ grading agrees with the following criteria:

- $\mathrm{SNR}>3, \delta t<0.8^{*} \operatorname{tlag}_{\max }\left(\operatorname{tlag}_{\max }\right.$ is the maximum $\delta t$ for grid search), maximum error in $\phi<25^{\circ}$, if it is not a null measurement, and if the measurement graded as a A or B cluster in the cluster grading. Note that the A grade criteria (a. SNR $>4$, b. $\delta t<0.8^{*} \operatorname{tlag}_{\max }$, c. not null and $d \phi<10^{\circ}$ ) are a subset of AB grade criteria, which use more narrow limits for SNR and $\mathrm{d} \phi$ compared to $\mathrm{AB}$ grade criteria. 
- Null measurements are those for which $\phi_{p}$ is within $20^{\circ}$ of parallel or perpendicular to $\phi$ (Fig. 3.13). Such measurements can lead to incorrect $\delta t$ (section 3.3.1).

- B cluster grade [Savage et al., 2010] has $\operatorname{var}(\mathrm{k})<5 * \operatorname{var}(\mathrm{k})_{\text {best }}$ and $\mathrm{N}_{\text {meas }}(\mathrm{k})$ $>\mathrm{N}_{\text {cmin(5) }}$ and also $\mathrm{t}_{\text {diff }}(\mathrm{k})>\operatorname{tlag}_{\max } / 8$ or $180^{\circ} / 8<\operatorname{phi}_{\text {diff }}(\mathrm{k})<7 * 180^{\circ} / 8$ (where $\mathrm{k}, \operatorname{var}(\mathrm{k}), \operatorname{var}(\mathrm{k})_{\text {best }}, \mathrm{N}_{\text {meas }}(\mathrm{k}), \mathrm{N}_{\text {cmin }(5)}, \mathrm{t}_{\text {diff }}(\mathrm{k})$ and phi $_{\text {diff }}(\mathrm{k})$ are the cluster number, average variance, variance of the best cluster, no of measurements in the cluster, minimum no of events per cluster $(=5)$, time difference, and angular distance respectively within cluster $\mathrm{k})$. Here, phi ${ }_{d i f f}=$ $\left|\operatorname{phi}(\mathrm{k})-\operatorname{phi}(\mathrm{k})_{\text {best }}\right|$ and $t_{\text {diff }}=\left|\mathrm{t}(\mathrm{k})-\mathrm{t}(\mathrm{k})_{\text {best }}\right|$. Note that the A grade cluster is a sub-cluster of the B grade cluster.

\section{Stable splitting measurements from different filters}

If a measurement agrees with the above quality scheme, one can extract the splitting measurements that give the most stable measurements for different filters. However, such a grading is not recommended for a study examining frequency dependent splitting using the same waveform, but different filters. This criterion extracts the measurements that give a similar answer, i.e. if the time difference $<\operatorname{tlag}_{\max } / 8$ or if the angular distance $<\pi / 8$, for each filter (refers to 'best filter measurements' in later sections).

\section{Highest quality splitting parameters}

This criterion allows us to extract very well constrained splitting measurements with dense error contours around the solution that satisfies the AB best filter grading criteria (as above). Error contours represent the contours of the minimum eigenvalues or the low energy surfaces. Savage et al. [2010] defines $\lambda_{\max }$ as the value that corresponds to maximum error contour when the contours around the $95 \%$ confidence level are re-scaled assuming that $95 \%$ confidence contour is 1 . The highest quality measurements defined in chapter 4 are those of which have A grade best filter 
measurements and $\lambda_{\max }>8$ (the highest quality). In chapter 5, measurements with $\lambda_{\max }>5$ are chosen as high quality measurements.

\section{Teanby et al. [2004b] and Mfast cluster analysis}

Teanby et al. [2004b] conducts shear-wave splitting measurements on several analysis windows $\left(N_{\text {total }}\right)$ that are defined using the set parameters in the configuration file. Mfast modified this method to generate a customized configuration file for each event considering the dominant frequency (section 3.2.3) of the $\mathrm{S}$ signal and the $\mathrm{S}$ arrival time.

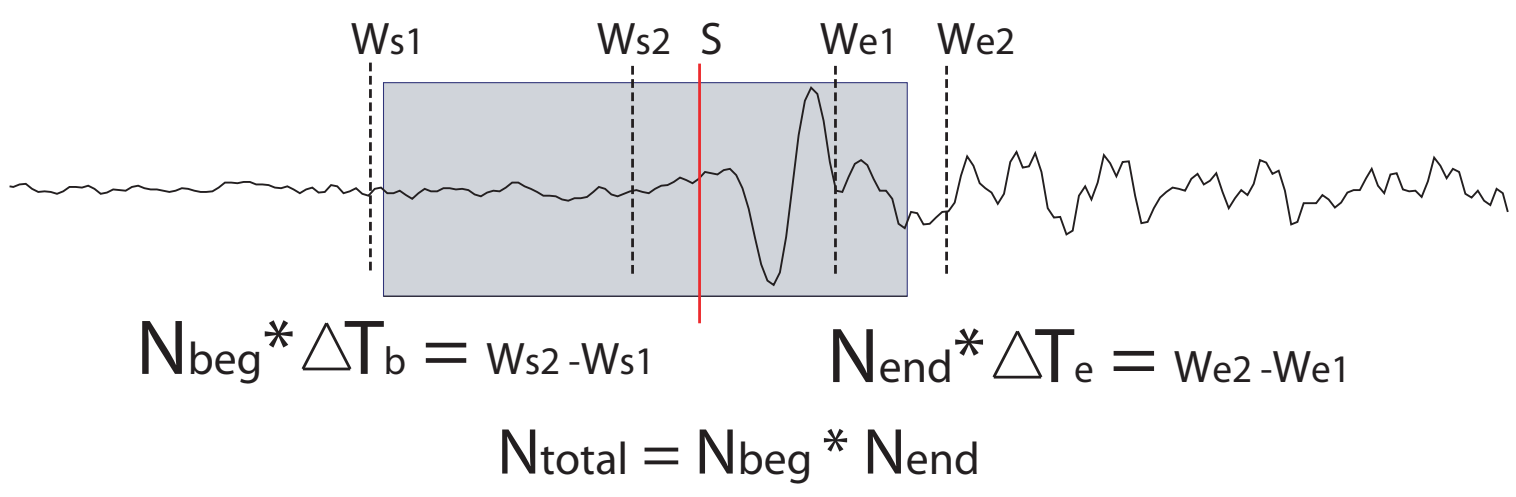

Figure 3.10 Defining a set of S-wave splitting analysis widows relative to S-arrival.

The number of start-time windows $\left(N_{b e g}\right)$ are defined between $W_{s 1}$ and $W_{s 2}$ with the steps of $\Delta T_{b}$ and the number of end-time $\left(N_{\text {end }}\right)$ windows are defined between $W_{e 1}$ and $W_{e 2}$ with $\Delta T_{e}$ steps relative to the S-pick (Fig. 3.10). The minimum and maximum window lengths are chosen to be $1 \mathrm{~s}\left(1 / f_{d}\right)$ and $2.5 \mathrm{~s}$ long respectively for local/regional splitting analysis (Fig. 3.10).

After measuring best splitting parameters on $N_{\text {total }}$ number of splitting analysis windows, clusters of similar splitting measurements are identified from the criteria based on the variance of clusters and the individual measurements [Teanby et al., 2004b]. These clusters can be identified on the $\phi$ versus $\delta t$ plots (measurement showen in Fig. 3.11-a has 6 clusters). The best cluster is identified as the cluster with 
2004.102.MLZ.2213304.0.2-3.fb1
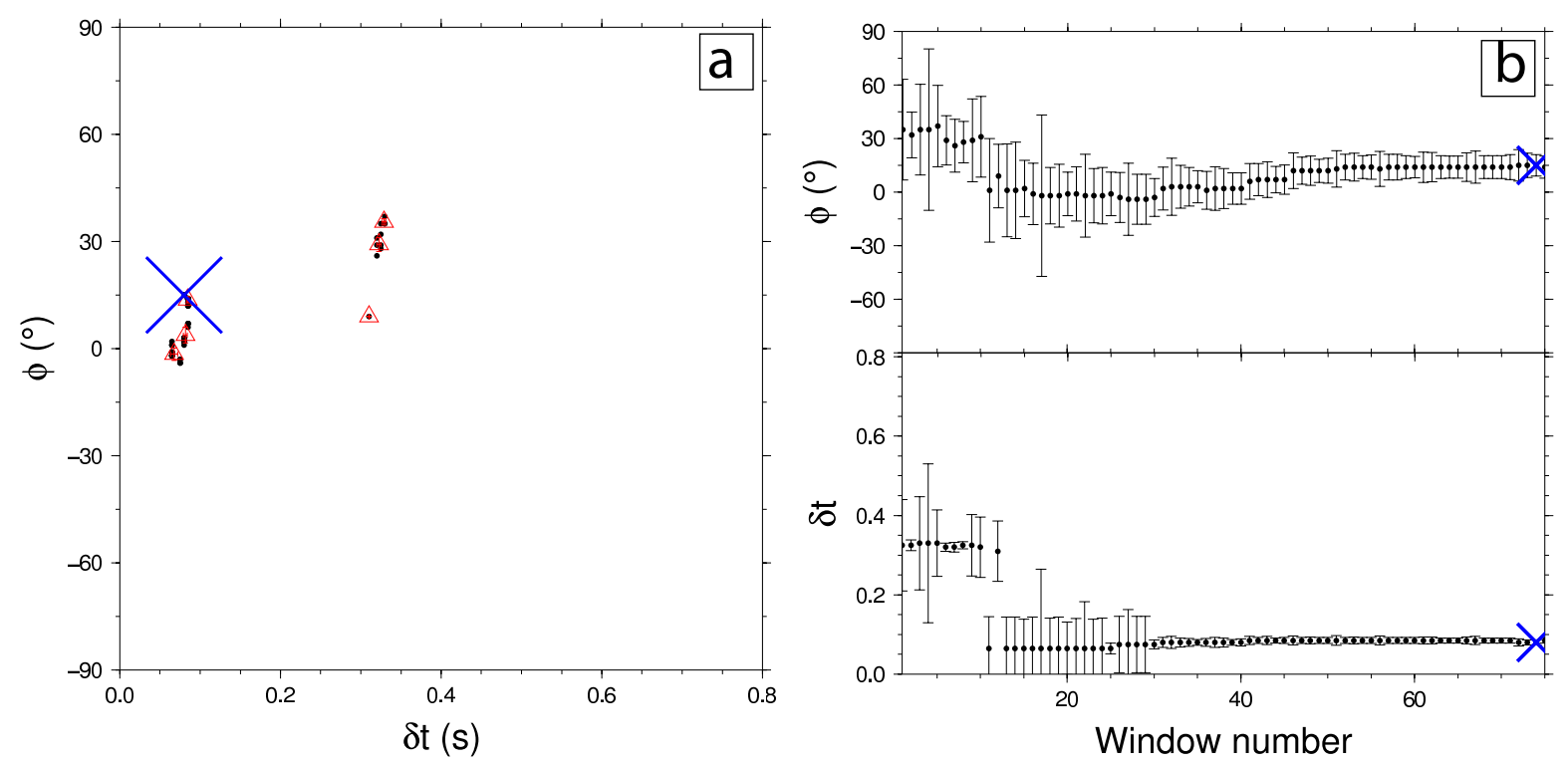

Figure 3.11 (a) Stable cluster regions (6 red triangles) that are determined from the cluster analysis [Teanby et al., 2004b]. The best cluster is marked by the blue cross. (b) Splitting parameters versus window number. The most stable measurement with in the best cluster has the minimum splitting errors compared to other measurements.

the lowest variance and low individual measurement errors. Thus, the best splitting measurements are extracted from the best cluster by finding the measurement with the smallest $\phi$ and $\delta t$ errors (Fig. 3.11-b).

Mfast incorporates the cluster analysis parameters of the best cluster and the other clusters (e.g. cluster number, average variance, variance of the best cluster, number of measurements in each cluster, time difference and angular distance between best cluster and the other clusters) to grade the splitting measurements of each event as one of the quality control steps (B cluster grade is explained in the section 3.2.3). This grading compares the properties of the best cluster with the other clusters and removes the measurements with similar cluster properties to the best cluster, but with different splitting measurements [Savage et al., 2010]. 


\subsection{Splitting parameters: limitations, quality and statistics}

Seismograms with clear S-wave onset are preferred to a obtain good splitting measurements. However, in most of the cases seismograms display complicated waveforms due to complexities in the subsurface structures and/or interaction of the wave forms at the interfaces of the layered media. Sometimes even if the seismogram has a clear S arrival, splitting measurements can be distorted by different other phenomena (section 3.3.1).

The polarization of an S-wave incident at the free surface is governed by many factors such as the angle of incidence, frequency of the incident wave, the source depth, and the layering of the crust [Booth and Crampin, 1985]. To minimize the effect of incidence angle on S-wave polarization, splitting analysis is usually carried out inside the shear-wave window (i.e. a few degrees less than critical incidence angle) [Evans, 1984, Nuttli, 1961]. Wave fronts with incident angles beyond the critical angle $\left(i_{c}=\sin ^{-1}\left[V_{s} / V_{p}\right]\right.$, where $V_{s}$ and $V_{p}$ are the surface $\mathrm{S}$ and $\mathrm{P}$ velocities) are likely to display complex particle motion due to phase conversions at the free surface. This will cause particle motion to be non-linear even in the absence of anisotropy [Nuttli, 1961] and make the interpretation of S-wave polarization complicated. Thus, splitting analysis is usually restricted to incident angles less than the $i_{c}$. However, the $i_{c}$ can be greater than $35^{\circ}$ due to curvature of the wave front of the local ray paths in the presence of low-velocity surface layer [Booth and Crampin, 1985]. In on-land investigation, we assume a low-velocity surface layer with $2.6 \mathrm{~km} / \mathrm{s}$ S velocity, by taking into account proposed velocity models for South Island [Eberhart-Phillips and Bannister, 2010, Eberhart-Phillips and Reyners, 2001]. In South Island, most of the seismicity is limited to the plate-boundary zone and this narrow distribution significantly reduces the number of local events that can be used to measure the crustal anisotropy far away from the plate-boundary. Thus, the average velocity structure allows us to obtain splitting parameters from ray paths 
with larger epicentral distances. Although these long ray paths of local/regional S-waves reach the surface sub-vertically, they are likely to attain a horizontal/subhorizontal propagation when considering the full curved ray-paths. These subhorizontal/horizontal ray segments can be sensitive to horizontal anisotropic layers with vertical symmetry axis [Paulssen, 2004], resulting in an integrated effect of anisotropy with different symmetry configurations along the ray paths.

As discussed in the section 3.1, ambiguous splitting parameters are primarily caused by the noise in the seimogram. The application of a proper filter can minimize the effect due to noise and enhance the SNR prior to splitting. However, there are some other cases when erroneous splitting parameters can occur. Such splitting measurements may lead to wrong interpretations. Thus evaluation of splitting parameters should be done with an extreme care to avoid ambiguous splitting measurements. Such measurements are visually clear in the diagnostic plots and can be minimized by applying automatic and manual grading criteria to splitting measurements or sometimes eliminating signals with poor quality waveforms prior to splitting analysis. The Mfast consists of such elimination criteria that allows us to extract robust splitting measurements. The following section explains how to diagnose good splitting measurements and different mechanisms that can produce erroneous measurements.

\subsubsection{Diagnosing robust splitting measurements}

The diagnostic plots that are produced during splitting can be used to identify the good quality splitting measurements, especially in the case of manual grading. Those plots also have a mathematical meaning that can be incorporated with the automatic grading. Quality measurements usually exhibit following characteristic properties: 


\section{Shear-wave analysis window}

The selected shear wave analysis window should have a clear S-wave onset with high SNR (SNR $\gtrsim 2$ ). Energy on both horizontal components is a clear indication of the S-wave splitting.

\section{Contour plots}

Contour plots are generated by contouring the second eigenvalues (or minimum energy) that are obtained from the covariance matrix between two horizontal components for different pairs of $\phi$ and $\delta t$ values in $\phi, \delta t$-space. The contours in those plots, therefore, indicate equal energy surfaces or eigenvalue surfaces. The best splitting parameters can be defined as the values correspond to the smallest second eigenvalue $\left(\lambda_{2(\min )}\right)$ and the location of this minimum is surrounded by several contours with clear minimum contour (contour plot in Fig. 3.8-3). Good splitting measurements have well defined ellipsoidal contours and a symmetric small $95 \%$ confidence area that indicates a well constrained splitting measurement. According to SC91, non-ellipsoidal contours indicate no splitting due to: $\delta t=0, \phi_{p}=\phi$, or $\phi_{p}$ $=\phi+90^{\circ}$.

\section{Wave form plots}

The waveform plots of the fast and slow components before and after correction for splitting can be used to examine how well they correlate each other (see Fig. 3.8-2). When fast and slow components of an original seismogram are rotated on to fast and slow directions by $\phi$ and $\phi+90$, waveform plots show a clear separation with $\delta t$ time lag between two components and when are they shifted by $\delta t$, the two waveforms must match well each other, indicating the unsplit stage of the S-wave.

Once the waveform is unsplit, energy of the waveform must be visible only on the component parallel to the initial polarization direction $\left(\phi_{p}\right)$ and energy on the other component must be minimized. To check this, corrected fast and slow components are rotated into $\phi_{p}$ and the component perpendicular to initial polarization direction 
must only display a very little energy that corresponds to the noise in the seismogram (Fig. 3.8-2).

\section{Particle motion plots}

Presence of energy on both fast and slow components denotes the split S-wave. The horizontal particle motions of the fast and slow components, thus display an elliptical particle motion as in the particle motion plot in Fig. 3.8. Once the energy on the component perpendicular to initial polarization direction is minimized or the covariance matrix is nearly singular, the particle motion of the two components is nearly linear. The eigenvector corresponding to the larger eigenvalue of the nearly singular covariance matrix therefore represents the particle motion in the horizontal plane, which is mostly linear (Fig. 3.8-2). The direction of this linear particle motion is equivalent to the $\phi_{p}$ direction of the unsplit S-wave.

\section{Cycle skipping}

Cycle skipping is caused by the mismatch between fast and slow components. This results in a shift in $\delta t$, giving either higher or smaller $\delta t$ compared to correctly fit wave-forms. Cycle skipping is often caused by the S-waves with narrow frequency ranges and/or filtering of the S-waves using narrow band-pass filters. Such a filtering, or the earth's natural filtering may result in sinusoidal waveforms, which are likely to cycle skip. Erroneous fit can happen either in half or full cycles of the wave period, so that the calculated $\delta t$ from cycle skipped splitting measurements $\delta t_{c y c}$ can be mathematically represented as [Gerst, 2003]: $\delta t_{c y c}=\delta t \pm n \cdot \frac{T}{2}$ where $\mathrm{T}$ is the period of the wave and $\mathrm{n}$ is an integer. As shown by the equation a mismatch may occur in two different ways:

1. Trying to fit the fast component with the wrong slow component that arrives one or several half-cycle periods later. This happens if the first arrival of the 
slow component has a weak onset. In this case $\delta t$ is enlarged by $n \cdot \frac{T}{2}$. Such a fitting will only produce error in $\delta t$, but not in $\phi$ (see Fig. 3.15).

2. Switching between slow and fast components. This is possible if the first arrival (fast wave) is weak and the slow component can be mistaken as the fast component. As a result, resolved initial polarization direction will have $90^{\circ}$ error and $\delta t$ will be reduced by $n \cdot \frac{T}{2}$. In such situation several minima can appear $90^{\circ}$ apart each other along the fast direction axis.
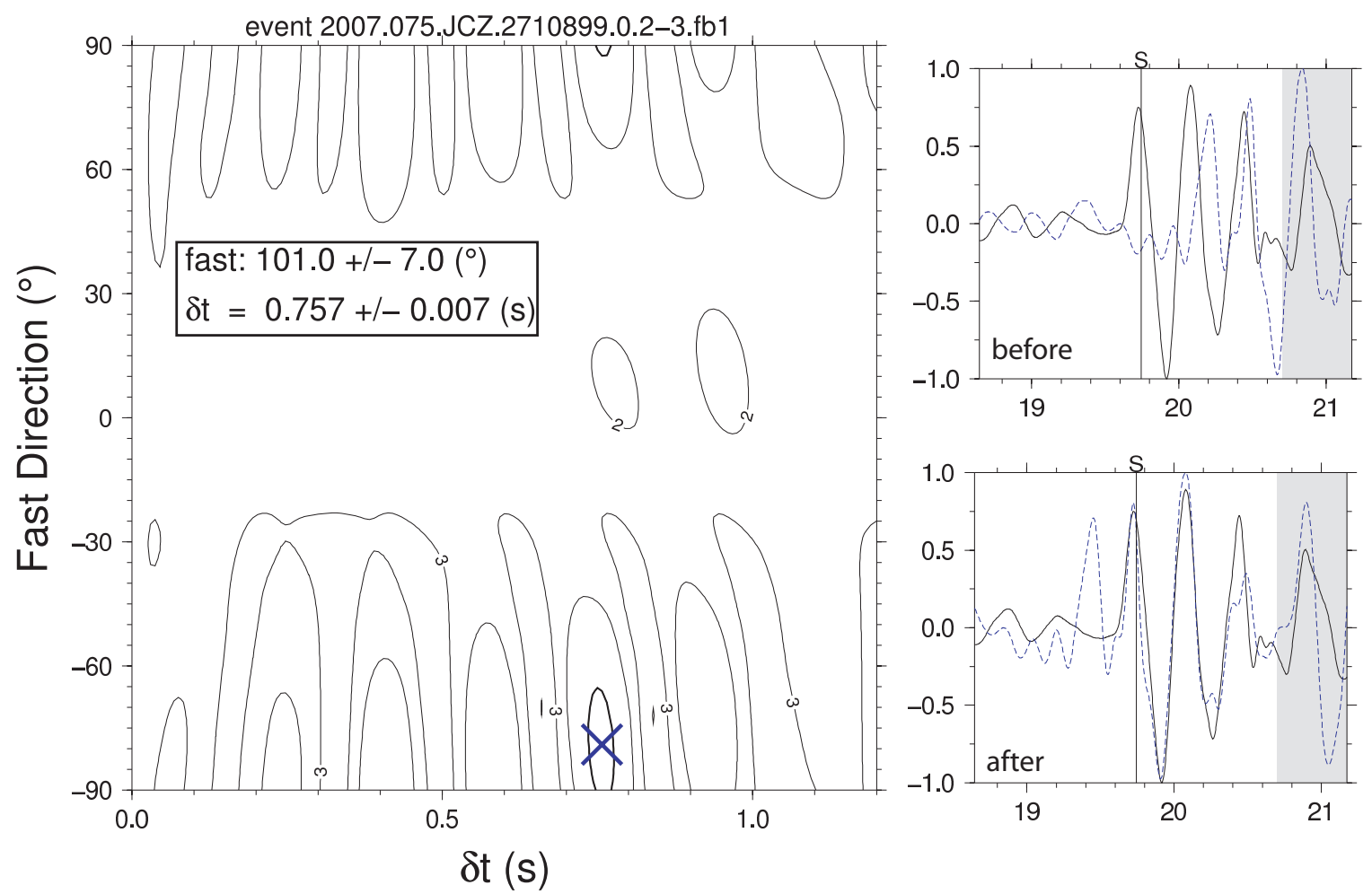

Figure 3.12 An example of cycle skipped measurement that explains case one (see text). Right: wave form plots of before and after correction for splitting, indicating a waveform mismatch and an enlarged $\delta t$. Left: contour plot of errors surfaces (gray contours) with the best splitting parameters (blue cross) and 95\% confidence limit (black contour) of the best splitting parameters.

In the case of addition of full or half cycles of wave-periods, $\delta t$ will display unusually high splitting delays. Hence, the elimination of splitting measurements with high $\delta t$ is one of the ways to alleviate ambiguities due to cycle skipping. Cycle skipping is quite obvious in the contour plots as they consist of several minima (or similar shape minimum contouring) that are separated by $n \cdot \frac{T}{2}$ along the $\delta t$ axis. In such cases, 
correct measurements are recognized as the minimum with the densest contours

\section{Null measurements}

Identifying null measurements is one of the main quality control steps, as those measurements may lead to an erroneous interpretations. Null measurements do not produce split S-waves. Such measurements are possible, if the S-wave satisfies the following conditions [Silver and Chan, 1991]:

1. If the initial polarization direction of the unsplit S-wave $\left(\phi_{p}\right)$ is aligned with either fast $\left(\phi_{p}=\phi \pm 180^{\circ}\right)$ or slow $\left(\phi_{p}=\phi \pm 90^{\circ}\right)$ polarization direction (see Fig. 13(a)).

2. If the media is isotropic or has a vertical axis of symmetry, vertically-traveling S-waves show no splitting and, thus, $\delta \mathrm{t}$ is zero.

If an S-phase satisfies either of the above conditions, the covariance matrix between two horizontal components will yield the same minimum $\lambda_{2}$ for the grid search pairs of $\phi$ with $\delta t=0$ and/or the pairs of different $\delta t$ with $\phi=\phi_{p} \pm 90^{\circ}$. The calculated minimum $\lambda_{2}$, therefore, only represents the correction of noise in the perpendicular component. Thus, $\delta t$ can not be determined accurately. Null measurements can be clearly recognized on the contour plots (Fig 3.13) with U shape contours [Gerst, 2003] and the wave-form plot with little or no energy on the direction perpendicular to $\phi$.

Mfast takes into account the cases where null measurements are likely and removes their effects by eliminating the measurements that agree with the above conditions. As explained in the null criteria, it retains only all the measurements with $\phi$ that agree with: $20^{\circ}<\left(\phi-\phi_{p}\right)<70^{\circ}$ (Fig. 3.13-b). And also, selecting measurements with high SNR ( $>3$ or 4 ) ensures that the measurement consists of a clear signal. 


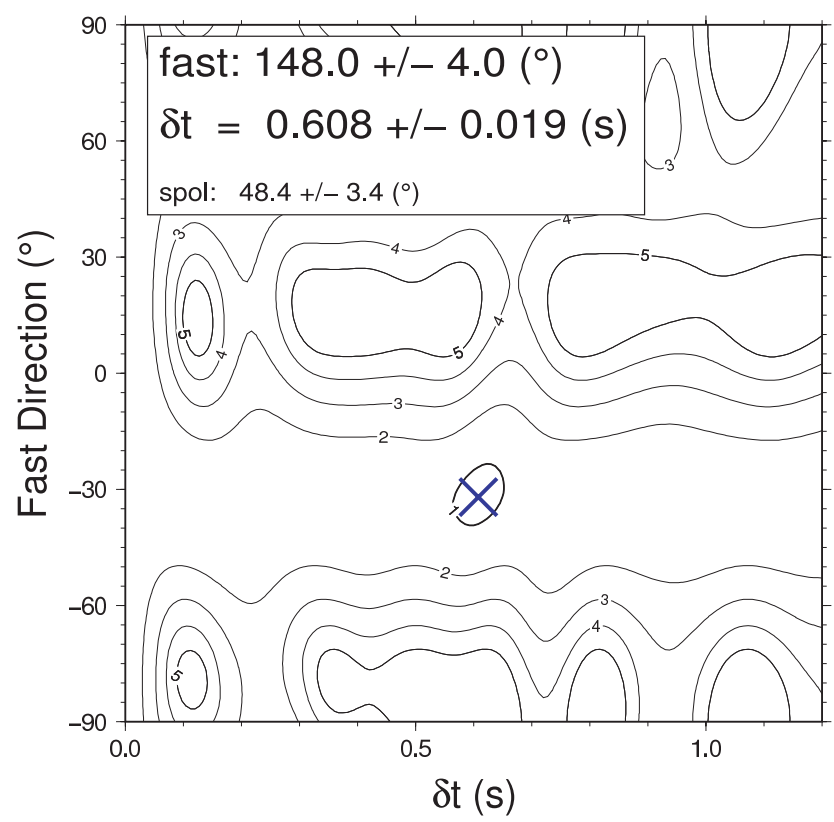

(a) The contour plot of the null measurement (blue cross) that agree with the first condition. Note the $\mathrm{U}$ shape contours and large $\delta t$ (p. 101).

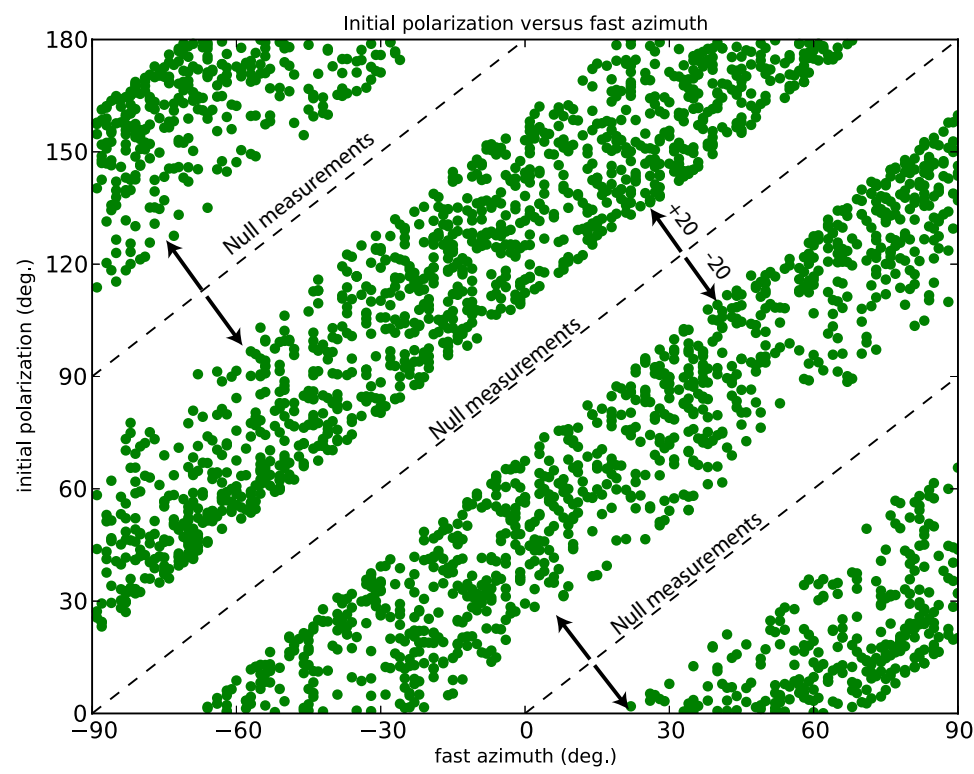

(b) Quality control step in Mfast to avoid possible null measurements.

Figure 3.13 Example of a null measurements (top) and application of the null-avoiding criteria to all on-land measurements (bottom). 


\section{Statistics of the splitting measurements}

The application of grading criteria to individual splitting measurements allows us to extract robust splitting parameters and thus, minimize the scatter in splitting measurements at an individual station. Nevertheless, some statistical parameters and analysis are important to explain the single station measurements. Directional data such as $\phi$ exhibit $180^{\circ}$ ambiguity and sometimes have more than a single mode of prominent azimuth within a single population. Therefore, both normal and directional statistics are important to determine statistical measures of the splitting measurements. Previous studies [e.g. Gerst and Savage, 2004, Savage et al., 2010, Roman et al., 2011] have successfully utilized such statistical calculations using the explanations given in Mardia [1972]. Mean $\phi$, length of the mean resultant vector of $\phi(\mathrm{R})$, circular standard deviation (CSD) and standard error (SE) are some of the directional statistics that have been used in this study.

\section{Mean $\phi$}

As $\phi$ is an angular data set, one cannot simply average $\phi$ measured at a station as in the normal statistics. To calculate mean angular direction, an individual fast azimuth $\left(\phi_{i}\right)$ should be first transformed into unit vectors in a rectangular coordinate system, so that they can be represented as a point on the 2-D plane. The unit vector $\left(\bar{r}_{i}\right)$ of a single measurement can be defined as: $\bar{r}_{i}=\left\{\cos f \phi_{i}, \sin f \phi_{i}\right\}$. The directional ambiguity associated with $\phi_{i}$ is removed by doubling the $\phi_{i}$ and thus $f=2$ [Jammalamadaka and Sengupta, 2001]. The mean resultant vector $\bar{r}$ is:

$$
\bar{r}=\left(\begin{array}{c}
\frac{1}{n} \sum_{i=1}^{n} \cos \left(2 \phi_{i}\right)=\bar{C} \\
\frac{1}{n} \sum_{i=1}^{n} \sin \left(2 \phi_{i}\right)=\bar{S}
\end{array}\right)
$$

The mean fast azimuth $\left(\phi_{s}\right)$ is, therefore equal to:

$$
\phi_{s}=\arctan (\bar{C} / \bar{S}) / 2
$$




\section{Mean resultant vector length $(\mathrm{R})$, Standard error (SE), and circular standard deviation (CSD)}

$\mathrm{R}$ is determined from:

$$
R=|\bar{r}|=\sqrt{\bar{C}^{2}+\bar{S}^{2}}
$$

$\mathrm{R}$ is a quantitative estimate of circular spread and it is used to determine both standard deviation of the $\phi_{s}(\mathrm{SE})$ and SCD of $\phi$. If the angular data set is more concentrated along one direction, $\mathrm{R}$ is close to 1 .

SE of $\phi_{s}$ is calculated using $R$ and the concentration parameter $(\kappa)$, which is a parameter of a Von Mises distribution $\left(V M_{\left(\phi_{s}, \kappa\right)}\right)$, and SE indicates the accuracy of the $\phi_{s} . \kappa$ can be estimated from $R . V M_{\left(\phi_{s}, \kappa\right)}$ is a continuous distribution on the range of $0-360^{\circ}$ and it is a probability density function that varies with $\phi_{s}$ and $\kappa\left[\right.$ Mardia, 1972]. Thus, $V M_{\left(\phi_{s}, \kappa\right)}$ is considered to be a circular equivalent of the normal distribution that measures the circular spread. Its probability density function is given by [Mardia, 1972, Philipp, 2009]:

$$
p_{\left(\phi_{i}, \phi_{s}, \kappa\right)}=\frac{1}{2 \pi I_{0}(\kappa)} \exp \left[\kappa \cos \left(\phi_{i}-\phi_{s}\right)\right]
$$

where, $I_{0}$ denotes the modified Bessel function of order zero. SE can be mathematically expressed as [Philipp, 2009]:

$$
S E=\frac{1}{f \sqrt{n R \kappa}}(f=2)
$$

Circular variance $(S=1-R)$ is also a measure of circular spread. $S$ of concentrated and scattered data will be close to 0 and 1 , respectively.

Circular standard deviation of the $\phi_{i}(\operatorname{SCD} \phi)$ determines the spread of the 
measurements around the $\phi_{s}$. SCD of $\phi$ is defined incorporating $S$ [Mardia, 1972] as:

$$
S C D_{\phi}=\sqrt{-2 \ln R} / f \quad(f=2)
$$

It is advised to carry out tests for randomness of the data, before analyzing the above statistical measurements. The most commonly used method is Rayleigh test, which is ideal to apply for $\phi$ with unimodal (only one significant $\phi$ ) distribution. This determines the threshold value for $\mathrm{R}$ to deviate from uniformity depending on the number of measurements and the significance level.

We have calculated most of the statistics discussed above from the $\phi$ of our local/regional shear wave splitting measurements. However, when analyzing splitting parameters spanning a large area and depth range, the above statistical estimations at single stations may not provide accurate constraints on the anisotropy as the scatter in splitting parameters or multi-mode distributions can be associated with the heterogeneous, spatial and depth varying anisotropy. In such cases, the elimination of station averages or measurements on the basis of statistical parameters is not suitable. 


\subsection{Direct estimations from splitting parameters}

Other than the alignment of the anisotropic medium, $\phi$ and $\delta t$ values can be used to obtain some quantitative estimations of the anisotropic medium. Some of the examples are given below:

\section{Anisotropic layer thickness:}

Assuming that the anisotropy is concentrated in a single homogeneous layer anisotropy, Vinnik et al. [1989a] and Silver and Chan [1991] (see equation 2.8) provide the following approximations to calculate the anisotropic layer thickness.:

$$
\frac{\delta t}{t} \simeq-\frac{\delta \beta}{\beta}=\frac{\delta t \cdot \beta_{0}}{L}
$$

Here, $\mathrm{t}$ denotes the average propagation time of the S-wave in the anisotropic medium with layer thickness L. $\delta \beta, \beta$ and $\beta_{0}$ represent velocity difference between fast and slow waves, the average and isotropic S-wave velocities respectively. Vinnik et al. [1989a] demonstrated that $70 \%$ anisotropic upper- mantle rock that contains $30 \%$ transversely isotropic olivine $(\delta \beta / \beta \simeq 0.02)$ should have $\sim 230 \mathrm{~km}$ layer anisotropic thickness to produce $1 \mathrm{~s} \delta t$.

\section{Crack density estimation:}

One common assumption is that anisotropy observed in the crust is solely due to crack induced anisotropy. In some tectonic settings such as volcanoes, geothermal areas and large-scale fracture zones, this assumption is useful to calculate crack density. Crack density of a medium can be dtermined the number of cracks $(\mathrm{N})$ ) with radius a in a unit Volume $(\mathrm{V}): \xi=N a^{3} / V$. Thus the normalized crack density of a unit cubic volume is equal to $a^{3}[H u d s o n, 1980]$. Crack density is equal to the $S W A / 100$ (section 2.4.1) and can also be expressed as : $\xi=\delta t . \beta_{0} / L[$ Peng and Ben-Zion, 2004, Hudson, 1980]. 


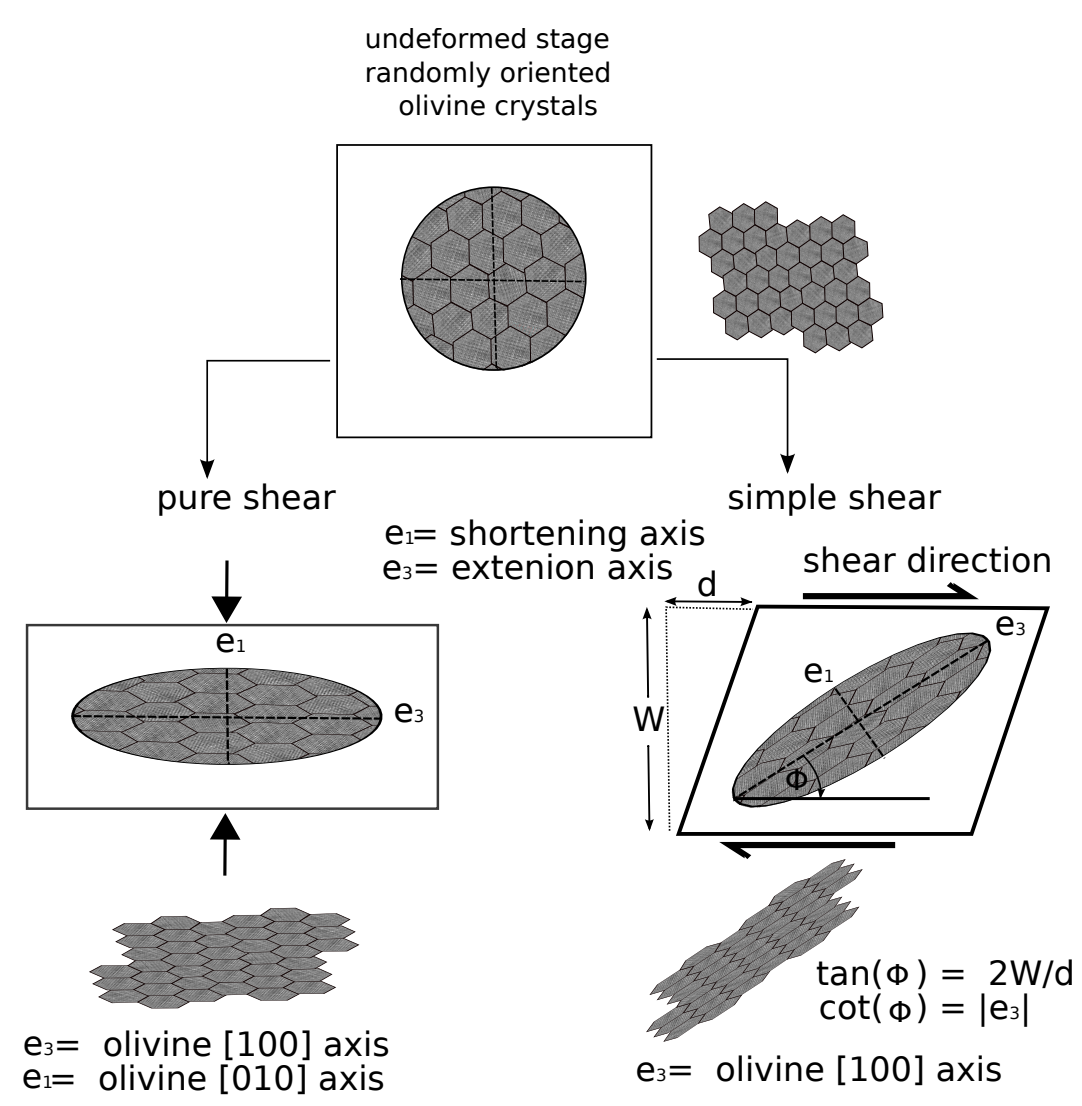

Figure 3.14 Olivine deformation under pure shear and simple shear conditions. Note that the extension axis $\left(e_{3}\right)$ is always perpendicular to shortening direction $\left(e_{1}\right)$ in the case of pure-shear deformation and it does not rotate with the increase in shear $(\Phi=0)$. But in the case of simple shear, extension axis rotates with respect to the shear plane when changing shear. The strain along the extension direction $\left(\left|e_{3}\right|\right)$ can be calculated from the equations given in the figure (also see text).

\section{Finite strain and shear-direction}

Splitting parameters from the S-phases that sample mantle anisotropy can be used to extract information on the magnitude $(|e|)$ and the direction of the strain $\left(e_{i}\right)$. Here, $i$ defines three principal strain directions: shortening $\left(e_{1}\right)$, intermediate $\left(e_{2}\right)$, and extension $\left(e_{3}\right)$. Naturally deformed Olivine [100] is predicted to align with the extension direction [Ribe, 1992]. This phenomenon can be used as a proxy for mantle deformation and flow due to relative motion of the plates (RPM) at plateboundaries and to determine shear or flow associated with absolute plate motion (i.e. differential motion between the overlying plate and mantle). These two processes are comparable with the progressive simple-shear type deformation.

In the case of pure shear deformation of Olivine, [010] is parallel to shortening axis 
and [100] is aligned with the extension direction, so that $\phi$ is parallel to extension. Extension via simple shear deformation is predicted to change the orientation of the extension axis, rotating towards the shear plane with increasing shear (or finite strain). As a result, the angle between the extension direction and shear plane $(\Phi$; see equation 1.1) becomes smaller [Ribe, 1992] with increasing finite strain. Finite strain associated with this extension (or progressive shear) at a given $\Phi$ can be estimated from the relationship : $\left|e_{3}\right|=\cot (\Phi)[$ Molnar et al., 1999].

\subsection{Characterizing splitting parameters}

It is important to extract the maximum possible information from the complex splitting data set to fully characterize the anisotropic structure. Complexities in splitting parameters are assumed to be caused by: 1) multiple layers of anisotropy,

2) frequency dependent splitting, 3) dipping anisotropic layers, and 4) presence of intrinsically anisotropic minerals with different crystal symmetry or crustal heterogeneities (mixed anisotropy). In some cases, observed splitting parameters can be modeled using forward modeling or inversion approaches to characterize such complexities.

\subsubsection{2-layer and multiple layer modeling}

The assumption of a single homogeneous anisotropic layer is sometimes not valid, especially in the cases of evaluating splitting parameters in complex tectonic settings. Such settings are likely characterized by layered anisotropic media. Silver and Savage [1994] introduced a forward modeling approach to explain the splitting parameters, where the anisotropic structure beneath the receiver is characterized by two or more layers of anisotropy with varying hexagonal symmetry orientations. 
Splitting analysis that assumes a single homogeneous anisotropic layer beneath the station will yield apparent splitting parameters if there is more than a single layer of anisotropy. In the presence of two layers of anisotropy, S-waves can be double-split by two layers [Yardley and Crampin, 1991]. In that case, splitting can be described using two splitting operators $\Gamma_{1}$ and $\Gamma_{2}$ that correspond to splitting at each layer (Fig. 3.15). Silver and Savage [1994] showed that the apparent splitting operator $\Gamma_{a}$ that is a function of both $\Gamma_{1}$ and $\Gamma_{2}$ can be used to characterize the splitting in each layer. They found that apparent splitting parameters from SKS phases exhibit $\pi / 2$ periodic patterns with the initial polarization direction $\left(\phi_{p}\right)$ (Fig. 3.15-right).
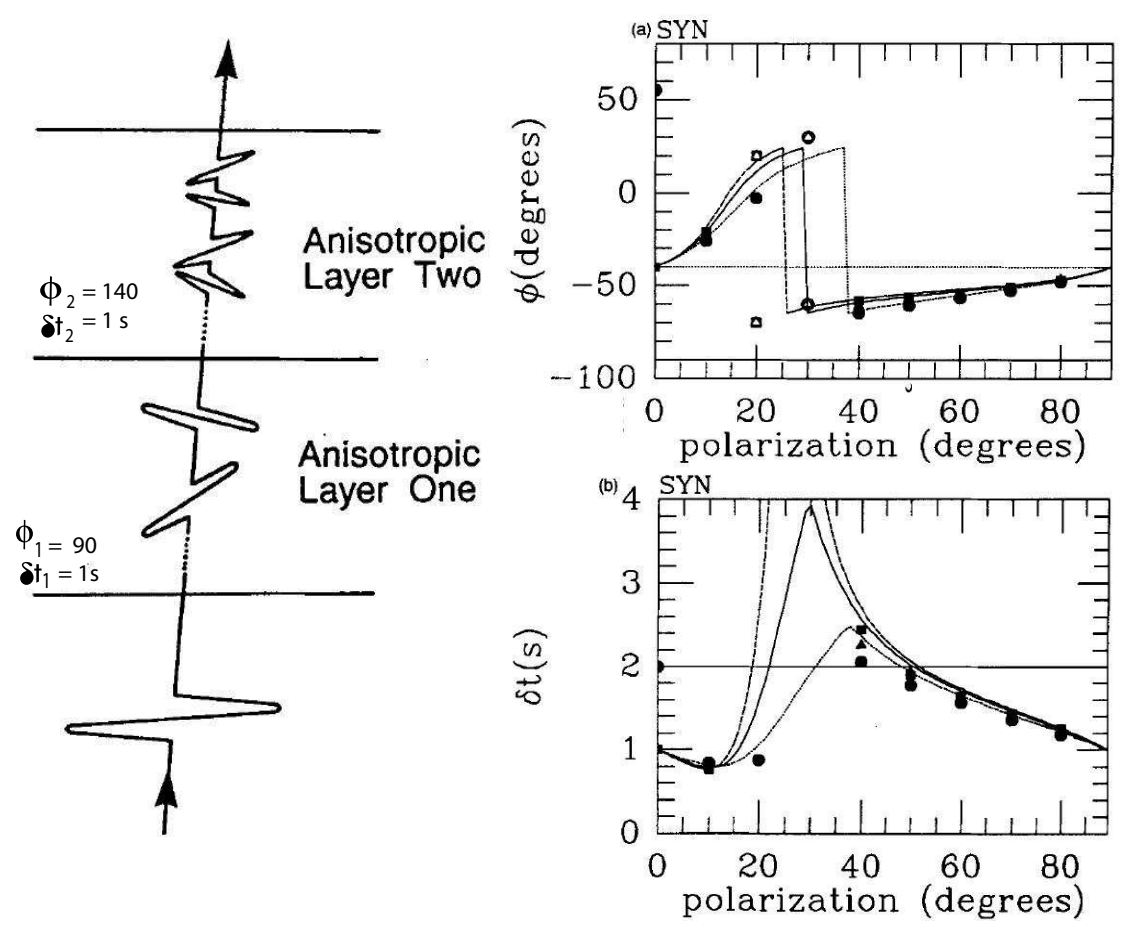

Figure 3.15 Modeled apparent splitting parameters in two-layer anisotropic media [after Silver and Savage, 1994]. Left: Double split S-phase from two anisotropic layers (top layer: $\phi_{2}=140^{\circ}, \delta t_{2}=1 \mathrm{~s}$; bottom layer: $\left.\phi_{1}=90, \delta t_{1}=1 \mathrm{~s}\right)$. Right(top/bottom): Variation of apparent splitting parameters with respect to $\phi_{p}$. Curved lines in Figs. $\mathbf{a}$ and $\mathbf{b}$ represent the predicted best-fitting splitting parameters from the 2-layer model at different S-wave periods (dotted line $-5 \mathrm{~s}$, solid line $-8 \mathrm{~s}$, and dashed $-20 \mathrm{~s}$ ).

However, such a method requires apparent splitting measurements from a wide range of backazimuths to constrain the splitting parameters accurately. Ozalaybey and Savage [1995] modified the same inversion approach used in SC91 by inverting single SKS/teleseismic S waveform with high SNR to determine splitting parameters 
of the two layers. Some studies have extended the 2-layer modeling approach to characterize the behavior of apparent splitting parameters in multiple-layered anisotropic media [e.g. Rumpker and Silver, 1998, Walker and Wookey, 2012]. Rumpker and Silver [1998] pointed out that the splitting parameters are biased towards the topmost layer anisotropy if short-period $(T / \delta t \leq 1)$ S-waves vertically propagate through a medium with smoothly varying anisotropic alignments in each layer.

\subsubsection{Frequency dependent splitting}

Frequency dependent S-wave splitting has been considered as another phenomenon that can be used to describe the complexities in anisotropic structure beneath a station. Favier and Chevrot [2003] adapted a finite-frequency approach, assuming the transverse component observed in SKS splitting represents the anisotropic perturbations of the elastic parameters and derived sensitivity kernels for $\delta t$ (or splitting intensity). They demonstrated that the size of the sensitivity kernel is proportional to the period of the S-wave (i.e., it is inversely proportional to the Swave frequency) in an anisotropic medium. Thus, if the medium consists of variable anisotropic structure with different length-scales, S-waves with different periods will extract different information about the anisotropic medium because those phases sample the medium differently.

As mentioned in the section 3.5.1, apparent splitting parameters from high frequency S-phases are mostly sensitive the topmost layer of anisotropy in the layered anisotropic medium. Thus, the higher frequency phases allow us to investigate the properties of the shallow anisotropic media (e.g. crustal anisotropy). Especially when utilizing deep high-frequency S-phases, it is important to clarify whether the splitting parameters actually agree with the deep anisotropy or if they represent the shallow structure. Recent studies investigating anisotropic structures beneath 
subduction zones found frequency dependent splitting [Wirth and Long, 2010, Long, 2010] and interpreted it as possibly multi-layer anisotropy in subduction tectonic settings [Huang et al., 2011a, Greve and Savage, 2009].

Marson-Pidgeon and Savage [1997] provide a set of splitting observation from SKS/ScS phases recorded in Wellington region, New Zealand that indicates frequency dependent shear-wave splitting. They noticed a clear increase in $\delta t$ with the periods of the S-waves and explained it in terms of three phenomena that might cause frequency dependent splitting: 1) the previously discussed dependency of size of the sensitivity kernel (splitting resolution) on the period; 2) multiple layers of anisotropy beneath the station; and/or 3) scattering of seismic waves by heterogeneous crustal structures. Frequency dependent splitting due to crustal structures have been investigated by several studies (e.g. Liu et al. [2003], Werner and Shapiro [1999], and Tod and Liu [2002]). As suggested by Marson-Pidgeon and Savage [1997] and Liu et al. [2003], frequency dependent splitting can be attributed to scattering of S-waves by large-scale crustal heterogeneities.

According to Sato and Fehler [1997], anisotropic heterogeneities can be examined as effective anisotropy only if the scale-lengths of heterogeneities are small enough to preclude scattering, so that the medium behaves as a quasi-homogeneous medium. In that case, an empirical relationship between wave-number $(k ; k=2 \pi / \lambda$, where $\lambda$ is wavelength) and the scale of heterogeneities (a) is given by: $k \mathbf{a}<0.01[W u$ and $A k i, 1988$ ]. For instance, a waveform with $5 \mathrm{~s}$ period (typical ScS period in our data set) requires heterogeneities with scale-lengths less than $\sim 38 \mathrm{~m}$ to observe effective anisotropy without scattering. For local S phases (period of $1 \mathrm{~s}$ ), the maximum scale length is around $5.2 \mathrm{~m}$. Thus, the large-scale heterogeneities will affect $\delta t$ due to scattering and can be seen as an effect of frequency dependency [Marson-Pidgeon and Savage, 1997]. We also noticed frequency-dependent splitting from both local and teleseismic S-phases and these results are discussed in sections 4.4.2, 6.2.1, and appendix C.5. 


\subsubsection{Dipping anisotropic layers}

Dipping anisotropic layers have been first investigated in central Europe by Babuska et al. [1984] from P-wave residuals analysis. The study found directional dependence of P-wave residuals and those directional variations are interpreted as dipping anisotropic structures that are caused by paleosubduction systems. In such domains, shear-wave splitting parameters from SKS phases are expected to show backazimuthal dependence [Levin et al., 1999] (Fig. 3.16) and will produce $2 \pi$ periodicity with BAZ [Silver and Savage, 1994].
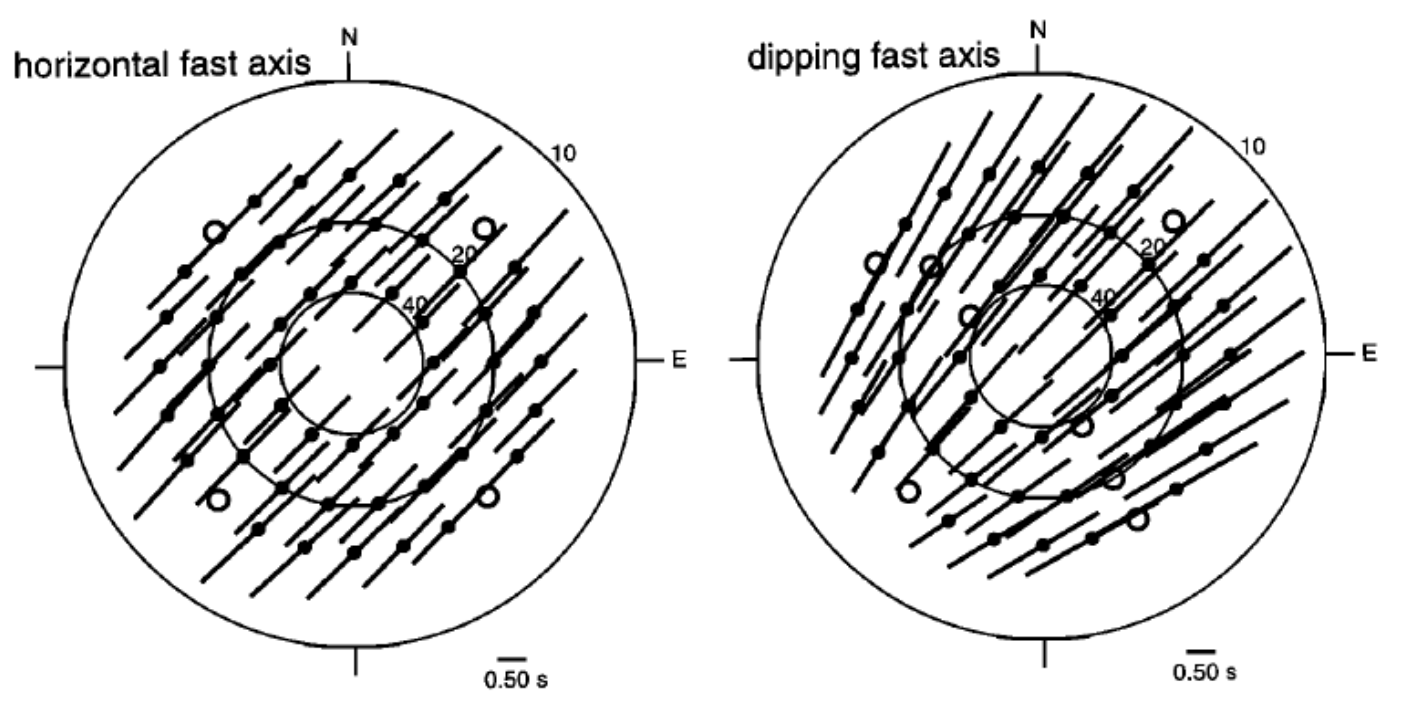

Figure 3.16 Modeled splitting parameters in systems that have a single hexagonally anisotropic layer with horizontal symmetry axes (left) and symmetry axes dipping at $45^{\circ}$ (right) [after Levin et al., 1999]. Note that the slowly (left) and rapidly (right) varying splitting parameters in two systems.

In the case of dipping anisotropic layers, the anisotropic tensor is inclined (inclined symmetry axis) and, thus, the plane perpendicular to symmetry axis also inclined. In such a system shear-wave splitting from vertical ray paths results in lower effective splitting anisotropy as in the two layer case. Sileny and Plomerova [1996] have modified Silver and Chan [1991] to measure S-wave splitting parameters 3dimensionally by calculating them in the plane perpendicular to the ray path. Splitting parameters are measured in the ray coordinate system (LQT), solving for the dip of anisotropic alignment. Here, the fast direction $(\psi)$ that is measured 
in the QT plane can be expressed using the fast azimuth $(\phi)$ in the North-EAST (NE) plane and its inclination $(\theta)$ on the vertical plane (Fig 3.17).

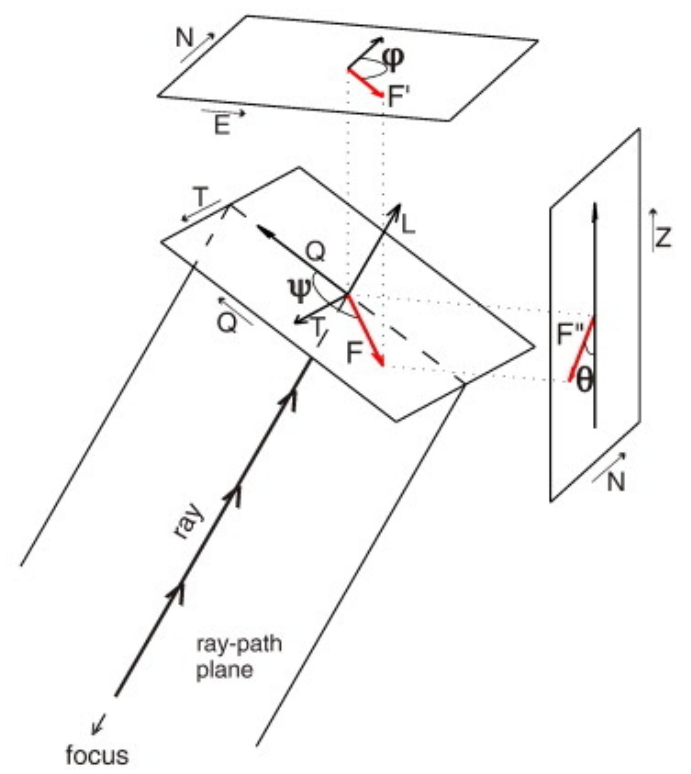

Figure 3.17 Splitting parameter estimations in the LQT-coordinate system. The fast polarization direction $(\psi)$ is now measured in the Q-T plane, which is perpendicular to the ray direction (L). $\psi$ can also be expressed by $\phi$ that is measured in horizontal plane (NE) with geographic coordinates $(\mathrm{N}-\mathrm{E})$ and $\theta$, which is a measure of inclination from vertical axis (Z) in the vertical plane (N-Z) [Figure after Vecsey et al., 2008].

The SPLITSHEAR package of Vecsey et al. [2008], which is based on the Sileny and Plomerova [1996] method, facilitates the measurement of splitting parameters in LQT coordinate system using three different techniques: 1) transverse energy, 2) eigenvalue minimization and 3) cross correlation techniques. The method calculates the misfit function between $\mathrm{Q}$ and $\mathrm{T}$ components that are corrected for splitting. The minimum of the misfit function, therefore, represents the best splitting parameters. The misfit function of the smaller eigenvalue of the correlation matrix is given by [Vecsey et al., 2008]:

$$
\lambda_{2}=\frac{1}{2}\left[\left(C_{f f}+C_{s s}\right)-\sqrt{\left(C_{f f}+C_{s s}\right)^{2}+4 C_{f s}^{2}}\right]
$$

where, $C_{f f}$ and $C_{s s}$ are auto-correlation of the rotated components and $C_{f s}$ is the cross-correlation. 


\subsection{Shear-wave splitting tomography}

Local splitting parameters measured at a single station from ray paths with different ray propagation directions (or BAZ) often yield complex splitting measurements. Those complexities can be attributed to spatially and/or depth varying anisotropy that the ray encounters when it traverses from source to station. 2-D delaytime tomography allows us to resolve spatially varying anisotropic structure. However, if the rays are contaminated with depth varying anisotropy (due to either anisotropic layers with variable symmetry axis or different sources of anisotropy) as in subduction zones, 3-D splitting tomography techniques are useful to resolve the complexities in splitting parameters.

\subsubsection{2-D delay time tomography and spatial averaging (TESSA)}

To examine spatial variations of $\delta t$ and $\phi$ associated with local splitting parameters, 2-D delay-time tomography and a spatial averaging technique (TESSA; Johnson et al. [2011]) has been utilized. Such an application allows us to obtain approximate constraints on regions with low and high anisotropy. $\delta t$ from a single measurement is an estimate of the anisotropic structure along the ray path from source to the station and the $\delta t$ is considered to be proportional to path length $(L)$ of the ray through the anisotropic medium [Silver, 1996]. TESSA is based on this simple linear relationship between $\delta t$ and $L$.

It assumes that $\delta t$ observed along the ray path $\delta t_{r}$ is the summation of $\delta t$ calculated for each grid block $\left(\delta t_{b}\right)$ with variable strength of anisotropy $\left(s_{b}\right)$ in 2-D space. First, straight-line ray tracing between station and events determines the lengths of ray segments $\left(L_{r b}\right)$ that lie on each grid block (Fig. 3.18). Then the total anisotropy 


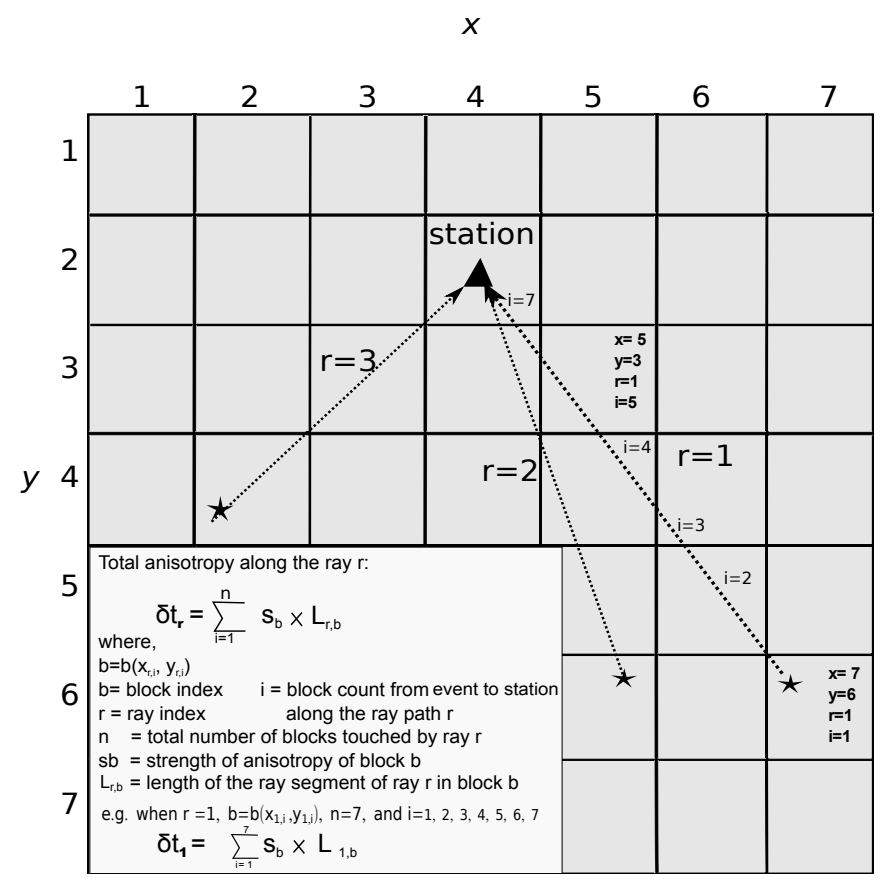

Figure 3.18 Schematic shows the calculation of total anisotropy $\left(\delta t_{r}\right)$ along a given ray path (r) in TESSA. Here, $\mathrm{b}$ denotes the block index that is a function of the ray index $(r$ and the block count $(i)$ from event to station along the ray path, $s_{b}$ strength of anisotropy in block b, and $L_{r b}$ is length of the ray segment in the block $b$.

measured at the station for a given event is calculated by,

$$
\delta t_{r}=\sum_{b=1}^{n}\left(s_{b} \times L_{r b}\right)
$$

The inversion part of TESSA determines the $s_{b}$ for resolved blocks and is carried out using the MATLAB medium-scale optimization inversion function that finds the linear least-squares solution of the problem. This solution is then used iteratively to find the model solution for the strength of anisotropy of the whole ray $\left(s_{r}\right)$ and this process is subjected to boundary conditions. As explained in Johnson et al. [2011], boundary constraints are applied in such a way that the minimum $s_{r}$ will always be greater than $0 \mathrm{~s} / \mathrm{km}$ and the maximum $s_{r}$ will not exceed $\delta t_{r(\max )} / L_{b}$, where $\delta t_{r(\max )}$ is the maximum $\delta t$ observed along a ray path and $L_{b}$ is the width of the grid block. Then the weighted model solution $(m)$ is:

$$
m=\left(G^{T} W_{d} G\right)^{-1} G^{T} W_{d} d \quad[\text { Stein and Wysession, 2009] }
$$


where $\mathrm{G}$ denotes the matrix consisting of $L_{r b}, d$ is the observed $\delta t$ from each ray path and $W_{d}$ is the weighting applied to data based on the errors. The covariance matrix $(\mathrm{C})$ of the measurement and inversion errors is used to define the weighting, so that $W_{d}=C^{-1}$. The diagonal components $C_{i i}$ and off diagonal components $C_{i j}$ of this matrix are given by:

$$
\begin{aligned}
C_{i i} & =\sigma_{d}^{2}(i)+L_{b}{ }^{2} \times \bar{\sigma}_{m}^{2} \times n_{b}(i) / n \\
C_{i j} & =\bar{\sigma}_{m}^{2} \times L_{b}{ }^{2} / n^{2}
\end{aligned}
$$

Here, $\sigma_{d}^{2}(i), \sigma_{m}^{2}$, and $n_{b}(i)$ indicate the squared standard error of the $i^{t h}$ measurement, mean variance of the solution when the data are weighted as $1 / \sigma_{d}(i)$ and the number of boxes that the $i^{t h}$ ray propagates through, respectively. Model resolution is constrained from the diagonal elements of the resolution matrix along with checkerboard tests.

In TESSA, spatial averages are calculated by averaging $\phi$ in a particular grid block once the $\phi$ calculated from each ray (event-station pair) is assigned to each ray segment in 2-D grid blocks between the event and station. Three different weighting schemes: $1 / d$ (where, $\mathrm{d}$ is distance from station to grid block), $1 / d^{2}$, and tomographic weighting $\left(\omega_{r b}\right)$, can be applied to individual $\phi$ before averaging in order to provide different constraints on the anisotropic structure of the region. For instance, to determine the spatial variations of $\phi$ close to the station, one can apply $1 / d^{2}$ weighting as it heavily weights the measurements closer to the station. $\omega_{r b}$ is suitable if the study aims to weight $\phi$ considering the delay-time tomography results. We use the tomographic weighting scheme presented in TESSA to determine average $\phi$ in grid blocks. Tomographic weighting is designed to take into account variations due to heterogeneous anisotropic structure. The tomographic weighting function $\left(\omega_{r b}\right)$ 
was the anisotropy strength profile determined for each grid block $\left(s_{b}\right)$ in the $\delta t$ tomography, so that more weighting is given to the regions with higher strength of anisotropy. $\omega_{r b}$ is defined as the strength profile of the each grid block, normalized by $\delta t_{r}[$ Johnson et al., 2011],

$$
\omega_{r b}=s_{b} / \delta t_{r}
$$

Then, the weighted mean $\phi$ of the grid block $b$ with $n$ number of rays is given by $\operatorname{azimuth}\left(\bar{\phi}_{b}\right)$,

$$
\bar{\phi}_{b}=\frac{1}{2} \arctan \left(\frac{\sum_{r=1}^{n} \cos \left(2 \phi_{r}\right) \times \omega_{r b}}{\sum_{r=1}^{n} \sin \left(2 \phi_{r}\right) \times \omega_{r b}}\right)
$$

where $b$ and $r$ denote the block and ray number, respectively.

\subsubsection{3-D splitting inversion: Abt and Fischer [2008]}

The shear wave splitting tomography (SWST) technique by Abt and Fischer [2008] can be used to resolve the 3-dimensionally distributed complex splitting parameters in terms of crystallographic orientations. This approach is useful for examining the geometry of the deep anisotropic structure (e.g. lower crust and upper mantle), where there is a good depth distribution of splitting parameters. The method neglects the anisotropy in the upper-most crust as it is mainly caused by SPO anisotropy and assumes that the observed local splitting is governed solely by the preferred alignment of the intrinsically anisotropic minerals (e.g. Olivine, Pyroxene). We apply this technique to resolve complex splitting parameters observed in our local-shear wave splitting estimation, assuming that the deep ray paths, especially in the two subduction zones, are mainly effected by the mineral alignment.

The method has three main steps: 1) model parametrization, 2) forward modeling of splitting and, 3) inversion of shear-wave splitting parameters. Here, we discuss how 
we adapt the above three steps to obtain the final model solution (section 5.3.3).

\section{Data and Model parameterization:}

We invert the local/regional S-wave splitting obtained from the on-land and off-shore studies. The model space of this data set is defined so that it includes all stations and events that were used to obtain shear-wave splitting parameters. We chose grid blocks with $25 \mathrm{~km}^{3}$ volume to characterize the $3-\mathrm{D}$ space [i.e., along the plate-boundary (400 km), across plate-boundary $(475 \mathrm{~km})$ and maximum depths of $250 \mathrm{~km}$. A total of 7020 ray paths $(2982$ events and 56 stations) were used for the inversion (Fig. 3.19). The scale of our study region is comparatively larger than the regions that have been investigated using this technique previously [Abt et al., 2009].
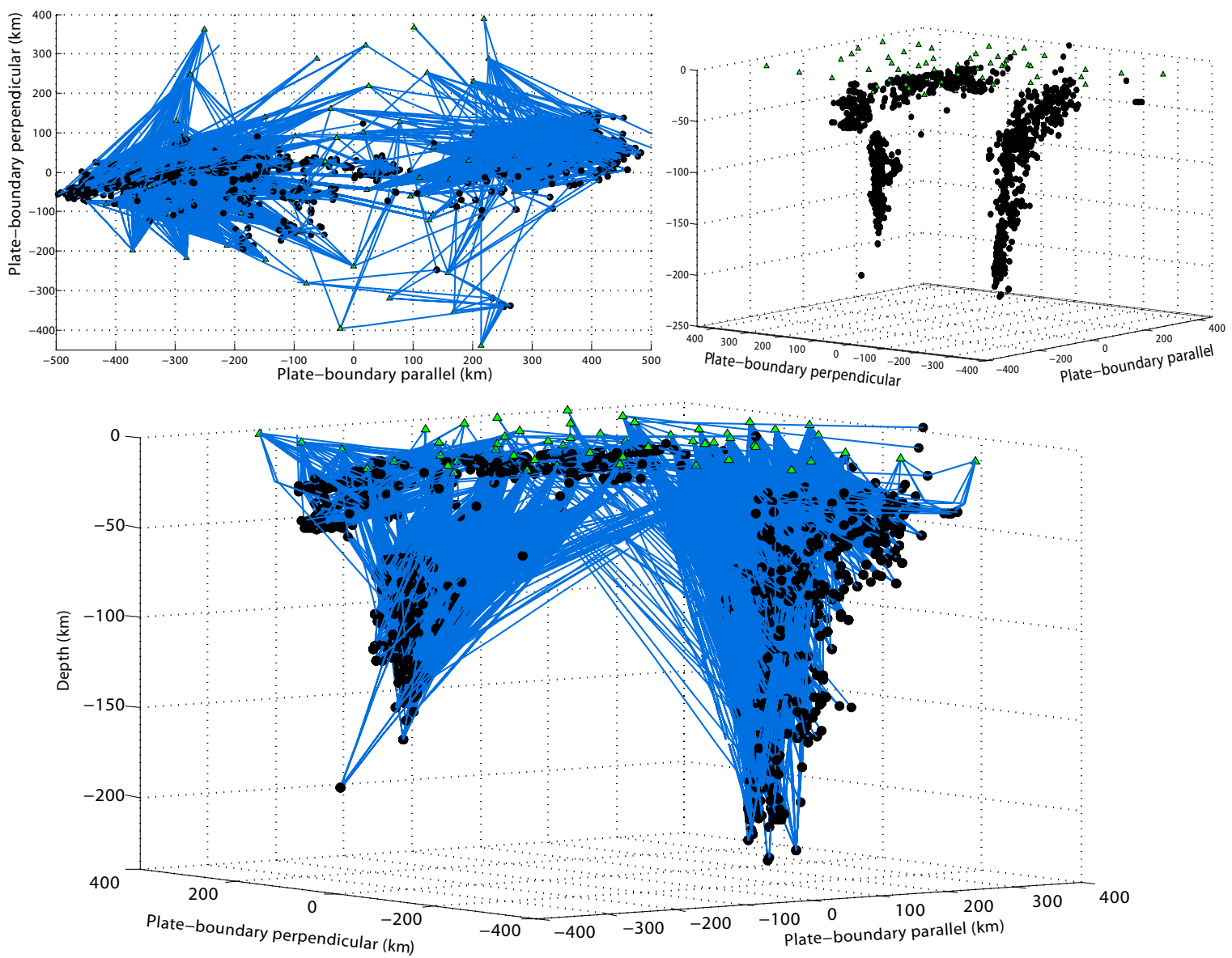

Figure 3.19 2-D (top-left) and 3-D view (bottom and top-right) of the model space (dotted lines), ray paths (blue), events (black circles) and stations (green triangles). Ray-tracing was done using 1-D velocity model of AK135 [Abt and Fischer, 2008]. 
The orientation of the crystallographic axis and the strength of anisotropy of each block in the model space are determined from the average starting model [see Abt and Fischer, 2008, appendix A]. We assume a hexagonal crystal symmetry that is simulated from the orthorhombic single-crystal elastic constants of olivine and ortho-pyroxene as described in Abt and Fischer [2008]. Thus, in each block, crystallographic orientations are defined by two angles

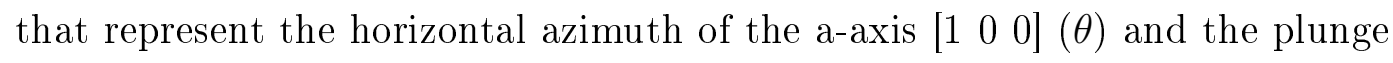
of the a-axis $(\psi)$. The strength of anisotropy $(\alpha)$ that is assigned for each block is a scalar parameter that ranges from 0-100\%. $\alpha$ takes into account the relatively high single crystal anisotropy of minerals (e.g. laboratory measurements) and reduces this high anisotropy to be comparable with $\delta t$ that is estimated from the in-situ shear-wave splitting.

\section{Forward modeling}

Forward modeling of splitting parameters (predicted splitting) is done with the particle motion perturbation method [Abt and Fischer, 2008] for each ray path in the model. The effect of 3-dimensionally varying anisotropic structure on the rays that travel from earthquakes to stations is determined by taking into account crystallographic orientations and the strength of anisotropy of each block that the ray propagates through. The Christoffel equation (see equation 2.7) is used to determine the polarization directions and the phase velocities (eigenvalues) in each block (as in section 2.2.2) using the initial model parameters. In each block, the two components perpendicular to the ray propagation are time lagged and rotated to add the effects of anisotropy in each block as the ray propagates. Thus, this calculation encounters the effects of S-wave splitting in the layered anisotropic media as the anisotropy in each block can be different from the others.

\section{Inversion}

Inversion allows us to find the the best-fit model with 3-dimensionally varying 
crystallographic orientations and the strength of anisotropy that best explains the observed splitting parameters. The method uses damped least-squares inversion iteratively to minimize the misfit between observed and predicted splitting parameters [e.g. Tarantola, 2005]. The improved model $\left(M^{i+1}\right)$ after $i^{\text {th }}$ iteration by perturbation of model parameters (in our case $\delta \theta, \delta \psi$, and $\delta \alpha$ ) in model $M^{i}$ is given by the following linear relationship:

$$
M^{i+1}=M^{i}+C_{m m} G^{T}\left[G C_{m m} G^{T}+C_{d d}\right]^{-1} \delta D^{i} \quad \text { Abt and Fischer }[2008]
$$

Here, $\delta D^{i}\left(=\left[d_{o b s}-d_{p r e d}\right]=\left[d_{o b s}-G M^{i}\right]\right)$ represents the data misfit. The G matrix consists of linear partial derivatives that are computed by finite difference [Abt and Fischer, 2008, equation 5]. This explains the variation in splitting parameters upon the perturbation of model parameters ( $5^{\circ}$ for $\theta, \psi$ and $5 \%$ for $\alpha$ ). During inversion, it recalculates the partial derivatives of the G matrix only if changes in orientations of the model parameters ( $\theta$ and $\psi$ ) between iterations $(i$ and $i+1)$ are exceeded by $1^{\circ}$. This allows the linearity of partial derivatives to be kept in the inversion problem and to obtain accurate solution. $C_{d d}$ denotes the diagonal component of the data covariance matrix $\left(d_{o b s}\right.$ and $\left.d_{\text {pred }}\right)$, which represents the sum of the squares of the errors. $C_{m m}$ is the a priori model covariance matrix and the diagonal component of $C_{m m}$ is used to damp the changes to model parameters to maintain the linearity of partial derivatives. Data misfits $\left(\delta D_{\phi, \delta t}\right)$ are weighted by the both predicted and observed splitting errors [see Abt and Fischer, 2008, equation 9].

Model resolution is determined using the resolution matrix $\left(R_{m}=C_{m m} G^{T}\right.$ $\left[G C_{m m} G^{T}+C_{d d}\right]^{-1} G ;$ Tarantola [2005]). The diagonal component of this matrix helps to determine well resolved model paramete s. $R_{m}$ varies between 0 and 1, indicating poorly and well resolved bloc s, respectively. This helps to find the regions with lower resolution $\left(R_{m}<0.25\right.$ from the final iteration as defined by [Abt and Fischer, 2008]), which is mainly due to low ray-coverage. 
In such regions, model resolution is improved by either combining model blocks into a larger block volumes or applying spatial smoothing [Abt and Fischer, 2008]. The blocks are combined part-way through the iterations once the solution is stabilized. The inversion also uses damping relaxation to test the model stability after initial model convergence. The relaxing damping will improve the model unless it achieved minimum data misfit. Preliminary results from the 3-D tomographic inversion are discussed in section 5.3.3. 


\title{
Chapter 4
}

\section{Seismic anisotropy and lithospheric deformation of the plate-boundary zone in South Island, New Zealand: inferences from local S-wave Splitting}

This chapter presents a published manuscript [Karalliyadda and Savage, 2013] that includes results of the on-land seismic anisotropy study. Some parts in the introduction and methodology sections may contain some repetitive sections from the previous chapters. A sub-study, which was completed after this publication is included in the section 5.3.1.

\begin{abstract}
Insights into plate-boundary deformation are gained from the shear-wave splitting of local S phases that originate within the lithosphere of the South Island, New Zealand. Analysis of the splitting parameters from land stations reveals changes in both delay times and fast azimuths with earthquake depth, earthquake-station back-azimuth and initial polarization azimuth, suggesting both laterally and depth varying anisotropy. When the average results are examined as a whole via tomographic inversion and spatial averaging, consistent patterns in delay times and fast azimuths exist. Spatially averaged fast azimuths reveal a localized high strain zone in the southern central region of the South Island. Based on fast azimuths observed above $100 \mathrm{~km}$ depth, we suggest that the plate-boundary sub-parallel anisotropy that is produced by pervasive shear is mainly distributed within a zone extending $\sim 130 \mathrm{~km}$ SE of the Alpine fault in southern South Island and is widely distributed in northern South Island. Average station delay times of $\sim 0.1-0.4 \mathrm{~s}$ compared to $1.7 \mathrm{~s}$ SKS delay times from previous studies in South Island further suggests a deeper seated
\end{abstract}


anisotropic zone in some areas, but in other regions frequency dependent anisotropy could explain the observed splitting.

\subsection{Introduction}

Anisotropy beneath plate-boundary zones provides indirect insights into the tectonic deformation associated with plate interactions. Among geophysical techniques, shear wave splitting (S-wave splitting) has been a well established and commonly used technique in exploring seismic anisotropy [Silver and Chan, 1991, Teanby et al., 2004b, Wustefeld et al., 2008]. Splitting tomography is another method that has been utilized to resolve the heterogeneous anisotropy structure from initial splitting parameters and to provide constraints on the distribution of seismic anisotropy [Abt and Fischer, 2008, Johnson et al., 2011]. Usage of such techniques is important in local and regional splitting studies, because it incorporates the changes in splitting due to various factors (e.g. back azimuth, depth, orientation of crystallographic axes) that could result in complex splitting. However, such methods are highly dependent on the station coverage and the earthquake distribution.

The crustal contributions of anisotropy are usually ignored by splitting analysis that investigates mantle anisotropy using SKS phases [Savage, 1999]. Yet crustal contributions might have significant effects on SKS measurements and result in complex patterns and small-scale lateral variations [Long and Silver, 2009, Mattatall and Fouch, 2007, Rumpker and Silver, 1998]. Thus, S-wave splitting of local and regional S-phases is vital for understanding the crustal and subcrustal contribution to total anisotropy in a given region. S-wave splitting using local events observed along the ray paths in the lithosphere is attributed to different sources of anisotropy that are developed from both shape preferred orientation and lattice preferred orientation. Seismic anisotropy at crustal depths is assumed to result from either stress-induced anisotropy or structural anisotropy [e.g., Boness and Zoback, 2006]. 
In the upper crust, fluid-filled microcracks or fractures and structural anisotropy (e.g. crustal faults, rock foliations or beddings) may affect S-wave splitting whereas in the lower crust and the upper mantle, splitting is mainly influenced by intrinsic anisotropy resulting from rock fabrics and preferred orientation of rock-forming minerals such as olivine in the upper mantle and mica and amphibole in the lower crust [Mainprice and Nicolas, 1989, Crampin et al., 2004, Silver, 1996]. As suggested by Wilson et al. [2004], sub-horizontal foliations with lineations (e.g. schist fabric) developed at lower crustal depths due to pervasive shear in strike-slip fault zones could also act as source of anisotropy.

The interpretation of local splitting is challenging because of the different factors and sources of anisotropy (e.g. tectonic processes) that control splitting. Lattice preferred orientation is mainly a result of finite strain [Nicolas and Christensen, 1987]. In the lower crust, mineral alignment is predicted to be parallel to the flow or shear direction and in the upper mantle, the [100] axis of olivine is fast and is parallel to the extension (maximum principal stretch) direction or to the flow direction if significant strain has developed in a dry regime [Nicolas and Christensen, 1987, Savage, 1999, Silver, 1996]. At shallow crustal depths, differential stress results in preferential closure of micro cracks perpendicular to the maximum stress direction creating an anisotropic medium [Crampin et al., 2004]. The fast azimuth of a split Swave in such a stress-aligned anisotropic medium is parallel to the cracks that remain open, hence, parallel to maximum principal stress direction [Nur and Simmons, 1969, Boness and Zoback, 2006].

Plate interactions at the plate-boundary zone in South Island, New Zealand are expected to result in deformation induced seismic anisotropy. Westward movement of the Pacific plate with respect to the Australian plate results in an obliquely convergent boundary across New Zealand (Fig. 4.1). The main surface manifestation of this plate-boundary, the Alpine fault, extends for about $460 \mathrm{~km}$, serving as an oblique transform fault that connects Puysegur and Hikurangi subduction zones with 
opposite polarities (Fig. 4.1). The structural evolution of South Island is marked by different tectonic episodes. Some of the key processes include rifting and the breakup of Gondwana in the late Cretaceous, evolution of the two subduction systems and the plate-boundary, the transition of the southern subduction system (Puysegur subduction) into the central Alpine fault zone during the Eocene to Quaternary periods and southward migration of the Hikurangi subduction system increasing the transpressional character of the central collisional (Alpine fault) system [Sutherland et al., 2000, Furlong, 2007, Cox and Sutherland, 2007]. Seismic anisotropy could remain from tectonic deformation during any of these episodes.

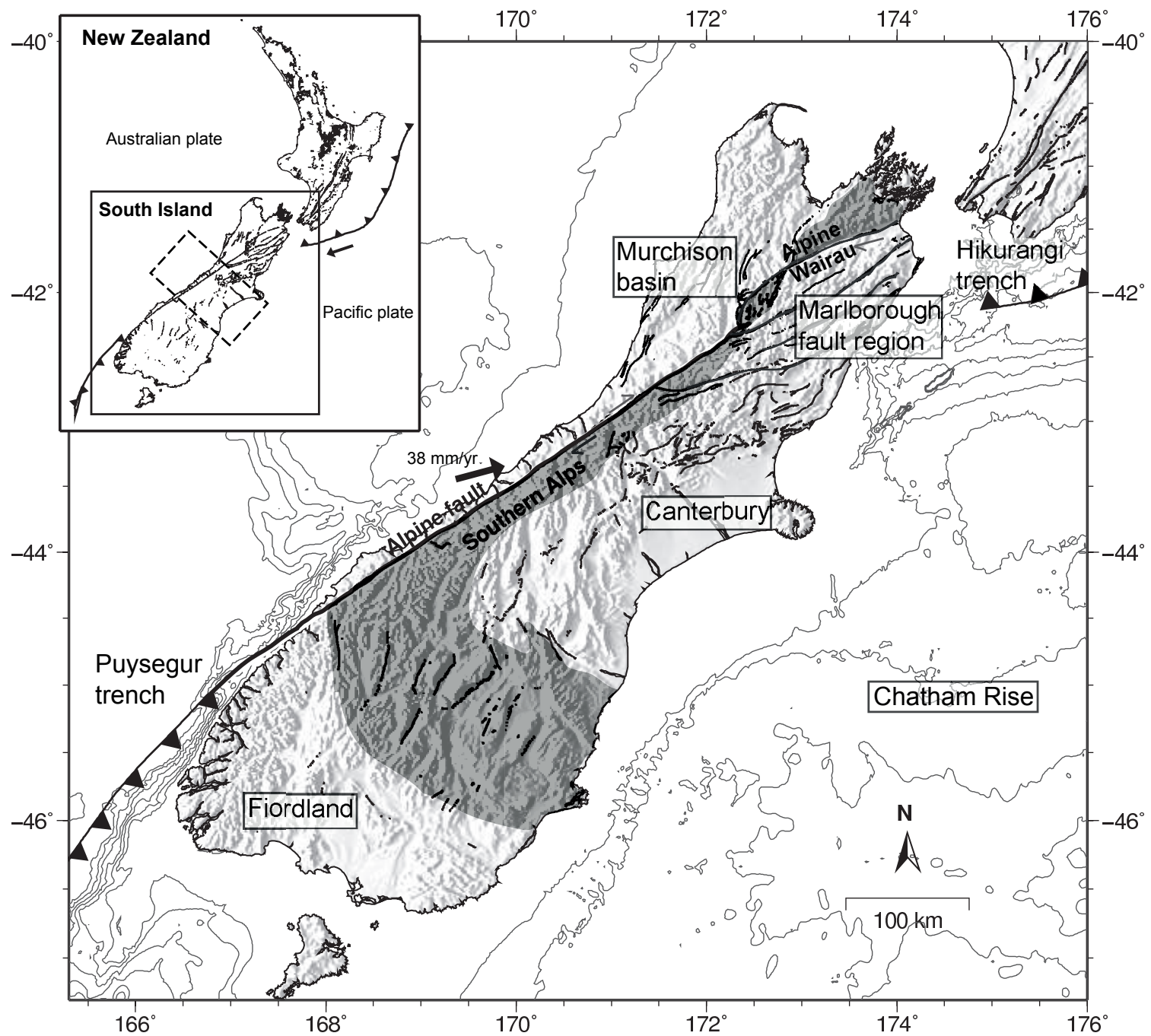

Figure 4.1 (a) Map of the tectonic setting of the South Island, New Zealand and the main structural features associated with the Australian-Pacific plate-boundary. Approximate location of the schist belt with high grade metamorphism is marked by the dark-gray shaded region. The black arrow denotes the relative plate motion direction $(\sim 38 \mathrm{~mm} / \mathrm{yr})$ between the Australian and Pacific plates [Walcott, 1998]. 
i. Distributed deformation / Thin viscous model

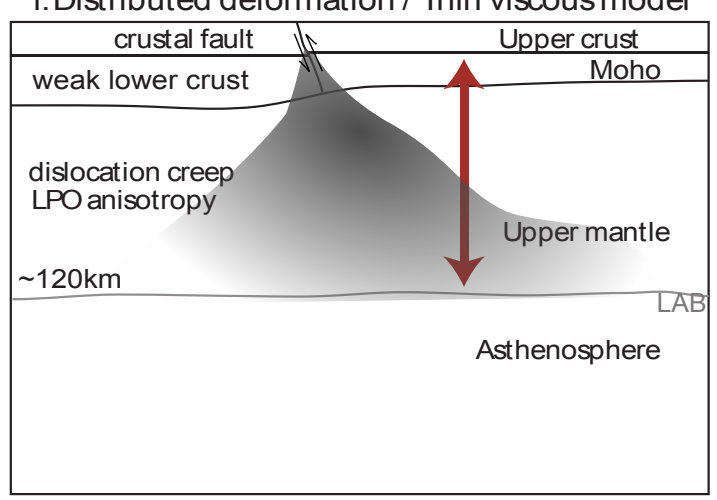

ii. Localized deformation / Fan shape model

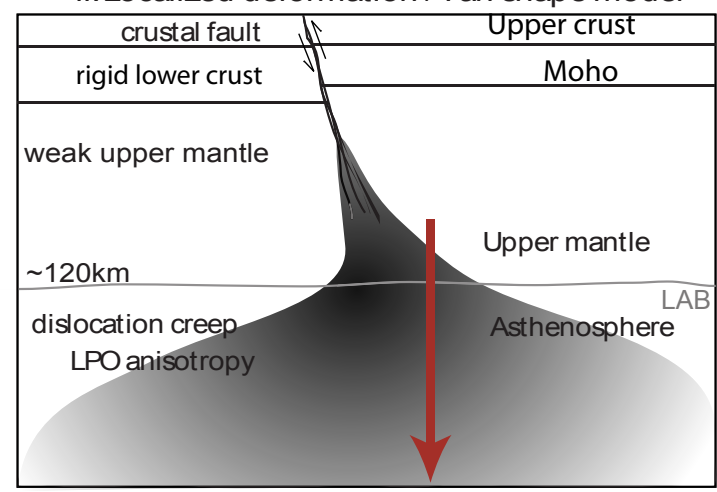

Figure 4.1 (b) Schematic diagrams (no vertical exaggeration) showing the proposed models of deformation for central South Island (dashed-line box in the inset of Figs. 4.1a). ( $i$ ) Pervasive shear or thin viscous sheet model proposed by Molnar et al. [1999]. (ii) Localized deformation model. LAB - Lithosephere-Asthenosphere Boundary.

Present deformation along the plate-boundary can also influence anisotropy. In the northern part of South Island, the relative plate motion $(\sim 38 \mathrm{~mm} / \mathrm{yr})$ between the Australian and Pacific plates is partitioned into a plate-boundary/Alpine fault parallel component (37 mm/yr strike-slip movement) and a plate convergent component $(11 \mathrm{~mm} / \mathrm{yr})$ orthogonal to the Alpine fault, resulting in significant transpression [Norris et al., 1990, Walcott, 1998]. Northern and north-eastern parts of this region are dominated by crustal faults (Fig. 4.1) that indicate transition from the Hikurangi subduction zone to the central collisional zone. Continental collision in the central region is marked by structural features such as the Southern Alps mountain range, the Alpine Fault and a crustal root $(\sim 37-42 \mathrm{~km})$ and the thickened mantle lithosphere $(\sim 150 \mathrm{~km})$ beneath the Southern Alps [Scherwath et al., 2002, Okaya et al., 2007]. In southern South Island, the Australian plate is being subducted obliquely underneath Fiordland, creating a relatively young subduction zone extending up to maximum depths of $\sim 150 \mathrm{~km}$ [Davey and Smith, 1983].

There have been two opposing hypotheses to explain the deformation in the central South Island: the pervasive shear model and the narrow/localized deformation model (Fig. 4.1b). Structural and geodetic data suggest the partition of total plate 
displacement to $480 \mathrm{~km}$ of right lateral slip on the Alpine fault and $\sim 200 \mathrm{~km}$ wide distributed deformation to the east of the fault [Norris et al., 1990]. Based on the consistent patterns of fast azimuths observed from SKS studies in South Island, Klosko et al. [1999] suggested the distributed deformation of right-lateral shear $(\sim 200 \mathrm{~km})$ within a 40-400 km depth range. Molnar et al. [1999] and Stern et al. [2000] argue that this distributed deformation zone is restricted to the first 100 kilometers of the upper mantle and extends in $\mathrm{a} \sim 100-150 \mathrm{~km}$ wide region either side of the Alpine fault. They proposed a thin viscous sheet model [Molnar et al., 1999, Wilson et al., 2004, Moore et al., 2002] that agrees with the pervasive shear model [Bourne et al., 1998] (Fig. 4.1b-i) to explain such deformation behavior in the presence of weak lower crust. Conversely, the localized deformation model (Fig. 4.1b-ii) assumes the presence of a weak upper mantle instead of a weak lower crust [Meade and Hager, 2005] and suggests that the strain is localized along the faults within the crustal and upper-mantle depths and is more distributed in the asthenosphere and/or deep mantle due to asthenospheric flow or shear [Avouac and Tapponnier, 1993, Devs et al., 2011].

Here we focus on splitting of local S waves that originate within the lithosphere of the South Island to determine how anisotropy is distributed laterally and vertically in and around the plate-boundary zone across the South Island. We consider the possible sources of anisotropy and the possible mechanisms/processes associated with the formation of anisotropy. In comparison to previous local splitting studies (section 4.4.4) in the northern most region of the South Island [Audoine et al., 2000, Balfour et al., 2005], we use a larger data set, covering the entire South Island and the complete depth range available, and apply a 2-D delay-time tomography/spatial averaging technique to resolve anisotropy. We compare our results with the available SKS splitting [Klosko et al., 1999, Savage et al., 2007a], finite strain calculations from GPS data and the structural geology data [Little and Mortimer, 2001, Little et al., 2002b] to examine how the upper lithospheric deformation varies from the 
asthenospheric deformation and to test the deformation models proposed for South Island.

\subsection{Data and method}

We use both local (epicentral distance $\leq \sim 100 \mathrm{~km}$ ) and regional ( $100 \mathrm{~km}>$ epicentral distance $>250 \mathrm{~km}$ ) earthquake data recorded on twenty six GeoNet permanent three component seismographs for the period of 2004-2010 and an eleven month deployment of four temporary broad band stations in the northern part of South Island to measure S-wave splitting parameters (Fig. 4.2). These four temporary PASSCAL seismographs were deployed by Victoria University of Wellington in collaboration with the University of Colorado to enhance the seismic coverage. The complete region has been divided into three geographic units: northern, central and southern South Island. Events in each block are collected from the stations in that particular block (Fig. 4.2b). Stations that are close to the borders (WKZ, MSZ, ODZ, CASS and LTZ) of a block provided events recorded from either side. This criterion is used to control the epicentral distances (see 3.1.1). To obtain good signalto-noise ratio, we use a threshold magnitude of 3.5 in northern and southern South Island. Due to the lack of seismicity and deep events in central South Island, the threshold magnitude of 3.0 is chosen to obtain more events. We utilize an automatic S-wave splitting (Mfast) technique [Wessel, 2010, Savage et al., 2010], which was developed on the basis of the eigen-value minimization method [Silver and Chan, 1991] and the cluster analysis method [Teanby et al., 2004b]. 


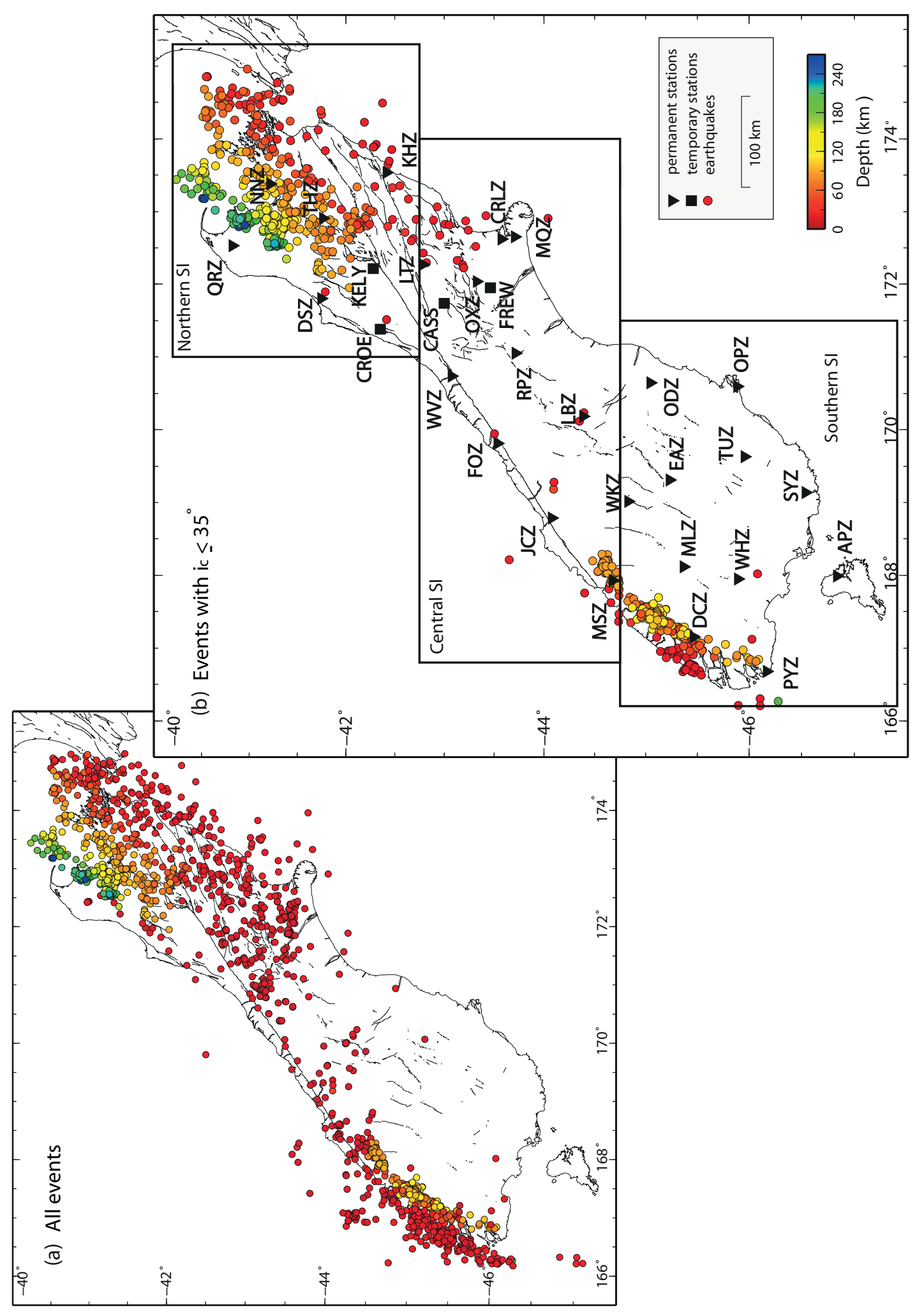

它吾至要

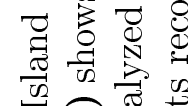

을

苛

↔

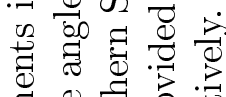

皇

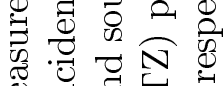

党.

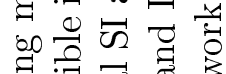

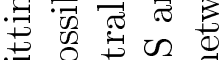

:

के

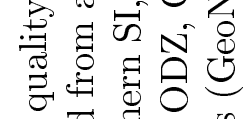

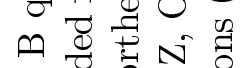

चี

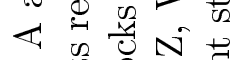

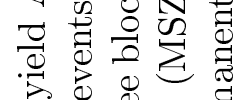

$\therefore 0$

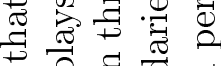

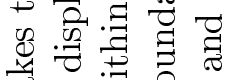

리워

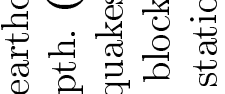

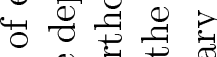

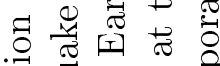

قี

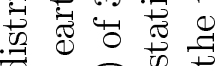

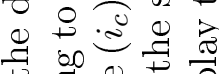

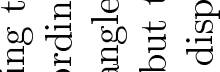

न

责

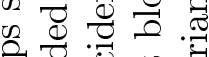

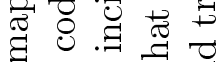

ลิ응

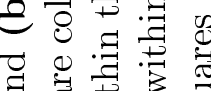

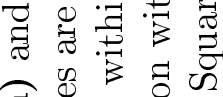

(ิ)

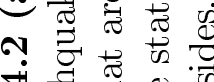

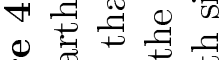

$\exists$ \& $\infty$

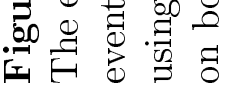


Splitting of shear waves into two quasi S-phases upon propagating through an anisotropic medium and the directional dependence of velocities of these split phases are the principal phenomena associated with the S-wave splitting method. The delay time $(\delta t)$ between fast and slow split components and the polarization azimuth of the fast split phase $(\phi)$ are the two main parameters that are determined during the splitting analysis. These parameters provide important information about the extent and degree of anisotropy and the orientation of the anisotropic medium and the past and/or present deformational processes [Silver and Chan, 1991, Crampin, 1994]. In an isotropic medium, the focal mechanism controls the S-wave particle motion, which is planar [e.g., Aki and Richards, 2002]. On entering an anisotropic medium, S waves obtain an elliptical particle motion (Fig. 4.3). Silver and Chan [1991] introduce an inverse splitting operator that removes the splitting from the S-waves to find the linearized particle motion. A grid search over all the possible pairs of fast azimuths (ranging from $-90^{\circ}$ to $90^{\circ}$ with $1^{\circ}$ increments) and delay times (ranging from 0 to $1 \mathrm{~s}$ at $0.01 \mathrm{~s}$ increments) is carried out. The values of $\phi$ and $\delta t$ are those that give the most linear incoming particle motion, as measured by minimizing the smaller eigenvalue $\left(\lambda_{2}\right)$ of the corrected two dimensional time-domain covariance matrix between the two horizontal components in the reference coordinate system (Fig. 4.3). Once the smaller eigenvalue is minimized, the corrected particle motion is linear and the eigenvector of the corresponding larger eigenvalue provides an approximate azimuth of the initial polarization or incoming polarization $\left(\phi_{p}\right)$ of the unsplit S-wave [Vidale, 1986]. This method uses the F-test to constrain the uncertainties of the splitting parameters by calculating the $95 \%$ confidence limits of the $\phi$ and $\delta t$ that correspond to the optimum solutions from the grid search analysis. 
(a) 2007.296.MSZ.2811684.0.2-3.fb1
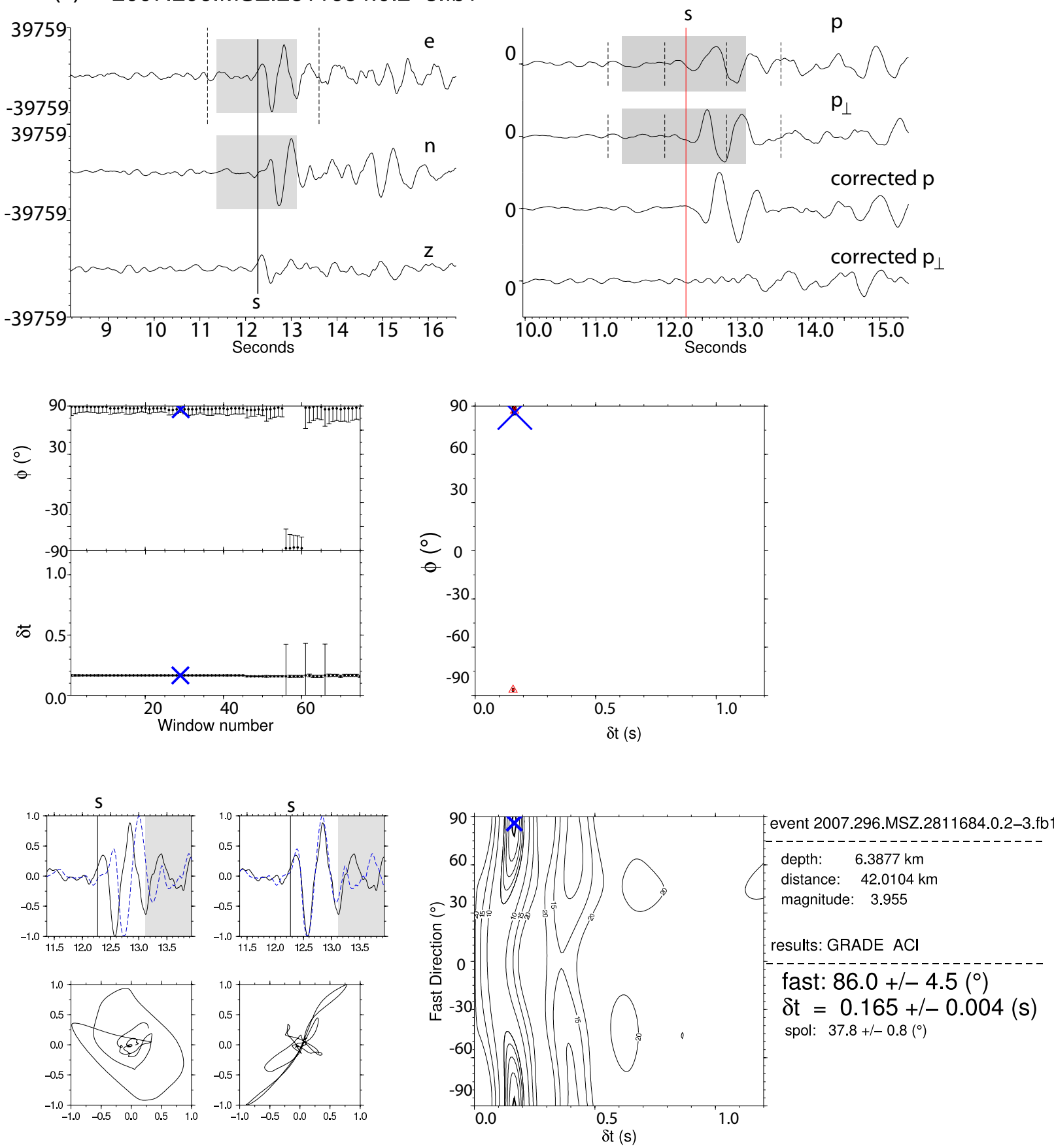

Figure 4.3 (a) Outputs from the S-wave splitting analysis using Mfast [Savage et al., 2010]. Top-left diagram shows the manual S pick (black line) on three components (e, $n$ and $\mathrm{z}$ ) of the seismograph. Dashed lines indicate the minimum starting time and maximum end time of the different $\mathrm{S}$-wave analysis windows. Top-right figure displays the rotated horizontal components before correction for splitting ( $p$ and $p_{\perp}$ ) and after correction for splitting (corrected $p$ and corrected $p_{\perp}$ ). Gray shaded regions in top figures indicate the selected S-wave analysis window. Dashed lines indicate minimum and maximum starting and ending windows. Two middle graphs show the splitting parameters (best is the blue cross) obtained from the Teanby et al. [2004b] cluster analysis. Bottom-left indicates the particle motion plots before and after correction for S-wave splitting. Here the gray region is the region not used in the analysis. Bottom-right plot is the contour diagram indicating the energy surfaces. The solution with the smallest second eigenvalue $\left(\lambda_{2}\right)$ is marked by blue cross and the $95 \%$ confidence limit of this solution is denoted by the dark black line. 
(b) 2008.250.DSZ.2964617.0.2-3.fb1
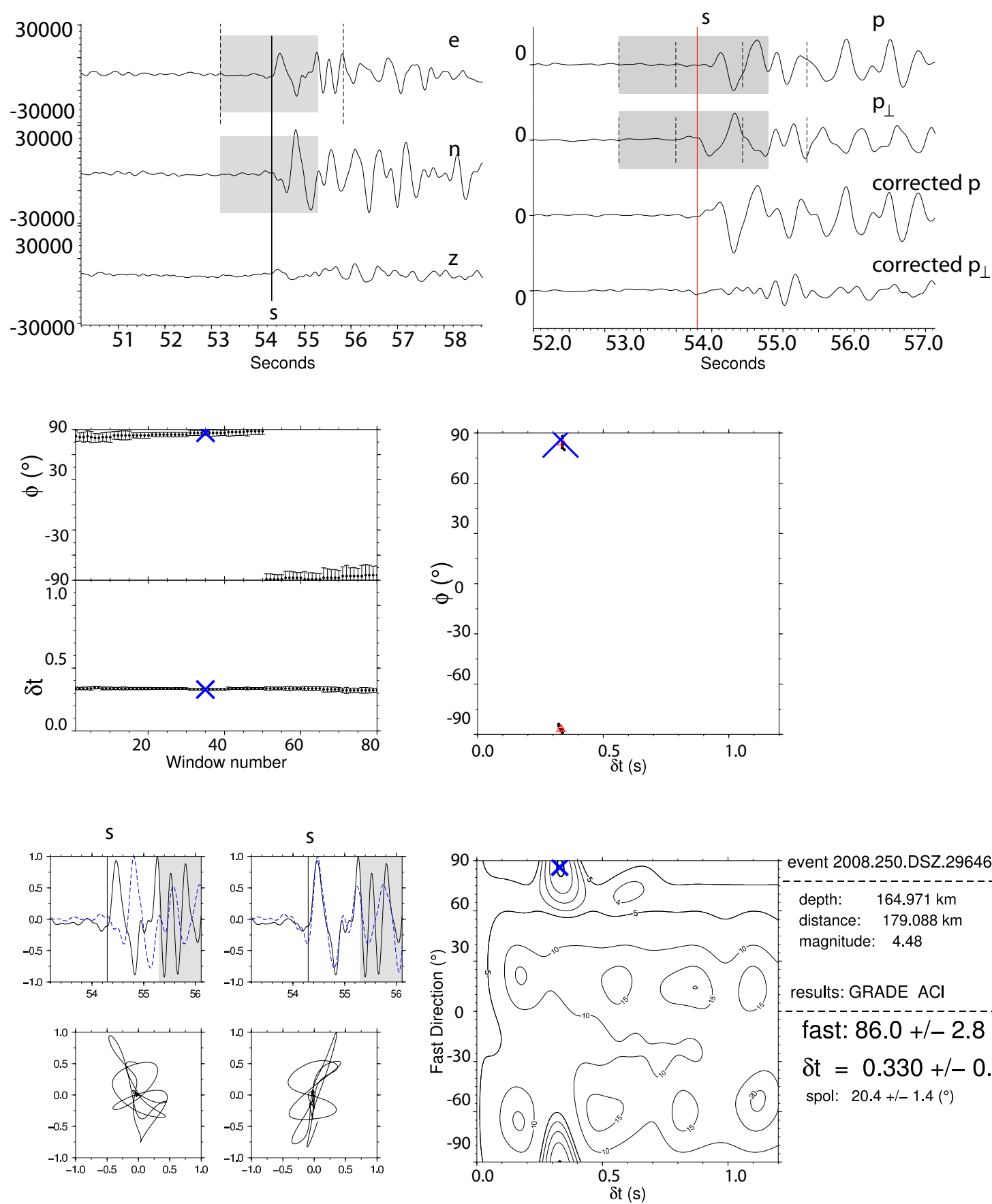

event 2008.250.DSZ.2964617.0.2-3.fb depth: $\quad 164.971 \mathrm{~km}$ distance: $179.088 \mathrm{~km}$ magnitude: 4.48
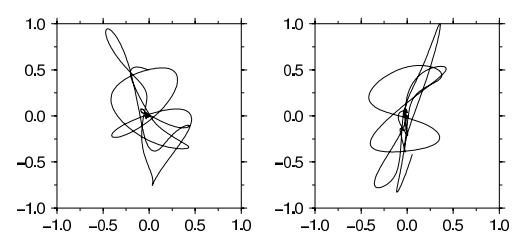

results: GRADE $A C$

fast: $86.0+/-2.8\left(^{\circ}\right)$ $\delta \mathrm{t}=0.330+/-0.006(\mathrm{~s})$ spol: $20.4+/-1.4\left(^{\circ}\right)$

Figure 4.3 (b) Same as figure caption in Fig. 4.3(a), but with an A grade measurement with higher $\delta t$ (see Figure 4.8). 
(c) 2004.003.MLZ.2165919.0.5-5.fb1
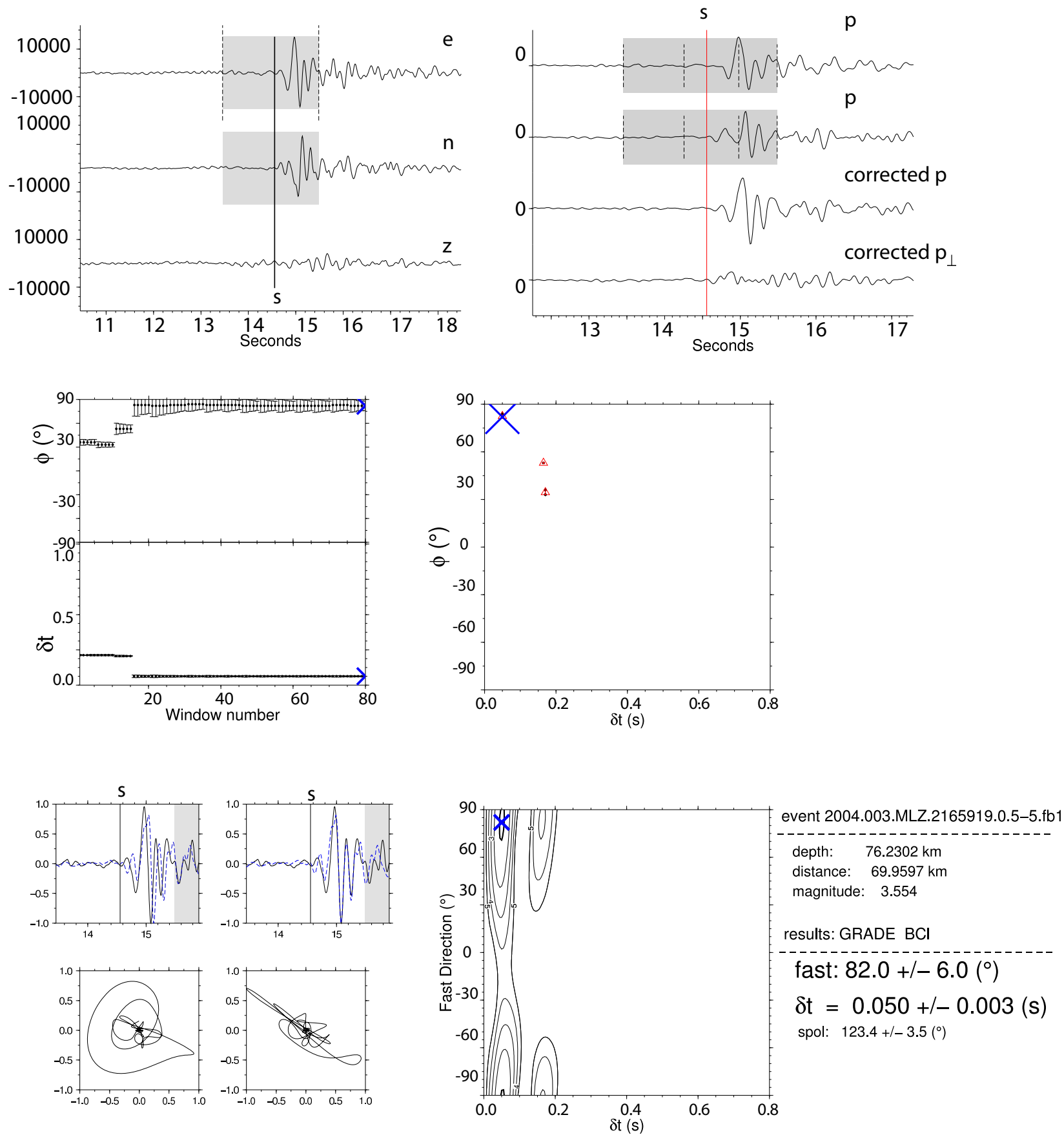

Figure 4.3 (c) Same as figure caption in Fig. 4.3(a), but showing a B grade splitting measurement.

Before processing data with the automatic method, all the events are visually inspected and S arrivals are hand picked. The automatic method involves searching over a given set of 14 band pass filters, which are two-pole Butterworth filters with frequency band-widths within the range of $0.4 \mathrm{~Hz}$ to $10 \mathrm{~Hz}$, and calculates 
signal-to-noise ratio (SNR) to find filters that give the highest SNR-bandwidth product $(\geq 3)$ for each earthquake-station pair. Then the signals obtained from the selected filters are used to estimate the splitting parameters. The dominant period of each S-phase is determined using the frequency that corresponds to the highest amplitude of the spectrum calculated from the three second window directly after the S-pick. Cluster analysis avoids uncertainties in the splitting parameters caused by the selection of S-wave analysis windows [Teanby et al., 2004b]. This method selects the analysis window that gives the most stable splitting parameters with small errors by performing splitting measurements through a given set of analysis windows (Fig. 4.3). We chose the splitting measurements that agree with the $\mathrm{A}$ and/or B grading criteria (here after AB grade) as good quality splitting measurements [Savage et al., 2010]. In Mfast, measurement attains AB grading if the measurement's SNR $>3, \delta t<0.8^{*} \operatorname{tag}_{\max }$ (tlag $\max _{\max }$ is the maximum $\delta t$ for grid search), maximum error in $\phi<25^{\circ}$, if it is not a null measurement, and if the measurement graded as a A or B cluster in the cluster grading (section 3.2.3). Note that the A grade criteria (a. SNR $>4$, b. $\delta t<0.8^{*} \operatorname{tlag}_{\max }$, c. not null and $d \phi<10^{\circ}$ ) are a subset of AB grade criteria, which use more narrow limits for SNR and $\mathrm{d} \phi$ compared to AB grade criteria. Null measurements are those for which $\phi_{p}$ is within $20^{\circ}$ of parallel or perpendicular to the fast azimuth, which can lead to incorrect $\delta t$. The splitting measurements from each event that meet the above quality scheme are again checked considering their uncertainties and errors for different filters. This final quality criterion extracts the measurements that give a similar answer, i.e. if the time difference $<\operatorname{tlag}_{\max } / 8$ or if the angular distance $<\pi / 8$, in each filter (refers to 'best filter measurements' in later sections). From the $\sim 1600$ AB quality splitting measurements [Savage et al., 2010] we selected the $\sim 850$ measurements that are within the incidence angle of $35^{\circ}$ (see section 4.2.1) for further analysis (Fig. 4.2b). The number of good splitting measurements made at each station varies depending on the seismicity distribution (Fig. 4.2) and the quality criteria used in the analysis. 


\subsubsection{Limitations and statistical analysis of the measure- ments}

The polarization of an S-wave incident at the free surface is governed by many factors such as the angle of incidence, frequency of the incident wave, the source depth, and the layering of the crust [Booth and Crampin, 1985]. To minimize the effect of incidence angle on S-wave polarization, splitting analysis is usually carried out inside the shear-wave window (i.e. a few degrees less than critical incidence angle) [Evans, 1984, Nuttli, 1961]. Wave fronts with incident angles beyond the critical angle $\left(i_{c}=\sin ^{-1}\left(V_{s} / V_{p}\right)\right.$, where $V_{s}$ and $V_{p}$ are $\mathrm{S}$ and $\mathrm{P}$ velocities respectively.) are likely to display complex particle motions due to phase conversions at the free surface. This will cause particle motion to be non-linear even in the absence of anisotropy [Nuttli, 1961] and make the interpretation of S-wave polarization complicated. To accurately calculate the incidence angle, we created a velocity model with average velocities that obtained from velocity-structure studies in South Island [EberhartPhillips and Bannister, 2010, Eberhart-Phillips and Reyners, 2001]. This average velocity model has an upper layer with $V_{s}$ (S wave velocity) and $V_{p}$ (P wave velocity) values of $2.6 \mathrm{~km} / \mathrm{s}$ and $4.4 \mathrm{~km} / \mathrm{s}$ respectively. The low velocity surface layer allows earthquake waves from a broad epicentral range to approach the surface within the S-wave window [Booth and Crampin, 1985]. In South Island, most of the seismicity is limited to the plate-boundary zone and this narrow distribution significantly reduces the number of events that can be used to measure the crustal anisotropy far away from the plate-boundary. Therefore, some of the stations in the eastern margin of the central and southern South Island (e.g. OPZ, ODZ, and SYZ) are only used for deep events (depth $\gtrsim 60 \mathrm{~km})$ (Fig. $4.4 \mathrm{~b})$.

In addition to calculating uncertainties and to grading individual splitting measurements, statistical parameters (e.g. mean, standard deviation, standard error) of the single station measurements are estimated (Table 4.1). We use directional statistics 

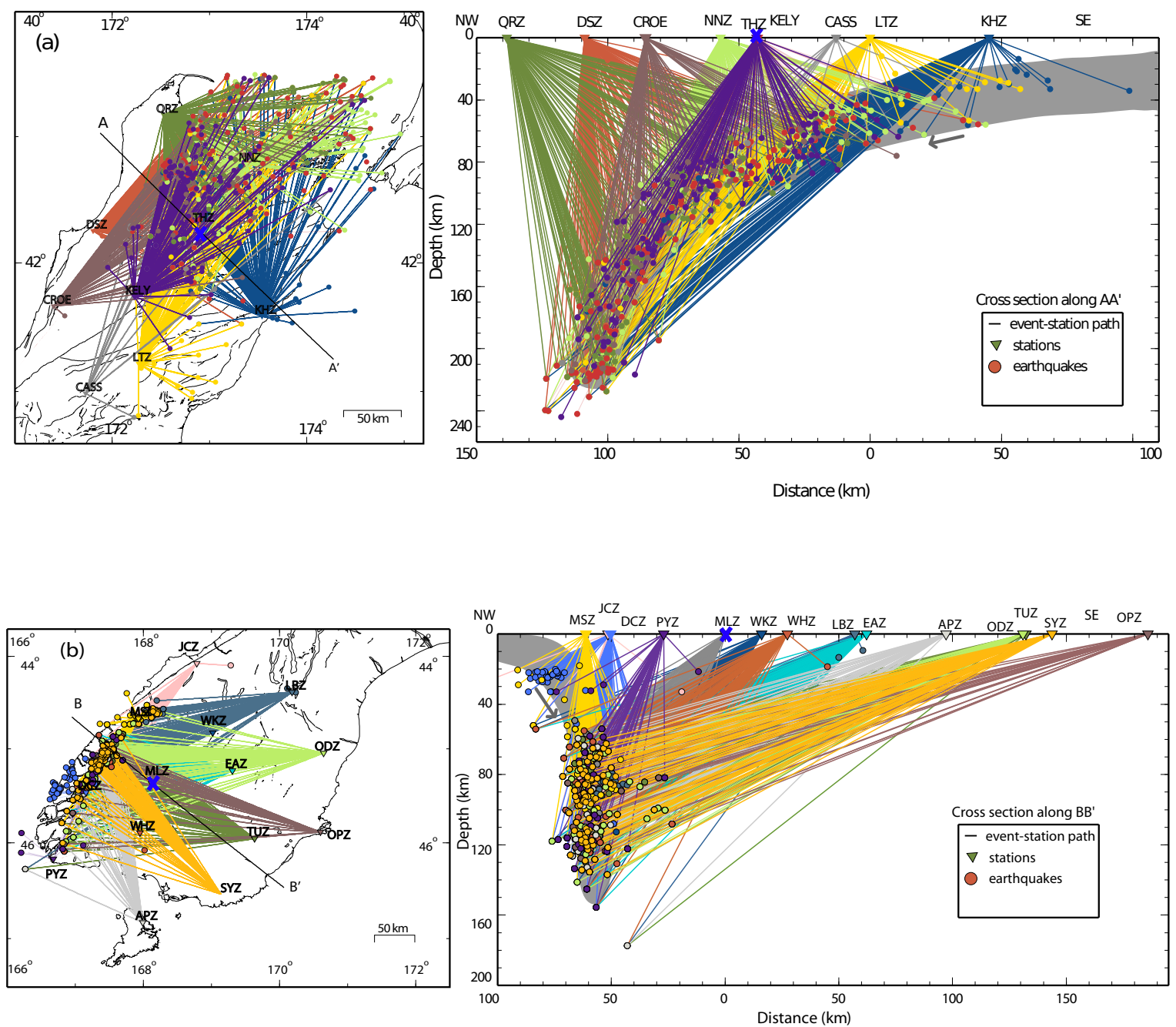

Figure 4.4 Straight-line ray paths showing the coverage in the subduction zones in northern South Island (a) and southern South Island (b). Right side maps show the cross-sections (depth profiles) along $\mathrm{AA}^{\prime}$ and $\mathrm{BB}^{\prime}$ lines that are marked on the left side figures. Events and ray paths corresponding to each station are color coded using the same color. The approximate slab positions are colored are in grey.

discussed in Mardia [1972] and Gerst and Savage [2004] for $\phi$. Directional statistics are necessary to determine the statistics of $\phi$ and to estimate errors and accuracy of the mean $\phi\left[\right.$ Roman et al., 2011]. Because of the $180^{\circ}$ ambiguity of angular data, directional statistics are different from normal statistics. Mean $\phi$, the length of the mean resultant vector of $\phi(\mathrm{R})$, circular standard deviation (CSD) and standard error (SE) are the statistical parameters that we estimate. $\mathrm{R}$ is a quantitative estimate of circular spread that is calculated from the mean angular azimuth $\left(\phi_{s}\right)$. SE of $\phi_{s}$ is calculated using $\mathrm{R}$ and the concentration parameter $(\kappa)$, which is a parameter of a Von Mises distribution $\left(V M_{\left(\phi_{s}, \kappa\right)}\right)$, and SE indicates the accuracy of the $\phi_{s} . V M_{\left(\phi_{s}, \kappa\right)}$ 
is a continuous distribution on the range of $0-360^{\circ}$ and it is a probability density function that varies with $\phi_{s}$ and $\kappa$. Thus, $V M_{\left(\phi_{s}, \kappa\right)}$ is considered to be a circular equivalent of the normal distribution that measures the circular spread [Mardia, 1972, Philipp, 2009]. Table 4.1(a) and Table 4.1(b) summarize the above statistical parameters that are calculated using splitting measurements made at each station and section 4.3 explains these results. We have also conducted a detailed analysis of splitting measurements considering the possible relationships between splitting parameters and other variables such as earthquake depth, earthquake-station back azimuth, average maximum frequency (or an average period) in the shear-wave analysis window and $\phi_{p}$ to provide constraints on both lateral distribution and depth extent of anisotropy (see section 4.4.1).

\subsubsection{Resolving the spatial heterogeneity of splitting mea- surements}

The heterogeneous anisotropic structure observed is further evaluated using 2-D delay-time tomography and a spatial averaging technique (TESSA) introduced by Johnson et al. [2011]. This allows us to obtain approximate constraints on regions with low and high anisotropy. A $\delta t$ from a single measurement is a quantitative estimation of the anisotropic structure along the ray path from source to the station and the $\delta t$ is taken to be proportional to path length $(L)$ of the ray through the anisotropic medium [Silver, 1996]. TESSA is based on a simplified and linear relationship between $\delta t$ and $(L)$. It assumes that the $\delta t_{r}$ observed along the ray path is the summation of $\delta t$ calculated for each grid block $\left(\delta t_{b}\right)$ with variable strength of anisotropy $\left(s_{b}\right)$ in 2-D space. First, straight-line ray tracing between station and events determines the lengths of ray segments $\left(L_{r b}\right)$ that lie on each grid block (Fig. 3.18). Then the total anisotropy measured at the station for a given event is 
calculated by,

$$
\delta t_{r}=\sum_{b=1}^{n}\left(s_{b} \times L_{r b}\right)
$$

The inversion part of TESSA determines the $s_{b}$ for resolved blocks and is carried out using the MATLAB medium-scale optimization inversion function that finds the linear least-square solution of the problem. This solution is then used iteratively to find the model solution for the strength of anisotropy of the whole ray $\left(s_{r}\right)$ and this process is subjected to boundary conditions. As explained in Johnson et al. [2011], boundary constraints are applied in such a way that the minimum $s_{r}$ will always be greater than $0 \mathrm{~s} / \mathrm{km}$ and the maximum $s_{r}$ will not exceed $\delta t_{r(\max )} / L_{b}$, where $\delta t_{r(\max )}$ is the maximum $\delta t$ observed along a ray path and $L_{b}$ is the width of the grid block. Tomography results discussed in section 4.4 .3 are obtained using regular gridding with $25 \mathrm{~km}$ square blocks. The minimum number of rays per block is set up to be 6 to obtain reliable results. Tomographic analysis only uses the AB grade measurements that are within the incidence angle of $35^{\circ}$ and that satisfy the best filter criteria. This enables us to avoid ambiguous splitting measurements as well as statistical uncertainties that could affect the final solution of the tomography and spatial averages.

We use the tomographic weighting scheme presented in TESSA to determine average $\phi$ in grid blocks. Tomographic weighting is designed to take into account variations due to heterogeneous anisotropic structure. The tomographic weighting function $\left(\omega_{r b}\right)$ that is applied in this averaging weights the $\phi$ considering the anisotropy strength profile determined for each grid block $\left(s_{b}\right)$ in the $\delta t$ tomography, so that more weighting is given to the regions with higher strength of anisotropy. $\omega_{r b}$ is defined as the strength profile of the each grid block, normalized by $\delta t_{r}$ [Johnson et al., 2011],

$$
\omega_{r b}=s_{b} / \delta t_{r}
$$

Then, the weighted mean $\phi$ of the grid block $b$ with $n$ number of rays is given by 
$\operatorname{azimuth}\left(\bar{\phi}_{b}\right)$,

$$
\bar{\phi}_{b}=\frac{1}{2} \arctan \left(\frac{\sum_{r=1}^{n} \cos \left(2 \phi_{r}\right) \times \omega_{r b}}{\sum_{r=1}^{n} \sin \left(2 \phi_{r}\right) \times \omega_{r b}}\right)
$$

where $b$ and $r$ denote the block and ray number respectively. Both tomography and spatial averaging results are given in the section 4.3.

\section{$4.3 \quad$ Results}

Single-station splitting parameters are mainly discussed with regard to two depth units; shallow $(\leq 100 \mathrm{~km})$ and deep $(>100 \mathrm{~km})$. The $100 \mathrm{~km}$ depth contour roughly represents the minimum depth limit of the lithosphere-asthenosphere boundary (LAB) in South Island [Stern et al., 2000, Scherwath et al., 2002]. Figs. 4.5(a) and $4.5(\mathrm{~b}) /(\mathrm{c} i / i i)$ show the normalized circular histograms of fast azimuths $(\phi)$ measured at each station using shallow and deep local earthquakes. These spatial maps represent $\phi$ of the high quality, A grade (section 4.2), measurements that were obtained from multiple filters (a maximum of three filters with $\mathrm{SNR} \geq 3$ for each event). Events that have the same $\phi$ for different filters are visually more prominent than those with varying azimuths for multiple filters. Table 4.1(a) and Table 4.1(b) summarize the average splitting parameters obtained at each station using deep and shallow earthquakes respectively. To calculate single station averages (Table 4.1a/b), we use an A-grade measurements that satisfy the best filter criterion (section 4.2) for each station-earthquake pair.

Some stations (e.g. MSZ, NNZ, KHZ, CROE) exhibit either large scattering or bimodal distribution of $\phi$ (or high standard error) (Fig. 4.5a and Table 4.1a/b). We attribute these complex patterns of $\phi$ to spatially varying anisotropy and depth dependent $\phi$ as seen in the detailed analysis of splitting parameters (Fig. 4.6). In general, $\phi$ observed from both shallow and deep events indicate significant lateral 
Table 4.1 (a) Summary statistics (see p105) of the splitting parameters from shallow ( $\leq$ $100 \mathrm{~km})$ events.

\begin{tabular}{|c|c|c|c|c|c|c|c|c|}
\hline station & no & $\phi_{s}^{a}$ & $\begin{array}{l}S E^{b} \text { of } \\
\phi_{s}\end{array}$ & $\begin{array}{l}C S D^{c} \\
\text { of } \phi \\
\end{array}$ & $R^{d}$ & $\begin{array}{l}\text { mean } \\
\delta t\end{array}$ & $\begin{array}{l}\mathbf{S E} \text { of } \\
\delta t\end{array}$ & $\begin{array}{l}S D^{e} \text { of } \\
\delta t\end{array}$ \\
\hline$\overline{\overline{\mathrm{DSZ}}}$ & 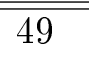 & 60.526 & 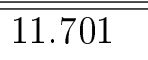 & 248.148 & 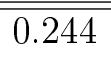 & "0.172 & 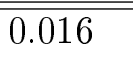 & 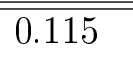 \\
\hline MQZ & 20 & -26.623 & 14.977 & 44.727 & 0.296 & 0.180 & 0.019 & 0.086 \\
\hline KHZ & 68 & -49.314 & 16.878 & 56.321 & 0.145 & 0.196 & 0.015 & 0.127 \\
\hline LTZ & 79 & -72.066 & 5.310 & 38.273 & 0.410 & 0.188 & 0.017 & 0.148 \\
\hline NNZ & 111 & -69.658 & 8.798 & 50.158 & 0.216 & 0.197 & 0.013 & 0.138 \\
\hline QRZ & 66 & 38.922 & 8.153 & 44.532 & 0.299 & 0.184 & 0.011 & 0.086 \\
\hline FOZ & 15 & -84.337 & 7.642 & 28.637 & 0.607 & 0.103 & 0.020 & 0.079 \\
\hline THZ & 58 & 11.330 & 17.411 & 55.619 & 0.152 & 0.285 & 0.019 & 0.146 \\
\hline CRLZ & 43 & -34.399 & 3.838 & 25.109 & 0.681 & 0.147 & 0.010 & 0.066 \\
\hline WKZ & 94 & 32.646 & 7.506 & 46.163 & 0.273 & 0.302 & 0.016 & 0.158 \\
\hline $\mathrm{JCZ}$ & 61 & -74.061 & 14.429 & 53.199 & 0.178 & 0.203 & 0.015 & 0.116 \\
\hline LBZ & 79 & 38.882 & 9.187 & 48.098 & 0.244 & 0.206 & 0.015 & 0.129 \\
\hline DCZ & 139 & -9.234 & 3.246 & 34.081 & 0.493 & 0.159 & 0.008 & 0.089 \\
\hline MSZ & 99 & 12.399 & 13.200 & 55.479 & 0.153 & 0.218 & 0.017 & 0.171 \\
\hline EAZ & 95 & 8.267 & 8.333 & 48.010 & 0.246 & 0.222 & 0.012 & 0.114 \\
\hline MLZ & 120 & 41.762 & 7.044 & 47.155 & 0.258 & 0.132 & 0.007 & 0.076 \\
\hline TUZ & 86 & -9.634 & 4.820 & 37.210 & 0.430 & 0.203 & 0.011 & 0.099 \\
\hline WHZ & 83 & -26.898 & 6.790 & 43.319 & 0.319 & 0.210 & 0.013 & 0.121 \\
\hline ODZ & 38 & -25.905 & 4.077 & 25.077 & 0.682 & 0.269 & 0.029 & 0.177 \\
\hline OXZ & 15 & -32.126 & 9.739 & 33.780 & 0.499 & 0.232 & 0.041 & 0.160 \\
\hline $\mathrm{APZ}$ & 38 & -83.567 & 6.099 & 33.713 & 0.500 & 0.263 & 0.020 & 0.124 \\
\hline $\mathrm{OPZ}$ & 13 & 15.006 & 19.602 & 45.660 & 0.281 & 0.182 & 0.022 & 0.080 \\
\hline PYZ & 24 & 22.666 & 16.154 & 47.578 & 0.252 & 0.229 & 0.025 & 0.121 \\
\hline SYZ & 26 & -9.093 & 11.187 & 41.845 & 0.344 & 0.108 & 0.010 & 0.053 \\
\hline CASS & 6 & 66.843 & 10.572 & 25.730 & 0.668 & 0.139 & 0.027 & 0.066 \\
\hline CROE & 51 & -57.412 & 8.198 & 42.323 & 0.336 & 0.153 & 0.012 & 0.086 \\
\hline FREW & 10 & -75.721 & 22.599 & 45.851 & 0.278 & 0.209 & 0.046 & 0.145 \\
\hline KELY & 107 & 8.748 & 6.321 & 44.303 & 0.302 & 0.176 & 0.011 & 0.114 \\
\hline RPZ & 29 & 25.981 & 11.223 & 42.899 & 0.326 & 0.177 & 0.016 & 0.088 \\
\hline
\end{tabular}

${ }^{a}$ mean fast azimuth

$b_{\text {standard error }}$

${ }^{c}$ circular standard deviation

$d_{\text {the length of the mean resultant vector } \phi}$

$e_{\text {standard deviation }}$ 
Table 4.1 (b) Summary statistics of the splitting parameters from deep ( $>100 \mathrm{~km})$ events.

\begin{tabular}{|c|c|c|c|c|c|c|c|c|}
\hline station & no & $\phi_{s}$ & $\begin{array}{l}\mathbf{S E} \\
\phi_{s}\end{array}$ & $\begin{array}{l}\text { CSD } \\
\phi\end{array}$ & $\mathbf{R}$ & $\delta t$ & $\begin{array}{l}\mathbf{S E} \text { of } \\
\delta t\end{array}$ & $\mathbf{S D} \delta t$ \\
\hline DSZ & 172 & -89.818 & 4.740 & 43.407 & 0.317 & 0.190 & 0.008 & 0.101 \\
\hline $\mathrm{KHZ}$ & 131 & 42.588 & 17.024 & 60.993 & 0.104 & 0.185 & 0.009 & 0.101 \\
\hline LTZ & 91 & -58.693 & 12.466 & 54.010 & 0.169 & 0.190 & 0.014 & 0.129 \\
\hline NNZ & 137 & 22.979 & 20.417 & 63.669 & 0.085 & 0.181 & 0.009 & 0.105 \\
\hline QRZ & 131 & -89.716 & 5.395 & 43.286 & 0.319 & 0.239 & 0.010 & 0.118 \\
\hline THZ & 103 & 7.699 & 5.271 & 40.653 & 0.365 & 0.185 & 0.012 & 0.122 \\
\hline WKZ & 36 & -8.316 & 12.335 & 46.449 & 0.269 & 0.259 & 0.027 & 0.161 \\
\hline $\mathrm{JCZ}$ & 43 & -60.998 & 2.761 & 18.326 & 0.815 & 0.219 & 0.009 & 0.059 \\
\hline LBZ & 124 & 38.022 & 1.786 & 20.145 & 0.781 & 0.165 & 0.006 & 0.064 \\
\hline DCZ & 91 & -12.607 & 4.413 & 36.018 & 0.454 & 0.162 & 0.010 & 0.096 \\
\hline MSZ & 84 & -67.490 & 11.236 & 51.816 & 0.195 & 0.257 & 0.013 & 0.115 \\
\hline EAZ & 53 & -11.328 & 22.251 & 58.472 & 0.125 & 0.259 & 0.024 & 0.173 \\
\hline MLZ & 84 & 59.590 & 9.536 & 49.211 & 0.229 & 0.193 & 0.013 & 0.119 \\
\hline TUZ & 74 & -41.503 & 5.457 & 38.171 & 0.412 & 0.206 & 0.018 & 0.154 \\
\hline WHZ & 79 & -9.397 & 11.475 & 51.666 & 0.197 & 0.178 & 0.015 & 0.129 \\
\hline ODZ & 36 & -20.020 & 8.517 & 39.788 & 0.381 & 0.228 & 0.026 & 0.156 \\
\hline OXZ & 20 & -34.412 & 20.972 & 50.346 & 0.213 & 0.308 & 0.027 & 0.120 \\
\hline $\mathrm{APZ}$ & 23 & -86.429 & 7.795 & 33.596 & 0.503 & 0.191 & 0.034 & 0.164 \\
\hline OPZ & 9 & 86.165 & 64.184 & 60.834 & 0.105 & 0.182 & 0.028 & 0.084 \\
\hline PYZ & 20 & -19.624 & 11.095 & 39.224 & 0.392 & 0.199 & 0.030 & 0.136 \\
\hline SYZ & 52 & 32.961 & 11.443 & 48.271 & 0.242 & 0.129 & 0.010 & 0.073 \\
\hline CASS & 3 & 49.081 & 35.524 & 43.222 & 0.320 & 0.230 & 0.084 & 0.146 \\
\hline CROE & 48 & -24.622 & 44.491 & 66.860 & 0.066 & 0.208 & 0.020 & 0.135 \\
\hline FREW & 23 & 21.890 & 18.091 & 49.093 & 0.230 & 0.247 & 0.037 & 0.178 \\
\hline KELY & 83 & 13.813 & 11.706 & 52.357 & 0.188 & 0.209 & 0.013 & 0.117 \\
\hline RPZ & 76 & -19.418 & 5.329 & 37.968 & 0.416 & 0.245 & 0.017 & 0.147 \\
\hline
\end{tabular}

variations compared to that of SKS azimuths (section 4.4.4), but measurements at some closely spaced stations tend to show regional consistencies over short distances suggesting coherent anisotropy in small regions (Fig. 4.5). Shallow events processed at stations in the northern South Island display two distinct fast polarization azimuths (Fig. 4.5b): sub-parallel to the Alpine fault and the Marlborough fault system, mainly in the western and central part of northern South Island (QRZ, KELY, DSZ and THZ); and approximate EW alignment at a high angle to the Alpine fault trace in the eastern side of the northern South Island (LTZ, CRLZ, OXZ and MQZ). $\phi$ also becomes approximately EW oriented from deeper earthquakes $(>100 \mathrm{~km})$ observed at coastal stations in northern South Island (DSZ,QRZ and 
KHZ) and the western part of southern South Island (MSZ and JCZ). For shallow earthquakes, NE oriented (plate-boundary sub-parallel) $\phi$ are observed at most of the stations in northern South Island and the central region of central South Island and southern South Island (e.g. CASS, RPZ, LBZ, WKZ, MLZ). However, the stations that are very close to the Alpine fault in central South Island (JCZ and FOZ) show either bimodal distributions or WNW-ESE fast azimuths at high angle to the fault (Fig. 4.5). This nearly fault-perpendicular azimuth also appears to be the prominent azimuth at depths $>100 \mathrm{~km}$ as seen in JCZ and MSZ (Figs. 4.5d and 4.6b), indicating a strong WNW-ESE alignment in anisotropy at subcrustal and/or upper mantle depths in the western side of southern South Island. 

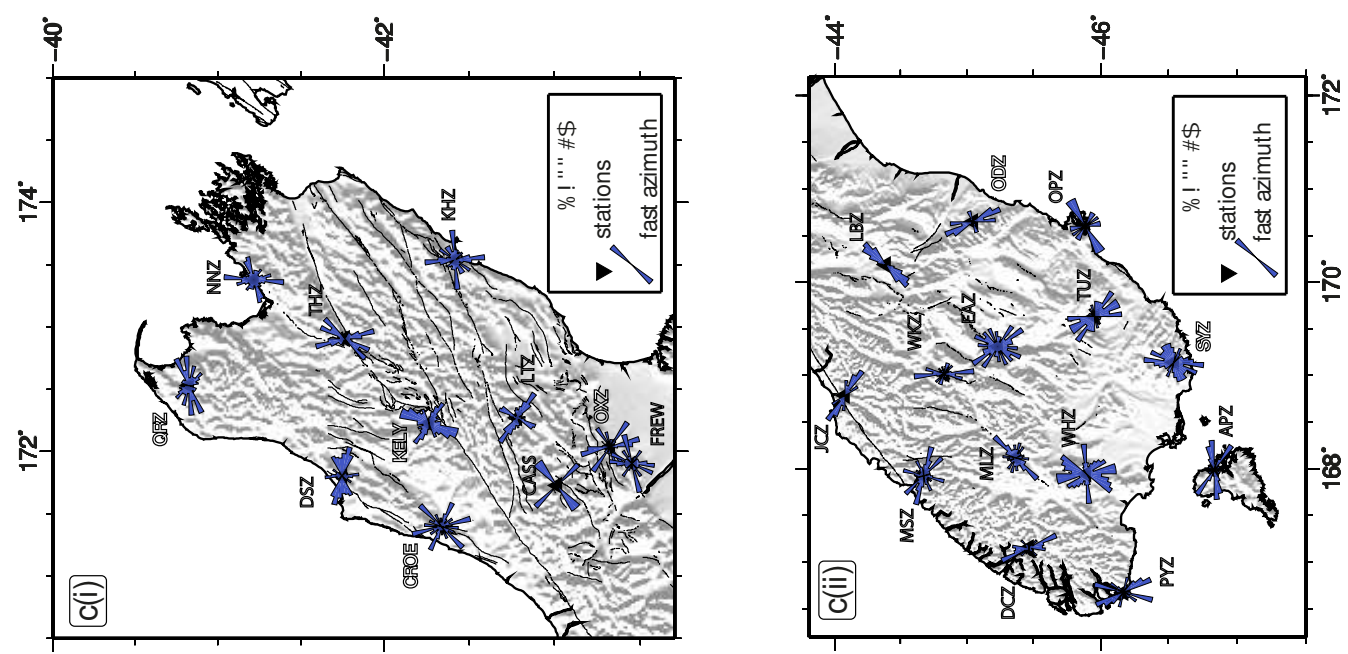

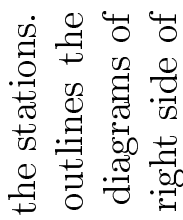

$* \approx 0 \%$

है मृ

के

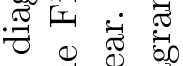

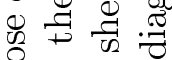

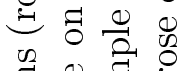

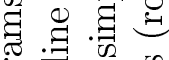

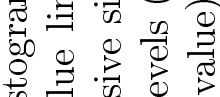

$\exists \bar{c}$

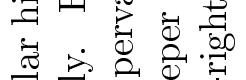

릴

.

๙

ग

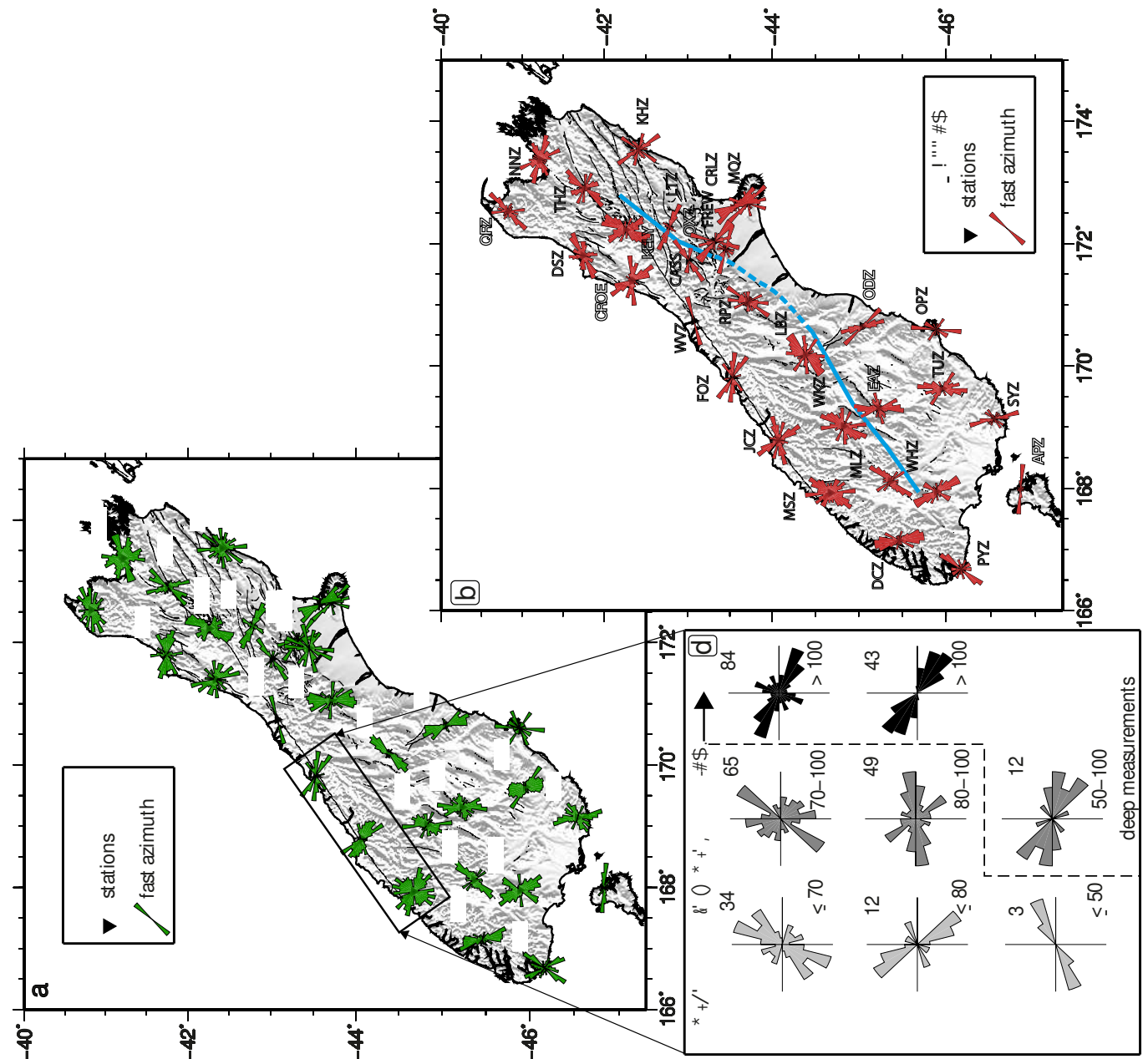

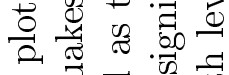

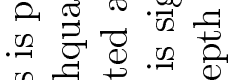

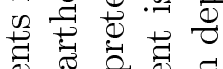

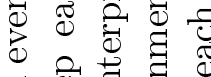

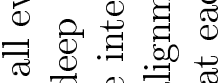

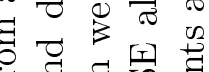

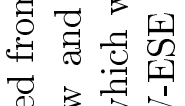

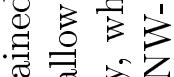

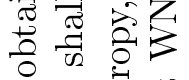

(1) छ

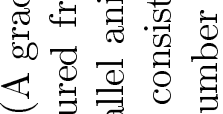

$\theta$ ब

廿 常

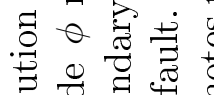

泀范蛋

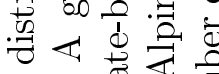

急焉

के

ป 궁

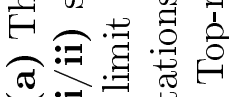

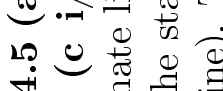

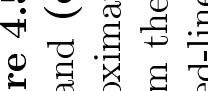

范

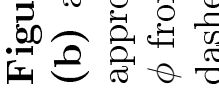




\subsection{Interpretations and discussion}

\subsubsection{Depth extent and backazimuthal variations of splitting parameters}

An analysis of the splitting parameters with respect to depth and backazimuth is carried out in order to understand the complex patterns of $\phi$ observed from the rose diagrams. This analysis is limited to northern and southern South Island where there is a good earthquake distribution with depth due to subducting-slab earthquakes (Fig. 4.4). Stations that sample different regions of the two subduction zones with considerable numbers of splitting measurements exhibit changes in $\phi$ with depth (e.g. DSZ, QRZ in Figs. 4.6a and JCZ, MLZ in Figs. 4.6b). These depth variations sometimes appear to be combined with lateral variation, indicated by a complex patterns of $\phi$ (Fig. 4.6).

We also search for variation in delay times $(\delta t)$ to determine the extent and the degree of the anisotropy. In the presence of homogeneous or slowly varying anisotropy, $\delta t$ accumulates along the earthquake-station ray path as a result of the difference in travel velocities of two split shear waves [Rumpker and Silver, 1998]. At most of the stations, $\delta t$ are within the range of $\sim 0.15-0.30 \mathrm{~s}$, but some stations recorded high $\delta t$ $(>0.4 \mathrm{~s})$ with $\phi$ sub-parallel to the Alpine fault. The west coast close to the Alpine fault in southern South Island is also characterized by varying anisotropic structure as shown in Fig. 4.6b (JCZ, WKZ and EAZ), with inconsistent patterns of $\phi$ and high $\delta t$. Such variations imply a complex tectonic setting in the Fiordland region. However, some clusters of closely spaced earthquakes give consistent readings at each station (e.g. Figs. 4.6 and A.2). 

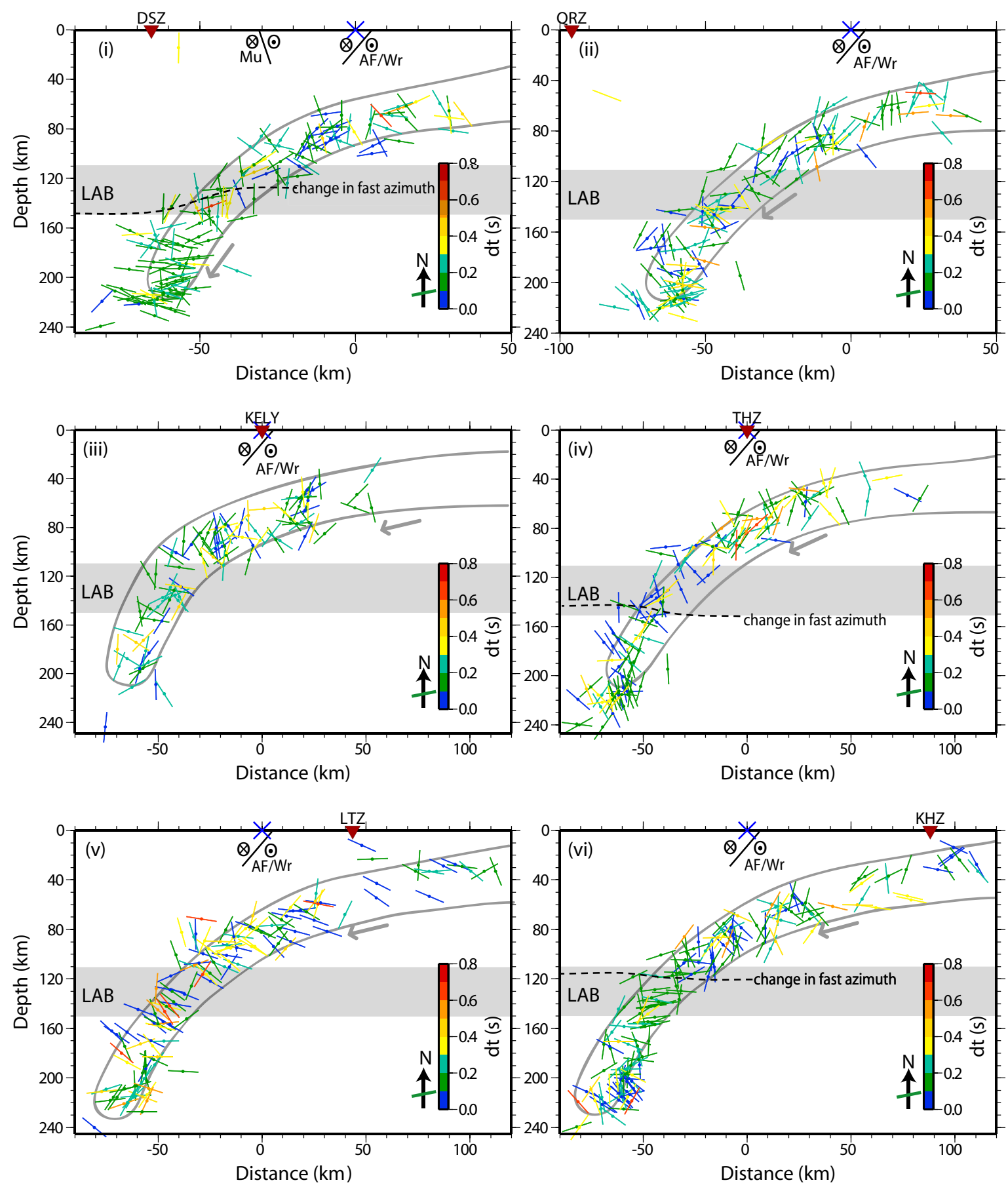

Figure 4.6 (a) Changes in $\phi$ with depth in northern South Island along the profile AA' (see Fig. 4.4a). Station measurements displayed in the upper, middle and bottom diagrams are located in western, central and eastern regions of the northern South Island respectively. Depth boundary where there is a visible change in $\phi$ with depth is marked with a black dashed line (i.e DSZ and THZ) and the colors of the bars denote the individual $\delta t$ measurements. Note that the $\phi$ are determined in the horizontal plane and are thus plotted relative to $\mathrm{N}$ as marked on the figures. The blue cross indicates the reference point of each profile that is used to calculate the relative distance to the station. The approximate position of the slab (gray line), possible depth range for the location of lithosphere-asthenosphere boundary (LAB - shaded gray region), and the location of the Alpine/Wairau (AF/Wr) and Murchison-basin $(\mathrm{Mu})$ faults are shown in each profile. The direction of the black bar next to each fault label indicates the approximate strike direction of the fault in map view (not the dip). 

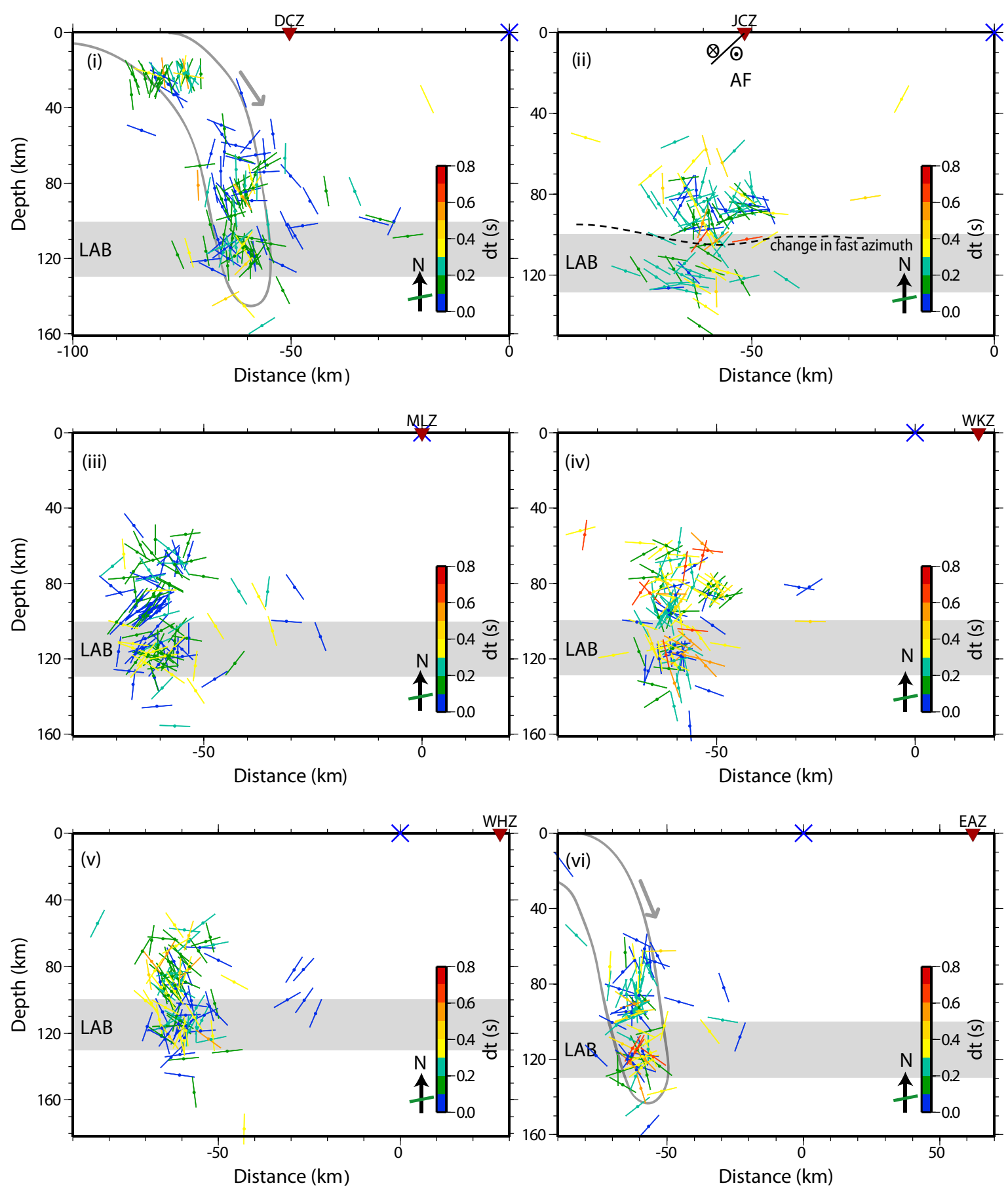

Figure 4.6 (b)Changes in splitting parameters with depth in southern South Island along the profile BB' (see Fig. 4.4b). Note the higher $\delta t$ and complex patterns of $\phi$ observed at the stations JCZ, WKZ and EAZ that are located in the northern region of the southern South Island close to the Alpine fault. Black dashed line (e.g. JCZ) indicates the depth boundary where there is a clear change in $\phi$ (colors of the bars denote the individual $\delta t$ measurements). 
$\phi$ perpendicular to the strike of the Alpine fault observed from the intermediate $(50-100 \mathrm{~km})$ to deep measurements $(>100 \mathrm{~km})$ that are made on some stations (e.g. JCZ, MSZ and FOZ) right above and close to the fault (Fig. 4.5d) could be explained by two alternative mechanisms. If the upper-most mantle $(\sim 30 \mathrm{~km}$ thick uppermantle layer) contributes most of the anisotropy, it is likely a product of deformation of olivine crystals under different physical and chemical conditions. As proposed by Karato et al. [2008], deformational fabrics of olivine could be influenced by stress, temperature and the water content of the material resulting in changes of olivine slip directions from typical [100] to [010] (B-type olivine). The subduction to collision tectonic setting in the western edge of the central and southern South Island could be a favorable place for high pressure and/or water rich environment that could change the orientation of $\phi$ from the expected fault sub-parallel $\phi$ and result the $\phi$ to be orthogonal to the plate-boundary and the Alpine fault. The other possibility is that the deep high-frequency phases are sensitive to crustal anisotropic structures [Audoine et al., 2000]. The prominent WNW-ESE direction at the above stations is parallel to the principal compressive direction $\left(\sim 115^{\circ}\right)$ of this region [Boese et al., 2012, Townend et al., 2012], suggesting the contribution of crustal stress controlled mechanisms.

Apparent spatial variations of $\phi$ can also be caused by the geometry of the anisotropic unit. Models of vertically varying structure with two [Silver and Savage, 1994] or more layers [Rumpker and Silver, 1998] of anisotropy that have different azimuths of horizontal symmetry or have plunging symmetry axes [Babuska et al., 1984, Babuska and Plomerova, 1993] yield complex but systematic patterns of splitting parameters (see section 4.4.2). However, modeling of these effects has been carried out mainly on SKS splitting studies focusing on the anisotropic structure of the upper mantle and models have limited applications for the high-frequency local S-wave splitting because of the crustal heterogeneities and scattering that also affect the splitting. Lack of strong correlation between splitting parameters and BAZ/incidence angle 
(Fig. A.2) in our splitting data set suggests splitting parameters are not affected solely by single dipping layers of anisotropy.

\section{Anisotropy in the two subduction systems}

According to the ray geometry, seismic anisotropy sampled by stations LTZ and KHZ in northern South Island and MSZ and DCZ in southern South Island are most likely coming from the slab and sub-slab regions of the Hikurangi subduction zone (southern most part) and the Fiordland subduction zone respectively (Figs. 4.4 and 4.7). LTZ and DCZ show approximately the same azimuths for most of the slab events (Fig. 4.6 and 4.5-a). MSZ and DCZ indicate a significant increase in $\delta t$ with depth (Fig. 4.7). Assuming $4.5 \mathrm{~km} / \mathrm{s}$ average S-wave velocity for a single anisotropic layer, we have calculated an approximate percent velocity anisotropy [Savage, 1999] in the full depth range $(\sim 0-240 \mathrm{~km}$ in Hikurangi subduction zone and $\sim 0-120 \mathrm{~km}$ Fiordland subduction zone) of the slab region (Fig. 4.7) using the highest quality splitting measurements (A grade, best filter and $\lambda_{\max }>8$; section 3.2.3). Most of the $\delta t$ used for this analysis are less than $0.3 \mathrm{~s}$, but a very few measurements are within the range of $0.3-0.5 \mathrm{~s}$. These higher $\delta t$ may indicate either a strong crustal contribution of anisotropy in the slab or the sub-slab contribution of anisotropy. Our calculations yield about average $0.14 \%( \pm 0.09 \%)$ shear-wave velocity anisotropy for the Hikurangi subducting slab region (Fig. 4.7a-ii) and $0.40 \%( \pm 0.135 \%)$ velocity anisotropy for the Fiordland subducting slab (Fig. 4.7b-ii). Slopes of the MSZ and the combined plots of MSZ and DCZ (Fig. 4.7b-i/ii) are statistically significant (table 4.2). Velocity anisotropy calculated for the Hikurangi subduction zone is within the error range of velocity anisotropy $(0.23 \% \pm 0.34 \%)$ found by Audoine et al. [2000] for the northern most region of northern South Island. Yet, they both are small compared to higher shear-wave velocity anisotropy $(3.1 \% \pm 2.2 \%)$ observed from $70 \mathrm{~km}$ deep zone in the Wellington region that is located further north in the Hikurangi subduction zone [Matcham et al., 2000]. The observed low velocity 
anisotropy implies the re-splitting of S-phases or sensitivity of S-phases to the uppermost crustal anisotropy. The ray paths suggest that deep slab events $(>70 \mathrm{~km})$ (Fig. 4.7) in northern South Island are most likely sampling the sub-slab region and only shallow events recorded on these stations should be sensitive to shallow slab anisotropy. When we only consider shallow slab events $(\leq 70 \mathrm{~km})$, our calculation yields $1.2 \pm 0.63 \%$ velocity anisotropy (Fig. A.3), within the error range of Matcham et al. [2000]. The anisotropy measured could be under estimated if the re-splitting has occurred (section 4.4.2 and 4.4.4), in which case the anisotropic region is smaller than the whole path.

Table 4.2 Regression coefficients and uncertainties of the Figure 4.7

\begin{tabular}{|llllll|}
\hline Plot & $\begin{array}{l}\text { Slope } \\
(\mathrm{s} / \mathrm{km})\end{array}$ & $\begin{array}{l}95 \% \text { C.I of slope } \\
(\mathrm{s} / \mathrm{km})\end{array}$ & $\begin{array}{l}\text { Intercept } \\
(\mathrm{s})\end{array}$ & $\begin{array}{l}95 \% \text { C.I of in- } \\
\text { tercept }(\mathrm{s})\end{array}$ & Vel.aniso.\% * \\
\hline \hline a(i) LTZ & 0.0003 & $-0.0004,0.0009$ & $0.12^{* *}$ & $0.0361,0.2003$ & $0.14( \pm 0.135)$ \\
a(ii) LTZ+KHZ & 0.0003 & $-0.0001,0.0007$ & $0.12^{* *}$ & $0.0654,0.1646$ & $0.14( \pm 0.090)$ \\
b(i) $\mathbf{M S Z}$ & $0.0019^{* *}$ & $0.0005,0.0033$ & 0.02 & $-0.1032,0.1458$ & $0.86( \pm 0.315)$ \\
b(ii) $\mathbf{M S Z}+\mathbf{D C Z}$ & $0.0009^{* *}$ & $0.0003,0.0016$ & $0.09^{* *}$ & $0.0311,0.1410$ & $0.40( \pm 0.135)$ \\
\hline
\end{tabular}

*Velocity anisotropy is determined from the slope assuming $4.5 \mathrm{~km} / \mathrm{s}$ S-wave velocity. The error of percent velocity anisotropy is calculated from the standard error (SE) of the slope.

${ }^{* *}$ statistically significant regression coefficients.

The steepness of the Fiordland subducting slab results in a narrow spatial extent of the subduction zone. Therefore, complexities due to anisotropy contributions from the mantle wedge and sub-slab zones are limited in this region. The velocity anisotropy calculated for the Fiordland subducting slab is higher than that calculated for southern most Hikurangi suducting slab (Fig. 4.7 and table 4.2). Shallow slab events $(\leq 100 \mathrm{~km})$ recorded on MSZ yield Alpine fault sub-parallel $\phi$ (Fig. 4.5b), suggesting fault induced anisotropy at crustal depths in Fiordland. Fairly low percent velocity anisotropy and $\phi$ that are parallel to structures (e.g. strike of the slab and faults) obtained for both subduction zones suggest that the anisotropy in the subducting slab could be overprinted by crustal anisotropy. The $\delta t$ measured at MSZ and LTZ are also sensitive to the average period of the shear-wave (Fig. 4.9). 

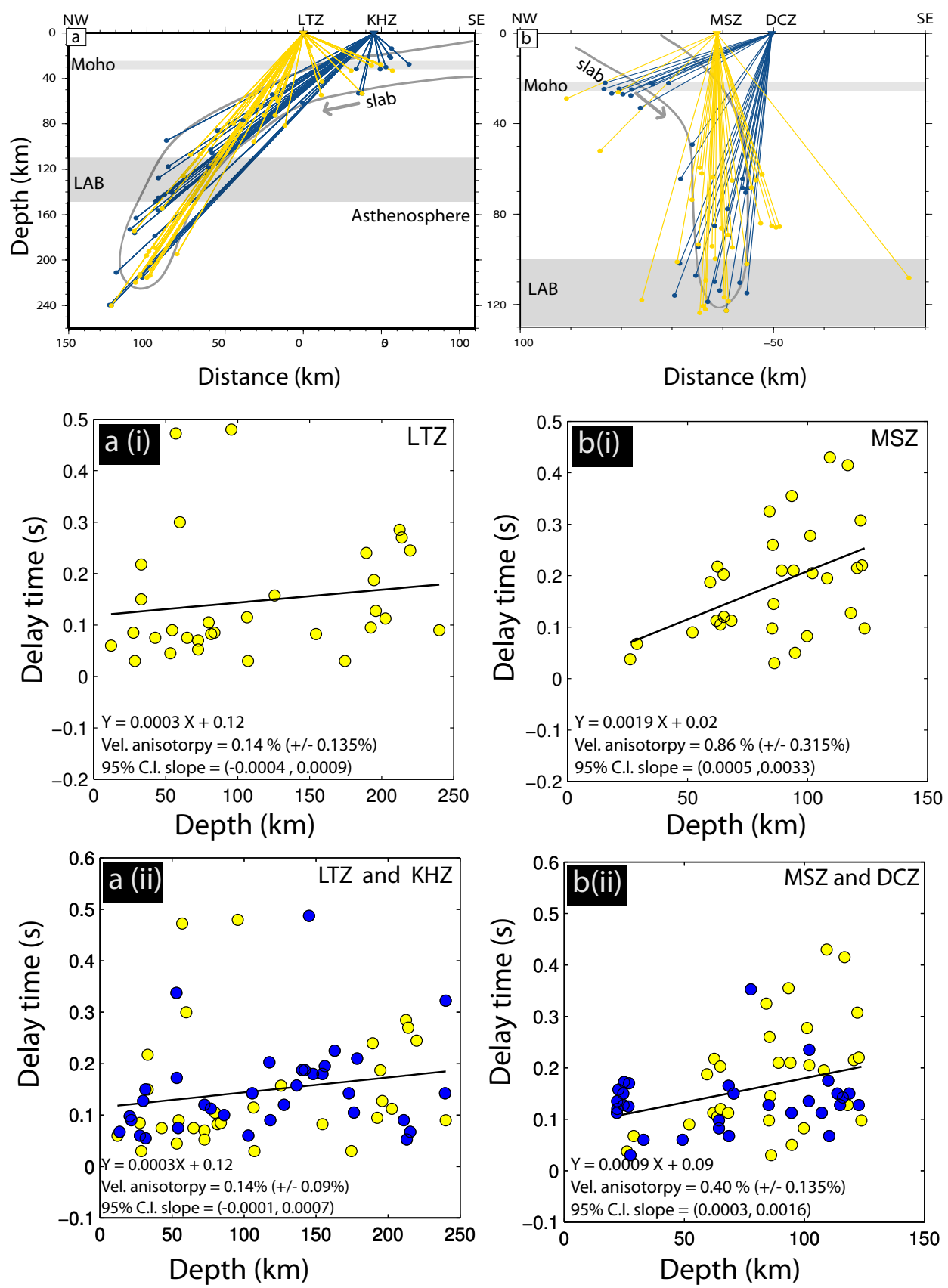

Figure 4.7 Anisotropy in the two subduction systems. Left and right hand plots show ray paths ( $\mathrm{a}$ and $\mathrm{b}$ ) and $\delta t$ measured from events originating in Hikurangi and Fiordland subducting slabs respectively. Possible depth range at which the Moho and lithosphereasthenosphere boundary (LAB) are located is shown by the gray shaded region. Here most of the ray paths sample the subducting slab region. Yellow (LTZ and MSZ) and blue (KHZ and DCZ) dots indicate $\delta t$ and the black line shows the linear regression fit that is used to calculate the velocity anisotropy of the two subduction zones. Regression coefficient uncertainties are given in the table 4.2 .

Therefore, the measured anisotropy from the slab events could be governed by more than a single source of anisotropy, such as crustal anisotropy or else the slab itself may consist of more than a single source of anisotropy (e.g. aligned micro-cracks, 
bending-induced faulting and mineral alignment), that could result in re-splitting of S-phases or frequency dependent splitting.

\subsubsection{Evidence for heterogeneous anisotropic structure}

Scatter in $\delta t$ and spatially varying $\phi$ imply complex anisotropic structure beneath South Island. Complex and/or multiple layered anisotropy can be investigated by studying changes in splitting parameters as a function of backazimuth (BAZ) and $\phi_{p}$ [Silver and Savage, 1994, Rumpker and Silver, 1998, Long and Silver, 2009] or with dominant period $(\mathrm{T})$ of the S-wave. $\phi_{p}$ is the polarization azimuth of the unsplit shear-wave before entering the anisotropic medium. In the case of re-splitting, this polarization azimuth is equivalent to the azimuth of the fast shear-wave that resulted from the preceding anisotropic medium. At DSZ in the western edge of the northern South Island, $\delta t$ varies with $\phi_{p}$ (Fig. 4.8b), and varies with depth (Fig. 4.8c), but other variables failed to yield strong correlation (e.g. backazimuth, magnitude, period of the S-wave).

Multiple-layered anisotropy with horizontal symmetry axes produce a characteristic pattern between $\phi_{p}$ and apparent splitting parameters with periodicity of $\pi / 2$ [Silver and Savage, 1994]. However, the patterns are only detectable with long-period ( $\mathrm{T} / \delta t$ $>5$ ) waves [Rumpker and Silver, 1998] from a wide range of $\phi_{p} . \mathrm{T} / \delta t$ values obtained from the local and regional S-phases used in this analysis are within the range of $\sim$ 0.3-20. Therefore, seismograms that have long period waveforms $(\sim 11 \%$ have $\mathrm{T} / \delta t$ $>5$ ) are likely yielding apparent splitting, but those with smaller ratios of $\mathrm{T} / \delta t \leq 1$ $(\sim 18 \%)$ probably measuring splitting parameters corresponding to the upper layer. We do not see a $\pi / 2$ periodic pattern in splitting parameters with respect to $\phi_{p}$ as observed in long period waveform studies [Silver and Savage, 1994], but instead we see a jump in $\delta t$ at about $\phi_{p}=80^{\circ}$ (Fig. $4.8 \mathrm{~b}$ ). Higher $\delta t$ tend to have $\phi_{p}$ between $20^{\circ}$ $80^{\circ}$ (blue) and the lower $\delta t$ have $\phi_{p}$ in the range of $80^{\circ}-170^{\circ}$ (red). At depths $>\sim 160$ 
$\mathrm{km}$ both sets of $\phi_{p}$ approximately give the same E-W $\phi$. But, at depths $\leq 160 \mathrm{~km}$, initial polarizations between $20^{\circ}-80^{\circ}$ yield N-S or NW-SE $\phi$, whereas $\phi$ with $80^{\circ}-170^{\circ}$ initial polarizations are mostly NE (see rose diagram of $\phi$ in Fig. 4.8c). Changes in both $\delta t$ with $\phi_{p}$ and $\phi$ with depth may result from a localized subcrustal anisotropic zone. Wannamaker et al. [2009] found that the Murchison basin area fault zone and Marlborough faults are characterized by moderately low resistivity structures from $\sim 25-50 \mathrm{~km}$. They infer that these structures are produced by fluid expelled from the deep $(\sim 100 \mathrm{~km})$ subduction interface and that they are dominated by fluid filled cracks and shear fabrics (Fig. 4.8e- $i$, Wannamaker et al. 2009 ), which should yield anisotropy parallel to the plate-boundary. Moderately high $V_{p} / V_{s}$ areas identified via tomographic inversion of local earthquake travel-times by Eberhart-Phillips and Bannister [2010] in the same region also suggest high fluid pressure (Fig. 4.8e-ii). Northwest of the major fault systems, low $V_{p} / V_{s}$ and high $V_{p}$ [Eberhart-Phillips and Bannister, 2010] in the upper mantle is interpreted to be strong Australian lithosphere, which may not be deformed by present processes [Eberhart-Phillips and Bannister, 2010]. Thus, the ray paths passing through different zones will measure different $\phi$.

We suggest that the waves from earthquakes deeper than $160 \mathrm{~km}$, which travel on the paths mostly from the NW of DSZ and display mainly E-W and WWN-EES $\phi$ (Figs. 4.8c and 4.8d), are passing through an anisotropic zone with E-W and/or WWN-EES fast orientation. This zone could be either in the mantle lithosphere or crust; if it is in the mantle lithosphere it could be left from the Gondwana Cretaceous deformation [Eberhart-Phillips and Bannister, 2010] and if it is in the crust it could be caused by the present stress orientation [Audoine et al., 2000]. A third possibility is that the E-W/WWN-EES alignment is a near source effect, because the earthquakes are within a region of high $V_{p} / V_{s}$ ratios at the deep slabedge (Fig. 4.8e-ii, Eberhart-Phillips and Bannister 2010). We favor the crustal stress interpretation as the $\phi$ is nearly parallel to the crustal stress direction (yellow bars in 

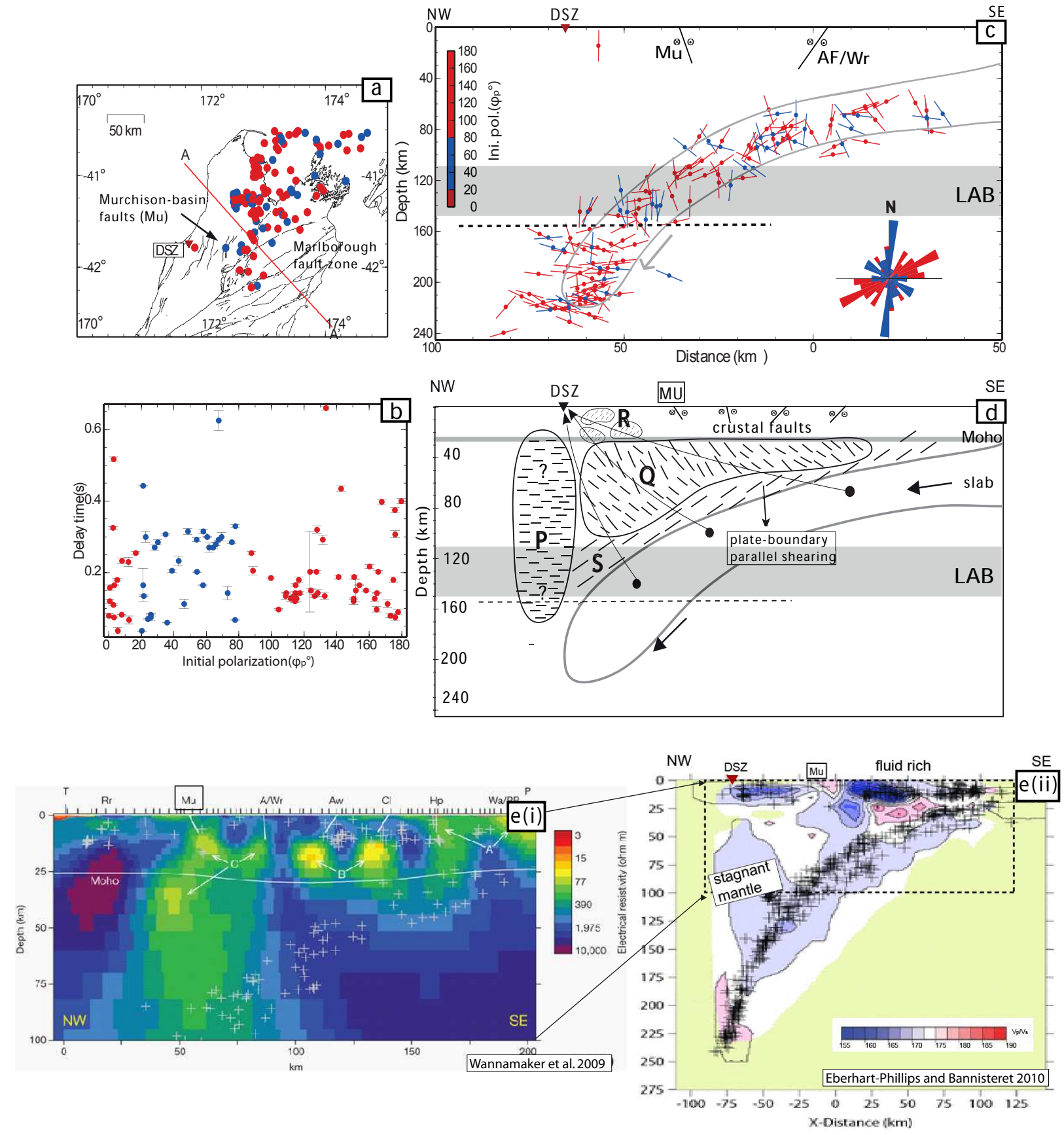

Figure 4.8 Changes in $\delta t$ with $\phi_{p}(\mathbf{b})$ and changes in $\phi$ with depth from events recorded at DSZ in Murcheson basin area (Fig. 4.1) in the western northern South Island. (a) map view of the events recorded. (c) depth profile of the $\phi$ along the line AA'. $\phi$ and the events are color coded according to $\phi_{p}$ and the dashed line indicates the change in $\phi$ with depth. Here the rose diagram shows the histogram of $\phi$ obtained from events $\leq$ $160 \mathrm{~km}$ and color coded according to $\phi_{p}$. (d) schematic diagram illustrating the different anisotropic zones ( $\mathrm{P}$ is a region with $\mathrm{E}-\mathrm{W}$ aligned anisotropy, $\mathrm{Q}$ is a layer with NW-SE anisotropy and R shows anisotropic patches with NE-SW orientation), which result in the observed patterns of $\phi$ in Fig. 4.8(c). (e-i) 2D inversion model of electrical resistivity for northern South Island [Wannamaker et al., 2009]. The low resistivity body in Murchison basin area and north of the Marlborough faults are marked by $\mathrm{C}$ and $\mathrm{B}$ respectively. (e-ii) Depth profile of the $V_{p} / V_{s}$ model for central northern South Island [Eberhart-Phillips and Bannister, 2010]. Dashed line box shows the rough location of the electrical resistivity model explained in Fig. 4.8(e- $\boldsymbol{i})$. 
Fig. 4.11, Townend et al. 2012), and short period waveforms are often most effected by the uppermost path. The shallower ray paths are in the lithospheric mantle and the crust to the SE of station DSZ, and pass through the Marlborough fault system, which has patches (zone c in Fig 4.8e- $i$ ) of high fluid content. These rays pass through a region in the lower crust/upper mantle that has roughly NE/SW oriented fast anisotropy induced by plate-boundary parallel shearing (zone $\mathrm{S}$ in Fig. 4.8d), and then pass through another layer with roughly NW/SE fast anisotropy (zone $\mathrm{Q}$ in Fig. 4.8d), which re-splits them. This NW/SE anisotropy could be generated by asthenospheric counterflow perpendicular to the plate-boundary or by some other processes. Some waves continue to DSZ without traveling through any other regions. They have $\phi$ between $\mathrm{N}$ and NW, $\delta t$ of about $0.3 \mathrm{~s}$ and NE/SW incoming polarizations obtained as they were traveling through the lowest layer as fast waves. Other waves pass through a third patch of NE/SW oriented $\phi$ (patchy area marked by R in Fig. 4.8d). They have NE/SW $\phi$ with shorter $\delta t$ of $\sim 0.1 \mathrm{~s}$, and NW/SE incoming polarizations (Fig. 4.8b and c). Perhaps these shallow patches are in the fault zone or are regions of schist. Cycle skipping could also produce a dependence between $\delta t$ and $\phi_{p}[$ Walsh, 2012]. However, the contour plots of the large $\delta t$ with $\sim 0.3-4 \mathrm{~s}$ (Figs. $4.3 \mathrm{~b}$ ) suggest this is not occurring.

Other than some stations (e.g. MSZ) that sample slab anisotropy, values of $\delta t$ show no clear increase with depth. Instead, there is a scatter in $\delta t$ that is measured on stations in the central northern South Island (e.g. THZ, KELY) and a few stations (e.g. WHZ, APZ) in southern South Island (Fig. A.4). Both scatter in $\delta t$ and changes in $\phi$ at nearby stations imply the presence of heterogeneous anisotropic structure. Regions where changes occur over short distances laterally and vertically are likely characterized by variable sources of anisotropy (e.g. shear-induced structures, microcracks, mineral alignment due to flow and large-scale structural fabric). 
Northern South Island
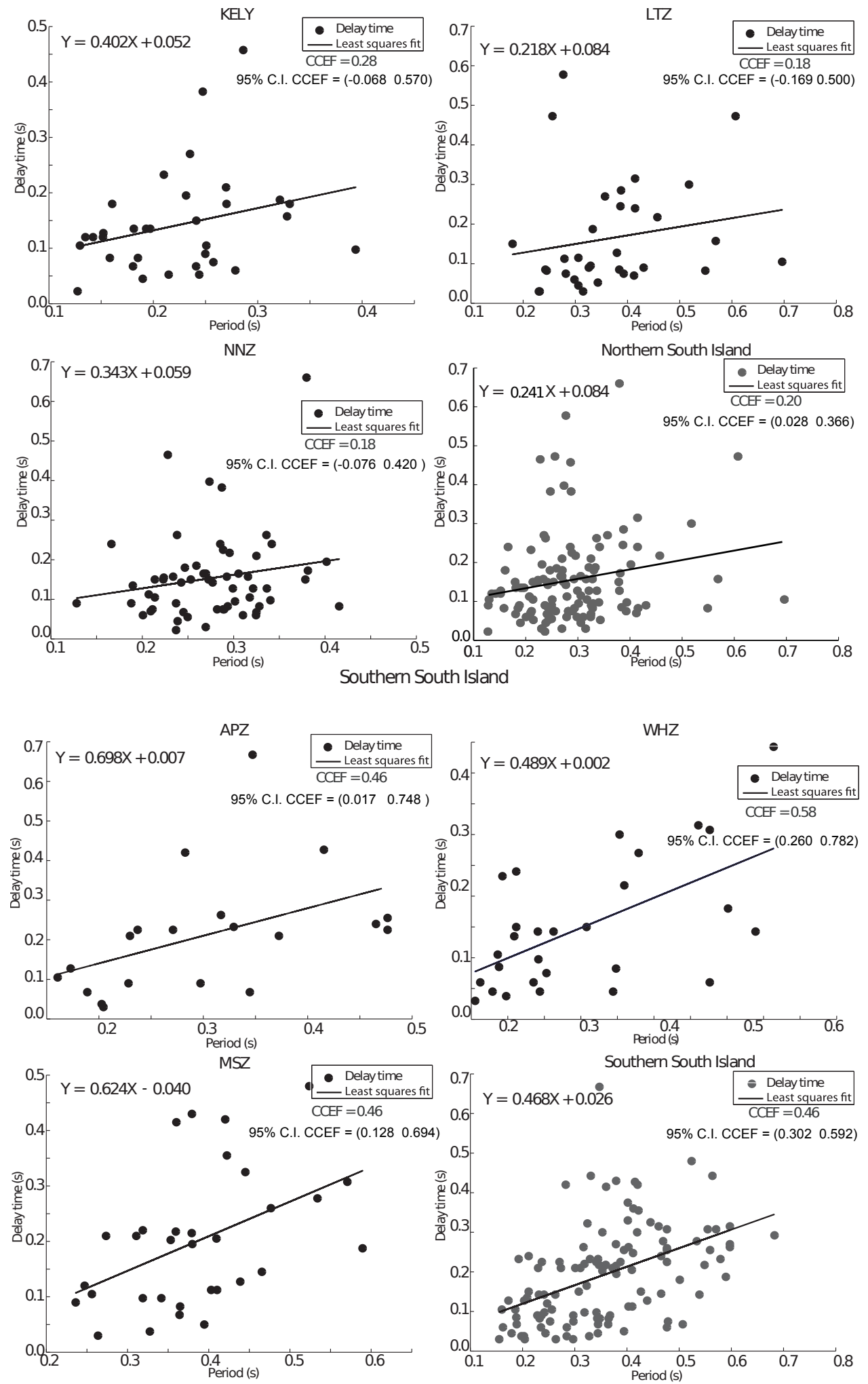

Figure 4.9 Examples of stations that show changes in $\delta t$ with period of the S-waves in northern and southern South Island. Black line indicates the least squares fit of the data. Two plots with gray circles show the combined plots of individual station data in the southern and northern South Island. Here the correlation coefficient (CCEF) and $95 \%$ confidence interval of the CCEF for each station are marked on the corresponding station plot. Note the moderately high CCEF $(>0.4)$ calculated at the southern South Island stations. 
Some studies suggest that different S-wave frequencies sample different scale-lengths of anisotropy, hence produce variable results depending on heterogeneity of the medium [Marson-Pidgeon and Savage, 1997, Wirth and Long, 2010]. We test this hypothesis by analyzing the highest quality splitting parameters (A grade, best filter and $\lambda_{\max }>8$; section 3.2.3) at each station (Fig. 4.9). The correlation coefficient $(\mathrm{CCEF})$ between $\delta t$ and period determined at northern South Island stations (KELY, LTZ and NNZ) are significant if they are combined (Fig. 4.9-top). In southern South Island, the individual CCEF values determined at stations MSZ, WHZ, APZ, and EAZ and CCEF of the pooled data set are significant (Fig. 4.9bottom). These stations and EAZ station in South Island show moderately high CCEF between period and $\delta t$, i.e., frequency dependent splitting (e.g. CCEF >0.4). The dependency of $\delta t$ on the period of the S-wave may reflect a heterogeneous anisotropic structure in the Fiordland subduction zone, and SKS splitting can be explained by shallow anisotropy in this area. But the lack of frequency dependent splitting at the other stations suggests the large SKS $\delta t$ with consistent $\phi[$ Klosko et al., 1999] can not solely be explained by frequency dependence and therefore require a deep seated source.

\subsubsection{Interpretations from 2-D delay-time tomography and spatial averages}

The inconsistency of measurements from closely spaced earthquakes at different stations suggests that lateral variations in anisotropy occur. This in turn suggests that the 2-D delay-time tomographic approach might be useful to determine the average structure. Fig. 4.10 illustrates the 2-D delay-time tomography results obtained using AB quality splitting parameters calculated from 2615 event-station pairs. Because of the depth dependence of splitting parameters, we produce 2-D distribution maps for two depth sections (depth $\leq 100 \mathrm{~km}$ and depth $>100 \mathrm{~km}$ ) 
and these maps provide average spatial variations of $\delta t$. In Fig. 4.10 (shallow/deep), warm colors (yellow to red) indicate the regions with high strength of anisotropy $(\gtrsim 0.004 \mathrm{~s} / \mathrm{km})$ compared to blue colors with weak strength of anisotropy $(\leq \sim 0.002$ $\mathrm{s} / \mathrm{km}$ ). The shaded regions Fig. 4.10 indicate the regions with low resolution and/or large residuals. Anisotropy strength profiles that are within the higher resolution region reasonably agree with the possible anisotropic sources in South Island. For depths less than $100 \mathrm{~km}$, crustal fault zones and the areas with schist rock (compare to Fig. 4.1) appear to be the regions with strong anisotropy. This is clear from the high anisotropy strength yields around the Alpine/Wairau faults, Marlborough fault zone, Murchison basin area faults in the northern South Island (Fig. 4.1) and some crustal faults in the southern South Island (Fig. 4.10-shallow). For the deep earthquakes $(>100 \mathrm{~km})$, the region with high strength of anisotropy in the northern part of the southern South Island (SE of the Alpine fault) coincides quite well with the region that has large crustal thickness $(\sim 48 \mathrm{~km})$ [Stern et al., 2000, Bourguignon et al., 2007a] and large negative Bouguer anomaly in and around the Southern Alps [Davey et al., 2007]. Deformation associated with crustal shortening and mantle lithosphere (upper $\sim 150 \mathrm{~km}$ ) thickening as a result of continental collision could be the main mechanisms that governs the anisotropic structure in this region. As suggested by Norris et al. [1990], this region (SE of the Alpine fault) might be dominated by distributed deformation that results from the plate motion mechanisms.

Weighted mean $\phi$ obtained for each grid block are shown in Fig. 4.11. Due to lack of spatial resolution of deep measurements (Fig. 4.4), spatial averaging is limited to depths $\leq 100 \mathrm{~km}$. Even at shallow depths of eastern-southern South Island, measurements are made in a limited region as a result of sparse distribution of ray paths and therefore, spatial averages are not well constrained at some locations (see Fig. 4.4 and Fig. 4.11). Spatial averages of $\phi$ obtained from shallow events display consistent regional patterns. $\phi$ in the red shaded regions are either sub- 
parallel to the plate-boundary or aligned parallel to the translational faults (e.g. Marlborough fault zone) in northern South Island. The consistent pattern of $\phi$ with NS and NNW alignment in the region surrounding the red-shaded zone in southern South Island agree with the SKS measurements that were made in those regions (red bars in Fig. 4.11, Savage et al. 2007a). Thus, it is possible that most of the anisotropy measured in this region is coming from the mantle lithosphere or from coupling between the crustal and the mantle-lithospheric anisotropy. But, the NESW alignment in the southern red-shaded zone shows a sharp change in $\phi$ from the general trend in this region and is aligned parallel to the $P_{n}$ anisotropy fast azimuth (blue bars in Fig. 4.11, Scherwath et al. 2002), the plate-boundary and the strike of the high topography (e.g. Southern Alps) of the region. This zone suggests an abrupt changes in strain field and is characterized by relatively high strength of anisotropy as seen in Fig. 4.10 (shallow).

Spatial averages in the northern part of the central South Island are poorly constrained due to lack of measurements. Standard error associated with most of the spatial averages in eastern and central part of the central South Island are greater than $25^{\circ}$, indicating more than a single mode of $\phi$. Although the prominent trend (WNW-ESE aligned gray bars in Fig. 4.11) is consistent with the horizontal maximum compressive direction $\left(S H_{\max }\right)$ of this region (yellow bars in Fig. 4.11, Townend et al. 2012), spatial averages do not provide robust estimations due to large errors. Single-station measurements at RPZ, LBZ and CASS (Figs. 4.5(b)) show that there is a plate-boundary sub-parallel trend in the central region, however this trend is masked by the WNW-ESE $\phi$ parallel to $S H_{\max }$ when considering spatial averages. Therefore, the contour line connecting the two red-shaded regions is determined comparing both Figs. 4.5(b) and Fig. 4.11 (see Fig. 4.11). 


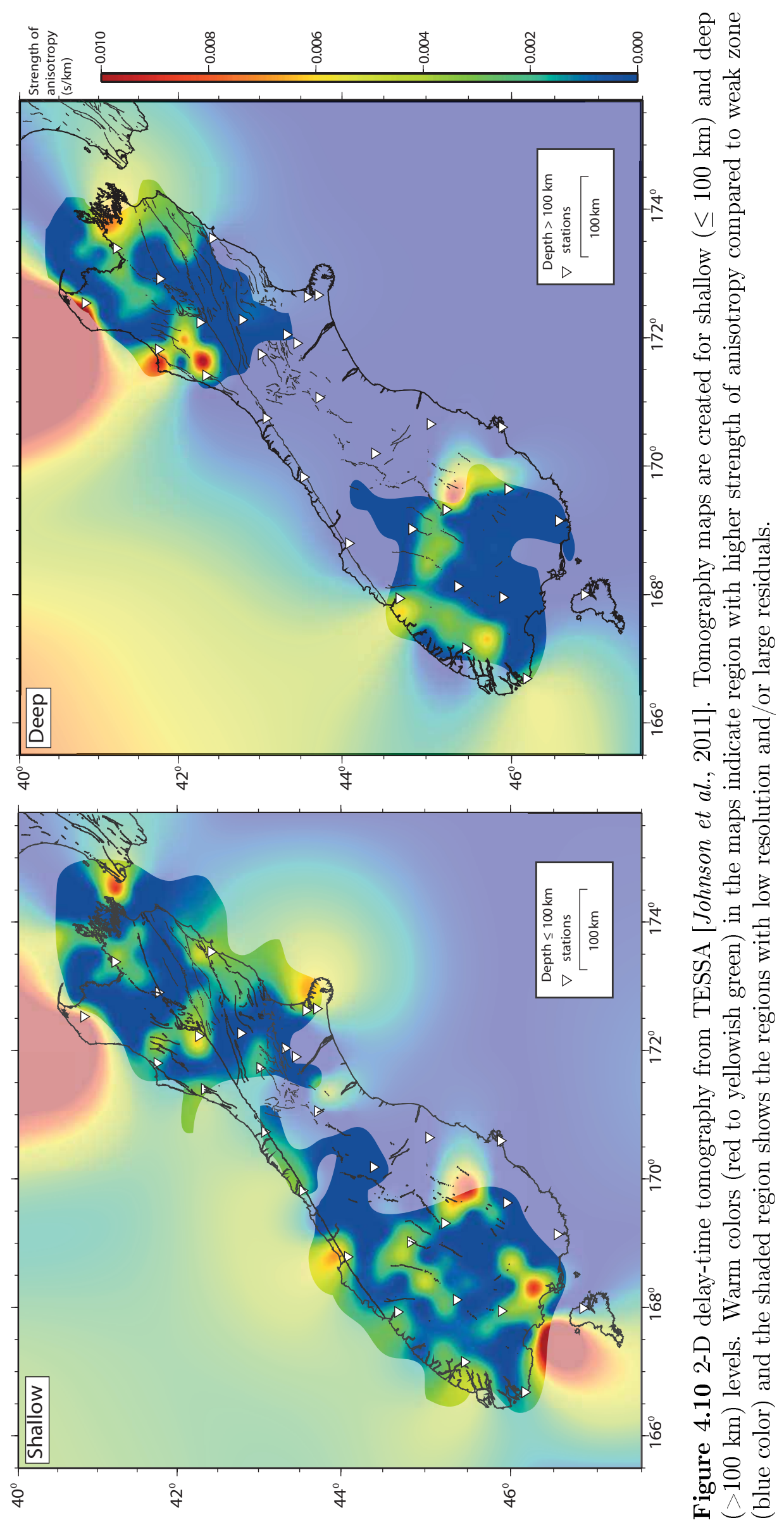




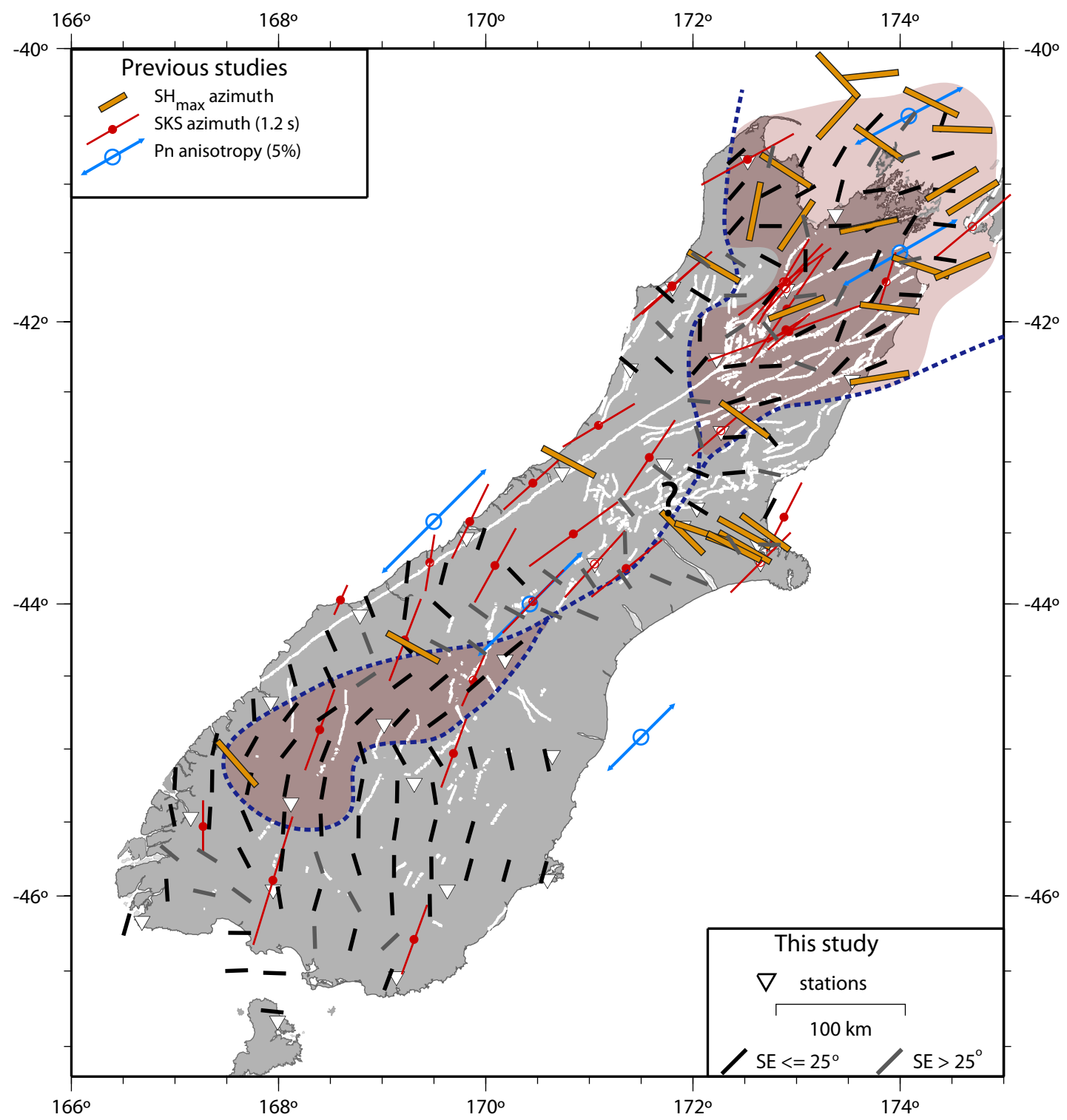

Figure 4.11 Spatially (25 km grid blocks) averaged fast azimuths ( $\bar{\phi}_{b}$, equation 4.3$)$ using TESSA. Map displays the spatial averages from shallow $(\leq 100 \mathrm{~km})$ events. Black color bars denote the $\bar{\phi}_{b}$ with standard error $(\mathrm{SE}) \leq \pm 25^{\circ}$ and the gray bars indicate the SE of $\bar{\phi}_{b}> \pm 25^{\circ}$. Regions with plate-boundary sub-parallel $\bar{\phi}_{b}$ are denoted by red shaded regions (note that the sharp change in $\phi$ from NW-SE trend to NE-SW trend in southern South Island is marked by the shaded red region in the south). Blue dashed line marks the boundary limits of the NE-SW trend that is aligned with strike of plate-boundary and the associated faults. Both individual station measurements (Fig. 4.5b) and spatial averages have been used to constrained these boundary limits. SKS [Savage et al., 2007a, Klosko et al., 1999], $P_{n}$ anisotropy [Bourguignon et al., 2007a] and $S H_{\max }$ [Townend et al., 2012] directions from previous studies are marked by red, blue and yellow bars respectively. White irregular lines indicate the fault structures in South Island. The question mark denotes the possible boundary limit of pervasive shear in the central South Island as noted by station $\phi$. 


\subsubsection{Comparison with previous anisotropy studies and im- plications for plate-boundary deformation}

Anisotropy in the crust and the lithospheric mantle in the Marlborough fault zone and the upper most region of the northern South Island was previously investigated by Balfour et al. [2005] and Audoine et al. [2000], but the spatial and the depth resolution of these studies are limited. Balfour et al. [2005] argued that the anisotropy in Marlborough is not controlled by the regional stress and both Balfour et al. [2005] and Audoine et al. [2000] found that $\phi$ in those regions are roughly parallel to the plate-boundary (NE-SW). In agreement with the above studies, we find a similar average $\phi$ (spatial) for the same regions in northern South Island (Fig. 4.11) from shallow events. Most of our deep events Figs. 4.6a yield the EW/WNW-ESE trend presented by Audoine et al. [2000] from deep events at some stations. As suggested in section 4.4.1, this E-W/WNW-ESE trend may result from either a deep anisotropic source (e.g. LPO fabrics in the upper mantle or lower crust) or a shallow stress-controlled source or the combination of both. The NE-SW trend is likely caused by pervasive shear associated with the plate-boundary deformation (Fig. 4.11).

As highlighted in New Zealand's terrane map (Fig. 1.4(b)), the basement terranes of South Island are aligned with the orientation of the ancient Gondwana margin (Fig. 1.3), however close to the plate-boundary these terranes have been bent and shifted by the present-day oblique strike-slip movement of the Alpine fault. In general most of the basement terrane rocks are weakly metamorphosed, but some Gneissic fabrics that indicate high-grade metamorphism is common in some western province rocks [Cox and Sutherland, 2007]. Overprinted metamorphic fabrics on the terranes adjacent to the plate-boundary (e.g. some parts of Rakaia terrane), increase in metamorphic grade and the bending of terranes towards the boundary in the eastern side of the Alpine fault reflect the increased deformation and change 
in state-of-deformation due to current plate-boundary activity. As explained in section 1.4, overprinted Haast-schist terrane consists of three distinct schist units (Otago, Alpine, and Marlborough Schists) with different deformation histories and structural orientations. Fabrics within those schist units and/or terranes manifest different deformation histories. In some regions of South Island, the individual station splittings, delay-time tomography and spatial averages of $\phi$ (e.g. Figs. 4.5, 4.10, and 4.11) correlate well with the above structural orientations and fabrics. Southeast of the South Island, about $60 \mathrm{~km}$ away from the Alpine fault, terrane boundaries and structures have a prominent NW-SE alignment, whereas close to the fault terranes have been truncated by the Alpine fault, resulting in bending and changing structural and terrane orientations to N-S, NNE-NNW and/or NE-SW (Fig. 1.4(b); Cox and Sutherland [2007]). Prominent NW-SE $\phi$ alignment in the southeast of central and southern South Island is in agreement with the structural trend in the southeast South Island (Fig. 4.5). The high strain zone with an abrupt change in $\phi$ from NW-SE to NE-SW in Fig. 4.11 coincides well with the region of dextral-bending of terranes occurs (Fig. 1.4(b)). The high strength of anisotropy detected from the delay-time tomography in the same region (around

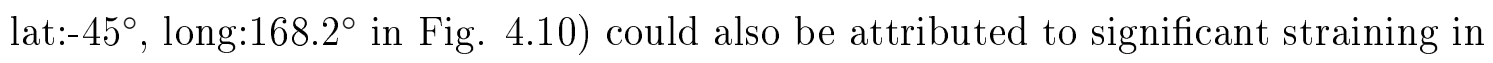
rock fabrics and heterogeneous anisotropic structure associated with the oroclinal bending associated with Cenozoic deformation.

When compared to SKS and $P_{n}$ anisotropy studies in South Island [Scherwath et al., 2002, Klosko et al., 1999], $\phi$ obtained from local/regional data are variable. But, the spatially averaged $\phi$ from earthquakes with depths $\leq 100 \mathrm{~km}$ (Fig. 4.11) are mostly consistent with the SKS and $P_{n}$ fast azimuths observed for the southern central South Island (regions where WKZ and LBZ are located), the eastern part of the southern South Island and northern South Island (compare red and black bars in Fig. 4.11). Our observations are also consistent with the crustal finite strain estimations from bending related geological markers in South Island [Little et al., 
2002b]. As suggested by Little et al. [2002b], the consistency indicates coupling of the upper mantle deformation (as inferred from SKS results) with crustal deformation more towards the Alpine fault and weak coupling in the eastern part of the central and southern South Island (Fig. 4.5). Based on the $\phi$ from shallow events $(\leq 100$ $\mathrm{km}$ ) (Figs. 4.5b and 4.11), we infer that the width of the plate-boundary sub-parallel $\phi$ that indicates pervasive simple shear [Molnar et al., 1999, Little et al., 2002b] is limited to about $130 \mathrm{~km}$ southeast from the Alpine fault in the southern part of central South Island (regions where WKZ and LBZ are located) and the northern region of southern South Island. A region with high geodetic shear strain rates in South Island as proposed by Beavan et al. [1999] also lies within the estimated width of the plate-boundary induced-deformation in our study. The western boundary limit of the near offshore region of northern and central South Island are not yet known. Also, the lack of deep events in the central South Island does not allow accurate constraints on the depth extent of this trend.

As proposed by Molnar et al. [1999], if the total strain produced by the pervasive shear is confined to the top $100 \mathrm{~km}$ of the upper mantle, a large portion of SKS $\delta t$ anisotropy should be accommodated in the lithosphere. But for local/regional events, even though the ray paths travel through the same region of the upper mantle, the observed station $\delta t$ average of local/regional splitting measurements is about $9-20 \%$ of that observed by SKS studies. Thus, small average station $\delta t(\sim$ $0.1-0.4 \mathrm{~s})$ suggest either: $(i)$ the majority of SKS $\delta t$ are from asthenosphere and/or deeper part of the lithosphere or (ii) SKS and local phases sample the same structure, but the latter have higher frequencies and are seeing smaller $\delta t$ due to frequency dependent splitting (Fig.4.9 and section 4.4.2) or (iii) higher frequency phases are more susceptible to re-splitting from small scale structures that are present at the last layer of a layered anisotropic media (e.g. Fig 4.8). Possibility (ii) appears to be a localized effect (section 4.4.2) and therefore either $(i)$ and/or (iii) can be used to explain the $\delta t$ discrepancies between local (this study) and SKS (previous work) 
splitting.

\subsection{Conclusions}

Using both local and regional earthquake data, we have obtained a S-wave splitting data set to provide constraints on the lateral and vertical distribution of seismic anisotropy in the lithosphere of the South Island. The application of quality criteria and statistical analysis allow us to extract well resolved splitting parameters and to provide more confident results from the complex splitting associated with local/regional S-wave splitting analysis. Therefore, the spatial and depth variations observed from the detailed splitting analysis and results from the tomography provide a good approximation for the spatial distribution of anisotropy (sections 4.4.1, 4.4.2, and 4.4.3). In general, splitting measurements in South Island show regional consistencies over short distances, which imply localized coherent anisotropy. Spatial and depth variations in splitting parameters suggest the existence of several deformation domains (e.g. Fig. 4.11 and Fig. 4.8d). Anisotropy in these domains appears to be controlled by different source mechanisms: shear induced fabrics related to present-day plate-boundary deformation (in northern and central-southern South Island), stress controlled anisotropy linked with prevailing stress (in eastern part of the central South Island) and anisotropy deeper than $50 \mathrm{~km}$ due to the mantle anisotropy within the lithosphere and/or asthenosphere that reflects the paleo-anisotropy from previous tectonic episodes or present asthenospheric flow (in southern and eastern parts of the Southern South Island).

2-D delay-time tomography and spatial averaging of $\phi$ give consistent patterns on a regional scale and suggest that the schist rock may also have a significant contribution to the total splitting $(\sim 0.1-0.4 \mathrm{~s})$ measured. A NE-SW trend of the spatial averages of northern South Island consistent with the strike of the plateboundary and the strikes of the crustal faults, suggests that the dominant source of 
anisotropy is plate-boundary induced structures. An abrupt variation in $\phi$ from NWSE to NE-SW in southern South Island (Fig. 4.11) suggests a high strain structure at crustal or subcrustal depths. This zone also indicates fairly high strength of anisotropy $(\sim 0.006 \mathrm{~s} / \mathrm{km})$ and resides in the region with high crustal thickness $(\sim 48 \mathrm{~km})$ and inferred thick lithosphere [Bourguignon et al., 2007a, Stern et al. , 2000]. The origin of this anisotropic structure may be related to pervasive shear from plate-boundary deformation.

Assuming that the plate-boundary sub-parallel $\phi$ is a product of distributed shear associated with plate-boundary deformation, we suggest that the zone of shear deformation extends $\sim 130 \mathrm{~km}$ from the Alpine fault in the northern part of the southern South Island (eastern boundary limit of the red shaded region in South), but in northern and central South Island this zone is more distributed than in southern South Island. Unless there is re-splitting due to misaligned anisotropic media, and/or frequency dependent splitting affecting the local/regional splitting, small $\delta t$ observed from local S-wave splitting compared to large $\delta t(\sim 1.7 \mathrm{~s})$ observed from SKS splitting suggest that most of splitting from SKS is accommodated either in the asthenosphere or in the deeper part of thickened mantle lithosphere (depths $\sim 150-250 \mathrm{~km}$ ) where we do not have enough depth coverage of splitting measurements. When considering upper lithospheric depths $(\sim 80-120 \mathrm{~km}$ thick lithosphere) in the central and upper-most southern South Island, our splitting results fit best with the localized deformation model (Fig. 4.1b). 


\section{Chapter 5}

\section{Shear-wave splitting anisotropy in the offshore plate-boundary zone, South Island}

This chapter is in the initial stage of manuscript preparation and this may contain some repetitive sections from the Introduction and Methodology chapters (1 and 3).

\subsection{Introduction}

The study of internal deformation at plate-boundaries enables us to understand how lithospheric blocks interact with each other. An oblique-convergent boundary between the Australian-Pacific (AUS-PAC) plates has evolved since Eocene ( 40 $\mathrm{Ma}$ ) in New Zealand (NZ), changing its tectonic character [Cox and Sutherland, 2007]. The plate-boundary was initiated as a passive margin and it was later transformed to a large strike-slip fault (Alpine fault) [Sutherland, 1999]. The Alpine Fault is the main structure in the AUS-PAC boundary in SI, NZ. The fault has accumulated an offset of $450 \mathrm{~km}$ over the last $25 \mathrm{My}$. During this time the fault movement has changed from pure strike-slip to oblique strike-slip in response to the changes in plate-boundary orientation. Consequently, the present-day plateboundary configuration in the SI is characterized by the transpressional Alpine fault that interlinks the Hikurangi and Puysegur subduction zones (Fig. 5.1a). The large-scale strike-slip faulting, transpressional behavior and relatively young tectonic history are some of the key features that make this plate-boundary an exceptional place to explore lithospheric deformation associated with continental margins. 


\subsubsection{Constraining plate-boundary deformation via shear- wave splitting}

Lattice preferred orientation (LPO) of minerals creates an anisotropic medium with directional variations of its physical properties. This has been considered as the main source of anisotropy in the upper mantle, asthenosphere and perhaps in the lower-crust [Savage, 1999]. Anisotropy in the upper-mid crust is largely due to stress controlled sources such as aligned micro-cracks, fluid-filled cracks; structural sources like macro-faults and the alignment of bedding planes and foliations [Boness and Zoback, 2004]. Anisotropy is an excellent indicator of strain (or deformation) distribution at the plate-boundaries [Skemer et al., 2012] and it helps to delineate deformation kinematics to understand the rheology of the system [Karato et al., 1989].

Vauchez and Nicolas [1991] hypothesized that large-scale displacement in plateboundary faults is likely associated with deformation in the whole lithosphere, and is characterized by broadly distributed shear deformation on either sides of the fault. Depth distribution of deformation, however, depends on the mechanical decoupling between layers [Flesch et al., 2005]. Shearing of intrinsically anisotropic minerals (e.g. olivine, pyroxene) in the lithosphere and asthenosphere in response to the fault displacement results in LPO anisotropy with horizontal mineral alignment on vertical flow planes (or steep foliations) that are parallel to the plate-boundary [Nicolas and Christensen, 1987]. The width of this LPO anisotropy zone is, therefore, a measure of distributed shear deformation associated with the transcurrent fault movement. As suggested by Otsuki [1978], 500 to $1000 \mathrm{~km}$ of fault displacement can produce deformation of a few kilometers to $\sim 40 \mathrm{~km}$ width in the lithospheric mantle and mid lower-crust. If this deformation is partitioned into several major shear zones in the mid-lower crustal depths, such zones will have a single large-scale shear zone in the mantle lithosphere and, therefore, distributed deformation $(\sim>100$ 
km) around the plate-boundary [Nicolas, 1993].

Previous studies of $P_{n}$ anisotropy [Scherwath et al., 2002, Baldock and Stern, 2005], P-wave delays [Molnar et al., 1999, Stern et al., 2000] and SKS splitting [Klosko et al., 1999] suggest that the mantle shear zone that is associated with the Alpine fault is distributed in a $\sim 200-300 \mathrm{~km}$ broad area around the plate-boundary and may extend into offshore regions of the SI. However, geodetic estimations and finite-element modeling suggest that the width of deformation is distributed only about 80-100 km zone from the fault [Beavan et al., 1999, Ellis et al., 2006, Moore et al., 2002]. Finite strain estimations from geological markers [Little et al., 2002b] also found more strain accumulation towards the plate-boundary (east to west), indicating crust-mantle coupling towards the fault. Because of the lack of depth resolution of the SKS splitting, such a study would not allow constraints on the lithospheric anisotropy using SKS phases. For this reason, Karalliyadda and Savage [2013] carried out a local shear-wave (S-wave) splitting investigation of on-land SI to examine lithospheric anisotropy. Despite the complex nature of splitting from high-frequency local S-waves, the study found that the mantle shear zone due to plate-boundary deformation is distributed within $\sim 130 \mathrm{~km}$ from the boundary in the central SI and may be more distributed in the northern SI than in the central SI. The study also noticed that high-frequency local S-phases are more sensitive to crustal stress in some regions in SI.

Klosko et al. [1999] found little anisotropy at Chatham Island station in the eastern off-shore SI compared to $1.7 \mathrm{~s}$ SKS $\delta t$ onland (Fig. 5.1-a). Based on the limit of low $P_{n}$ velocity $(\sim 7.95 \pm 0.05 \mathrm{~km} / \mathrm{s})$ across the central SI, Baldock and Stern [2005] suggested the width of distributed shear is less than $235 \mathrm{~km}$ east of the plateboundary. However, $P_{n}$ anisotropy has a limited depth constraint (i.e. just below Moho depth). Comparing the above findings with petrophysical analysis, Duclos et al. [2005] interpreted this anisotropic zone as an asymmetric body that fans out of the plate-boundary, so that the upper-limit of this body is shallower in the western 
side in agreement with Scherwath et al. [2002] and deeper in the eastern side to explain 0\% $P_{n}$ anisotropy in the central-east offshore SI [Baldock and Stern, 2005]. Both local S-wave splitting delays (maximum $\sim 0.3 \mathrm{~s}$ station average and 0.6-0.7 $\mathrm{s}$ individual delay times) [Karalliyadda and Savage, 2013] and petrophysical results [Duclos et al., 2005, Godfrey et al., 2000] have suggested that the depth extent of this anisotropic body is not only limited to the shallowest upper-mantle as argued by Molnar et al. [1999], but extends into the deep upper-mantle, possibly down to the asthenosphere. This has also been suggested by the recent geodetic studies by Houlié and Stern [2012], at least in the southern-central and southern SI.

We investigate seismic anisotropy in the previously unexplored offshore regions of SI using local/regional S-wave splitting. S-wave birefringence (i.e. splitting of S-wave into two quasi S-phases) is a characteristic property of S-waves propagating in an anisotropic medium. Splitting occurs in such a way that the polarization direction of the faster S-wave (fast direction- $\phi$ ) is governed by the alignment of the anisotropic medium and the slower S-wave is orthogonal to the polarization direction of the fast S-wave. The time separation (delay time- $\delta t$ ) acquired by the two quasi S-phases upon propagating in the anisotropic medium gives information on the strength and spatial extent of anisotropy. We estimate these S-wave splitting parameters (i.e. $\phi$ and $\delta t$ ) from regional and local events recorded on the ocean-bottom seismographs to constrain the spatial distribution of splitting parameters. We also aim to address the question of whether the local/regional splitting in this region is entirely governed by the plate-boundary deformation or by other means. 


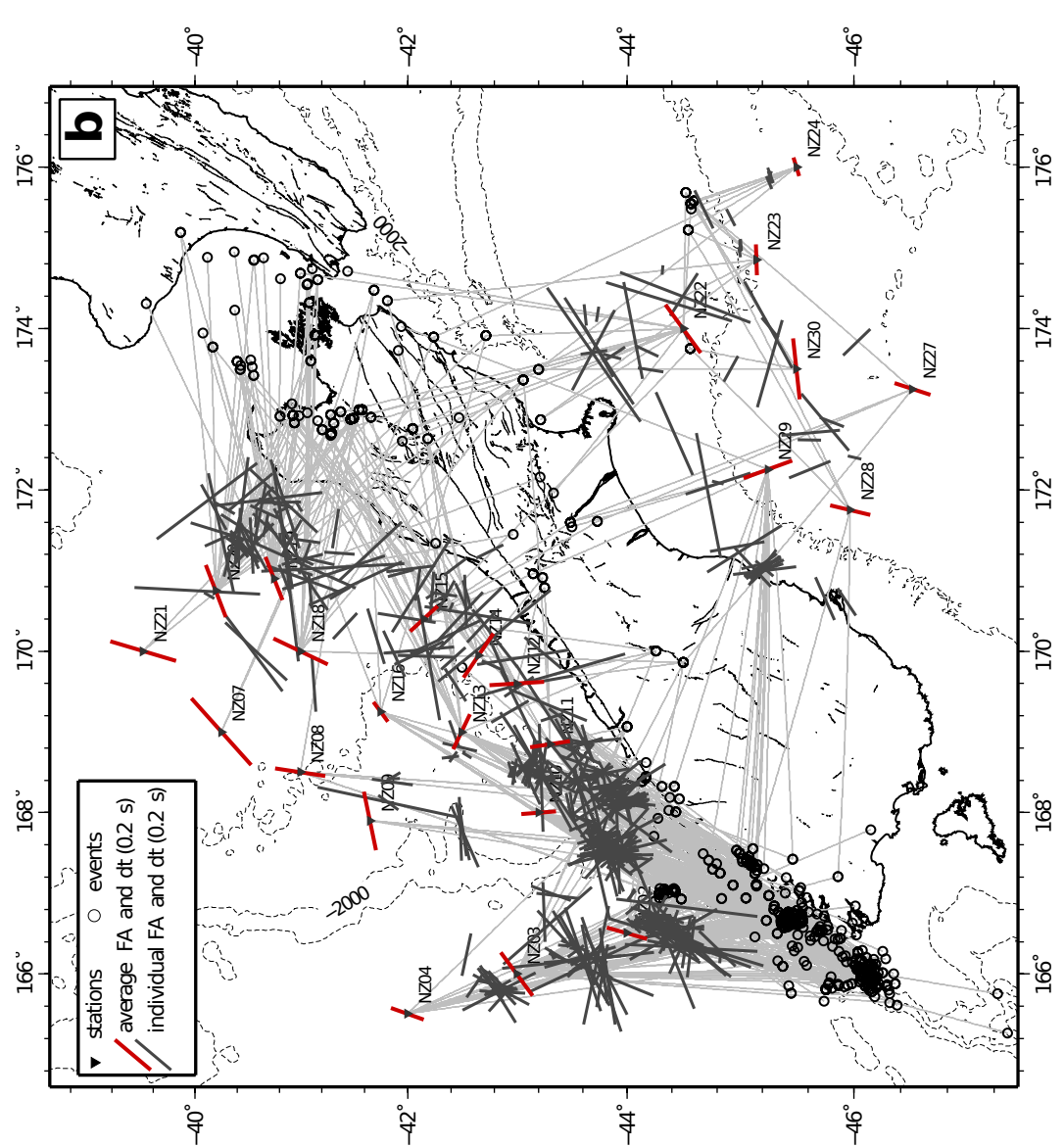

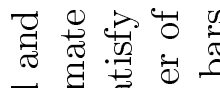

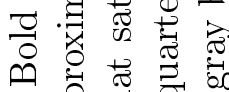

$\oplus$.

ลิ

i

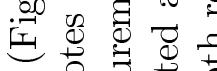

पै

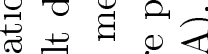

节寻

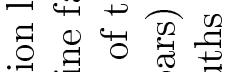

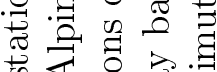

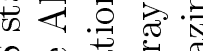

भि

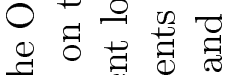

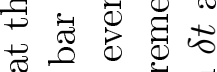

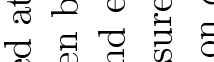

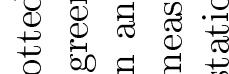

2. $8.0 \%$

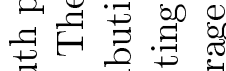

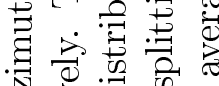

สู

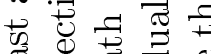

要

的完泀

\& $\dot{2}$ \&

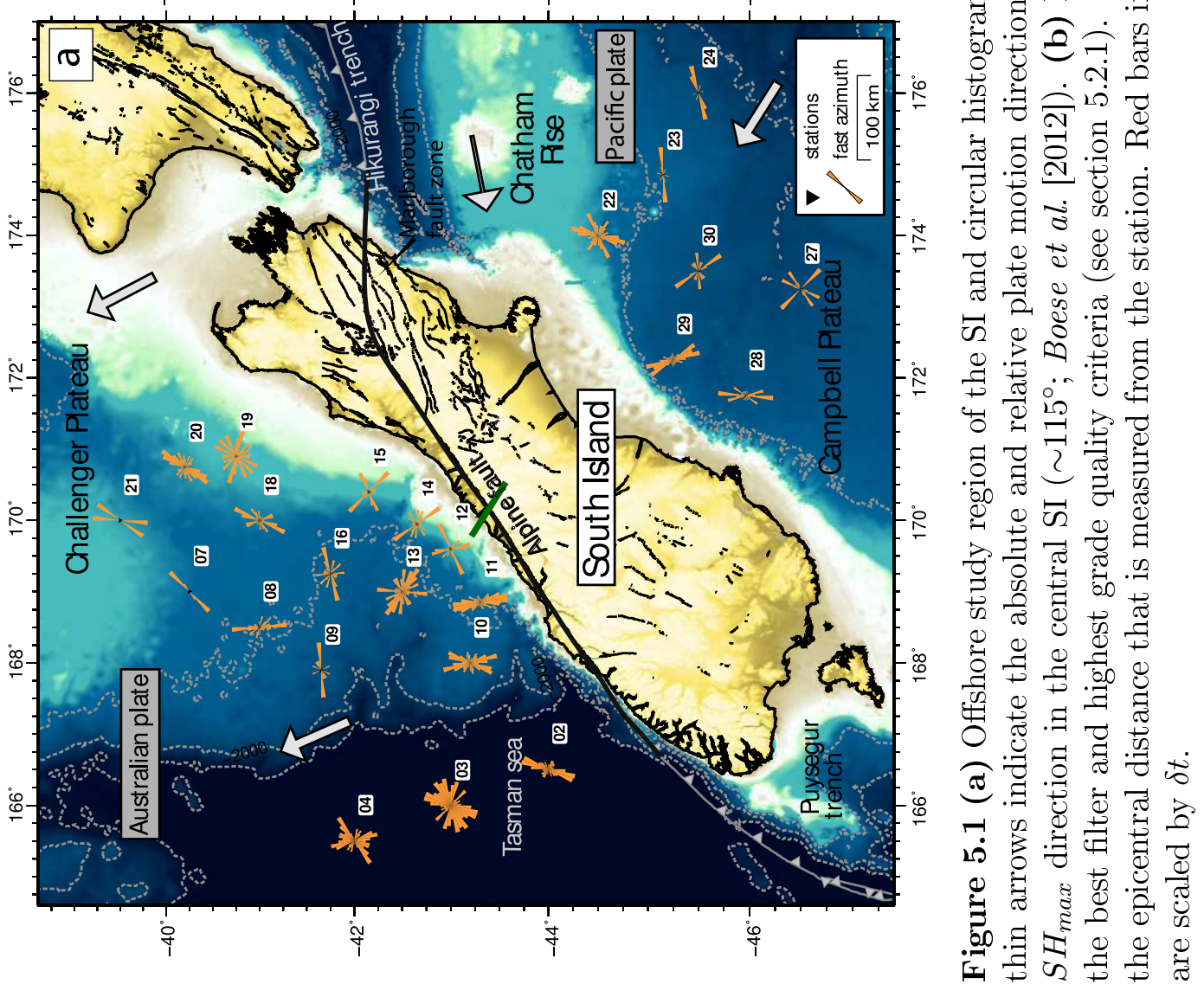




\subsection{Splitting parameters from OBS data}

\subsubsection{Data and method}

Twenty eight broadband Ocean Bottom Seismographs (OBS) that were deployed in eastern and western offshore regions of the SI with the MOANA (Marin Observation of Anisotropy Near Aotearoa) experiment [Collins et al., 2010, Yang et al., 2012] were used to retrieve S-phases from local and regional events. OBS on the western side were distributed in both the shallow (challenger Plateau) and deep ocean (Tasman sea). Eastern side OBS were located on the Campbell Plateau and Chatham Rise (Fig. 5.1-a). Seismicity in the area was mostly distributed outside of the OBS network, roughly centering between the western and eastern OBS deployments (open circles in Fig. 5.1-b show the events that yield good quality splitting measurements). We used the GeoNet catalog for the period from February 2009 - January 2010 to determine events and to extract the events from OBS records. Waveforms recorded on OBS stations were corrected for the OBS rotations using the rotation angles estimations by Stachnik et al. [2012]. We examined S-wave splitting on $\sim 2000$ Sphases from events with magnitude $\geq 3.0$ in the eastern and $\geq 3.5$ in the western side, respectively. Hand picked S-phases were analyzed for splitting with the automated Swave splitting technique of Mfast [Wessel, 2010, Savage et al., 2010]. Mfast uses the eigenvalue minimization and cluster analysis methods introduced by Silver and Chan [1991] and Teanby et al. [2004b], respectively. S-waves were subjected to filtering using a range of two-pole Butterworth bandpass filters (frequency band-widths between $0.4 \mathrm{~Hz}$ and $10 \mathrm{~Hz}$ frequencies). Filters were selected so that the S-wave analysis window had the highest signal-to-noise (SNR) ratio bandwidth product. We eliminated poor quality events and/or measurements in three steps: (i) events with ambiguous and/or distorted S-wave impulses were avoided prior to splitting analysis, (ii) signals with low SNR $(\leq 2)$ were avoided during the analysis and, (iii) poor quality measurements were eliminated by either visual inspection of splitting 
parameters on diagnostic plots (e.g. particle motion plots, contour diagrams and wave-form plots) or applying the highest quality grading criteria that comes with the Mfast program (section 3.2.3). We noticed that waveforms and/or splitting measurements of the farthest stations (NZ05, NZ06, and NZ 26) do not satisfy the above quality criteria and, thus, those stations do not yield any acceptable splitting measurements.

Volti et al. [2005] emphasized that the S-wave window (SWW) used for splitting in the sea-floor can be deviated from the limits of usual SWW (incidence angle $\leq$ $35^{\circ}$ ) that applies in the onshore splitting analysis. As a result of the low-velocity unconsolidated sediment layers in the sea floor [Tsuji et al., 2011], high-frequency local and regional S-waves attain almost vertical incidence as they reach the seafloor [Volti et al., 2005] and, therefore, splitting measurements are unaffected by the distorted S-arrivals owing to phase conversions [Booth and Crampin, 1985, Nuttli, 1961] at the sea-floor. With careful inspection of splitting measurements from Swaves that have both large $\left(>35^{\circ}\right)$ and small $\left(\leq 35^{\circ}\right)$ incidence, we adapted the same approach to extract an unambiguous set of splitting parameters that have been determined from the earthquakes with a large range of epicentral distances. Long rays may attain horizontal propagation in some parts of the ray path, depending on the ray geometry. Such a horizontal propagation (especially shallow ray paths) could be sensitive to transversely isotropic media with vertical symmetry axis (e.g. horizontal foliations and sedimentary bedding). These types of media most likely to occur in the eastern offshore, where there is an oceanic-plateau with shallow bathymetry, thick continental crust and less tectonic activity [Davy and Uruski, 2002]. As found by Paulssen [2004], the fast-split S-wave of a wave with horizontal propagation is usually horizontally polarized in the transversely isotropic media and thus, the horizontal projections of ray path and $\phi$ are orthogonal to each other. 


\subsubsection{Results and interpretations}

About 1540 measurements were graded as $\mathrm{AB}$ quality, but the spatial variations of splitting parameters were evaluated from 1300 measurements, which satisfied the high quality grading criteria (table 5.1). The selected measurements were non-null measurements with relatively dense error contours $\left(\lambda_{\max }>5\right)$ and small splitting errors (see grading in section 3.2.3). In Fig. 5.1-a, fast azimuths $(\phi)$ measured at stations in the western and eastern offshore regions are represented as rose diagrams. We allowed the program to calculate at least three splitting measurements for each event to examine the sensitivity of splitting parameters to different filter bandwidths. Individual rose diagrams are weighted by the number of measurements with that particular azimuth and therefore, the longer the rose segment the higher the number of measurements in that direction. The rose diagrams are not normalized according to the number of measurements at each station, but information can be found in tables 5.2 and 5.1. Because of the configuration of the OBS network and earthquake distribution, splitting measurements on western and eastern OBS mainly sample the anisotropy in the Australian and Pacific lithosphere respectively (Fig. 5.1-a).

Table 5.1 The number of measurements from different selection criteria.

\begin{tabular}{ll}
\hline criteria* & no.measurements \\
\hline AB grade $/$ all $* *$ & 2076 \\
$\mathrm{AB}$ grade $/ \lambda_{\max }>5 / \delta t \leq 0.7 \mathrm{~s}$ & 1300 \\
$\mathrm{AB}$ grade $/$ best-filter $/ \delta t \leq 0.7 \mathrm{~s}$ & 1390 \\
$\mathrm{AB}$ grade $/$ best-filter $/ \lambda_{\max }>5 / \delta t \leq 0.7 \mathrm{~s}$ & 547 \\
\hline
\end{tabular}

**measurements from all filters with SNR $>3$.

*see appendix B.2 for more information on the selection criteria. 
We extracted a single pair of splitting parameters for each event by using the best filter criterion. The best filter criterion compares the splitting measurements from different filters for each event and chooses a single set of splitting parameters if the splitting measurements are similar for all different filters [Savage et al., 2010]. Figures 5.1, 5.2-A/B and table 5.2 show these best filter splitting measurements and their station averages. In general, individual splitting delay times $(\delta \mathrm{t})$ from both sides range from $\sim 0.02-0.5 \mathrm{~s}$ (Fig. 5.2-B) with an average value of $0.25 \mathrm{~s} . \phi$ are not spatially consistent throughout the whole region, but patches of spatial consistencies can be seen in the western offshore with a rapid change in $\phi$ from plate-boundary parallel in the far west to plate-boundary perpendicular at about $150 \mathrm{~km}$ from the plate-boundary (Fig. 5.1a/b). Section 5.2.2 discusses the splitting estimations and gives possible interpretations for the observed patterns in the western and eastern offshore regions. 

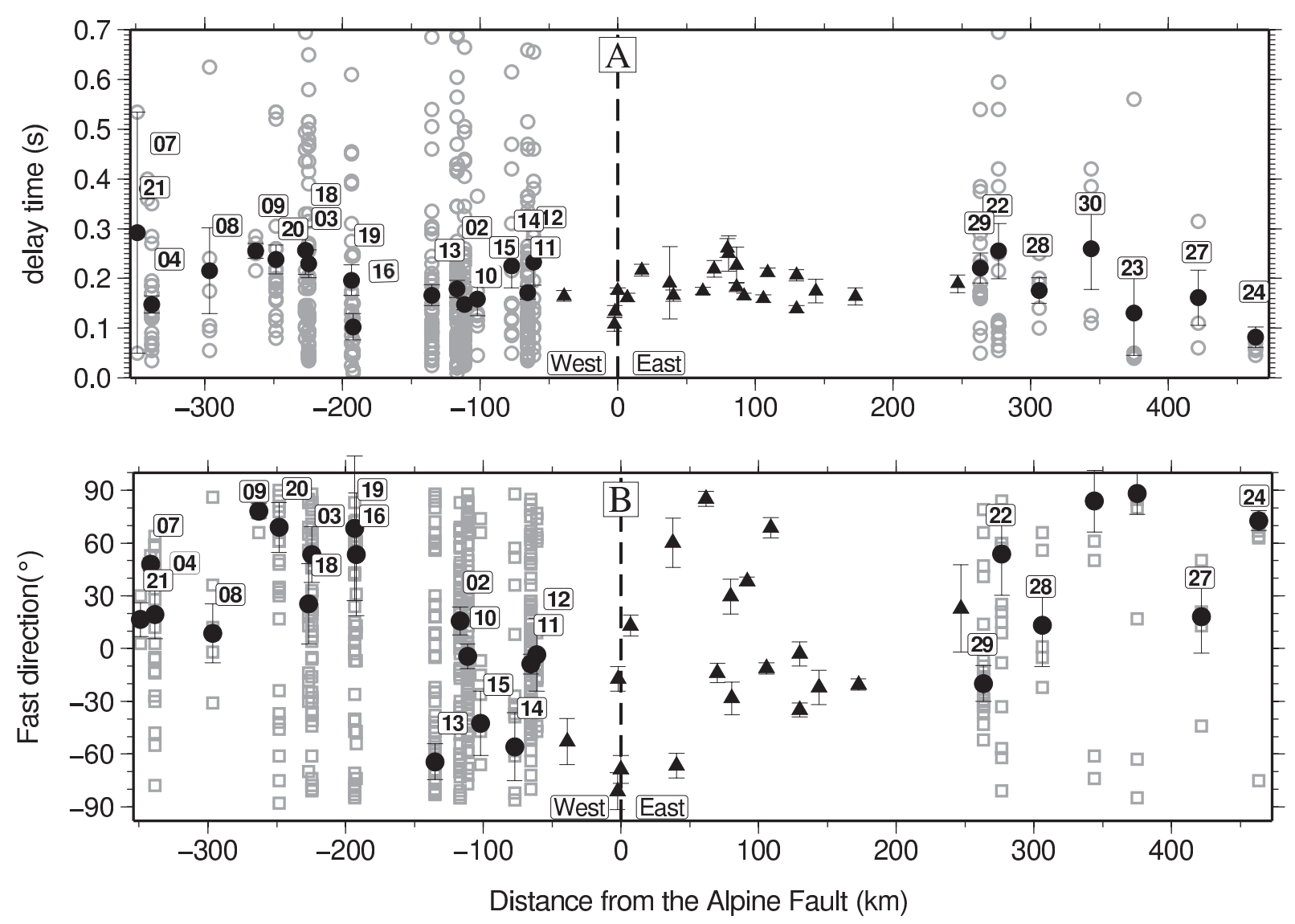

Figure $5.2 \phi$ (bottom-B) and $\delta$ t(top-A) variations with distance from the Alpine fault (gray open circles and open boxes). Mean splitting parameters at each station are marked by the black circles. Black triangles denote the average $\delta t$ and $\phi$ estimated at the inland stations [Karalliyadda and Savage, 2013], which are located in the central and northern SI. Error bars indicate the standard errors of the mean splitting parameters at each station. 


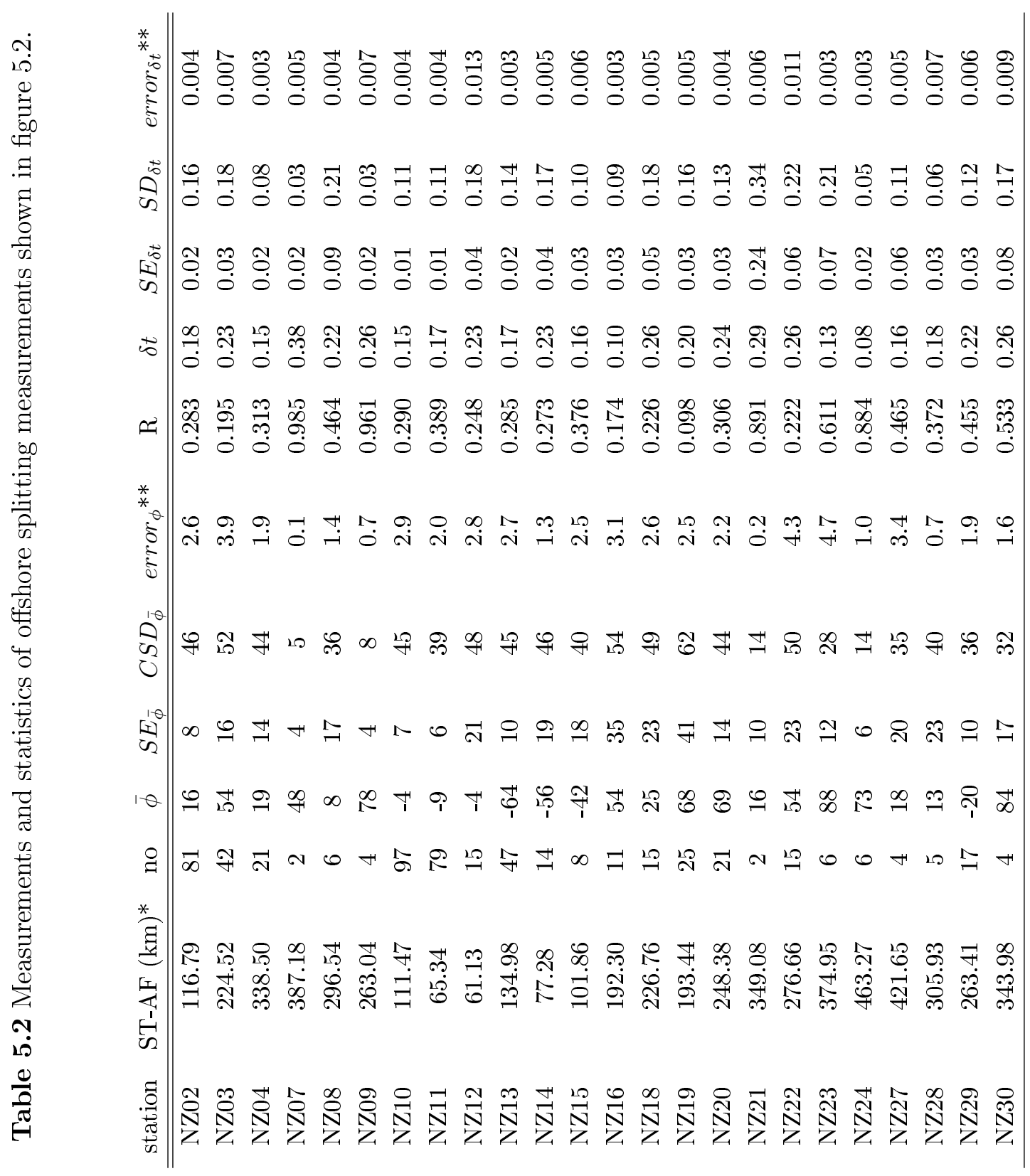




\section{Splitting estimation in western offshore}

Western OBS are distributed up to $\sim 350 \mathrm{~km}$ distance from the Alpine fault. Stations are located in three distinct zones (Fig. 5.1): 1) deep ocean basin (Tasman sea) with oceanic crust close to the Puysegur subduction (NZ02, NZ03, and NZ04), 2) central region (southernmost part of the Challenger plateau) with continentalcontinental collisional zone near the Alpine fault (where NZ11, NZ10, NZ12, NZ13, NZ14, and NZ15 are located), and 3) northeast and central parts of the Challenger

Plateau with paleo-rift systems [Wood, 1991]. In the northeast Challenger Plateau (NZ18, NZ20, NZ21, and NZ07), $\phi$ show prominent plate-boundary parallel $\left(30-70^{\circ}\right)$ alignment compared to $\phi$ perpendicular to the plate-boundary in the central offshore region. OBS in the deep sea and near by regions (NZ02, NZ03, NZ04, and NZ10) yield bimodal distribution of $\phi$ with one mode parallel to the NE-SW alignment. This azimuth is also consistent with the $\phi$ at stations in the northeast Challenger Plateau (NZ18, NZ07, NZ20). The other mode is roughly parallel to the NWSE or NNW-SSE direction and it is consistent with the predominant azimuth of the two-modes observed at the central stations (NZ10, NZ11, NZ13, NZ14, NZ15). NNW-SSE correlates well with the absolute plate motion (APM) in the Tasman sea region, indicating the possibility of asthenospheric flow-governed anisotropy in the region.

It is not clear if the NW-SE $\phi$ mode in the central stations (NZ15, NZ14, NZ13, NZ12) is a product of asthenospheric flow or has a stress controlled source (e.g. fluid-filled cracks and/or grain-boundary aligned consolidation). The maximum horizontal compressive stress $\left(S H_{\max }\right)$ direction of the adjacent onshore region is $\sim 115^{\circ}$ (Fig. 5.1-a) [Boese et al., 2012], which is consistent with the NW-SE trend. If the brittle deformation ( e.g. cracks and fractures) associated with the Alpine fault extends into offshore regions, relatively high compressive stress in this region may have an effect on crustal anisotropy. A rather diffuse, but fault parallel mode 
(NE-SW) observed at NZ12, NZ13, NZ15 and NZ14 in central offshore suggests that S-wave splitting for some paths in this region is also sensitive to anisotropy due to plate-boundary parallel shear. However, high frequency local/regional S-phases tend to be more biased towards shallow-stress controlled anisotropy. NE-SW $\phi$ at stations in the northeast Challenger Plateau agrees well with $\phi$ patterns in the onshore regions of the northern SI (see section 5.2.3; Karalliyadda and Savage [2013]). This implies that the deformation around the transform-translational fault zone in the northern SI is distributed in a broader region. The other possible explanation for NE-SW $\phi$ in this region is that fast anisotropic directions align parallel to the paleorifting (parallel to rift axis; Vauchez and Nicolas [1991]), which are assumed to be preserved throughout the Challenger Plateau [Wood, 1991]. The average $\delta t$ varies between 0.1-0.4 s in western offshore regions (Fig. 5.2-A). When considering the $\delta t$ variations across the Alpine fault, there is a slight increase in average station $\delta \mathrm{t}$ (from about $0.17 \pm 0.02 \mathrm{~s}$ to $0.26 \pm 0.04$; table 5.2 ) after the $150 \mathrm{~km}$ boundary-limit from the Alpine fault (Fig. 5.2-B). This could be due to accumulation of the $\delta$ t along the ray path [Silver and Chan, 1991, Rumpker and Silver, 1998] and/or sampling of a more pronounced zone of anisotropy at depth by steeply curved ray paths. Except for NZ08, $\phi$ display a NE-SW azimuth that is parallel to the plate-boundary (Fig. $5.2-\mathrm{A})$ beyond the $150 \mathrm{~km}$ boundary limit.

\section{Splitting parameter estimation in eastern offshore}

OBS on the eastern side are mainly located on the Chatham Rise and northernmost part of the Campbell Plateau; these regions are considered as continental extensions of the eastern SI [Sutherland, 1999]. Stations in the eastern offshore are far away from most of the earthquakes and, thus, only a few S-phases yield acceptable quality splitting parameters (Fig. 5.1). Splitting analysis of SKS phases on Chatham Island in the Chatham Rise inferred that the region is almost isotropic [Klosko et al., 1999]. 


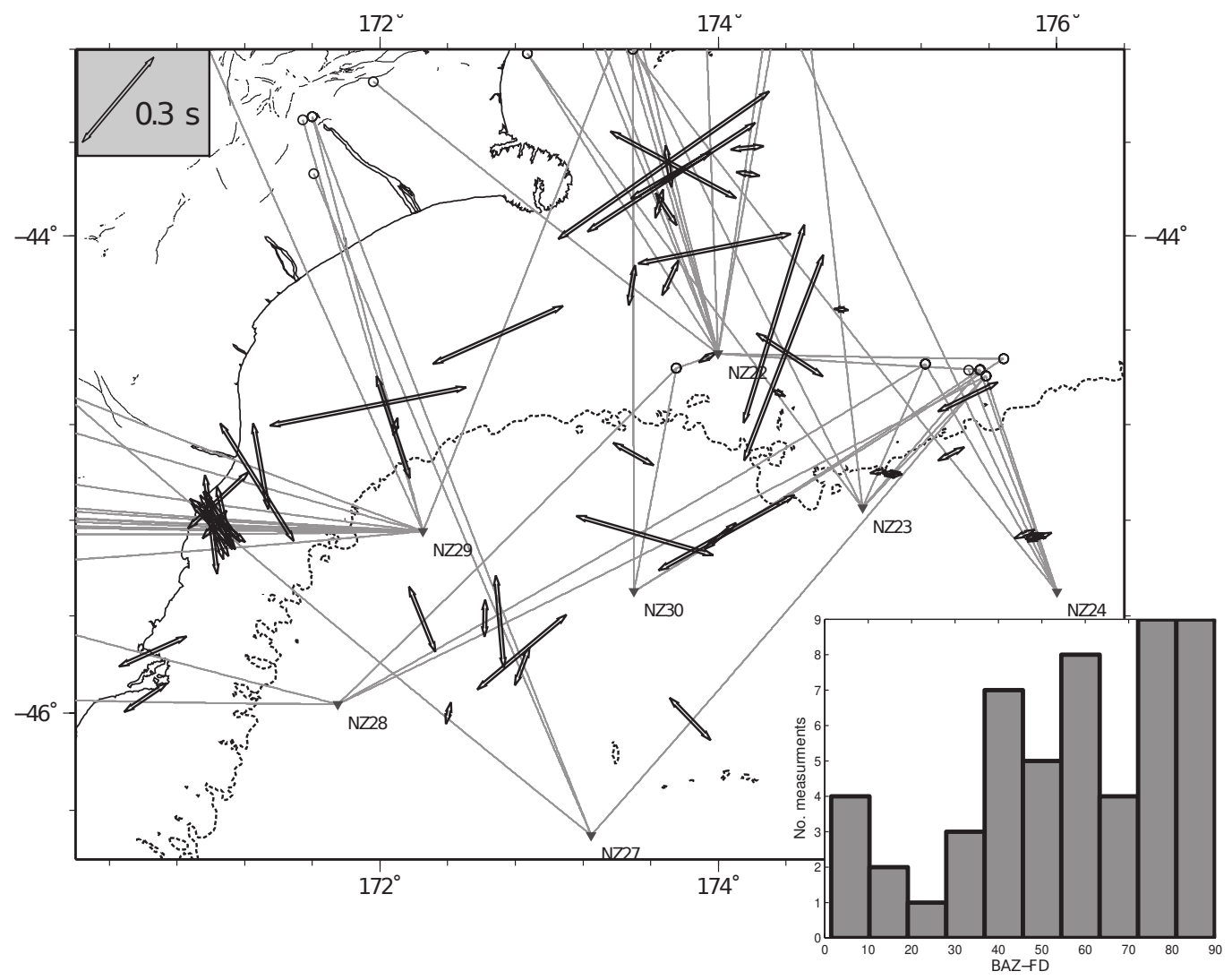

Figure 5.3 Zoomed-in view of the individual measurements (good quality) in eastern offshore SI. Measurements are plotted at one-quarter of the epicentral distance from the station to avoid overlapping. Histogram show the number of measurements versus an acute-angle between BAZ and $\phi(\mathrm{FD})$. Note that about $40 \%$ of the $\phi$ are perpendicular or high-angle $\left(70^{\circ}-90^{\circ}\right)$ to the horizontal projection of the ray paths (gray lines).

The station $\delta t$ averages range from $0.1-0.3 \mathrm{~s}$ (Fig. 5.2-B) and these values are most likely representing a shallow crustal source. NZ29 and NZ28 show NW-SE $\phi$ that are consistent with the $\phi$ from adjacent onshore stations (section 5.2.3). Swaves could also be sensitive to anisotropy due to horizontal layering if the ray paths attain a longer horizontal propagation due to ray-curvature from source to station [Paulssen, 2004]. $\phi$ perpendicular to ray paths as observed on some event-station pairs in the eastern offshore (Fig. 5.3) may indicate such an effect $(\sim 40 \%$ of the total $\phi$ within are $70^{\circ}-90^{\circ}$ of the back azimuth (BAZ), Fig. 5.3). Chatham Rise stations (NZ23 and NZ24) have smaller average delays ( $\sim 0.1 \mathrm{~s}$ or less) compared to those measured at other stations (Fig. 5.1-b). Most of the measurements made at these stations have $\phi$ nearly perpendicular to the ray paths. This suggests that they may sample the local/shallow anisotropy that represents a crustal source such 
as shallow sedimentary layers. Therefore, complex fast azimuth patterns in eastern offshore (Fig. 5.3) most likely reflect varying anisotropic sources or geometry (i.e. changing symmetry axis orientation) of the anisotropy. Low $\delta t(\sim 0.2 \mathrm{~s})$ can be attributed to either cancellation of anisotropy due to changes in the orientation of the symmetry axis, and flipping between fast and slow waves along the ray path or to crustal re-splitting owing to shallow upper-crustal anisotropy.

\subsubsection{Comparison with SKS splitting measurements}

We compare fast split azimuths with the recent SKS splitting analysis by Zietlow et al. [2013]. This was a parallel study that focused on the deep anisotropy in the off-shore region using the same OBS network, and also re-analyzed on-land SKS splitting measurements using a recent data set. We infer that the small observed $\delta t$ may be caused by the re-splitting due to multi-layer anisotropy or upper-most layer crustal anisotropy and/or frequency-dependent splitting (see 3.5). If the observed anisotropy is due to multi-layer anisotropy and the medium close to the station is nearly isotropic, the observed $\phi$ may represent the anisotropic alignment of the most prominent source along the ray path. SKS measurements show clear a deflection in the alignment of $\phi$ from fault-parallel (NE-SW) near the fault to N-S or NWSE (at high angle to the fault) alignment in the central-east and southeast of the SI (Fig. 5.4-bottom). This change reflects the change in orientation of the deep anisotropy. A similar trend can be observed from the local/regional splitting, but only in the eastern side of the plate-boundary. The prominent $\phi$ from the local events observed at stations NZ30, NZ29 and eastern on-land SI stations (WHZ, EAZ, ODZ, TUZ, SYZ) display APM sub-parallel $\phi$ and they are also consistent with the SKS azimuths. This implies that the long ray paths of the S-waves may have propagated through the lithosphere and/or asthenosphere and captured the anisotropy due to flow in the asthenosphere or fossilized anisotropy in the deep- 

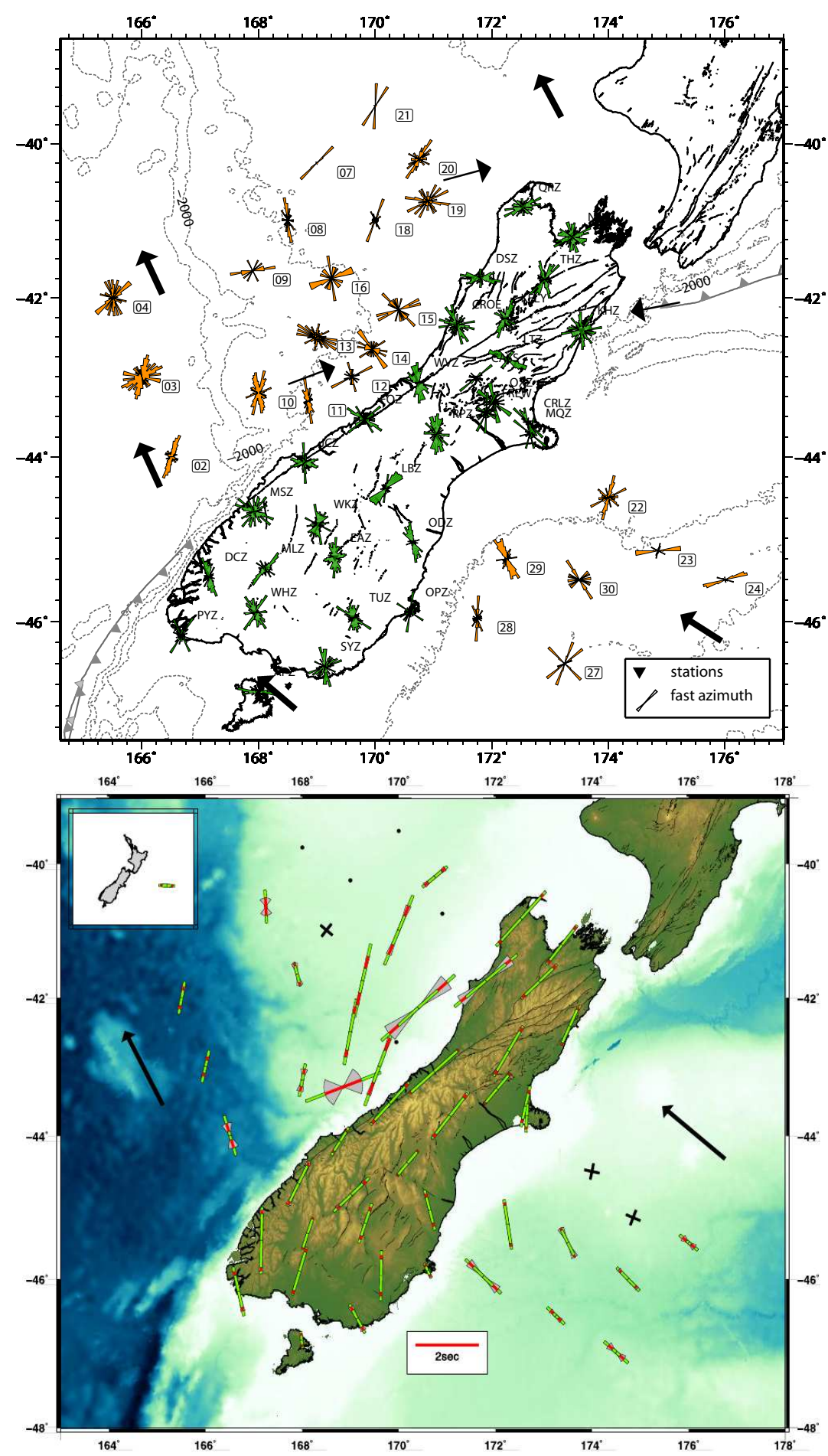

Figure 5.4 A comparison of local/regional splitting (top) and SKS splitting measurements [Zietlow et al., 2013] on-land (re-analysis) and offshore (bottom). Green and yellow rose diagrams in the top figure indicate the on-land and offshore splitting measurements, respectively. Thick arrows indicate APM direction and thin arrows indicate the RPM directions. Green bars in the bottom figure display the SKS fast azimuths, and the size of the bars are proportional to the $\delta t$. The length of the pink bars and circular uncertainties of gray wedges denote $\delta t$ and $\phi$ errors respectively. Crosses and dots represent the null measurements and stations with no measurement, respectively. 
lithosphere. SKS $\phi$ azimuths attain plate-boundary parallel/subparallel direction (also RPM direction) within $\sim 80-90 \mathrm{~km}$ distance east of the boundary, indicating anisotropy due to plate-boundary parallel shear anisotropy. Although this is not clear from the local $\phi$ at stations on the Alpine fault due to multi-mode $\phi$, this change is obvious at MLZ, WKZ, LBZ, RPZ stations and is consistent with SKS $\phi$. This shear-zone is considered to be much broader in northern and northern-central SI than in the southern SI [Karalliyadda and Savage, 2013, Houlié and Stern, 2012, Wilson et al., 2004]. This is clear offshore in the west as well, from the prominent local $\phi$ at stations NZ18, NZ07, and NZ20 that show RPM and SKS parallel fast azimuths (except NZ19). In the central west-offshore, local $\phi$ are different from the SKS fast-azimuth, but NZ12 shows one $\phi$ mode that is parallel to the fault. This mode can be attributed to either structure or to shear induced anisotropy. The prominent $\phi$ at other stations in central-offshore (NZ14, NZ13, and NZ11) are oblique to the SKS fast trends at those stations.

\subsection{Integration of on-shore and offshore splitting measure- ments}

We combined the splitting measurements from offshore OBS stations (this study), and on-shore local splitting measurements from Karalliyadda and Savage [2013], and an additional study (below) in central SI to examine the spatial patterns of $\phi$ in the entire plate-boundary zone of the South Island. 


\subsubsection{Additional land measurements: splitting measurements in the schist belt of Central SI}

The work included in this section was carried out at on-land stations to improve the spatial constrains on splitting parameters in the areas dominated by schist rock in the central SI. This study was completed after the on-land manuscript publication (chapter 4).

The events analyzed for the splitting of S-phases in the central SI in previous study (chapter 4) had threshold magnitude of 3.0 and the maximum earthquake depth of $90 \mathrm{~km}$. The measurements used in chapter 4 were also restricted to (section 4.2) the incidence angle $\left(i_{c}\right)$ of $35^{\circ}$. The above event and measurements selection criteria significantly reduced the number of measurements close to the station and, therefore, measurements presented in chapter 4 had a limited splitting resolution at shallow depths close to the station (Fig. 4.2). Because of the lack of measurements to provide information on the in-situ anisotropy of the schist rock in the central SI, splitting measurements were made using shallow $(<40 \mathrm{~km})$ and smaller earthquakes (threshold magnitude of 2.0) than in the earlier study. This data set enables us to characterize the shallow anisotropy close to the station (within $60 \mathrm{~km}$ epicentral distance) with an enhanced data set. Event selection criteria can be found in section 3.1.1. Splitting measurements displayed in figure 5.5 and table 5.3 are AB grade and no-null measurements that were made from 1-5 Hz bandpass filtered data.

Circular histograms in Fig. 5.5 display fast azimuths $(\phi)$ measured at stations. The prominent $\phi$ observed at stations MLZ, EAZ, and LBZ show a clear change in orientation compared to $\phi$ observed from events with larger spatial and depth distribution (compare with Fig. 4.5). This suggests that the measured anisotropy at those stations is mainly controlled by a shallow source (crustal contribution) close to the station. These prominent $\phi$ are also apparent in the circular histograms of Fig. 5.5 as secondary-modes, suggesting depth varying or spatial effects of anisotropy. 
$\phi$ of the off-fault stations (except WKZ) show no clear alignment with the plateboundary (Fig. 5.5), suggesting that shallow anisotropy (depth $<40 \mathrm{~km}$ ) about 100 $\mathrm{km}$ away from the boundary is not controlled by the plate-boundary shear. $\phi$ at ODZ and LBZ are aligned with the strike of the geologic contact and fault structures in the area. The prominent $\phi$ measured at the station in the southeast of central SI are consistent with NW-SE aligned basement terranes (Fig. 5.5) and the structures within those terranes [Cox and Sutherland, 2007] suggesting the crustal origin of anisotropy. WKZ shows NNE-SSW $\phi$ that are parallel to local faulting and subparallel to the plate-boundary. It is possible that some of the anisotropy measured at WKZ is sampling the western side (as the maximum epicentral distance is 60 $\mathrm{km})$, so that S-phases are sensitive to plate-boundary shear. Nevertheless, it is also possible that the observed anisotropy at WKZ is biased towards the near-surface structural anisotropy as it is located on top of the NE-SW aligned fault (Fig. 5.5). Alternatively a series of NE-SW striking (sub-parallel to the plate-boundary) faults in this region could have well-developed shear-zones beneath them, and if those shear zones are linked at depth with the plate-boundary shear-zone, producing a single large shear-zone [Otsuki, 1978]. If that is the case, $\phi$ at WKZ likely represent the alignment of both structural and shear-zone anisotropy. LBZ shows plate-boundary parallel $\phi$ from the splitting of S-phases with depths $\leq 100 \mathrm{~km}$ (Fig. 4.5-b), but not with depths $\leq 40 \mathrm{~km}$ (Fig. 5.5). This implies that the plate-boundary shear-zone at depths $40 \mathrm{~km}$ and shallower than $40 \mathrm{~km}$ is not as broad as at depths $40-100 \mathrm{~km}$. An absence of plate-boundary parallel $\phi$ at both LBZ and EAZ at shallow depths (Fig. 5.5) suggests that shear-deformation is likely distributed within at least $\sim 30$ $\mathrm{km}$ (distance from plate-boundary to WKZ is about $90 \mathrm{~km}$ and maximum epicentral distance is $60 \mathrm{~km})$ distance from the plate-boundary at shallow depths $(<40 \mathrm{~km})$. The maximum limit of the distributed shear could be $\sim 90 \mathrm{~km}$ from the fault at depths $\leq 40 \mathrm{~km}$. Beyond that distance limit, anisotropy may reflect structuralinduced anisotropy. The stations that are adjacent to plate-boundary (JCZ, FOZ and WVZ) show a bimodal distribution of fast azimuth, but at WVZ both modes 
have approximately similar azimuths (NNW-SSE and NW-SE). One fast mode at FOZ and JCZ is plate-boundary sub-parallel, which could be attributed to localized shear at sub-crutsal depths and/or due to fault structure. NNW-SSE and NW-SE modes at FOZ and WVZ are consistent with the $S H_{\max }$ orientation in the region (compare with Fig. 4.11). Table 5.3 summarizes the average splitting measurements and their statistics as discussed in section 3.4.

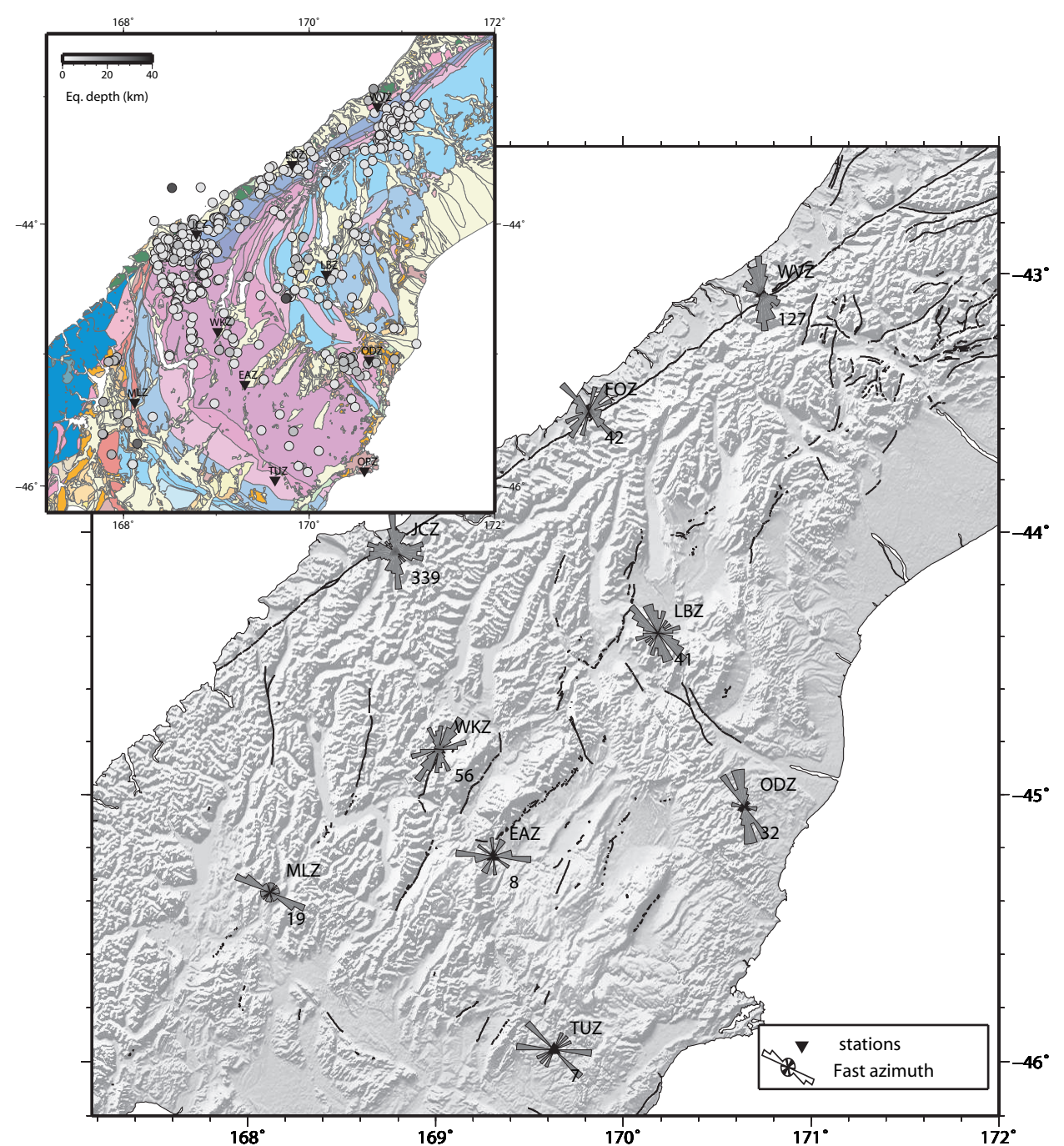

Figure 5.5 $\phi$ from the shallow S-phases recorded at the stations in schist rock of the central SI. $\phi$ at stations display as circular histograms. Inset figure shows the geology of the area and events used for this analysis. Purple to pink colors marks schist rock with increase in metamorphism. 
Table 5.3 All measurements and statistics of the shallow $(\leq 40 \mathrm{~km})$ splitting measurements in central SI.

\begin{tabular}{llllllll} 
station & no. & mean $\delta t$ & error $\delta t$ & mean $\phi$ & error $\phi$ & SE of $\phi$ & CSD of $\phi$ \\
\hline \hline WKZ & 56 & 0.20 & 0.007 & 26 & 2.6 & 9 & 44 \\
JCZ** & 339 & 0.16 & 0.005 & 43 & 2.5 & 39 & 76 \\
LBZ & 41 & 0.15 & 0.005 & -37 & 1.9 & 15 & 50 \\
WVZ & 127 & 0.14 & 0.007 & -24 & 2.6 & 3 & 34 \\
EAZ & 8 & 0.14 & 0.006 & 81 & 1.1 & 25 & 45 \\
MLZ & 19 & 0.10 & 0.006 & -57 & 3.0 & 14 & 43 \\
TUZ & 7 & 0.12 & 0.010 & 86 & 2.8 & 28 & 47 \\
ODZ & 32 & 0.10 & 0.004 & -17 & 2.5 & 5 & 29 \\
FOZ** & 42 & 0.15 & 0.005 & 79 & 2.0 & 33 & 62 \\
\hline \multicolumn{7}{r}{ **stations with clear double-modes of $\phi$}
\end{tabular}

Delay time variations across the plate-boundary zone and Schist rock in the central SI are analyzed from the AB graded (no-null) splitting measurements with dense error contours $\left(\lambda_{\max } \geq 5\right.$; section 3.2 .3$)$ and $\delta t \leq 0.6$ s. Figure 5.6 shows the individual (top-gray circles) and average (bottom/top - black circles) $\delta t$ that are calculated from those measurements as a function of distance from the Alpine Fault (dashed line in Figure 5.6). The two stations that are located further south, away from the central plate-boundary zone are marked in black squares. Except MLZ that is located further south and does not lie on the schist rock, the other stations show an increase in average $\delta t$ towards the plate-boundary (the Alpine fault). Although individual measurements are a bit scattered, this trend is also visible in the individual station measurements (Fig. 5.6-bottom). Individual measurements also show an increase in scatter towards the plate-boundary, implying a more complex anisotropic structure towards the plate-boundary. A similar increase in velocity anisotropy had been determined by the petrophysical studies in the same schist terrane of the central SI by Okaya et al. [1995], and this was attributed to an increase in metamorphic grade towards the Alpine fault (section 1.5). Local S-phases recorded on the stations that are close to the fault (e.g. JCZ, FOZ) could sample anisotropy as high as $\sim 0.5 \mathrm{~s}$ and as low as $\sim 0.05 \mathrm{~s}$. These splitting measurements were obtained from high-frequency S-phases (filtered at 1-5 Hz), thus 

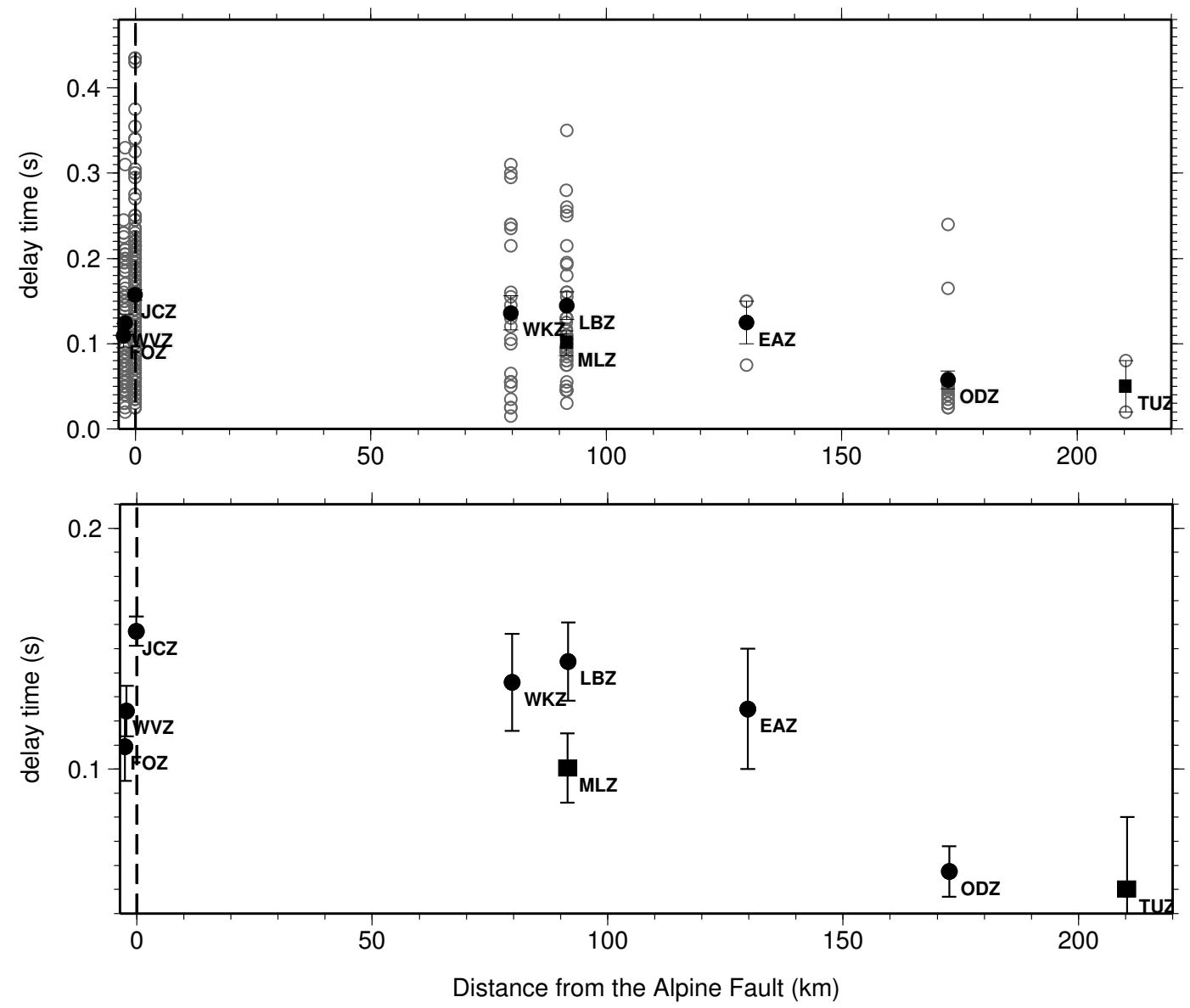

Figure 5.6 Top: The Averaged (black) and individual (gray-open) $\delta t$ (measurements with $\lambda_{\max } \geq 5$ and $\delta t \leq 0.6 \mathrm{~s}$ ) variations across the schist rock in central SI from shallow $\mathrm{S}$-waves $(<40 \mathrm{~km})$. Distance to station is measured from the Alpine Fault (dashed line). Error bars in both plots denote the standard error of the mean station $\delta t$. Note that MLZ and TUZ (black squares) are located in the southernmost region of Central SI. Bottom: Same as top figure, but zoomed-in view of the average $\delta t$ variations across the schist rock in central SI. Note a moderate increase in $\delta t$ at the central SI stations from east to west.

we could minimize the effects due to frequency-dependence, but smaller delays could be caused by the re-split S-phases from upper-most crustal anisotropy. Pulford et al. [2003] found very little splitting anisotropy (0 - $0.08 \mathrm{~s})$ from high-frequency S-phases from active source experiment across the Alpine Fault. As determined by the petrophysical studies in the same area, variable delays can also be caused by changing ray propagation with respect to the symmetry of the anisotropic system [Godfrey et al., 2000] or the orientation of the foliations planes [Barruol, 1993]. According to figure 1.6, such a variation can produce a range of $\delta t(\sim 0-0.9 \mathrm{~s})$, as seen in our in-situ data set. Thus, unless there is crustal re-splitting we favor the 
proposed idea by Godfrey et al. [2000] to explain the range of delays from shallow local-splitting. The increase in $\delta t$ from TUZ to JCZ possibly reflects the increased metaphoric grade, finite strain, and hence, the strength of anisotropy towards the plate-boundary in agreement with the petrophysical studies [Okaya et al., 1995] and finite strain estimations from bending related geological markers [Little et al., 2002b]. Strain may accumulate as crustal shear-zones with foliations parallel to the plate boundary. Thus, the splitting at shallow depths in the Hasst schist of the central SI suggests that such a shear-zone is likely extends $\sim 30-90 \mathrm{~km}$ distances from the plate-boundary at shallow depths.

\subsubsection{2-D $\delta t$ tomography and spatial averages of $\phi$}

Karalliyadda and Savage [2013] attributed variable splitting parameters from the onshore experiment to stress and mantle-shear induced anisotropy in different regions. However, the spatial extent of these anisotropic sources is not well resolved. Thus, we attempted to constrain the boundary limits of the observed patterns using the enhanced data set, which includes OBS measurements and additional central SI measurements (appendix B.4). Because of this enhanced data set, we expect some discrepancies between the solutions obtained from the on-land tomography/spatial averaging and this combined analysis. As there are limited measurements in offshore, this analysis assumes a single anisotropic layer at depth, but this assumption may not be true especially in on-land as the depth varying anisotropy is obvious throughout the region [Karalliyadda and Savage, 2013]. Thus this study will give us a first-order approximation on the spatial patterns of anisotropy.

We used three runs(processing cycles) with different parameters as summarized in table 5.4 and $\operatorname{run}_{3}$ is used to determine both tomography and spatial averages. The results from $\mathrm{run}_{3}$ are discussed in this section. run 1 is only used to obtain the spatial averages of $\phi$ (Fig. 5.9) using quadtree gridding with maximum $50 \mathrm{~km}$ grid-sizes 
Table 5.4 Parameters used for the delay-time tomography and/or spatial averaging

\begin{tabular}{lccccl}
\hline run & block size $(\mathbf{k m})$ & grid & no paths & weighting & grade \\
\hline \hline run $_{1}$ & 50 & quadtree & 7035 & no-weighting & $\begin{array}{l}\text { AB-grade/best- } \\
\text { filter }\end{array}$ \\
run $_{2}$ & 50 & regular & 4838 & no-weighting & $\begin{array}{l}\text { AB-grade/good* } \\
/ \text { best-filter }\end{array}$ \\
run $_{3}$ & 25 & regular & 4838 & tomo.weighting & $\begin{array}{l}\text { AB-grade/good* } \\
\text { /best-filter }\end{array}$ \\
\hline
\end{tabular}

*best filter measurements with dense error contours $\left(\lambda_{\max }>5\right)$. See appendix B.4.

and to compare with the spatial averages from run $_{3}$ (Fig. 5.8) with $25 \mathrm{~km}$ regular grids.

The final solution from run $_{3}$ is constrained from 4838 ray paths, 2130 events and 56 stations from $25 \mathrm{~km}$ regular gridding. Log resolution contours displayed in figure 5.7(a) are used to determined the well constrained solution. By adding OBS splitting measurements and enhancing the splitting data set in the central SI, we have increased the spatial resolution of the tomographic solution and minimized uncertainties of the spatial averages in the central SI data. Comparing to figure 4.10, most of the features resolved by the on-land tomography remain consistent, but combined analysis has a better spatial constraint on those features. For example, high strength of anisotropy (SA) along the central part of the Alpine fault is more resolved than that in figure 4.10. This feature does not show a broadly distributed character within the depth resolution $(\lesssim 60 \mathrm{~km})$ of the central SI data. Relatively high SA in the southern west-offshore SI is likely associated with the oceanic crust (Fig. 5.1-a). In the northern SI, high strength of anisotropy in both on-land and adjacent offshore (northeast) regions appears to correlate with the complexities associated with the subduction-collision transition. The region where the Alpine Fault terminates into the Marlborough fault zone (transition faults) appears to have a high strength of anisotropy (about lat:- $42^{\circ}$ and lon:171.5 $5^{\circ}$. Note that the high strength of anisotropy observed in some regions (e.g. far-west offshore) exists in 
the regions with low resolution (Fig. 5.7-a; $\log _{10}$ resolution $\lesssim 4$ ) and may indicate artifacts in the solution.

Both regular (Fig. 5.8-left) and quadtree (Fig. 5.9-bottom) gridding with tomographic weighting and without weighting respectively yield similar spatial patterns. According to spatial averages of $\phi$ (Fig. 5.8-left), the spatial distribution of anisotropic structure appears to be characterized by three distinct anisotropic sources. The region with yellow bars: $\phi$ are parallel/sub-parallel to the relative plate motion $\left(\mathrm{RPM}=\sim 70^{\circ} ;\right.$ DeMets et al. [1994b]) direction (thin arrows in Fig. $5.8)$ and and strike of the Alpine Fault $\left(\sim 55^{\circ}\right)$; the region with blue bars: $\phi$ (outermost- $\sim 330^{\circ} \pm 45^{\circ}$ are consistent with the absolute plate motion (APM; Gripp and Gordon [2002]) direction (bold blue arrows in Fig. 5.8). The zone with red bars: $\phi\left(\sim 115^{\circ} \pm 45^{\circ}\right)$ are consistent with $S H_{\max }$ azimuth (bold-gray bars; Townend et al. [2012]). Except in the $S H_{\max }$ governed zone, local/regional $\phi$ are mostly aligned with the SKS fast azimuths (gray bars with closed circles in Fig. 5.8) in other regions, implying either a deep source of anisotropy or crust-mantle coupled anisotropy. Yellow bars are distributed in a wide region across the upper-central and northern SI (Fig. 5.9-bottom). However, in the central SI, the plate-boundary parallel zone tends to be masked by a zone with a plate-boundary oblique azimuth (zone with red bars) that is aligned with the $S H_{\max }$ direction in that region [Townend et al., 2012] or the axis of shortening [Houlié and Stern, 2012]. The outer-most zone with blue bars has a very diffuse pattern, but it is likely controlled by the presentday asthenospheric flow or preserved fabrics in the deep upper-lithosphere (Fig. 5.9-bottom).

Adding the OBS splitting measurements and additional inland measurement to the previous inland data set, we have extended delay time tomographic solution in Fig.4.10 into near offshore regions and the quality of the solution in central SI is improved. The offshore portion of the tomographic solution is only well constrained on the western side within $\sim 120 \mathrm{~km}$ of the plate-boundary. In the region with high 


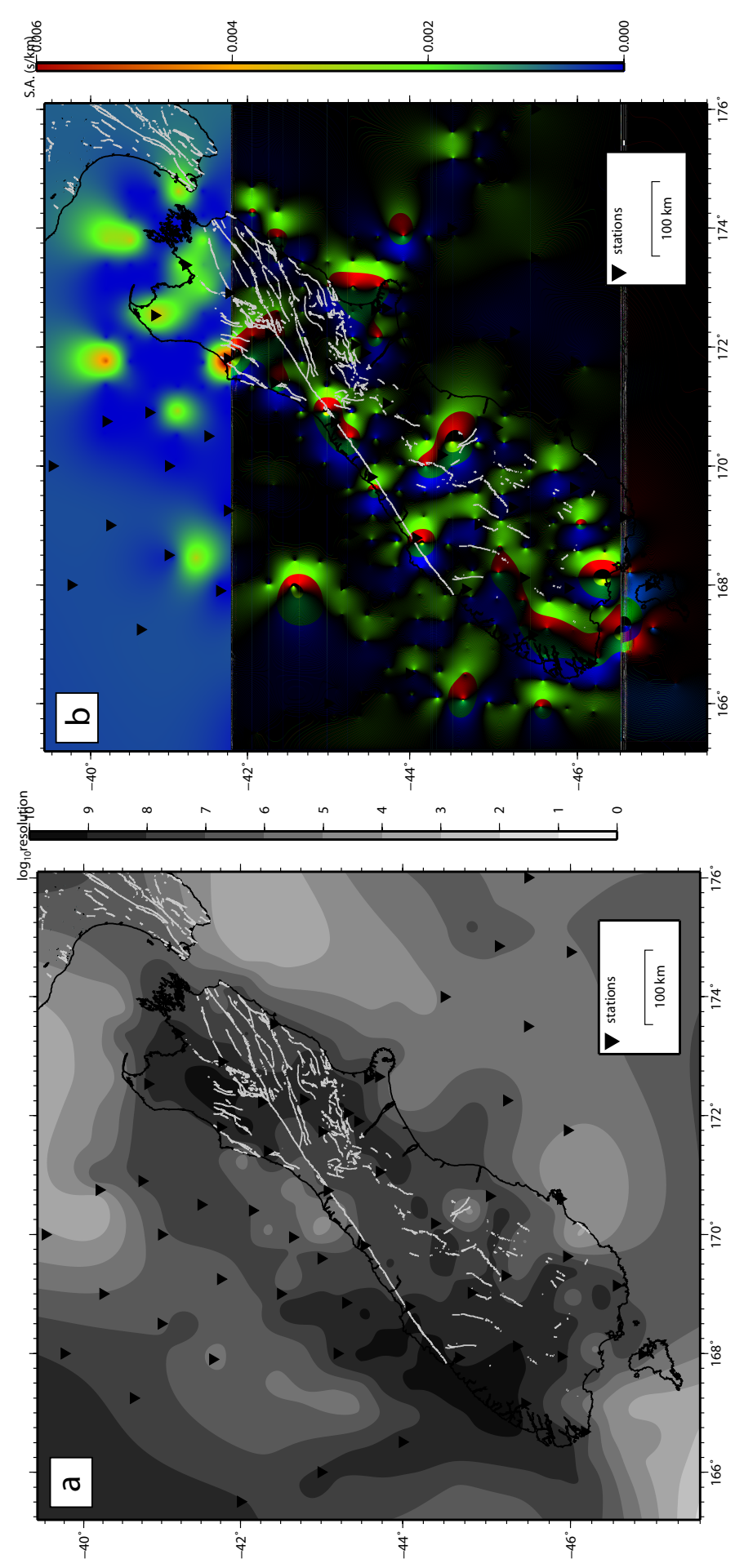

\begin{tabular}{l}
0 \\
$\bar{\sigma}$ \\
\multirow{\sigma}{*}{} \\
$\approx$ \\
.$\Xi$
\end{tabular}

窝

施色

ఠี

㮃

कृ

孛莡

त)

50

링.

\pm สี

चี

.

离

范范

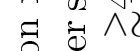

壱

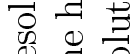

此

迹高

毒 0

की

$\exists=$

宵

용

疍

0.

ज.

进焉它

둥

$\circ .0$

पे

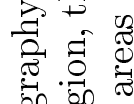

द्व

옾

원

寻它

完焉

$\therefore \dot{0}$

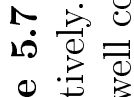

일

$\begin{array}{ccc}3 & 0 & 0 \\ 0 & 0 & 0 \\ 0 & 0 & 0 \\ 1 & 0 & 0\end{array}$ 


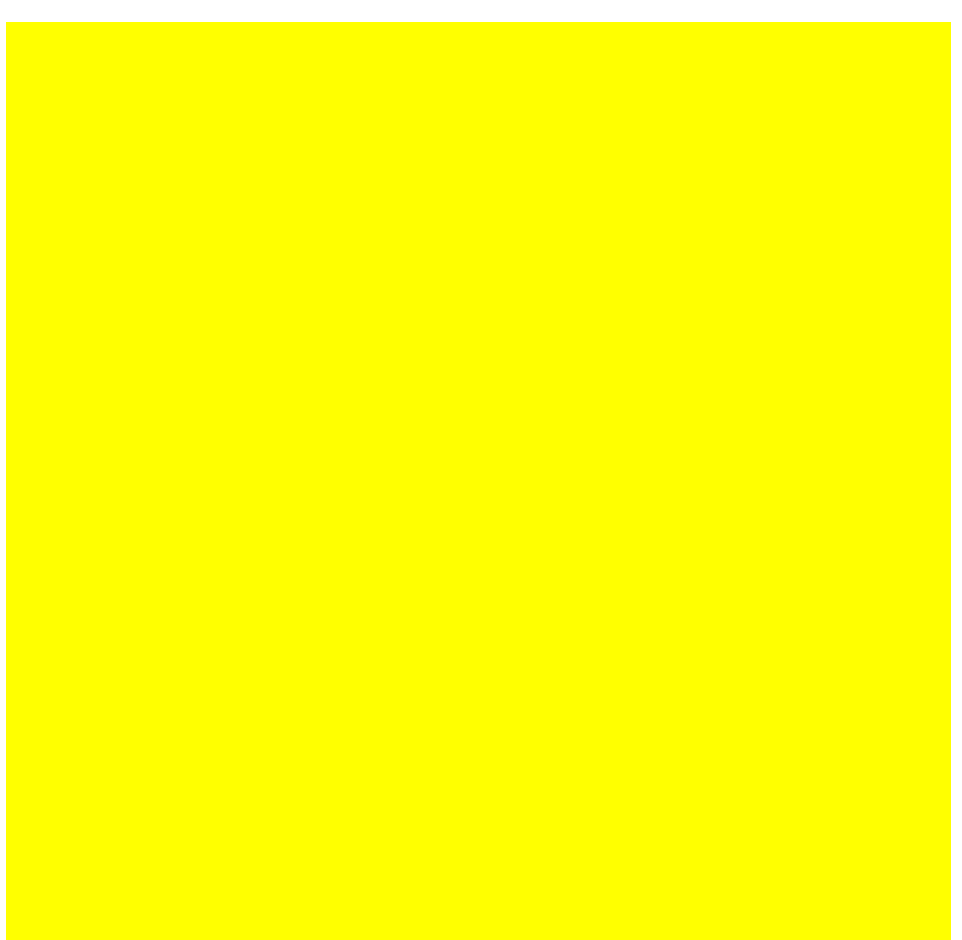

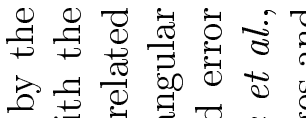

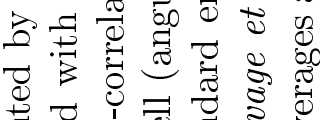

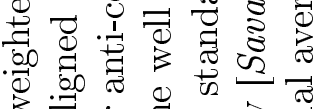

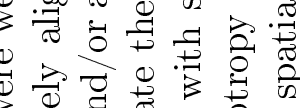
$Q$ .

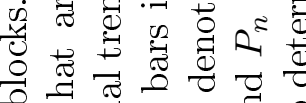
Ð

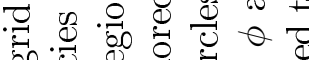

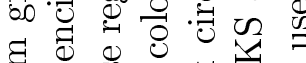

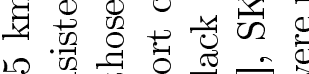

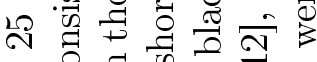

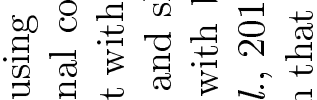
क द्व व 0 व

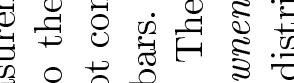
ชิ

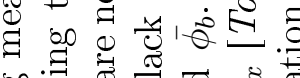

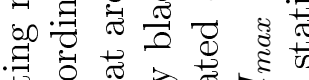

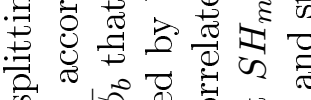
क

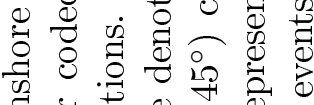
官苍芯

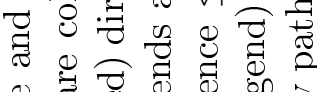

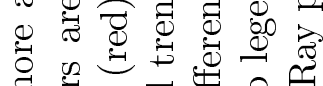

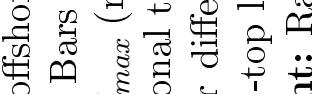

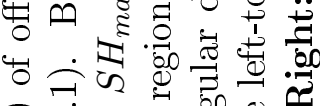
ڤே Q $.0 \%$

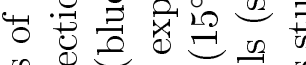

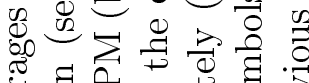

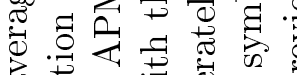

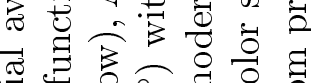
要

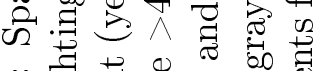

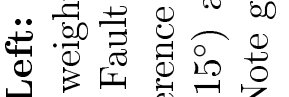

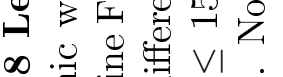

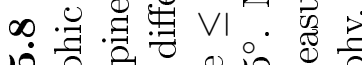

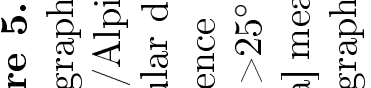
㟧

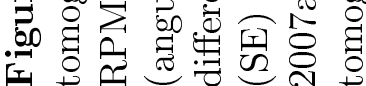



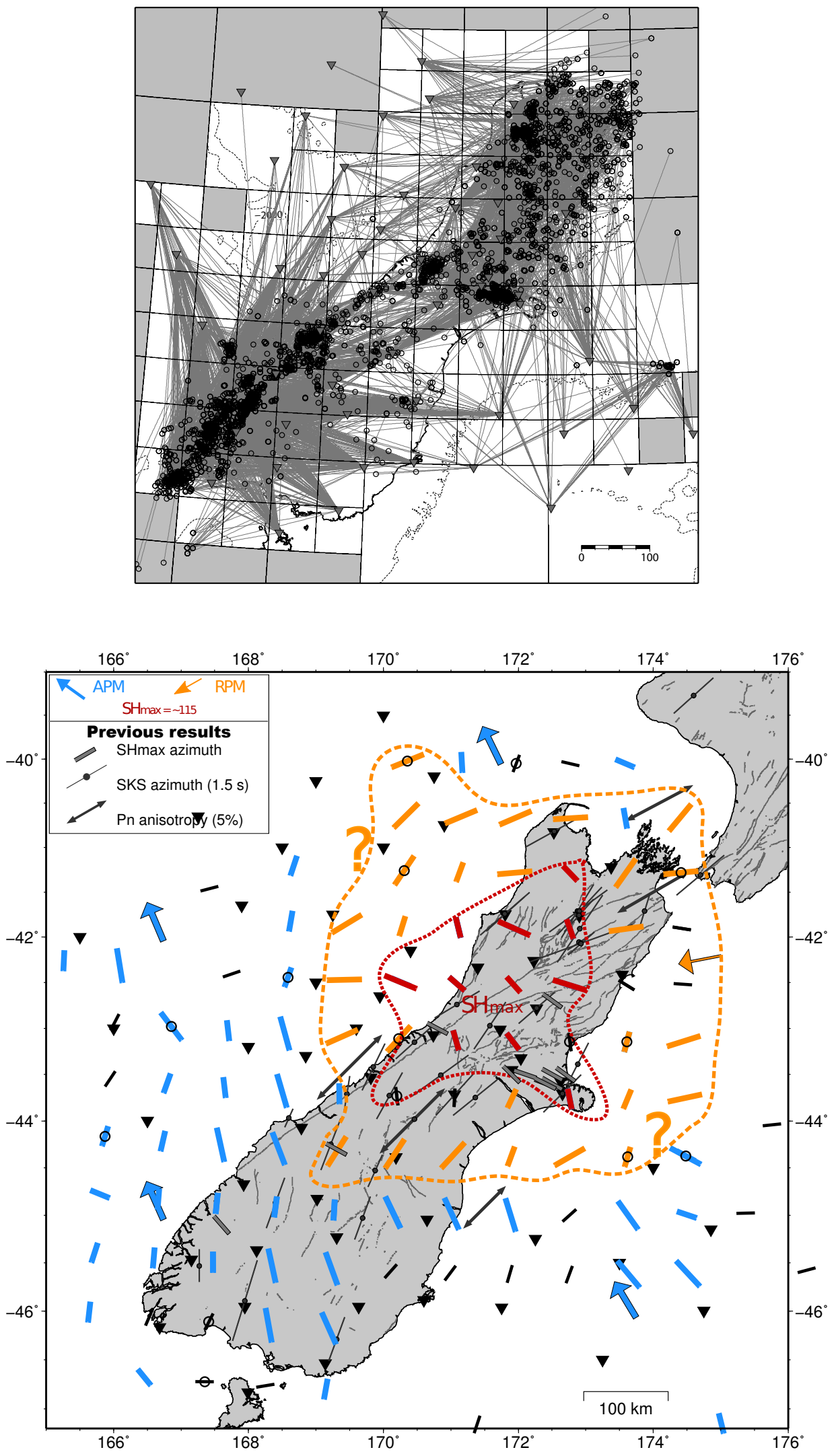

Figure 5.9 The colors and symbols are the same as figure 5.8(left), but AB-grade bestfilter (run $_{1}$ in table 5.4) measurements (top-ray paths) are spatially averaged (bottom) from quadtree griding with $50 \mathrm{~km}$ maximum grid size. 
resolution, the patches of relatively high strength of anisotropy can be detected in the two-subduction zones (see Fig. 5.7). The inland spatial patterns of $\phi$ with the improved data set (Fig. 5.8) are better constrained compared to the patterns observed from the initial data set (see section 4.4.3). For instance, the zones of plate-boundary parallel $\phi$ detected in the northern and southern central SI (Fig. 4.11) are likely linked at depth as a single shear zone, which is produced by the present-day plate-boundary deformation. This zone is broader in the northern SI and extends into offshore regions than in the southern South Island (see Fig. 5.8). In addition to this 2-D spatial analysis, we used 3-D tomographic analysis (SWST) that allows us to characterize the 3-D distribution of anisotropy in terms of mineral alignment especially in the deeper part ( $\gtrsim 12 \mathrm{~km})$. The methodology used and the preliminary results of this sub-study are given in sections 3.6.2 and section 5.3.3 respectively.

\subsubsection{Preliminary results from 3-D tomography}

We used the AB-grade (best filter) splitting data set that was used in the combined analysis of 2-D delay time tomography (section 5.3.2) for a 3-D inversion. Tomography code (SWST) by Abt and Fischer [2008] is used for the analysis (section 3.6.2). The model space is defined so that the $x$-axis is aligned with the plateboundary and the $y$-axis is perpendicular to the plate-boundary. Because of the extreme computation time (approximately 10 days for 100 iterations), we have limited this analysis to one main run $\left(\right.$ run $\left._{3}\right)$, We performed some test runs prior to the main run in order to select the inversion and model parameters. The table 5.5 summarizes the model-space,model and inversion parameters that were used to obtain the inversion solution.

Abt and Fischer [2008] noticed that the final inversion solution depends on the starting model. In their test runs with splitting from known structures and different 
starting models, the final model obtained from the average starting model produced the splitting parameters that fit well with the observations in both well-resolved areas and under-sampled regions. Final models determined using the other starting models did not yield a better-fit in the under-sampled regions because of the nonlinearity of the problem. Therefore, we used the average starting model for this analysis. The average starting model is determined from averaging the observed splitting parameters in the 3-D model space after ray tracing through the model blocks [see Abt and Fischer, 2008, appendix A].

We used the plotting options given in the 3 -D tomography code to illustrate the final model with the resolved a-axis azimuths (fast direction). Figures 5.10 and 5.11 display the $2-\mathrm{D}$ views (map views) of the $3-\mathrm{D}$ models with a-axis azimuths. In figure 5.11, the independently well-resolved model (Fig. 10(b)) is divided in to several depth-layers.

Table 5.5 Model-space, model and inversion parameters

\begin{tabular}{|c|c|c|}
\hline \multicolumn{3}{|c|}{ Model-space setup } \\
\hline parameter & value & variable $^{a}$ \\
\hline block size & $25 \mathrm{~km}$ & $\operatorname{MTP}(8)$ \\
\hline no. of blocks & 12160 & nblks \\
\hline blocks per layer & 1216 & nblyr \\
\hline no. events & 2982 & nevents \\
\hline no. rays & 7020 & nrys \\
\hline \multicolumn{3}{|c|}{ Model } \\
\hline parameter & value & variable $^{a}$ \\
\hline run no. & 3 & run \\
\hline crystal symmetry & hexagonal & $\operatorname{MTP}(2)=2$ \\
\hline \multicolumn{3}{|c|}{ Inversion } \\
\hline parameter & value & variable $^{a}$ \\
\hline type & real & $\operatorname{RTP}(1)=0$ \\
\hline iterations & 100 & $\operatorname{ITP}(3)$ \\
\hline starting model & average & $\operatorname{ITP}(2)=1$ \\
\hline $\begin{array}{l}\text { no model parame- } \\
\text { ters to solve }\end{array}$ & $\mathrm{np}=\theta, \psi, \alpha$ & $\operatorname{MTP}(1)=3$ \\
\hline larger-volumes & yes & $\operatorname{ITP}(5)=70$ \\
\hline smoothing & no & $\operatorname{ITP}(7)=0$ \\
\hline
\end{tabular}




\section{Main features in the independently resolved model}

This section discusses the main features that have been identified in the independently well-resolved model (Figs. 5.10 and 5.11-d, e, \& f). Note that Abt and Fischer [2008] use a-axis orientation (olivine) to explain the alignment of fast direction $(\phi)$. The overall well-resolved model suggests (Fig. 5.10(b)) that the plate-boundary parallel shear dominates close to the plate-boundary. $\phi$ are either parallel (red) or sub-parallel (magenta) in the near by regions of the plate boundary. Well resolved plate-boundary parallel azimuths in the south-east of the Alpine fault agree well with the schist-band in the region, suggesting a high-strained zone possibly linked with the plate-boundary shear. Most of the resolved structures also agree with the 2-D spatial averages of $\phi$ (compare Fig. 5.10(b) with 5.8 and Fig.5.9). Further investigation into inversion modeling of this data set would involve exploring smoothing and damping parameters, comparison with models obtained assuming orthorhombic symmetry and testing the stability of the inversion solution with different starting models. The final model solution may vary with the selection of different starting models and crystal symmetry (or elastic coefficients). Thus, the tectonic interpretations based on the final model solution given here, which is constrained from the average starting model with hexagonal symmetry, could be preliminary. The following section summarizes main features resolved at different depths of the independently well-resolved model (Fig. 5.10(b)). 


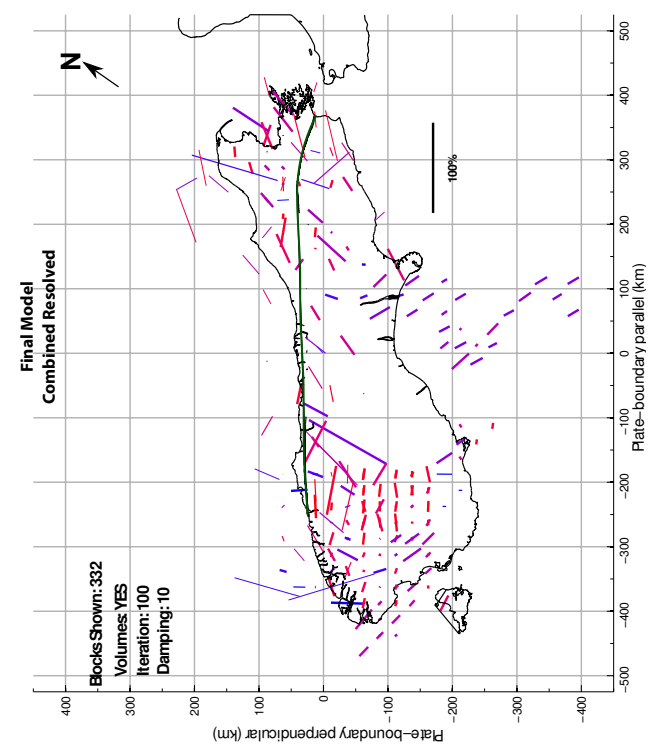

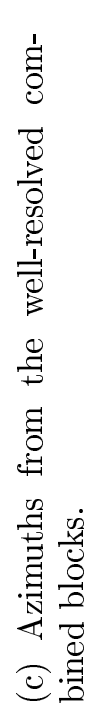

菏岁要这

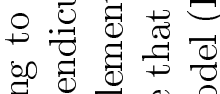

$\exists$ 임

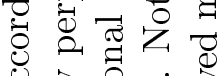

¿

च

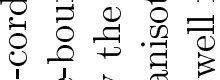

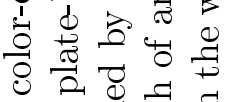

0

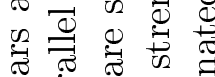

ஸี

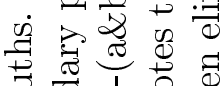

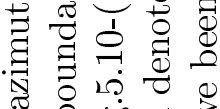

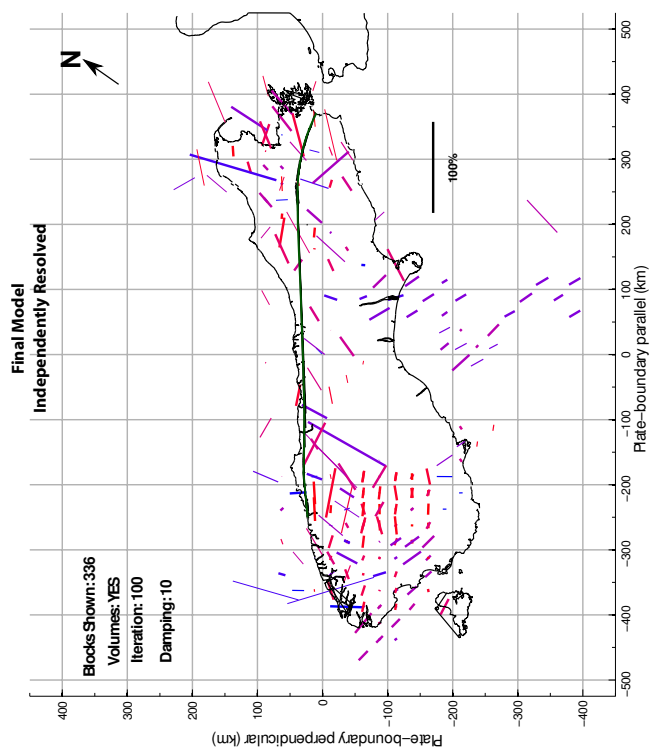

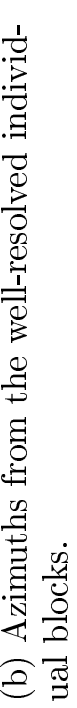

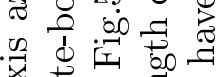

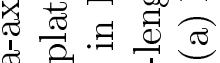

플

$3 \infty$ 灵

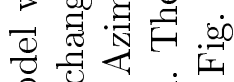

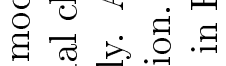

ค

$\rightarrow$ b 0 仓

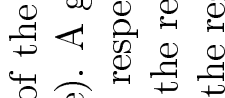

पे

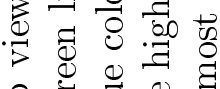

శ.

$\exists \gtrsim \circ$ की

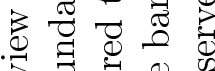

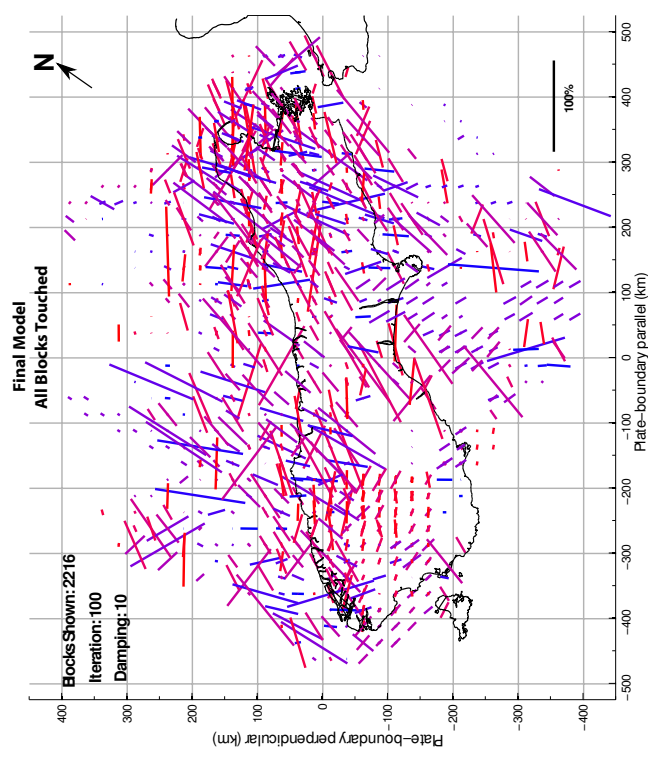

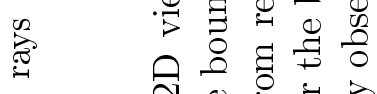

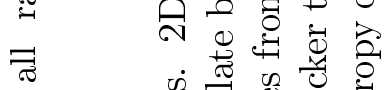

过范

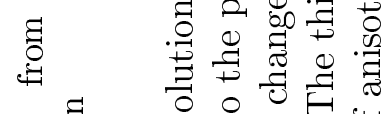

ช 00 क

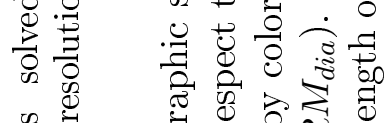

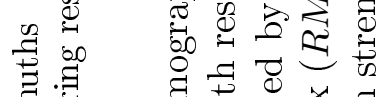

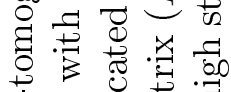

สี

.

ค

○䒕艺

i⿱

ம.

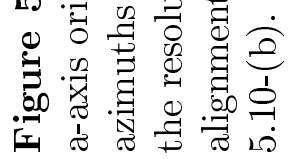



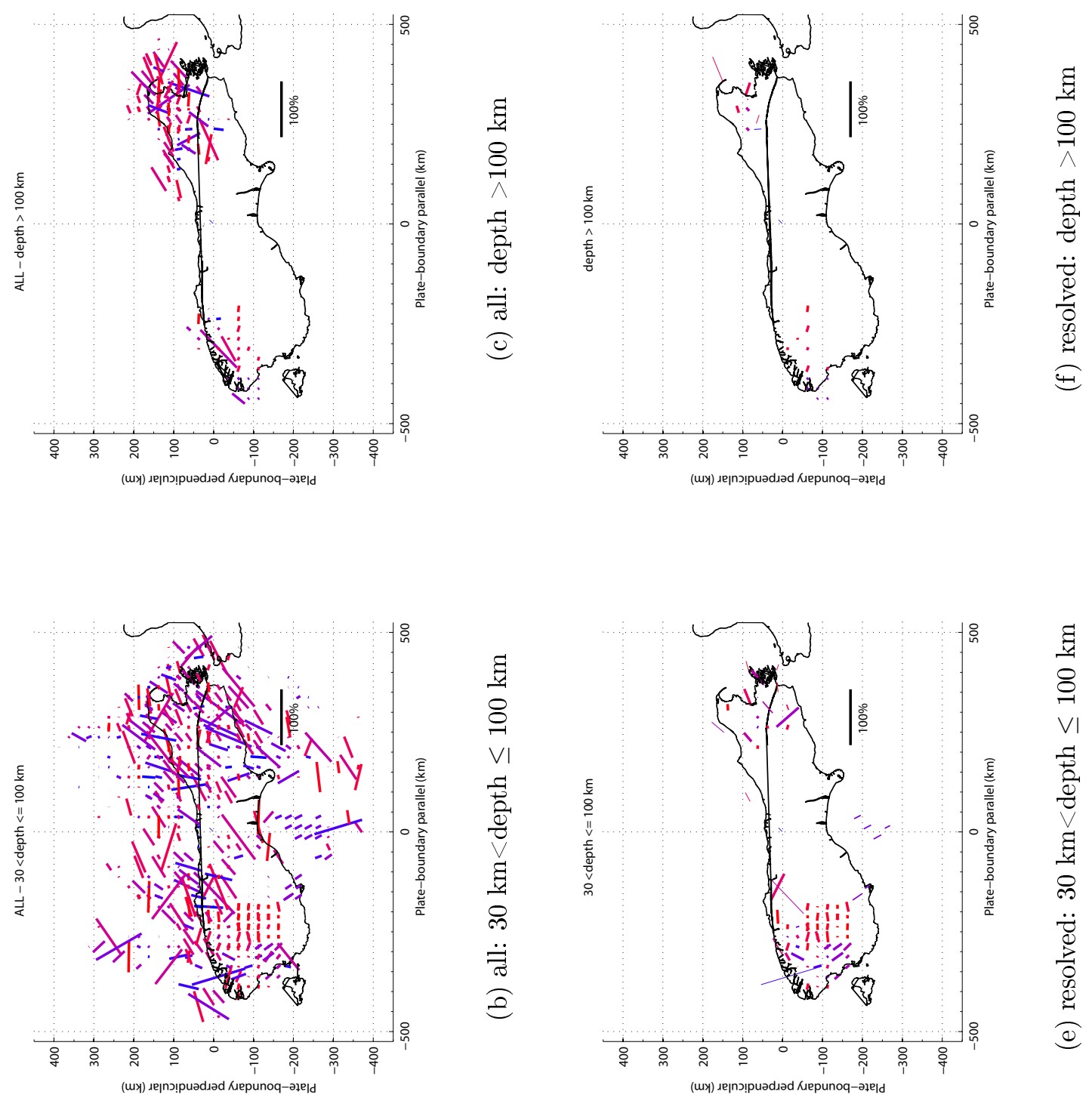

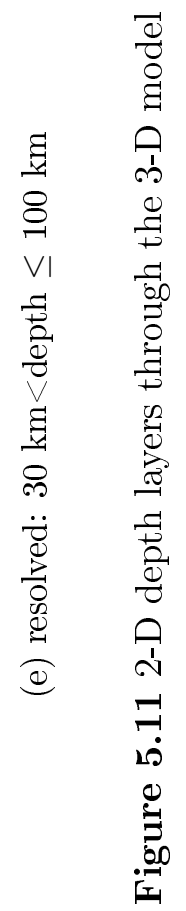
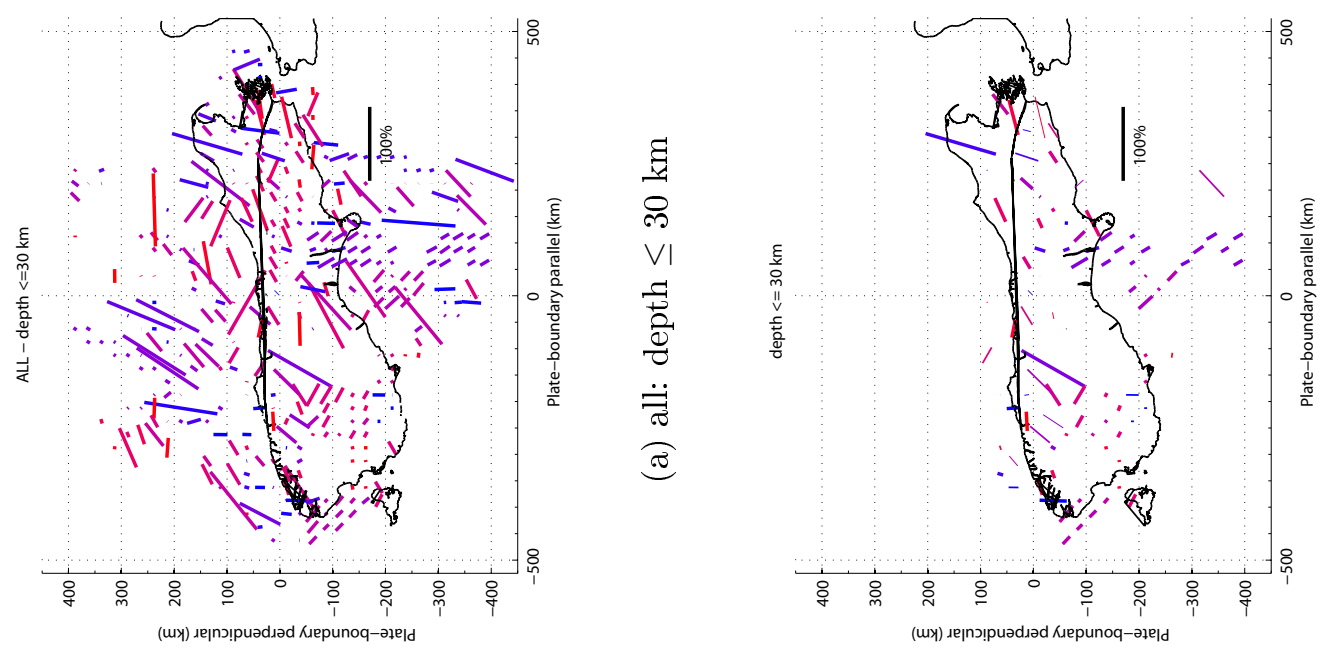

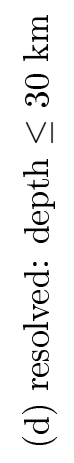




\section{Resolved features:}

- Fig. 5.11(d): $\phi$ orientations at depths $\leq 30 \mathrm{~km}$ suggest that the plateboundary parallel/sub-parallel ( $\sim 30^{\circ}$ from the plate-boundary) shear could occur in a $\sim 40-80 \mathrm{~km}$ wide zone around the plate-boundary. This zone is narrower $(\sim 40 \mathrm{~km})$ towards southern SI compared to northern $\mathrm{SI}$ and is characterized by a high strength of anisotropy compared to other well-resolved features. Consistent $\phi$ with low-strength of anisotropy that is oblique to the plate-boundary is significant in the eastern offshore and south of the Fiordland subduction zone. This feature could be related to the preserved anisotropic fabrics from the ancient Gondwana-subduction or be representative of the deeper flow in the asthenosphere (APM direction in Fig. 5.8).

- Fig. $\mathbf{5 . 1 1}(\mathbf{e})$ : depths $30 \mathrm{~km}<$ depth $\leq 100 \mathrm{~km}$, the well-resolved features are mainly located in the southern SI. A broad plate-boundary parallel zone seems to correlate with the schist-band in the southern SI (Fig. 1.4). We do not know the depth extent of this schist-band, thus this alignment could represent the straining in the schist-band itself and/or deep extension of the plate-boundary shear zone that fans out from the plate-boundary.

- Fig. 5.11(f): Anisotropic alignment in the two subduction zones is well resolved, indicating the fore-arc mantle wedge in both Hikurangi and Fiordland subduction zones are characterized by the trench-parallel flow or shear at depths $>100 \mathrm{~km}$. The resolution in the central SI is poor, therefore we could not constrain the deep extension of the plate-boundary shear. 


\subsection{Discussion and conclusions}

Local/regional events recorded on the OBS network on offshore SI enabled us to enhance the spatial resolution of anisotropic structure in the AUS-PAC plateboundary zone in SI. Both long and short S-wave paths used in this analysis are likely sensitive to different structures at depth. Schematic 5.12 shows the possible ray paths that could sample different sources of anisotropy in the central SI plateboundary zone. If we assume a broad zone of an asymmetric lithospheric shearzone parallel to the plate-boundary as predicted by Duclos et al. [2005] (possibly extending down to the asthenosphere) (Fig. 5.12), the majority of S-waves of deep or long ray paths are likely to sample the anisotropy of this shear-zone (red ray paths in Fig. 5.12). Thus, they will yield plate-boundary parallel $\phi$ unless anisotropy at shallow depth is strong. Near vertical shear-planes, which are in the vicinity of the plate-boundary, can be tilted to produce dipping anisotropy or can be horizontal away from the plate-boundary. In such cases the strength of splitting may vary. This may also be the case if ray segments are tilted (i.e. not near vertical) in vertically aligned media (Fig. 1.6). In the regions where this shear is not distributed or strong, S-phases of long and/or deep paths will be sensitive to present-day asthenospheric flow-derived or preserved anisotropy from the asthenospheric flow in the deep lithosphere, resulting in APM parallel/subparallel anisotropy. In the southern SI especially in offshore and eastern on-land this could be the case (Fig. 5.9). S-phases from shallow and shorter ray paths mostly reside in the shallow crust and they will be sensitive to crustal anisotropy (blue ray paths in Fig. 5.12). In the central SI, the majority of events occur at shallow crustal depths. Thus, those Sphases can be sensitive to both shallow shear-induced anisotropy and crustal stress or/structure induced anisotropy, depending on the geometry of ray propagation.

We have identified a zone with $S H_{\max }$ parallel $\phi$ in the northern-central/central SI, where we do not have a good depth resolution (section 5.3.2). High-stress conditions 


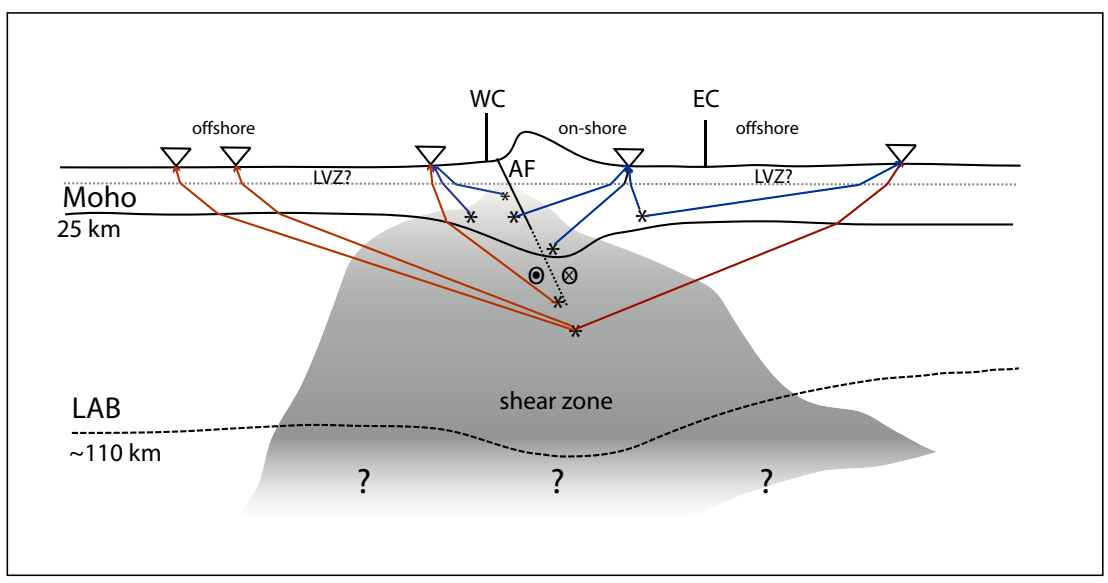

Figure 5.12 Schematic explaining the ray configuration and sensitivity of ray paths to different anisotropic zones. Red-colored ray paths likely sample the plate-boundary parallel shear-zone, whereas blue ray paths may be sensitive to more shallow sources (e.g. crustal anisotropy).

in the shallow depths of the Alpine fault zone may have a strong control over the anisotropy at shallow depths. This effect diminishes in the southern-central and the southern SI, and plate-boundary parallel $\phi$ or APM sub-parallel $\phi$ become dominant (Fig. 5.8). This observation could be partly due to presence of deep ray paths that could sample deep anisotropy. It is possible that there is a plate-boundary induced shear zone beneath the shallow stress-induced anisotropic zone in the central SI. This zone is likely distributed in the west-offshore at lithospheric depths and not extended into east-offshore at lithospheric depths (Fig. 5.12). This type of an asymmetric anisotropic shear is possible due to the oblique strike-slip nature of the plate-boundary. However, it is also possible that this zone is broadly distributed in the deep-lithosphere (deeper than $90 \mathrm{~km}$ ), where we do not have ray-coverage, and extends into the asthenosphere. Nevertheless, SKS splitting suggests that such a shear-induced body is absent in the eastern offshore regions at deeper depths and is mainly governed by the present-day asthenospheric flow (Zietlow et al. [2013] and Fig. 5.4). The northern SI is mainly characterized by a broad zone ( $\sim 200 \mathrm{~km}$ wide $)$ of shear beneath the plate-boundary and the transitional fault zone.

We think that long S-wave paths possibly encounter more than a single layer of anisotropy, or plunging anisotropic layers, when they reach the station. Even if 
the anisotropy is characterized by a horizontal symmetry axis, the S-phases may not sample the full effect of the properties of the anisotropic medium because of the ray geometry (e.g. curved ray paths). At least some parts of the long and shallow ray paths are likely sensitive to transverse isotropy with vertical symmetry axis. In that case, observed variable $\phi$ can be attributed to the effects of both transverse isotropy with vertical symmetry and horizontal symmetry (i.e. azimuthally anisotropy). Depending on how rays propagate in the layered media and the anisotropic symmetry, splitting may reflect an integrated effect at the receiver. Measured low $\delta t$, therefore, probably represents either the anisotropy of the upper most layer (crustal source) or the integrated effect of the variable anisotropy along the ray path. This integrated effect can be addressed by 3D splitting tomography (section 5.3.3).

It is hard to conclude whether this entire region agrees with either localized deformation or the thin-viscous sheet model based only on the observed patterns of $\phi$ and $\delta t$ of the local/regional S-wave splitting. These measurements, however, suggest that at least in the northern SI the shear-zones associated with the plateboundary zone is broad $(\sim 100-150 \mathrm{~km})$, as predicted by the thin-viscous sheet model. In the northern-central SI, this deformation is not well constrained due to lack of depth resolution and shallow stress-induced anisotropy. S-wave splitting from shallow earthquakes $(<40 \mathrm{~km})$ in the central SI suggests that plate-boundary shear is most likely to occur up to $30-90 \mathrm{~km}$ distance east of the plate-boundary within the shallow depths (section 5.3.1). Thus, in the southern SI and the lowercentral SI, shear induced deformation is narrower $(\lesssim 80 \mathrm{~km})$ east of the Alpine fault than the northern SI. In the rest of the southern SI, anisotropy is governed by the asthenospheric flow at deeper levels. 


\section{Chapter 6}

\section{Deep anisotropic structure beneath the Hikurangi subduction zone}

This chapter presents the results from a deep anisotropy investigation in the southern North Island (NI) from SKS, ScS, and teleseismic S (Tel. S) splittings on the SAHKE transect (Fig. 3.6). Previous work around the study area is discussed in section 1.6. Data and techniques that have been used during this analysis are explained in sections 3.1.3 and 3.2.2. SKS splitting was determined from the transverse energy minimization method and ScS/Tel. S splitting was estimated from the eigenvalue minimization method (Fig. 6.1). Splitting parameters are then analyzed for frequency-dependent (section 6.2.1) splitting to extract more information about the anisotropic structure in the region. All the good/fair quality splitting measurements were also re-analyzed with the method by Vecsey et al. [2008] to examine splitting parameters in the ray-coordinate (LQT) system. Finally, we combined and compared our splitting measurements with the previously published data in order to fully understand the lateral anisotropic structure in the southernmost Hikurangi subduction zone.

\subsection{Splitting parameters from SKS, ScS and Tel. S phases}

We assessed 95 well resolved SKS, ScS and Tel. S splitting measurements from 27 events (see Appendix C.2) to understand the mantle anisotropy in the east of the southern NI. Teleseisms with magnitudes $\geq 6$ were used to extract SKS, ScS and teleseismic S arrivals (see table 3.2 for event selection criteria). The events of selected ScS and Tel. S splitting measurements have a minimum source-side 


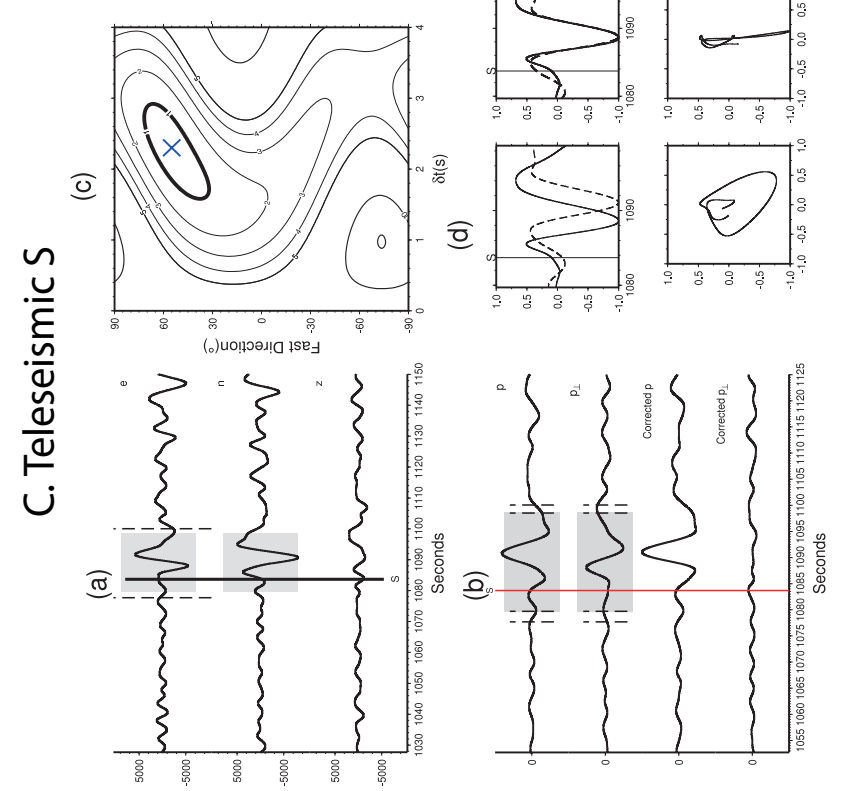

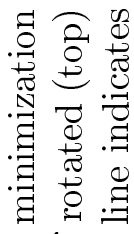

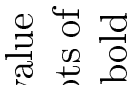
过 $.8 \overrightarrow{0}$ ह

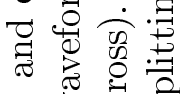
का की ฮิ 2.

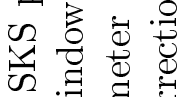
需荡

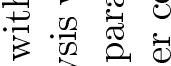

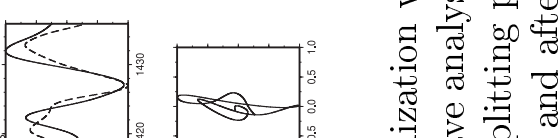
$\begin{array}{lll}0 & 0 \\ \pi & 0 & 0 \\ 3 & 0 & 0 \\ 3 & 0 & 0\end{array}$

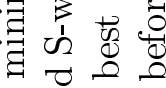

호

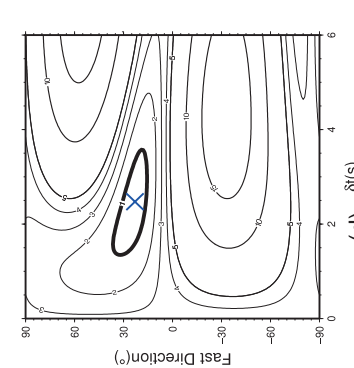

$\frac{n}{\sim}$
$\infty$
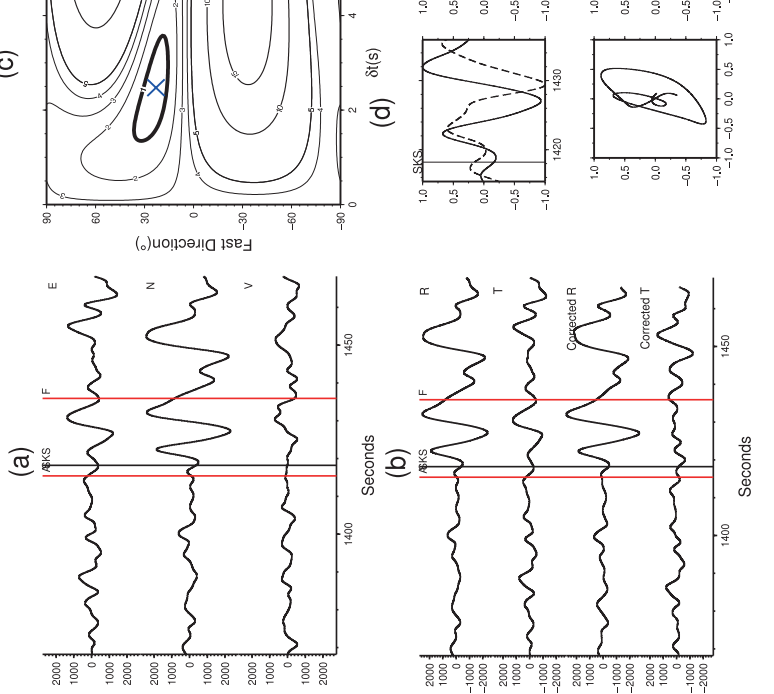

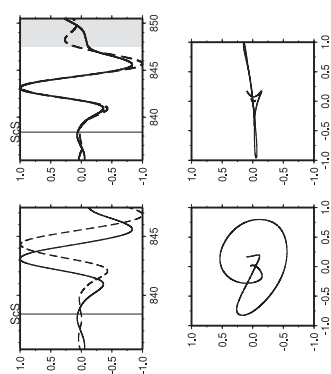

苞焉焉 㲾 घี 를 눙 눙 的 的范。 so पु 3 व ज व व 50 द्व $\circlearrowright$ 要 严 ฮ త so 0 $\stackrel{\infty}{g} \backsim$

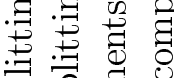
की की क थ

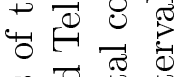

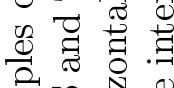
द्व
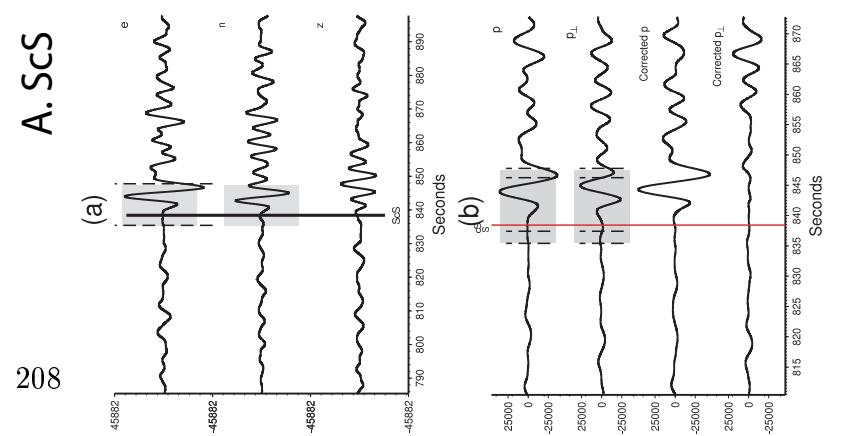
다의 [I 돔 둥

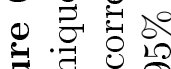

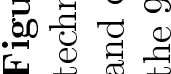




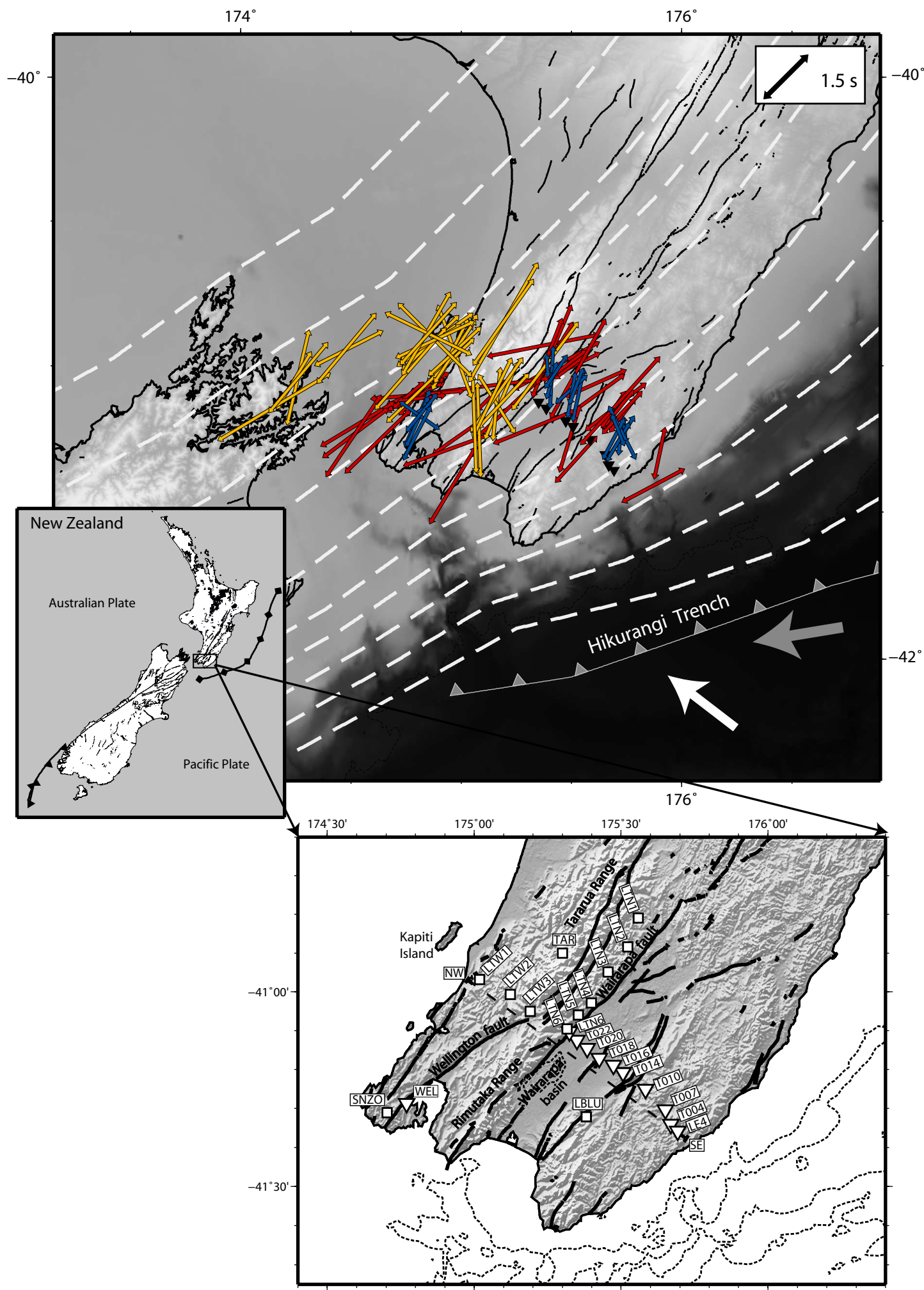

Figure 6.2 SAHKE network, tectonic settings and splitting parameters. The double headed arrows that are plotted at $100 \mathrm{~km}$ ray piercing points denote the $\phi$ from ScS (blue), Tel.S (yellow) and SKS (red) phases and their lengths indicate the $\delta t$. The gray $\left(80.2^{\circ} \mathrm{NE}\right)$ and white $\left(52.8^{\circ} \mathrm{NW}\right)$ arrows indicate the relative (RPM; DeMets et al. [1994b]) and absolute plate motion (APM relative to hotspot reference frame; Gripp and Gordon [2002]) directions. The shallow slab model [Ansell and Bannister, 1996] is denoted by the dashed-white lines. LTW1-3 and LTN1-6 (squares) are the Tararua array [Stuart et al., 1995]. 
depth of $400 \mathrm{~km}$ and $450 \mathrm{~km}$, respectively (table 3.2). The study region is located in the southern most extension of the Hikurangi subduction zone (Fig. 6.2). Splitting measurements are determined from the events recorded on both temporary stations that are located on the NW-SE oriented transect and one permanent station (WEL) in Wellington region (section 3.1.3). Splitting measurements at Wellington station provide a complementary measurement set. Event and measurement information are given in table 6.1 (good and fair measurement) and table C.3 (null measurements). We considered the measurement as null if $12^{\circ} \geq\left|\phi-\phi_{p}\right|$ and $80^{\circ} \leq\left|\left(\phi+90^{\circ}\right)-\phi_{p}\right|$, where, $\phi_{p}$ is initial polarization direction. All SKS and ScS phases have almost vertical incidence $\left(i_{c} \leq 12^{\circ}\right)$, and $i_{c}$ of the Tel. seismic waves range between $16-22^{\circ}$ (Fig. 6.3 and section 6.2.3).

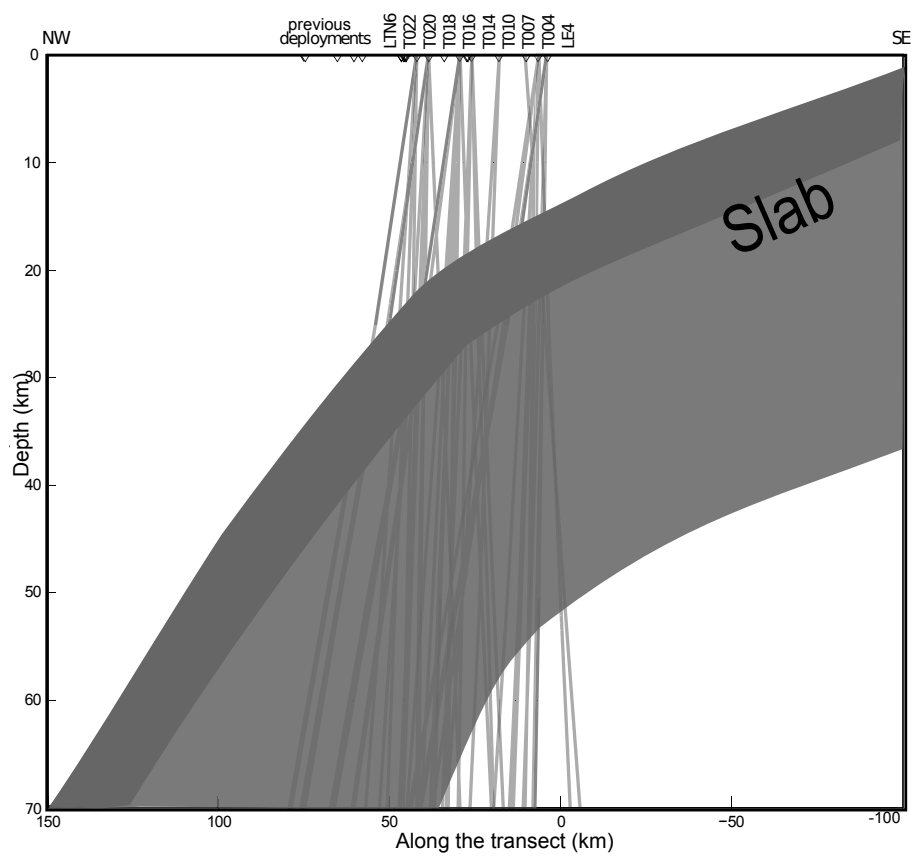

Figure 6.3 2-D cross-section of the slab, station locations, and ray paths along the SE-NW profile in figure 6.2. The stations along the profile are denoted by inverted triangles. Ray paths are obtained from the ray trace [Abt and Fischer, 2008] through the 1-D (AK135) velocity model without encountering the velocity gradient in the slab. Note the near vertical incidence of most of the rays and Tel. S arrivals with slightly higher $i_{c}$ angles. Also, note the vertical exaggeration.

About $93 \%$ of the measurements display NE-SW $\phi$, which is aligned with the strike of the Hikurangi trench, the subducting slab and the faulting in the overriding Australian plate. NE-SW $\phi$ varies between $5-85^{\circ}$ and $\delta t$ values ranges from $\sim 0.5$ 

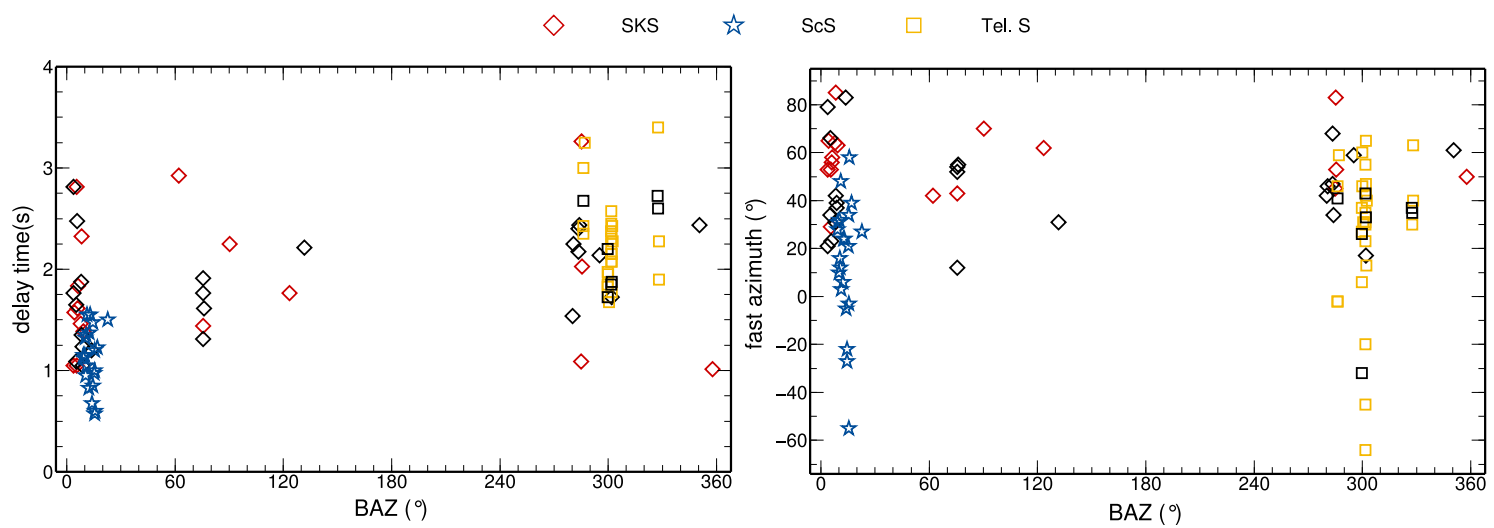

Figure 6.4 $\delta t$ versus BAZ (a) and Fast azimuth $(\phi)$ versus BAZ. Fair quality measurements (non-null, but error $\delta t>0.5 \mathrm{~s}$ and/or error $\phi>20^{\circ}$ ) are marked in black symbols.

- 3.0 s (Figs. 6.2 and 6.4). Compared to SKS and Tel. S, ScS phases yield NNESSW and low $\delta t$ (ranging from 0.5 to $1.5 \mathrm{~s}$ ) at most of the stations. Measurements from Tel. S and ScS are limited to $280-340^{\circ}$ and $10-30^{\circ}$ BAZ, respectively, and sample the fore-arc and/or mantle wedge region (Fig. 6.4). Although most of the $\phi$ are NE-SW aligned parallel to the trench, $\phi$ of a few well resolved measurements from ScS and Tel. S are oriented oblique $\left(-20\right.$ to $\left.60^{\circ}\right)$ to the trench and consistent with the absolute plate motion (APM direction) (Fig. 6.2). It is hard to provide accurate constraints on the backazimuthal variations of splitting parameters from ScS and Tel. S phases as the arrivals are limited to a small range of azimuths. SKS phases, have relatively good coverage, but show no clear BAZ dependency of splitting parameters (Fig. 6.4 and section 6.2.3 ). However, both ScS and some SKS phases from $0^{\circ}-30^{\circ} \mathrm{BAZ}$ tend to show relatively smaller $\delta t$ (ranging from $0.5-1.5$ ) compared to that from other BAZ (Fig. 6.4).

In general, the estimated splitting parameters from the three different phases are in agreement with the previous splitting anisotropy studies in the western side (Taraua array and LBLU) and in Wellington [Marson-Pidgeon and Savage, 1997, MarsonPidgeon et al., 1999, Gledhill and Gubbins, 1996]. However, they show a wide range of values as found by Marson-Pidgeon and Savage [2004b], implying lateral variations in the anisotropic structure in the south of Hikurangi subduction zone. 
Table 6.1 Measurement information of the splitting of SKS ScS and Tel. S phases on SAHKE transect.

\begin{tabular}{|c|c|c|c|c|c|c|c|c|}
\hline \multirow[t]{2}{*}{ station } & event & event & phase & $\delta t(\mathbf{s})$ & error & $\phi^{\circ}$ & $\operatorname{error} \phi^{\circ}$ & $\operatorname{grade}^{\S}$ \\
\hline & lat. ${ }^{\circ}$ & lon. ${ }^{\circ}$ & & & $\delta t(\mathbf{s})$ & & & \\
\hline
\end{tabular}

$\begin{array}{lllllllll}\text { LE4 } & -169.848 & 52.876 & \text { SKS } & 1.050 & 0.544 & 37 & 18.000 & \text { fair } \\ \text { T004 } & -169.848 & 52.876 & \text { SKS } & 1.238 & 0.919 & 39 & 21.500 & \text { fair } \\ \text { T004 } & -97.782 & 16.396 & \text { SKS } & 1.312 & 0.694 & 12 & 15.750 & \text { fair } \\ \text { T004 } & -175.870 & 51.451 & \text { SKS } & 1.087 & 1.003 & 34 & 20.500 & \text { fair } \\ \text { T004 } & 63.951 & 28.777 & \text { SKS } & 1.538 & 0.628 & 42 & 17.250 & \text { fair } \\ \text { T004 } & 96.548 & 33.165 & \text { SKS } & 1.725 & 1.378 & 17 & 23.250 & \text { fair } \\ \text { T007 } & -68.328 & -22.227 & \text { SKS } & 1.762 & 0.450 & 62 & 8.750 & \text { good } \\ \text { T010 } & -175.870 & 51.451 & \text { SKS } & 1.650 & 0.525 & 66 & 12.750 & \text { fair } \\ \text { T010 } & 93.471 & 11.132 & \text { SKS } & 2.175 & 1.322 & 68 & 23.250 & \text { fair } \\ \text { T014 } & -97.782 & 16.396 & \text { SKS } & 1.440 & 0.340 & 43 & 10.500 & \text { good } \\ \text { T014 } & -175.870 & 51.451 & \text { SKS } & 2.812 & 0.309 & 29 & 3.250 & \text { good } \\ \text { T014 } & -109.159 & 24.690 & \text { SKS } & 2.925 & 0.403 & 42 & 4.000 & \text { good } \\ \text { T016 } & -171.836 & 52.050 & \text { SKS } & 1.462 & 0.319 & 64 & 11.000 & \text { good } \\ \text { T016 } & 159.843 & 52.498 & \text { SKS } & 2.438 & 0.591 & 61 & 6.750 & \text { fair } \\ \text { T016 } & -97.782 & 16.396 & \text { SKS } & 1.913 & 0.506 & 52 & 9.000 & \text { fair } \\ \text { T016 } & -175.870 & 51.451 & \text { SKS } & 1.050 & 0.178 & 53 & 11.000 & \text { good } \\ \text { T016 } & -178.649 & 51.423 & \text { SKS } & 1.050 & 0.375 & 53 & 15.750 & \text { good } \\ \text { T016 } & 93.471 & 11.132 & \text { SKS } & 2.400 & 0.900 & 47 & 15.500 & \text { fair } \\ \text { T020 } & -171.836 & 52.050 & \text { SKS } & 1.875 & 0.562 & 31 & 11.500 & \text { fair } \\ \text { T020 } & -175.361 & 51.374 & \text { SKS } & 2.475 & 0.553 & 23 & 5.250 & \text { fair } \\ \text { T020 } & -97.782 & 16.396 & \text { SKS } & 1.762 & 0.628 & 54 & 10.000 & \text { fair } \\ \text { T020 } & -178.649 & 51.423 & \text { SKS } & 1.762 & 0.806 & 21 & 9.750 & \text { fair } \\ \text { T020 } & 92.831 & 13.667 & \text { SKS } & 1.087 & 0.338 & 45 & 12.750 & \text { good } \\ \text { T022 } & -178.649 & 51.423 & \text { SKS } & 2.812 & 0.562 & 79 & 3.750 & \text { fair } \\ \text { T022 } & 92.831 & 13.667 & \text { SKS } & 3.263 & 0.487 & 83 & 3.500 & \text { good } \\ & & & & & & & & \end{array}$

\begin{tabular}{lllllllll}
\hline \hline LE4 & 123.519 & 6.218 & Tel.S & 2.075 & 0.294 & 23 & 16.500 & good \\
LE4 & 122.974 & 4.195 & Tel.S & 1.825 & 0.287 & 6 & 14.500 & good \\
LE4 & 123.039 & 4.077 & Tel.S & 1.725 & 0.319 & -32 & 22.000 & fair \\
LE4 & 123.467 & 6.486 & Tel.S & 1.850 & 0.662 & 43 & 24.250 & fair \\
LE4 & 116.720 & -6.873 & Tel.S & 2.350 & 0.275 & -2 & 11.000 & good \\
T004 & 123.519 & 6.218 & Tel.S & 2.250 & 0.275 & 35 & 15.500 & good \\
T004 & 122.974 & 4.195 & Tel.S & 2.200 & 0.487 & 26 & 24.000 & fair \\
T004 & 116.720 & -6.873 & Tel.S & 2.425 & 0.244 & -2 & 10.000 & good \\
T004 & 139.869 & 26.978 & Tel.S & 2.725 & 0.562 & 37 & 4.750 & fair \\
T016 & 139.154 & 28.360 & Tel.S & 3.400 & 0.431 & 30 & 5.000 & good \\
T016 & 123.519 & 6.218 & Tel.S & 2.150 & 0.119 & 31 & 5.500 & good \\
T016 & 123.247 & 6.528 & Tel.S & 2.450 & 0.156 & -20 & 8.000 & good \\
T016 & 122.974 & 4.195 & Tel.S & 1.850 & 0.269 & 37 & 4.250 & good \\
T016 & 123.039 & 4.077 & Tel.S & 1.975 & 0.069 & 27 & 4.250 & good \\
T016 & 116.720 & -6.873 & Tel.S & 3.000 & 0.263 & 46 & 16.500 & good \\
T016 & 139.869 & 26.978 & Tel.S & 2.600 & 0.569 & 35 & 7.500 & fair \\
T016 & 123.409 & 6.718 & Tel.S & 1.875 & 0.656 & 33 & 18.000 & fair \\
T016 & 123.467 & 6.486 & Tel.S & 1.775 & 0.163 & 47 & 9.250 & good \\
T020 & 123.519 & 6.218 & Tel.S & 2.300 & 0.325 & 55 & 9.750 & good \\
T020 & 123.247 & 6.528 & Tel.S & 2.575 & 0.244 & -45 & 8.000 & good \\
T020 & 122.974 & 4.195 & Tel.S & 1.950 & 0.487 & 46 & 12.000 & good \\
Continued & on next page... & & & & & &
\end{tabular}


Table 6.1 - Continued

\begin{tabular}{|c|c|c|c|c|c|c|c|c|}
\hline \multirow[t]{2}{*}{ station } & event & event & phase & $\delta t(\mathbf{s})$ & error & $\phi^{\circ}$ & $\operatorname{error} \phi^{\circ}$ & $\operatorname{grade}^{\S}$ \\
\hline & lat. ${ }^{\circ}$ & lon. ${ }^{\circ}$ & \multicolumn{6}{|c|}{$\delta t \mathbf{( s )}$} \\
\hline T020 & 123.039 & 4.077 & Tel.S & 1.725 & 0.244 & 60 & 11.000 & good \\
\hline T020 & 116.720 & -6.873 & Tel.S & 2.675 & 0.444 & 41 & 27.500 & fair \\
\hline T020 & 123.467 & 6.486 & Tel.S & 2.150 & 0.225 & 65 & 5.000 & good \\
\hline T022 & 123.519 & 6.218 & Tel.S & 2.375 & 0.431 & 30 & 14.500 & good \\
\hline T022 & 123.247 & 6.528 & Tel.S & 2.375 & 0.175 & -64 & 7.500 & good \\
\hline T022 & 123.467 & 6.486 & Tel.S & 2.075 & 0.188 & 42 & 4.000 & good \\
\hline LE4 & 179.116 & -23.307 & $\mathrm{ScS}$ & 1.325 & 0.044 & 32 & 7.500 & good \\
\hline LE4 & 179.760 & -23.784 & $\mathrm{ScS}$ & 0.825 & 0.188 & 6 & 6.750 & good \\
\hline LE4 & -178.425 & -17.794 & $\mathrm{ScS}$ & 0.675 & 0.094 & -5 & 11.250 & good \\
\hline LE4 & 178.394 & -26.142 & $\mathrm{ScS}$ & 1.125 & 0.031 & 31 & 3.500 & good \\
\hline LE4 & -178.826 & -20.799 & $\mathrm{ScS}$ & 1.475 & 0.219 & -27 & 10.000 & good \\
\hline T004 & 178.394 & -26.142 & $\mathrm{ScS}$ & 1.150 & 0.044 & 28 & 3.000 & good \\
\hline T004 & -178.826 & -20.799 & $\mathrm{ScS}$ & 0.850 & 0.188 & -22 & 15.250 & good \\
\hline T014 & 179.116 & -23.307 & $\mathrm{ScS}$ & 1.350 & 0.056 & 12 & 4.250 & good \\
\hline T016 & 179.116 & -23.307 & $\mathrm{ScS}$ & 1.050 & 0.225 & 48 & 16.000 & good \\
\hline T016 & 178.394 & -26.142 & $\mathrm{ScS}$ & 1.150 & 0.087 & 10 & 7.250 & good \\
\hline T016 & -178.826 & -20.799 & $\mathrm{ScS}$ & 0.975 & 0.113 & 21 & 6.000 & good \\
\hline T020 & 179.116 & -23.307 & $\mathrm{ScS}$ & 1.550 & 0.100 & 3 & 8.000 & good \\
\hline T020 & 178.394 & -26.142 & $\mathrm{ScS}$ & 0.950 & 0.025 & 16 & 3.000 & good \\
\hline T020 & -178.826 & -20.799 & $\mathrm{ScS}$ & 1.000 & 0.106 & 34 & 9.500 & good \\
\hline T022 & -178.826 & -20.799 & $\mathrm{ScS}$ & 0.575 & 0.169 & -3 & 17.000 & good \\
\hline
\end{tabular}

Lateral variations of splitting in the northern Hikurangi subduction zone across the CVR (Fig.1.1) had been investigated by Greve and Savage [2009] via finite difference and analytical modeling, assuming an anisotropic model with a constant average isotropic velocity. The study found a model with a highly anisotropic mantlewedge (15\% S-wave anisotropy), subslab region with $3.5 \%$ anisotropy, and isotropic slab and back-arc region as the best-fit (forward) model that explains the observed measurements. High anisotropy in the mantle wedge is attributed to aligned-melt bands/cracks together with mineral alignment. Because of the shallow subduction below our array in the southern NI and the absence of arc activity, we do not expect to see such strong wedge contribution of anisotropy as in the model proposed for

\footnotetext{
${ }^{\S}$ grade of the non-null measurements. good: error $\delta t \leq 0.5$ s and error $\phi \leq 20^{\circ}$.
} 
the northern NI. However, lateral variations in splitting parameters could be caused by other phenomena (e.g. isotropic velocity variations, the upper-plate structures, properties in the shallow-wedge, and properties within the slab). To investigate whether the scatter in splitting parameters is due to any spatial effect we analyzed splitting parameters along the profile across the subduction zone, combining our splitting measurements with the previous splitting data. The detailed description of the lateral variations is given in section 6.2.2.

\subsection{Synthesis of splitting parameters}

The most recent studies across Tararua array and modeling of splitting parameters at SNZO in the Wellington region suggested laterally varying anisotropy and complex geometry beneath the SNZO station [Marson-Pidgeon and Savage, 2004a,b]. The main findings of these studies was the NE-SW aligned anisotropic domains beneath the Tararua array (NE of SNZO) that possibly extends towards the Wellington region. The SAHKE transect is aligned with the Tararua array, but extends further SE from the SE end of the NW-SE aligned arm of the Tararua array (Fig. 6.2). The NE-SW aligned anisotropic domain that was constrained by Marson-Pidgeon and Savage [2004b] lies in the northwest end of the Tararua array and our splitting data set does not provide any constraints on that zone, but we compare it to see whether that zone is just a localized feature across the Hikurangi subduction zone and how anisotropy in the southwest region varies compared to northeast along the profile (Fig. 6.2).

Since we use three different phases for the anisotropy investigation, it is important to check whether these phases are sensitive to the same anisotropic zones at depth. This allows us to determine lateral variations with more confidence. Marson-Pidgeon and Savage [1997] found heterogeneous anisotropic media as inferred by frequency dependent splitting of SKS, ScS and S phases in the Wellington region SW of the 
SAHKE transect. Therefore, we check for the sensitivity of splitting parameters to the period of the waves before discussing variations along the transect.

\subsubsection{Frequency dependence and evidence for multi-layer anisotropy}

Periods of the ScS, Tel. S, and SKS phases used in this study vary within the ranges of $\sim 3-7 \mathrm{~s}, \sim 8-13 \mathrm{~s}$, and $\sim 9-15 \mathrm{~s}$, respectively. Only the SKS and Tel. S consist of long period energy. Filter bandwidths used to filter SKS and Tel. S range from $0.04-0.1 \mathrm{~Hz}$, whereas ScS phases are band-passed at 0.1 and $0.3 \mathrm{~Hz}$. MarsonPidgeon and Savage [1997] noticed that ScS splitting recorded in Wellington region are independent of the filter. Because $\mathrm{ScS}$ phases have little long period energy we cannot use the same long period filters that we use for SKS/Tel.S phases.

Figure $6.5 \mathrm{~b}-i / i i$ shows the $\delta t$ variations with respect to the period of the S-phases recorded at SAHKE transect stations separately, and figure 6.5a-i/ii shows transect measurements together with the measurements at WEL station (Fig. 6.2). In both cases, $\delta t$ of the good quality $\mathrm{ScS}$ and Tel. S phases show a clear increase in $\delta t$ with periods up to $11 \mathrm{~s}$. $\phi$ show no significant variation with the period (not shown). Frequency dependency is also not sensitive to the BAZ of the phases (Fig. 6.5a/b-ii). Tel.S phases with period $\gtrsim 11 \mathrm{~s}$ and SKS phases show no clear evidence for frequency dependent splitting, suggesting that $\mathrm{ScS}(\mathrm{T} \lesssim 7 \mathrm{~s})$ and $\mathrm{Tel} . \mathrm{S}(\mathrm{T} \lesssim 10 \mathrm{~s})$ phases with relatively short period energy are either sensitive to different length-scales of anisotropy or scattering by large-scale crustal heterogeneities (section 3.5.2; Wu and Aki [1988]). Varying anisotropic length-scales can distribute laterally and/or at depths. Modeling of the multi-layer anisotropy can be used to extract information on depth varying anisotropic layers, but this can be complicated by the lateral variations in the anisotropic structure. Fresnel zone arguments have been used by many studies (e.g. Wirth and Long [2010], Rumpker et al. [2003], Marson-Pidgeon and Savage 
Table 6.2 Fresnel zone (FZ) approximations for the average frequencies of ScS, Tel. S and SKS phases at different depths (DP).

\begin{tabular}{|c|c|c|c|}
\hline phase & $\mathrm{ScS}$ & Tel. S & SKS \\
\hline avg.freq..$^{\dagger}(\mathrm{Hz})$ & 0.2 & 0.1 & 0.07 \\
\hline \multicolumn{2}{|l|}{ depth (km) } & \multicolumn{2}{|c|}{ FZ width $(\mathrm{km})$} \\
\hline 5 & 15 & 25 & 32 \\
\hline 10 & 24 & 36 & 46 \\
\hline 100 & 70 & 100 & 122 \\
\hline
\end{tabular}

[2004b]) to estimate the highest length-scale of anisotropy at a given depth that could be sampled by S-phases with different frequencies to produce frequency dependency. Fresnel zone (FZ) defines the boundary limits of the area at depth (L) from the receiver, where seismic waves are sensitive to subsurface structures. For plane waves this can be approximated from [Gudmundsson, 1996]: $F Z=\sqrt{2 \lambda L+(\lambda)^{2} / 4}$. If the different length-scales of anisotropy are smaller than FZ, then frequency dependent splitting is possible [Eakin and Long, 2013-in revision]. S-waves with short-period energy will be sensitive to smaller area than those with long-period energy, and this provides approximate constraints on the lateral variations in anisotropy. The approximate Fresnel zones widths at different depth for ScS, Tel. S and SKS phases that have been used in this study are given in table 6.2.

ScS phases, which have short period energy, consistently yield NNE-SSW fast orientation with smaller $\delta t$ at all of the SAHKE stations and WEL station compared to SKS and Tel. S. This result is consistent with the previous observations in the surrounding region (section 6.2.2). It is likely that the fore-arc region of the southernmost Hikurangi subduction zone is characterized by a shallow crustal-anisotropic layer perhaps due to fluid-induced cracks or clay mineralization in the crust/subcrust of the overriding Australian plate, and short-period ScS phases are sensitive to that structure. Clear frequency dependent anisotropy from both ScS and Tel. S with periods less than $\sim 10 \mathrm{~s}$ suggests that this crustal anisotropy is most likely 
characterized by different length-scales. According to table 6.2, ScS phases can be sensitive to structure with length scales as high as $24 \mathrm{~km}$ and Tel. S phases can be sensitive to the maximum length of $\sim 36 \mathrm{~km}$ at crustal depths. This suggests that medium is most likely characterized by small-scale heterogeneities with maximum length-scales up to $\sim 36 \mathrm{~km}$. Nevertheless, the lack of frequency dependence from SKS suggests that those phases are mostly sensitive to the large-scale anisotropic structure (length-scale $>100$ s of $\mathrm{km}$ ) that is possibly distributed in the upper-mantle depths and are less effected by the small length-scales of anisotropy.

0.5 - $1.5 \mathrm{~s}$ ScS delays are relatively large to be caused solely by a shallow-crustal anisotropic source. As suggested by Gledhill and Gubbins [1996], 1 - 2 s $\delta t$ measured in the region north of Wellington and west of the SHAKE transect (Fig. 6.2) from Tararua array arises from $\sim 300 \mathrm{~km}$ thick trench-parallel flow-governed anisotropic zone in the sub-slab region. Therefore, some of the ScS phases appear to reflect the typical upper-mantle anisotropy $(\delta t \sim 1-1.5 \mathrm{~s})$ and the smaller delays $(\sim 0.5$ - 1 s) could be due to frequency dependence and/or crustal re-splitting (section 3.5.1). Slight changes in $\phi$ (from NNE-SSW to NNW-SSE) may also be effects of these two scenarios (Fig. 6.7). If this is the case, large $\delta t(>2 \mathrm{~s})$ from long period SKS and Tel. S phases likely reflect an integrated effect of anisotropy along the ray path. However, the range of $\delta t(1.8-3 \mathrm{~s})$ from long period SKS phases implies that not all of the ray paths show the same cumulative effect of anisotropy. This suggests laterally varying or localized anisotropic zone/s perhaps above the subslab region (assuming that the slab itself is also governed by lateral variations in anisotropy). The majority of $\phi$ are oriented NE-SW $\left(10^{\circ}-80^{\circ}\right)$ throughout the area and thus it is possible that S-waves, which sample the anisotropic layers with approximately similar orientation of axis of symmetry, integrate the $\delta t$ along the ray path rather than cancel out as a result of the path integration with competing fabric. The lack of frequency dependent anisotropy at long period phases (period $>11 \mathrm{~s}$ ) could also be an effect of this integration. The splitting sensitivity of smaller 
periods (period $\leq 11 \mathrm{~s}$ ) also suggests depth localization of anisotropy (or multi-layer anisotropy). If this is the case, layers with different anisotropic length-scales can be distributed at depths to produce frequency dependent splitting. The resolved multilayer models only have moderate fits to the observed splitting (section C.4), suggesting lateral inconsistencies within the stratified anisotropic structure or a more complex behavior.
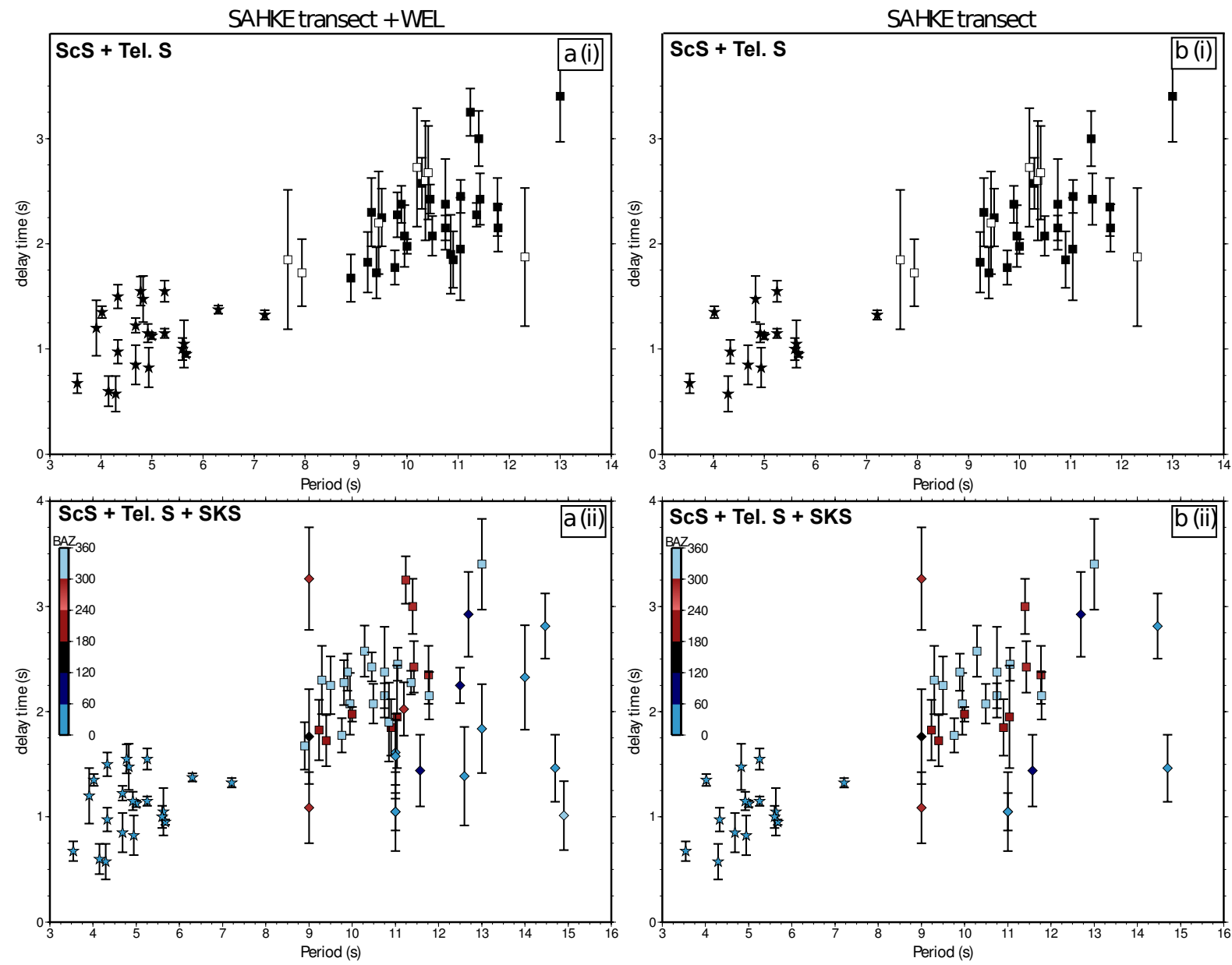

Figure 6.5 $\delta t$ as a function of the period of the different S-phases (stars - ScS, squares - Tel.S, diamonds - SKS). Figure 6.5a(i) and (ii) include the $\delta t$ measurements at WEL station, whereas figure $6.5 \mathrm{~b}(\mathrm{i})$ and (ii) exclude the $\delta t$ measurements at WEL. Figures 6.5 $\mathrm{a}(\mathrm{i})$ and $\mathrm{b}(\mathrm{i})$ only display the $\delta t$ variations with periods of the ScS and Tel.S phases. Good quality measurements are marked in black color and uncolored (open) symbols denote fair measurements. $\delta t$ measurements in figures 6.5 a(ii) and b(ii) are color coded according to BAZ to show that the observed frequency dependent splitting is not a function of BAZ. 


\subsubsection{Lateral variations of splitting along the transect}

The period versus $\delta t$ analysis in section 6.2.1 (Fig. 6.5) and moderately resolved multilayer models (section C.4) imply stratified anisotropic structure with lateral complexities is plausible. The lack of frequency dependence from long period SKS and a wide range of $\delta t(1.8-3 \mathrm{~s})$ even from phases with similar periods also suggest that waves are not only sensitive to the multilayer anisotropy, but also to lateral variations in the anisotropic structure. Therefore, splitting parameters along the transect across the Hikurangi subduction zone were analyzed to detect lateral variations using the measurements made on the SAHKE transect and previous deployments that are within the $30 \mathrm{~km}$ distance either side of NW-SE aligned profile (Fig. 6.2).
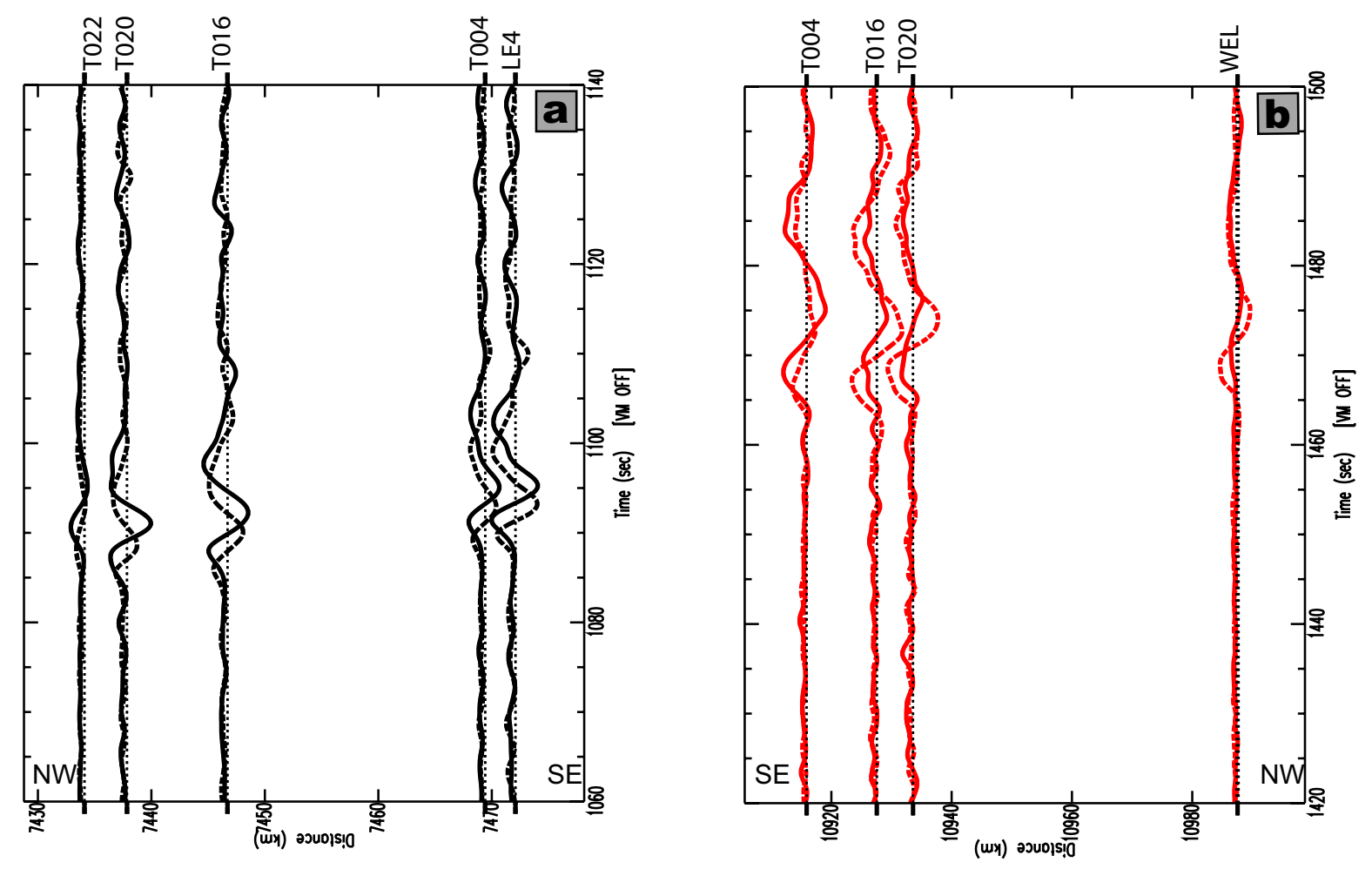

Figure 6.6 Record sections of the filtered (bandpass at $0.04-0.1 \mathrm{~Hz}$ ) Tel. S (a event:10205053) and SKS (b - event:10181072) arrivals at different stations across the Hikurangi subduction zone. Phases are rotated into their fast (dashed-line) and slow polarization azimuths to display $\delta t$ variations. Note that the SKS arrival (red in Fig.b) at T004 displays a smaller lag-time compared to T016 and T014 on the SHAKE transect, however Tel. S arrivals acquire approximately the same high $\delta t$ along the transect. 
In a few cases, the same events recorded at different stations yield good quality splitting measurements. The example record sections of Tel. S and SKS phases that are rotated into their individual fast and slow components are are given in Figure 6.6(a\&b) for comparison. We do not have an SKS-phase from the same event recorded on all transect stations that we could used to compare the $\delta t$ variations along the transect in the record section, however Figure 6.6(b) indicates that the time lag between fast (red dashed-line) and slow (red bold-line) are smaller at T004 than at T016 and T020 (also see section 6.2.2). Tel. S phases do not show any lateral variations along the SAHKE transect (Fig. 6.6(a)).

Map views of the measurements made at each station are displayed in Figure 6.7. Measurements from ScS (blue), SKS (red), and Tel. S (yellow) are shown in the separate maps, and previous measurements (SKS-Gledhill and Gubbins [1996], ScS/Tel.S-Marson-Pidgeon et al. [1999]; Marson-Pidgeon and Savage [2004b], and Greve et al. [2008]) are marked in purple color. Circular histograms in each plot represent the $\phi$ measured at all the stations. ScS phases generally show consistent $\phi$ (NNE-SSW or NE-SW) and small $\delta t$ at all the stations. The ScS delays also agree with the previous measurements at Tararua array stations (Fig. 6.8-A) and SNZO station (Fig. 6.7a), suggesting $\mathrm{ScS}$ phases are sensitive to a coherent anisotropic layer that is spatially distributed throughout the area. But there is a slight change in $\phi$ between stations in the eastern and western side of the Wairarapa fault. Most of the ScS measurements made in the eastern side are more NNE-SSW compared to NE-SW trend in the western stations (Fig. 6.7a), suggesting only wave-polarizations are slightly effected by the complexities in the region (Fig. 6.8-A).

Because of the consistency in $\delta t$ and relatively small delays from ScS phases (Fig. 6.8A), we infer ScS splitting represents either background anisotropy or a coherent anisotropic layer in the lithosphere, and it is only slightly affected by the previously suggested lateral variations. 

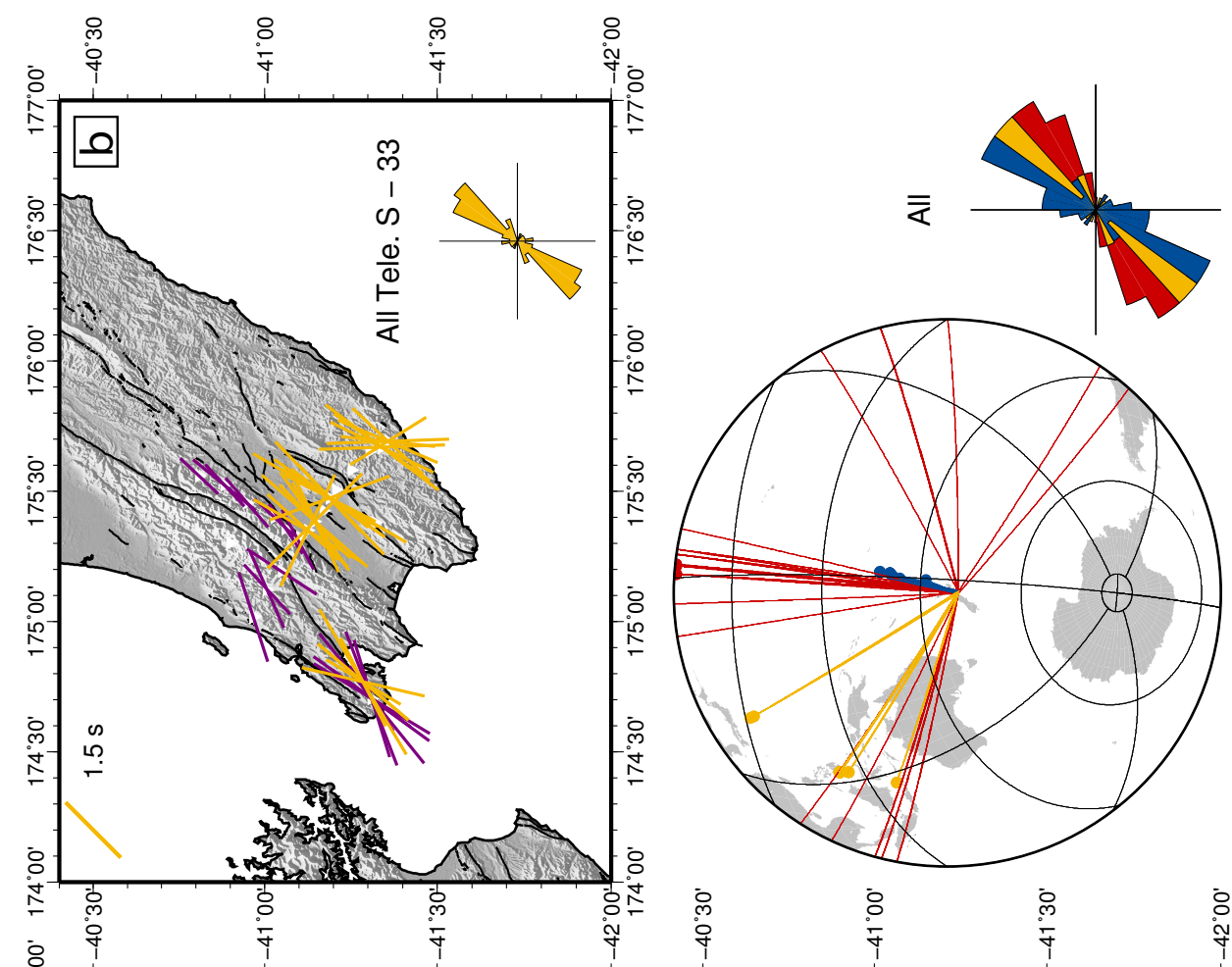

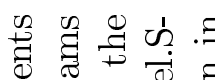

पू

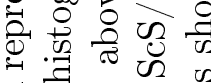

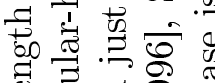

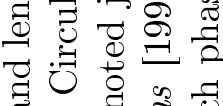

त

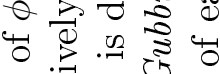

: 1000

屯 छ

$\exists-\frac{1}{0} \approx 0$

需

品

काष

की

政 0

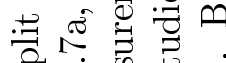

की

శే

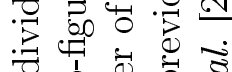

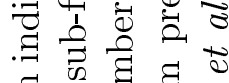

专.

घं

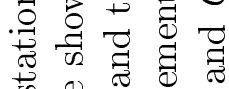
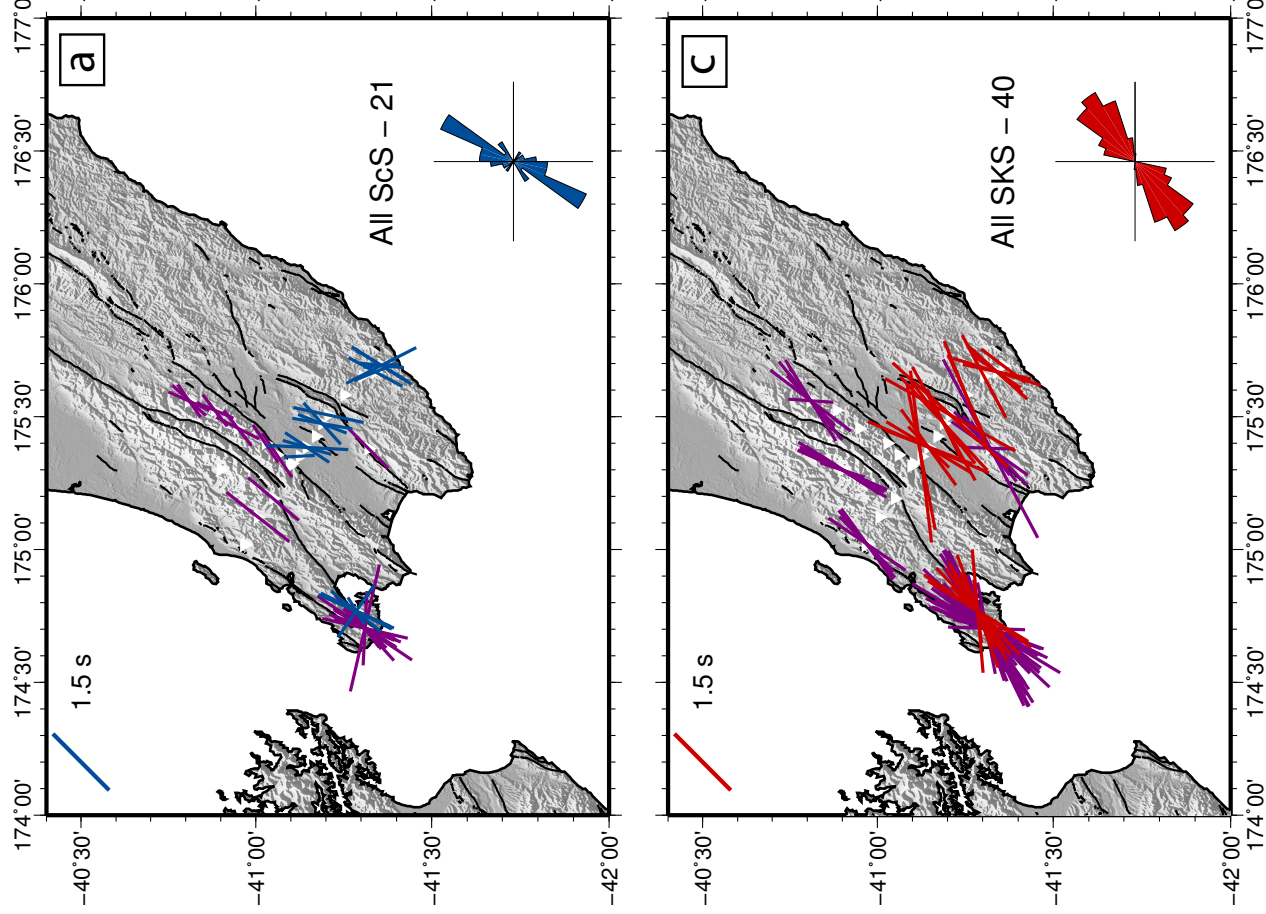

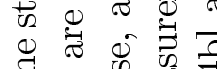

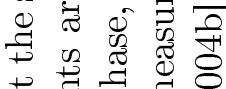

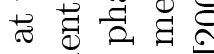

ఫ

을 च

考

ซ

¿

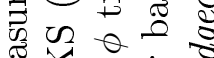

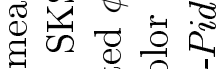

$\infty \vec{\sigma})$

$\exists$ డ.$\exists \circlearrowright$

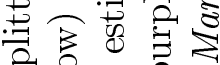

की

卷

廿

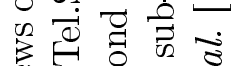

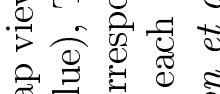

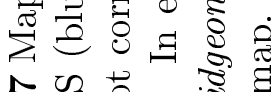

유 范

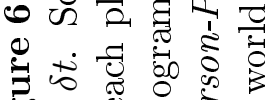

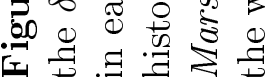


The sensitivity of $\mathrm{ScS}$ phases to a coherent anisotropic layer with the different lengthscale of anisotropy is possible due to smaller periods $(\lesssim 6 \mathrm{~s})$ of the ScS phases compared to SKS and Tel. S phases (Fig. 6.5). However, SKS and Tel. S seem to sample both background anisotropy and spatially varying anisotropic structure producing the range of $\delta t$ values, which include typical upper mantle values mostly to the west $(\sim 1-1.5 \mathrm{~s})$ and unusually high $\delta t(\gtrsim 2 \mathrm{~s}$; Figs. $6.8 \mathrm{~B} / \mathrm{C})$, from both phases in the central-east. Figures $6.8 \mathrm{C}$ and $6.8 \mathrm{~B}$ display the SKS and Tel. S measurements along the NW-SE aligned profile parallel to the SAHKE transect. Measurements from the Tararua array and LBLU stations (stations within $30 \mathrm{~km}$ distance from the transect) (circles in figures 6.8-A,B,C) are also plotted to examine the variations along the line.

Tel.S phases show an abrupt variation in $\delta t$ (about $1.2 \mathrm{~s} \delta t$ change within $\sim 5$ $\mathrm{km}$ distance) between T022 and LTN6, dividing the profile into two anisotropic domains with distinct anisotropic properties (Fig. 6.8B). Note LTN6 and T022 stations are located in and around the Wairarapa fault (Fig. 6.2). Stations west of this anisotropic transition zone have an average Tel. S $\delta t$ of $1.38 \mathrm{~s} \pm 0.091 \mathrm{~s}$, and the stations on the eastern side indicate an average Tel. S $\delta t$ of $2.25 \mathrm{~s} \pm 0.078 \mathrm{~s}$ average Tel.S $\delta t$ (Fig. 6.10). Both SAHKE and previous measurements from Tel.S phases have the same backazimuthal coverage $\left(280-340^{\circ}\right)$, which rules out the effect of the BAZ (Fig. 6.8D) and minimizes the path effects. Marson-Pidgeon and Savage [2004b] modified the SC91 method to perform splitting from ScS / Tel S phases using transverse minimization method by calculating $\phi_{p}$. They showed that the splitting parameters from both the modified method and eigenvalue minimization method give the same results regardless of the filtering. This confirms that the splitting variation along the transect profile is not due to different techniques used. It is also unlikely that the observed discrepancy between the eastern and western side of the Wairarapa fault is a measuring/processing artifact because: the previous SKS measurements at SNZO and LBLU are consistent with the SKS measurements at 

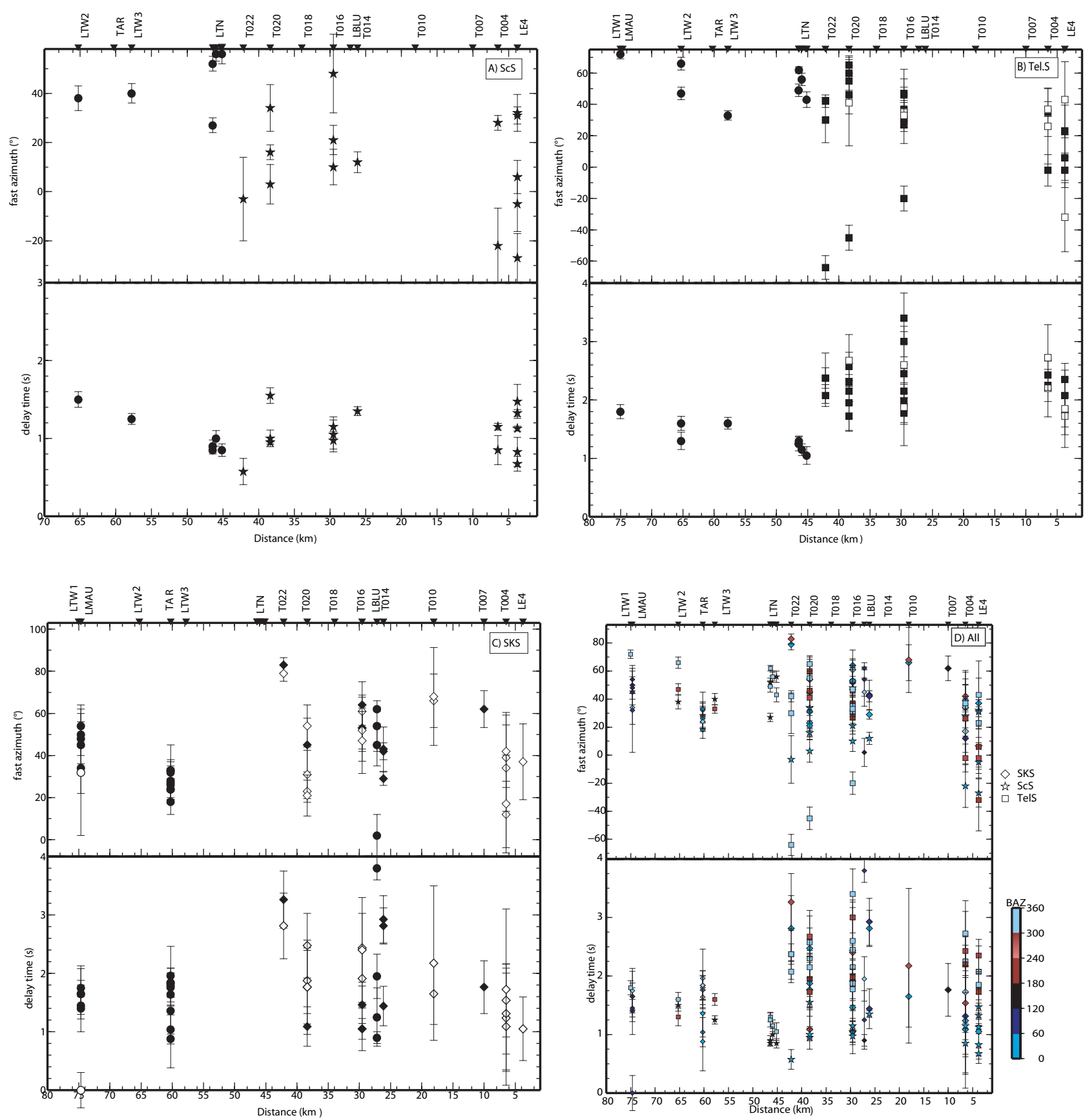

Figure 6.8 Lateral variation in splitting parameters along the transect. Figures 6.8-A,B,C and D display the projected splitting measurement on to the SE-NW oriented profile line (Fig. 6.2) that is approximately parallel to the SAHKE transect and the SE-NW Tararua arm. Stations within $30 \mathrm{~km}$ distance from the profile have been used. Figures $6.8-\mathrm{A}, \mathrm{B}$, and C display the variations from ScS, Tel.S and SKS phases separately. Circles in those plots represent the measurements from previous studies and fair measurements are indicated by the open-symbols. Note an abrupt change in Tel.S delays around LTN stations. Figure 6.8-D shows all the measurements, color coded according to event BAZ. Relatively small symbols represent the previous measurements. 
nearby station WEL and the SAHKE stations on the eastern side (Fig. 6.7-c, Fig. 6.8-C), $\mathrm{ScS} \delta t$ are as same as previous $\delta t$ estimations (Fig. 6.7-a, Fig. 6.8-A), and our splitting estimations in the LQT coordinate system using a different method yield similar splitting parameters (see section 6.2.3). Most of the broadband sensors used for the SAHKE experiments were CMG-40T seismometers with the standard response of $30 \mathrm{~s}-50 \mathrm{~Hz}$ and two sites (LTN6 and T004) had CMG-3ESP sensors with the same standard response (table C.1). Previous Tararua array consisted CMG-3T broadband sensors with the standard response of $120 \mathrm{~s}-50 \mathrm{~Hz}$. Thus, all the seismometers across the region had a flat response over the $1-15 \mathrm{~s}$ band that is strong in the phases used for this analysis (Fig. 6.5). Both previous and SAHKE sensors are broadband. We do not see a significant change in ScS splitting parameters between previous and SAHKE splitting data sets (Fig. 6.8-A). And also, SKS measurements in the southeastern end of SAHKE transect agree well with the previous SKS measurements in northwest (Fig. 6.8-C), implying that there is no significant effect due to two different instruments of the previous and SAHKE experiments. Therefore, instrument responses cannot explain the abrupt variation in $\delta t$, the increase in average station $\delta t$ of SKS splitting (Fig. 6.9), and the scatter in the individual $\delta t$ of SKS and Tel. S phases (Fig. 6.8-C\&B) towards the NW end of the SAHKE transect, suggesting the presence of laterally varying anisotropic structure.

It is hard to provide accurate constraints on such a boundary zone from SKS phases as there are no $\delta t$ measurements available at individual LTN stations and there are a limited number of previous measurements in the northwest profile (Fig. 6.8-D). SKS phases recorded on the SAHKE transect arrive at a range of BAZ (Figs. 6.7 and 6.4) and give a wide range of station delays (1 - 3.4 s) especially towards the NW end of the transect (Fig. 6.8-C), where the Wairarapa basin is located. T022, which is located in the vicinity of the Wairarapa fault, yields anomalously high delays from two measurements $(2.8-3.2 \mathrm{~s})$, but one of those measurements is rated 
as marginal by the error criteria. When those measurements are averaged together with Tel. S measurements, the average $\delta t$ recorded at T022 is about $2.6 \mathrm{~s}$ (Fig. 6.10) and this value is quite high to be caused only by a crustal or upper mantle (above and below the slab) source. We think that such a high value can be a product of coupled anisotropy from mantle-flow, lithospheric shear beneath the fault zone and the fault structure itself. Both Wairarapa fault and Wellington fault, which is located to the west of the Wairarapa fault (Fig. 6.2), are large-scale strike-slip faults in the dextral shear-belt of NI. A reflection study by Henrys et al. [2013] along the same transect line found that the Wairarapa fault structure is more prominent than the Wellington fault. The Wairarapa fault acts as the western boundary limit of the Wairarapa basin. Gravity anomaly detected in Wairarapa region is interpreted as mainly due to density variation between Cenozoic sediments accumulated in the Wairarapa basin and Mesozoic basement rocks [Hicks and Woodward, 1978]. As constrained by these models, the thickness of the sediments overlying the Mesozoic basement reaches a maximum of $\sim 3.2 \mathrm{~km}$ at the western edge of the basin close to Wairarapa fault (i.e., the basin becomes deeper towards the Fault). Well-developed sedimentary beddings within the basin can generate structural anisotropy in the shallow crust. If we assume $1.5 \mathrm{~s} \delta t$ is accumulated within the sub-slab region [Gledhill and Gubbins, 1996] and/or the upper-mantle above the slab, then $\sim 1.1 \mathrm{~s}$ must have come from the anisotropic source that resides in the slab and/or crust. Splitting $\delta t$ in the crust usually varies between $0.1-0.3 \mathrm{~s}$ [Silver, 1996, Karalliyadda and Savage, 2013]. In our case, an additional $\sim 0.8 \mathrm{~s} \delta t$ must, therefore, be produced within the crustal depths or in the slab. Crust beneath a large strike-slip fault like the Wairarapa fault can be characterized by steeply dipping foliations, and such vertical foliations, can produce large delays up to $0.5 \mathrm{~s}$ (Barruol et al. [1997] and Lloyd et al. [2009]; section 2.4.1). 


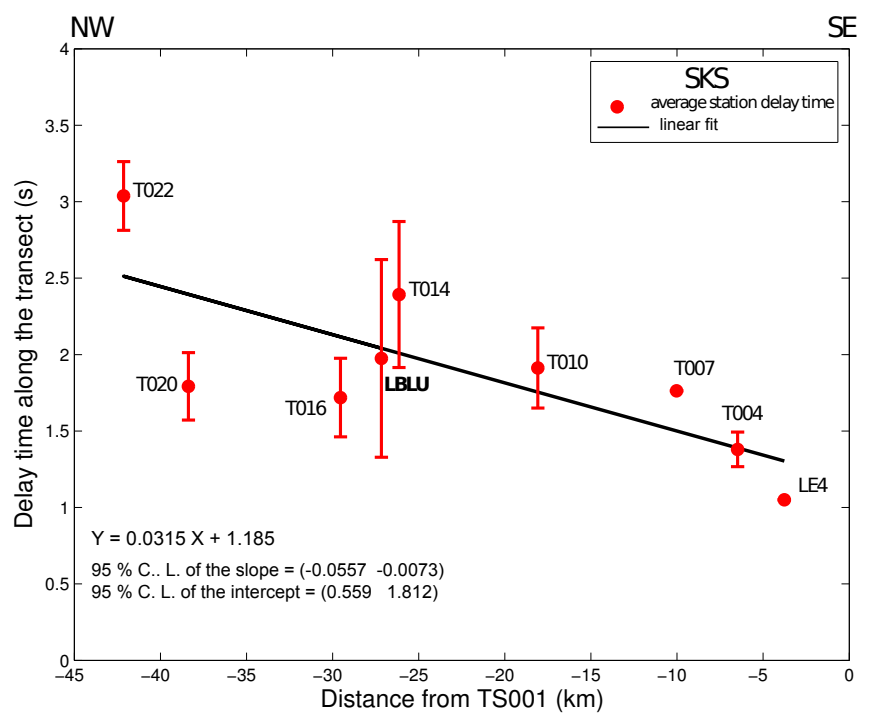

Figure 6.9 SKS $\delta t$ variation along the SAHKE transect. The average station $\delta t$ are plotted as red circles at the station position in the SE-NW profile (Fig. 6.2). The error bars denote the standard error of average $\delta t$ at each station. Measurement at LBLU indicates the averages $\delta t$ of previous study. The black line denotes the linear-fit to the data. Note the slight increase (statistically significant) in $\delta t$ from SE to NW.

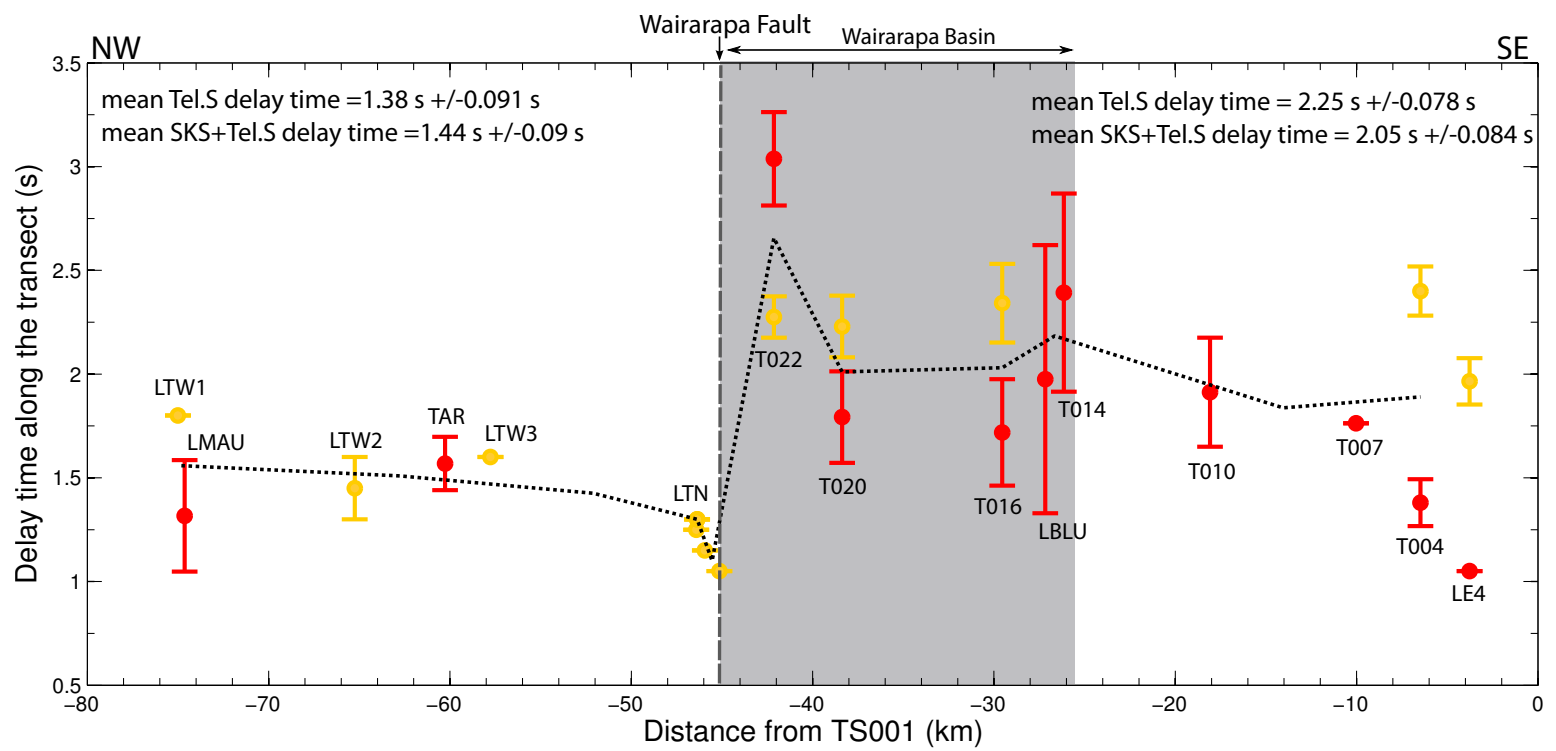

Figure 6.10 $\delta t$ variation along the profile from Tel.S and SKS phases. SKS and Tel. $\mathrm{S}$ station averages are marked in red and yellow circles. The black-dotted line indicates the binned ( $\sim 3.6 \mathrm{~km}$ wide) averages of the individual $\delta t$ along the profile. The error bars denote the standard error of average $\delta t$ at each station. The mean $\delta t$ of SKS and SKS+Tel. $\mathrm{S}$ in the western and eastern sides of the Wairarapa fault are marked on the plot. The gray shaded area and the gray-dashed line indicate the Wairarapa basin and the fault, respectively. 
If the anisotropy contribution from the slab is maximum $0.3 \mathrm{~s}$ and the orientation of the axis of symmetry in each layer is approximately similar, then the average $\delta t$ of $\sim 2.6 \mathrm{~s}$ at T022 (Fig 6.10) can be well explained by the different possible anisotropic contributions at depths in the region, and can be approximated with the model of vertical coherent deformation [Silver, 1996]. It is also possible that the adjacent Wairarapa basin is characterize by highly foliated structures, sedimentary beddings and/or in-filled materials with different anisotropic properties (e.g. clay minerals) that could produce high crustal contribution of anisotropy. As revealed by Kaviani et al. [2011], the basin structure itself and other small scale heterogeneities within the basin can create laterally varying structure (or isotropic velocity) that could affect the split S-waves differently, producing effective anisotropy. To produce effective anisotropy, length-scale of heterogeneities should be less than the dominant $\mathrm{S}$ wavelength. Therefore, the observed $\delta t$ variation could be due to a laterally varying structure (or isotropic velocity) across the study region. Unlike Tel.S, SKS phases do not show a striking $\delta t$ difference between the west and east side of the transition zone, as inferred by Tel.S splitting. There are not enough SKS splitting measurements available at LTN6 and LTW3 stations to provide robust constraints on such a variation. The slight increase in station averages of SKS $\delta t$ (Fig. 6.9) and individual measurements from certain BAZ (Fig. 6.8-D) suggest some SKS delays are affected by both backazimuthal variations and the complexities beneath the station.

Figure 6.10 summarizes the average $\delta t$ measurements at each station along the profile from SKS (red) and Tel. S phases (orange). The black-dotted line indicates the binned ( $\sim 3.6 \mathrm{~km}$ wide) averages of the individual $\delta t$ along the profile. There is a sharp change in $\delta t$ around the NE-SW aligned Wairarapa fault. From SW to $\mathrm{NE}$ along the profile line this change is visible as a sharp increment in $\delta t(\sim 1.2 \mathrm{~s}$ increment within $\sim 3 \mathrm{~km}$ distance) and the average $\delta t$ appears to remain $\sim 0.6 \mathrm{~s}$ larger east $(2.05 \mathrm{~s} \pm 0.084 \mathrm{~s})$ of the fault than to the west $(1.44 \mathrm{~s} \pm 0.09 \mathrm{~s})$. A similar 
type of $\delta t$ variation has been detected by [Rumpker et al., 2003], across the Avara fault (transform fault) in the Dead Sea region from long-period (5-7 s) SKS phases. They noticed enlarged delays of $0.3-0.5 \mathrm{~s}$ within $\sim 30 \mathrm{~km}$ distance of the fault. Rumpker et al. [2003] used the finite-difference approach by Rumpker and Ryberg [2000] to model the 2D anisotropic structure along the profile, inverting the observed wave field and optimizing the fit of observed and synthetic splitting parameters. The best fitting model suggests the variation is assumed to be caused by sub-horizontal mantle flow in $\mathrm{a} \sim 20 \mathrm{~km}$ localized area around the deep extension of the fault. Rumpker and Ryberg [2000] constrained the Fresnel zones (wave-sensitivity range) of teleseismic-waves with various periods at different depths using finite-difference modeling. According to Rumpker and Ryberg [2000], waves with 10 s period would have a wave-sensitivity range of $\sim 100 \mathrm{~km}$ at $50 \mathrm{~km}$ and $\sim 175 \mathrm{~km}$ at $400 \mathrm{~km}$ depth [see Rumpker and Ryberg, 2000, Fig.4]. In our case, the $\delta t$ variation that is constrained from Tel. S waves $(T \simeq 10 \mathrm{~s}$ ) appears to be more abrupt. A change of $1.2 \mathrm{~s}$ in $\delta t$ occurs within a distance as short as $\sim 5 \mathrm{~km}$ (compare Tel.S $\delta t$ at T022 and LTN in Fig. 6.10), suggesting a very shallow source of anisotropy and/or small-scale lateral variation in the anisotropic parameters [Favier and Chevrot, 2003].

As pointed out by Kaviani et al. [2011], short-scale variation in $\delta t$ can also be due to spatially varying isotropic velocity and, thus, providing an interpretation based only on variation in anisotropic structure may be misleading. There is evidence for such velocity variations (e.g. high velocity lid on the subducting slab) as found by Gubbins and Snieder [1991] in the Wellington region. Therefore, future work on this study needs to be focused on the finite-difference modeling of the observed $\delta t$ variations considering both anisotropic structure and isotropic velocity variations in order to provide accurate constraints on the shallow structure in the fore-arc region of the southernmost Hikurangi subduction zone. Also, to obtain robust constraints on these variations, previous data sets need to be re-evaluated as those splitting measurements were obtained from a different set of instruments using a small number 
of events.

If this $\delta t$ variation is solely due to lateral variation in anisotropic structure, it is most likely caused by the shallow (upper most crust) anisotropic structure that lies east of the Wairarapa fault and it may extend further east towards the trench as the observed $\delta t$ in the east is relatively higher than the western side. Because of the slight increase in delays from SE to NW along the SAHKE line and high delays close to the fault (Fig. 6.9), it is also possible that most of the anisotropy is localized towards the NW end of the profile where the Wairarapa basin and fault are located. As detected by the stacked reflections from active-source first-arrivals recorded on the SAHKE I/II transect [Henrys et al., 2013], the Wairarapa fault has been interpreted as a listric strike-slip (strike of $N 45^{\circ}$ ) fault with small dips $\left(15^{\circ}\right)$ at deeper levels $(\sim 15 \mathrm{~km})$, but is exposed at the surface (east of the Tararua range) as a vertically dipping fault $\left(\operatorname{dip}>60^{\circ}\right)$. Henrys et al. [2013] also noticed several splay faults that are branching out from the deeper part of the Wairarapa fault beneath the Tararua range and are exposed in the Wairarapa basin area and around the east coast. The $10 \mathrm{~km}$ shot point separation around the Wairarapa fault, however, did not allow them to characterize detailed structure in the very shallow crust $(\leq 5$ $\mathrm{km})$. The Wairarapa basin itself also contains several active dextral-faults that are aligned with the trend of the Wairarapa fault [Beanland, 1995].

The observed abrupt $\delta t$ variation across the forearc Hikurangi subduction zone (Fig. 6.10) also correlates well with the terrane boundary between Pahau and Rakaia terranes, suggesting different deformation histories and/or anisotropic properties associated with the two terranes. This boundary is characterized by Esk Head Mèlange that resulted from intense deformation of accretionary sediments [Wandres and Bradshaw, 2005] and coincides with the dextral Wairarapa fault. Both Pahau and Rakaia terranes consist of Torlesse quartzofelspathic greywacke, but Pahau terrane is a younger Torlesse-greywacke terrane than Rakaia terrane. Pahau terrane mostly consists of Rakaia sediments recycled during the Cenozoic [Wandres and 
Bradshaw, 2005]. As explained above, this region is in general characterized by dextral faulting (e.g. Wellington, Wairarapa, Dry river faults) and fault controlled basin structures and uplifted mountain ranges (e.g. Rimutaka and Tarua) (Fig. 6.2). The eastern boundary of the Rimutaka-Tararua ranges is marked by the Wairarapa fault and the ranges are cross-cut by Wellington fault to the west of Wairarapa fault (Fig. 6.2).

Considering all of these facts, it is plausible that the region east of the Wairarapa fault and the basin is governed by shallow structural anisotropy due to faulting and/or uplifted sedimentary bedding. Large scale vertically dipping strike-slip faults like Wairarapa may accommodate intensely developed foliations at crustal depths. Therefore, relatively large $\delta t$ in the east of the Wairarapa region can be attributed to structural anisotropy due to intense foliations around the fault zones [Bastow et al., 2007], sedimentary bedding and to the fault zones themselves. The scatter in $\delta t$ in and around the Wairarapa basin may reflect the structural complexities and/or isotropic velocity variations of the material within the basin. However, there could also be other minor effects from deeper contributions such as the nature of the upper-plate and the accretionary sediments above the slab [Eberhart-Phillips et al., 2005], changing slab dip, and bending related faulting structures within the slab. Eberhart-Phillips et al. [2005] noticed a sharp change in $\mathrm{Vp} / \mathrm{Vs}$ ratios around the Wairarapa fault, as well as high Vp/Vs and low Qp at 0-20 km depths in the eastern side of the fault [see Eberhart-Phillips et al., 2005, Fig. 7d] that they interpreted as over-pressurized subducting sediments. High $\mathrm{Vp} / \mathrm{Vs}$ ratios may also reflect an intense clay (e.g. talc, chlorite) mineralization, and such clay rich zones can be highly anisotropic (see section 2.7.2). We think that a shallow crustal source with high anisotropy is responsible for the observed rapid change in split delay times across this region. If it's due to clay minerals or sedimentary bedding within the Wairarapa basin, the thickness of this anisotropic zone could be approximately equal the thickness of the sediment infill $(\sim 3.2 \mathrm{~km})$ of the basin. 


\subsubsection{Possibility of dipping anisotropic layers?}

Splitting parameters have also been estimated in the ray coordinate (LQT) system (section 3.5.3) in order to compare the measurements on the horizontal plane and the inclined plane [Babuska and Plomerova, 1993, Sileny and Plomerova, 1996, Vecsey et al., 2008]. If the geometry of the anisotropic layers are inclined and the ray paths have large incident angles, such a comparison would result in significant variation between two sets of splitting parameters. Modeling of splitting parameters at the SNZO station [Marson-Pidgeon and Savage, 2004a] in the Wellington region suggested the observed splitting fits better with a model with horizontal axis of symmetry that consists of two or more layers of anisotropy. Our comparison of splitting measurements in the LQT with the geographic coordinate sytem (RTZ) showed no significant variation in splitting parameters from the two methods (Fig. 6.11). The plunge of fast direction of all most all of the measurements are greater than $80^{\circ}$. This implies a nearly horizontal axis of symmetry (Fig. 6.12). 

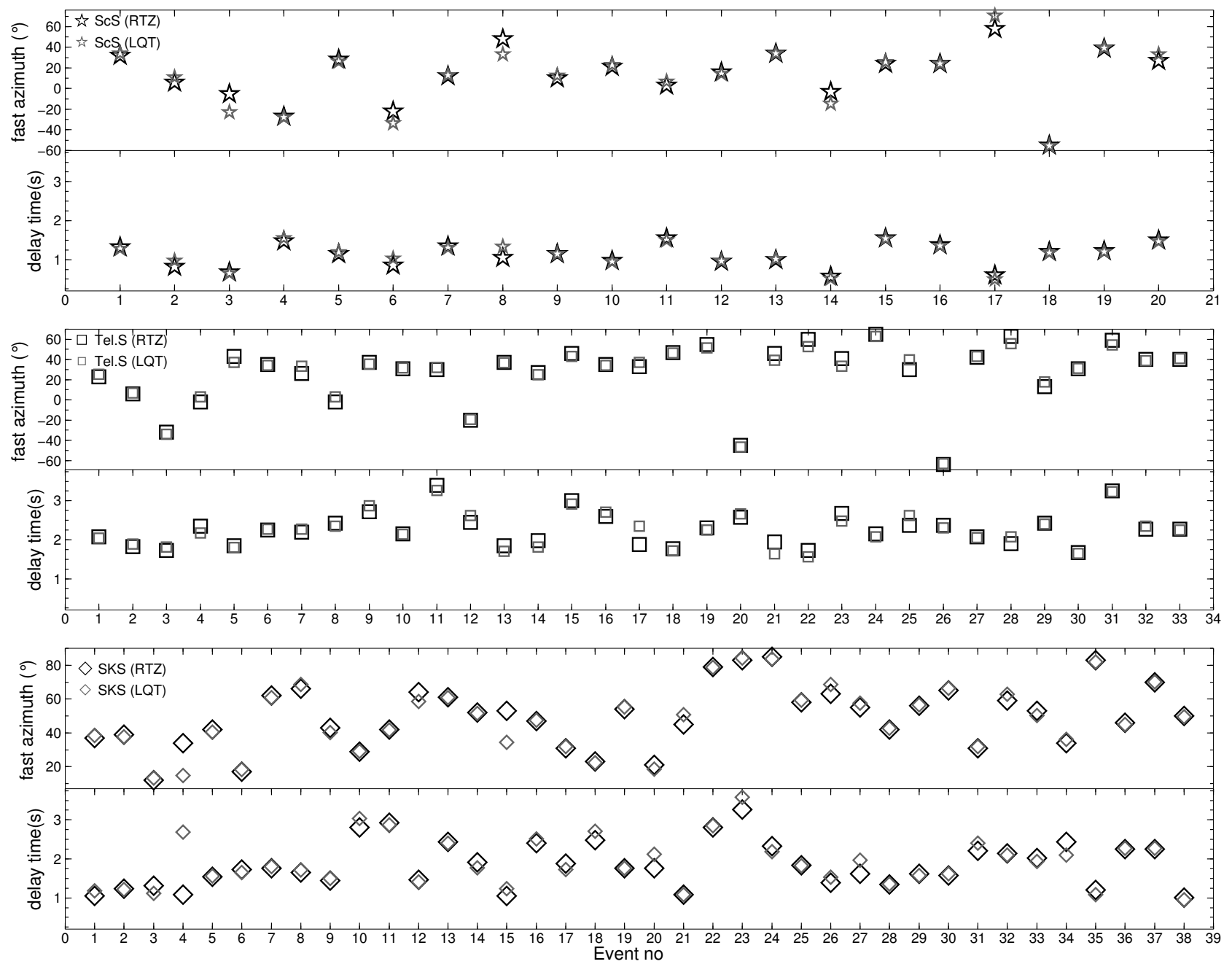

Figure 6.11 Comparison of splitting measurements from ScS (top), Tel.S (middle) and SKS (bottom) phases in RTZ (black) and LQT (gray) coordinate systems. The splitting parameters of ScS and Tel.S phases have been estimated from the eigenvalue minimization method, whereas SKS splitting parameters were determined from transverse minimization method. 


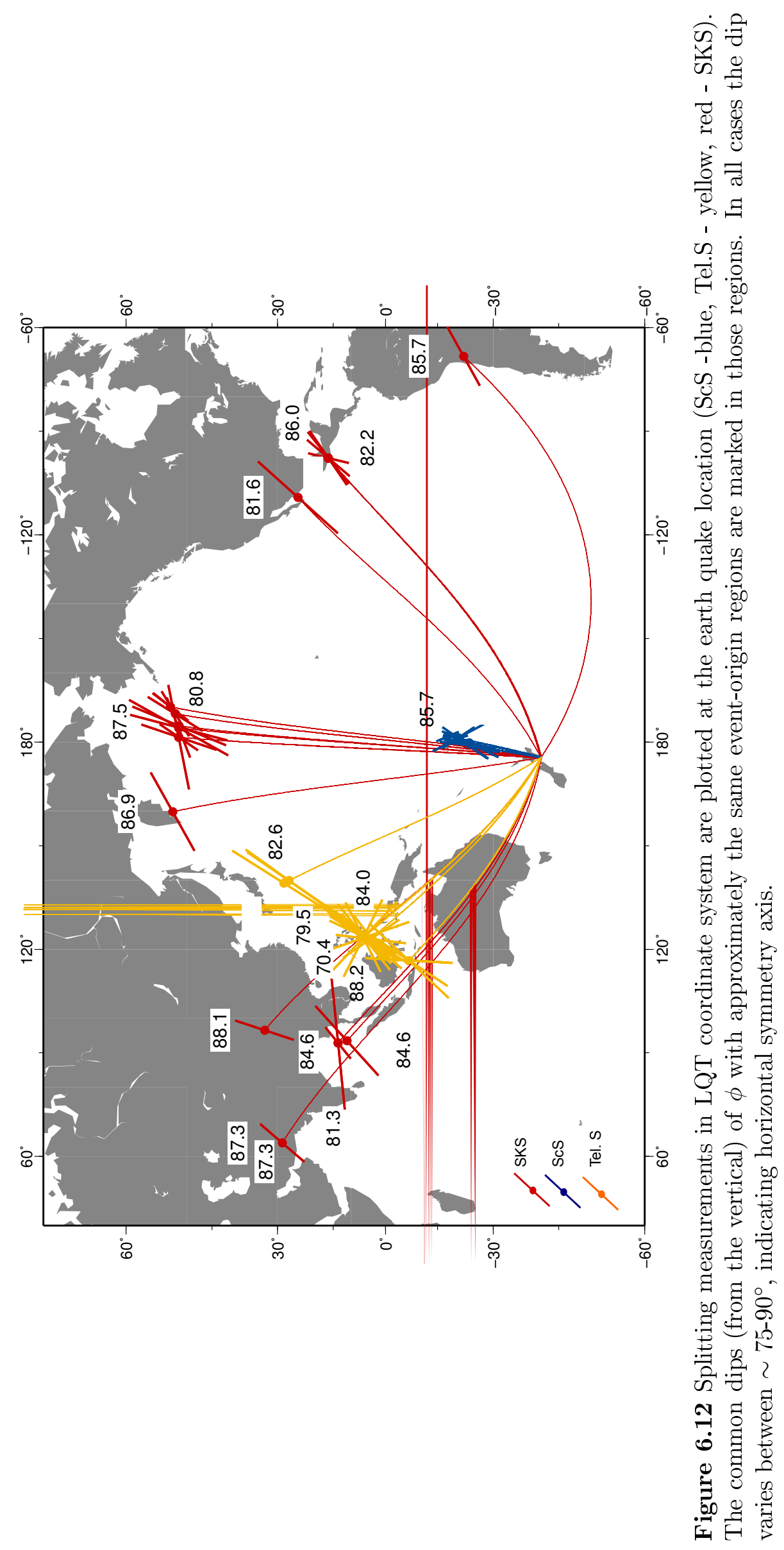


Song and Kawakatsu [2012] attempted to explain the splitting parameters observed in the Hikurangi subduction zone using slab-coupled asthenospheric flow model. This model determines the possible splitting variations in the presence of dipping anisotropy, presumably oceanic asthenosphere with orthorombic symmetry. The predictions based on such a model can be more accurate and clear in the shallow subduction zones with low dipping angles $\left(\leq 15^{\circ}\right)$. The model that they proposed for NZ (Fig. 6.13b/c) should accurately reproduce the observed splitting in at least the shallow subduction in the northern NI, if such dipping layers exist. We compare our splitting parameters plotted with respect to BAZ and incident angle (Fig. 6.13d) to check whether such a model can be used to explain the $\delta t$ variations and the trench-orthogonal splitting.

Neither $\phi$ nor $\delta t$ agrees with the model predictions, especially from the events that arrive at $30-210^{\circ}$ BAZ. Previous measurements [Marson-Pidgeon et al., 1999, Greve and Savage, 2009] from the same BAZ range also do not fully correlate with the model predictions. $\phi$ measured from $210-30^{\circ}$ appear to fit with the predictions, but those directions are also in agreement with the directions that one would expect from the fore-arc and/or sub-slab trench parallel flow models. The few trench oblique $\phi$ in our splitting data set are unlikely an effect of dipping anisotropy as the observations strongly disagree with the model predictions in those particular regions (Fig. 6.13b). However, to test this proposed model precisely [Song and Kawakatsu, 2012], we need more measurements from $90-210^{\circ} \mathrm{BAZs}$ and with higher incidence angles $(\sim 20$ $\left.-35^{\circ}\right)$ 
a

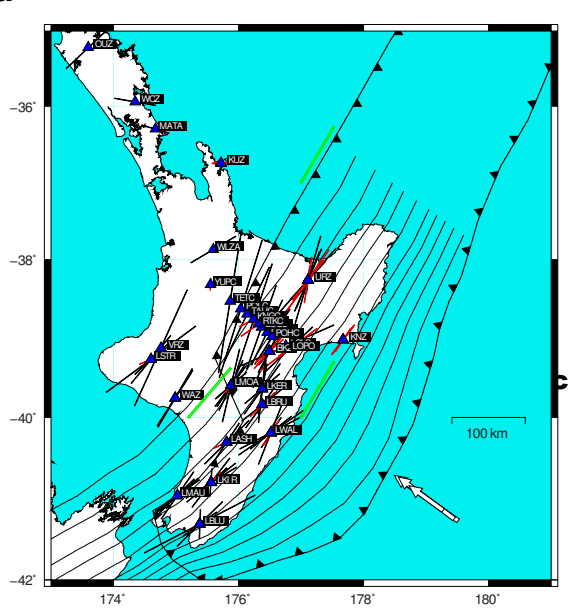

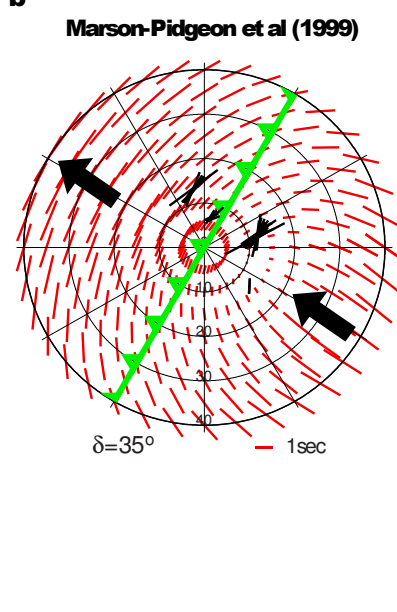

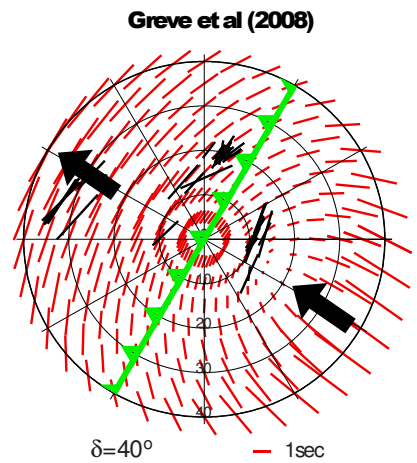

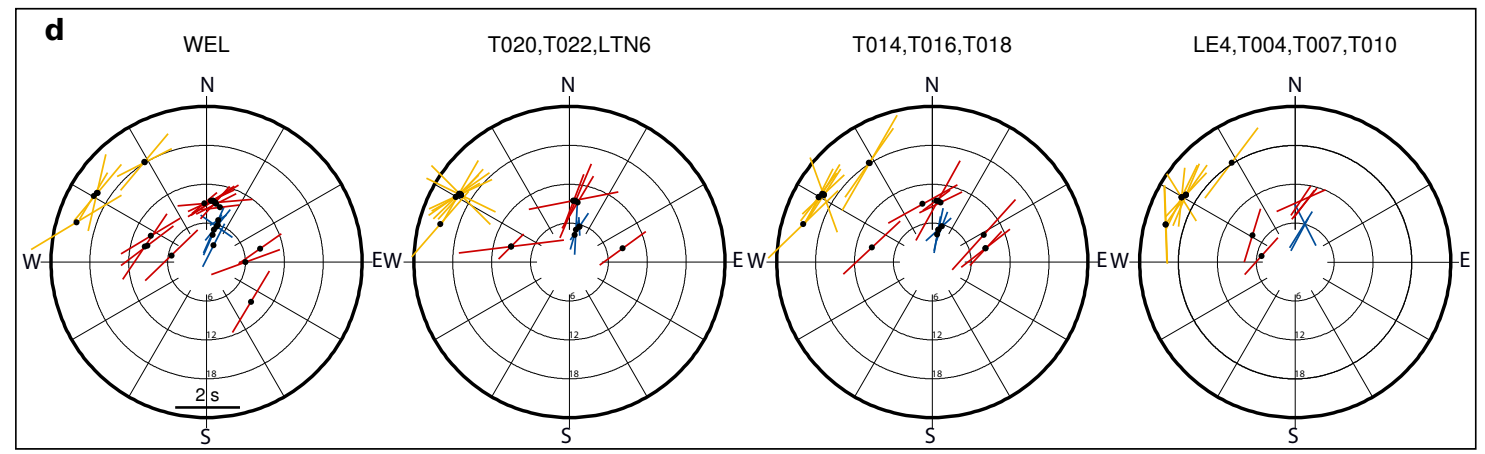

Figure 6.13 Predicted splitting parameters (red bars in b\&c) from the slab-coupled asthenospheric flow model [Song and Kawakatsu, 2012] and observed splitting (black bars in b\&c) from Greve and Savage [2009] and Marson-Pidgeon et al. [1999]. Black arrow indicates the APM direction. (d) measurements from this study are grouped as the station position along the profile. WEL station data is shown separately. Variation in splitting parameters from SKS (red), Tel.S (yellow) and ScS (blue) phases with respect to BAZ and incidence angle $\left(\mathrm{i}_{c}\right)$ are plotted in the equidistant circular diagram. BAZ represents the radial position and $i_{c}$ represents radius segments of the circular diagram. Note: $\delta t$ measurements in $\mathbf{d}$ and $\mathbf{b} \& \mathbf{c}$ are scaled differently. 


\subsection{Conclusions}

Shear-wave splitting anisotropy across the Hikurangi subduction zone in the southern NI from SKS, ScS and Tel.S reveal short-scale lateral variations in the anisotropic structure. The general trend of splitting anisotropy, however, agrees well with the previous studies around this area, implying trench parallel flow in the south of the Hikurangi subduction zone. A wide range of splitting $\delta t$ and small scale inconsistencies in $\phi$ are evidence for spatially varying anisotropic structure across the fore-arc subduction settings. An increase in average SKS $\delta t$ along the SE-NW profile and scatter in delays from both SKS and Tel.S phases towards the SW end of the SAHKE profile in the Wairarapa fault and basin area suggest an additional shallow anisotropy source(s) other than sub-slab mantle flow. The proposed model for the northern NI, which consists of highly anisotropic mantle wedge due to presence of melt-bands [Greve and Savage, 2009], cannot be adequately used to explain the observed lateral variations of the Hikurangi subduction zone in the southern NI. The $\delta t$ values of $2 \mathrm{~s}-3 \mathrm{~s}$ from Tel. $\mathrm{S}$ and SKS phases just east of the CVR in the northern NI (Fig. 1.1) [Greve et al., 2008] is consistent with the $\delta t$ variations in the NW end of the SAHKE transect in the southeast of the NI.

We think that an enhanced anisotropic zone in crustal and/or sub-crustal depths is responsible for the observations in the southern NI. This zone is likely characterized by a crustal origin and associated with intense foliations around the Wairarapa fault, sedimentary layers with in the Wairarapa basin and/or clay mineralization beneath the fault zones and basin area (Fig. 6.14). To produce large delays (2 s), these different sources of anisotropy must be coupled and deform in a coherent manner. The range of delays $(\sim 1.0 \mathrm{~s}-3 \mathrm{~s})$ also suggests the anisotropic body itself may contain heterogeneous anisotropy with different length scales, so that frequency dependent splitting is plausible. Multilayer modeling of the anisotropy suggests that these anisotropic heterogeneities can also be segregated into multiple layers, and 
anisotropy in the individual-layer could be enhanced by anisotropy associated with the fault zones in that region. As constrained by Tel.S splitting, an abrupt variation in $\delta t$ around the Wairarapa fault is presumably caused by such enhanced crustal and sub-crustal anisotropy around the fault, coupling with the multilayer anisotropy (section C.4). This $\delta t$ change may also reflect the deferent deformation histories associated with the two different terranes either side of the Fault. Whether this structure is actually characterized by the higher strength of anisotropy compared to surrounding material or isotropic velocity variation due to laterally varying structure is yet to be investigated via finite-difference modeling.

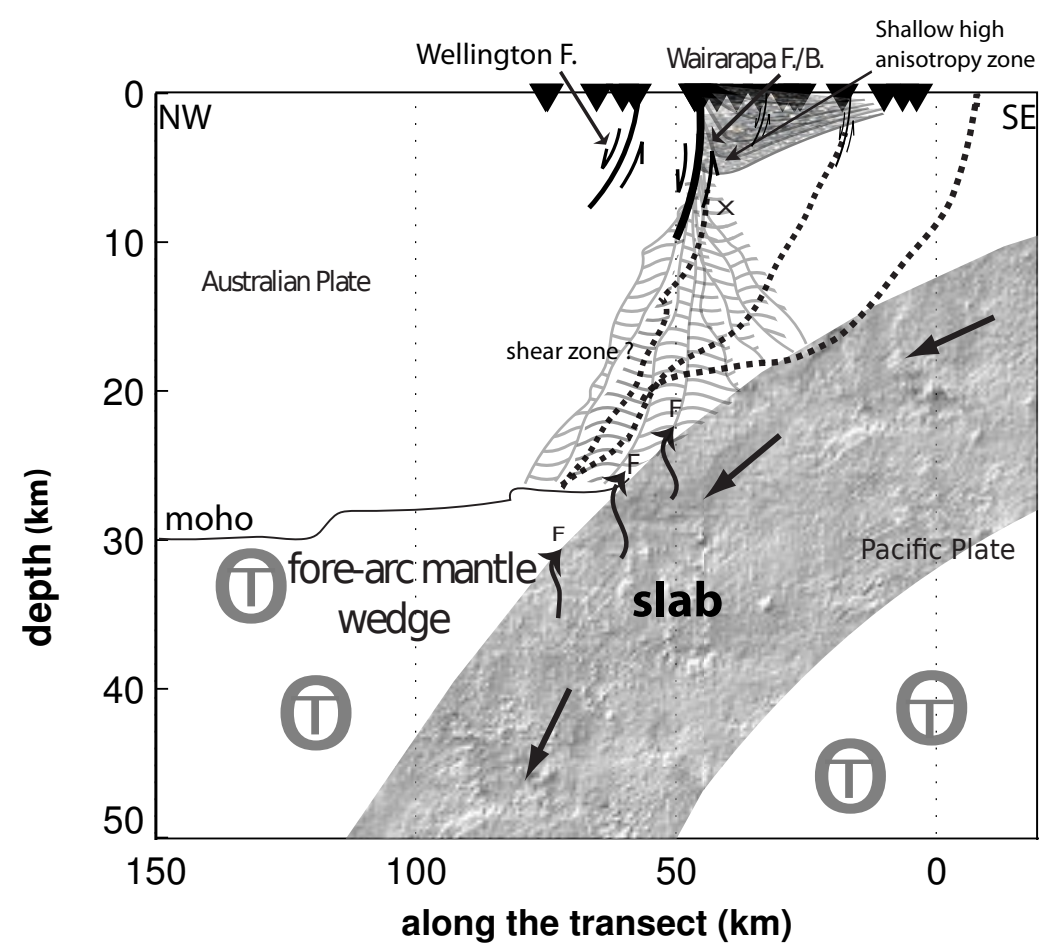

Figure 6.14 Schematic diagram showing the possible anisotropic structure in the region. Here, the crustal faulting and high anisotropy that may be associated with faulting (e.g. shear zone), clay mineralization and/or layered sediment deposits just above the slab are coupled with the sub-slab mantle flow (circled T) to produce high anisotropy in the eastern side of the fault. The Wairarapa fault geometry is obtained from Henrys et al. [2013]. Note the vertical exaggeration.

Our study provides no direct evidence for plunging anisotropic structures, and supports the previous anisotropy modeling prediction of axis with horizontal symmetry anisotropy. The slab-coupled asthenospheric flow model [Song and Kawakatsu, 2012] is not entirely explained by our measurements in the southern 
NI. Thus, the few well-constrained measurements that yield trench oblique (nearly trench orthogonal) directions, which are roughly parallel to APM, are unlikely a result of such a plunging structure. Departure from strong trench parallel splitting may reflect either source-side complexities, or far-field anisotropy that aligns with the APM direction (Fig. 6.2).

Splitting measurements on the SAHKE transect along with previous results suggest trench parallel flow in the sub-slab and perhaps in the fore-arc mantle wedge. NESW aligned upper-plate faulting, that is possibly associated with the transpressional deformation in the oblique subduction appears to be coupled with the trench parallel flow in the sub-slab mantle (Fig. 6.14). The NE-SW aligned Wairarapa fault and basin act as a main anisotropic boundary in the region. The fault itself and its splay faults that are distributed in the east of the fault may have a significant effect on the observed high $\delta t$ by enhancing shallow structural anisotropy and/or shear zones beneath the faulting region. 


\section{Chapter 7}

\section{Concluding remarks}

\subsection{South Island on-land and offshore experiment}

This study provides an extensive analysis of local/regional S-wave splitting parameters in the upper lithosphere of the transpressional plate-boundary zone in SI and its near offshore regions. The analysis used splitting parameters that were determined from a new seismic data set recorded on an enhanced seismograph network that consisted of both land and offshore seismographs. Estimated splitting measurements and the analysis provide a first-order constraint on the spatially and depth-varying anisotropic structure in to lithospheric depths of the SI.

Despite the complex nature of the local/regional splitting of high-frequency Sphases, grading criteria that have been applied to individual measurements, spatial averaging and delay time tomography allowed us to determine regions with spatial consistencies. These spatially consistent regions can be attributed to coherent sources of anisotropy. However, the limited depth-resolution and inhomogeneous distribution of seismicity and ray paths greatly influence the final solution of the anisotropic patterns. Thus the consistent patterns from spatial averages appear to correlate with the possible depth contribution of anisotropy in the region and the extent of those sources. To minimize such limitations, we have used different event selection criteria and additional studies to obtain information on the anisotropic structure.

Both on-land and offshore splitting measurements suggest that the anisotropy at shallow depths of the plate-boundary zone is not entirely controlled by the shearinduced anisotropy that is associated with the plate-boundary fault at depth, but it is 
also governed by structure and stress-induced anisotropy. These different anisotropic sources appear to dominate in tectonically distinct regimes throughout SI, depending on the regional tectonics and depth distribution of seismicity (Fig. 4.11 and Fig. 5.8). In the northern SI, shear-wave splitting anisotropy is primarily controlled by shear-induced fabrics that are aligned parallel/sub-parallel to the plate-boundary and transitional faults, suggesting a broad zone $(\sim 100-150 \mathrm{~km})$ of lithospheric shear at depths as predicted by the thin viscous sheet model [Molnar et al., 1999] and previous studies [Audoine et al., 2000, Balfour et al., 2005]. Because of the limited depth extent of earthquakes, the anisotropic structure resolved at some stations in the central SI was limited to maximum depths of $\sim 70 \mathrm{~km}$. Based on the spatially averaged $\phi$, which are parallel to $S H_{\max }$, we suggest that the upper-crustal anisotropy in the southern part of the northern SI and part of the central SI is mainly controlled by stress-induced anisotropy. This zone also extends into near offshore regions of the upper-west central SI. However, $\phi$ from deep Swaves (deeper than crustal depths) associated with Fiordland subduction suggest that the southern-central region is most likely a high-strain zone that is parallel to the plate-boundary (Fig. 4.11). This high strain zone has been interpreted as a lithospheric-shear zone that represents the deep extension of the plate-boundary fault zone [Karalliyadda and Savage, 2013]. If such a shear-zone exists, it should extend northwards, connecting the pervasive-shear zone in the northern SI and, therefore, likely underlies the shallow stress-induced anisotropy in the central SI.

From the spatially averaged $\phi$ at depths above $100 \mathrm{~km}$, we suggest that this shearinduced zone could extend to a maximum $\sim 130 \mathrm{~km}$ east of the plate-boundary at depths above $100 \mathrm{~km}$ in the central SI and north of the southern SI (4.4.4). As inferred from the seismic anisotropy at shallow depths $(<40 \mathrm{~km})$ in schist rock of the central SI, this shear-zone can be as narrow as $\sim 30 \mathrm{~km}$ at crustal depths, suggesting that the width of this shear zone is most likely fanning out from the plate-boundary at depth. Because of the lack of deep seismicity in the central SI, our results do 
not provide constraints on the depth extent of this shear zone. However, an average station $\delta t$ of $\sim 0.1-0.4 \mathrm{~s}$ (maximum $\sim 0.7$ individual delays) compared to $1.7 \mathrm{~s}$ SKS $\delta t$ from previous studies in SI implies that this shear zone may penetrate down to the asthenosphere.

Varying delays and/or relatively low delays may result from frequency-dependent splitting due to variable length-scale anisotropy, variable anisotropic orientations (complex structure) with respect to S-wave propagation, or re-splitting of S-phases due to multiple-layers anisotropy and/or upper-crustal heterogeneities. We have provided evidence to support some of these proposed ideas, suggesting a complex and heterogeneous anisotropic structure in the region (section 4.4 .2 and C.5). In addition to the above scenarios, we also speculate that the change in deformation kinematics to diffusion creep at upper lithospheric depths especially in the regions far from the plate-boundary, where absolute plate motion (APM) governed anisotropy dominates, is another possible explanation for the observed low delays and absence of plate-boundary shear throughout the SI. Dislocation creep typically activates in regimes with high stress/strain, high temperature conditions and large grain sizes. However, the activation of diffusion creep could result in little or no anisotropy. Stress conditions at plate boundaries are usually high $(\geq 10 \mathrm{MPa})$, but if low temperature, low strain conditions prevail and/or the grain size of the medium is small, diffusion creep becomes more favorable [Poirier, 1990, Kameyama et al., 1999].

Beyond the shear and stress induced anisotropic domains at shallow depths of the central South Island, splitting appears to correlate with the structural imprints from the tectonic episodes prior to Cenozoic deformation. Deep upper-lithospheric anisotropy in the southern South Island (both offshore and onshore) is consistent with the APM direction (NW-SE) away from the Fiordland subduction zone, but regions close to the subduction zone show a slight rotation in $\phi$ with N-S alignment, suggesting anisotropy is likely controlled by the combined effects of trench-parallel 
flow/shear and flow in the asthenosphere (Figs. 5.9 and 5.12). In the eastern-part of the SI, splitting reflects asthenospheric flow derived anisotropy (either present-day flow or preserved).

The spatial analysis of OBS splitting measurements along with land measurements suggests that there is a broad zone of pervasive shear in the plate-boundary fault at least in the central-north SI and northern SI. Because of the limited deep earthquakes in the central South Island and possible frequency dependent and/or multilayer effects, the depth extent of this shear zone is not well constrained by both land and inland splitting measurements. Nevertheless, plate boundary parallel $\phi$ at some stations far way $(\sim 200 \mathrm{~km})$ from the plate-boundary suggest, steeply curved ray paths from deep events $(50-90 \mathrm{~km})$ are likely sensitive to a shear zone in the upper lithosphere (above $\sim 90 \mathrm{~km}$ ). This implies that deformation distribution around the plate-boundary zone in South Island agrees with the deformation distribution predicted by the thin viscous sheet model (Fig. 1.2-i). However, detailed ray tracing needs to be done in order to fully discriminate between the thin viscous sheet and localized deformation models (Fig. 1.2) proposed for South Island.

Future splitting-anisotropy studies in this region need to consider the affects of dipping anisotropic layers on the observed anisotropy. Comprehensive modeling approaches can be used to demonstrate how ray geometry and the complex orientations of anisotropic structures can influence the in-situ splitting anisotropy. Our data do not provide constraints on the splitting anisotropy deeper than $\sim$ $70 \mathrm{~km}$ in the on-land central SI, due to limitations in incidence angles and Swaves with long ray paths. However, a small-scale study can be used to determine the deeper structure in the central SI only using deep S-waves $(>100 \mathrm{~km})$ from the two subduction systems unless those phases are sensitive to the uppermost anisotropic structure. If those S-phases consist of both long period and highfrequency energy, such a study will also be able to test the proposed ideas of frequency dependent splitting or re-splitting of S-phases in the layered media. The 
model-solution from the preliminary 3-D inversion study (section 5.3.3) can be improved by using different starting models, applying smoothing, and changing damping parameters. To understand the overall anisotropic structure of SI, this model can also be extended, including upper-most crack-induced anisotropy and S-wave splitting from core and regional S-phases.

\subsection{Southern North Island experiment}

We have explored the deep anisotropic structure in the south-east of the Hikurangi subduction zone in NI using different S-phases that sample the receiver-side anisotropy. The measurements have been integrated with the previous splitting parameters in the same region to examine the lateral variation in anisotropy across the inland fore-arc region of the Hikurangi subduction.

The results suggest that the general trend of $\phi$ are consistent with trench-parallel flow in the upper-mantle and in agreement with the previous measurements in the surrounding regions. The range of $\delta t(\sim 0.5-3.0 \mathrm{~s})$ and slightly varying $\phi$ across the south of NI suggest a laterally varying anisotropic structure. As inferred from the splitting variations across the profile, the upper-plate Wairarapa fault and basin area appear to be characterized by a distinct anisotropic structure that is possibly localized at crustal depths. Abrupt change in delays around this fault zone divides the region in to two distinct domains of eastern and western sides and the average $\delta t$ in eastern side is $\sim 0.6 \mathrm{~s}$ higher than that measured in the western side of the Wairarapa fault. This sharp $\delta t$ change at the Wairarapa fault also coincides well with the terrane boundary between Pahau and Rakaia terranes, suggesting contrasting deformational or structural fabrics in two terranes. Stress and strain around the Wairarapa fault can be distributed asymmetrically owing to contrasting rock fabrics either sides of the fault. Thick sediment infill at crustal depths $(\leq 3.2 \mathrm{~km})$ in the western end of the Wairarapa basin in Pahau terrane is the most likely to produce 
contrasting anisotropic (or deformational) fabric compared to Mesozoic Greywake basement terrane in Rakaia terrane.

These lateral variations and observed frequency dependence of splitting suggest that the shear wave splitting in the southern NI is governed in part by the laterally varying crustal contribution of anisotropy or velocity variations within the shallow crust. This shallow anisotropy together with the deep anisotropy may reflect multiple layers of anisotropy with small variations in $\phi$, but with different length-scales. We find no clear evidence for dipping anisotropic layers within the subduction structure. Deep anisotropic layers may be characterized by lithospheric shear beneath faulting and mantle flow parallel to the trench. The observed large splitting $(\delta t>2 \mathrm{~s})$ and similar $\phi$ imply crust-mantle coupling of anisotropy in the region.

The observed lateral variation needs to be tested in the future using modeling approaches to understand the configuration of the observed anisotropic structure. The 2-D finite-difference method [Kaviani et al., 2011, Rumpker and Ryberg, 2000], which can model both variations in anisotropic parameters and isotropic velocity variations in the crust and mantle due to heterogeneous structure, appears to be a good way to obtain quantitative and accurate information (e.g. location, size, strength etc.) on the structure that is responsible for this change. With careful inspection of the data and the correction for instruments responses, teleseismic Sphases recorded on the 3-component short period array/transect across the south of NI can also be used to obtain a denser splitting data set along the transect. Such a dense splitting data set will provide robust constraints on the observed changes and simplify the finite difference modeling. 


\section{Appendix A}

\section{On-land investigation}

\section{A.1 Station information}

On-land experiments retrieved data from both the GeoNet permanent network (26 stations) and four temporary deployments (table A.1). All the seismographs used were broadband sensors. Temporary deployments consisted of IRIS-PASSCAL instruments, and they were deployed by VUW in collaboration with the University of Colorado. Raw data was processed with the PASSCAL routine, and event were extracted from the SAC-records using the GeoNet catalog from 2009-2010. Antelope software was used to archive data and this was sent to the IRIS data center.

Problem: Polarity change in the N-component (horizontal) of the DCZ station in the GeoNet network was detected through SKS splitting analysis by Zietlow et al. [2013]. Events from this station were reanalyzed to account for this error. We noticed that there was $\sim 40^{\circ}$ clockwise rotation in the general trend of the uncorrected $\phi$. The comparison between before and after correction of the measurements is given in figure A.1.

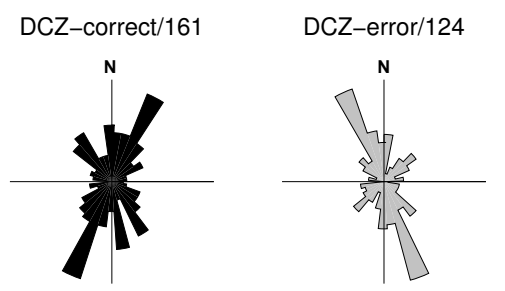

Figure A.1 The comparison of $\phi$ change before (error) and after (correct) correction for the polarity flip at DCZ. Note that the general trend of NNW is rotated into NNE $\left(\sim 40^{\circ}\right.$ clockwise from the NNW direction) after correcting for the polarity flip. 


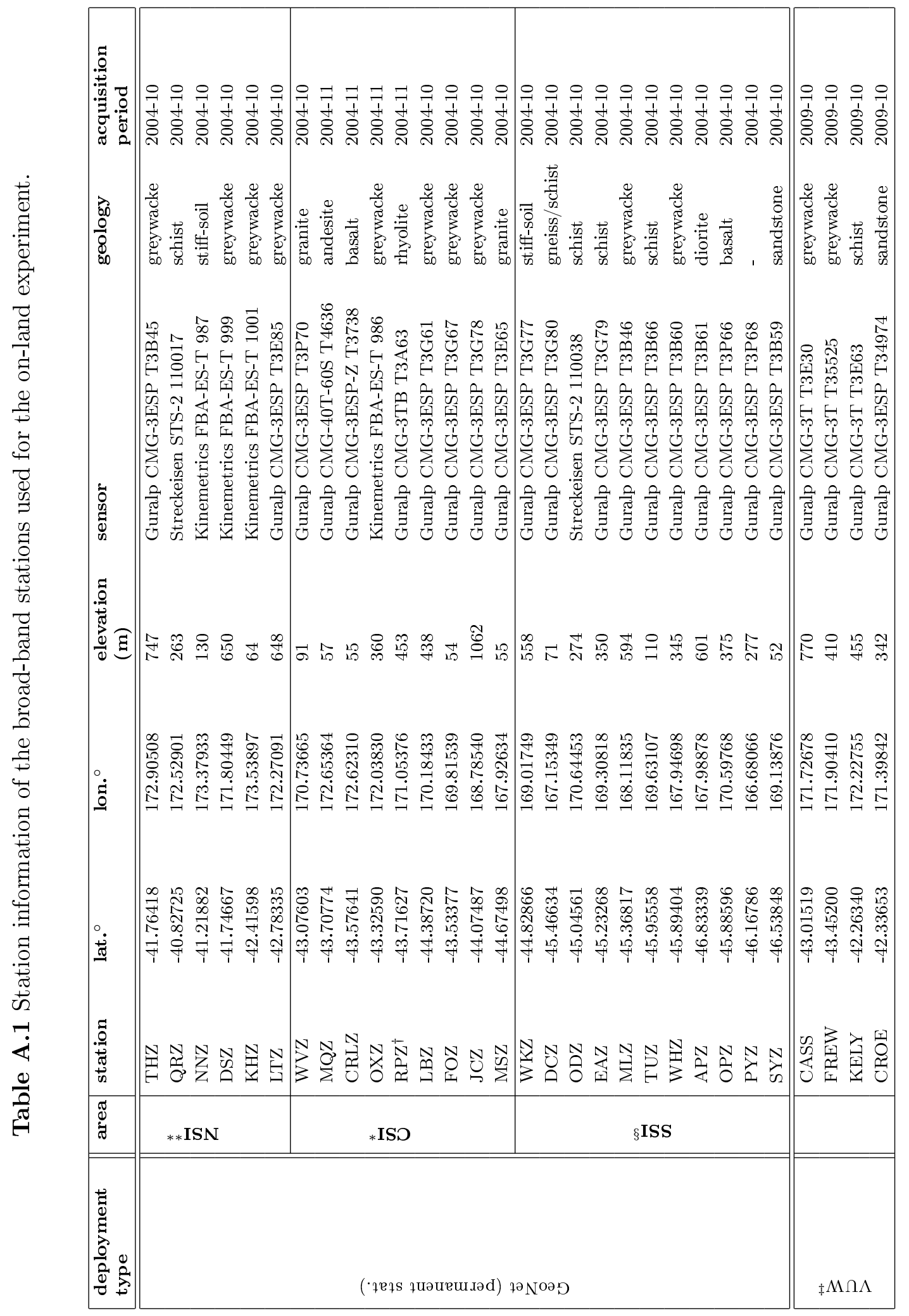




\section{A.2 Event information of the A-grade splitting parameters}

Event information of the A-grade best filter measurements are given in the table

A.2. A-grade selection criteria and cluster analysis criteria are explained in section 3.2.3 and Savage et al. [2010].

Table A.2 Events yield A-grade best filter measurement**.

\begin{tabular}{|c|c|c|c|c|c|c|}
\hline cuspid & year & jul.day & lat. $^{\circ}$ & lon. ${ }^{\circ}$ & depth & mag. \\
\hline 2165241 & 2004 & 001 & -41.213 & 172.544 & 219.765 & 4.3 \\
\hline 2166031 & 2004 & 003 & -43.159 & 170.603 & 8.085 & 3.8 \\
\hline 2166422 & 2004 & 004 & -41.369 & 173.372 & 93.746 & 3.3 \\
\hline 2166642 & 2004 & 005 & -41.342 & 173.052 & 93.820 & 3.1 \\
\hline 2166804 & 2004 & 005 & -45.604 & 167.031 & 9.000 & 4.0 \\
\hline 2167639 & 2004 & 007 & -45.083 & 167.425 & 93.908 & 4.2 \\
\hline 2168610 & 2004 & 009 & -43.434 & 170.389 & 5.000 & 4.1 \\
\hline 2168698 & 2004 & 010 & -44.580 & 168.766 & 5.000 & 3.3 \\
\hline 2169096 & 2004 & 011 & -40.647 & 173.867 & 85.919 & 3.3 \\
\hline 2169162 & 2004 & 011 & -41.324 & 172.415 & 5.000 & 3.8 \\
\hline 2169838 & 2004 & 013 & -42.339 & 174.037 & 12.000 & 3.5 \\
\hline 2169945 & 2004 & 013 & -45.248 & 167.022 & 11.653 & 3.6 \\
\hline 2171083 & 2004 & 016 & -41.858 & 174.126 & 12.716 & 3.9 \\
\hline 2171148 & 2004 & 016 & -42.973 & 172.393 & 5.000 & 3.5 \\
\hline 2172290 & 2004 & 019 & -42.081 & 173.787 & 31.550 & 3.1 \\
\hline 2172542 & 2004 & 020 & -42.366 & 174.084 & 7.337 & 3.7 \\
\hline 2172782 & 2004 & 021 & -45.224 & 166.916 & 26.699 & 3.8 \\
\hline 2173116 & 2004 & 021 & -42.226 & 173.055 & 11.994 & 3.3 \\
\hline 2174766 & 2004 & 024 & -42.300 & 174.018 & 39.201 & 3.3 \\
\hline 2175242 & 2004 & 025 & -45.435 & 167.156 & 103.872 & 3.7 \\
\hline 2175527 & 2004 & 026 & -44.625 & 168.189 & 85.499 & 3.5 \\
\hline 2176121 & 2004 & 027 & -43.654 & 173.423 & 17.183 & 3.1 \\
\hline 2176328 & 2004 & 028 & -41.640 & 174.782 & 31.094 & 3.1 \\
\hline 2176454 & 2004 & 028 & -45.151 & 167.103 & 13.158 & 3.6 \\
\hline 2176479 & 2004 & 028 & -44.615 & 167.289 & 16.160 & 3.1 \\
\hline 2177341 & 2004 & 029 & -42.452 & 172.943 & 5.000 & 3.2 \\
\hline 2177405 & 2004 & 029 & -43.508 & 172.668 & 8.419 & 3.1 \\
\hline 2177589 & 2004 & 029 & -41.724 & 172.618 & 89.887 & 3.0 \\
\hline 2179414 & 2004 & 031 & -45.181 & 166.992 & 25.744 & 3.7 \\
\hline 2179877 & 2004 & 032 & -42.954 & 171.931 & 12.000 & 3.2 \\
\hline 2180565 & 2004 & 034 & -45.582 & 167.072 & 11.251 & 4.2 \\
\hline 2180670 & 2004 & 034 & -41.010 & 172.853 & 216.869 & 3.6 \\
\hline 2180744 & 2004 & 034 & -42.636 & 173.172 & 33.000 & 3.0 \\
\hline 2181094 & 2004 & 035 & -41.386 & 173.071 & 97.351 & 3.3 \\
\hline 2183311 & 2004 & 041 & -45.088 & 167.040 & 19.148 & 4.1 \\
\hline 2183966 & 2004 & 041 & -45.219 & 166.960 & 26.935 & 3.6 \\
\hline 2184019 & 2004 & 042 & -45.609 & 167.120 & 84.066 & 3.8 \\
\hline 2184403 & 2004 & 042 & -42.193 & 174.772 & 33.785 & 4.6 \\
\hline 2184814 & 2004 & 043 & -45.275 & 166.939 & 24.493 & 3.7 \\
\hline 2185761 & 2004 & 045 & -43.079 & 173.369 & 23.454 & 3.2 \\
\hline 2185957 & 2004 & 046 & -44.541 & 168.674 & 12.000 & 3.0 \\
\hline 2187002 & 2004 & 048 & -45.305 & 166.963 & 24.012 & 3.9 \\
\hline 2187366 & 2004 & 049 & -44.995 & 167.541 & 129.781 & 3.7 \\
\hline 2187501 & 2004 & 049 & -43.198 & 173.488 & 12.000 & 3.0 \\
\hline 2187515 & 2004 & 049 & -40.848 & 174.257 & 61.025 & 3.1 \\
\hline 2187735 & 2004 & 050 & -43.415 & 173.134 & 15.447 & 3.4 \\
\hline 2188069 & 2004 & 050 & -44.531 & 168.051 & 18.045 & 3.1 \\
\hline 2190336 & 2004 & 055 & -42.058 & 173.164 & 9.675 & 3.7 \\
\hline 2191681 & 2004 & 058 & -45.379 & 167.208 & 108.854 & 4.1 \\
\hline 2191915 & 2004 & 058 & -45.067 & 167.429 & 97.054 & 3.7 \\
\hline 2192455 & 2004 & 059 & -45.243 & 166.929 & 23.402 & 4.9 \\
\hline 2192669 & 2004 & 060 & -45.238 & 166.938 & 24.664 & 4.4 \\
\hline 2192783 & 2004 & 060 & -41.142 & 173.001 & 129.925 & 3.7 \\
\hline 2192878 & 2004 & 060 & -45.242 & 166.919 & 24.545 & 3.6 \\
\hline 2193185 & 2004 & 061 & -42.029 & 171.586 & 12.000 & 3.1 \\
\hline
\end{tabular}


Table A.2 - Continued

\begin{tabular}{|c|c|c|c|c|c|c|}
\hline cuspid & year & jul.day & lat. $^{\circ}$ & lon. ${ }^{\circ}$ & depth & mag. \\
\hline 2197549 & 2004 & 070 & -42.723 & 172.800 & 33.000 & 3.1 \\
\hline 2197714 & 2004 & 071 & -45.129 & 167.438 & 80.991 & 3.6 \\
\hline 2198118 & 2004 & 071 & -44.033 & 169.618 & 33.000 & 4.1 \\
\hline 2198446 & 2004 & 072 & -43.160 & 173.825 & 21.924 & 3.2 \\
\hline 2198750 & 2004 & 073 & -40.978 & 174.399 & 53.554 & 3.2 \\
\hline 2198958 & 2004 & 073 & -44.181 & 168.598 & 5.000 & 3.1 \\
\hline 2199054 & 2004 & 074 & -45.269 & 166.685 & 19.673 & 4.0 \\
\hline 2199408 & 2004 & 074 & -42.297 & 173.343 & 38.842 & 3.0 \\
\hline 2200334 & 2004 & 076 & -41.891 & 174.017 & 19.128 & 4.5 \\
\hline 2200400 & 2004 & 076 & -41.887 & 174.012 & 18.021 & 3.0 \\
\hline 2200660 & 2004 & 077 & -42.469 & 173.850 & 27.597 & 3.5 \\
\hline 2201439 & 2004 & 079 & -44.045 & 168.492 & 5.000 & 3.0 \\
\hline 2201726 & 2004 & 079 & -45.200 & 167.349 & 119.236 & 3.6 \\
\hline 2201956 & 2004 & 080 & -45.231 & 166.958 & 22.207 & 3.5 \\
\hline 2202855 & 2004 & 082 & -43.526 & 171.187 & 5.000 & 3.1 \\
\hline 2205598 & 2004 & 088 & -41.654 & 173.875 & 15.683 & 3.7 \\
\hline 2207974 & 2004 & 092 & -45.173 & 167.069 & 23.692 & 3.8 \\
\hline 2208003 & 2004 & 092 & -45.196 & 167.530 & 130.698 & 3.5 \\
\hline 2208493 & 2004 & 009 & -43.460 & 170.389 & 5.000 & 4.4 \\
\hline 2208792 & 2004 & 093 & -45.130 & 167.405 & 117.216 & 4.8 \\
\hline 2210424 & 2004 & 096 & -41.389 & 172.853 & 115.079 & 3.6 \\
\hline 2210987 & 2004 & 097 & -42.636 & 172.155 & 7.476 & 4.4 \\
\hline 2213304 & 2004 & 102 & -45.241 & 166.924 & 23.010 & 4.2 \\
\hline 2216109 & 2004 & 109 & -41.463 & 174.974 & 25.104 & 3.6 \\
\hline 2217019 & 2004 & 111 & -41.359 & 174.198 & 35.148 & 3.1 \\
\hline 2217222 & 2004 & 111 & -41.291 & 174.773 & 47.265 & 3.4 \\
\hline 2219145 & 2004 & 115 & -41.394 & 173.284 & 85.142 & 3.1 \\
\hline 2219507 & 2004 & 116 & -44.624 & 168.237 & 71.484 & 3.4 \\
\hline 2219741 & 2004 & 117 & -45.141 & 167.322 & 120.638 & 3.8 \\
\hline 2220857 & 2004 & 117 & -41.788 & 171.898 & 14.416 & 3.3 \\
\hline 2221137 & 2004 & 118 & -45.043 & 167.424 & 93.405 & 3.9 \\
\hline 2221191 & 2004 & 118 & -44.086 & 168.577 & 12.000 & 3.0 \\
\hline 2222177 & 2004 & 119 & -41.148 & 172.534 & 5.000 & 3.3 \\
\hline 2222853 & 2004 & 121 & -42.376 & 174.494 & 34.109 & 3.1 \\
\hline 2226065 & 2004 & 127 & -41.419 & 172.971 & 106.561 & 3.6 \\
\hline 2226081 & 2004 & 127 & -45.133 & 167.546 & 90.905 & 3.6 \\
\hline 2226251 & 2004 & 127 & -45.174 & 167.014 & 20.000 & 3.5 \\
\hline 2229236 & 2004 & 133 & -44.978 & 167.489 & 121.836 & 3.7 \\
\hline 2230096 & 2004 & 135 & -41.624 & 172.857 & 86.515 & 4.0 \\
\hline 2230781 & 2004 & 136 & -41.730 & 171.984 & 7.500 & 4.1 \\
\hline 2232751 & 2004 & 139 & -40.608 & 173.854 & 94.019 & 3.3 \\
\hline 2233748 & 2004 & 141 & -40.822 & 173.715 & 95.601 & 3.4 \\
\hline 2234048 & 2004 & 141 & -45.154 & 167.462 & 112.262 & 3.8 \\
\hline 2235298 & 2004 & 144 & -42.023 & 172.830 & 81.578 & 3.5 \\
\hline 2235722 & 2004 & 144 & -42.739 & 172.832 & 15.580 & 3.0 \\
\hline 2236446 & 2004 & 146 & -44.950 & 167.595 & 67.560 & 4.2 \\
\hline 2237498 & 2004 & 148 & -45.061 & 167.026 & 12.000 & 3.8 \\
\hline 2237811 & 2004 & 148 & -40.836 & 172.964 & 221.514 & 4.1 \\
\hline 2238221 & 2004 & 149 & -44.521 & 168.288 & 5.000 & 3.0 \\
\hline 2238715 & 2004 & 150 & -41.344 & 174.375 & 42.394 & 3.4 \\
\hline 2238854 & 2004 & 150 & -45.241 & 167.345 & 102.035 & 3.9 \\
\hline 2240587 & 2004 & 153 & -45.149 & 167.290 & 114.989 & 4.0 \\
\hline 2241544 & 2004 & 155 & -41.408 & 172.890 & 98.179 & 3.6 \\
\hline 2241772 & 2004 & 155 & -45.082 & 167.383 & 104.494 & 4.6 \\
\hline 2242087 & 2004 & 156 & -41.456 & 172.836 & 99.662 & 3.2 \\
\hline 2243719 & 2004 & 159 & -43.561 & 172.220 & 20.095 & 3.5 \\
\hline 2248250 & 2004 & 167 & -41.250 & 172.648 & 202.426 & 3.9 \\
\hline 2252524 & 2004 & 175 & -42.171 & 173.926 & 31.620 & 3.5 \\
\hline 2252734 & 2004 & 175 & -45.064 & 167.414 & 115.608 & 3.6 \\
\hline 2255507 & 2004 & 181 & -43.330 & 171.866 & 9.007 & 3.1 \\
\hline 2256302 & 2004 & 182 & -44.934 & 167.560 & 77.679 & 3.8 \\
\hline 2256750 & 2004 & 183 & -45.232 & 166.912 & 12.000 & 3.8 \\
\hline 2257665 & 2004 & 185 & -45.087 & 167.023 & 20.000 & 4.4 \\
\hline 2257908 & 2004 & 186 & -45.300 & 167.246 & 68.488 & 3.6 \\
\hline 2258122 & 2004 & 186 & -42.203 & 173.886 & 19.785 & 4.0 \\
\hline 2263205 & 2004 & 194 & -42.822 & 172.443 & 9.272 & 3.4 \\
\hline 2265342 & 2004 & 197 & -42.816 & 171.360 & 5.000 & 3.1 \\
\hline 2265509 & 2004 & 198 & -45.058 & 167.113 & 5.000 & 3.8 \\
\hline 2266037 & 2004 & 199 & -40.962 & 174.926 & 31.764 & 3.3 \\
\hline 2266184 & 2004 & 200 & -41.212 & 173.381 & 94.991 & 3.4 \\
\hline
\end{tabular}


Table A.2 - Continued

\begin{tabular}{|c|c|c|c|c|c|c|}
\hline cuspid & year & jul.day & lat. $^{\circ}$ & lon. ${ }^{\circ}$ & depth & mag \\
\hline 2267941 & 2004 & 203 & -43.358 & 171.930 & 9.457 & 3.4 \\
\hline 2268502 & 2004 & 204 & -40.564 & 174.290 & 74.992 & 3.4 \\
\hline 2268698 & 2004 & 204 & -41.252 & 172.640 & 192.408 & 3.7 \\
\hline 2269062 & 2004 & 205 & -40.530 & 173.674 & 127.693 & 4.6 \\
\hline 2269844 & 2004 & 207 & -42.697 & 173.667 & 35.883 & 3.1 \\
\hline 2271646 & 2004 & 210 & -44.563 & 168.363 & 5.000 & 3.2 \\
\hline 2272438 & 2004 & 212 & -41.153 & 174.598 & 60.205 & 4.6 \\
\hline 2272755 & 2004 & 213 & -41.810 & 172.737 & 87.062 & 3.2 \\
\hline 2272849 & 2004 & 213 & -41.647 & 173.935 & 8.502 & 3.1 \\
\hline 2302197 & 2004 & 276 & -42.268 & 172.738 & 11.423 & 3.5 \\
\hline 2302434 & 2004 & 276 & -42.333 & 173.145 & 5.000 & 3.4 \\
\hline 2303253 & 2004 & 278 & -42.383 & 172.818 & 12.136 & 3.4 \\
\hline 2303625 & 2004 & 279 & -41.508 & 173.386 & 69.272 & 3.1 \\
\hline 2304495 & 2004 & 281 & -45.143 & 166.954 & 25.343 & 3.8 \\
\hline 2306884 & 2004 & 286 & -45.054 & 167.858 & 12.000 & 4.1 \\
\hline 2307072 & 2004 & 287 & -44.266 & 168.661 & 5.000 & 3.1 \\
\hline 2307605 & 2004 & 288 & -40.518 & 174.306 & 86.071 & 3.3 \\
\hline 2307840 & 2004 & 288 & -45.151 & 167.348 & 109.969 & 3.5 \\
\hline 2308266 & 2004 & 289 & -42.578 & 171.911 & 5.000 & 3.3 \\
\hline 2308271 & 2004 & 289 & -40.702 & 174.566 & 72.498 & 3.1 \\
\hline 2308546 & 2004 & 290 & -41.405 & 173.709 & 58.294 & 3.4 \\
\hline 2310945 & 2004 & 295 & -43.173 & 173.513 & 22.207 & 3.4 \\
\hline 2311679 & 2004 & 297 & -41.256 & 172.700 & 185.281 & 4.2 \\
\hline 2311774 & 2004 & 297 & -45.182 & 167.303 & 125.062 & 3.7 \\
\hline 2312015 & 2004 & 298 & -45.346 & 166.817 & 12.000 & 3.7 \\
\hline 2312381 & 2004 & 298 & -42.189 & 174.228 & 33.000 & 3.6 \\
\hline 2312482 & 2004 & 299 & -40.686 & 173.916 & 89.276 & 3.2 \\
\hline 2313500 & 2004 & 301 & -44.311 & 168.346 & 20.613 & 3.1 \\
\hline 2313713 & 2004 & 302 & -45.126 & 167.057 & 20.000 & 3.9 \\
\hline 2314163 & 2004 & 303 & -41.282 & 172.569 & 206.230 & 3.5 \\
\hline 2315087 & 2004 & 305 & -43.068 & 172.036 & 5.000 & 3.1 \\
\hline 2315598 & 2004 & 306 & -43.006 & 172.825 & 27.545 & 3.6 \\
\hline 2316723 & 2004 & 308 & -45.159 & 167.260 & 114.188 & 3.6 \\
\hline 2316812 & 2004 & 308 & -40.925 & 172.903 & 208.856 & 3.5 \\
\hline 2318753 & 2004 & 312 & -40.504 & 173.467 & 142.411 & 4.3 \\
\hline 2318769 & 2004 & 312 & -40.647 & 173.628 & 110.160 & 3.8 \\
\hline 2320355 & 2004 & 316 & -41.723 & 172.201 & 94.633 & 3.4 \\
\hline 2320849 & 2004 & 317 & -44.528 & 167.397 & 5.000 & 3.3 \\
\hline 2320957 & 2004 & 317 & -41.012 & 173.790 & 83.954 & 3.1 \\
\hline 2321673 & 2004 & 318 & -41.664 & 174.242 & 9.413 & 3.4 \\
\hline 2321906 & 2004 & 319 & -40.998 & 172.434 & 5.000 & 3.4 \\
\hline 2321935 & 2004 & 319 & -41.546 & 173.511 & 64.303 & 3.5 \\
\hline 2322081 & 2004 & 319 & -44.595 & 168.214 & 85.843 & 3.9 \\
\hline 2322299 & 2004 & 320 & -41.208 & 174.004 & 53.002 & 3.2 \\
\hline 2323155 & 2004 & 321 & -45.639 & 166.985 & 9.000 & 3.6 \\
\hline 2324239 & 2004 & 324 & -40.966 & 174.639 & 61.547 & 3.1 \\
\hline 2324418 & 2004 & 324 & -44.589 & 168.240 & 83.787 & 3.6 \\
\hline 2325873 & 2004 & 327 & -45.206 & 166.960 & 12.623 & 4.0 \\
\hline 2325951 & 2004 & 327 & -45.069 & 166.963 & 5.000 & 4.1 \\
\hline 2326696 & 2004 & 023 & -41.401 & 172.222 & 5.000 & 3.1 \\
\hline 2328987 & 2004 & 333 & -42.605 & 173.536 & 39.294 & 3.7 \\
\hline 2329087 & 2004 & 333 & -40.985 & 172.432 & 5.000 & 3.8 \\
\hline 2329697 & 2004 & 334 & -41.278 & 172.661 & 174.165 & 3.7 \\
\hline 2329763 & 2004 & 334 & -41.014 & 172.916 & 186.242 & 3.7 \\
\hline 2329949 & 2004 & 335 & -44.081 & 168.754 & 8.169 & 3.1 \\
\hline 2331522 & 2004 & 337 & -43.061 & 170.847 & 5.957 & 3.3 \\
\hline 2336444 & 2004 & 346 & -45.127 & 167.415 & 124.185 & 4.5 \\
\hline 2337155 & 2004 & 347 & -41.705 & 172.847 & 79.358 & 3.4 \\
\hline 2337190 & 2004 & 347 & -41.497 & 174.130 & 35.995 & 3.0 \\
\hline 2337656 & 2004 & 348 & -40.955 & 174.601 & 42.325 & 3.6 \\
\hline 2338463 & 2004 & 349 & -41.647 & 174.276 & 14.946 & 3.1 \\
\hline 2340108 & 2004 & 352 & -44.038 & 172.908 & 26.398 & 3.6 \\
\hline 2340467 & 2004 & 353 & -45.066 & 166.818 & 5.000 & 4.0 \\
\hline 2341023 & 2004 & 355 & -44.691 & 167.957 & 94.786 & 3.3 \\
\hline 2341413 & 2004 & 356 & -44.523 & 168.465 & 5.000 & 3.1 \\
\hline 2341695 & 2004 & 356 & -42.412 & 172.301 & 5.000 & 3.2 \\
\hline 2341801 & 2004 & 357 & -45.144 & 167.370 & 112.310 & 4.7 \\
\hline 2342689 & 2004 & 359 & -45.227 & 170.068 & 5.000 & 3.8 \\
\hline 2342705 & 2004 & 359 & -45.163 & 167.463 & 109.112 & 3.7 \\
\hline 2342814 & 2004 & 359 & -42.873 & 172.940 & 14.281 & 3.4 \\
\hline
\end{tabular}


Table A.2 - Continued

\begin{tabular}{|c|c|c|c|c|c|c|}
\hline cuspid & year & jul.day & lat. ${ }^{\circ}$ & lon. ${ }^{\circ}$ & depth & mag. \\
\hline 2343092 & 2004 & 360 & -45.166 & 167.347 & 83.699 & 3.7 \\
\hline 2343259 & 2004 & 360 & -44.091 & 168.797 & 8.681 & 3.0 \\
\hline 2343713 & 2004 & 362 & -44.949 & 167.089 & 5.000 & 3.5 \\
\hline 2344142 & 2004 & 363 & -44.410 & 168.200 & 5.000 & 3.6 \\
\hline 2344228 & 2004 & 363 & -41.290 & 172.562 & 212.870 & 3.8 \\
\hline 2344284 & 2004 & 363 & -43.978 & 169.321 & 5.000 & 3.9 \\
\hline 2344960 & 2004 & 364 & -45.101 & 167.026 & 5.000 & 3.5 \\
\hline 2345202 & 2004 & 365 & -45.057 & 167.432 & 109.337 & 4.7 \\
\hline 2345474 & 2004 & 366 & -41.253 & 174.507 & 55.374 & 3.0 \\
\hline 2345598 & 2004 & 366 & -41.311 & 172.773 & 136.783 & 3.7 \\
\hline 2348362 & 2005 & 007 & -42.815 & 173.229 & 33.000 & 3.5 \\
\hline 2348447 & 2005 & 007 & -43.505 & 170.717 & 6.288 & 3.5 \\
\hline 2352265 & 2005 & 016 & -44.625 & 166.971 & 5.000 & 3.7 \\
\hline 2352652 & 2005 & 017 & -45.445 & 166.825 & 20.946 & 3.5 \\
\hline 2352729 & 2005 & 017 & -45.001 & 167.479 & 97.802 & 4.0 \\
\hline 2353253 & 2005 & 018 & -45.090 & 167.427 & 86.901 & 4.2 \\
\hline 2355829 & 2005 & 024 & -45.129 & 166.917 & 20.000 & 4.6 \\
\hline 2355961 & 2005 & 024 & -41.307 & 172.719 & 146.361 & 3.6 \\
\hline 2360692 & 2005 & 035 & -44.587 & 168.173 & 84.153 & 3.9 \\
\hline 2361453 & 2005 & 037 & -41.410 & 172.962 & 111.823 & 3.5 \\
\hline 2361698 & 2005 & 038 & -45.027 & 167.540 & 77.697 & 4.0 \\
\hline 2363065 & 2005 & 041 & -47.039 & 166.319 & 33.000 & 4.1 \\
\hline 2364350 & 2005 & 044 & -45.330 & 166.922 & 21.376 & 5.1 \\
\hline 2366807 & 2005 & 049 & -41.423 & 172.861 & 104.594 & 3.9 \\
\hline 2366974 & 2005 & 050 & -45.229 & 167.312 & 61.399 & 3.7 \\
\hline 2367019 & 2005 & 050 & -45.123 & 167.392 & 87.299 & 3.7 \\
\hline 2367324 & 2005 & 051 & -45.155 & 167.382 & 122.686 & 3.5 \\
\hline 2368297 & 2005 & 053 & -40.830 & 173.155 & 172.635 & 3.5 \\
\hline 2369901 & 2005 & 057 & -40.515 & 173.549 & 144.554 & 4.2 \\
\hline 2371160 & 2004 & 109 & -44.554 & 167.763 & 11.212 & 3.8 \\
\hline 2372178 & 2005 & 062 & -40.720 & 173.244 & 170.675 & 3.5 \\
\hline 2373267 & 2005 & 065 & -41.297 & 172.806 & 138.617 & 3.7 \\
\hline 2373531 & 2005 & 065 & -41.289 & 172.519 & 212.966 & 3.7 \\
\hline 2374145 & 2004 & 114 & -44.959 & 167.093 & 22.682 & 3.6 \\
\hline 2375614 & 2005 & 070 & -45.082 & 167.538 & 114.894 & 3.5 \\
\hline 2376410 & 2005 & 072 & -45.072 & 167.488 & 115.635 & 3.5 \\
\hline 2376477 & 2005 & 072 & -41.267 & 172.591 & 202.700 & 3.8 \\
\hline 2378677 & 2005 & 078 & -45.312 & 166.999 & 22.063 & 3.7 \\
\hline 2379429 & 2005 & 079 & -41.292 & 172.576 & 192.780 & 4.0 \\
\hline 2382229 & 2005 & 085 & -45.001 & 167.526 & 93.320 & 4.1 \\
\hline 2388715 & 2004 & 127 & -45.050 & 167.581 & 114.912 & 3.7 \\
\hline 2388847 & 2005 & 101 & -41.650 & 174.550 & 34.607 & 4.8 \\
\hline 2391405 & 2005 & 106 & -44.350 & 169.530 & 11.858 & 4.8 \\
\hline 2393740 & 2005 & 111 & -45.153 & 167.377 & 116.756 & 3.8 \\
\hline 2395344 & 2005 & 115 & -45.878 & 166.865 & 86.948 & 5.3 \\
\hline 2397298 & 2005 & 119 & -44.093 & 169.183 & 58.931 & 3.2 \\
\hline 2397632 & 2005 & 120 & -40.992 & 172.889 & 192.719 & 3.6 \\
\hline 2398948 & 2005 & 123 & -43.932 & 168.970 & 12.000 & 3.5 \\
\hline 2402919 & 2005 & 132 & -41.416 & 172.809 & 109.481 & 3.6 \\
\hline 2403463 & 2005 & 133 & -45.014 & 167.427 & 84.950 & 4.2 \\
\hline 2403620 & 2005 & 133 & -44.944 & 167.458 & 100.371 & 3.7 \\
\hline 2404541 & 2005 & 135 & -45.075 & 167.395 & 118.371 & 3.9 \\
\hline 2407258 & 2005 & 141 & -45.311 & 166.874 & 27.534 & 3.8 \\
\hline 2408156 & 2005 & 143 & -41.451 & 174.368 & 30.826 & 4.4 \\
\hline 2411621 & 2004 & 186 & -45.142 & 166.927 & 12.000 & 3.9 \\
\hline 2412564 & 2005 & 153 & -44.608 & 168.209 & 86.236 & 3.5 \\
\hline 2418341 & 2005 & 166 & -44.626 & 168.283 & 86.070 & 0.0 \\
\hline 2419267 & 2005 & 168 & -41.242 & 172.662 & 218.667 & 3.8 \\
\hline 2421529 & 2005 & 173 & -41.336 & 172.539 & 197.634 & 3.8 \\
\hline 2422085 & 2005 & 174 & -41.982 & 173.892 & 12.000 & 3.5 \\
\hline 2422240 & 2005 & 175 & -44.853 & 167.347 & 54.200 & 3.8 \\
\hline 2422650 & 2005 & 175 & -41.309 & 172.551 & 200.614 & 3.7 \\
\hline 2425170 & 2004 & 201 & -41.708 & 172.475 & 11.515 & 3.5 \\
\hline 2426106 & 2005 & 182 & -45.103 & 167.454 & 119.185 & 3.9 \\
\hline 2432882 & 2005 & 196 & -41.099 & 173.512 & 105.670 & 4.2 \\
\hline 2432954 & 2005 & 196 & -42.406 & 173.890 & 16.441 & 3.9 \\
\hline 2433378 & 2005 & 197 & -44.571 & 168.291 & 87.890 & 0.0 \\
\hline 2433564 & 2005 & 198 & -45.045 & 167.403 & 86.817 & 3.6 \\
\hline 2438565 & 2005 & 208 & -41.791 & 172.395 & 89.152 & 3.6 \\
\hline 2439182 & 2005 & 210 & -45.065 & 167.380 & 88.450 & 4.0 \\
\hline
\end{tabular}


Table A.2 - Continued

\begin{tabular}{|c|c|c|c|c|c|c|}
\hline cuspid & year & jul.day & lat. ${ }^{\circ}$ & lon. ${ }^{\circ}$ & depth & mag. \\
\hline 2439371 & 2005 & 210 & -41.004 & 173.832 & 68.969 & 4.1 \\
\hline 2439596 & 2005 & 211 & -47.108 & 166.217 & 33.000 & 3.5 \\
\hline 2439652 & 2005 & 211 & -41.457 & 174.194 & 35.214 & 4.0 \\
\hline 2442391 & 2005 & 217 & -41.568 & 173.068 & 77.608 & 3.7 \\
\hline 2446969 & 2005 & 227 & -44.481 & 168.199 & 87.498 & 3.2 \\
\hline 2448668 & 2005 & 231 & -41.673 & 172.818 & 79.869 & 3.8 \\
\hline 2449310 & 2005 & 233 & -41.550 & 174.763 & 29.484 & 3.7 \\
\hline 2452869 & 2005 & 241 & -41.257 & 172.626 & 209.444 & 3.8 \\
\hline 2463808 & 2005 & 266 & -44.594 & 168.177 & 85.846 & 3.2 \\
\hline 2466872 & 2005 & 274 & -43.265 & 170.785 & 5.000 & 3.7 \\
\hline 2470940 & 2004 & 276 & -42.336 & 173.137 & 5.000 & 3.8 \\
\hline 2472594 & 2005 & 286 & -44.733 & 167.364 & 28.880 & 0.0 \\
\hline 2474138 & 2005 & 290 & -41.292 & 173.992 & 48.391 & 3.9 \\
\hline 2475493 & 2005 & 293 & -45.139 & 167.513 & 123.548 & 3.5 \\
\hline 2475730 & 2005 & 294 & -45.943 & 166.973 & 100.194 & 3.5 \\
\hline 2477338 & 2005 & 297 & -45.880 & 166.897 & 84.383 & 4.7 \\
\hline 2477589 & 2005 & 298 & -41.341 & 172.893 & 125.677 & 3.6 \\
\hline 2478135 & 2005 & 299 & -44.976 & 167.503 & 141.369 & 4.2 \\
\hline 2480719 & 2005 & 305 & -42.193 & 173.468 & 33.000 & 3.7 \\
\hline 2481459 & 2005 & 307 & -45.213 & 167.364 & 74.090 & 3.7 \\
\hline 2484883 & 2005 & 314 & -40.992 & 172.947 & 185.232 & 3.5 \\
\hline 2485606 & 2005 & 316 & -41.240 & 172.853 & 150.868 & 3.5 \\
\hline 2486372 & 2005 & 317 & -46.092 & 166.841 & 108.171 & 4.0 \\
\hline 2486404 & 2005 & 318 & -45.300 & 167.289 & 68.224 & 3.5 \\
\hline 2486477 & 2005 & 318 & -41.330 & 172.562 & 188.748 & 3.9 \\
\hline 2487262 & 2005 & 319 & -41.549 & 174.316 & 27.022 & 3.9 \\
\hline 2488793 & 2005 & 323 & -44.863 & 167.684 & 77.032 & 3.5 \\
\hline 2489083 & 2005 & 324 & -42.680 & 172.906 & 33.000 & 0.0 \\
\hline 2489325 & 2005 & 324 & -42.682 & 172.875 & 33.000 & 0.0 \\
\hline 2490144 & 2005 & 326 & -44.735 & 167.891 & 99.940 & 0.0 \\
\hline 2491398 & 2005 & 329 & -44.632 & 168.206 & 86.610 & 0.0 \\
\hline 2492944 & 2005 & 332 & -45.450 & 166.768 & 22.023 & 3.8 \\
\hline 2494342 & 2005 & 335 & -45.139 & 167.444 & 113.861 & 3.7 \\
\hline 2495389 & 2005 & 338 & -41.788 & 173.836 & 37.268 & 3.6 \\
\hline 2497818 & 2005 & 343 & -41.431 & 173.311 & 79.862 & 4.2 \\
\hline 2498619 & 2005 & 345 & -41.016 & 172.846 & 204.702 & 4.0 \\
\hline 2499328 & 2005 & 347 & -41.096 & 174.862 & 29.746 & 4.6 \\
\hline 2501023 & 2005 & 351 & -41.244 & 172.627 & 215.232 & 3.6 \\
\hline 2501759 & 2005 & 353 & -45.108 & 167.453 & 118.745 & 3.9 \\
\hline 2503622 & 2005 & 357 & -45.251 & 166.899 & 23.898 & 4.5 \\
\hline 2506620 & 2005 & 364 & -41.184 & 173.267 & 106.339 & 4.2 \\
\hline 2509731 & 2006 & 005 & -44.738 & 167.808 & 76.154 & 3.2 \\
\hline 2510085 & 2006 & 006 & -41.103 & 174.670 & 32.864 & 4.0 \\
\hline 2514232 & 2006 & 016 & -40.480 & 173.428 & 154.684 & 4.6 \\
\hline 2516214 & 2006 & 021 & -41.049 & 173.942 & 63.417 & 3.7 \\
\hline 2518655 & 2006 & 026 & -45.987 & 166.864 & 99.089 & 3.5 \\
\hline 2519069 & 2006 & 027 & -41.015 & 174.087 & 59.417 & 3.8 \\
\hline 2520364 & 2006 & 031 & -45.469 & 167.052 & 68.482 & 3.5 \\
\hline 2520592 & 2006 & 031 & -41.102 & 174.682 & 32.430 & 3.6 \\
\hline 2520889 & 2006 & 032 & -41.056 & 173.528 & 118.189 & 3.9 \\
\hline 2521211 & 2006 & 033 & -41.120 & 172.960 & 155.757 & 3.5 \\
\hline 2522760 & 2006 & 037 & -41.279 & 172.529 & 213.133 & 3.6 \\
\hline 2522859 & 2006 & 037 & -45.771 & 167.051 & 105.195 & 4.4 \\
\hline 2526910 & 2006 & 047 & -41.421 & 174.656 & 53.036 & 4.0 \\
\hline 2528587 & 2006 & 051 & -45.108 & 167.394 & 66.537 & 4.2 \\
\hline 2529755 & 2006 & 054 & -41.430 & 173.972 & 43.275 & 3.7 \\
\hline 2533155 & 2006 & 061 & -41.715 & 174.650 & 28.259 & 3.9 \\
\hline 2533834 & 2006 & 063 & -45.026 & 167.422 & 112.797 & 4.7 \\
\hline 2534340 & 2006 & 064 & -44.878 & 167.603 & 73.675 & 3.6 \\
\hline 2536786 & 2006 & 069 & -41.230 & 172.589 & 201.429 & 3.6 \\
\hline 2537149 & 2006 & 069 & -45.289 & 167.183 & 85.405 & 4.6 \\
\hline 2538232 & 2006 & 072 & -40.681 & 174.466 & 79.016 & 3.5 \\
\hline 2538892 & 2006 & 073 & -41.612 & 173.586 & 58.367 & 3.9 \\
\hline 2540415 & 2006 & 076 & -44.727 & 167.901 & 97.290 & 3.2 \\
\hline 2543160 & 2006 & 082 & -45.110 & 167.415 & 113.802 & 3.5 \\
\hline 2543194 & 2006 & 082 & -40.898 & 174.468 & 47.491 & 4.1 \\
\hline 2544672 & 2006 & 085 & -44.567 & 168.137 & 82.278 & 3.6 \\
\hline 2546014 & 2006 & 088 & -42.552 & 173.714 & 24.512 & 3.2 \\
\hline 2546560 & 2006 & 089 & -41.389 & 174.654 & 53.828 & 3.7 \\
\hline 2547781 & 2006 & 092 & -41.207 & 174.777 & 52.669 & 4.4 \\
\hline
\end{tabular}


Table A.2 - Continued

\begin{tabular}{|c|c|c|c|c|c|c|}
\hline cuspid & year & jul.day & lat. $^{\circ}$ & lon. ${ }^{\circ}$ & depth & mag. \\
\hline 2549731 & 2006 & 097 & -41.117 & 173.032 & 156.406 & 4.0 \\
\hline 2551363 & 2006 & 099 & -41.270 & 172.781 & 142.044 & 3.7 \\
\hline 2551460 & 2006 & 100 & -41.245 & 172.790 & 148.244 & 3.6 \\
\hline 2555839 & 2006 & 109 & -45.394 & 166.874 & 24.911 & 4.3 \\
\hline 2558232 & 2006 & 113 & -45.126 & 167.358 & 84.280 & 4.3 \\
\hline 2558585 & 2006 & 114 & -45.280 & 167.194 & 110.134 & 3.9 \\
\hline 2559763 & 2006 & 117 & -43.299 & 170.508 & 5.000 & 4.2 \\
\hline 2559903 & 2006 & 117 & -43.298 & 170.506 & 5.000 & 4.1 \\
\hline 2562489 & 2006 & 123 & -45.108 & 167.494 & 116.478 & 3.7 \\
\hline 2563468 & 2006 & 124 & -45.158 & 167.391 & 118.291 & 3.7 \\
\hline 2565311 & 2006 & 128 & -45.117 & 167.397 & 125.476 & 4.3 \\
\hline 2567466 & 2006 & 133 & -44.953 & 167.538 & 97.879 & 3.6 \\
\hline 2567689 & 2006 & 134 & -41.113 & 174.566 & 40.056 & 3.7 \\
\hline 2567873 & 2006 & 134 & -40.943 & 174.454 & 59.406 & 5.0 \\
\hline 2568379 & 2006 & 135 & -41.306 & 172.815 & 140.379 & 3.8 \\
\hline 2568890 & 2006 & 137 & -40.605 & 174.353 & 67.052 & 3.8 \\
\hline 2570686 & 2006 & 141 & -41.367 & 173.122 & 95.110 & 3.7 \\
\hline 2572359 & 2006 & 144 & -45.072 & 167.474 & 111.482 & 3.5 \\
\hline 2573232 & 2006 & 146 & -45.063 & 167.439 & 114.176 & 3.6 \\
\hline 2573533 & 2006 & 147 & -45.085 & 167.420 & 90.624 & 3.9 \\
\hline 2574820 & 2006 & 149 & -45.077 & 167.458 & 84.538 & 3.9 \\
\hline 2576189 & 2006 & 152 & -44.843 & 167.702 & 72.853 & 3.5 \\
\hline 2578100 & 2006 & 156 & -44.606 & 168.180 & 81.094 & 4.1 \\
\hline 2579538 & 2006 & 159 & -40.293 & 173.522 & 174.344 & 3.9 \\
\hline 2580443 & 2006 & 161 & -40.892 & 174.603 & 46.415 & 4.7 \\
\hline 2586781 & 2006 & 175 & -41.771 & 173.089 & 95.596 & 3.6 \\
\hline 2592468 & 2006 & 187 & -45.167 & 167.292 & 114.247 & 4.3 \\
\hline 2597464 & 2006 & 198 & -45.131 & 167.430 & 65.116 & 3.6 \\
\hline 2598293 & 2006 & 200 & -44.666 & 167.937 & 95.734 & 3.6 \\
\hline 2598839 & 2006 & 201 & -44.392 & 170.237 & 9.498 & 3.4 \\
\hline 2599831 & 2006 & 204 & -40.600 & 173.286 & 172.748 & 5.5 \\
\hline 2599966 & 2006 & 204 & -41.277 & 172.773 & 142.145 & 3.7 \\
\hline 2600075 & 2006 & 204 & -45.127 & 167.395 & 132.827 & 4.0 \\
\hline 2600789 & 2006 & 206 & -41.273 & 172.810 & 128.647 & 3.5 \\
\hline 2601210 & 2006 & 206 & -45.102 & 167.417 & 110.845 & 4.0 \\
\hline 2602165 & 2006 & 208 & -42.662 & 173.195 & 33.000 & 3.5 \\
\hline 2602486 & 2006 & 209 & -41.202 & 174.615 & 57.818 & 3.6 \\
\hline 2603539 & 2006 & 211 & -40.397 & 173.244 & 213.894 & 3.9 \\
\hline 2608311 & 2006 & 221 & -45.122 & 167.322 & 94.803 & 4.3 \\
\hline 2609143 & 2006 & 223 & -41.463 & 174.209 & 21.458 & 3.7 \\
\hline 2609766 & 2006 & 225 & -41.764 & 172.652 & 94.128 & 5.9 \\
\hline 2613953 & 2006 & 233 & -44.471 & 168.286 & 5.000 & 3.8 \\
\hline 2615935 & 2006 & 237 & -43.597 & 170.621 & 5.000 & 3.6 \\
\hline 2616404 & 2006 & 238 & -45.062 & 167.458 & 129.042 & 3.6 \\
\hline 2616532 & 2006 & 239 & -46.079 & 168.019 & 18.577 & 3.5 \\
\hline 2619948 & 2006 & 246 & -41.015 & 172.872 & 214.607 & 3.6 \\
\hline 2623315 & 2006 & 254 & -41.271 & 172.709 & 183.045 & 5.2 \\
\hline 2624333 & 2006 & 256 & -41.187 & 173.465 & 94.649 & 4.2 \\
\hline 2624977 & 2006 & 257 & -42.011 & 174.089 & 21.373 & 3.9 \\
\hline 2625245 & 2006 & 258 & -40.965 & 174.478 & 48.504 & 5.1 \\
\hline 2625655 & 2006 & 259 & -45.149 & 167.359 & 92.730 & 4.0 \\
\hline 2628986 & 2006 & 266 & -40.964 & 174.820 & 32.156 & 3.9 \\
\hline 2630049 & 2006 & 268 & -41.215 & 172.836 & 170.210 & 3.9 \\
\hline 2630581 & 2006 & 269 & -41.360 & 172.565 & 189.341 & 4.2 \\
\hline 2630758 & 2006 & 270 & -45.374 & 167.287 & 108.065 & 3.5 \\
\hline 2632675 & 2006 & 274 & -41.245 & 172.732 & 143.735 & 3.5 \\
\hline 2634680 & 2006 & 279 & -41.240 & 172.750 & 167.250 & 3.7 \\
\hline 2635139 & 2006 & 280 & -45.095 & 167.435 & 112.653 & 3.6 \\
\hline 2637385 & 2006 & 284 & -44.718 & 167.870 & 96.248 & 3.1 \\
\hline 2639387 & 2006 & 288 & -44.847 & 166.920 & 33.000 & 4.0 \\
\hline 2641098 & 2006 & 292 & -41.184 & 172.791 & 178.461 & 3.7 \\
\hline 2643586 & 2006 & 297 & -45.341 & 167.161 & 96.678 & 4.2 \\
\hline 2647980 & 2006 & 307 & -43.655 & 169.695 & 5.000 & 3.9 \\
\hline 2648753 & 2006 & 309 & -47.034 & 166.211 & 33.000 & 3.5 \\
\hline 2653841 & 2006 & 319 & -45.101 & 167.340 & 84.302 & 4.3 \\
\hline 2654099 & 2006 & 320 & -41.218 & 172.695 & 202.560 & 3.6 \\
\hline 2654530 & 2006 & 320 & -41.037 & 174.173 & 57.658 & 4.6 \\
\hline 2654849 & 2006 & 321 & -41.062 & 174.334 & 71.594 & 5.5 \\
\hline 2655095 & 2006 & 322 & -41.364 & 172.789 & 131.216 & 3.6 \\
\hline 2655189 & 2006 & 322 & -43.318 & 172.523 & 35.182 & 3.2 \\
\hline
\end{tabular}


Table A.2 - Continued

\begin{tabular}{|c|c|c|c|c|c|c|}
\hline cuspid & year & jul.day & lat. $^{\circ}$ & lon. ${ }^{\circ}$ & depth & mag \\
\hline 2655520 & 2006 & 322 & -42.988 & 172.102 & 5.000 & 4.2 \\
\hline 2664838 & 2006 & 341 & -44.660 & 167.621 & 26.118 & 3.3 \\
\hline 2666076 & 2006 & 344 & -45.031 & 167.512 & 72.623 & 3.5 \\
\hline 2666824 & 2006 & 346 & -45.153 & 167.383 & 124.553 & 4.1 \\
\hline 2667016 & 2006 & 346 & -44.713 & 168.043 & 83.294 & 3.3 \\
\hline 2672673 & 2006 & 357 & -44.825 & 167.643 & 82.576 & 3.7 \\
\hline 2674538 & 2006 & 361 & -45.184 & 167.321 & 114.743 & 3.5 \\
\hline 2676137 & 2006 & 364 & -43.394 & 172.548 & 22.401 & 3.0 \\
\hline 2676169 & 2006 & 364 & -44.602 & 168.137 & 81.162 & 3.6 \\
\hline 2678505 & 2007 & 005 & -41.256 & 172.733 & 156.048 & 4.4 \\
\hline 2679334 & 2007 & 007 & -44.599 & 168.028 & 92.554 & 3.3 \\
\hline 2680518 & 2007 & 009 & -44.581 & 168.181 & 87.087 & 3.0 \\
\hline 2681421 & 2007 & 011 & -45.074 & 167.442 & 116.071 & 4.1 \\
\hline 2685645 & 2007 & 021 & -45.416 & 167.205 & 102.262 & 3.7 \\
\hline 2690111 & 2007 & 031 & -45.072 & 167.137 & 29.157 & 3.6 \\
\hline 2692934 & 2007 & 038 & -41.016 & 174.477 & 51.571 & 4.7 \\
\hline 2698711 & 2007 & 049 & -45.446 & 167.045 & 75.588 & 3.7 \\
\hline 2699618 & 2007 & 051 & -41.128 & 174.145 & 49.820 & 4.4 \\
\hline 2699849 & 2007 & 052 & -45.323 & 167.251 & 118.705 & 4.7 \\
\hline 2702307 & 2007 & 057 & -40.921 & 173.820 & 108.029 & 4.5 \\
\hline 2704689 & 2007 & 062 & -45.549 & 166.971 & 68.565 & 4.3 \\
\hline 2707519 & 2007 & 068 & -46.003 & 166.818 & 100.045 & 3.7 \\
\hline 2707727 & 2007 & 069 & -41.196 & 173.030 & 132.392 & 3.9 \\
\hline 2711310 & 2007 & 076 & -41.303 & 172.566 & 190.406 & 3.7 \\
\hline 2713754 & 2007 & 081 & -40.383 & 173.470 & 177.212 & 4.0 \\
\hline 2713982 & 2007 & 081 & -42.007 & 172.714 & 78.945 & 4.7 \\
\hline 2718373 & 2007 & 090 & -44.623 & 168.175 & 79.191 & 3.3 \\
\hline 2720328 & 2007 & 095 & -41.251 & 172.603 & 192.920 & 3.6 \\
\hline 2722537 & 2007 & 099 & -44.738 & 167.718 & 58.479 & 3.3 \\
\hline 2724187 & 2007 & 103 & -45.095 & 167.436 & 122.909 & 4.2 \\
\hline 2724360 & 2007 & 103 & -44.703 & 167.222 & 29.669 & 3.4 \\
\hline 2726034 & 2007 & 107 & -45.162 & 167.314 & 94.971 & 3.9 \\
\hline 2726331 & 2007 & 107 & -44.817 & 167.326 & 21.166 & 4.4 \\
\hline 2726729 & 2007 & 108 & -41.232 & 172.521 & 231.010 & 3.6 \\
\hline 2727588 & 2007 & 110 & -41.246 & 172.550 & 212.435 & 3.6 \\
\hline 2727967 & 2007 & 111 & -45.135 & 167.237 & 52.040 & 4.3 \\
\hline 2732430 & 2007 & 121 & -40.695 & 173.213 & 176.194 & 4.0 \\
\hline 2733458 & 2007 & 123 & -45.370 & 167.130 & 83.072 & 3.7 \\
\hline 2734475 & 2007 & 125 & -41.381 & 172.930 & 116.220 & 3.6 \\
\hline 2734605 & 2007 & 126 & -40.565 & 173.267 & 193.383 & 3.5 \\
\hline 2735669 & 2007 & 128 & -44.588 & 168.171 & 86.900 & 3.5 \\
\hline 2737094 & 2007 & 131 & -42.350 & 173.641 & 28.166 & 4.4 \\
\hline 2737975 & 2007 & 133 & -45.371 & 167.150 & 100.502 & 3.5 \\
\hline 2737984 & 2007 & 133 & -41.217 & 173.549 & 89.750 & 5.4 \\
\hline 2741903 & 2007 & 142 & -41.017 & 172.877 & 206.570 & 3.5 \\
\hline 2741903 & 2007 & 142 & -41.017 & 172.877 & 206.820 & 3.5 \\
\hline 2743833 & 2007 & 146 & -45.021 & 167.565 & 88.321 & 3.8 \\
\hline 2743899 & 2007 & 146 & -41.242 & 172.610 & 197.143 & 3.6 \\
\hline 2744487 & 2007 & 147 & -41.034 & 172.868 & 202.298 & 3.5 \\
\hline 2747840 & 2007 & 155 & -45.000 & 167.513 & 59.995 & 3.5 \\
\hline 2748555 & 2007 & 156 & -44.626 & 168.200 & 81.052 & 4.2 \\
\hline 2748555 & 2007 & 156 & -44.626 & 168.200 & 88.683 & 4.2 \\
\hline 2751226 & 2007 & 162 & -41.417 & 174.690 & 56.064 & 3.8 \\
\hline 2754371 & 2007 & 169 & -45.076 & 167.449 & 114.475 & 3.6 \\
\hline 2754669 & 2007 & 170 & -43.186 & 172.267 & 28.144 & 3.3 \\
\hline 2757147 & 2007 & 175 & -41.598 & 174.035 & 38.907 & 4.0 \\
\hline 2758600 & 2007 & 178 & -41.999 & 174.376 & 31.184 & 3.5 \\
\hline 2760737 & 2007 & 183 & -45.053 & 167.393 & 49.226 & 3.7 \\
\hline 2762590 & 2007 & 187 & -46.029 & 167.118 & 21.397 & 3.8 \\
\hline 2763016 & 2007 & 188 & -45.214 & 167.321 & 82.588 & 4.5 \\
\hline 2768313 & 2007 & 200 & -45.181 & 167.337 & 73.925 & 3.9 \\
\hline 2769800 & 2007 & 203 & -41.219 & 172.586 & 217.863 & 3.7 \\
\hline 2770871 & 2007 & 206 & -41.298 & 172.569 & 188.682 & 3.5 \\
\hline 2775327 & 2007 & 216 & -44.458 & 168.361 & 5.000 & 3.7 \\
\hline 2778812 & 2007 & 223 & -41.319 & 173.652 & 72.117 & 3.9 \\
\hline 2780655 & 2007 & 227 & -42.430 & 173.649 & 29.924 & 3.6 \\
\hline 2780734 & 2007 & 227 & -40.937 & 173.357 & 130.358 & 5.1 \\
\hline 2780735 & 2007 & 227 & -40.943 & 173.442 & 136.420 & 4.8 \\
\hline 2780892 & 2007 & 228 & -41.052 & 174.194 & 57.323 & 4.2 \\
\hline 2782916 & 2007 & 232 & -44.402 & 167.755 & 20.508 & 3.4 \\
\hline
\end{tabular}


Table A.2 - Continued

\begin{tabular}{|c|c|c|c|c|c|c|}
\hline cuspid & year & jul.day & lat. ${ }^{\circ}$ & lon. ${ }^{\circ}$ & depth & mag. \\
\hline 2783015 & 2007 & 232 & -42.688 & 172.626 & 42.533 & 3.1 \\
\hline 2783557 & 2007 & 233 & -41.174 & 173.073 & 152.386 & 3.7 \\
\hline 2784297 & 2007 & 235 & -45.516 & 166.986 & 110.364 & 3.8 \\
\hline 2784513 & 2007 & 236 & -42.582 & 172.847 & 5.745 & 4.0 \\
\hline 2786597 & 2007 & 240 & -41.639 & 172.793 & 94.768 & 4.1 \\
\hline 2786597 & 2007 & 240 & -41.651 & 172.814 & 85.143 & 4.1 \\
\hline 2788623 & 2007 & 245 & -44.663 & 167.925 & 94.201 & 3.5 \\
\hline 2788623 & 2007 & 245 & -44.731 & 167.930 & 93.617 & 3.6 \\
\hline 2790417 & 2007 & 249 & -45.322 & 166.609 & 20.453 & 4.6 \\
\hline 2791597 & 2007 & 251 & -40.646 & 173.016 & 219.333 & 3.5 \\
\hline 2791978 & 2007 & 252 & -41.404 & 173.193 & 85.918 & 3.7 \\
\hline 2791978 & 2007 & 252 & -41.405 & 173.205 & 87.662 & 3.6 \\
\hline 2794086 & 2007 & 257 & -45.177 & 167.293 & 134.334 & 3.6 \\
\hline 2795163 & 2007 & 259 & -40.951 & 172.797 & 225.224 & 3.7 \\
\hline 2795420 & 2007 & 260 & -45.067 & 167.450 & 85.148 & 3.5 \\
\hline 2796284 & 2007 & 262 & -44.592 & 168.109 & 82.181 & 4.5 \\
\hline 2797472 & 2007 & 265 & -43.420 & 172.942 & 32.885 & 3.1 \\
\hline 2801221 & 2007 & 273 & -45.032 & 166.837 & 19.037 & 3.9 \\
\hline 2802758 & 2007 & 276 & -42.258 & 173.066 & 92.390 & 4.0 \\
\hline 2802763 & 2007 & 276 & -42.143 & 172.825 & 72.516 & 3.6 \\
\hline 2802792 & 2007 & 276 & -42.151 & 172.870 & 61.711 & 3.7 \\
\hline 2802991 & 2007 & 277 & -45.176 & 167.378 & 120.162 & 3.7 \\
\hline 2803171 & 2007 & 277 & -42.100 & 172.850 & 58.189 & 4.0 \\
\hline 2803171 & 2007 & 277 & -42.154 & 172.842 & 59.814 & 4.0 \\
\hline 2804091 & 2007 & 279 & -42.201 & 172.933 & 70.369 & 4.8 \\
\hline 2804091 & 2007 & 279 & -42.202 & 172.904 & 67.840 & 4.7 \\
\hline 2804454 & 2007 & 280 & -44.882 & 167.675 & 78.063 & 3.6 \\
\hline 2805690 & 2007 & 282 & -41.258 & 172.605 & 195.854 & 3.7 \\
\hline 2806153 & 2007 & 283 & -42.151 & 172.838 & 58.841 & 3.7 \\
\hline 2806153 & 2007 & 283 & -42.160 & 172.818 & 51.075 & 3.7 \\
\hline 2809105 & 2007 & 290 & -44.996 & 167.557 & 86.156 & 3.5 \\
\hline 2809382 & 2007 & 290 & -41.404 & 172.848 & 129.651 & 3.7 \\
\hline 2810009 & 2007 & 292 & -44.727 & 167.445 & 19.387 & 3.2 \\
\hline 2811409 & 2007 & 295 & -40.694 & 173.804 & 107.215 & 4.8 \\
\hline 2812268 & 2007 & 297 & -44.880 & 167.285 & 17.337 & 4.5 \\
\hline 2814738 & 2007 & 303 & -44.750 & 167.454 & 19.281 & 3.2 \\
\hline 2815104 & 2007 & 303 & -44.797 & 167.382 & 17.578 & 3.7 \\
\hline 2815873 & 2007 & 305 & -44.778 & 167.416 & 20.827 & 4.4 \\
\hline 2815887 & 2007 & 305 & -45.280 & 167.253 & 80.174 & 3.7 \\
\hline 2816258 & 2007 & 306 & -45.115 & 167.369 & 118.740 & 3.5 \\
\hline 2818146 & 2007 & 310 & -42.100 & 172.893 & 57.038 & 5.4 \\
\hline 2818174 & 2007 & 310 & -42.123 & 172.875 & 58.925 & 4.5 \\
\hline 2819042 & 2007 & 313 & -45.549 & 166.965 & 70.506 & 3.6 \\
\hline 2820110 & 2007 & 315 & -44.498 & 167.854 & 15.364 & 3.5 \\
\hline 2821549 & 2007 & 318 & -44.486 & 166.930 & 20.833 & 3.3 \\
\hline 2823057 & 2007 & 320 & -44.406 & 168.380 & 23.384 & 3.1 \\
\hline 2826859 & 2007 & 328 & -41.880 & 172.844 & 93.681 & 3.7 \\
\hline 2827926 & 2007 & 330 & -45.041 & 167.511 & 83.621 & 4.1 \\
\hline 2829153 & 2007 & 333 & -45.064 & 167.257 & 117.077 & 5.9 \\
\hline 2829912 & 2007 & 335 & -45.333 & 167.261 & 58.636 & 3.7 \\
\hline 2841741 & 2007 & 359 & -40.969 & 173.188 & 155.055 & 3.5 \\
\hline 2842767 & 2007 & 361 & -40.926 & 172.878 & 220.807 & 4.3 \\
\hline 2842976 & 2007 & 362 & -45.149 & 167.023 & 28.565 & 4.2 \\
\hline 2847841 & 2008 & 008 & -44.740 & 167.466 & 52.052 & 3.4 \\
\hline 2848597 & 2008 & 010 & -42.219 & 172.807 & 65.233 & 4.3 \\
\hline 2848762 & 2008 & 010 & -42.281 & 172.998 & 65.980 & 3.7 \\
\hline 2849290 & 2008 & 012 & -40.317 & 173.335 & 176.305 & 4.3 \\
\hline 2849572 & 2008 & 012 & -45.450 & 167.076 & 104.781 & 3.5 \\
\hline 2850317 & 2008 & 013 & -41.229 & 172.542 & 217.440 & 3.6 \\
\hline 2855227 & 2008 & 024 & -45.075 & 167.392 & 119.892 & 3.6 \\
\hline 2855529 & 2008 & 024 & -44.961 & 167.463 & 101.868 & 3.9 \\
\hline 2856129 & 2008 & 026 & -41.086 & 174.248 & 54.669 & 3.7 \\
\hline 2859104 & 2008 & 032 & -45.063 & 167.392 & 107.260 & 3.7 \\
\hline 2859712 & 2008 & 033 & -44.915 & 167.393 & 118.057 & 4.5 \\
\hline 2859889 & 2008 & 034 & -45.220 & 167.337 & 128.290 & 3.7 \\
\hline 2859915 & 2008 & 034 & -41.271 & 172.625 & 213.545 & 3.7 \\
\hline 2860839 & 2008 & 036 & -41.569 & 173.528 & 65.788 & 4.3 \\
\hline 2862831 & 2008 & 040 & -40.511 & 173.183 & 239.539 & 3.8 \\
\hline 2863084 & 2008 & 040 & -45.205 & 167.345 & 118.213 & 3.5 \\
\hline 2864471 & 2008 & 043 & -40.676 & 174.560 & 59.173 & 4.0 \\
\hline
\end{tabular}


Table A.2 - Continued

\begin{tabular}{|c|c|c|c|c|c|c|}
\hline cuspid & year & jul.day & lat. $^{\circ}$ & lon. ${ }^{\circ}$ & depth & mag \\
\hline 2865339 & 2008 & 045 & -45.075 & 166.463 & 21.367 & 3.7 \\
\hline 2866423 & 2008 & 047 & -41.250 & 172.945 & 194.620 & 3.7 \\
\hline 2867818 & 2008 & 050 & -45.123 & 167.696 & 122.296 & 3.9 \\
\hline 2867952 & 2008 & 050 & -44.978 & 167.423 & 120.998 & 3.7 \\
\hline 2869115 & 2008 & 053 & -40.948 & 172.880 & 220.531 & 3.7 \\
\hline 2869234 & 2008 & 053 & -45.098 & 167.450 & 58.094 & 3.7 \\
\hline 2871428 & 2008 & 057 & -45.523 & 167.067 & 66.863 & 4.0 \\
\hline 2872889 & 2008 & 060 & -40.673 & 174.052 & 92.549 & 3.5 \\
\hline 2876720 & 2008 & 068 & -45.191 & 167.349 & 117.612 & 3.6 \\
\hline 2877311 & 2008 & 069 & -40.895 & 172.852 & 216.311 & 4.2 \\
\hline 2880560 & 2008 & 076 & -40.699 & 173.786 & 97.901 & 4.6 \\
\hline 2880972 & 2008 & 077 & -45.141 & 166.752 & 22.439 & 3.5 \\
\hline 2884471 & 2008 & 084 & -41.187 & 172.735 & 174.637 & 3.5 \\
\hline 2885017 & 2008 & 084 & -43.050 & 172.629 & 19.317 & 3.3 \\
\hline 2887310 & 2008 & 090 & -44.674 & 167.921 & 99.741 & 3.3 \\
\hline 2888755 & 2008 & 093 & -45.012 & 167.560 & 89.280 & 3.9 \\
\hline 2889129 & 2008 & 094 & -45.011 & 167.462 & 94.652 & 4.4 \\
\hline 2889513 & 2008 & 095 & -41.266 & 173.815 & 64.932 & 3.5 \\
\hline 2889830 & 2008 & 096 & -40.822 & 174.895 & 38.275 & 3.5 \\
\hline 2890301 & 2008 & 097 & -43.844 & 173.328 & 25.204 & 3.2 \\
\hline 2891738 & 2008 & 099 & -45.063 & 167.477 & 92.569 & 3.6 \\
\hline 2892435 & 2008 & 101 & -44.597 & 168.126 & 91.573 & 3.0 \\
\hline 2892935 & 2008 & 102 & -44.905 & 167.603 & 72.297 & 4.0 \\
\hline 2893405 & 2008 & 103 & -45.154 & 167.360 & 145.167 & 3.3 \\
\hline 2894271 & 2008 & 105 & -40.550 & 174.394 & 59.177 & 3.9 \\
\hline 2894504 & 2008 & 105 & -45.486 & 167.129 & 108.723 & 3.9 \\
\hline 2897192 & 2008 & 111 & -41.177 & 173.596 & 93.843 & 3.9 \\
\hline 2897985 & 2008 & 113 & -41.233 & 172.564 & 213.022 & 4.0 \\
\hline 2897985 & 2008 & 113 & -41.233 & 172.564 & 215.676 & 4.0 \\
\hline 2901496 & 2008 & 120 & -44.860 & 167.512 & 26.972 & 2.1 \\
\hline 2902528 & 2008 & 122 & -43.225 & 171.019 & 5.000 & 4.6 \\
\hline 2904522 & 2006 & 324 & -40.970 & 172.850 & 192.007 & 3.5 \\
\hline 2904865 & 2008 & 127 & -44.530 & 168.053 & 81.539 & 3.0 \\
\hline 2907267 & 2008 & 132 & -45.256 & 167.229 & 56.734 & 4.5 \\
\hline 2910138 & 2008 & 139 & -44.614 & 168.032 & 73.617 & 3.2 \\
\hline 2911911 & 2008 & 143 & -44.627 & 168.032 & 92.807 & 3.1 \\
\hline 2914692 & 2006 & 349 & -41.409 & 172.952 & 115.340 & 3.6 \\
\hline 2918151 & 2008 & 156 & -40.576 & 174.583 & 91.385 & 3.6 \\
\hline 2920375 & 2008 & 160 & -41.246 & 172.610 & 214.473 & 3.6 \\
\hline 2920666 & 2008 & 161 & -45.472 & 167.049 & 64.402 & 3.5 \\
\hline 2923267 & 2008 & 166 & -41.175 & 174.417 & 39.559 & 3.7 \\
\hline 2925442 & 2008 & 170 & -42.605 & 172.713 & 54.512 & 3.5 \\
\hline 2926002 & 2008 & 172 & -41.281 & 172.570 & 227.521 & 3.6 \\
\hline 2929377 & 2008 & 178 & -45.461 & 166.907 & 51.086 & 3.6 \\
\hline 2929831 & 2008 & 179 & -40.553 & 174.292 & 64.570 & 3.9 \\
\hline 2931647 & 2008 & 183 & -40.646 & 173.290 & 162.902 & 4.6 \\
\hline 2936807 & 2008 & 193 & -42.717 & 172.889 & 33.000 & 3.0 \\
\hline 2936916 & 2008 & 193 & -45.382 & 167.066 & 67.627 & 3.7 \\
\hline 2936936 & 2008 & 194 & -45.791 & 166.804 & 102.662 & 6.0 \\
\hline 2938906 & 2008 & 198 & -40.938 & 174.653 & 68.084 & 4.2 \\
\hline 2944056 & 2008 & 208 & -41.750 & 174.333 & 55.651 & 3.5 \\
\hline 2944471 & 2008 & 209 & -43.506 & 169.944 & 10.561 & 3.2 \\
\hline 2946142 & 2008 & 212 & -41.222 & 173.857 & 63.434 & 3.7 \\
\hline 2950527 & 2008 & 221 & -45.002 & 167.516 & 80.035 & 4.4 \\
\hline 2950769 & 2008 & 222 & -40.533 & 173.176 & 240.017 & 3.5 \\
\hline 2951523 & 2008 & 223 & -42.891 & 172.881 & 28.597 & 3.1 \\
\hline 2952161 & 2008 & 225 & -45.066 & 167.469 & 81.384 & 3.8 \\
\hline 2953023 & 2008 & 226 & -44.616 & 168.154 & 85.148 & 3.3 \\
\hline 2954005 & 2008 & 228 & -45.081 & 167.346 & 109.954 & 4.1 \\
\hline 2956873 & 2008 & 234 & -41.204 & 172.518 & 213.742 & 4.4 \\
\hline 2961674 & 2008 & 244 & -41.219 & 172.757 & 147.890 & 3.8 \\
\hline 2961889 & 2008 & 237 & -41.202 & 172.904 & 156.906 & 3.7 \\
\hline 2962981 & 2008 & 246 & -41.398 & 172.841 & 109.057 & 3.7 \\
\hline 2963425 & 2008 & 247 & -41.479 & 173.517 & 62.070 & 4.5 \\
\hline 2964617 & 2008 & 250 & -40.585 & 173.284 & 164.971 & 4.5 \\
\hline 2966534 & 2008 & 253 & -42.090 & 173.003 & 81.566 & 3.9 \\
\hline 2967823 & 2008 & 256 & -44.989 & 167.472 & 54.182 & 3.5 \\
\hline 2968431 & 2008 & 257 & -45.007 & 167.560 & 70.056 & 4.5 \\
\hline 2969446 & 2008 & 259 & -41.212 & 172.605 & 206.442 & 3.6 \\
\hline 2970989 & 2008 & 262 & -41.832 & 172.632 & 83.879 & 3.6 \\
\hline
\end{tabular}


Table A.2 - Continued

\begin{tabular}{|c|c|c|c|c|c|c|}
\hline cuspid & year & jul.day & lat. $^{\circ}$ & lon. ${ }^{\circ}$ & depth & mag. \\
\hline 2972380 & 2008 & 265 & -42.957 & 172.673 & 33.000 & 3.2 \\
\hline 2973670 & 2008 & 268 & -42.025 & 173.922 & 21.118 & 3.6 \\
\hline 2974157 & 2008 & 269 & -42.284 & 173.887 & 20.249 & 4.6 \\
\hline 2974665 & 2008 & 270 & -42.309 & 173.923 & 23.894 & 5.2 \\
\hline 2974715 & 2008 & 270 & -42.286 & 173.889 & 21.674 & 3.8 \\
\hline 2976419 & 2008 & 273 & -44.968 & 167.408 & 81.125 & 4.1 \\
\hline 2977473 & 2008 & 275 & -41.289 & 172.656 & 179.403 & 3.9 \\
\hline 2978678 & 2008 & 278 & -40.236 & 173.441 & 164.403 & 3.5 \\
\hline 2979524 & 2008 & 270 & -42.279 & 173.887 & 20.545 & 3.7 \\
\hline 2979619 & 2008 & 280 & -41.737 & 173.143 & 69.120 & 3.7 \\
\hline 2980909 & 2008 & 282 & -42.803 & 172.719 & 30.422 & 3.1 \\
\hline 2985839 & 2008 & 292 & -41.251 & 172.810 & 135.250 & 3.7 \\
\hline 2985966 & 2008 & 292 & -42.035 & 172.620 & 76.907 & 4.0 \\
\hline 2987302 & 2008 & 295 & -40.653 & 174.201 & 67.895 & 3.5 \\
\hline 2987304 & 2008 & 295 & -45.123 & 166.864 & 26.745 & 4.1 \\
\hline 2993491 & 2008 & 307 & -41.024 & 174.571 & 39.025 & 3.7 \\
\hline 2993608 & 2008 & 307 & -45.029 & 167.455 & 73.528 & 3.6 \\
\hline 2993623 & 2008 & 307 & -45.491 & 167.010 & 69.198 & 4.0 \\
\hline 2993712 & 2008 & 308 & -45.109 & 167.404 & 92.821 & 3.9 \\
\hline 2995312 & 2008 & 310 & -41.093 & 174.581 & 60.404 & 3.6 \\
\hline 2996979 & 2008 & 314 & -45.161 & 167.306 & 122.081 & 5.0 \\
\hline 2997750 & 2008 & 315 & -41.741 & 174.391 & 55.840 & 4.0 \\
\hline 2998010 & 2008 & 316 & -44.963 & 167.499 & 133.586 & 3.7 \\
\hline 2999479 & 2008 & 319 & -41.815 & 172.701 & 87.947 & 4.3 \\
\hline 2999492 & 2008 & 319 & -45.083 & 167.424 & 91.125 & 3.8 \\
\hline 2999830 & 2008 & 320 & -41.953 & 174.343 & 24.764 & 3.5 \\
\hline 3000748 & 2008 & 321 & -40.838 & 172.832 & 210.909 & 4.8 \\
\hline 3001721 & 2008 & 323 & -44.371 & 169.835 & 5.000 & 3.9 \\
\hline 3002448 & 2008 & 324 & -45.221 & 167.123 & 28.573 & 4.0 \\
\hline 3003193 & 2008 & 326 & -43.575 & 171.461 & 20.444 & 3.1 \\
\hline 3003350 & 2008 & 326 & -41.146 & 172.722 & 200.846 & 3.7 \\
\hline 3004305 & 2008 & 328 & -40.804 & 174.473 & 52.653 & 3.8 \\
\hline 3005451 & 2008 & 330 & -45.322 & 167.064 & 30.274 & 4.2 \\
\hline 3008283 & 2008 & 336 & -45.013 & 167.492 & 77.751 & 3.9 \\
\hline 3008539 & 2008 & 336 & -44.349 & 170.116 & 13.324 & 4.1 \\
\hline 3010647 & 2008 & 340 & -42.695 & 171.278 & 5.000 & 3.6 \\
\hline 3011535 & 2008 & 342 & -44.955 & 167.429 & 70.787 & 3.7 \\
\hline 3011793 & 2008 & 343 & -44.663 & 168.120 & 85.215 & 3.6 \\
\hline 3013837 & 2008 & 347 & -44.099 & 169.282 & 33.000 & 3.1 \\
\hline 3019466 & 2008 & 358 & -45.365 & 167.250 & 123.754 & 4.2 \\
\hline 3019527 & 2008 & 358 & -45.344 & 167.185 & 103.036 & 4.1 \\
\hline 3021098 & 2008 & 361 & -40.554 & 174.867 & 52.144 & 5.1 \\
\hline 3021243 & 2008 & 362 & -41.933 & 174.408 & 33.000 & 3.6 \\
\hline 3024412 & 2009 & 002 & -45.409 & 167.026 & 55.084 & 4.0 \\
\hline 3025017 & 2009 & 003 & -40.561 & 174.858 & 49.503 & 3.8 \\
\hline 3025488 & 2009 & 004 & -44.737 & 167.803 & 62.004 & 3.7 \\
\hline 3028730 & 2009 & 011 & -41.582 & 173.238 & 99.906 & 4.6 \\
\hline 3028783 & 2009 & 010 & -41.302 & 172.594 & 208.350 & 4.1 \\
\hline 3030153 & 2009 & 014 & -44.870 & 167.555 & 116.042 & 3.7 \\
\hline 3035179 & 2009 & 024 & -45.081 & 167.429 & 90.692 & 4.2 \\
\hline 3035714 & 2009 & 025 & -45.146 & 167.398 & 109.224 & 3.6 \\
\hline 3041931 & 2009 & 038 & -41.233 & 172.566 & 213.946 & 3.6 \\
\hline 3042565 & 2009 & 039 & -45.537 & 167.071 & 76.165 & 3.9 \\
\hline 3042860 & 2009 & 040 & -41.668 & 173.420 & 54.237 & 3.6 \\
\hline 3046321 & 2009 & 047 & -42.148 & 172.848 & 54.321 & 2.2 \\
\hline 3047203 & 2009 & 049 & -42.838 & 172.948 & 30.006 & 2.6 \\
\hline 3047204 & 2009 & 049 & -41.708 & 173.094 & 90.129 & 2.5 \\
\hline 3047503 & 2009 & 049 & -45.096 & 167.389 & 117.456 & 3.9 \\
\hline 3049306 & 2009 & 053 & -42.736 & 172.847 & 5.000 & 2.6 \\
\hline 3050923 & 2009 & 056 & -43.504 & 171.544 & 12.000 & 3.5 \\
\hline 3051399 & 2009 & 057 & -41.221 & 172.725 & 201.834 & 3.4 \\
\hline 3051685 & 2009 & 058 & -43.063 & 173.362 & 26.056 & 3.8 \\
\hline 3051705 & 2009 & 058 & -43.084 & 173.428 & 33.000 & 2.7 \\
\hline 3052037 & 2009 & 059 & -42.086 & 172.753 & 58.838 & 2.5 \\
\hline 3052463 & 2009 & 059 & -43.208 & 171.474 & 5.000 & 2.8 \\
\hline 3053423 & 2009 & 061 & -41.742 & 173.968 & 31.649 & 2.3 \\
\hline 3053698 & 2009 & 062 & -42.735 & 173.341 & 33.000 & 2.6 \\
\hline 3053791 & 2009 & 062 & -41.594 & 172.722 & 100.908 & 2.5 \\
\hline 3054051 & 2009 & 063 & -41.856 & 173.107 & 55.935 & 2.5 \\
\hline 3054325 & 2009 & 063 & -43.214 & 172.156 & 5.000 & 4.1 \\
\hline
\end{tabular}


Table A.2 - Continued

\begin{tabular}{|c|c|c|c|c|c|c|}
\hline cuspid & year & jul.day & lat. $^{\circ}$ & lon. ${ }^{\circ}$ & depth & mag. \\
\hline 3054326 & 2009 & 063 & -43.211 & 172.167 & 5.000 & 3.3 \\
\hline 3055648 & 2009 & 066 & -43.609 & 171.143 & 5.000 & 2.4 \\
\hline 3055781 & 2009 & 066 & -41.400 & 172.991 & 104.129 & 2.8 \\
\hline 3056338 & 2009 & 067 & -41.169 & 172.869 & 141.322 & 3.1 \\
\hline 3057524 & 2009 & 069 & -42.968 & 171.446 & 5.000 & 3.5 \\
\hline 3057877 & 2009 & 070 & -41.688 & 174.477 & 54.052 & 4.3 \\
\hline 3058398 & 2009 & 071 & -42.147 & 172.811 & 49.076 & 2.7 \\
\hline 3058975 & 2009 & 072 & -45.095 & 167.398 & 117.789 & 3.8 \\
\hline 3059515 & 2009 & 073 & -40.542 & 173.522 & 158.489 & 4.1 \\
\hline 3060955 & 2009 & 076 & -42.632 & 172.026 & 21.210 & 2.1 \\
\hline 3061005 & 2009 & 076 & -40.434 & 173.543 & 158.239 & 3.6 \\
\hline 3061192 & 2009 & 076 & -41.232 & 172.593 & 215.319 & 3.5 \\
\hline 3061664 & 2009 & 077 & -41.405 & 173.401 & 64.561 & 2.6 \\
\hline 3062588 & 2009 & 079 & -41.830 & 173.260 & 50.894 & 2.6 \\
\hline 3062982 & 2009 & 080 & -40.968 & 172.762 & 186.497 & 3.3 \\
\hline 3064185 & 2009 & 082 & -41.302 & 172.804 & 131.611 & 2.4 \\
\hline 3065595 & 2009 & 085 & -45.061 & 167.422 & 97.395 & 4.7 \\
\hline 3066315 & 2009 & 086 & -44.256 & 169.993 & 14.145 & 4.8 \\
\hline 3066448 & 2009 & 086 & -42.705 & 172.346 & 11.069 & 2.5 \\
\hline 3066652 & 2009 & 087 & -44.264 & 170.012 & 14.891 & 3.2 \\
\hline 3066767 & 2009 & 087 & -42.893 & 173.163 & 33.000 & 2.1 \\
\hline 3067191 & 2009 & 088 & -42.426 & 172.797 & 52.821 & 2.5 \\
\hline 3067364 & 2009 & 088 & -41.739 & 172.521 & 86.280 & 2.6 \\
\hline 3068099 & 2009 & 090 & -41.499 & 172.888 & 123.965 & 4.6 \\
\hline 3068852 & 2009 & 091 & -45.025 & 167.393 & 125.892 & 3.6 \\
\hline 3069262 & 2009 & 092 & -43.132 & 171.461 & 5.000 & 2.3 \\
\hline 3069643 & 2009 & 093 & -43.007 & 172.992 & 33.000 & 2.9 \\
\hline 3071401 & 2009 & 096 & -41.660 & 172.901 & 91.317 & 4.5 \\
\hline 3073195 & 2009 & 100 & -40.979 & 172.875 & 200.923 & 3.1 \\
\hline 3074691 & 2009 & 103 & -41.469 & 172.876 & 121.837 & 3.9 \\
\hline 3074989 & 2009 & 103 & -41.072 & 172.880 & 181.175 & 2.7 \\
\hline 3075089 & 2009 & 103 & -43.519 & 171.640 & 5.000 & 2.5 \\
\hline 3075740 & 2009 & 105 & -43.486 & 171.607 & 5.000 & 2.3 \\
\hline 3076083 & 2009 & 104 & -41.959 & 172.196 & 84.238 & 2.5 \\
\hline 3076229 & 2009 & 106 & -42.928 & 173.063 & 33.000 & 3.3 \\
\hline 3077046 & 2009 & 107 & -42.540 & 171.933 & 5.000 & 2.1 \\
\hline 3077049 & 2009 & 107 & -41.171 & 173.220 & 109.017 & 3.1 \\
\hline 3077937 & 2009 & 109 & -40.402 & 173.590 & 154.805 & 3.7 \\
\hline 3079510 & 2009 & 112 & -40.741 & 174.077 & 96.377 & 3.0 \\
\hline 3079670 & 2009 & 112 & -41.231 & 172.704 & 146.663 & 2.6 \\
\hline 3080021 & 2009 & 113 & -41.377 & 172.966 & 116.455 & 3.7 \\
\hline 3080313 & 2009 & 113 & -41.314 & 172.707 & 216.569 & 2.3 \\
\hline 3080807 & 2009 & 114 & -41.284 & 172.678 & 208.877 & 3.8 \\
\hline 3084351 & 2009 & 121 & -41.187 & 174.805 & 30.875 & 4.1 \\
\hline 3084790 & 2009 & 122 & -44.259 & 170.003 & 14.189 & 3.6 \\
\hline 3086566 & 2009 & 125 & -41.357 & 173.115 & 92.435 & 2.9 \\
\hline 3086578 & 2009 & 125 & -42.503 & 172.979 & 12.000 & 3.2 \\
\hline 3087732 & 2009 & 128 & -43.152 & 170.960 & 5.000 & 4.0 \\
\hline 3088222 & 2009 & 129 & -43.233 & 171.603 & 5.000 & 2.2 \\
\hline 3088333 & 2009 & 129 & -41.245 & 173.869 & 55.237 & 3.0 \\
\hline 3090764 & 2009 & 133 & -42.643 & 171.961 & 17.417 & 2.7 \\
\hline 3090908 & 2009 & 134 & -42.153 & 173.842 & 33.000 & 2.5 \\
\hline 3091002 & 2009 & 134 & -43.113 & 171.023 & 5.000 & 3.0 \\
\hline 3091380 & 2009 & 134 & -41.374 & 174.118 & 39.063 & 4.5 \\
\hline 3091508 & 2009 & 135 & -43.395 & 171.445 & 5.000 & 2.5 \\
\hline 3091656 & 2009 & 135 & -41.134 & 173.919 & 67.400 & 3.7 \\
\hline 3091866 & 2009 & 135 & -41.815 & 172.450 & 84.029 & 2.6 \\
\hline 3093057 & 2009 & 137 & -41.283 & 172.799 & 129.064 & 3.0 \\
\hline 3093437 & 2009 & 138 & -43.179 & 171.559 & 5.000 & 2.0 \\
\hline 3093885 & 2009 & 139 & -41.795 & 173.216 & 87.277 & 2.7 \\
\hline 3094669 & 2009 & 140 & -41.974 & 173.359 & 30.572 & 2.5 \\
\hline 3096079 & 2009 & 143 & -45.410 & 167.196 & 136.906 & 3.6 \\
\hline 3096312 & 2009 & 143 & -44.179 & 171.186 & 12.000 & 2.3 \\
\hline 3096871 & 2009 & 144 & -45.158 & 167.356 & 120.805 & 3.9 \\
\hline 3097684 & 2009 & 146 & -41.534 & 173.302 & 86.897 & 2.6 \\
\hline 3097924 & 2009 & 146 & -41.077 & 174.582 & 38.910 & 3.5 \\
\hline 3099415 & 2009 & 149 & -42.956 & 172.413 & 12.000 & 2.4 \\
\hline 3101119 & 2009 & 152 & -40.324 & 173.314 & 172.081 & 4.2 \\
\hline 3101277 & 2009 & 152 & -41.335 & 172.735 & 103.093 & 2.3 \\
\hline 3101867 & 2009 & 153 & -41.252 & 172.589 & 195.736 & 3.4 \\
\hline
\end{tabular}


Table A.2 - Continued

\begin{tabular}{|c|c|c|c|c|c|c|}
\hline cuspid & year & jul.day & lat. $^{\circ}$ & lon. ${ }^{\circ}$ & depth & mag. \\
\hline 3101969 & 2009 & 153 & -42.951 & 172.422 & 17.954 & 2.6 \\
\hline 3102158 & 2009 & 154 & -43.238 & 172.012 & 10.210 & 2.5 \\
\hline 3102845 & 2009 & 155 & -41.060 & 174.540 & 44.080 & 4.0 \\
\hline 3103763 & 2009 & 157 & -42.133 & 172.787 & 57.840 & 2.8 \\
\hline 3104152 & 2009 & 158 & -41.474 & 173.899 & 42.670 & 2.8 \\
\hline 3104352 & 2009 & 158 & -41.071 & 174.557 & 43.478 & 3.9 \\
\hline 3105343 & 2009 & 160 & -42.592 & 172.863 & 5.000 & 2.5 \\
\hline 3106393 & 2009 & 162 & -41.708 & 172.189 & 102.730 & 2.6 \\
\hline 3106824 & 2009 & 163 & -42.045 & 172.356 & 76.179 & 3.3 \\
\hline 3107010 & 2009 & 163 & -43.672 & 171.604 & 5.000 & 3.3 \\
\hline 3107189 & 2009 & 163 & -41.414 & 172.504 & 118.326 & 3.0 \\
\hline 3107602 & 2009 & 164 & -45.214 & 167.304 & 105.020 & 3.8 \\
\hline 3108186 & 2009 & 165 & -44.827 & 167.250 & 33.000 & 3.7 \\
\hline 3108837 & 2009 & 166 & -40.555 & 173.419 & 165.474 & 4.1 \\
\hline 3109454 & 2009 & 167 & -42.038 & 172.925 & 75.936 & 3.3 \\
\hline 3110536 & 2009 & 169 & -41.113 & 172.970 & 160.957 & 3.1 \\
\hline 3111177 & 2009 & 171 & -42.115 & 173.337 & 75.988 & 3.0 \\
\hline 3112187 & 2009 & 172 & -42.042 & 172.837 & 62.720 & 2.3 \\
\hline 3112232 & 2009 & 155 & -43.608 & 171.514 & 5.000 & 2.2 \\
\hline 3112571 & 2009 & 173 & -43.130 & 172.329 & 27.711 & 2.1 \\
\hline 3112716 & 2009 & 173 & -40.943 & 172.834 & 193.891 & 3.9 \\
\hline 3113703 & 2009 & 175 & -41.181 & 173.016 & 128.605 & 2.3 \\
\hline 3114247 & 2009 & 176 & -43.218 & 172.297 & 17.991 & 2.0 \\
\hline 3114306 & 2009 & 176 & -41.241 & 172.603 & 198.377 & 3.1 \\
\hline 3114457 & 2009 & 176 & -42.429 & 173.047 & 20.000 & 2.3 \\
\hline 3114581 & 2009 & 177 & -43.477 & 171.467 & 5.000 & 2.3 \\
\hline 3115714 & 2009 & 178 & -42.502 & 169.801 & 33.000 & 4.8 \\
\hline 3115973 & 2009 & 179 & -42.693 & 173.438 & 33.000 & 3.6 \\
\hline 3118326 & 2009 & 183 & -41.550 & 172.995 & 91.464 & 4.2 \\
\hline 3118345 & 2009 & 183 & -41.578 & 172.993 & 91.361 & 4.6 \\
\hline 3119086 & 2009 & 185 & -43.394 & 172.451 & 12.000 & 2.9 \\
\hline 3119449 & 2009 & 185 & -40.854 & 172.931 & 210.082 & 3.4 \\
\hline 3119699 & 2009 & 186 & -42.069 & 172.923 & 80.538 & 2.9 \\
\hline 3120449 & 2009 & 187 & -41.264 & 172.776 & 144.004 & 2.5 \\
\hline 3120467 & 2009 & 188 & -43.022 & 172.722 & 25.211 & 3.0 \\
\hline 3120521 & 2009 & 188 & -41.431 & 172.987 & 92.780 & 2.7 \\
\hline 3120655 & 2009 & 188 & -43.416 & 172.538 & 5.000 & 2.9 \\
\hline 3120890 & 2009 & 188 & -44.728 & 167.851 & 96.719 & 3.0 \\
\hline 3121142 & 2009 & 189 & -41.289 & 172.777 & 146.310 & 2.8 \\
\hline 3121525 & 2009 & 180 & -44.284 & 171.890 & 33.000 & 2.3 \\
\hline 3121535 & 2009 & 190 & -41.234 & 172.641 & 195.414 & 2.7 \\
\hline 3122125 & 2009 & 191 & -41.489 & 172.996 & 78.819 & 2.2 \\
\hline 3122292 & 2009 & 191 & -40.985 & 172.916 & 174.270 & 4.2 \\
\hline 3123338 & 2009 & 193 & -40.917 & 173.065 & 172.778 & 3.5 \\
\hline 3123875 & 2009 & 194 & -41.262 & 172.635 & 172.555 & 2.6 \\
\hline 3123888 & 2009 & 194 & -41.327 & 173.373 & 70.068 & 2.3 \\
\hline 3124008 & 2009 & 194 & -42.240 & 173.892 & 33.000 & 3.7 \\
\hline 3124085 & 2009 & 194 & -41.295 & 172.602 & 190.798 & 3.4 \\
\hline 3124516 & 2009 & 195 & -40.893 & 172.790 & 243.850 & 3.4 \\
\hline 3124878 & 2009 & 196 & -46.278 & 166.267 & 177.420 & 5.2 \\
\hline 3125061 & 2009 & 196 & -45.521 & 167.033 & 65.012 & 5.1 \\
\hline 3125201 & 2009 & 197 & -45.897 & 166.211 & 33.000 & 5.4 \\
\hline 3125559 & 2009 & 198 & -45.702 & 166.529 & 19.531 & 4.6 \\
\hline 3125643 & 2009 & 198 & -42.763 & 171.934 & 5.000 & 3.2 \\
\hline 3125686 & 2009 & 198 & -45.727 & 166.531 & 22.396 & 3.9 \\
\hline 3125689 & 2009 & 198 & -45.395 & 166.706 & 21.864 & 4.9 \\
\hline 3125707 & 2009 & 198 & -45.384 & 166.703 & 21.886 & 3.5 \\
\hline 3125797 & 2009 & 198 & -45.557 & 166.564 & 20.887 & 4.3 \\
\hline 3125822 & 2009 & 198 & -45.494 & 166.621 & 21.520 & 3.9 \\
\hline 3125829 & 2009 & 198 & -46.019 & 166.482 & 32.839 & 3.8 \\
\hline 3125831 & 2009 & 198 & -45.428 & 166.625 & 21.897 & 4.3 \\
\hline 3125939 & 2009 & 198 & -45.420 & 166.701 & 21.574 & 4.5 \\
\hline 3125991 & 2009 & 198 & -45.529 & 166.558 & 21.431 & 4.4 \\
\hline 3126025 & 2009 & 199 & -45.363 & 166.658 & 21.554 & 4.8 \\
\hline 3126043 & 2009 & 199 & -45.459 & 166.600 & 22.887 & 3.7 \\
\hline 3126108 & 2009 & 199 & -45.523 & 166.679 & 21.855 & 3.5 \\
\hline 3126142 & 2009 & 199 & -45.398 & 166.675 & 23.063 & 3.6 \\
\hline 3126218 & 2009 & 199 & -45.863 & 166.345 & 21.578 & 4.1 \\
\hline 3126262 & 2009 & 199 & -45.490 & 166.553 & 20.181 & 3.6 \\
\hline 3126393 & 2009 & 199 & -45.501 & 166.542 & 21.470 & 4.3 \\
\hline
\end{tabular}


Table A.2 - Continued

\begin{tabular}{|c|c|c|c|c|c|c|}
\hline cuspid & year & jul.day & lat. $^{\circ}$ & lon. ${ }^{\circ}$ & depth & mag. \\
\hline 3126549 & 2009 & 200 & -43.101 & 171.003 & 5.000 & 2.4 \\
\hline 3126568 & 2009 & 200 & -45.682 & 166.528 & 21.560 & 4.1 \\
\hline 3126670 & 2009 & 200 & -45.714 & 166.493 & 20.943 & 3.5 \\
\hline 3126729 & 2009 & 200 & -45.545 & 166.654 & 22.410 & 4.4 \\
\hline 3126734 & 2009 & 200 & -43.056 & 171.245 & 5.000 & 2.9 \\
\hline 3126801 & 2009 & 200 & -45.673 & 166.529 & 18.602 & 3.7 \\
\hline 3126843 & 2009 & 200 & -45.437 & 166.663 & 21.870 & 3.5 \\
\hline 3127061 & 2009 & 201 & -45.464 & 166.746 & 21.983 & 3.6 \\
\hline 3127203 & 2009 & 201 & -45.470 & 166.631 & 21.900 & 3.8 \\
\hline 3127714 & 2009 & 202 & -45.881 & 166.438 & 26.520 & 4.0 \\
\hline 3127867 & 2009 & 203 & -43.039 & 173.246 & 20.779 & 3.1 \\
\hline 3128341 & 2009 & 204 & -42.668 & 172.595 & 33.000 & 3.3 \\
\hline 3128712 & 2009 & 204 & -45.409 & 166.730 & 22.087 & 3.8 \\
\hline 3128785 & 2009 & 204 & -45.376 & 166.704 & 24.653 & 3.7 \\
\hline 3128864 & 2009 & 204 & -45.460 & 166.673 & 23.413 & 4.3 \\
\hline 3129136 & 2009 & 205 & -45.393 & 166.722 & 23.897 & 4.6 \\
\hline 3129198 & 2009 & 205 & -44.615 & 168.159 & 80.070 & 3.2 \\
\hline 3129333 & 2009 & 205 & -45.390 & 166.591 & 19.728 & 3.6 \\
\hline 3129804 & 2009 & 207 & -41.290 & 172.857 & 144.939 & 3.2 \\
\hline 3129834 & 2009 & 207 & -41.420 & 172.992 & 79.919 & 2.3 \\
\hline 3129894 & 2009 & 207 & -41.292 & 172.704 & 158.588 & 3.6 \\
\hline 3130125 & 2009 & 207 & -45.889 & 166.258 & 26.656 & 4.8 \\
\hline 3130282 & 2009 & 208 & -45.056 & 166.940 & 33.000 & 3.7 \\
\hline 3130541 & 2009 & 208 & -45.499 & 166.588 & 22.036 & 4.6 \\
\hline 3130656 & 2009 & 208 & -45.454 & 166.648 & 23.256 & 3.8 \\
\hline 3130676 & 2009 & 209 & -45.471 & 166.657 & 21.433 & 3.6 \\
\hline 3131488 & 2009 & 210 & -45.548 & 166.632 & 20.584 & 5.1 \\
\hline 3131501 & 2009 & 211 & -45.539 & 166.646 & 20.917 & 4.0 \\
\hline 3131736 & 2009 & 211 & -45.618 & 166.469 & 19.518 & 3.7 \\
\hline 3131896 & 2009 & 211 & -45.646 & 166.448 & 21.977 & 4.7 \\
\hline 3132013 & 2009 & 212 & -45.535 & 166.611 & 20.605 & 3.8 \\
\hline 3132372 & 2009 & 212 & -45.446 & 166.710 & 24.893 & 4.3 \\
\hline 3132601 & 2009 & 213 & -44.971 & 167.535 & 127.785 & 3.6 \\
\hline 3132839 & 2009 & 213 & -41.766 & 173.048 & 90.793 & 2.5 \\
\hline 3133043 & 2009 & 214 & -45.464 & 166.759 & 22.554 & 4.6 \\
\hline 3133200 & 2009 & 214 & -46.105 & 166.305 & 28.945 & 3.9 \\
\hline 3133354 & 2009 & 214 & -45.681 & 166.642 & 22.519 & 3.8 \\
\hline 3133815 & 2009 & 215 & -46.161 & 166.277 & 33.000 & 4.2 \\
\hline 3133914 & 2009 & 215 & -46.248 & 166.279 & 33.000 & 3.8 \\
\hline 3134050 & 2009 & 215 & -41.460 & 172.926 & 111.391 & 3.2 \\
\hline 3134115 & 2009 & 216 & -41.811 & 174.345 & 29.308 & 4.2 \\
\hline 3134378 & 2009 & 216 & -45.498 & 166.660 & 22.454 & 3.8 \\
\hline 3134387 & 2009 & 216 & -44.593 & 168.208 & 81.415 & 3.0 \\
\hline 3134616 & 2009 & 200 & -45.413 & 166.714 & 20.706 & 4.0 \\
\hline 3135253 & 2009 & 218 & -45.442 & 166.676 & 21.755 & 3.5 \\
\hline 3135313 & 2009 & 218 & -45.476 & 166.746 & 21.993 & 3.8 \\
\hline 3135487 & 2009 & 200 & -46.106 & 166.206 & 32.296 & 3.6 \\
\hline 3135552 & 2009 & 219 & -45.858 & 166.425 & 39.614 & 3.7 \\
\hline 3135754 & 2009 & 219 & -45.551 & 166.658 & 20.658 & 4.5 \\
\hline 3136366 & 2009 & 220 & -41.314 & 173.396 & 106.713 & 3.0 \\
\hline 3136653 & 2009 & 221 & -41.067 & 174.027 & 60.141 & 3.5 \\
\hline 3136713 & 2009 & 221 & -45.542 & 166.627 & 23.347 & 4.8 \\
\hline 3137032 & 2009 & 198 & -45.491 & 166.569 & 21.123 & 3.6 \\
\hline 3137460 & 2009 & 223 & -45.963 & 166.434 & 30.215 & 3.7 \\
\hline 3137567 & 2009 & 223 & -41.497 & 172.590 & 105.322 & 2.3 \\
\hline 3137861 & 2009 & 198 & -45.380 & 166.703 & 21.864 & 3.7 \\
\hline 3138040 & 2009 & 224 & -42.249 & 173.018 & 63.885 & 2.3 \\
\hline 3138587 & 2009 & 225 & -45.339 & 166.767 & 26.962 & 3.7 \\
\hline 3138705 & 2009 & 225 & -41.370 & 172.838 & 122.640 & 3.0 \\
\hline 3139106 & 2009 & 226 & -43.256 & 170.790 & 3.000 & 4.1 \\
\hline 3139431 & 2009 & 227 & -41.293 & 172.778 & 139.405 & 2.6 \\
\hline 3139540 & 2009 & 227 & -41.497 & 174.177 & 61.478 & 3.1 \\
\hline 3139605 & 2009 & 227 & -43.207 & 170.921 & 5.000 & 2.6 \\
\hline 3139869 & 2009 & 228 & -41.362 & 172.767 & 136.649 & 3.1 \\
\hline 3139883 & 2009 & 228 & -45.668 & 166.651 & 21.164 & 3.5 \\
\hline 3140195 & 2009 & 229 & -45.552 & 166.656 & 22.020 & 4.1 \\
\hline 3140201 & 2009 & 229 & -42.800 & 172.885 & 33.000 & 2.9 \\
\hline 3140802 & 2009 & 230 & -43.260 & 170.919 & 12.000 & 2.9 \\
\hline 3141083 & 2009 & 231 & -44.642 & 168.156 & 81.365 & 3.5 \\
\hline 3141134 & 2009 & 231 & -41.739 & 172.297 & 96.294 & 2.6 \\
\hline
\end{tabular}


Table A.2 - Continued

\begin{tabular}{|c|c|c|c|c|c|c|}
\hline cuspid & year & jul.day & lat. $^{\circ}$ & lon. ${ }^{\circ}$ & depth & mag. \\
\hline 3141338 & 2009 & 231 & -40.530 & 173.610 & 118.346 & 3.8 \\
\hline 3141375 & 2009 & 231 & -41.726 & 172.188 & 100.391 & 3.3 \\
\hline 3141471 & 2009 & 231 & -42.992 & 172.660 & 12.000 & 2.4 \\
\hline 3141478 & 2009 & 231 & -46.032 & 166.272 & 33.000 & 3.6 \\
\hline 3141703 & 2009 & 232 & -44.594 & 168.173 & 80.351 & 3.3 \\
\hline 3141850 & 2009 & 232 & -41.714 & 172.304 & 91.897 & 2.4 \\
\hline 3142187 & 2009 & 233 & -42.078 & 172.786 & 56.183 & 2.2 \\
\hline 3142436 & 2009 & 234 & -42.713 & 171.693 & 5.000 & 2.7 \\
\hline 3142644 & 2009 & 234 & -41.161 & 172.859 & 145.078 & 3.5 \\
\hline 3143358 & 2009 & 199 & -45.475 & 166.638 & 22.118 & 4.1 \\
\hline 3143553 & 2009 & 236 & -41.621 & 172.818 & 86.083 & 2.7 \\
\hline 3143670 & 2009 & 199 & -45.747 & 166.529 & 22.234 & 3.5 \\
\hline 3143705 & 2009 & 237 & -45.150 & 167.280 & 121.351 & 3.8 \\
\hline 3144863 & 2009 & 199 & -45.496 & 166.634 & 19.625 & 3.7 \\
\hline 3145196 & 2009 & 239 & -41.434 & 174.702 & 33.979 & 4.3 \\
\hline 3145383 & 2009 & 240 & -45.499 & 166.789 & 17.847 & 4.0 \\
\hline 3145405 & 2009 & 240 & -43.399 & 170.791 & 5.000 & 2.8 \\
\hline 3145684 & 2009 & 240 & -45.510 & 166.481 & 19.859 & 4.0 \\
\hline 3146145 & 2009 & 241 & -40.910 & 172.832 & 214.090 & 3.5 \\
\hline 3146444 & 2009 & 242 & -45.539 & 166.552 & 20.120 & 4.9 \\
\hline 3146610 & 2009 & 242 & -45.429 & 166.770 & 23.174 & 3.9 \\
\hline 3147147 & 2009 & 243 & -41.603 & 173.409 & 76.593 & 2.9 \\
\hline 3147878 & 2009 & 244 & -41.844 & 172.722 & 94.546 & 2.7 \\
\hline 3148257 & 2009 & 228 & -41.314 & 172.584 & 183.094 & 3.4 \\
\hline 3148278 & 2009 & 245 & -45.438 & 166.582 & 22.305 & 4.0 \\
\hline 3148588 & 2009 & 245 & -40.926 & 172.922 & 187.429 & 4.0 \\
\hline 3148730 & 2009 & 228 & -41.431 & 173.680 & 50.641 & 2.5 \\
\hline 3148981 & 2009 & 246 & -41.681 & 172.714 & 77.311 & 2.0 \\
\hline 3149453 & 2009 & 247 & -46.261 & 166.292 & 33.000 & 4.9 \\
\hline 3149488 & 2009 & 247 & -42.136 & 172.758 & 66.106 & 2.6 \\
\hline 3150135 & 2009 & 249 & -40.915 & 172.912 & 220.487 & 3.4 \\
\hline 3150506 & 2009 & 249 & -41.255 & 172.764 & 111.313 & 2.1 \\
\hline 3150827 & 2009 & 250 & -41.309 & 172.827 & 139.828 & 3.8 \\
\hline 3150963 & 2009 & 250 & -42.060 & 172.849 & 74.711 & 3.0 \\
\hline 3151539 & 2009 & 252 & -45.535 & 166.695 & 23.847 & 4.0 \\
\hline 3151678 & 2009 & 252 & -41.292 & 172.719 & 156.583 & 3.3 \\
\hline 3151852 & 2009 & 252 & -45.546 & 166.690 & 20.138 & 4.9 \\
\hline 3152083 & 2009 & 253 & -40.725 & 174.347 & 72.184 & 4.1 \\
\hline 3152416 & 2009 & 254 & -41.781 & 172.626 & 80.212 & 2.7 \\
\hline 3152666 & 2009 & 254 & -43.066 & 173.530 & 12.000 & 2.5 \\
\hline 3152685 & 2009 & 254 & -40.813 & 174.620 & 44.762 & 3.7 \\
\hline 3153108 & 2009 & 255 & -41.311 & 174.546 & 41.211 & 3.6 \\
\hline 3153511 & 2009 & 256 & -43.053 & 172.816 & 29.129 & 3.4 \\
\hline 3153542 & 2009 & 256 & -41.280 & 172.554 & 148.831 & 2.7 \\
\hline 3154121 & 2009 & 247 & -42.717 & 171.684 & 5.000 & 2.6 \\
\hline 3155299 & 2009 & 260 & -45.094 & 167.368 & 123.759 & 4.2 \\
\hline 3155307 & 2009 & 260 & -41.679 & 172.516 & 84.173 & 2.1 \\
\hline 3156245 & 2009 & 262 & -41.262 & 172.576 & 193.485 & 3.3 \\
\hline 3156489 & 2009 & 262 & -42.761 & 171.933 & 5.000 & 3.1 \\
\hline 3157895 & 2009 & 265 & -41.724 & 173.195 & 73.163 & 3.0 \\
\hline 3158405 & 2009 & 266 & -41.014 & 173.225 & 129.210 & 3.3 \\
\hline 3158502 & 2009 & 267 & -41.748 & 172.473 & 80.029 & 3.2 \\
\hline 3158781 & 2009 & 267 & -42.740 & 171.980 & 12.000 & 3.2 \\
\hline 3159511 & 2009 & 269 & -41.727 & 172.215 & 100.475 & 3.1 \\
\hline 3159733 & 2009 & 270 & -45.709 & 166.541 & 16.656 & 3.8 \\
\hline 3159932 & 2009 & 270 & -41.424 & 174.243 & 63.695 & 3.3 \\
\hline 3160158 & 2009 & 270 & -41.331 & 173.291 & 78.377 & 3.5 \\
\hline 3160218 & 2009 & 271 & -43.024 & 172.569 & 26.633 & 2.2 \\
\hline 3160832 & 2009 & 272 & -41.447 & 173.271 & 82.509 & 2.6 \\
\hline 3160872 & 2009 & 272 & -43.245 & 171.020 & 5.000 & 2.8 \\
\hline 3161447 & 2009 & 273 & -45.305 & 166.844 & 27.355 & 3.7 \\
\hline 3161988 & 2009 & 274 & -45.392 & 166.700 & 21.507 & 3.6 \\
\hline 3162137 & 2009 & 275 & -44.592 & 168.173 & 81.789 & 3.1 \\
\hline 3162238 & 2009 & 275 & -42.984 & 171.469 & 5.000 & 3.5 \\
\hline 3162524 & 2009 & 275 & -41.448 & 172.821 & 110.288 & 2.4 \\
\hline 3163469 & 2009 & 278 & -40.966 & 173.431 & 89.585 & 3.1 \\
\hline 3163716 & 2009 & 278 & -41.718 & 172.277 & 98.095 & 2.6 \\
\hline 3163741 & 2009 & 278 & -45.440 & 166.627 & 21.401 & 3.5 \\
\hline 3164576 & 2009 & 280 & -42.114 & 172.815 & 55.253 & 2.9 \\
\hline 3164688 & 2009 & 280 & -41.218 & 172.628 & 212.621 & 3.1 \\
\hline
\end{tabular}


Table A.2 - Continued

\begin{tabular}{|c|c|c|c|c|c|c|}
\hline cuspid & year & jul.day & lat. $^{\circ}$ & lon. ${ }^{\circ}$ & depth & mag \\
\hline 3165024 & 2009 & 281 & -45.561 & 166.723 & 21.242 & 4.8 \\
\hline 3165121 & 2009 & 281 & -41.458 & 172.827 & 89.094 & 2.4 \\
\hline 3165297 & 2009 & 281 & -41.525 & 172.760 & 97.597 & 2.8 \\
\hline 3166123 & 2009 & 283 & -43.191 & 170.807 & 5.000 & 2.6 \\
\hline 3166147 & 2009 & 283 & -41.274 & 172.647 & 177.074 & 3.0 \\
\hline 3166368 & 2009 & 262 & -43.040 & 172.670 & 5.000 & 2.1 \\
\hline 3166997 & 2009 & 285 & -45.062 & 167.386 & 92.545 & 3.6 \\
\hline 3167025 & 2009 & 285 & -41.714 & 172.265 & 100.618 & 2.4 \\
\hline 3167546 & 2009 & 286 & -41.269 & 173.251 & 111.029 & 2.9 \\
\hline 3167757 & 2009 & 287 & -41.256 & 172.772 & 137.411 & 2.9 \\
\hline 3168364 & 2009 & 288 & -43.174 & 171.586 & 5.000 & 2.3 \\
\hline 3168423 & 2009 & 288 & -45.604 & 166.766 & 18.208 & 3.5 \\
\hline 3168594 & 2009 & 288 & -41.258 & 172.742 & 154.633 & 2.8 \\
\hline 3168595 & 2009 & 280 & -41.247 & 172.618 & 154.227 & 2.4 \\
\hline 3170003 & 2009 & 290 & -42.735 & 172.420 & 5.000 & 2.6 \\
\hline 3170073 & 2009 & 290 & -45.083 & 167.454 & 94.385 & 4.1 \\
\hline 3170768 & 2009 & 292 & -43.311 & 171.030 & 5.000 & 2.6 \\
\hline 3170794 & 2009 & 292 & -41.404 & 174.285 & 61.820 & 3.0 \\
\hline 3171001 & 2009 & 292 & -45.148 & 167.089 & 33.000 & 4.3 \\
\hline 3171353 & 2009 & 293 & -41.460 & 173.537 & 70.743 & 2.7 \\
\hline 3171506 & 2009 & 293 & -41.706 & 172.205 & 102.110 & 2.5 \\
\hline 3171550 & 2009 & 293 & -45.665 & 166.876 & 62.468 & 3.6 \\
\hline 3171667 & 2009 & 294 & -40.715 & 174.451 & 67.502 & 3.1 \\
\hline 3171768 & 2009 & 294 & -42.879 & 171.606 & 5.000 & 2.6 \\
\hline 3171833 & 2009 & 294 & -41.064 & 172.958 & 155.883 & 3.6 \\
\hline 3172135 & 2009 & 274 & -41.201 & 172.595 & 211.171 & 3.0 \\
\hline 3172220 & 2009 & 295 & -41.285 & 172.933 & 117.325 & 3.7 \\
\hline 3172297 & 2009 & 295 & -42.414 & 171.514 & 12.000 & 2.5 \\
\hline 3172529 & 2009 & 295 & -41.096 & 173.597 & 92.472 & 3.5 \\
\hline 3172864 & 2009 & 296 & -41.865 & 172.449 & 79.415 & 2.6 \\
\hline 3173133 & 2009 & 297 & -41.283 & 172.720 & 147.248 & 3.0 \\
\hline 3173202 & 2009 & 297 & -41.749 & 173.399 & 44.254 & 2.1 \\
\hline 3173371 & 2009 & 297 & -41.133 & 173.188 & 113.744 & 3.0 \\
\hline 3174442 & 2009 & 299 & -45.134 & 167.331 & 114.108 & 3.7 \\
\hline 3176325 & 2009 & 303 & -40.537 & 173.355 & 179.809 & 3.4 \\
\hline 3176331 & 2009 & 303 & -43.103 & 172.098 & 5.000 & 2.1 \\
\hline 3177164 & 2009 & 305 & -43.198 & 172.225 & 29.387 & 2.1 \\
\hline 3177784 & 2009 & 306 & -41.141 & 173.411 & 95.189 & 2.7 \\
\hline 3178098 & 2009 & 307 & -40.969 & 173.820 & 79.762 & 3.1 \\
\hline 3178429 & 2009 & 308 & -42.048 & 172.761 & 89.719 & 4.5 \\
\hline 3178945 & 2009 & 309 & -41.694 & 172.060 & 23.009 & 2.5 \\
\hline 3181530 & 2009 & 314 & -42.476 & 172.891 & 42.801 & 3.5 \\
\hline 3182026 & 2009 & 315 & -40.537 & 173.544 & 153.829 & 3.4 \\
\hline 3182458 & 2009 & 316 & -45.463 & 166.746 & 21.672 & 4.0 \\
\hline 3183648 & 2009 & 318 & -41.814 & 174.435 & 20.547 & 3.7 \\
\hline 3185157 & 2009 & 321 & -41.215 & 172.635 & 129.567 & 2.0 \\
\hline 3185640 & 2009 & 322 & -41.389 & 172.348 & 159.390 & 2.7 \\
\hline 3186228 & 2009 & 323 & -43.218 & 172.871 & 33.000 & 4.2 \\
\hline 3187010 & 2009 & 325 & -45.021 & 167.431 & 89.211 & 3.5 \\
\hline 3187561 & 2009 & 326 & -42.530 & 171.929 & 5.000 & 2.6 \\
\hline 3188308 & 2009 & 328 & -44.603 & 168.197 & 88.074 & 3.0 \\
\hline 3188343 & 2009 & 328 & -43.531 & 173.028 & 32.755 & 2.9 \\
\hline 3188409 & 2009 & 197 & -45.510 & 166.549 & 16.951 & 4.2 \\
\hline 3190570 & 2009 & 332 & -43.339 & 171.961 & 8.759 & 3.5 \\
\hline 3190843 & 2009 & 333 & -45.258 & 166.970 & 33.000 & 3.8 \\
\hline 3202873 & 2009 & 336 & -44.941 & 167.103 & 20.512 & 3.7 \\
\hline 3202903 & 2009 & 336 & -42.964 & 172.437 & 5.000 & 3.2 \\
\hline 3204025 & 2009 & 339 & -40.805 & 172.910 & 209.131 & 4.0 \\
\hline 3204627 & 2009 & 340 & -41.359 & 173.801 & 53.861 & 3.3 \\
\hline 3205351 & 2009 & 342 & -46.011 & 166.840 & 81.918 & 4.1 \\
\hline 3205715 & 2009 & 342 & -40.562 & 174.853 & 48.911 & 5.1 \\
\hline 3206052 & 2009 & 343 & -45.117 & 167.373 & 95.279 & 3.8 \\
\hline 3206940 & 2009 & 345 & -41.083 & 174.318 & 67.175 & 3.7 \\
\hline 3207677 & 2009 & 346 & -43.139 & 172.290 & 30.905 & 2.3 \\
\hline 3211310 & 2009 & 353 & -45.000 & 167.494 & 97.385 & 3.6 \\
\hline 3213039 & 2009 & 357 & -41.838 & 172.885 & 85.668 & 2.3 \\
\hline 3213667 & 2009 & 359 & -44.533 & 167.708 & 8.983 & 3.0 \\
\hline 3213731 & 2009 & 359 & -41.760 & 172.748 & 87.916 & 2.5 \\
\hline 3215575 & 2009 & 363 & -43.136 & 170.989 & 5.000 & 2.8 \\
\hline 3219434 & 2009 & 361 & -41.950 & 173.029 & 48.971 & 2.2 \\
\hline
\end{tabular}


Table A.2 - Continued

\begin{tabular}{|c|c|c|c|c|c|c|}
\hline cuspid & year & jul.day & lat. $^{\circ}$ & lon. ${ }^{\circ}$ & depth & mag \\
\hline 3219727 & 2010 & 006 & -45.066 & 167.404 & 88.520 & 3.8 \\
\hline 3228164 & 2010 & 008 & -45.133 & 167.244 & 84.824 & 3.8 \\
\hline 3229793 & 2010 & 011 & -45.101 & 167.342 & 116.846 & 3.7 \\
\hline 3229901 & 2010 & 012 & -44.167 & 168.445 & 5.000 & 3.7 \\
\hline 3239754 & 2010 & 013 & -43.084 & 171.430 & 5.000 & 3.4 \\
\hline 3241870 & 2010 & 014 & -44.133 & 168.387 & 5.000 & 4.6 \\
\hline 3241943 & 2010 & 014 & -42.998 & 171.442 & 9.424 & 2.9 \\
\hline 3242948 & 2010 & 016 & -44.306 & 168.080 & 5.000 & 3.4 \\
\hline 3243091 & 2010 & 016 & -43.200 & 173.495 & 33.000 & 3.8 \\
\hline 3244016 & 2010 & 019 & -45.251 & 167.310 & 155.558 & 3.7 \\
\hline 3245798 & 2010 & 022 & -44.186 & 168.443 & 2.000 & 3.1 \\
\hline 3245861 & 2010 & 022 & -45.479 & 166.694 & 12.000 & 4.6 \\
\hline 3245867 & 2010 & 022 & -45.465 & 166.761 & 12.000 & 4.1 \\
\hline 3245913 & 2010 & 022 & -44.164 & 168.423 & 5.000 & 4.1 \\
\hline 3246039 & 2010 & 023 & -41.203 & 172.747 & 169.818 & 3.5 \\
\hline 3246628 & 2010 & 024 & -46.067 & 166.815 & 81.818 & 3.8 \\
\hline 3253233 & 2010 & 037 & -44.570 & 168.146 & 87.847 & 3.6 \\
\hline 3253712 & 2010 & 038 & -41.258 & 172.602 & 211.170 & 3.6 \\
\hline 3254077 & 2010 & 039 & -45.364 & 166.700 & 12.000 & 3.7 \\
\hline 3254472 & 2010 & 040 & -41.952 & 172.605 & 78.788 & 4.4 \\
\hline 3255116 & 2010 & 041 & -42.525 & 172.660 & 5.000 & 3.2 \\
\hline 3259829 & 2010 & 042 & -45.149 & 167.237 & 77.023 & 4.0 \\
\hline 3260765 & 2010 & 044 & -45.368 & 167.022 & 63.231 & 3.6 \\
\hline 3260895 & 2010 & 044 & -46.092 & 166.213 & 12.000 & 4.7 \\
\hline 3261607 & 2010 & 046 & -45.430 & 166.678 & 12.000 & 3.7 \\
\hline 3261808 & 2010 & 046 & -40.915 & 172.911 & 220.784 & 3.6 \\
\hline 3267369 & 2010 & 057 & -44.843 & 167.314 & 5.000 & 3.6 \\
\hline 3267529 & 2010 & 058 & -44.839 & 167.320 & 5.000 & 3.8 \\
\hline 3267555 & 2010 & 058 & -44.838 & 167.298 & 5.000 & 3.8 \\
\hline 3267698 & 2010 & 058 & -46.020 & 166.884 & 5.000 & 4.4 \\
\hline 3268042 & 2010 & 059 & -45.684 & 166.965 & 89.415 & 5.0 \\
\hline 3269429 & 2010 & 062 & -45.772 & 166.555 & 5.000 & 3.6 \\
\hline 3270055 & 2010 & 063 & -44.298 & 167.032 & 5.000 & 3.7 \\
\hline 3270861 & 2010 & 065 & -45.492 & 166.730 & 9.120 & 3.7 \\
\hline 3273161 & 2010 & 069 & -44.815 & 167.423 & 5.000 & 3.5 \\
\hline 3274567 & 2010 & 072 & -44.287 & 166.955 & 2.000 & 3.5 \\
\hline 3274724 & 2010 & 072 & -45.529 & 166.659 & 12.000 & 4.9 \\
\hline 3275824 & 2010 & 075 & -45.430 & 166.957 & 59.410 & 4.0 \\
\hline 3275857 & 2010 & 075 & -43.062 & 173.006 & 33.000 & 3.6 \\
\hline 3275957 & 2010 & 075 & -42.120 & 173.836 & 12.000 & 4.0 \\
\hline 3276884 & 2010 & 077 & -45.646 & 167.213 & 5.000 & 3.5 \\
\hline 3277280 & 2010 & 077 & -41.243 & 172.766 & 144.901 & 3.9 \\
\hline 3277943 & 2010 & 079 & -45.074 & 167.436 & 63.685 & 4.3 \\
\hline 3278236 & 2010 & 079 & -44.257 & 167.739 & 2.000 & 3.1 \\
\hline 3281249 & 2010 & 085 & -45.109 & 167.480 & 114.562 & 3.7 \\
\hline 3283355 & 2010 & 089 & -44.362 & 167.067 & 12.000 & 3.2 \\
\hline 3284490 & 2010 & 091 & -40.817 & 172.956 & 215.673 & 3.6 \\
\hline 3285425 & 2010 & 093 & -43.240 & 172.746 & 33.000 & 3.8 \\
\hline 3287387 & 2010 & 097 & -44.869 & 170.940 & 12.000 & 3.6 \\
\hline 3287792 & 2010 & 098 & -41.370 & 172.856 & 115.590 & 4.0 \\
\hline 3288443 & 2010 & 099 & -44.609 & 168.120 & 91.969 & 3.2 \\
\hline 3291715 & 2010 & 105 & -40.653 & 173.334 & 144.549 & 3.5 \\
\hline 3293147 & 2010 & 108 & -45.109 & 167.462 & 70.767 & 4.5 \\
\hline 3295053 & 2010 & 112 & -40.575 & 174.168 & 80.485 & 3.5 \\
\hline 3296413 & 2010 & 115 & -45.092 & 167.437 & 102.686 & 3.5 \\
\hline 3296708 & 2010 & 115 & -42.123 & 173.804 & 12.000 & 3.9 \\
\hline 3299011 & 2010 & 119 & -44.173 & 168.389 & 2.000 & 3.1 \\
\hline 3299270 & 2010 & 120 & -41.262 & 172.831 & 117.499 & 4.2 \\
\hline 3299486 & 2010 & 120 & -40.765 & 173.915 & 110.232 & 4.4 \\
\hline 3301720 & 2010 & 125 & -40.690 & 174.121 & 85.442 & 3.7 \\
\hline 3301978 & 2010 & 125 & -45.230 & 166.946 & 12.000 & 3.5 \\
\hline 3302061 & 2010 & 125 & -45.157 & 166.801 & 12.000 & 4.5 \\
\hline 3302132 & 2010 & 125 & -44.346 & 168.120 & 5.000 & 3.2 \\
\hline 3302261 & 2010 & 126 & -45.073 & 167.610 & 121.619 & 3.7 \\
\hline 3302929 & 2010 & 127 & -44.593 & 168.119 & 88.338 & 3.2 \\
\hline 3303093 & 2010 & 127 & -44.874 & 167.477 & 5.000 & 3.6 \\
\hline 3306416 & 2010 & 133 & -42.787 & 172.431 & 23.211 & 3.1 \\
\hline 3306930 & 2010 & 134 & -44.583 & 168.114 & 91.003 & 3.1 \\
\hline 3309345 & 2010 & 139 & -45.111 & 167.226 & 39.702 & 3.7 \\
\hline 3310436 & 2010 & 141 & -41.253 & 172.834 & 141.856 & 3.6 \\
\hline
\end{tabular}


Table A.2 - Continued

\begin{tabular}{|c|c|c|c|c|c|c|}
\hline cuspid & year & jul.day & lat. ${ }^{\circ}$ & lon. ${ }^{\circ}$ & depth & mag. \\
\hline 3312931 & 2010 & 145 & -44.611 & 168.125 & 88.426 & 3.5 \\
\hline 3313020 & 2010 & 146 & -43.380 & 172.533 & 12.000 & 3.1 \\
\hline 3315096 & 2010 & 150 & -42.575 & 172.916 & 12.000 & 3.9 \\
\hline 3315329 & 2010 & 150 & -43.598 & 170.629 & 5.000 & 3.0 \\
\hline 3316380 & 2010 & 152 & -44.670 & 168.120 & 89.393 & 3.0 \\
\hline 3316474 & 2010 & 152 & -42.476 & 173.329 & 53.212 & 4.3 \\
\hline 3316477 & 2010 & 152 & -42.550 & 173.352 & 50.625 & 3.4 \\
\hline 3317333 & 2010 & 154 & -44.188 & 168.348 & 5.000 & 3.2 \\
\hline 3317665 & 2010 & 155 & -41.155 & 173.446 & 110.390 & 3.6 \\
\hline 3322168 & 2010 & 163 & -44.400 & 168.288 & 5.000 & 3.3 \\
\hline 3323536 & 2010 & 166 & -41.286 & 172.596 & 189.853 & 3.6 \\
\hline 3326488 & 2010 & 171 & -45.088 & 167.332 & 126.430 & 3.8 \\
\hline 3326729 & 2010 & 171 & -45.139 & 167.311 & 85.557 & 3.6 \\
\hline 3327324 & 2010 & 173 & -45.120 & 167.328 & 88.927 & 3.7 \\
\hline 3327568 & 2010 & 173 & -44.154 & 168.585 & 2.000 & 3.3 \\
\hline 3327688 & 2010 & 173 & -46.838 & 166.324 & 33.000 & 3.8 \\
\hline 3327812 & 2010 & 173 & -45.135 & 167.368 & 85.541 & 3.8 \\
\hline 3327852 & 2010 & 174 & -45.429 & 166.753 & 12.000 & 3.9 \\
\hline 3328970 & 2010 & 176 & -45.685 & 166.634 & 5.000 & 3.5 \\
\hline 3329809 & 2010 & 177 & -42.707 & 171.705 & 5.000 & 3.5 \\
\hline 3332362 & 2010 & 182 & -44.233 & 171.569 & 12.000 & 3.1 \\
\hline 3336735 & 2010 & 190 & -45.724 & 166.534 & 12.000 & 3.5 \\
\hline 3338289 & 2010 & 193 & -40.869 & 172.860 & 218.186 & 4.2 \\
\hline 3338766 & 2010 & 194 & -40.512 & 173.562 & 142.791 & 4.9 \\
\hline 3339606 & 2010 & 125 & -45.153 & 166.759 & 12.000 & 3.9 \\
\hline 3341085 & 2010 & 198 & -43.675 & 168.284 & 5.000 & 3.5 \\
\hline 3341372 & 2010 & 199 & -41.239 & 172.799 & 154.449 & 3.6 \\
\hline 3341682 & 2010 & 200 & -45.213 & 167.239 & 76.964 & 4.4 \\
\hline 3344300 & 2010 & 205 & -42.819 & 172.298 & 12.000 & 3.0 \\
\hline 3346364 & 2010 & 208 & -45.075 & 167.317 & 101.166 & 4.6 \\
\hline 3346371 & 2010 & 208 & -45.036 & 167.412 & 116.906 & 3.7 \\
\hline 3346616 & 2010 & 209 & -45.164 & 167.340 & 118.585 & 4.0 \\
\hline 3348741 & 2010 & 213 & -46.020 & 166.288 & 20.000 & 3.5 \\
\hline 3349104 & 2010 & 214 & -41.264 & 172.950 & 114.212 & 4.3 \\
\hline 3350372 & 2010 & 216 & -45.139 & 167.390 & 87.999 & 4.4 \\
\hline 3352922 & 2010 & 221 & -45.039 & 167.476 & 94.879 & 4.5 \\
\hline 3353879 & 2010 & 223 & -45.106 & 167.147 & 33.000 & 3.4 \\
\hline 3358415 & 2010 & 232 & -40.617 & 173.204 & 162.130 & 3.7 \\
\hline 3358526 & 2010 & 232 & -45.200 & 166.868 & 33.000 & 3.7 \\
\hline 3358599 & 2010 & 232 & -42.825 & 171.810 & 5.000 & 3.7 \\
\hline 3362156 & 2010 & 239 & -43.210 & 171.991 & 5.000 & 3.3 \\
\hline 3363606 & 2010 & 241 & -41.166 & 173.969 & 61.378 & 4.0 \\
\hline 3364383 & 2010 & 243 & -41.273 & 172.551 & 209.676 & 3.6 \\
\hline 3364515 & 2010 & 243 & -44.171 & 168.609 & 5.000 & 3.3 \\
\hline 3365555 & 2010 & 245 & -44.304 & 168.091 & 5.000 & 3.4 \\
\hline 3365975 & 2010 & 246 & -44.489 & 168.143 & 12.000 & 3.7 \\
\hline 3366206 & 2010 & 246 & -43.603 & 172.385 & 5.000 & 3.9 \\
\hline 3366305 & 2010 & 246 & -43.604 & 172.346 & 5.000 & 3.9 \\
\hline 3366329 & 2010 & 246 & -43.546 & 172.374 & 5.000 & 3.9 \\
\hline 3366506 & 2010 & 247 & -43.548 & 172.422 & 12.000 & 3.8 \\
\hline 3366542 & 2010 & 247 & -43.748 & 172.310 & 5.000 & 3.9 \\
\hline 3366601 & 2010 & 247 & -41.190 & 172.573 & 215.865 & 3.8 \\
\hline 3366669 & 2010 & 247 & -43.507 & 172.201 & 5.947 & 3.2 \\
\hline 3366754 & 2010 & 247 & -43.595 & 172.190 & 12.646 & 4.1 \\
\hline 3366806 & 2010 & 247 & -43.579 & 171.963 & 5.000 & 3.7 \\
\hline 3366920 & 2010 & 248 & -43.563 & 171.928 & 3.117 & 3.8 \\
\hline 3367084 & 2010 & 248 & -43.552 & 171.916 & 10.244 & 3.3 \\
\hline 3367309 & 2010 & 248 & -43.596 & 171.852 & 5.000 & 3.3 \\
\hline 3367331 & 2010 & 248 & -43.609 & 172.219 & 5.000 & 3.2 \\
\hline 3367453 & 2010 & 248 & -43.468 & 172.166 & 7.223 & 3.5 \\
\hline 3367923 & 2010 & 249 & -43.566 & 171.880 & 7.858 & 3.5 \\
\hline 3367932 & 2010 & 249 & -41.260 & 172.622 & 213.314 & 3.7 \\
\hline 3368008 & 2010 & 249 & -43.526 & 171.879 & 10.020 & 3.9 \\
\hline 3368099 & 2010 & 250 & -41.346 & 174.529 & 40.330 & 3.8 \\
\hline 3368110 & 2010 & 250 & -41.353 & 174.535 & 39.662 & 3.7 \\
\hline 3368872 & 2010 & 251 & -45.147 & 167.349 & 91.204 & 3.8 \\
\hline 3369037 & 2010 & 251 & -43.634 & 171.975 & 5.000 & 3.0 \\
\hline 3369555 & 2010 & 252 & -43.484 & 172.122 & 5.000 & 3.0 \\
\hline 3369677 & 2010 & 252 & -43.587 & 172.025 & 9.251 & 3.5 \\
\hline 3370794 & 2010 & 254 & -43.540 & 171.876 & 10.464 & 4.1 \\
\hline
\end{tabular}


Table A.2 - Continued

\begin{tabular}{|c|c|c|c|c|c|c|}
\hline cuspid & year & jul.day & lat. $^{\circ}$ & lon. ${ }^{\circ}$ & depth & mag. \\
\hline 3370904 & 2010 & 255 & -43.453 & 172.121 & 6.311 & 3.3 \\
\hline 3371267 & 2010 & 255 & -43.599 & 172.107 & 5.000 & 3.2 \\
\hline 3371307 & 2010 & 255 & -42.450 & 173.696 & 10.157 & 3.6 \\
\hline 3372347 & 2010 & 258 & -42.450 & 173.706 & 9.925 & 3.7 \\
\hline 3372458 & 2010 & 258 & -42.441 & 173.696 & 13.599 & 3.7 \\
\hline 3375021 & 2010 & 246 & -43.652 & 172.413 & 5.000 & 4.5 \\
\hline 3375052 & 2010 & 248 & -43.493 & 172.170 & 6.004 & 3.1 \\
\hline 3375623 & 2010 & 248 & -43.285 & 171.942 & 7.918 & 3.8 \\
\hline 3375633 & 2010 & 248 & -43.296 & 171.935 & 8.206 & 3.3 \\
\hline 3375648 & 2010 & 249 & -43.430 & 172.139 & 5.000 & 3.2 \\
\hline 3376108 & 2010 & 264 & -40.873 & 172.909 & 215.607 & 3.6 \\
\hline 3376255 & 2010 & 250 & -43.549 & 171.936 & 5.000 & 3.1 \\
\hline 3376734 & 2010 & 248 & -43.590 & 172.178 & 13.465 & 3.7 \\
\hline 3376937 & 2010 & 266 & -45.342 & 166.857 & 12.000 & 3.6 \\
\hline 3376944 & 2010 & 248 & -43.616 & 172.607 & 5.000 & 3.4 \\
\hline 3377329 & 2010 & 248 & -43.563 & 172.387 & 5.000 & 3.1 \\
\hline 3378555 & 2010 & 248 & -43.625 & 172.387 & 5.000 & 3.1 \\
\hline 3379185 & 2010 & 270 & -42.479 & 173.035 & 9.697 & 4.3 \\
\hline 3379423 & 2010 & 248 & -43.623 & 172.240 & 5.000 & 3.1 \\
\hline 3381332 & 2010 & 274 & -41.630 & 174.092 & 12.000 & 3.6 \\
\hline 3384822 & 2010 & 281 & -41.336 & 173.723 & 73.492 & 4.0 \\
\hline 3386185 & 2010 & 248 & -43.586 & 172.375 & 5.000 & 3.2 \\
\hline 3387174 & 2010 & 285 & -45.043 & 167.401 & 111.853 & 4.7 \\
\hline 3389489 & 2010 & 248 & -43.558 & 171.891 & 5.000 & 3.4 \\
\hline 3390981 & 2010 & 248 & -43.572 & 172.385 & 5.000 & 3.3 \\
\hline 3392078 & 2010 & 248 & -43.583 & 172.186 & 5.000 & 3.0 \\
\hline 3392092 & 2010 & 248 & -43.543 & 171.887 & 8.899 & 3.7 \\
\hline 3392139 & 2010 & 248 & -43.621 & 172.418 & 5.000 & 3.4 \\
\hline 3392641 & 2010 & 248 & -43.606 & 172.068 & 5.000 & 3.0 \\
\hline 3392673 & 2010 & 293 & -41.237 & 173.319 & 102.931 & 4.3 \\
\hline 3393152 & 2010 & 247 & -43.659 & 172.440 & 5.000 & 3.0 \\
\hline 3393206 & 2010 & 294 & -45.536 & 166.645 & 12.000 & 3.5 \\
\hline 3394547 & 2010 & 297 & -44.954 & 167.473 & 64.402 & 3.5 \\
\hline 3400618 & 2010 & 305 & -42.480 & 172.962 & 12.000 & 3.6 \\
\hline 3401807 & 2010 & 307 & -40.952 & 172.813 & 241.865 & 3.6 \\
\hline 3405049 & 2010 & 314 & -45.164 & 167.378 & 118.524 & 3.7 \\
\hline 3407572 & 2010 & 319 & -45.698 & 166.461 & 5.000 & 3.9 \\
\hline 3408796 & 2010 & 321 & -40.903 & 172.897 & 220.197 & 3.6 \\
\hline 3412101 & 2010 & 328 & -45.390 & 166.669 & 26.097 & 3.7 \\
\hline 3413579 & 2010 & 331 & -45.714 & 166.573 & 5.000 & 3.7 \\
\hline 3414693 & 2010 & 333 & -45.231 & 167.334 & 70.062 & 3.8 \\
\hline 3415424 & 2010 & 335 & -45.176 & 167.353 & 135.442 & 4.1 \\
\hline 3416605 & 2010 & 337 & -41.436 & 173.335 & 82.453 & 3.7 \\
\hline 3417589 & 2010 & 339 & -41.175 & 174.639 & 33.000 & 3.7 \\
\hline 3420141 & 2010 & 345 & -46.285 & 166.178 & 12.000 & 4.0 \\
\hline 3420248 & 2010 & 345 & -45.451 & 167.107 & 53.942 & 4.0 \\
\hline 3420825 & 2010 & 346 & -45.113 & 167.384 & 97.743 & 4.3 \\
\hline 3421891 & 2010 & 349 & -41.753 & 172.210 & 100.618 & 3.5 \\
\hline 3422970 & 2010 & 349 & -41.266 & 172.563 & 225.037 & 3.5 \\
\hline 3437269 & 2010 & 360 & -40.624 & 173.301 & 174.468 & 3.5 \\
\hline 3438304 & 2010 & 362 & -40.541 & 174.377 & 78.451 & 3.7 \\
\hline 3438312 & 2010 & 362 & -45.334 & 166.761 & 12.000 & 4.3 \\
\hline
\end{tabular}

\footnotetext{
${ }^{* *}$ measurements within the incidence angle $\leq 35^{\circ}$ are given. Note that events with magnitude $<3$ were used in the schist anisotropy study. All measurements and event information can be obtained on request.
} 


\section{A.3 Supplementary figures of chapter 4}

Fig. A.2 Equal area sterographs display backazimuthal variations in splitting parameters (the best filter measurements) at some stations. The radial position of these plots indicates the incidence angle, which ranges from $0-40^{0}$. Split bars are plotted in the direction of BAZ and at the incidence angle. Note that splitting parameters from near by stations show no systematic variations with either BAZ or incidence angle.

Fig. A.3 displays the percent velocity anisotropy estimation from shallow events (depths $\leq 70 \mathrm{~km}$ ) in southernmost part of the Hikurangi subduction zone using the $\delta t$ measured at LTZ (yellow) and KHZ (blue).

Fig. A.4 shows some examples of stations that show scatter in $\delta t$ with depth in northern and southern South Island. 


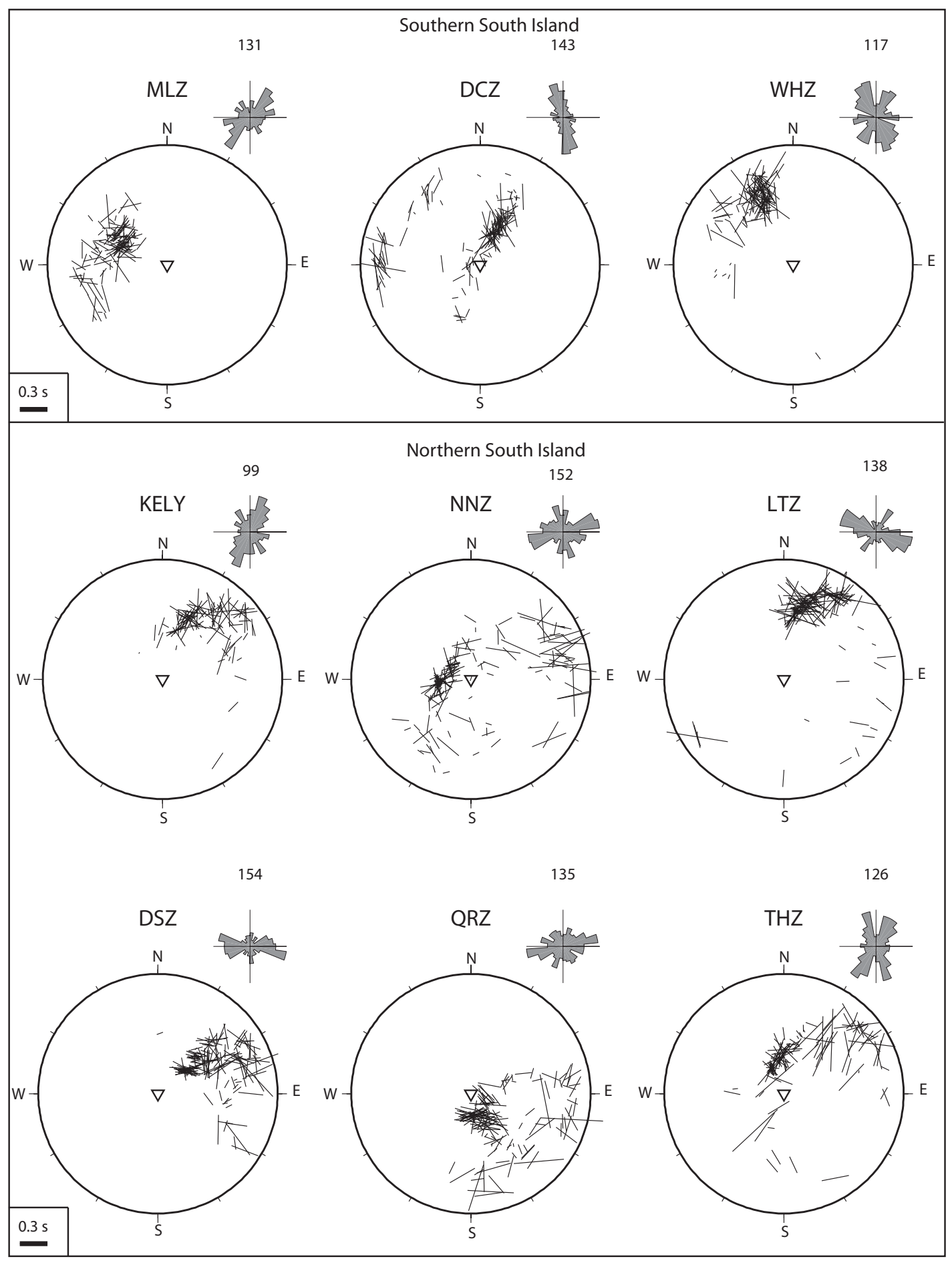

Figure A.2 Examples of the equal area sterographs display variations in station splitting parameters (best filter splitting measurements) with BAZ and incidence angle. The radial position of these plots indicates the incidence angle, which ranges from $0-40^{\circ}$. Split bars are plotted in the direction of BAZ and at the incidence angle. The direction of each bar denotes the $\phi$ and the size corresponds to the $\delta t$. The upper right circular histogram of each plot shows all the individual measurements together and the number indicates the number of measurements that satisfy the best filter criterion (see section 4.2). 


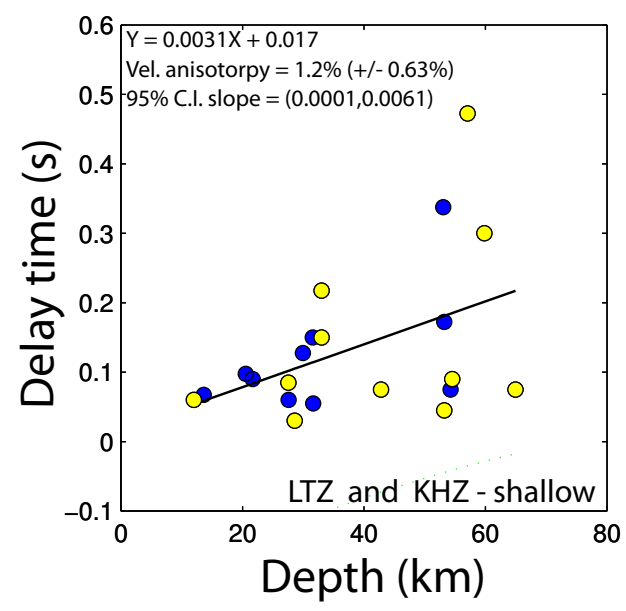

Figure A.3 Same as figure caption in Fig. 4.7, but velocity anisotropy is calculated from shallow slab events in the northern South Island. Blue and yellow color dots denote $\delta$ measurements at KHZ and LTZ respectively.
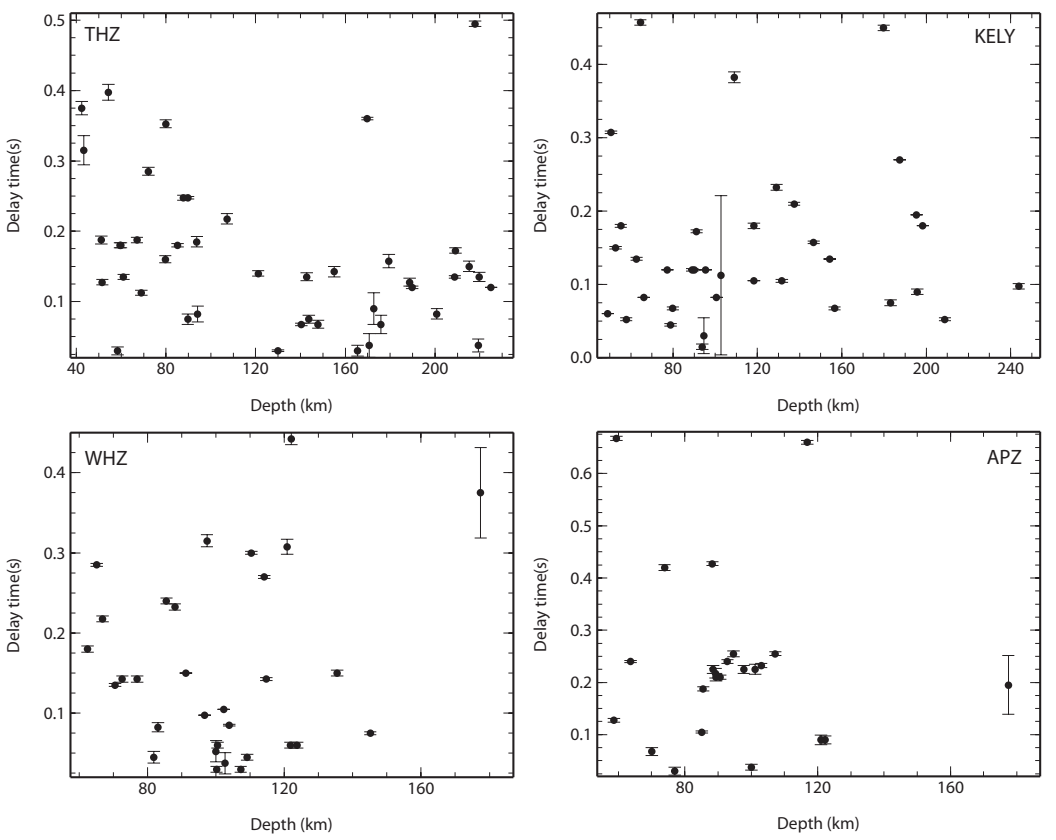

Figure A.4 Scatter in $\delta t$ with depth observed at some stations around subduction zones in northern and southern South Island. 


\section{Appendix B}

\section{Offshore investigation}

\section{B.1 Station information}

Table B.1 Station information of the OBS deployments.

\begin{tabular}{|c|c|c|c|c|c|}
\hline station & sensor & st.lat $^{\circ}$ & st.lon. ${ }^{\circ}$ & $\begin{array}{l}\text { bathymetry } \\
\text { (m) }\end{array}$ & $\begin{array}{l}\text { sensor } \\
\text { orie. }\left({ }^{\circ}\right)^{\S}\end{array}$ \\
\hline NZ01 & S116 & -44.498 & 165.002 & 4682 & $-* *$ \\
\hline NZ02 & $\mathrm{S} 128$ & -43.997 & 166.508 & 3816 & 36.3 \\
\hline NZ03 & S132 & -43.002 & 165.999 & 4225 & 292.5 \\
\hline NZ04 & $\mathrm{S} 142$ & -41.999 & 165.508 & 4470 & 205.7 \\
\hline NZ05 & $\mathrm{S} 125$ & -40.644 & 167.250 & 1188 & 264.3 \\
\hline NZ06 & S113 & -44.986 & 168.001 & 859 & 197.5 \\
\hline NZ07 & $\mathrm{S} 115$ & -40.250 & 169.000 & 852 & 89.0 \\
\hline NZ08 & S098 & -40.998 & 168.501 & 995 & 355.3 \\
\hline NZ09 & S112 & -41.651 & 167.900 & 1335 & 315.3 \\
\hline NZ10 & S082 & -43.201 & 168.000 & 1193 & 40.6 \\
\hline NZ11 & $\mathrm{S} 124$ & -43.305 & 168.850 & 911 & 174.5 \\
\hline NZ12 & $\mathrm{S} 127$ & -43.002 & 169.598 & 625 & 116.4 \\
\hline NZ13 & $\mathrm{S} 134$ & -42.499 & 168.999 & 1165 & 93.0 \\
\hline NZ14 & S114 & -42.651 & 169.950 & 633 & 346.5 \\
\hline NZ15 & S119 & -42.151 & 170.400 & 561 & 249.5 \\
\hline NZ16 & S096 & -41.749 & 169.249 & 1063 & 54.8 \\
\hline NZ17 & S118 & -41.500 & 170.500 & 595 & $-* *$ \\
\hline NZ18 & S097 & -41.000 & 170.000 & 805 & 321.7 \\
\hline NZ19 & $\mathrm{S} 120$ & -40.000 & 170.900 & 549 & 243.2 \\
\hline NZ20 & $\mathrm{S} 122$ & -40.200 & 170.750 & 714 & 154.2 \\
\hline NZ21 & S080 & -39.501 & 170.000 & 676 & 237.7 \\
\hline NZ22 & $\mathrm{S} 135$ & -44.501 & 173.998 & 776 & 233.5 \\
\hline NZ23 & $\mathrm{S} 126$ & -45.149 & 174.852 & 1091 & 13.6 \\
\hline NZ24 & S131 & -45.499 & 176.003 & 1507 & 283.0 \\
\hline NZ25 & S117 & -45.998 & 174.750 & 1727 & 36.6 \\
\hline NZ26 & S121 & -46.998 & 174.498 & 1316 & 108.8 \\
\hline NZ27 & $\mathrm{S} 123$ & -46.501 & 173.249 & 1544 & 216.8 \\
\hline NZ28 & $\mathrm{S} 133$ & -45.964 & 171.751 & 1328 & 330.1 \\
\hline NZ29 & S130 & -45.247 & 172.254 & 1383 & 43.9 \\
\hline NZ30 & S129 & -45.498 & 173.501 & 1416 & 57.4 \\
\hline
\end{tabular}

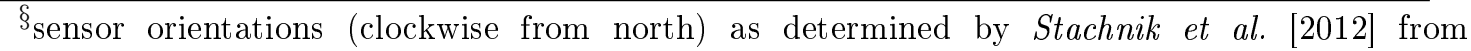
rayleigh-wave particle motion analysis

${ }^{* *}$ missing sensors 


\section{B.2 Measurements selection criteria}

AB-grade splitting parameters were used for the analysis (AB-grade and best filter criteria are given in section 3.2.3). Splitting parameters are graded as good quality if the $\lambda_{\max }>5$. Here, $\lambda_{\max }$ denotes the number of scaled energy contours around the best resolved splitting parameters (section 3.2.3). Measurements with $\delta t \leq 0.7 \mathrm{~s}$ were selected to avoid ambiguous splitting parameters.

\section{B.3 Events with AB-grade measurements}

Table B.2 Events with AB quality high-grade measurement ${ }^{\ddagger}$.

\begin{tabular}{|c|c|c|c|c|c|c|}
\hline cuspid & year & jul.day & lat. $^{\circ}$ & lon. $^{\circ}$ & depth & mag. \\
\hline 3041931 & 2009 & 038 & -41.233 & 172.566 & 213.946 & 3.6 \\
\hline 3042565 & 2009 & 039 & -45.537 & 167.071 & 76.165 & 3.9 \\
\hline 3043284 & 2009 & 041 & -44.798 & 167.367 & 5.000 & 3.7 \\
\hline 3045760 & 2009 & 046 & -45.589 & 166.945 & 12.000 & 3.7 \\
\hline 3047503 & 2009 & 049 & -45.096 & 167.389 & 117.456 & 3.9 \\
\hline 3049002 & 2009 & 052 & -46.305 & 165.643 & 12.000 & 4.1 \\
\hline 3049393 & 2009 & 053 & -45.128 & 166.627 & 12.000 & 4.0 \\
\hline 3049778 & 2009 & 054 & -41.915 & 173.728 & 40.573 & 3.6 \\
\hline 3050923 & 2009 & 056 & -43.504 & 171.544 & 12.000 & 3.5 \\
\hline 3051685 & 2009 & 058 & -43.063 & 173.362 & 26.056 & 3.8 \\
\hline 3052092 & 2009 & 059 & -41.007 & 174.766 & 32.583 & 3.3 \\
\hline 3054325 & 2009 & 063 & -43.214 & 172.156 & 5.000 & 4.1 \\
\hline 3055693 & 2009 & 066 & -45.466 & 167.424 & 11.641 & 3.7 \\
\hline 3057072 & 2009 & 069 & -44.970 & 167.536 & 57.478 & 3.3 \\
\hline 3057524 & 2009 & 069 & -42.968 & 171.446 & 5.000 & 3.5 \\
\hline 3057877 & 2009 & 070 & -41.688 & 174.477 & 54.052 & 4.3 \\
\hline 3058975 & 2009 & 072 & -45.095 & 167.398 & 117.789 & 3.8 \\
\hline 3059063 & 2009 & 072 & -41.958 & 174.110 & 15.880 & 3.3 \\
\hline 3059454 & 2009 & 073 & -44.735 & 167.889 & 96.807 & 3.1 \\
\hline 3059515 & 2009 & 073 & -40.542 & 173.522 & 158.489 & 4.1 \\
\hline 3059728 & 2009 & 074 & -44.347 & 170.115 & 12.755 & 3.1 \\
\hline 3060059 & 2009 & 074 & -45.106 & 167.483 & 114.984 & 3.1 \\
\hline 3060760 & 2009 & 076 & -39.533 & 174.311 & 235.339 & 4.2 \\
\hline 3061005 & 2009 & 076 & -40.434 & 173.543 & 158.239 & 3.6 \\
\hline 3062725 & 2009 & 079 & -44.950 & 167.370 & 33.000 & 3.1 \\
\hline 3063032 & 2009 & 080 & -40.309 & 174.092 & 117.688 & 3.7 \\
\hline 3063549 & 2009 & 081 & -40.431 & 173.491 & 155.490 & 4.9 \\
\hline 3064359 & 2009 & 083 & -45.150 & 167.110 & 12.000 & 4.5 \\
\hline 3064368 & 2009 & 083 & -41.205 & 173.755 & 70.267 & 3.3 \\
\hline 3064812 & 2009 & 083 & -41.788 & 173.841 & 15.392 & 3.3 \\
\hline 3065595 & 2009 & 085 & -45.061 & 167.422 & 97.395 & 4.7 \\
\hline 3066319 & 2009 & 086 & -44.254 & 170.018 & 12.000 & 3.1 \\
\hline 3066543 & 2009 & 087 & -45.211 & 167.277 & 100.059 & 3.5 \\
\hline 3066555 & 2009 & 087 & -45.069 & 167.453 & 110.902 & 3.0 \\
\hline 3067162 & 2009 & 088 & -44.310 & 168.326 & 5.000 & 3.7 \\
\hline 3067199 & 2009 & 088 & -44.269 & 170.010 & 14.379 & 3.2 \\
\hline 3068099 & 2009 & 090 & -41.499 & 172.888 & 123.965 & 4.6 \\
\hline 3068852 & 2009 & 091 & -45.025 & 167.393 & 125.892 & 3.6 \\
\hline 3069768 & 2009 & 093 & -44.824 & 167.658 & 85.710 & 3.2 \\
\hline 3070857 & 2009 & 095 & -42.456 & 172.979 & 8.880 & 3.2 \\
\hline 3071236 & 2009 & 096 & -42.794 & 173.136 & 5.000 & 3.2 \\
\hline 3071401 & 2009 & 096 & -41.660 & 172.901 & 91.317 & 4.5 \\
\hline 3072643 & 2009 & 098 & -44.210 & 168.412 & 5.000 & 3.7 \\
\hline
\end{tabular}


Table B.2 - Continued

\begin{tabular}{|c|c|c|c|c|c|c|}
\hline cuspid & year & jul.day & lat. $^{\circ}$ & lon. ${ }^{\circ}$ & depth & mag \\
\hline 3072794 & 2009 & 099 & -44.563 & 173.752 & 33.000 & 3.8 \\
\hline 3073334 & 2009 & 100 & -45.029 & 167.537 & 127.050 & 3.5 \\
\hline 3074226 & 2009 & 102 & -44.401 & 169.595 & 12.000 & 3.0 \\
\hline 3074364 & 2009 & 102 & -40.172 & 173.769 & 161.285 & 3.8 \\
\hline 3074557 & 2009 & 102 & -45.035 & 167.465 & 98.712 & 3.1 \\
\hline 3074691 & 2009 & 103 & -41.469 & 172.876 & 121.837 & 3.9 \\
\hline 3074697 & 2009 & 103 & -43.490 & 171.600 & 5.000 & 4.3 \\
\hline 3075621 & 2009 & 104 & -46.143 & 167.789 & 12.000 & 4.5 \\
\hline 3077937 & 2009 & 109 & -40.402 & 173.590 & 154.805 & 3.7 \\
\hline 3080021 & 2009 & 113 & -41.377 & 172.966 & 116.455 & 3.7 \\
\hline 3080807 & 2009 & 114 & -41.284 & 172.678 & 208.877 & 3.8 \\
\hline 3083936 & 2009 & 120 & -40.371 & 173.383 & 192.020 & 3.6 \\
\hline 3084790 & 2009 & 122 & -44.259 & 170.003 & 14.189 & 3.6 \\
\hline 3087732 & 2009 & 128 & -43.152 & 170.960 & 5.000 & 4.0 \\
\hline 3088615 & 2009 & 129 & -42.479 & 174.703 & 33.000 & 3.1 \\
\hline 3088974 & 2009 & 130 & -40.075 & 173.943 & 162.557 & 3.9 \\
\hline 3090296 & 2009 & 133 & -45.859 & 167.204 & 12.000 & 4.3 \\
\hline 3091656 & 2009 & 135 & -41.134 & 173.919 & 67.400 & 3.7 \\
\hline 3092792 & 2009 & 137 & -42.260 & 171.335 & 5.000 & 3.6 \\
\hline 3095999 & 2009 & 143 & -40.555 & 175.458 & 34.813 & 3.9 \\
\hline 3096079 & 2009 & 143 & -45.410 & 167.196 & 136.906 & 3.6 \\
\hline 3096871 & 2009 & 144 & -45.158 & 167.356 & 120.805 & 3.9 \\
\hline 3097975 & 2009 & 146 & -44.504 & 169.860 & 5.000 & 4.3 \\
\hline 3099203 & 2009 & 149 & -41.944 & 174.027 & 19.783 & 3.5 \\
\hline 3102845 & 2009 & 155 & -41.060 & 174.540 & 44.080 & 4.0 \\
\hline 3104352 & 2009 & 158 & -41.071 & 174.557 & 43.478 & 3.9 \\
\hline 3106816 & 2009 & 163 & -40.375 & 174.950 & 5.000 & 3.7 \\
\hline 3107010 & 2009 & 163 & -43.672 & 171.604 & 5.000 & 3.3 \\
\hline 3107602 & 2009 & 164 & -45.214 & 167.304 & 105.020 & 3.8 \\
\hline 3107978 & 2009 & 165 & -43.734 & 171.607 & 5.000 & 3.7 \\
\hline 3108186 & 2009 & 165 & -44.827 & 167.250 & 33.000 & 3.7 \\
\hline 3108837 & 2009 & 166 & -40.555 & 173.419 & 165.474 & 4.1 \\
\hline 3109454 & 2009 & 167 & -42.038 & 172.925 & 75.936 & 3.3 \\
\hline 3112716 & 2009 & 173 & -40.943 & 172.834 & 193.891 & 3.9 \\
\hline 3115714 & 2009 & 178 & -42.502 & 169.801 & 33.000 & 4.8 \\
\hline 3118326 & 2009 & 183 & -41.550 & 172.995 & 91.464 & 4.2 \\
\hline 3118345 & 2009 & 183 & -41.578 & 172.993 & 91.361 & 4.6 \\
\hline 3119748 & 2009 & 186 & -45.036 & 167.393 & 110.561 & 3.5 \\
\hline 3120467 & 2009 & 188 & -43.022 & 172.722 & 25.211 & 3.0 \\
\hline 3122085 & 2009 & 181 & -42.026 & 173.927 & 19.720 & 3.4 \\
\hline 3122205 & 2009 & 191 & -43.239 & 170.909 & 5.000 & 3.5 \\
\hline 3122292 & 2009 & 191 & -40.985 & 172.916 & 174.270 & 4.2 \\
\hline 3123338 & 2009 & 193 & -40.917 & 173.065 & 172.778 & 3.5 \\
\hline 3124008 & 2009 & 194 & -42.240 & 173.892 & 33.000 & 3.7 \\
\hline 3124791 & 2009 & 196 & -40.179 & 173.556 & 167.322 & 5.4 \\
\hline 3124795 & 2009 & 196 & -46.035 & 165.883 & 12.000 & 5.3 \\
\hline 3124798 & 2009 & 196 & -45.835 & 166.435 & 5.000 & 5.6 \\
\hline 3124807 & 2009 & 196 & -46.196 & 165.844 & 33.000 & 5.0 \\
\hline 3124856 & 2009 & 196 & -46.114 & 165.803 & 12.000 & 3.9 \\
\hline 3124997 & 2009 & 196 & -45.450 & 166.641 & 5.000 & 4.8 \\
\hline 3125012 & 2009 & 196 & -46.036 & 166.087 & 12.000 & 3.9 \\
\hline 3125061 & 2009 & 196 & -45.521 & 167.033 & 65.012 & 5.1 \\
\hline 3125075 & 2009 & 197 & -45.640 & 166.535 & 12.000 & 4.3 \\
\hline 3125083 & 2009 & 197 & -46.288 & 165.867 & 5.000 & 5.5 \\
\hline 3125111 & 2009 & 197 & -45.770 & 166.544 & 5.000 & 4.9 \\
\hline 3125112 & 2009 & 197 & -45.400 & 167.000 & 12.000 & 5.1 \\
\hline 3125133 & 2009 & 197 & -46.370 & 165.613 & 12.000 & 5.0 \\
\hline 3125179 & 2009 & 197 & -45.704 & 166.590 & 12.000 & 4.7 \\
\hline 3125184 & 2009 & 197 & -46.246 & 166.014 & 15.852 & 4.8 \\
\hline 3125200 & 2009 & 197 & -45.702 & 166.551 & 5.000 & 5.6 \\
\hline 3125201 & 2009 & 197 & -45.897 & 166.211 & 33.000 & 5.4 \\
\hline 3125217 & 2009 & 197 & -46.154 & 165.936 & 12.000 & 4.6 \\
\hline 3125237 & 2009 & 197 & -45.544 & 166.888 & 12.000 & 3.8 \\
\hline 3125242 & 2009 & 197 & -45.587 & 166.106 & 12.000 & 3.9 \\
\hline 3125250 & 2009 & 197 & -46.110 & 165.876 & 19.889 & 3.5 \\
\hline 3125262 & 2009 & 197 & -45.685 & 166.550 & 4.515 & 4.4 \\
\hline 3125394 & 2009 & 197 & -46.167 & 166.100 & 30.000 & 5.3 \\
\hline 3125459 & 2009 & 197 & -46.184 & 165.925 & 5.000 & 4.4 \\
\hline 3125523 & 2009 & 197 & -46.186 & 166.121 & 33.000 & 5.6 \\
\hline 3125534 & 2009 & 197 & -46.067 & 165.971 & 12.000 & 4.3 \\
\hline
\end{tabular}


Table B.2 - Continued

\begin{tabular}{|c|c|c|c|c|c|c|}
\hline cuspid & year & jul.day & lat. $^{\circ}$ & lon. ${ }^{\circ}$ & depth & mag. \\
\hline 3125543 & 2009 & 197 & -46.099 & 165.846 & 12.000 & 3.6 \\
\hline 3125554 & 2009 & 197 & -45.556 & 166.577 & 22.278 & 3.9 \\
\hline 3125563 & 2009 & 198 & -46.121 & 166.060 & 26.758 & 3.8 \\
\hline 3125571 & 2009 & 198 & -46.108 & 165.931 & 12.000 & 4.8 \\
\hline 3125584 & 2009 & 198 & -45.464 & 166.614 & 19.540 & 4.8 \\
\hline 3125593 & 2009 & 198 & -46.217 & 165.795 & 33.000 & 4.0 \\
\hline 3125608 & 2009 & 198 & -46.139 & 166.065 & 31.528 & 3.5 \\
\hline 3125633 & 2009 & 198 & -46.207 & 166.013 & 30.580 & 4.7 \\
\hline 3125673 & 2009 & 198 & -46.310 & 165.873 & 33.000 & 3.5 \\
\hline 3125686 & 2009 & 198 & -45.727 & 166.531 & 22.396 & 3.9 \\
\hline 3125689 & 2009 & 198 & -45.395 & 166.706 & 21.864 & 4.9 \\
\hline 3125691 & 2009 & 198 & -45.331 & 166.502 & 12.000 & 5.6 \\
\hline 3125693 & 2009 & 198 & -45.374 & 166.602 & 21.326 & 3.6 \\
\hline 3125699 & 2009 & 198 & -45.394 & 166.573 & 20.240 & 3.8 \\
\hline 3125707 & 2009 & 198 & -45.384 & 166.703 & 21.886 & 3.5 \\
\hline 3125714 & 2009 & 198 & -45.367 & 166.626 & 21.089 & 4.4 \\
\hline 3125782 & 2009 & 198 & -45.646 & 166.967 & 8.729 & 3.6 \\
\hline 3125784 & 2009 & 198 & -45.503 & 165.727 & 33.000 & 3.8 \\
\hline 3125829 & 2009 & 198 & -46.019 & 166.482 & 32.839 & 3.8 \\
\hline 3125831 & 2009 & 198 & -45.428 & 166.625 & 21.897 & 4.3 \\
\hline 3125892 & 2009 & 198 & -46.180 & 165.717 & 33.000 & 3.9 \\
\hline 3125939 & 2009 & 198 & -45.420 & 166.701 & 21.574 & 4.5 \\
\hline 3125976 & 2009 & 198 & -41.574 & 173.590 & 63.677 & 3.2 \\
\hline 3126009 & 2009 & 199 & -45.812 & 166.344 & 19.473 & 3.9 \\
\hline 3126016 & 2009 & 199 & -45.370 & 166.637 & 21.158 & 4.8 \\
\hline 3126021 & 2009 & 199 & -46.084 & 166.098 & 27.012 & 4.0 \\
\hline 3126025 & 2009 & 199 & -45.363 & 166.658 & 21.554 & 4.8 \\
\hline 3126068 & 2009 & 199 & -46.033 & 166.262 & 28.432 & 3.6 \\
\hline 3126070 & 2009 & 199 & -46.070 & 166.294 & 31.831 & 3.7 \\
\hline 3126085 & 2009 & 199 & -46.307 & 165.755 & 33.000 & 3.7 \\
\hline 3126095 & 2009 & 199 & -46.149 & 166.008 & 33.000 & 3.8 \\
\hline 3126108 & 2009 & 199 & -45.523 & 166.679 & 21.855 & 3.5 \\
\hline 3126113 & 2009 & 199 & -45.496 & 166.677 & 21.684 & 3.8 \\
\hline 3126121 & 2009 & 199 & -46.242 & 165.973 & 29.645 & 3.6 \\
\hline 3126128 & 2009 & 199 & -45.465 & 166.694 & 20.259 & 3.6 \\
\hline 3126147 & 2009 & 199 & -45.478 & 166.285 & 12.000 & 3.8 \\
\hline 3126167 & 2009 & 199 & -46.017 & 166.481 & 32.855 & 4.5 \\
\hline 3126201 & 2009 & 199 & -45.652 & 166.500 & 20.362 & 4.5 \\
\hline 3126239 & 2009 & 199 & -46.169 & 166.184 & 32.848 & 4.0 \\
\hline 3126249 & 2009 & 199 & -46.154 & 166.049 & 30.066 & 4.3 \\
\hline 3126312 & 2009 & 199 & -45.548 & 166.645 & 21.462 & 4.5 \\
\hline 3126393 & 2009 & 199 & -45.501 & 166.542 & 21.470 & 4.3 \\
\hline 3126413 & 2009 & 199 & -46.150 & 165.816 & 12.000 & 3.5 \\
\hline 3126418 & 2009 & 199 & -45.481 & 166.611 & 21.308 & 3.7 \\
\hline 3126438 & 2009 & 200 & -46.155 & 165.883 & 12.000 & 3.7 \\
\hline 3126520 & 2009 & 200 & -45.379 & 166.097 & 12.000 & 5.2 \\
\hline 3126535 & 2009 & 200 & -46.136 & 166.094 & 29.870 & 3.7 \\
\hline 3126568 & 2009 & 200 & -45.682 & 166.528 & 21.560 & 4.1 \\
\hline 3126570 & 2009 & 200 & -44.522 & 175.686 & 33.000 & 3.9 \\
\hline 3126592 & 2009 & 200 & -45.337 & 166.164 & 12.000 & 5.2 \\
\hline 3126670 & 2009 & 200 & -45.714 & 166.493 & 20.943 & 3.5 \\
\hline 3126686 & 2009 & 200 & -45.396 & 166.722 & 17.842 & 3.6 \\
\hline 3126710 & 2009 & 200 & -45.602 & 165.763 & 33.000 & 3.5 \\
\hline 3126729 & 2009 & 200 & -45.545 & 166.654 & 22.410 & 4.4 \\
\hline 3126801 & 2009 & 200 & -45.673 & 166.529 & 18.602 & 3.7 \\
\hline 3126817 & 2009 & 200 & -46.121 & 165.975 & 31.502 & 3.7 \\
\hline 3126909 & 2009 & 201 & -46.192 & 165.861 & 12.000 & 3.6 \\
\hline 3127061 & 2009 & 201 & -45.464 & 166.746 & 21.983 & 3.6 \\
\hline 3127091 & 2009 & 201 & -44.684 & 175.528 & 33.000 & 3.4 \\
\hline 3127103 & 2009 & 201 & -45.422 & 166.585 & 22.206 & 3.8 \\
\hline 3127139 & 2009 & 201 & -46.169 & 166.143 & 27.544 & 3.6 \\
\hline 3127319 & 2009 & 202 & -46.042 & 166.170 & 27.031 & 3.7 \\
\hline 3127345 & 2009 & 202 & -45.864 & 165.851 & 33.000 & 4.5 \\
\hline 3127489 & 2009 & 202 & -46.086 & 166.071 & 29.436 & 4.0 \\
\hline 3127579 & 2009 & 202 & -47.313 & 165.269 & 33.000 & 4.1 \\
\hline 3127607 & 2009 & 202 & -46.015 & 166.148 & 28.158 & 4.1 \\
\hline 3127631 & 2009 & 202 & -46.192 & 165.820 & 12.000 & 4.5 \\
\hline 3127703 & 2009 & 202 & -45.432 & 165.852 & 33.000 & 4.1 \\
\hline 3127714 & 2009 & 202 & -45.881 & 166.438 & 26.520 & 4.0 \\
\hline 3127740 & 2009 & 202 & -46.173 & 166.047 & 33.000 & 4.0 \\
\hline
\end{tabular}


Table B.2 - Continued

\begin{tabular}{|c|c|c|c|c|c|c|}
\hline cuspid & year & jul.day & lat. $^{\circ}$ & lon. ${ }^{\circ}$ & depth & mag. \\
\hline 3127929 & 2009 & 203 & -45.425 & 166.627 & 12.000 & 3.8 \\
\hline 3128006 & 2009 & 203 & -46.353 & 165.878 & 33.000 & 4.5 \\
\hline 3128140 & 2009 & 203 & -45.473 & 166.753 & 22.952 & 3.6 \\
\hline 3128237 & 2009 & 203 & -46.216 & 165.875 & 33.000 & 3.8 \\
\hline 3128341 & 2009 & 204 & -42.668 & 172.595 & 33.000 & 3.3 \\
\hline 3128439 & 2009 & 204 & -44.569 & 175.549 & 33.000 & 3.9 \\
\hline 3128653 & 2009 & 204 & -45.739 & 165.664 & 12.000 & 3.9 \\
\hline 3128712 & 2009 & 204 & -45.409 & 166.730 & 22.087 & 3.8 \\
\hline 3128728 & 2009 & 204 & -46.099 & 165.821 & 12.000 & 3.7 \\
\hline 3128836 & 2009 & 204 & -45.410 & 166.728 & 25.015 & 3.9 \\
\hline 3128844 & 2009 & 204 & -45.807 & 165.821 & 33.000 & 4.0 \\
\hline 3128890 & 2009 & 205 & -46.136 & 166.246 & 33.000 & 3.5 \\
\hline 3128927 & 2009 & 205 & -46.156 & 165.805 & 33.000 & 4.3 \\
\hline 3128996 & 2009 & 205 & -45.656 & 166.484 & 16.840 & 3.5 \\
\hline 3129028 & 2009 & 205 & -45.805 & 165.747 & 33.000 & 3.8 \\
\hline 3129136 & 2009 & 205 & -45.393 & 166.722 & 23.897 & 4.6 \\
\hline 3129173 & 2009 & 205 & -46.156 & 165.882 & 33.000 & 3.7 \\
\hline 3129198 & 2009 & 205 & -44.615 & 168.159 & 80.070 & 3.2 \\
\hline 3129235 & 2009 & 205 & -44.564 & 175.549 & 33.000 & 3.3 \\
\hline 3129381 & 2009 & 206 & -45.399 & 166.612 & 19.687 & 3.6 \\
\hline 3129388 & 2009 & 206 & -45.385 & 166.591 & 19.743 & 3.8 \\
\hline 3129805 & 2009 & 207 & -45.868 & 166.278 & 21.556 & 3.7 \\
\hline 3129848 & 2009 & 207 & -46.130 & 165.798 & 12.000 & 4.6 \\
\hline 3129894 & 2009 & 207 & -41.292 & 172.704 & 158.588 & 3.6 \\
\hline 3130140 & 2009 & 207 & -45.356 & 166.722 & 22.340 & 3.5 \\
\hline 3130239 & 2009 & 208 & -44.575 & 175.493 & 33.000 & 3.4 \\
\hline 3130282 & 2009 & 208 & -45.056 & 166.940 & 33.000 & 3.7 \\
\hline 3130415 & 2009 & 208 & -46.047 & 166.050 & 30.909 & 3.5 \\
\hline 3130486 & 2009 & 208 & -45.734 & 166.409 & 20.095 & 3.6 \\
\hline 3130487 & 2009 & 208 & -44.374 & 168.030 & 5.000 & 4.1 \\
\hline 3130648 & 2009 & 208 & -45.754 & 165.818 & 12.000 & 3.8 \\
\hline 3130676 & 2009 & 209 & -45.471 & 166.657 & 21.433 & 3.6 \\
\hline 3130851 & 2009 & 209 & -42.158 & 173.721 & 40.950 & 3.0 \\
\hline 3131288 & 2009 & 210 & -45.117 & 167.204 & 33.000 & 3.2 \\
\hline 3131425 & 2009 & 210 & -46.048 & 166.161 & 33.000 & 5.2 \\
\hline 3131438 & 2009 & 210 & -46.169 & 165.899 & 33.000 & 5.0 \\
\hline 3131449 & 2009 & 210 & -46.168 & 165.884 & 33.000 & 3.9 \\
\hline 3131501 & 2009 & 211 & -45.539 & 166.646 & 20.917 & 4.0 \\
\hline 3131547 & 2009 & 211 & -45.623 & 166.417 & 22.697 & 5.0 \\
\hline 3131612 & 2009 & 211 & -45.657 & 166.461 & 19.171 & 4.8 \\
\hline 3131648 & 2009 & 211 & -46.046 & 165.904 & 27.275 & 3.6 \\
\hline 3131736 & 2009 & 211 & -45.618 & 166.469 & 19.518 & 3.7 \\
\hline 3131885 & 2009 & 211 & -46.169 & 165.950 & 29.097 & 4.3 \\
\hline 3131896 & 2009 & 211 & -45.646 & 166.448 & 21.977 & 4.7 \\
\hline 3132026 & 2009 & 212 & -39.862 & 175.192 & 66.499 & 3.7 \\
\hline 3132105 & 2009 & 212 & -45.520 & 166.691 & 23.874 & 3.8 \\
\hline 3132302 & 2009 & 212 & -45.430 & 166.756 & 24.235 & 4.7 \\
\hline 3132372 & 2009 & 212 & -45.446 & 166.710 & 24.893 & 4.3 \\
\hline 3132552 & 2009 & 213 & -46.063 & 165.971 & 28.807 & 3.6 \\
\hline 3132601 & 2009 & 213 & -44.971 & 167.535 & 127.785 & 3.6 \\
\hline 3132894 & 2009 & 213 & -45.776 & 165.865 & 33.000 & 3.5 \\
\hline 3133200 & 2009 & 214 & -46.105 & 166.305 & 28.945 & 3.9 \\
\hline 3133476 & 2009 & 214 & -44.584 & 175.504 & 33.000 & 3.5 \\
\hline 3133651 & 2009 & 198 & -46.145 & 166.126 & 33.787 & 4.1 \\
\hline 3133655 & 2009 & 198 & -46.147 & 166.110 & 32.452 & 4.4 \\
\hline 3133914 & 2009 & 215 & -46.248 & 166.279 & 33.000 & 3.8 \\
\hline 3133965 & 2009 & 215 & -45.134 & 166.458 & 12.000 & 5.0 \\
\hline 3134000 & 2009 & 215 & -45.479 & 166.620 & 20.410 & 3.8 \\
\hline 3134001 & 2009 & 215 & -45.799 & 165.965 & 32.123 & 3.6 \\
\hline 3134115 & 2009 & 216 & -41.811 & 174.345 & 29.308 & 4.2 \\
\hline 3134119 & 2009 & 200 & -45.453 & 165.762 & 33.000 & 4.1 \\
\hline 3134155 & 2009 & 216 & -45.382 & 166.696 & 22.819 & 4.3 \\
\hline 3134378 & 2009 & 216 & -45.498 & 166.660 & 22.454 & 3.8 \\
\hline 3134481 & 2009 & 216 & -44.437 & 168.008 & 5.000 & 3.5 \\
\hline 3134616 & 2009 & 200 & -45.413 & 166.714 & 20.706 & 4.0 \\
\hline 3134710 & 2009 & 217 & -45.508 & 166.328 & 12.000 & 5.2 \\
\hline 3134768 & 2009 & 217 & -46.146 & 166.117 & 27.712 & 4.8 \\
\hline 3134829 & 2009 & 217 & -45.564 & 166.156 & 12.000 & 4.8 \\
\hline 3135253 & 2009 & 218 & -45.442 & 166.676 & 21.755 & 3.5 \\
\hline 3135313 & 2009 & 218 & -45.476 & 166.746 & 21.993 & 3.8 \\
\hline
\end{tabular}


Table B.2 - Continued

\begin{tabular}{|c|c|c|c|c|c|c|}
\hline cuspid & year & jul.day & lat. $^{\circ}$ & lon. ${ }^{\circ}$ & depth & mag. \\
\hline 3135487 & 2009 & 200 & -46.106 & 166.206 & 32.296 & 3.6 \\
\hline 3135552 & 2009 & 219 & -45.858 & 166.425 & 39.614 & 3.7 \\
\hline 3135583 & 2009 & 202 & -46.091 & 166.006 & 29.150 & 4.4 \\
\hline 3135671 & 2009 & 219 & -46.080 & 165.917 & 25.205 & 3.6 \\
\hline 3135754 & 2009 & 219 & -45.551 & 166.658 & 20.658 & 4.5 \\
\hline 3135791 & 2009 & 219 & -45.551 & 166.667 & 18.191 & 4.8 \\
\hline 3135990 & 2009 & 219 & -46.028 & 166.051 & 26.633 & 3.9 \\
\hline 3136205 & 2009 & 220 & -46.142 & 165.870 & 33.000 & 4.3 \\
\hline 3136426 & 2009 & 198 & -46.168 & 165.997 & 28.758 & 3.5 \\
\hline 3136489 & 2009 & 221 & -44.571 & 175.546 & 33.000 & 4.0 \\
\hline 3136656 & 2009 & 221 & -45.236 & 166.664 & 22.950 & 4.7 \\
\hline 3136713 & 2009 & 221 & -45.542 & 166.627 & 23.347 & 4.8 \\
\hline 3136769 & 2009 & 221 & -46.229 & 166.073 & 33.000 & 3.9 \\
\hline 3136860 & 2009 & 221 & -45.754 & 165.807 & 12.000 & 4.2 \\
\hline 3137460 & 2009 & 223 & -45.963 & 166.434 & 30.215 & 3.7 \\
\hline 3137692 & 2009 & 223 & -45.391 & 166.702 & 21.414 & 3.5 \\
\hline 3137861 & 2009 & 198 & -45.380 & 166.703 & 21.864 & 3.7 \\
\hline 3138383 & 2009 & 198 & -46.299 & 165.797 & 33.000 & 3.9 \\
\hline 3138587 & 2009 & 225 & -45.339 & 166.767 & 26.962 & 3.7 \\
\hline 3139054 & 2009 & 226 & -45.061 & 167.400 & 112.849 & 3.3 \\
\hline 3139106 & 2009 & 226 & -43.256 & 170.790 & 3.000 & 4.1 \\
\hline 3139357 & 2009 & 209 & -44.545 & 175.224 & 12.000 & 3.7 \\
\hline 3139393 & 2009 & 198 & -46.101 & 165.916 & 26.398 & 3.6 \\
\hline 3139869 & 2009 & 228 & -41.362 & 172.767 & 136.649 & 3.1 \\
\hline 3140195 & 2009 & 229 & -45.552 & 166.656 & 22.020 & 4.1 \\
\hline 3140272 & 2009 & 229 & -46.015 & 166.108 & 28.419 & 3.9 \\
\hline 3140382 & 2009 & 229 & -42.722 & 173.910 & 12.000 & 3.7 \\
\hline 3140483 & 2009 & 229 & -45.923 & 165.779 & 29.158 & 5.1 \\
\hline 3140527 & 2009 & 229 & -45.915 & 165.840 & 12.000 & 4.4 \\
\hline 3141039 & 2009 & 230 & -44.423 & 168.327 & 5.000 & 4.0 \\
\hline 3141091 & 2009 & 231 & -44.534 & 175.380 & 33.000 & 3.3 \\
\hline 3141178 & 2009 & 231 & -46.200 & 165.846 & 33.000 & 3.7 \\
\hline 3141301 & 2009 & 231 & -44.586 & 175.530 & 33.000 & 3.1 \\
\hline 3141338 & 2009 & 231 & -40.530 & 173.610 & 118.346 & 3.8 \\
\hline 3141375 & 2009 & 231 & -41.726 & 172.188 & 100.391 & 3.3 \\
\hline 3141436 & 2009 & 231 & -45.682 & 166.458 & 20.648 & 4.8 \\
\hline 3141439 & 2009 & 231 & -45.685 & 166.487 & 19.718 & 3.6 \\
\hline 3141478 & 2009 & 231 & -46.032 & 166.272 & 33.000 & 3.6 \\
\hline 3141540 & 2009 & 232 & -40.338 & 174.038 & 127.364 & 3.5 \\
\hline 3141684 & 2009 & 232 & -45.980 & 165.880 & 33.000 & 4.9 \\
\hline 3141695 & 2009 & 232 & -46.067 & 166.166 & 30.888 & 4.5 \\
\hline 3141703 & 2009 & 232 & -44.594 & 168.173 & 80.351 & 3.3 \\
\hline 3141789 & 2009 & 232 & -44.595 & 175.581 & 33.000 & 3.6 \\
\hline 3142644 & 2009 & 234 & -41.161 & 172.859 & 145.078 & 3.5 \\
\hline 3142845 & 2009 & 235 & -46.151 & 165.778 & 33.000 & 3.5 \\
\hline 3142936 & 2009 & 235 & -44.983 & 167.458 & 87.478 & 3.5 \\
\hline 3143207 & 2009 & 235 & -45.946 & 165.858 & 29.908 & 3.7 \\
\hline 3143313 & 2009 & 217 & -44.580 & 175.597 & 33.000 & 3.3 \\
\hline 3143345 & 2009 & 236 & -46.279 & 165.848 & 33.000 & 4.4 \\
\hline 3143358 & 2009 & 199 & -45.475 & 166.638 & 22.118 & 4.1 \\
\hline 3143705 & 2009 & 237 & -45.150 & 167.280 & 121.351 & 3.8 \\
\hline 3143806 & 2009 & 237 & -46.115 & 166.047 & 26.287 & 5.5 \\
\hline 3143822 & 2009 & 237 & -46.119 & 166.114 & 30.687 & 4.6 \\
\hline 3144142 & 2009 & 237 & -46.117 & 166.091 & 29.162 & 4.3 \\
\hline 3145159 & 2009 & 239 & -41.442 & 174.709 & 33.910 & 5.2 \\
\hline 3145245 & 2009 & 239 & -45.100 & 167.368 & 112.461 & 3.1 \\
\hline 3145383 & 2009 & 240 & -45.499 & 166.789 & 17.847 & 4.0 \\
\hline 3145684 & 2009 & 240 & -45.510 & 166.481 & 19.859 & 4.0 \\
\hline 3146008 & 2009 & 241 & -45.461 & 166.346 & 19.526 & 4.2 \\
\hline 3146016 & 2009 & 241 & -44.933 & 167.482 & 74.659 & 3.0 \\
\hline 3146610 & 2009 & 242 & -45.429 & 166.770 & 23.174 & 3.9 \\
\hline 3147149 & 2009 & 243 & -46.020 & 166.186 & 29.116 & 3.5 \\
\hline 3147189 & 2009 & 243 & -45.681 & 166.495 & 21.568 & 4.3 \\
\hline 3147386 & 2009 & 243 & -41.847 & 173.309 & 84.966 & 3.1 \\
\hline 3147530 & 2009 & 244 & -44.483 & 166.926 & 5.000 & 4.0 \\
\hline 3148588 & 2009 & 245 & -40.926 & 172.922 & 187.429 & 4.0 \\
\hline 3150084 & 2009 & 249 & -44.169 & 168.623 & 5.000 & 3.9 \\
\hline 3150087 & 2009 & 249 & -46.150 & 165.812 & 30.569 & 3.5 \\
\hline 3150137 & 2009 & 249 & -44.991 & 167.590 & 69.385 & 3.2 \\
\hline 3150190 & 2009 & 249 & -44.240 & 167.708 & 12.000 & 3.6 \\
\hline
\end{tabular}


Table B.2 - Continued

\begin{tabular}{|c|c|c|c|c|c|c|}
\hline cuspid & year & jul.day & lat. $^{\circ}$ & lon. ${ }^{\circ}$ & depth & mag \\
\hline 3150361 & 2009 & 249 & -45.985 & 166.143 & 29.931 & 3.8 \\
\hline 3150770 & 2009 & 250 & -45.628 & 167.294 & 11.769 & 3.3 \\
\hline 3150827 & 2009 & 250 & -41.309 & 172.827 & 139.828 & 3.8 \\
\hline 3151327 & 2009 & 251 & -46.070 & 165.976 & 29.168 & 4.5 \\
\hline 3151404 & 2009 & 251 & -41.227 & 172.680 & 139.723 & 3.0 \\
\hline 3151539 & 2009 & 252 & -45.535 & 166.695 & 23.847 & 4.0 \\
\hline 3151852 & 2009 & 252 & -45.546 & 166.690 & 20.138 & 4.9 \\
\hline 3152083 & 2009 & 253 & -40.725 & 174.347 & 72.184 & 4.1 \\
\hline 3152626 & 2009 & 254 & -46.123 & 165.837 & 33.000 & 4.1 \\
\hline 3152685 & 2009 & 254 & -40.813 & 174.620 & 44.762 & 3.7 \\
\hline 3153108 & 2009 & 255 & -41.311 & 174.546 & 41.211 & 3.6 \\
\hline 3153511 & 2009 & 256 & -43.053 & 172.816 & 29.129 & 3.4 \\
\hline 3153651 & 2009 & 243 & -46.343 & 166.004 & 33.000 & 3.7 \\
\hline 3153729 & 2009 & 257 & -45.481 & 166.733 & 21.285 & 3.5 \\
\hline 3154556 & 2009 & 258 & -45.302 & 166.797 & 12.000 & 4.4 \\
\hline 3154683 & 2009 & 259 & -45.985 & 166.632 & 19.576 & 3.6 \\
\hline 3154988 & 2009 & 259 & -46.201 & 165.958 & 33.000 & 4.1 \\
\hline 3155299 & 2009 & 260 & -45.094 & 167.368 & 123.759 & 4.2 \\
\hline 3156081 & 2009 & 262 & -44.843 & 166.935 & 12.000 & 3.9 \\
\hline 3156088 & 2009 & 262 & -45.118 & 167.554 & 5.000 & 3.8 \\
\hline 3157492 & 2009 & 265 & -40.651 & 174.874 & 41.600 & 3.6 \\
\hline 3157795 & 2009 & 265 & -40.117 & 174.882 & 17.602 & 3.7 \\
\hline 3157830 & 2009 & 265 & -45.058 & 167.476 & 117.902 & 3.5 \\
\hline 3158054 & 2009 & 266 & -43.651 & 168.212 & 33.000 & 3.1 \\
\hline 3158128 & 2009 & 266 & -44.277 & 167.929 & 12.000 & 4.4 \\
\hline 3158502 & 2009 & 267 & -41.748 & 172.473 & 80.029 & 3.2 \\
\hline 3159659 & 2009 & 269 & -44.466 & 168.171 & 12.000 & 4.5 \\
\hline 3159733 & 2009 & 270 & -45.709 & 166.541 & 16.656 & 3.8 \\
\hline 3160421 & 2009 & 271 & -46.125 & 166.093 & 27.798 & 4.2 \\
\hline 3161106 & 2009 & 272 & -44.572 & 175.481 & 33.000 & 3.7 \\
\hline 3161447 & 2009 & 273 & -45.305 & 166.844 & 27.355 & 3.7 \\
\hline 3161601 & 2009 & 274 & -46.089 & 166.004 & 25.565 & 3.6 \\
\hline 3161674 & 2009 & 274 & -46.088 & 166.032 & 24.827 & 4.2 \\
\hline 3161700 & 2009 & 274 & -46.144 & 165.923 & 12.000 & 4.7 \\
\hline 3161868 & 2009 & 274 & -46.108 & 165.941 & 26.416 & 4.1 \\
\hline 3161988 & 2009 & 274 & -45.392 & 166.700 & 21.507 & 3.6 \\
\hline 3162137 & 2009 & 275 & -44.592 & 168.173 & 81.789 & 3.1 \\
\hline 3162293 & 2009 & 275 & -46.065 & 165.923 & 29.578 & 3.8 \\
\hline 3163125 & 2009 & 277 & -44.577 & 175.344 & 33.000 & 3.2 \\
\hline 3163469 & 2009 & 278 & -40.966 & 173.431 & 89.585 & 3.1 \\
\hline 3164709 & 2009 & 280 & -46.088 & 166.244 & 26.738 & 3.7 \\
\hline 3164782 & 2009 & 280 & -42.325 & 173.660 & 58.120 & 3.1 \\
\hline 3165019 & 2009 & 281 & -45.606 & 166.775 & 16.462 & 4.7 \\
\hline 3165850 & 2009 & 283 & -46.035 & 165.916 & 28.392 & 3.8 \\
\hline 3165869 & 2009 & 283 & -41.162 & 174.602 & 41.898 & 4.8 \\
\hline 3166610 & 2009 & 284 & -45.545 & 166.745 & 20.234 & 3.6 \\
\hline 3166997 & 2009 & 285 & -45.062 & 167.386 & 92.545 & 3.6 \\
\hline 3168258 & 2009 & 288 & -44.761 & 167.299 & 5.000 & 3.6 \\
\hline 3168423 & 2009 & 288 & -45.604 & 166.766 & 18.208 & 3.5 \\
\hline 3170073 & 2009 & 290 & -45.083 & 167.454 & 94.385 & 4.1 \\
\hline 3171001 & 2009 & 292 & -45.148 & 167.089 & 33.000 & 4.3 \\
\hline 3171550 & 2009 & 293 & -45.665 & 166.876 & 62.468 & 3.6 \\
\hline 3171833 & 2009 & 294 & -41.064 & 172.958 & 155.883 & 3.6 \\
\hline 3172220 & 2009 & 295 & -41.285 & 172.933 & 117.325 & 3.7 \\
\hline 3172529 & 2009 & 295 & -41.096 & 173.597 & 92.472 & 3.5 \\
\hline 3173062 & 2009 & 297 & -46.116 & 165.796 & 12.000 & 4.0 \\
\hline 3174178 & 2009 & 299 & -45.889 & 166.541 & 18.847 & 3.6 \\
\hline 3174442 & 2009 & 299 & -45.134 & 167.331 & 114.108 & 3.7 \\
\hline 3176786 & 2009 & 304 & -44.001 & 169.068 & 12.000 & 4.4 \\
\hline 3178306 & 2009 & 307 & -42.187 & 172.632 & 2.000 & 4.2 \\
\hline 3178429 & 2009 & 308 & -42.048 & 172.761 & 89.719 & 4.5 \\
\hline 3178435 & 2009 & 308 & -45.552 & 166.825 & 19.317 & 3.5 \\
\hline 3179193 & 2009 & 309 & -45.457 & 166.706 & 22.504 & 3.7 \\
\hline 3181530 & 2009 & 314 & -42.476 & 172.891 & 42.801 & 3.5 \\
\hline 3182457 & 2009 & 316 & -45.486 & 166.755 & 21.047 & 3.7 \\
\hline 3182458 & 2009 & 316 & -45.463 & 166.746 & 21.672 & 4.0 \\
\hline 3183512 & 2009 & 318 & -46.087 & 166.049 & 27.984 & 3.8 \\
\hline 3185298 & 2009 & 321 & -41.261 & 173.149 & 122.563 & 3.2 \\
\hline 3185741 & 2009 & 322 & -44.680 & 167.492 & 5.000 & 3.7 \\
\hline 3186184 & 2009 & 323 & -45.161 & 168.875 & 5.000 & 3.7 \\
\hline
\end{tabular}


Table B.2 - Continued

\begin{tabular}{|c|c|c|c|c|c|c|}
\hline cuspid & year & jul.day & lat. $^{\circ}$ & lon. ${ }^{\circ}$ & depth & mag. \\
\hline 3186228 & 2009 & 323 & -43.218 & 172.871 & 33.000 & 4.2 \\
\hline 3186604 & 2009 & 324 & -47.230 & 165.755 & 33.000 & 4.2 \\
\hline 3187010 & 2009 & 325 & -45.021 & 167.431 & 89.211 & 3.5 \\
\hline 3188308 & 2009 & 328 & -44.603 & 168.197 & 88.074 & 3.0 \\
\hline 3188423 & 2009 & 197 & -45.861 & 165.870 & 12.000 & 3.7 \\
\hline 3188874 & 2009 & 197 & -45.512 & 166.612 & 19.765 & 4.1 \\
\hline 3188888 & 2009 & 329 & -46.120 & 166.051 & 25.584 & 4.3 \\
\hline 3190183 & 2009 & 331 & -46.059 & 165.976 & 23.551 & 3.6 \\
\hline 3190570 & 2009 & 332 & -43.339 & 171.961 & 8.759 & 3.5 \\
\hline 3190843 & 2009 & 333 & -45.258 & 166.970 & 33.000 & 3.8 \\
\hline 3191284 & 2009 & 334 & -45.741 & 166.717 & 12.000 & 3.6 \\
\hline 3202873 & 2009 & 336 & -44.941 & 167.103 & 20.512 & 3.7 \\
\hline 3203616 & 2009 & 338 & -42.700 & 175.697 & 33.000 & 3.1 \\
\hline 3204025 & 2009 & 339 & -40.805 & 172.910 & 209.131 & 4.0 \\
\hline 3204445 & 2009 & 340 & -45.082 & 167.322 & 117.553 & 3.5 \\
\hline 3204538 & 2009 & 340 & -42.061 & 174.443 & 28.002 & 3.0 \\
\hline 3205351 & 2009 & 342 & -46.011 & 166.840 & 81.918 & 4.1 \\
\hline 3205412 & 2009 & 342 & -41.633 & 172.342 & 106.367 & 3.3 \\
\hline 3205715 & 2009 & 342 & -40.562 & 174.853 & 48.911 & 5.1 \\
\hline 3205722 & 2009 & 342 & -45.783 & 165.893 & 33.000 & 4.3 \\
\hline 3206052 & 2009 & 343 & -45.117 & 167.373 & 95.279 & 3.8 \\
\hline 3206940 & 2009 & 345 & -41.083 & 174.318 & 67.175 & 3.7 \\
\hline 3207634 & 2009 & 346 & -40.555 & 174.844 & 48.993 & 4.2 \\
\hline 3207977 & 2009 & 347 & -46.073 & 165.961 & 12.000 & 4.4 \\
\hline 3209307 & 2009 & 349 & -44.383 & 168.188 & 5.000 & 4.6 \\
\hline 3210836 & 2009 & 353 & -44.385 & 168.190 & 2.000 & 3.2 \\
\hline 3211310 & 2009 & 353 & -45.000 & 167.494 & 97.385 & 3.6 \\
\hline 3211830 & 2009 & 354 & -45.733 & 166.221 & 2.000 & 4.0 \\
\hline 3212649 & 2009 & 356 & -40.997 & 174.683 & 43.612 & 4.3 \\
\hline 3212678 & 2009 & 356 & -44.344 & 167.020 & 12.000 & 3.9 \\
\hline 3212744 & 2009 & 357 & -44.285 & 166.995 & 12.000 & 3.7 \\
\hline 3212774 & 2009 & 357 & -44.418 & 167.058 & 12.000 & 4.1 \\
\hline 3213027 & 2009 & 357 & -45.464 & 166.649 & 5.000 & 3.7 \\
\hline 3215170 & 2009 & 362 & -45.693 & 166.242 & 5.000 & 3.8 \\
\hline 3215421 & 2009 & 362 & -44.432 & 167.017 & 12.000 & 3.9 \\
\hline 3215677 & 2009 & 363 & -44.404 & 167.038 & 5.000 & 4.6 \\
\hline 3215699 & 2009 & 363 & -44.427 & 167.040 & 5.000 & 4.1 \\
\hline 3215741 & 2009 & 363 & -44.400 & 167.025 & 12.000 & 3.5 \\
\hline 3215786 & 2009 & 363 & -44.249 & 166.901 & 12.000 & 3.9 \\
\hline 3215798 & 2009 & 363 & -44.400 & 167.040 & 5.000 & 4.1 \\
\hline 3215901 & 2009 & 363 & -44.289 & 167.009 & 5.000 & 3.7 \\
\hline 3215902 & 2009 & 363 & -44.370 & 167.004 & 12.000 & 3.7 \\
\hline 3218624 & 2010 & 004 & -44.286 & 166.981 & 12.000 & 3.7 \\
\hline 3218632 & 2010 & 004 & -44.326 & 166.986 & 12.000 & 4.1 \\
\hline 3218873 & 2010 & 004 & -44.350 & 167.035 & 12.000 & 3.6 \\
\hline 3218875 & 2010 & 004 & -44.288 & 166.942 & 5.000 & 3.5 \\
\hline 3218911 & 2010 & 004 & -44.415 & 167.036 & 5.000 & 3.7 \\
\hline 3219107 & 2010 & 005 & -44.336 & 167.049 & 12.000 & 3.5 \\
\hline 3219127 & 2010 & 005 & -44.330 & 167.007 & 12.000 & 3.8 \\
\hline 3219727 & 2010 & 006 & -45.066 & 167.404 & 88.520 & 3.8 \\
\hline 3226288 & 2010 & 007 & -43.589 & 168.092 & 5.000 & 3.0 \\
\hline 3227595 & 2010 & 007 & -40.379 & 174.225 & 90.911 & 4.4 \\
\hline 3228164 & 2010 & 008 & -45.133 & 167.244 & 84.824 & 3.8 \\
\hline 3228266 & 2010 & 008 & -41.112 & 174.739 & 32.995 & 3.7 \\
\hline 3229512 & 2010 & 011 & -45.771 & 166.593 & 5.000 & 3.6 \\
\hline 3229793 & 2010 & 011 & -45.101 & 167.342 & 116.846 & 3.7 \\
\hline 3229901 & 2010 & 012 & -44.167 & 168.445 & 5.000 & 3.7 \\
\hline 3239748 & 2010 & 013 & -44.351 & 167.030 & 5.000 & 3.6 \\
\hline 3239807 & 2010 & 013 & -44.292 & 167.057 & 5.000 & 4.0 \\
\hline 3241870 & 2010 & 014 & -44.133 & 168.387 & 5.000 & 4.6 \\
\hline 3243091 & 2010 & 016 & -43.200 & 173.495 & 33.000 & 3.8 \\
\hline 3243510 & 2010 & 017 & -45.138 & 167.411 & 109.896 & 3.1 \\
\hline 3244402 & 2010 & 019 & -44.877 & 167.654 & 78.381 & 3.5 \\
\hline 3244959 & 2010 & 021 & -46.050 & 169.862 & 14.461 & 3.2 \\
\hline 3245861 & 2010 & 022 & -45.479 & 166.694 & 12.000 & 4.6 \\
\hline 3245913 & 2010 & 022 & -44.164 & 168.423 & 5.000 & 4.1 \\
\hline 3246039 & 2010 & 023 & -41.203 & 172.747 & 169.818 & 3.5 \\
\hline 3248116 & 2010 & 027 & -44.370 & 168.003 & 5.000 & 3.9 \\
\hline 3248616 & 2010 & 028 & -41.242 & 172.757 & 146.179 & 3.2 \\
\hline 3249826 & 2010 & 031 & -41.984 & 173.206 & 55.878 & 3.1 \\
\hline
\end{tabular}


Table B.2 - Continued

\begin{tabular}{|lllllll|}
\hline cuspid & year & jul.day $^{\text {jut. }}{ }^{\circ}$ & lon. & & depth & mag. \\
\hline 3250965 & 2010 & 033 & -46.273 & 166.185 & 23.050 & 3.8 \\
3251871 & 2010 & 035 & -44.938 & 167.685 & 72.093 & 3.1 \\
3252436 & 2010 & 036 & -44.977 & 167.512 & 63.735 & 3.2 \\
3252455 & 2010 & 036 & -44.726 & 167.410 & 5.000 & 5.0 \\
3254472 & 2010 & 040 & -41.952 & 172.605 & 78.788 & 4.4 \\
3260100 & 2010 & 043 & -40.983 & 173.886 & 70.729 & 3.4 \\
3314094 & 2009 & 196 & -46.032 & 166.085 & 12.000 & 5.2 \\
3316575 & 2009 & 196 & -45.485 & 166.643 & 12.000 & 5.2 \\
3321339 & 2009 & 196 & -46.067 & 166.077 & 20.000 & 4.7 \\
3324631 & 2009 & 196 & -45.932 & 166.149 & 12.000 & 4.3 \\
3327447 & 2009 & 196 & -46.024 & 166.055 & 12.000 & 4.1 \\
3328516 & 2009 & 196 & -45.546 & 166.634 & 12.000 & 4.8 \\
3330099 & 2009 & 196 & -45.680 & 166.740 & 22.207 & 3.6 \\
3333207 & 2009 & 196 & -46.043 & 166.085 & 20.000 & 3.7 \\
3340769 & 2009 & 196 & -45.627 & 166.716 & 5.000 & 4.4 \\
3341255 & 2009 & 196 & -46.035 & 166.093 & 12.000 & 4.9 \\
3348155 & 2009 & 196 & -46.046 & 165.989 & 12.000 & 3.9 \\
3348162 & 2009 & 196 & -46.033 & 166.168 & 12.000 & 3.5 \\
3348710 & 2009 & 196 & -45.924 & 166.223 & 12.000 & 3.9 \\
3350237 & 2009 & 196 & -46.007 & 166.108 & 12.000 & 4.9 \\
3350700 & 2009 & 196 & -45.424 & 166.649 & 12.000 & 4.3 \\
3350709 & 2009 & 196 & -46.016 & 166.182 & 20.000 & 4.7 \\
\hline
\end{tabular}

\section{B.4 Combined analysis of splitting tomography and spatial averaging}

The splitting data set used for this analysis consists of all AB-grade best filter measurements with $\delta t \leq 0.7 \mathrm{~s}$ (see the electronic supplement provided with this thesis for measurement information).

Table B.3 Measurement information

\begin{tabular}{|lc|}
\hline \multicolumn{2}{|c|}{ combined analysis } \\
\hline stations & 56 \\
no. events & 2984 \\
event-station pairs & 7035 \\
\hline
\end{tabular}

${ }^{\ddagger}$ high grade measurements are defined as $\mathrm{AB}$ grade measurements with $\lambda_{\max }>5$. Measurements with $\delta t>0.7$ s have been eliminated. 


\section{Appendix C}

\section{Southern NI investigation}

\section{C.1 Station information}

Table C.1 Station information of the SAHKE transect in southern NI

\begin{tabular}{|l|l|l|l|l|l|l|}
\hline station & st.lat $^{\circ}$ & st.lon. $^{\circ}$ & $\begin{array}{l}\text { elevation } \\
(\mathbf{m})\end{array}$ & $\begin{array}{l}\text { logger } \\
\text { type }\end{array}$ & $\begin{array}{l}\text { logger } \\
\text { no. }\end{array}$ & $\begin{array}{l}\text { sensor } \\
\text { type }\end{array}$ \\
\hline \hline LE4 & -41.3579 & 175.6919 & 197 & Reftek & 9298 & $40 \mathrm{~T}$ \\
LTN6 & -41.1033 & 175.3238 & 103 & Reftek & 9926 & $3 \mathrm{ESP}$ \\
T004 & -41.3403 & 175.6688 & 47 & Reftek & AC13 & $3 \mathrm{ESP}$ \\
T007 & -41.3041 & 175.6513 & 48 & - & AUS & $40 \mathrm{~T}$ \\
T010 & -41.2520 & 175.5825 & 111 & - & AUS & $40 \mathrm{~T}$ \\
T014 & -41.2075 & 175.5063 & 145 & - & AUS & $40 \mathrm{~T}$ \\
T016 & -41.1893 & 175.4737 & 39 & Reftek & AC40 & $40 \mathrm{~T}$ \\
T018 & -41.1715 & 175.4257 & 56 & - & AUS & $40 \mathrm{~T}$ \\
T020 & -41.1461 & 175.3850 & 23 & Reftek & 9F96 & $40 \mathrm{~T}$ \\
T022 & -41.1251 & 175.3497 & 31 & - & AUS & $40 \mathrm{~T}$ \\
\hline
\end{tabular}

C.2 Event information of the acceptable quality splitting measurements 


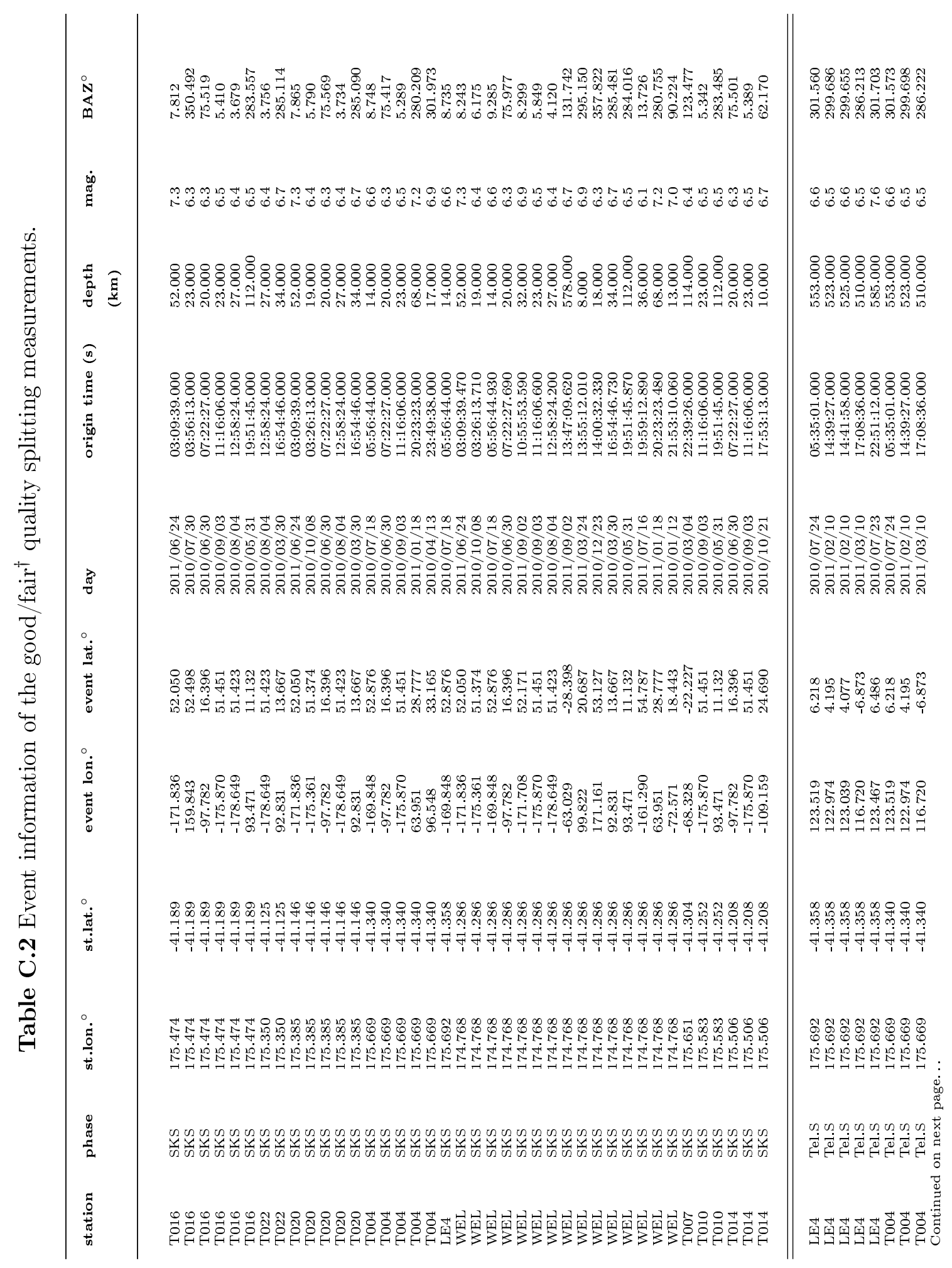




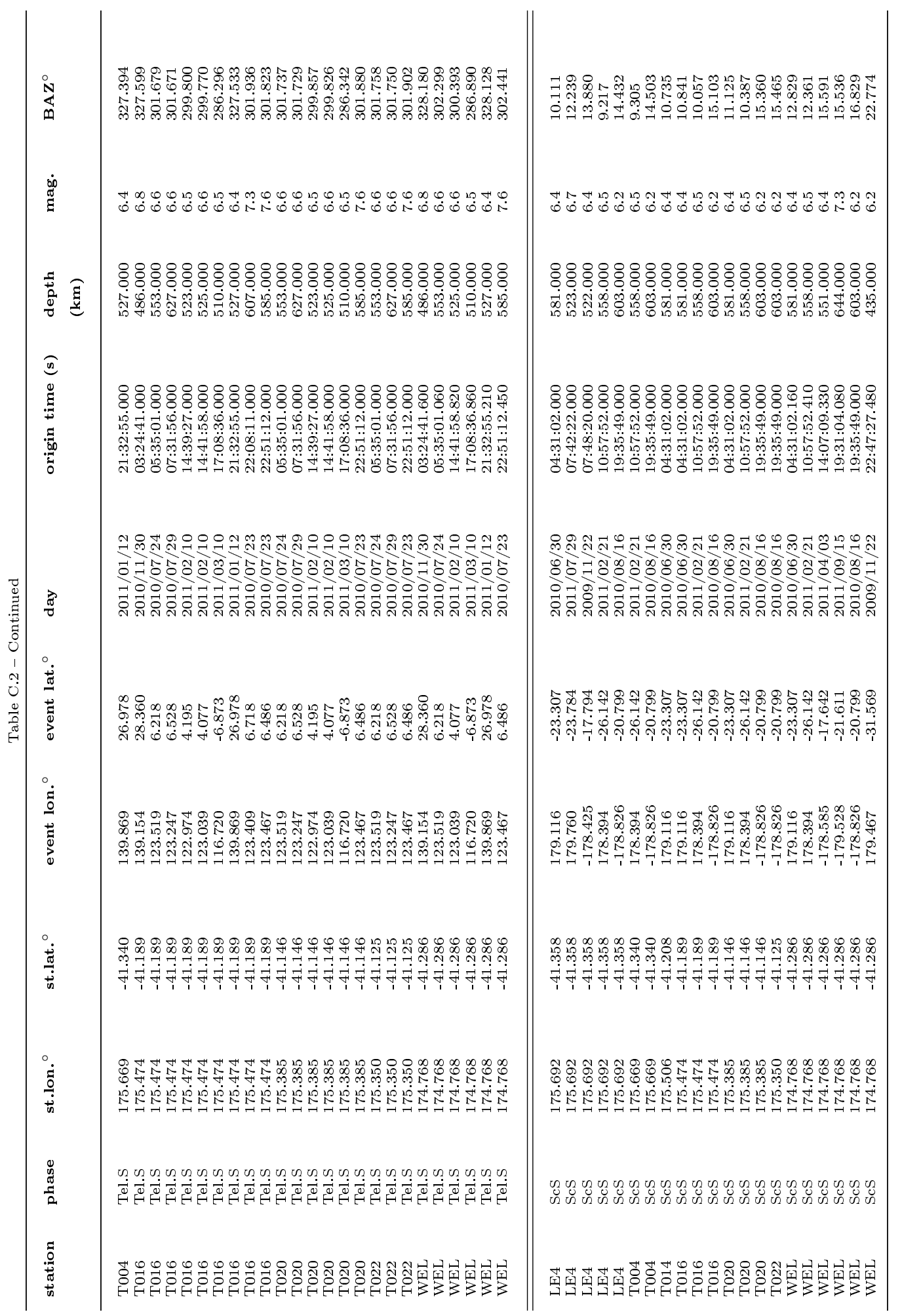




\section{C.3 Null-measurement information}

Table C.3 Information of the null-splitting ${ }^{\dagger}$ measurements.

\begin{tabular}{|c|c|c|c|c|c|c|c|}
\hline \multirow[t]{2}{*}{ station } & event & event & phase & $\delta t(\mathbf{s})$ & error & $\phi^{\circ}$ & $\operatorname{error} \phi^{\circ} \quad \phi_{p} *$ \\
\hline & lat. ${ }^{\circ}$ & lon. ${ }^{\circ}$ & & & $\delta t(\mathbf{s})$ & & \\
\hline
\end{tabular}

\begin{tabular}{|c|c|c|c|c|c|c|c|c|}
\hline LE4 & -171.836 & 52.050 & SKS & 5.888 & 1.425 & 12.000 & 16.250 & 7.680 \\
\hline LE4 & -63.029 & -28.398 & SKS & 6.000 & 1.500 & -51.000 & 45.250 & 131.119 \\
\hline LE4 & 99.822 & 20.687 & SKS & 5.700 & 0.591 & 34.000 & 3.250 & 294.555 \\
\hline LE4 & -68.228 & -21.701 & SKS & 5.438 & 1.378 & -46.000 & 14.750 & 123.165 \\
\hline T004 & -68.216 & -22.146 & SKS & 5.662 & 1.453 & -53.000 & 24.750 & 123.489 \\
\hline T004 & -171.836 & 52.050 & SKS & 6.000 & 0.356 & 13.000 & 1.000 & 7.694 \\
\hline T004 & -77.306 & -1.266 & SKS & 5.925 & 1.500 & 15.000 & 45.250 & 102.341 \\
\hline T004 & 99.822 & 20.687 & SKS & 5.287 & 0.384 & 33.000 & 1.250 & 294.570 \\
\hline T004 & -68.228 & -21.701 & SKS & 6.000 & 1.500 & -57.000 & 45.250 & 123.180 \\
\hline T007 & 92.831 & 13.667 & SKS & 3.487 & 1.031 & 18.000 & 1.000 & 284.909 \\
\hline Т014 & -169.848 & 52.876 & SKS & 6.400 & 0.200 & 4.000 & 22.500 & 8.844 \\
\hline T014 & -178.649 & 51.423 & SKS & 6.400 & 0.370 & -88.000 & 22.500 & 3.659 \\
\hline T014 & 92.831 & 13.667 & SKS & 3.337 & 0.947 & 23.000 & 23.250 & 285.008 \\
\hline T016 & -175.361 & 51.374 & SKS & 3.300 & 1.481 & -90.000 & 22.000 & 5.735 \\
\hline T016 & -169.848 & 52.876 & SKS & 4.013 & 0.506 & -90.000 & 1.750 & 8.863 \\
\hline T016 & -77.306 & -1.266 & SKS & 5.625 & 0.656 & -70.000 & 2.250 & 102.444 \\
\hline T016 & 99.822 & 20.687 & SKS & 5.550 & 1.500 & 21.000 & 45.250 & 294.703 \\
\hline T016 & -68.228 & -21.701 & SKS & 3.413 & 1.500 & 30.000 & 45.250 & 123.305 \\
\hline T016 & 92.831 & 13.667 & SKS & 2.700 & 0.956 & 23.000 & 4.250 & 285.030 \\
\hline T020 & -77.306 & -1.266 & SKS & 2.288 & 1.500 & -72.000 & 45.250 & 102.497 \\
\hline T020 & -68.228 & -21.701 & SKS & 2.850 & 0.366 & 25.000 & 1.500 & 123.363 \\
\hline T020 & 96.548 & 33.165 & SKS & 3.000 & 1.500 & -55.000 & 45.250 & 302.172 \\
\hline T022 & -175.361 & 51.374 & SKS & 4.950 & 1.500 & 5.000 & 45.250 & 5.812 \\
\hline T022 & -169.848 & 52.876 & SKS & 6.000 & 0.684 & -88.000 & 24.750 & 8.936 \\
\hline T022 & -175.870 & 51.451 & SKS & 3.562 & 1.378 & 85.000 & 24.000 & 5.486 \\
\hline T022 & -77.306 & -1.266 & SKS & 6.000 & 0.525 & 90.000 & 24.750 & 102.517 \\
\hline T022 & 96.548 & 33.165 & SKS & 5.438 & 1.031 & -69.000 & 24.750 & 302.196 \\
\hline WEL & -70.547 & -18.058 & SKS & 6.000 & 0.938 & 21.000 & 24.500 & 119.715 \\
\hline WEL & -63.136 & -26.803 & SKS & 3.487 & 0.947 & 33.000 & 2.500 & 130.652 \\
\hline WEL & -94.338 & 17.208 & SKS & 3.750 & 0.966 & 65.000 & 5.250 & 77.505 \\
\hline WEL & -71.558 & -8.087 & SKS & 6.000 & 1.022 & 12.000 & 4.750 & 112.015 \\
\hline WEL & -68.228 & -21.701 & SKS & 3.150 & 1.087 & 25.000 & 4.750 & 123.796 \\
\hline WEL & -65.089 & -15.364 & SKS & 2.513 & 1.181 & 26.000 & 5.000 & 121.780 \\
\hline WEL & 96.548 & 33.165 & SKS & 2.250 & 1.462 & -68.000 & 24.750 & 302.489 \\
\hline T004 & 139.154 & 28.360 & Tel. & 1.975 & 0.863 & -73.000 & 18.500 & 32.058 \\
\hline T004 & 139.869 & 26.978 & Tel. & 2.725 & 0.562 & 37.000 & 4.750 & 204.416 \\
\hline T020 & 139.869 & 26.978 & Tel. & 0.900 & 0.594 & -75.000 & 16.750 & 30.494 \\
\hline Т020 & 123.409 & 6.718 & Tel. & 2.900 & 1.000 & 45.000 & 45.250 & 139.682 \\
\hline WEL & 122.974 & 4.195 & Tel. & 3.225 & 0.875 & 40.000 & 16.500 & 142.929 \\
\hline WEL & 123.409 & 6.718 & Tel. & 4.000 & 1.000 & 34.000 & 45.250 & 125.664 \\
\hline LE4 & -179.528 & -21.611 & $\mathrm{ScS}$ & 2.475 & 0.531 & -40.000 & 29.250 & 4.660 \\
\hline T004 & -178.585 & -17.642 & $\mathrm{ScS}$ & 1.925 & 0.356 & 14.000 & 22.750 & 111.951 \\
\hline
\end{tabular}


Table C.3 - Continued

\begin{tabular}{lllllllll}
\hline station & event & event & phase & $\delta t(\mathbf{s})$ & error & $\phi^{\circ}$ & error $\phi^{\circ} \phi_{p}^{*}$ \\
& lat. $^{\circ}$ & lon. $^{\circ}$ & & & $\delta t(\mathbf{s})$ & & & \\
\hline T016 & 179.760 & -23.784 & ScS & 2.800 & 0.219 & -63.000 & 22.000 & -49.668 \\
T020 & -178.585 & -17.642 & ScS & 2.925 & 0.125 & 90.000 & 2.750 & 258.106 \\
WEL & 179.760 & -23.784 & ScS & 1.375 & 0.950 & 16.000 & 16.250 & 96.065 \\
WEL & -178.425 & -17.794 & ScS & 1.575 & 0.281 & 43.000 & 4.250 & 143.270 \\
\hline
\end{tabular}

*initial polarization direction. In the case of SKS, $\phi_{p}=\mathrm{BAZ}$

${ }^{\dagger}$ measurements are considered to be null if the $\left|\phi-\phi_{p}\right| \leq 12^{\circ}$ or $\left|\phi+90^{\circ}-\phi_{p}\right| \geq 80^{\circ}$ 


\section{C.4 Multilayer analysis}

We have detected lateral variations in splitting parameters (Fig. 6.8) along the SAHKE profile and delay times from $\mathrm{S}$ phases also exhibit frequency dependency throughout the southern Hikurangi subduction zone (Fig. 6.5 and Marson-Pidgeon and Savage [1997]). These observations suggest that lateral and/or depth varying anisotropic structure is plausible in the region. Since the frequency dependent splitting is obvious throughout the region, it is clear that this is not caused by a localized source of anisotropy. Anisotropy might, therefore, be characterized by a multilayer structure. To explain the observed lateral variations in anisotropy, this multilayer structure must contain layers with laterally varying anisotropy. To explain the frequency dependent splitting, this multilayer structure should accommodate anisotropy with varying length scales. It is possible these different length-scales of anisotropy are either localized in a single layer or distributed in two or more layers, mixing with different length scales of anisotropy.

Initial polarization $\left(\phi_{p}\right)$ versus splitting parameter analysis is used to determine the best-fitting 2-layer and 3-layer (section 3.5.1) models for the observed anisotropy in the study region. We use 2-layer and N-layer analysis codes provided by Wustefeld et al. [2008] and Walker and Wookey [2012] for this analysis. Both these codes use the Silver and Savage [1994] method to determine multilayer anisotropy. Our aim is to find whether the observed frequency dependent splitting and/or lateral variations are due to depth varying multilayer structure. Because of the lateral variations in splitting parameters, this modeling approach is applied to three distinct regions (regions E, C and S; Fig. C.1) that have been subdivided based on the lateral variations in splitting parameters and the spatial distribution of stations. Each model starts with a set of initially defined multi layer structures. We used the assumed anisotropic contributions in the region (e.g. absolute, and relative plate and upper-plate fault orientations; Fig. 6.2) to define anisotropic layers in our initial 


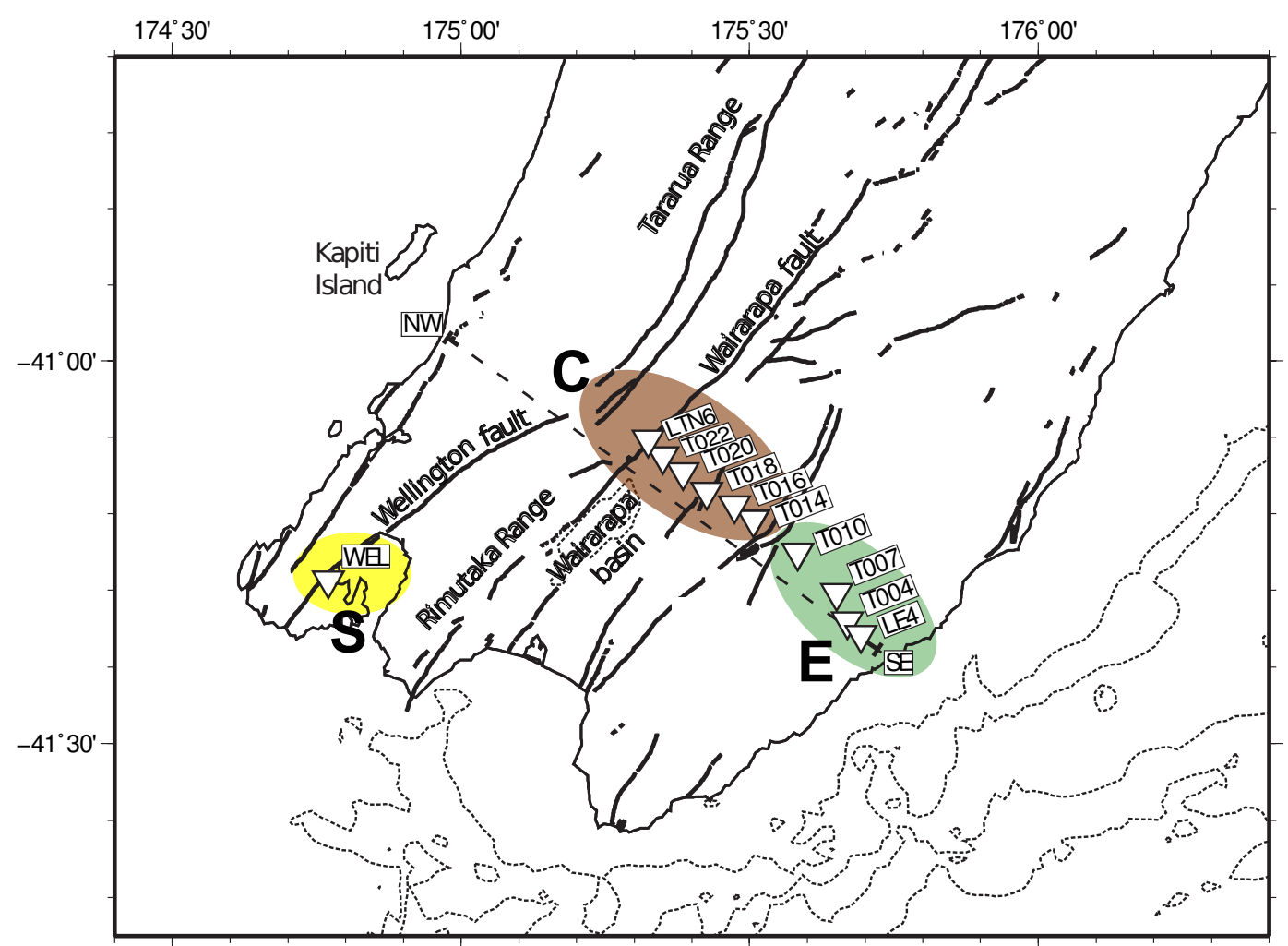

Figure C.1 Map showing the spatial division of stations that two and three layer analysis. Stations and models in green, brown and yellow zones are named as region $\mathbf{E}$, region $\mathbf{C}$ and $\mathbf{S}$ in the text respectively.

structures. In each layer, $\delta t$ and $\phi$ are set to vary within a range of values (table C.4) and a best-fitting model is determined for that particular structure. In two layer case the best fitting model is found by minimizing the total normalized sumsquared residuals of $\phi$ and $\delta t$. The total sum-squared residuals of $\phi$ and $\delta t$ are normalized by the maximum total sum-squared of $\phi$ and $\delta t$, respectively. Then the best fit 2-layer model is selected by finding the model with the minimum total $(\phi$, $\delta t)$ normalized sum-squared residuals (table C.5). Next we visually compared the best-fitting models obtained from different initially predicted structures to find the most appropriate model to explain the observed splitting parameters. Because of the short (5 s; ScS) and long period (11 s; SKS and Tel. S) S-phases Fig. 6.5) used in this study, some of the models are tested for different frequencies. However, due to the limited observed initial polarization directions for the short period phases $(\mathrm{ScS})$, it is hard to explain whether relatively smaller delays and frequency dependent splitting from $\mathrm{ScS}$ phases are caused by a spatial or a depth effect. At least three possible 
Table C.4 $\delta t$ and $\phi$ search ranges in each layer of the different 2-layer models.

\begin{tabular}{|l|ll||ll|}
\hline \multicolumn{5}{|c|}{ 2-layer search ranges } \\
\hline model & $\delta$-top(s) & $\delta$-bot. $(\mathrm{s})$ & $\phi$-top $\left(^{\circ}\right)$ & $\phi$-bot. $\left(^{\circ}\right)$ \\
\hline \hline C1 & $1-2.5$ & $0.8-1.2$ & $70-90$ & $-10-(-80)$ \\
C2 & $0.8-1.2$ & $1-2.5$ & $-10-(-80)$ & $70-90$ \\
C3 & $0.7-1$ & $1-1.5$ & $10-40$ & $70-85$ \\
\hline \hline E1 & $1-2.5$ & $0.8-1.2$ & $50-90$ & $-10-(-80)$ \\
E2 & $0.7-1$ & $1-1.5$ & $10-40$ & $70-85$ \\
\hline \hline S1 & $1-2.5$ & $0.8-1.2$ & $70-90$ & $-10-(-80)$ \\
S3 & $0.7-1$ & $1-1.5$ & $10-40$ & $70-85$ \\
\hline
\end{tabular}

sources could control the extent and alignment of anisotropic structure in the region (i.e. faulting in the upper plate, relative and absolute plate motions). Therefore, we have extended our two-layer analysis to a three-layer case. Best-fitting two-layer models are modified by adding another layer and by changing $\phi$ and $\delta t$ in a small range around the best-fit values of the resolved 2-layer model. In the following sections, we discuss the best resolved two and selected 3-layer models in each of the regions separately.

\section{C.4.1 Layered anisotropic structure in region $\mathrm{C}$}

This region lies in the Western end of the SAHKE transect (region C-brown zone in Fig. C.1). Splitting parameters from Tel. S and SKS (long period) phases in this region show a higher variability $(\sim 1-3.4 \mathrm{~s})$ and relatively large $\delta t(3.4 \pm 0.43 \mathrm{~s})$ (Fig. 6.8) compared to eastern side $(\sim 2.2 \pm 0.3 \mathrm{~s})$ (region E-brown; green zone in Fig. C.1). Figure C.4 shows the best fitting models resolved from three different multi layer structures (note the initial structural configuration of the 2-layers displays at the top of the each model).

The model $C_{1}$ appears to fit reasonably well with the observed data, suggesting the possibility of 2-layer anisotropic structure that is characterized by lower APM 
controlled anisotropy and upper RPM controlled anisotropy. The top layer of $C_{1}$ has high $\delta t(2.5 \mathrm{~s})$. This implies either higher strength of anisotropy or more unmodeled sub-layers of anisotropy in the upper layer, so that cumulative anisotropy can be as high as $2.5 \mathrm{~s}$. The mantle-wedge contribution must be negligible as the subduction is very shallow in this region. But, subducting slab-lithosphere ( $\sim 60 \mathrm{~km}$ thick),

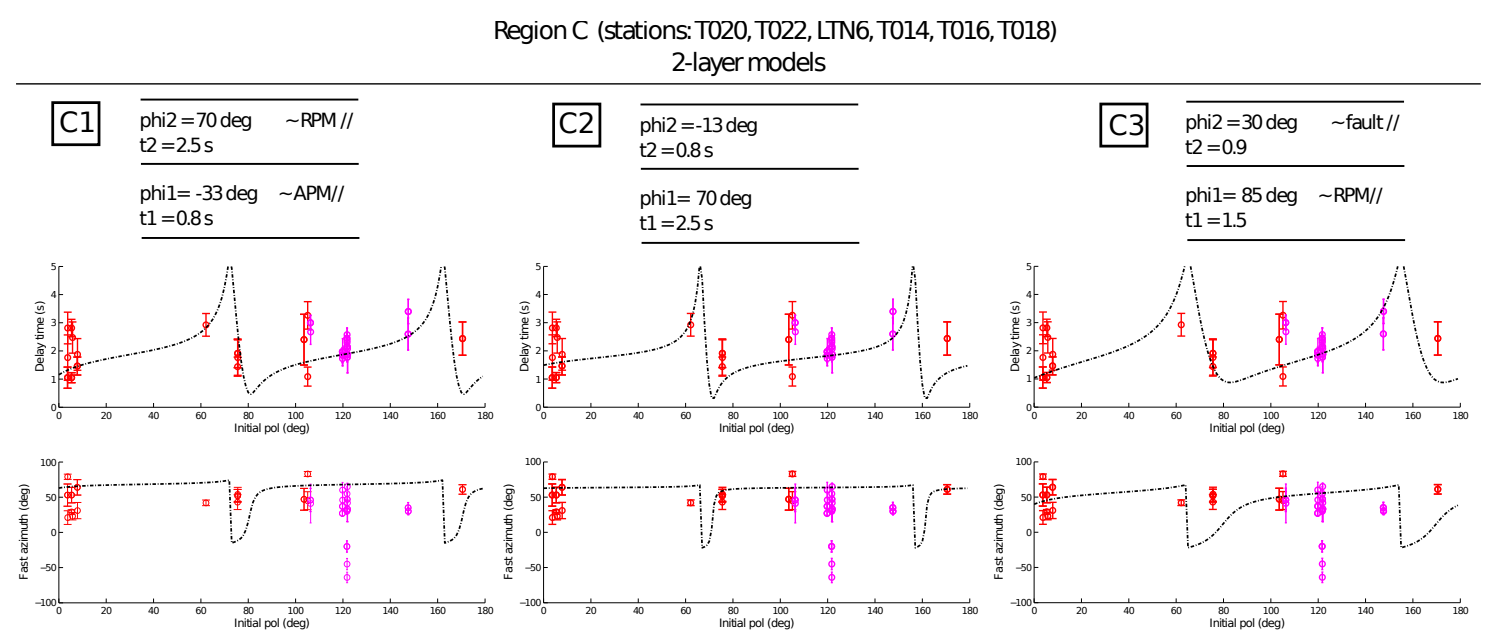

Figure C.2 Best fitting 2-layer models obtained for three different structures in the central part of southern NI (region $\mathbf{C}$ ). The best-fitting structural configuration with 2-layers displays top of the each model. Red and magenta symbols indicate the measured SKS and Tel. Splitting parameters respectively. The best fitting 2-layer model is marked in dashed-dotted line.

Region C (stations: T020, T022, LTN6, T014, T016, T018) 3-layer models

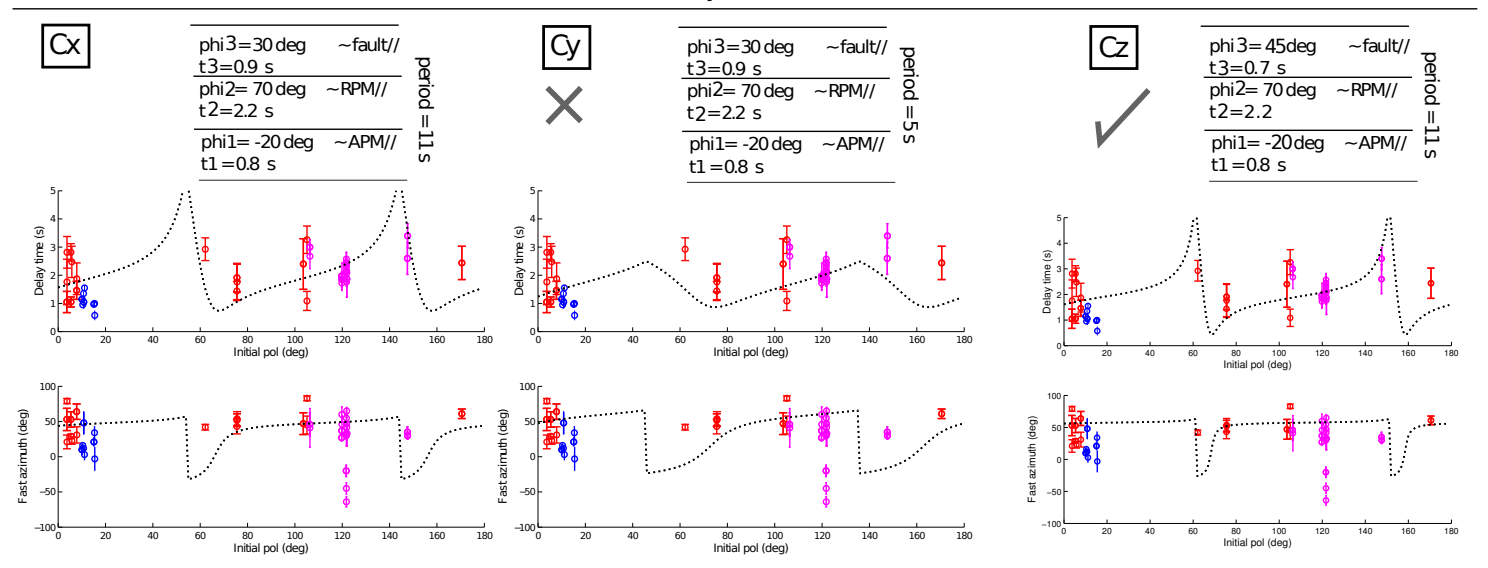

Figure C.3 Selected 3-layer models obtained for the extended versions of the best fitting two-layer structures in the central part of southern NI (region $\mathbf{C}$ ). The resolved structural configuration with 3-layers is shown top of the each model. Red, magenta and blue symbols indicate the measured SKS, Tel. S and ScS splitting parameters respectively. The best resolved 3-layer model is marked by the dotted line. Correct mark denotes the selected model that agree with the majority of the data points. 
Table C.5 Misfits ${ }^{\dagger}$ of the best-fit 2-layer and selected 3-layer models.

\begin{tabular}{|c|l|l|l||l|l|l|}
\cline { 2 - 7 } \multicolumn{1}{c|}{} & \multicolumn{3}{c||}{$\begin{array}{c}\text { 2-layer } \\
\text { normalized misfit }\end{array}$} & \multicolumn{3}{c|}{ 3-layer } \\
\hline region & 1 & 2 & 3 & $\mathrm{x}$ & $\mathrm{y}$ & $\mathrm{z}$ \\
\hline $\mathrm{C}$ & 0.21 & 0.21 & 1.04 & 4.77 & 5.65 & $\mathbf{4 . 7 3}$ \\
& & & & 246.35 & 265.32 & $\mathbf{2 4 2 . 3 6}$ \\
\hline \hline $\mathrm{E}$ & 0.11 & 0.69 & - & 7.41 & 9.31 & $\mathbf{4 . 7 7}$ \\
\hline \hline $\mathrm{S}$ & & & & 227.60 & 268.35 & $\mathbf{1 6 4 . 5 9}$ \\
\hline
\end{tabular}

${ }^{\dagger}$ minimum misfits was estimated from the normalized sum-squared residuals of $\phi$ and $\delta t$ in 2-layer case. In 3-layer case, the misfits of $\phi$ and $\delta t$ are determined separately by calculating the square root of sum-squared residuals of $\phi$ and $\delta t$. In 3 -layer case we did not determine the best-fit, thus $\phi$ and $\delta t$ are not normalized.

including its sub-slab region and upper-plate can act as two separate anisotropic layers (sub-layers) within the upper-layer of the well resolved 2-layer model. 2.5 s $\delta t$ can be produced by these two sub-layers, if the anisotropy in the layers are coupled. However, small-scale variations in the alignment of anisotropy in each layer are possible. Based on this possibility, we have tested another 2-layer case that is characterized by upper moderately high anisotropic zone (maximum up to 1 $\mathrm{s})$ and lower high anisotropic zone (maximum up to $1.5 \mathrm{~s}$ ). Anisotropic orientations are allowed to vary around RPM direction (table C.4) in the lower layer and fault parallel direction in the upper layer (e.g. best fitting model $C_{3}$ in Fig. C.2). Model $C_{3}$ shows the best-fit found for the above approximation and this also fit fairly well with the observations. Thus, we extended out 2-layer case to 3-layer case combining the best fit models of $C_{1}$ and $C_{3}$.

The initial 3-layer models are defined so that the top two layers are comparable with the resolved anisotropic layers by the best-fitting two layer models (Fig. C.2 ), but a third layer $\left(\phi=-20^{\circ}, \delta t=0.8 \mathrm{~s}\right)$ is added to represent potential APM-derived anisotropy (Fig. C.3). Figure C.3 (bottom) shows the 3-layer models $\left(C_{x}, C_{y}\right.$, and $C_{z}$ ) that are obtained from extended two-layer structures. We choose $C_{z}$ as the best-resolved 3-layer model that agrees well with our splitting data set. $C_{x}$ also 
shows a good fit to data, but we think that the upper $\delta t$ of $0.9 \mathrm{~s}$ is too high to occur within the upper-most layer. The selected 3-layer model $\left(C_{z}\right)$ (Table C.5), consists of layers that are approximately aligned (from deep to shallow) with the APM, RPM and general trend of the upper-plate faulting from bottom to top respectively. The $\delta t$ value of $2.2 \mathrm{~s}$ is constrained in the middle layer. Anisotropy responsible for such high $\delta t$ could be caused by a the thick anisotropic layer and/or a source with high strength of anisotropy. This model has a top most layer anisotropy of $0.7 \mathrm{~s}$, which is considerably higher than the typical crustal anisotropy $(\sim 0.2 \mathrm{~s})$. However, high delays in the top two layers can be explained by anisotropic structure associated with the high strength of anisotropy (e.g. fault structure with intense foliations and a broad shear-zone). This structure could be vertically localized in the upper most two layers, coupled with the background layer anisotropy (i.e. typical anisotropy of the layer). Therefore, high delays in the topmost two layers can be explained by this argument. Since this region underlies the large-scale dextral fault (Wairarapa fault), it is likely characterized by intense foliations and a broad shear-zone. We think that the uppermost of the three layers represents crust with high structural anisotropy (e.g. upper-plate crust with fault structures). The middle layer could have anisotropic contributions from the lower crustal, upper-mantle (sub-slab) and subducting-slab lithosphere, but this anisotropy could be enhanced by the vertically extending fault shear zone in order to produce $2.2 \mathrm{~s} \delta t$. Depth extent of this fault zone is hard to constrain accurately, but when compared with lower $\delta t(0.8 \mathrm{~s})$ and APM parallel $\phi$ from the bottom most layer, we argue that the shear zone below the fault does not extend beyond the sub-slab region. It is possible that this fault shear zone penetrates into the subducting-slab lithosphere, and possibly down to sub-slab region. About 2.8 - $3 \mathrm{~s}$ large $\delta t$ observed from few ray paths close to the Wairarapa fault may indicate the cumulative affect of anisotropy of top-most two layers, suggesting anisotropy due to foliations and shear zone and background anisotropy of the layer are vertically coupled. If we test the same layered structures for region E (Fig. C.1); we will find a good fit to data from a 3 layer structure, but 
with lower split $\delta t$ for the topmost two layers. This may be due to lack of large-scale faulting in the eastern part (see section C.4.2).

\section{C.4.2 Layered anisotropic structure in region $\mathrm{E}$}

Region E (green zone in Fig. C.1) includes the eastern part of the SAHKE profile. This region does not contain large-scale fault traces on the surface, but a few splayfaults of the Wairarapa fault are predicted to exposed in this region [Henrys et al., 2013]. We used the same approach we applied to region $\mathrm{C}$ to find the multilayer structure that could explain the observed splitting parameters. We have found two best fitting two layer models (Table C.5) with different layer approximations and they match fairly well with the observed splitting parameters. However, we prefer model $E_{2}$ as it fits well with the observed $\delta t$ (Figs. C.4). Since there are two different best fitting models (Table C.5) that explain two different layered structure, we searched for the 3-layer case by introducing third layer to both best-fitting two layer models and considering the possible depth distribution of anisotropic layers in the region. We have selected $E_{z}$ as the well resolved 3-layer model that explains the observations. This model also agrees with the 3-layer model resolved for the region $C_{z}$. It has the uppermost layer $\delta t$ anisotropy of $0.5 \mathrm{~s}$ that is aligned about $12^{\circ} \mathrm{NE}$, middle layer with $1.6 \mathrm{~s}$ with RPM sub-parallel $\left(71^{\circ}\right)$ anisotropy, and the lower layer of $0.8 \mathrm{~s}$ delay with approximately APM parallel anisotropy $\left(-20^{\circ} \mathrm{NW}\right)$.

When comparing the best constrained 3-layer models for regions $C$ and $E$ along the SAHKE profile, there is a clear discrepancy between $\delta t$ measured in the top two layers of the $C_{z}(\mathrm{top}=0.7 \mathrm{~s}$, middle $=2.2 \mathrm{~s})$ and $E_{z}(\mathrm{top}=0.5 \mathrm{~s}$, middle $=1.6 \mathrm{~s})$. Both areas have the lowermost layer anisotropy of 0.8 s roughly parallel to APM direction $\left(20^{\circ}\right)$. Although the general trend of $\phi$ in these two layers are NE-SW aligned, $\phi$ in $C_{z}$ is more NE-SW aligned $\left(45^{\circ}\right)$ compared to NNE-SSW $\left(12^{\circ}\right)$ alignment found for region $\mathbf{E}$. Higher $\delta t$ in the top two layers in $C_{z}$ compared to that in $E_{z}$ is evidence 


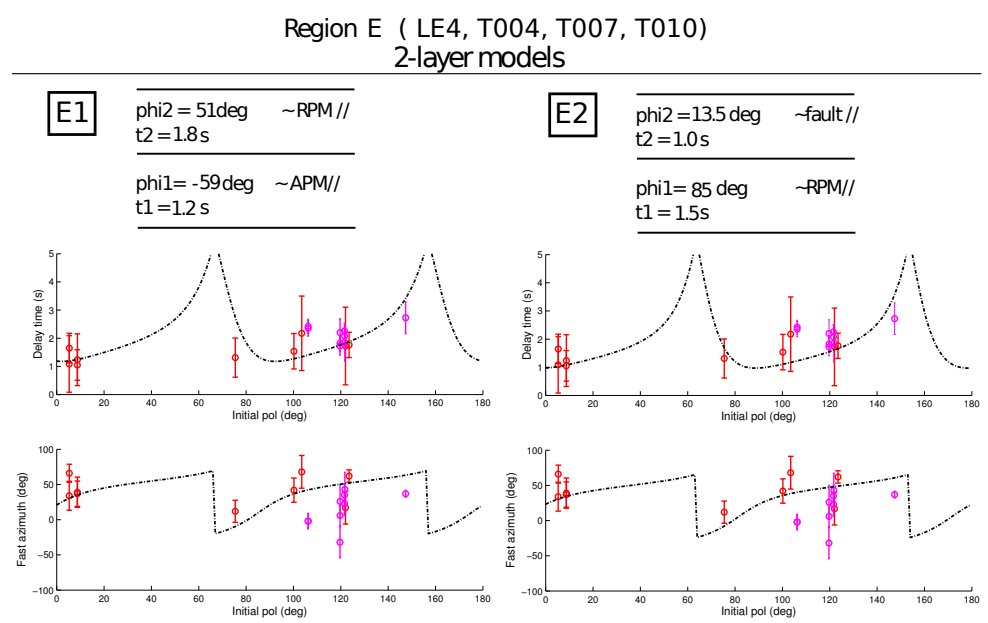

Figure C.4 Best fitting 2-layer models obtained for two different structures in eastern part of southern NI (region $\mathbf{E}$ ). The best-fitting structural configuration with 2-layers displays top of the each model. Red and magenta symbols indicate the measured SKS and Tel. Splitting parameters respectively. The best fitting two layer model is marked in dash-dot line.

Region E ( LE4, T004, T007, T010)

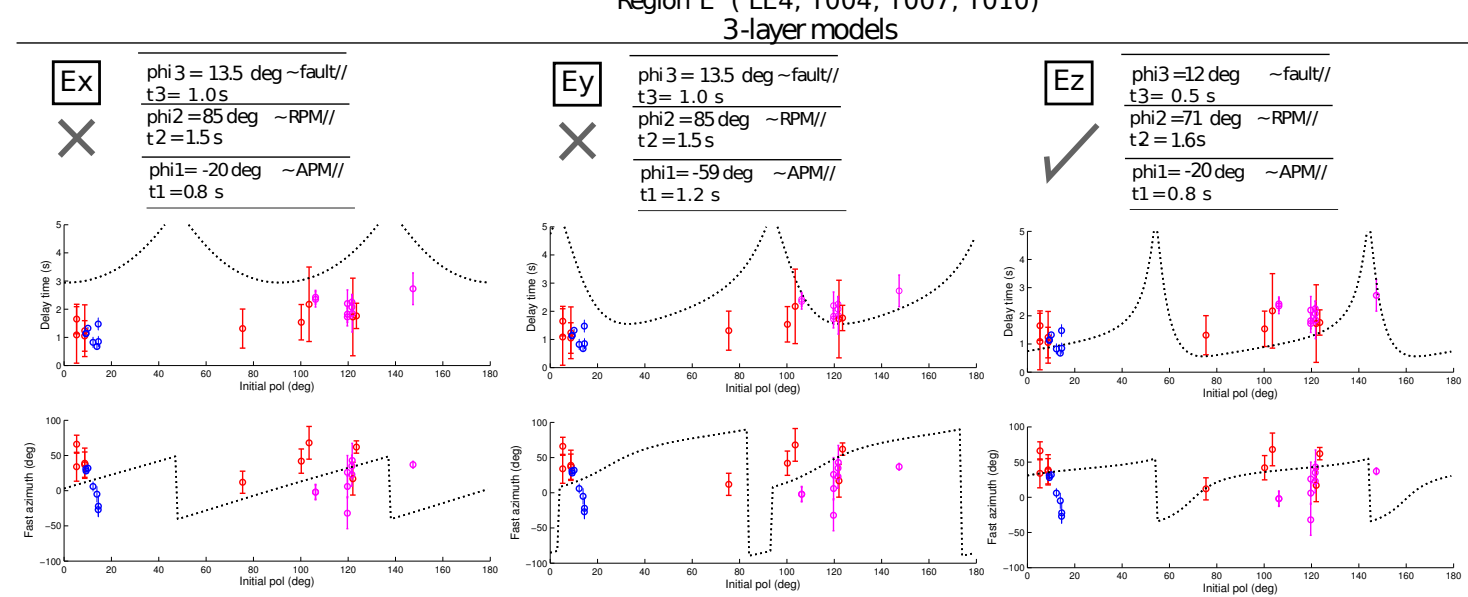

Figure C.5 Well resolved 3-layer models obtained for the extended versions of the best fitting two-layer structures in the eastern part of southern NI (region $\mathbf{C}$ ). The resolved structural configuration with 3-layers displays top of the each model. Red, magenta and blue symbols indicate the measured SKS, Tel. S and ScS splitting parameters respectively. The best fitting two layer model is marked by the dotted line. Correct mark denotes the selected model that agree with the majority of the data points.

for a predicted vertically extending high anisotropic structure, cross-cutting at least the topmost two layers. We think that this structure likely represents the Wairarapa fault and its associated shear-zone. Both crustal and sub-crustal layer anisotropy in region $\mathrm{C}$ appears to be enhanced by this structure. The lateral variation in $\delta t$ along the profile (Fig. 6.9) implies that the spatial extent of the shear zone at depth may be asymmetric and distributed in the Wairarapa basin area, but may not extend as 
far as region E from the fault.

\section{C.4.3 Layered anisotropic structure in region $\mathrm{S}$}

We used splitting measurements obtained from WEL station, which is located in region S, (Fig. C.1) to determine a multilayer structure in the southwest of the SAHKE profile. This region is also located around a large dextral strike-slip fault zone (Wellington fault). Anisotropy in this region was previously approximated with a 2-layer structure by using a different set of stations [Gledhill and Gubbins, 1996, Marson-Pidgeon and Savage, 2004a]. Dipping-anisotropy layer study by MarsonPidgeon and Savage [2004a] suggested a complex anisotropic structure in the region. Here, we analyze a new splitting data set with a better backazimuthal coverage that is measured on the GeoNet permanent station (WEL).

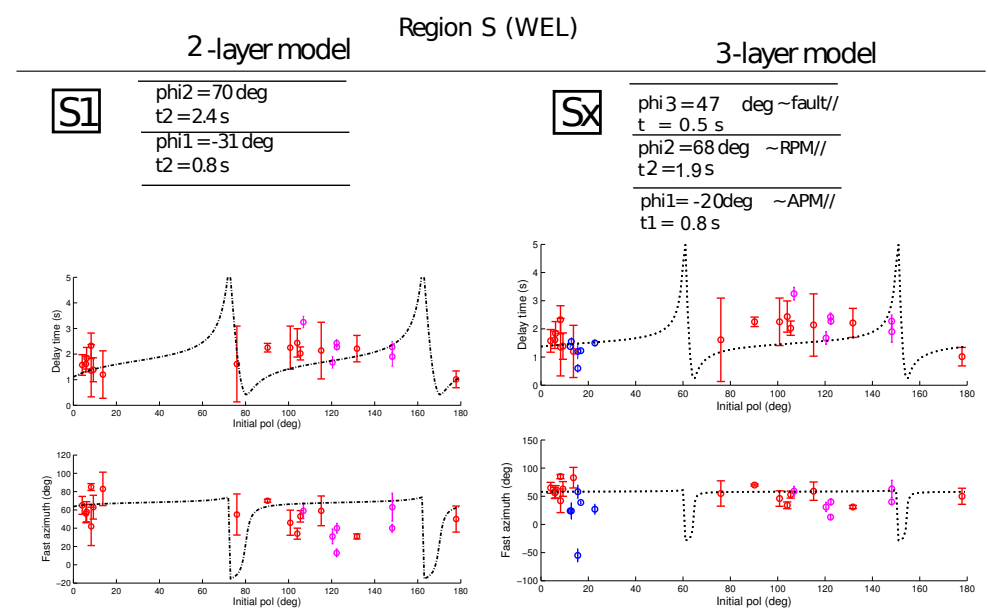

Figure C.6 3-layer and 2-layer models determined for the southwest region of the SHAKE profile (region $\mathbf{S}$ ). The figure symbols are same as in figures C.2 and C.3.

Both best fitting 2-layer $\left(S_{1}\right)$ and expanded 3-layer structure $\left(S_{x}\right)$ that are determined from the splitting measurements at WEL are shown in figure left $\left(S_{1}\right)$ and right $\left(S_{x}\right)$ respectively. Both models do not fit-well with the observations (Table C.5), suggesting a more complex anisotropic structure that could not be explained by a simple 2- or 3- layer model. 


\section{C.4.4 Overall structure}

The anisotropic structure of the southern North Island is likely stratified, but in complex manner. It is hard to distinguish between the best-fit models from different structures that are initially proposed for each region. Multilayer models suggest that the anisotropy of the individual layers can be enhanced by localized anisotropic zones that are possibly at shallow depths around faults, creating a more complex anisotropic structure and producing the observed lateral variation. Thus, this laterally varying anisotropy should be investigated using a different approach. These multilayer modeling results provide a first-order approximation on the possible layered anisotropic structure, however they do not fully explain the observations. 


\section{C.5 Combined analysis of short and long period frequency- dependent splitting}

Figure C.7 shows the frequency-dependent estimations in the Hikurangi subduction zone using both local and teleseismic $\delta t$. The extrapolated least-squares fits of both local and teleseismic measurements fit well with the observed measurements (Fig. C.7), suggesting S-phase with different periods will be sensitive to different anisotropic length scales and, thus, give a range of $\delta t$.

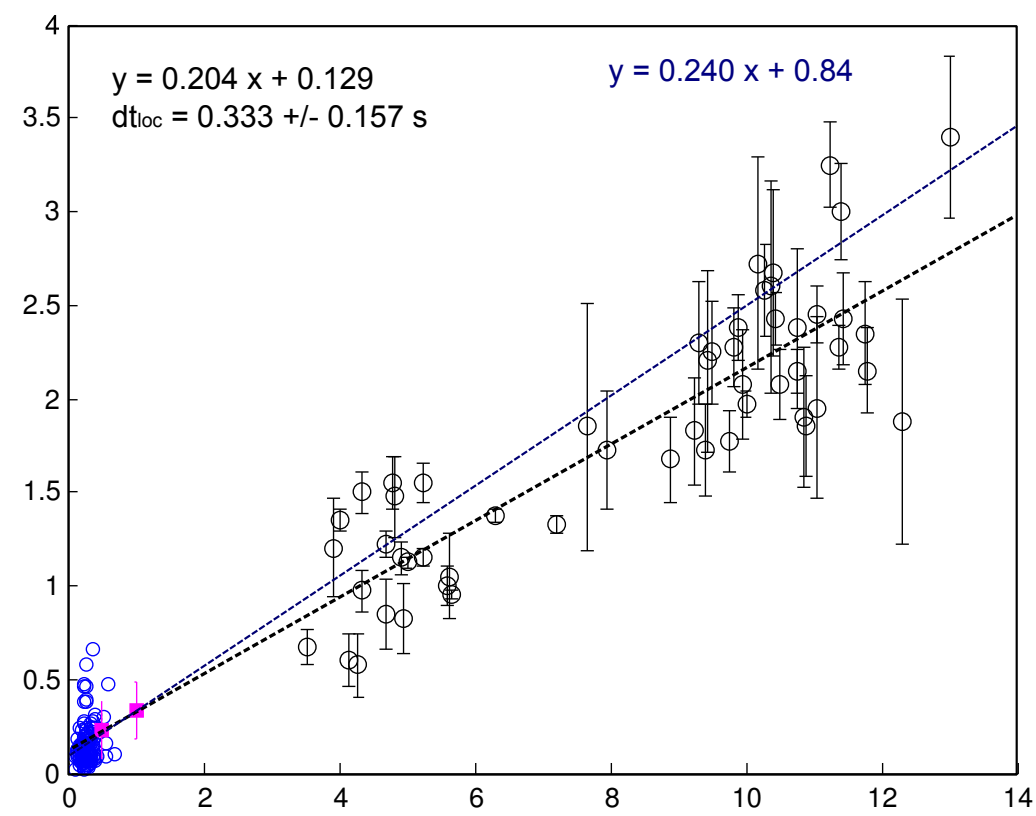

Figure C.7 An integration of frequency-dependent $\delta t$ from local splitting (blue circles) and teleseismic (ScS and Tel. S) splitting (black circles) in the Hikurangi subduction zone. Blue circles denotes the frequency-dependent local $\delta t$ that are determined at the northern SI stations (NSI in Fig. 4.9). Black circles represents frequency-dependent splitting observed in the southern SI (Fig. 6.5). Black and blue dashed-lines indicate the least-squares fit of the teleseismic and local data, respectively. Two pink squares denote the predicted local $\delta t$ measurements at periods $1 \mathrm{~s}$ and $0.5 \mathrm{~s}$ based on the teleseismic least-squares fit.

Predicted teleseismic $\delta t$ from the least-squares fit of local $\delta t$ versus period plots in figure Fig. 4.9 have been compared with the in-situ teleseismic $\delta t$ in SI. The average period of $6 \mathrm{~s}$ is assumed in the predictions. For large periods, local models seem to over-predict teleseismic $\delta t$ (Fig. 4.9).

According to Rumpker and Silver [1998], the measurements that lie in the region 


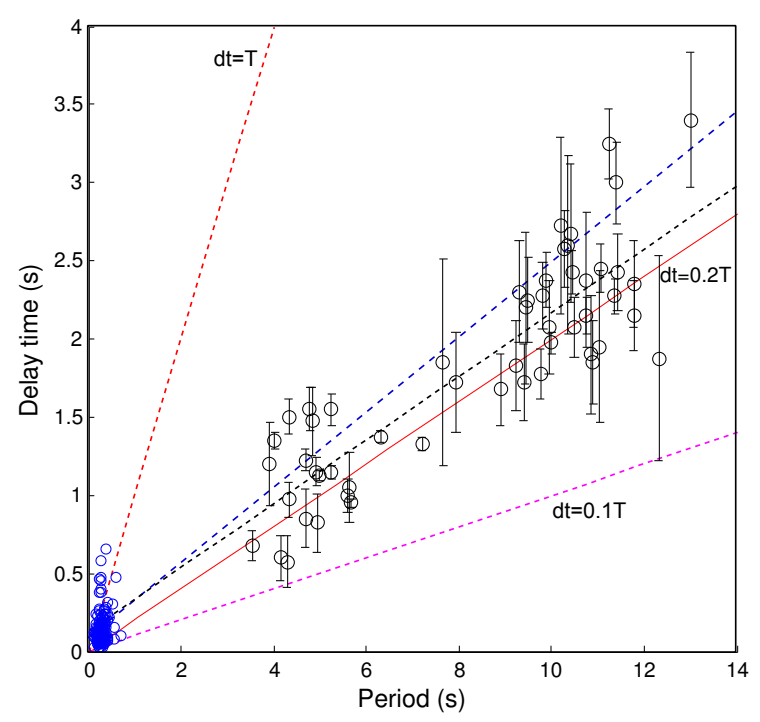

Figure C.8 Same as figure caption in Fig. C.7, but the frequency dependence is compared with the modeling approximations (red-bold and red-dashed lines) by Rumpker and Silver [1998]. The red-bold line represents: $\delta t=0.2 \mathrm{~T}$, the red-dashed line indicates: $\delta t=\mathrm{T}$ and the magenta-dashed line denotes: $\delta t=0.1 \mathrm{~T}$.

$\mathrm{T} / \delta t \lesssim 1$ (i.e. region above the red-dashed line) are biased towards the upper-most layer anisotropy. Only high frequency measurements (blue circles) agree with this model. The measurements with $\mathrm{T} / \delta t \gtrsim 5$ in figure C.8 (i.e. region below the red-bold line) likely represent the apparent splitting parameters from an underlying multilayered anisotropy. The majority of estimated $\delta t$ from both high-frequency and long-period S-waves splitting are less than 1/10 of a corresponding S-wave period (i.e. region above the magenta-dashed line).

Table C.6 Comparison between predicted SKS $\delta \mathrm{t}_{\text {tel }}$ at $6 \mathrm{~s}$ period from the local least-squares fits in figure 4.9 and measured $\delta \mathrm{t}_{t e l}$ values from previous SKS studies in approximately the same locations in SI [Savage et al., 2007a, Duclos et al., 2005].

\begin{tabular}{ccc}
\hline station & predicted $\delta \mathbf{t}_{t e l}(\mathbf{s})$ & in-situ $\delta \mathbf{t}_{t e l}(\mathbf{s})$ \\
\hline Northen South Island & $1.392 \pm 1.328$ & $1.76 \pm 0.72$ \\
LTZ & $2.461 \pm 1.527$ & $1.4 \pm 0.20$ \\
KELY & $2.115 \pm 1.530$ & - \\
NNZ & $1.445 \pm 0.088$ & - \\
\hline Average NSI $^{a}$ & & - \\
\hline Southern South Island $_{\text {APZ }}$ & $4.188 \pm 2.026$ & $2.95 \pm 0.55$ \\
WHZ & $2.937 \pm 0.859$ & - \\
MSZ & $3.702 \pm 1.421$ & - \\
Average SSI & & \\
\hline Average South Island & $2.834 \pm 0.540$ & $1.6 \pm 0.2$
\end{tabular}

${ }^{a}$ Northern South Island

${ }^{b}$ Southern South Island 


\section{Appendix D}

\section{Data-processing and archiving information}

- Data from the temporary deployments were archived using PASSCALPASSOFT and Antelope softwares. The basic steps involved with this process are documented and located in the archived folder ${ }^{\ddagger}$ on the Geophysics network, and these have also been provided to the Geophysics data unit. This information and data can be obtained on request.

- Sac-file records of the OBS and SAHKE data are placed in the archived folder

- An integrated method was implemented to merge hour-long miniseed files, to generate continuous sac records from the Earth-Data, and to extract events depending on the origin-time and phase arrivals. This method used PASSCAL(mseed2sac, mseedmod), SEISAN and SAC softwares. Processing steps and scripts related to this raw-data conversion can be found in the archived folder ${ }^{\ddagger}$ on the Geophysics network and these documents can be obtained on request.

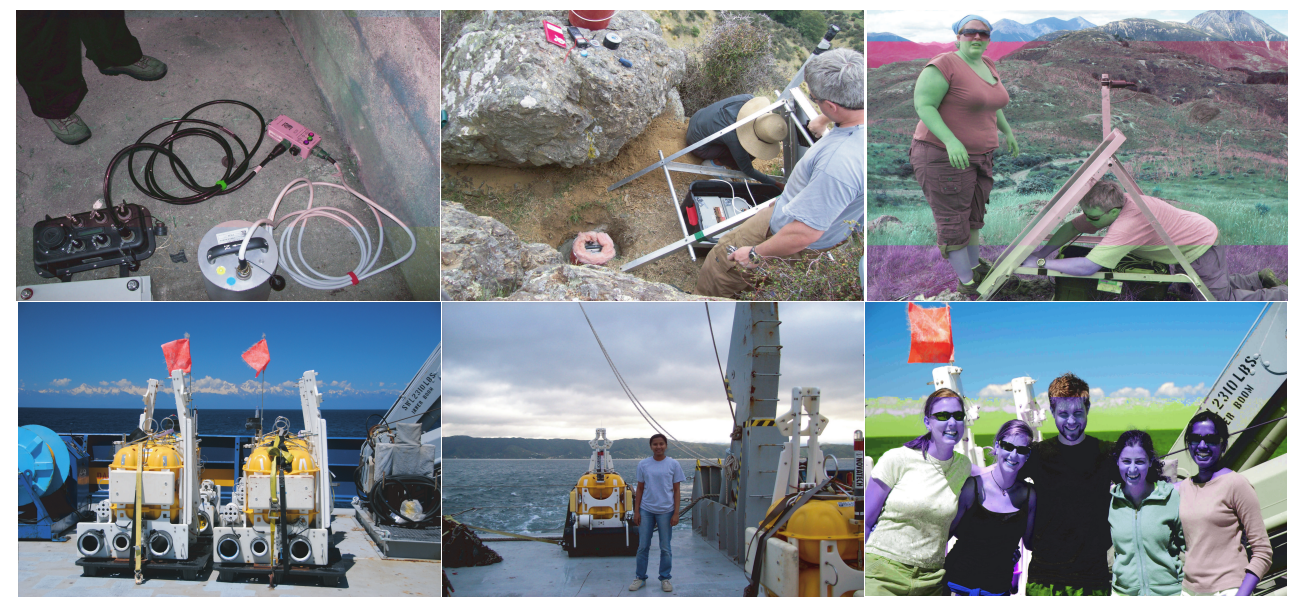

"Experience is the universal mother of sciences."

\footnotetext{
${ }^{\ddagger}$ path: /Volumes/GeoPhysics_07/users-data/karallsa
} 


\section{Bibliography}

Abt, D. L., and K. M. Fischer (2008), Resolving three-dimensional anisotropic structure with shear wave splitting tomography, Geophysical Journal International, $173,859-886$.

Abt, D. L., K. M. Fischer, G. A. Abers, W. Strauch, J. M. Protti, and V. Gonzlez (2009), Shear wave anisotropy beneath nicaragua and costa rica: Implications for flow in the mantle wedge, Geochemistry, Geophysics, Geosystems, 10(5).

Aki, K., and P. G. Richards (2002), Quantitative Seismology, 2 ed., University Science Books.

Ansell, J. H., and S. C. Bannister (1996), Shallow morphology of the subducted pacific plate along the hikurangi margin, new zealand, Physics of the Earth and Planetary Interiors, 93(1-2), 3-20.

Audoine, E., M. K. Savage, and K. Gledhill (2000), Seismic anisotropy from local earthquakes in the transition region from a subduction to a strike-slip plate boundary, new zealand, Journal of Geophysical Research-Solid Earth, 105(B4), 8013-8033.

Avouac, J., and P. Tapponnier (1993), Kinematic model of active deformation in central asia, Geophys. Res. Lett., $20(10), 895-898$.

Babuska, V., and M. Cara (1991), Seismic ansiotropy in the earth, vol. 10, Kluwer Academic Publishers.

Babuska, V., and J. Plomerova (1993), Lithospheric thickness and velocity anisotropy - seismological and geothermal aspects, Tectonophysics, 225(1-2), 7989.

Babuska, V., J. Plomerova, and J. Sileny (1984), Large-scale oriented structures in the subcrustal lithosphere of central europe, Ann Geophys, 2, 649-662.

Baldock, G., and T. Stern (2005), Width of mantle deformation across a continental transform: Evidence from upper mantle (pn) seismic anisotropy measurements, Geology, 33(9), 741-744.

Balfour, N. J., M. K. Savage, and J. Townend (2005), Stress and crustal anisotropy in marlborough, new zealand: evidence for low fault strength and structurecontrolled anisotropy, Geophysical Journal International, 163(3), 1073-1086.

Barruol, G., P. G. Silver, and A. Vauchez (1997), Seismic anisotropy in the eastern united states: Deep structure of a complex continental plate, Journal of Geophysical Research-Solid Earth, 102(B4), 8329-8348.

Barruol, M. D., G. (1993), A quantitative evaluation of the contribution of crustal rocks to the shear-wave splitting of teleseismic sks waves, Physics of the Earth and Planetary Interiors, 78(3-4), 281-300. 
Bastow, I. D., T. J. Owens, G. Helffrich, and J. H. Knapp (2007), Spatial and temporal constraints on sources of seismic anisotropy: Evidence from the scottish highlands, Geophysical Research Letters, 34.

Beanland, S. (1995), The north island dextral fault belt, Ph.D. thesis, Victoria University of Wellington.

Beavan, J., et al. (1999), Crustal deformation during 1994-1998 due to oblique continental collision in the central southern alps, new zealand, and implications for seismic potential of the alpine fault, Journal of Geophysical Research-Solid Earth, 104(B11), 25,233-25,255.

Beavan, J., D. Matheson, P. Denys, M. Denham, T. Herring, B. Hager, and P. Molnar (2004), A vertical deformation profile across the southern alps, new zealand, from 3.5 years of continuous gps data, in Proceedings of the Workshop-The State of GPS Vertical Positioning Precision: Separation of Earth Processes by Space Geodesy, 23, 111-123.

Boese, C. M., J. Townend, E. G. C. Smith, and T. A. Stern (2012), Microseismicity and stress in the vicinity of the alpine fault, central southern alps, new zealand, Journal of Geophysical Research, 117(BO2302).

Boness, N. L., and M. D. Zoback (2004), Stress-induced seismic velocity anisotropy and physical properties in the safod pilot hole in parkfield, ca, Geophysical Research Letters, $31(15),-$

Boness, N. L., and M. D. Zoback (2006), Mapping stress and structurally controlled crustal shear velocity anisotropy in california, Geology, 34(10), 825-828.

Booth, D., and S. Crampin (1985), Shear-wave polarizations on a curved wavefront at an isotropic free-surface, Geophysical Journal of the Royal Astronomical Society, 83(1), 31-45.

Boudier, F., A. Baronnet, and D. Mainprice (2010), Serpentine mineral replacements of natural olivine and their seismic implications: Oceanic lizardite versus subduction-related antigorite, Journal of Petrology, 51 (1-2), 495-512.

Bourguignon, S., T. A. Stern, and M. K. Savage (2007a), Crust and mantle thickening beneath the southern portion of the southern alps, new zealand, Geophysical Journal International, 168(2), 681-690.

Bourguignon, S., M. K. Savage, and T. A. Stern (2007b), Crustal thickness and pn anisotropy beneath the southern alps oblique collision, new zealand, New Zealand American Geophysical Union Geophysical Monograph, 175, 115-121.

Bourne, S. J., P. C. England, and B. Parsons (1998), The motion of crustal blocks driven by flow of the lower lithosphere and implications for slip rates of continental strike-slip faults, Nature, 391 (0028-0836), 655-659, doi:doi.org/10.1038/35556.

Bowman, J. R., and M. Ando (1987), Shear-wave splitting in the upper-mantle wedge above the tonga subduction zone, Geophysical Journal of the Royal Astronomical Society, 88(1), 25-41, doi:10.1111/j.1365-246X.1987.tb01367.x. 
Brisbourne, A., G. Stuart, and J. M. Kendall (1999), Anisotropic structure of the hikurangi subduction zone, new zealand - integrated interpretation of surface-wave and body-wave observations, Geophysical Journal International, 137, 214-230.

Burgmann, R., and G. Dresen (2008), Rheology of the lower crust and upper mantle: Evidence from rock mechanics, geodesy, and field observations, Annual Review of Earth and Planetary Sciences, 36, 531-567.

Bystricky, M., K. Kunze, L. Burlini, and J. P. Burg (2000), High shear strain of olivine aggregates: Rheological and seismic consequences, Science, 290(5496), $1564-1567$.

Cande, S. C., and J. M. Stock (2004), Pacific-antarctic-australia motion and the formation of the macquarie plate, Geophysical Journal International, 157(1), 399414 .

Cerveny, V. (2001), Seismic ray theory, Cambridge University Press.

Chevrot, S. (2000), Multichannel analysis of shear wave splitting, Journal of Geophysical Research-Solid Earth, 105(B9), 21,579-21,590.

Collins, J., A. F. Sheehan, P. Molnar, and O. cruise (2010), Moana recovery cruise report.

Cox, S., and R. Sutherland (2007), Regional geological framework of south island, new zealand, and its significance for understanding the active plate boundary, American Geophysical Union. Geophysical monograph, 175, 19-46.

Crampin, S. (1978), Seismic-wave propagation through a cracked solid: polarization as a possible dilatancy diagnostic, Geophysical Journal of the Royal Astronomical Society, 53(3), 467-496, doi:10.1111/j.1365-246X.1978.tb03754.x.

Crampin, S. (1994), The fracture criticality of crustal rocks, Geophysical Journal International, 118(2), 428-438.

Crampin, S., and J. H. Lovell (1991), A decade of shear-wave splitting in the earth's crust: what does it mean? what use can we make of it? and what should we do next?, Geophysical Journal International, 107(3), 387-407, doi: 10.1111/j.1365-246X.1991.tb01401.x.

Crampin, S., Y. A. Ga, and T. Volti (2004), Earthquake stress-forecasting: A new understanding of rock deformation, Earthquake: Hazard, Risk, and Strong Ground Motion, pp. 95-106 486.

Crotwell, H. P., T. J. Owens, and J. Ritsema (1999), The TauP Toolkit: Flexible seismic travel-time and ray-path utilities, Seismological Research Letters, 70, 154160, doi:10.1785/gssrl.70.2.154.

Davey, F. J., and E. G. C. Smith (1983), The tectonic setting of the fiordland region, southwest new-zealand, Geophysical Journal of the Royal Astronomical Society, 72(1), 23-38. 
Davey, F. J., D. Eberhart-Phillips, M. D. Kohler, S. Bannister, G. Caldwell, S. Henrys, M. Scherwath, T. Stern, and H. V. Avendonk (2007), Geophysical structure of the southern alps orogen, south island, new zealand, in $A$ continental plate boundary: tectonics at South Island, New Zealand, edited by D. Okaya, T. Stern, and F. Davey, Geophysical monograph, pp. 47-73, American Geophysical Union.

Davy, T., and C. . Uruski (2002), Results of the 2001 deep seismic survey of the chatham rise and hikurangi plateau and implication for petroleum exploration, in Proc. New Zealand Petroleum Conference Proceedings February-2002.

DeMets, C., R. G. Gordon, D. F. Argus, and S. Stein (1994a), Effect of recent revisions to the geomagnetic reversal time scale on estimates of current plate motions, Geophysical research letters, 21(20), 2191-2194.

DeMets, C., R. G. Gordon, D. F. Argus, and S. Stein (1994b), Effect of recent revisions to the geomagnetic reversal time scale on estimates of current plate motions, Geophysical research letters, 21 (20), 2191-2194.

Devès, M., G. C. King, Y. Klinger, and A. Agnon (2011), Localised and distributed deformation in the lithosphere: Modelling the dead sea region in 3 dimensions, Earth and Planetary Science Letters, 308, 172-184, doi:10.1016/j.epsl.2011.05.044.

Duclos, M., M. K. Savage, A. Tommasi, and K. R. Gledhill (2005), Mantle tectonics beneath new zealand inferred from sks splitting and petrophysics, Geophysical Journal International, 163(2), 760-774.

Eakin, M. C., and M. D. Long (2013-in revision), Complex anisotropy beneath the peruvian flat-slab from frequency dependent, multi-phase shear wave splitting analysis, Journal of Geophysical Research,.

Eberhart-Phillips, D., and S. Bannister (2010), 3-d imaging of marlborough, new zealand, subducted plate and strike-slip fault systems, Geophysical Journal International, 182(1), 73-96.

Eberhart-Phillips, D., and C. M. Henderson (2004), Including anisotropy in 3d velocity inversion and application to marlborough, new zealand, Geophysical Journal International, 156(2), 237-254, doi:10.1111/j.1365-246X.2003.02044.x.

Eberhart-Phillips, D., and M. Reyners (2001), A complex, young subduction zone imaged by three-dimensional seismic velocity, fiordland, new zealand, Geophysical Journal International, 146(3), 731-746.

Eberhart-Phillips, D., M. Reyners, M. Chadwick, and J. M. Chiu (2005), Crustal heterogeneity and subduction processes: 3-d vp, vp/vs and $\mathrm{q}$ in the southern north island, new zealand, Geophysical Journal International, 162, 270-288.

Ellis, S., J. Beavan, and D. Eberhart-Phillips (2006), Bounds on the width of mantle lithosphere flow derived from surface geodetic measurements: application to the central southern alps, new zealand, Geophysical Journal International, 166(1), 403-417. 
England, P., G. Houseman, and L. Sonder (1985), Length scales for continental deformation in convergent, divergent, and strike-slip environments: Analytical and approximate solutions for a thin viscous sheet model, Journal of geophysical research, 90(B5), 3551-3557.

England, P. C., and J. Jackson (1989), Active deformation of the continents, Annual Review of Earth and Planetary Sciences, 17(1589), 197-226.

Evans, R. (1984), Effects of the free-surface on shear wavetrains, Geophysical Journal of the Royal Astronomical Society, 76, 165-172.

Faccenda, M., L. Burlini, T. V. Gerya, and D. Mainprice (2008), Fault-induced seismic anisotropy by hydration in subducting oceanic plates, Nature, 455, 1097$\mathrm{U} 8$.

Favier, N., and S. Chevrot (2003), Sensitivity kernels for shear wave splitting in transverse isotropic media, Geophysical Journal International, 153(1).

Fischer, K. M., E. M. Parmentier, A. R. Stine, and E. R. Wolf (2000), Modeling anisotropy and plate-driven flow in the tonga subduction zone back arc, Journal of Geophysical Research-Solid Earth, 105(B7), 16,181-16,191.

Flesch, L. M., W. E. Holt, P. G. Silver, M. Stephenson, C. Y. Wang, and W. W. Chan (2005), Constraining the extent of crust-mantle coupling in central asia using gps, geologic, and shear wave splitting data, Earth and Planetary Science Letters, 238(1-2), 248-268.

Fukao, Y. (1984), Evidence from core-reflected shear-waves for anisotropy in the earths mantle, Nature, 309(5970), 695-698.

Furlong, P. K. (2007), Locating the deep extent of the plate boundary along the alpine fault zone, new zealnd: Implications for patterns of exhumation in the southern alps, Geological Society of America Special Papers, 434, 1-14.

Galea, P. (1993), Upper mantle anisotropy in the sw pacific from earthquake traveltime analysis, Physics of the Earth and Planetary Interiors, 76(3-4), 229-239.

Gerst, A. (2003), Temporal changes in seismic anisotropy as a new eruption forecasting tool, Awarded research masters thesis, School of Geography, Environment and Earth Sciences, Victoria University of Wellington.

Gerst, A., and M. K. Savage (2004), Seismic anisotropy beneath ruapehu volcano: A possible eruption forecasting tool, Science, 306(5701), 1543-1547.

Gledhill, K., and D. Gubbins (1996), Sks splitting and the seismic anisotropy of the mantle beneath the hikurangi subduction zone, new zealand, Physics of the Earth and Planetary Interiors, 95(3-4), 227-236.

Gledhill, K., and G. Stuart (1996), Seismic anisotropy in the fore-arc region of the hikurangi subduction zone, new zealand, Physics of the Earth and Planetary Interiors, 95(3-4), 211-225. 
Godfrey, N. J., N. I. Christensen, and D. A. Okaya (2000), Anisotropy of schists: Contribution of crustal anisotropy to active source seismic experiments and shear wave splitting observations, Journal of Geophysical Research-Solid Earth, 105(B12), 27,991-28,007.

Greve, S. M., and M. K. Savage (2009), Modelling seismic anisotropy variations across the Hikurangi subduction margin, New Zealand, Earth and Planetary Science Letters, 285, 16-26.

Greve, S. M., M. K. Savage, and S. D. Hofmann (2008), Strong variations in seismic anisotropy across the hikurangi subduction zone, north island, new zealand, Tectonophysics, 462(1-4), 7-21.

Gripp, A. E., and R. G. Gordon (2002), Young tracks of hotspots and current plate velocities, Geophysical Journal International, 150(2), 321-361.

Gubbins, D., and R. Snieder (1991), Dispersion of p waves in subducted lithosphere: Evidence for an eclogite layer, Journal of Geophysical Research: Solid Earth (1978-2012), 96(B4), 6321-6333.

Gudmundsson, O. (1996), On the effect of diffraction on traveltime measurements, Geophysical Journal International, 124(1), 304-314.

Hall, L. S., S. H. Lamb, and C. Mac Niocaill (2004), Cenozoic distributed rotational deformation, south island, new zealand, Tectonics, 23(2).

Henrys, S., et al. (2013), Sahke geophysical transect reveals crustal and subduction zone structure at the southern hikurangi margin, new zealand, Geochemistry, Geophysics, Geosystems.

Hess, H. H. (1964), Sesimic anisotropy of the uppermost mantle under oceans, Nature, 203, 629-631.

Hicks, S., and D. Woodward (1978), Gravity models of the wairarapa region, new zealand, New Zealand journal of geology and geophysics, 21, 539-544.

Holtzman, B. K., and J. M. Kendall (2010), Organized melt, seismic anisotropy, and plate boundary lubrication, Geochemistry Geophysics Geosystems, 11.

Holtzman, B. K., D. L. Kohlstedt, M. E. Zimmerman, F. Heidelbach, T. Hiraga, and J. Hustoft (2003), Melt segregation and strain partitioning: Implications for seismic anisotropy and mantle flow, Science, 301 (5637), 1227-1230.

Houlié, N., and T. Stern (2012), A comparison of gps solutions for strain and sks fast directions: Implications for modes of shear in the mantle of a plate boundary zone, Earth and Planetary Science Letters, 345, 117-125.

Huang, Z., D. Zhao, and L. Wang (2011a), Frequency-dependent shear-wave splitting and multilayer anisotropy in northeast japan, Geophysical Research Letters, 38.

Huang, Z. C., D. P. Zhao, and L. S. Wang (2011b), Shear wave anisotropy in the crust, mantle wedge, and subducting pacific slab under northeast japan, Geochemistry Geophysics Geosystems, 12. 
Hudson, J. A. (1980), Overall properties of a cracked solid, Mathematical Proceedings of the Cambridge Philosophical Society, 88(SEP), 371-384.

Ismail, W. B., and D. Mainprice (1998), An olivine fabric database: an overview of upper mantle fabrics and seismic anisotropy, Tectonophysics, 296, 145 - 157, doi:10.1016/S0040-1951(98)00141-3.

Jammalamadaka, S. R., and A. Sengupta (2001), Topics in Circular Statistics, World Scientific Pub Co Inc.

Ji, S., A. Li, Q. Wang, C. Long, H. Wang, D. Marcotte, and M. Salkisbury (2013), Seismic velocities, anisotropy and shear-wave splitting of antigorite serpentinites and tectonic implications for subduction zones, Journal of Geophysical Research: Solid Earth.

Johnson, J. H., M. K. Savage, and J. Townend (2011), Distinguishing between stress-induced and structural anisotropy at mount ruapehu volcano, new zealand, Journal of Geophysical Research: Solid Earth (1978-2012), 116(B12).

Jung, H., W. Mo, and H. W. Green (2009), Upper mantle seismic anisotropy resulting from pressure-induced slip transition in olivine, Nature Geoscience, 2(1), $73-77$.

Kameyama, M., D. A. Yuen, and S.-I. Karato (1999), Thermal-mechanical effects of low-temperature plasticity (the peierls mechanism) on the deformation of a viscoelastic shear zone, Earth and Planetary Science Letters, 168(1), 159-172.

Karalliyadda, S. C., and M. K. Savage (2013), Seismic anisotropy and lithospheric deformation of the plate-boundary zone in south island, new zealand: inferences from local s-wave splitting, Geophysical Journal International, doi:10.1093/gji/ ggt022.

Karato, S. (1998), Seismic anisotropy in the deep mantle, boundary layers and the geometry of mantle convection, in Geodynamics of Lithosphere and Earths Mantle, pp. 565-587, Springer.

Karato, S., and P. Wu (1993), Rheology of the upper mantle - a synthesis, Science, 260(5109), 771-778.

Karato, S., H. Jung, I. Katayama, and P. Skemer (2008), Geodynamic significance of seismic anisotropy of the upper mantle: New insights from laboratory studies, Annual Review of Earth and Planetary Sciences, 36(1), 59-95.

Karato, S., et al. (1989), Seismic anisotropy: Mechanisms and tectonic implications, Rheology of Solids and of the Earth, pp. 393-422.

Kaviani, A., G. Ruempker, M. Weber, and G. Asch (2011), Short-scale variations of shear-wave splitting across the dead sea basin: Evidence for the effects of sedimentary fill, Geophysical Research Letters, 38.

Kendall, J. M. (2000), Seisimic anisotropy in the boundary layers of the mantle, Geophysical Monograph, 117, 133-157. 
Kern, H., and H. R. Wenk (1990), Fabric-related velocity anisotropy and shearwave splitting in rocks from the santa rosa mylonite zone, california, Journal of Geophysical Research-Solid Earth and Planets, 95(B7), 11,213-11,223.

Kirby, S. H., and A. K. Kronenberg (1987), Rheology of the lithosphere - selected topics, Reviews of Geophysics, 25(6), 1219-1244.

Klosko, E. R., F. T. Wu, H. J. Anderson, D. Eberhart-Phillips, T. V. McEvilly, E. Audoine, M. K. Savage, and K. R. Gledhill (1999), Upper mantle anisotropy in the new zealand region, Geophysical Research Letters, 26 (10), 1497-1500.

Lamb, S. (2011), Cenozoic tectonic evolution of the new zealand plate-boundary zone: A paleomagnetic perspective, Tectonophysics, In Press, Corrected Proof,--, doi:DOI:10.1016/j.tecto.2011.06.005.

Leary, P. C., S. Crampin, and T. V. McEvilly (1990), Seismic fracture anisotropy in the earth's crust: An overview, J. Geophys. Res., 95, 11,105-11,114, doi:DOI: 10.1029/JB095iB07p11105.

Lebrun, J., G. Lamarche, and J. Collot (2003), Subduction initiation at a strikeslip plate boundary: The cenozoic pacific-australian plate boundary, south of new zealand, Journal of Geophysical Research: Solid Earth (1978-2012), 108(B9).

Levin, V., W. Menke, and J. Park (1999), Shear wave splitting in the appalachians and the urals: A case for multilayered anisotropy, Journal of Geophysical Research-Solid Earth, 104 (B8), 17,975-17,993.

Little, T., R. Holcombe, and B. Ilg (2002a), Kinematics of oblique collision and ramping inferred from microstructures and strain in middle crustal rocks, central southern alps, new zealand, Journal of Structural Geology, 24(1), 219 - 239, doi: 10.1016/S0191-8141(01)00060-8.

Little, T. A., and N. Mortimer (2001), Rotation of ductile fabrics across the alpine fault and cenozoic bending of the new zealand orocline, Journal of the Geological Society, 158, 745-756.

Little, T. A., M. K. Savage, and B. Tikoff (2002b), Relationship between crustal finite strain and seismic anisotropy in the mantle, pacific-australia plate boundary zone, south island, new zealand, Geophysical Journal International, 151 (1), 106116.

Little, T. A., S. Cox, J. K. Vry, and G. Batt (2005), Variations in exhumation level and uplift rate along the oblique-slip alpine fault, central southern alps, new zealand, Geological Society of America Bulletin, 117(5-6), 707-723.

Liu, E., J. H. Queen, X. Y. Li, M. Chapman, S. Maultzsch, H. B. Lynn, and E. M. Chesnokov (2003), Observation and analysis of frequency-dependent anisotropy from a multicomponent vsp at bluebell-altamont field, utah, Journal of Applied Geophysics, 54(3-4). 
Lloyd, G. E., R. W. Butler, M. Casey, and D. Mainprice (2009), Mica, deformation fabrics and the seismic properties of the continental crust, Earth and Planetary Science Letters, 288, 320 - 328, doi:10.1016/j.epsl.2009.09.035.

Long, M. D. (2010), Frequency-dependent shear wave splitting and heterogeneous anisotropic structure beneath the gulf of california region, Physics of the Earth and Planetary Interiors, 182(1-2), 59-72.

Long, M. D. (2013), Constraints on subduction geodynamics from seismic anisotropy, Reviews of Geophysics.

Long, M. D., and P. G. Silver (2008), The subduction zone flow field from seismic anisotropy: A global view, Science, 319(5861), 315-318.

Long, M. D., and P. G. Silver (2009), Shear wave splitting and mantle anisotropy: Measurements, interpretations, and new directions, Surveys in Geophysics, 30(45), 407-461.

Love, A. E. H. (1944), A Treatise on the Mathematical Theory of Elasticiity, Dover, New York.

Lynner, C., and M. D. Long (2013), Sub-slab seismic anisotropy and mantle flow beneath the caribbean and scotia subduction zones: Effects of slab morphology and kinematics, Earth and Planetary Science Letters, 361, 367-378.

Mainprice, D., and B. Ildefonse (2009), Seismic anisotropy of subduction zone minerals-contribution of hydrous phases, Subduction Zone Geodynamics, pp. 6384.

Mainprice, D., and A. Nicolas (1989), Development of shape and lattice preferred orientations: application to the seismic anisotropy of the lower crust, Journal of Structural Geology, 11(1/2), 175 - 189, doi:10.1016/0191-8141(89)90042-4.

Mainprice, D., G. Barruol, and W. Ben Ismail (2000), The seismic anisotropy of the earth's mantle: from single crystal to polycrystal, Geophysical Monograph, 117, $237-264$.

Mardia, K. V. (1972), Statistics of directional data, Academic Press (London and New York).

Marson-Pidgeon, K., and M. K. Savage (1997), Frequency-dependent anisotropy in wellington, new zealand, Geophysical Research Letters, 24 (24), 3297-3300.

Marson-Pidgeon, K., and M. K. Savage (2004a), Modelling shear wave splitting observations from wellington, new zealand, Geophysical Journal International, $157(2), 853-864$.

Marson-Pidgeon, K., and M. K. Savage (2004b), Shear-wave splitting variations across an array in the southern north island, new zealand, Geophysical Research Letters, $31(21)$. 
Marson-Pidgeon, K., M. K. Savage, K. Gledhill, and G. Stuart (1999), Seismic anisotropy beneath the lower half of the north island, new zealand, Journal of Geophysical Research-Solid Earth, 104(B9), 20,277-20,286.

Matcham, I., M. K. Savage, and K. R. Gledhill (2000), Distribution of seismic anisotropy in the subduction zone beneath the wellington region, new zealand, Geophysical Journal International, 140(1), 1-10, doi:10.1046/j.1365-246x.2000. 00928.x.

Mattatall, L. R., and M. J. Fouch (2007), Small-scale variations in sks splitting near parkfield, california, AGU Fall Meeting Abstracts, pp. -.

McKenzie, D., and J. Jackson (1983), The relationship between strain rates, crustal thickening, palaeomagnetism, finite strain and fault movements within a deforming zone, Earth and Planetary Science Letters, 65(1), 182 - 202, doi: 10.1016/0012-821X(83)90198-X.

Meade, B. J., and B. H. Hager (2005), Block models of crustal motion in southern california constrained by gps measurements, J. Geophys. Res., 110 (B03403), doi: 10.1029/2004JB003209.

Mehl, L., B. R. Hacker, G. Hirth, and P. B. Kelemen (2003), Arc-parallel flow within the mantle wedge: Evidence from the accreted talkeetna arc, south central alaska, Journal of Geophysical Research-Solid Earth, 108(B8).

Miller, V., and M. Savage (2001), Changes in seismic anisotropy after volcanic eruptions: evidence from mount ruapehu, Science, 293(5538), 2231-2233.

Molnar, P., and P. Tapponnier (1975), Cenozoic tectonics of asia: Effects of a continental collision, Science, 189 (4201), 419-426.

Molnar, P., et al. (1999), Continuous deformation versus faulting through the continental lithosphere of new zealand, Science, 286(5439), 516-519.

Montagner, J., and L. Guillot (2002), Geophysical structure of the southern alps orogen, south island, new zealand, Plastic deformation of minerals and rocks, pp. 353-385, Mineralogical Society of America and Geochemical Society, Washington, DC.

Moore, M., P. England, and B. Parsons (2002), Relation between surface velocity field and shear wave splitting in the south island of new zealand, J. Geophys. Res., 107(2198), 0148-0227, doi:doi:10.1029/97TC03148.

Morley, A. M., G. W. Stuart, J. M. Kendall, and M. Reyners (2006), Mantle wedge anisotropy in the hikurangi subduction zone, central north island, new zealand, Geophys. Res. Lett., 33(5), -, doi:doi:10.1029/2005GL024569.

Mortimer, N. (2004), New zealand's geological foundations, Gondwana Research, 7, 261-272, doi:10.1016/s1342-937x(05)70324-5. 
Mueller, M. C. (1991), Prediction of lateral variability in fracture intensity using multicomponent shear-wave surface seismic as a precursor to horizontal drilling in the Austin Chalk, Geophysical Journal International, 10\%, 409-415, doi:10. 1111/j.1365-246X.1991.tb01402.x.

Nicolas, A. (1993), Why fat polarization direction of sks seismic waves are parallel to mountain belts, Physics of the Earth and Planetary Interiors, 78, 337-342.

Nicolas, A., and N. I. Christensen (1987), Formation of anisotropy in upper mantle peridotite: a review, AGU Geodyn. Monog. Series, 16, 111-123.

Norris, R. J., P. O. Koons, and A. F. Cooper (1990), The obliquely-convergent plate boundary in the south island of new-zealand - implications for ancient collision zones, Journal of Structural Geology, 12(5-6), 715-725.

Nur, A. (1971), Effects of stress on velocity anisotropy in rocks with cracks, Journal of Geophysical Research, 76(8), 2022.

Nur, A., and G. Simmons (1969), Stress-induced velocity anisotropy in rock: An experimental study, J. Geophys. Res., 74(27), 6667-6674, doi:doi:10.1029/ JB074i027p06667.

Nuttli, O. (1961), The effect of the earth's surface on the s wave particle motion, Bulletin of the Seismological Society of America, 51 (2), 237-246.

Okaya, D., N. Christensen, D. Stanley, and T. Stern (1995), Crustal anisotropy in the vicinity of the alpine fault zone, south island, new zealand, New Zealand Journal of Geology and Geophysics, 38(4), 579-583.

Okaya, D., T. Stern, F. Davey, S. Henrys, and S. Cox (2007), Continent-continent collision at the pacific/indo-australian plate boundary: Background, motivation, and principal results, New Zealand American Geophysical Union Geophysical Monograph, 175, 1-18.

Otsuki, K. (1978), On the relationship between the width of shear zone and the displacement along fault, J. Geol. Soc. Jpn, 84, 661-669.

Ozalaybey, S., and M. K. Savage (1995), Shear-wave splitting beneath western united-states in relation to plate-tectonics, Journal of Geophysical Research-Solid Earth, 100(B9).

Paulssen, H. (2004), Crustal anisotropy in southern california from local earthquake data, Geophysical Research Letters, 31 (1).

Peacock, S., S. Crampin, D. C. Booth, and J. B. Fletcher (1988), Shear-wave splitting in the anza seismic gap, southern-california - temporal variations as possible precursors, Journal of Geophysical Research-Solid Earth and Planets, 93(B4), 3339-3356.

Peng, Z., and Y. Ben-Zion (2004), Systematic analysis of crustal anisotropy along the karadere-düzce branch of the north anatolian fault, Geophysical Journal International, 159, 253-274. 
Philipp, B. (2009), Circstat: A matlab toolbox for circular statistics, Journal of Statistical Software, 31 (10), 1-21.

Poirier, J. (1985), Cambridge University Press.

Poirier, J. P. (1990), Rheology of solids and of the earth, Science, 248(4961), 14301430.

Pulford, A., M. Savage, and T. Stern (2003), Absent anisotropy: The paradox of the southern alps orogen, Geophysical Research Letters, 30(20), -.

Reyners, M., R. Robinson, A. Pancha, and P. McGinty (2002), Stresses and strains in a twisted subduction zone-fiordland, new zealand, Geophysical Journal International, $148(3), 637-648$.

Ribe, N. M. (1992), On the relation between seismic anisotropy and finite strain, Journal of Geophysical Research-Solid Earth, 97(B6), 8737-8747, doi:10.1029/ 92jb00551, ribe, nm.

Roman, D. C., M. K. Savage, R. Arnold, J. L. Latchman, and S. De Angelis (2011), Analysis and forward modeling of seismic anisotropy during the ongoing eruption of the soufriẤre hills volcano, montserrat, 19968211;2007, J. Geophys. Res., 116 (B3), B03,201.

Rumpker, G., and T. Ryberg (2000), New fresnel-zone estimates for shear-wave splitting observations from finite-difference modeling, Geophysical research letters, $27(13), 2005-2008$.

Rumpker, G., and P. G. Silver (1998), Apparent shear-wave splitting parameters in the presence of vertically varying anisotropy, Geophysical Journal International, 135.

Rumpker, G., T. Ryberg, G. Bock, and G. Desert Seismology (2003), Boundary-layer mantle flow under the dead sea transform fault inferred from seismic anisotropy, Nature, 425(6957), 497-501.

Russo, R. M. (2012), Source-side shear-wave splitting and upper-mantle flow beneath the arakan slab, india-asia-sundalind triple junction, Geosphere, 8(1), 158-178.

Russo, R. M., and P. G. Silver (1994), Trench-parallel flow beneath the nazca plate from seismic anisotropy, Science, 263(5150), 1105-1111.

Sato, H., and M. C. Fehler (1997), Seismic Wave Propagation and Scattering in the Heterogenous Earth, Springer-Verlag, Berlin.

Savage, J. C., and R. O. Burford (1970), Accumulation of tectonic strain in california, Bulletin of the Seismological Society of America, 60 (6), 1877.

Savage, K., M. Duclos, and K. Marson-Pidgon (2007a), Seisimic anisotropy in south island, new zealand, Geophysical Monograph Series 175.

Savage, M. K. (1999), Seismic anisotropy and mantle deformation: What have we learned from shear wave splitting?, Reviews of Geophysics, 37(1), 65-106. 
Savage, M. K., J. Park, and H. Todd (2007), Velocity and anisotropy structure at the hikurangi subduction margin, new zealand from receiver functions, Geophysical Journal International, 168 (3), 1034-1050.

Savage, M. K., T. Ohminato, Y. Aoki, H. Tsuji, and S. M. Greve (2010), Stress magnitude and its temporal variation at mt. asama volcano, japan, from seismic anisotropy and gps, Earth and Planetary Science Letters, 290(3-4), 403-414.

Scherwath, M., T. Stern, A. Melhuish, and P. Molnar (2002), Pn anisotropy and distributed upper mantle deformation associated with a continental transform fault, Geophysical Research Letters, 29(8), -.

Scherwath, M., T. Stern, F. Davey, D. Okaya, W. S. Holbrook, R. Davies, and S. Kleffmann (2003), Lithospheric structure across oblique continental collision in new zealand from wide-angle p wave modeling, Journal of Geophysical ResearchSolid Earth, 108(B12).

Seward, S. H. M. S. R., A.and Henrys, M. Stern, T.and Savage, K. Mochizuki, H. Sato, T. Iwasaki, C. Kennedy, and D. Bassett (2010), Seismic array hikurangi experiment (sahke); field acquisition report, gns science report.

Shearer, P., and J. Orcutt (1985), Anisotropy in the oceanic lithosphere - theory and observations from the ngendei seismic refraction experiment in the southwest pacific, Geophysical Journal of the Royal Astronomical Society, 80(2), 493-526.

Shih, X. R., R. P. Meyer, and J. F. Schneider (1989), An automated, analytical method to determine shear-wave splitting, Tectonophysics, 165(1), 271-278.

Sileny, J., and J. Plomerova (1996), Inversion of shear-wave splitting parameters to retrieve three-dimensional orientation of anisotropy in continental lithosphere, Physics of the Earth and Planetary Interiors, 95(3-4), 277-292.

Silver, P. (2002), The mantle flow field beneath western north, Science, $1066878(1054), 295$.

Silver, P. G. (1996), Seismic anisotropy beneath the continents: Probing the depths of geology, Annual Review of Earth and Planetary Sciences, 24, 385-432.

Silver, P. G., and W. W. Chan (1988), Implications for continental structure and evolution from seismic anisotropy, Nature, 335(6185), 34-39.

Silver, P. G., and W. W. Chan (1991), Shear-wave splitting and subcontinental mantle deformation, Journal of Geophysical Research-Solid Earth, 96(B10), $16,429-16,454$.

Silver, P. G., and M. D. Long (2011), The non-commutivity of shear wave splitting operators at low frequencies and implications for anisotropy tomography, Geophysical Journal International, $184(3), 1415-1427$.

Silver, P. G., and M. K. Savage (1994), The interpretation of shear-wave splitting parameters in the presence of two anisotropic layers, Geophysical Journal International, 119 (3), 949-963, doi:10.1111/j.1365-246X.1994.tb04027.x. 
Skemer, P., J. M. Warren, and G. Hirth (2012), The influence of deformation history on the interpretation of seismic anisotropy, Geochemistry, Geophysics, Geosystems, 13(3).

Smith, G. P., D. A. Wiens, K. M. Fischer, L. M. Dorman, S. C. Webb, and J. A. Hildebrand (2001), A complex pattern of mantle flow in the lau backarc, Science, 292(5517), 713-716.

Song, T. R. A., and H. Kawakatsu (2012), Subduction of oceanic asthenosphere: Evidence from sub-slab seismic anisotropy, Geophysical Research Letters, 39.

Stachnik, J., A. Sheehan, D. Zietlow, Z. Yang, J. Collins, and A. Ferris (2012), Determination of new zealand ocean bottom seismometer orientation via rayleighwave polarization, Seismol. Res. Lett., 83, 704-712, doi:doi:10.1785/0220110128.

Stein, S., and M. Wysession (2009), An introduction to seismology, earthquakes, and earth structure, Wiley-Blackwell.

Stern, T., P. Molnar, D. Okaya, and D. Eberhart-Phillips (2000), Teleseismic p wave delays and modes of shortening the mantle lithosphere beneath south island, new zealand, Journal of Geophysical Research-Solid Earth, 105 (B9), 21,615-21,631.

Stern, T., D. Okaya, S. Kleffmann, M. Scherwath, S. Henrys, and F. Davey (2007), Geophysical exploration and dynamics of the alpine fault zone, A Continental Plate Boundary: Tectonics at South Island, New Zealand, pp. 207-233.

Stuart, G., D. Francis, D. Gubbins, and G. Smith (1995), Tararua broadband array, north island, new zealand, Bulletin of the Seismological Society of America, 85(1), $325-333$.

Sutherland, R. (1999), Basement geology and tectonic development of the grater new zealand region: interpretation from regional magnetic data, Tectonophysics, 308, 341-362.

Sutherland, R., F. Davey, and J. Beavan (2000), Plate boundary deformation in south island, new zealand, is related to inherited lithospheric structure, Earth and Planetary Science Letters, 177(3-4), 141-151.

Tarantola, A. (2005), Inverse problem theory and methods for model parameter estimation, Society for Industrial and Applied.

Teanby, N. A., J. M. Kendall, and M. Van der Baan (2004b), Automation of shearwave splitting measurements using cluster analysis, Bulletin of the Seismological Society of America, 94 (2), 453-463.

Thatcher, W. (2009), How the continents deform: The evidence from tectonic geodesy, Annu. Rev. Earth Planet, Sci., 37, 237-62.

Tod, S. R., and E. Liu (2002), Frequency-dependent anisotropy due to fluid flow in bed limited cracks, Geophysical Research Letters, $29(15)$. 
Toussaint, G., E. Burov, and J. Avouac (2004), Tectonic evolution of a continental collision zone: A thermomechanical numerical model, Tectonics, 23(6).

Townend, J., S. Sherburn, R. Arnold, C. Boese, and L. Woods (2012), Threedimensional variations in present-day tectonic stress along the australia-pacific plate boundary in new zealand, Earth and Planetary Science Letters, 353-354, 47 - 59, doi:10.1016/j.epsl.2012.08.003.

Tsuji, T., J. Dvorkin, G. Mavko, N. Nakata, T. Matsuoka, A. Nakanishi, S. Kodaira, and O. Nishizawa (2011), Vp/vs ratio and shear-wave splitting in the nankai trough seismogenic zone: Insights into effective stress, pore pressure, and sediment consolidation, Geophysics, 76 (3), WA71-WA82.

Van Avendonk, H., W. Holbrook, J. Austin, D. Okaya, S. Group, et al. (2000), Seismic velocity and wide-angle reflectivity structure of the australian-pacific plate boundary, south island, new zealand, Eos Trans. AGU, 80, 46.

van den Beukel, J., and R. Wortel (1987), Temperatures and shear stresses in the upper part of a subduction zone, Geophysical Research Letters, 14 (10), 1057-1060.

Vauchez, A., and A. Nicolas (1991), Mountain building strike-parallel motion and mantle anisotropy, Tectonophysics, 185, 183-201.

Vauchez, A., and A. Tommasi (2003), Wrench faults down to the asthenosphere: Geological and geophysical evidence and thermo-mechanical effects, London, Geological Society of London, 210, 15-34.

Vecsey, L., J. Plomerova, and V. Babuska (2008), Shear-wave splitting measurements-problems and solutions, Tectonophysics, 462, 178 - 196, doi: 10.1016/j.tecto.2008.01.021.

Vidale, J. E. (1986), Complex polarization analysis of particle motion, Bulletin of the Seismological Society of America, 76 (5), 1393-1405.

Vinnik, L. P., V. Farra, and B. Romanowicz (1989a), Azimuthal anisotropy in the earth from observations of sks at geoscope and nars broad-band stations, Bulletin of the Seismological Society of America, 79 (5), 1542-1558.

Vinnik, L. P., R. Kind, G. L. Kosarev, and L. I. Makeyeva (1989b), Azimuthal anisotropy in the lithosphere from observations of long-period s-waves, Geophysical Journal International, 99(3), 549-559.

Volti, T., Y. Kaneda, S. Zatsepin, and S. Crampin (2005), An anomalous spatial pattern of shear-wave splitting observed in ocean bottom seismic data above a subducting seamount in the nankai trough, Geophysical Journal International, $163(1), 252-264$.

Walcott, R. I. (1998), Modes of oblique compression: Late cenozoic tectonics of the south island of new zealand, Reviews of Geophysics, 36(1), 1-26.

Walker, A. M., and J. Wookey (2012), Msat-a new toolkit for the analysis of elastic and seismic anisotropy, Computers \& Geosciences, 49, 81-90. 
Walsh, E. (2012), Measuring shear wave splitting using the silver and chan method, Master's thesis.

Walsh, E., R. Arnold, and M. Savage (), Silver and chan revisited, Journal of Geophysical Research, submitted.

Wandres, A., and J. Bradshaw (2005), New zealand tectonostratigraphy and implications from conglomeratic rocks for the configuration of the sw pacific margin of gondwana, Geological Society, London, Special Publications, 246, 179216.

Wannamaker, P. E., T. G. Caldwell, G. R. Jiracek, V. Maris, G. J. Hill, Y. Ogawa, H. M. Bibby, S. L. Bennie, and W. Heise (2009), Fluid and deformation regime of an advancing subduction system at marlborough, new zealand, Nature, 460 (7256), 733-737, doi:10.1038/nature08204.

Wellman, H. (1979), An uplift map for the south island of new zealand, and a model for uplift of the southern alps, Bull. R. Soc. NZ, 18, 13-20.

Werner, U., and S. A. Shapiro (1999), Frequency-dependent shear-wave splitting in thinly layered media with intrinsic anisotropy, Geophysics, 64(2), 604-608, doi:10.1190/1.1444567.

Wessel, A. (2010), Automatic shear wave splitting measurements at mt. ruapehu volcano, new zealand, Awarded research masters thesis, School of Geography, Environment and Earth Sciences, Victoria University of Wellington.

Wilson, C. K., C. H. Jones, P. Molnar, A. F. Sheehan, and O. S. Boyd (2004), Distributed deformation in the lower crust and upper mantle beneath a continental strike-slip fault zone: Marlborough fault system, south island, new zealand, Geology, 32(10), 837-840, doi:10.1130/G20657.

Wirth, E., and M. D. Long (2010), Frequency-dependent shear wave splitting beneath the japan and izu-bonin subduction zones, Physics of the Earth and Planetary Interiors, $181(3-4), 141-154$.

Wood, R. (1991), Structure and seismic stratigraphy of the western challenger plateau, New Zealand Journal of Geology and Geophysics, 34, 1-9.

Wu, R. S. (1982), Attenuation of short-period seismic-waves due to scattering, Geophysical Research Letters, 9(1).

Wu, R. S., and K. Aki (1988), Seismic wave scattering in the three-dimensionally heterogeneous earth, PAGEOPH, 128.

Wustefeld, A., G. Bokelmann, C. Zaroli, and G. Barruol (2008), Splitlab: A shearwave splitting environment in matlab, Computers Geosciences, 34(5), 515-528.

Xie, J. (1992), Shear-wave splitting near guam, Physics of the Earth and Planetary Interiors, 72(3-4), 211-219, doi:10.1016/0031-9201(92)90202-7. 
Yang, Z., A. Sheehan, J. A. Collins, and G. Laske (2012), The character of the seafloor ambient noise recorded offshore new zealand: Results from the moana ocean bottom seismic experiment, Geochemistry Geophysics Geosystems, 13(10), $1-10$.

Yardley, G. S., and S. Crampin (1991), Extensive-dilatancy anisotropy - relative information in vsps and reflection surveys, Geophysical Prospecting, 39(3).

Zhang, J., Y. Wang, and Z. Jin (2008), Cpo-induced seismic anisotropy in uhp eclogites, Science in China Series D: Earth Sciences, 51(1), 11-21.

Zhang, S. Q., and S. Karato (1995), Lattice preferred orientation of olivine aggregates deformed in simple shear, Nature, 375(6534), 774-777.

Zietlow, D. W., A. F. Sheehan, P. H. Molnar, M. K. Savage, and J. Collins (2013), Distributed lithospheric deformation versus asthenospheric shear at a strike-slip fault: Mantle seismic anisotropy under south island, new zealand, Journal of Geophysical Research, doi:10.1002/2013JB010676. 